\title{
Temporal integration of loudness as a function of level
}

\author{
Florentine, Mary; Buus, Søren; Poulsen, Torben
}

Published in:

Acoustical Society of America. Journal

Link to article, DOI:

10.1121/1.411567

Publication date:

1996

Document Version

Publisher's PDF, also known as Version of record

Link back to DTU Orbit

Citation (APA):

Florentine, M., Buus, S., \& Poulsen, T. (1996). Temporal integration of loudness as a function of level. Acoustical Society of America. Journal, 99(3), 1633-1644. https://doi.org/10.1121/1.411567

\section{General rights}

Copyright and moral rights for the publications made accessible in the public portal are retained by the authors and/or other copyright owners and it is a condition of accessing publications that users recognise and abide by the legal requirements associated with these rights.

- Users may download and print one copy of any publication from the public portal for the purpose of private study or research.

- You may not further distribute the material or use it for any profit-making activity or commercial gain

- You may freely distribute the URL identifying the publication in the public portal

If you believe that this document breaches copyright please contact us providing details, and we will remove access to the work immediately and investigate your claim. 
The 129th Meeting of the Acoustical Society of America

\title{
Renaissance Washington, DC Hotel $\bullet$ Washington, DC $\bullet 30$ May-3 June 1995
}

NOTE: All Journal articles and Letters to the Editor are peer reviewed before publication. Program abstracts, however, are not reviewed before publication, since we are prohibited by time and schedule.

\section{Session 1aAO}

\section{Acoustical Oceanography: Ocean Acoustic Tomography: Observing the Ocean in the 1990s I}

\author{
Walter Munk, Cochair \\ Scripps Institution of Oceanography, University of California, San Diego, La Jolla, California 92093 \\ Brian D. Dushaw, Cochair \\ Applied Physics Laboratory, University of Washington, Seattle, Washington 98105
}

Chair's Introduction-8:00

\section{Invited Paper}

8:05

\begin{abstract}
1aAO1. Acoustic thermometry of ocean climate (ATOC). Walter H. Munk (Scripps Inst. of Oceanog., IGPP-0225, UCSD, La Jolla, CA 92093)

The rocky road followed in the 1990 s leading to the present status of acoustic thermometry of ocean climate (ATOC) will be reviewed.
\end{abstract}

\section{Contributed Papers}

8:30

1aAO2. A comparison of measured and predicted broadband acoustic arrival patterns out to $10-\mathrm{Mm}$ range during the ATOC Acoustic Engineering Test. Bruce M. Howe, Brian D. Dushaw, James A. Mercer, Robert I. Odom, Robert C. Spindel (Appl. Phys. Lab., Univ. of Washington, Seattle, WA 98105), Peter F. Worcester, John Colosi, Bruce D. Comuelle, Matthew Dzieciuch (Scripps Inst. of Oceanog., Univ. of Califormia at San Diego, La Jolla, CA 92093), Arthur B. Baggeroer (MIT, Cambridge, MA 02139), Ted Birdsall, Kurt Metzger (Univ. of Michigan, Ann Arbor, MI 48109), Gary Bold, Sze Tan, Chris Tindle (Univ. of Auckland, Auckland, New Zealand), and Michael Guthrie (Defense Scientific Establishment, Auckland, New Zealand)

A low-frequency acoustic source suspended from R/P FLIP approximately 340 nautical miles WSW of San Diego transmitted to receivers 90 to $10000 \mathrm{~km}$ distant during the Acoustic Engineering Test of the Acoustic Thermometry of Ocean Climate (ATOC) Program. The source was suspended for 7 days during November 1994 near the depth of the sound channel axis (about $650 \mathrm{~m}$ ) in water over $4000 \mathrm{~m}$ deep, in order to avoid near-source bottom interactions. The source transmitted a phase-coded $m$-sequence with a center frequency of $75 \mathrm{~Hz}$ and a digit length of $27 \mathrm{~ms}$ [Metzger et al., this meeting]. Measured receptions on five bottommounted SOSUS receivers at ranges from $300-4000 \mathrm{~km}$, on two vertical line array receivers at ranges of 90 and $3300 \mathrm{~km}$, and on a sonobuoy modified to have the hydrophone on the sound channel axis at about $10000-\mathrm{km}$ range, are compared with ray theoretic, adiabatic normal mode, and broadband parabolic equation predictions. [Work supported by the Strategic Environmental Research and Development Program through ARPA.] 
1aA03. Signal generation and processing for the R/P Flip ATOC transmissions. Kurt Metzger and Ted Birdsall (CSPL, Univ. of Michigan, Ann Arbor, MI 48109-2122)

The waveforms transmitted during the November 1994 Acoustic Thermometry of Ocean Climate (ATOC) project R/P Flip measurement used an $m$-sequence phase modulated $75-\mathrm{Hz}$ carrier, two carrier cycles per digit. Because the source had an inherent $Q$ of 5 , the drive waveform was modified in order to produce a two cycle per digit output. This paper describes the basic characteristics of the source, the procedure used to broaden the source bandwidth, the enforcement of a source peak power limit, and shows the resulting waveforms. The received waveforms possessed small amounts of Doppler "shift" caused by the motion of Flip on its mooring. Doppler limits the amount of time over which receptions can be integrated. Doppler normally must be treated as time compression/ expansion. However, if the Doppler is sufficiently small it can be treated as simple time shift. This paper compares the results of using both approaches to account for the motion of Flip. [Work supported by the Strategic Environmental Research and Development Program through ARPA.]

9:00

1aA04. The response of acoustic measurements to anthropogenic climate change. Matthew A. Dzieciuch (Scripps Inst. of Oceanog., IGPP-0225, UCSD, La Jolla, CA 92093)

Anthropogenic $\mathrm{CO} 2$ in the atmosphere is expected to result in increased ocean heating. The output of a coupled ocean-atmosphere general circulation model was used to simulate to expected climate change scenario. The model was run with no $\mathrm{CO} 2$ increase and with $\mathrm{CO} 2$ doubling. The spatial properties of the $\mathrm{CO} 2$ signal are nonuniform and show that heating is not confined to the ocean surface. The ambient climate variability shows a different spatial structure that is more emphasized at the surface. The GCM output was then used as an input to an acoustic propagation model. The acoustic time series can then be analyzed for climatic trends. The control run is used to estimate the ambient noise processes on long-term scales. Maps of the anthropogenic signal to the ambient noise ratio of the climate system can then be constructed. Acoustic thermometry measures integrals of heat content through these maps. A network of sources and receivers can then be designed to efficiently monitor anthropogenic climate change. Work is in progress to compare the efficiency of different measurement systems such as satellite altimeters, drifters, and traditional measurements to that of acoustic thermometry.

\section{9:15}

1aAO5. Stochastic modeling and global warming trend extraction for ocean acoustic travel times. Steven Bottone (Mission Res. Corp., P. O. Drawer 719, Santa Barbara, CA 93102-0719), Henry L. Gray, and Wayne A. Woodward (Southern Methodist Univ., Dallas, TX 75275)

A possible indication of the existence of global climate warming is a negative trend for the travel time of an acoustic pulse along a fixed long path, or paths, in the ocean over a period of many years. Statistical methods for determining whether a significant long term trend is present in a given set of time series data of acoustic propagation times have been developed and, for illustration, applied to some specific travel-time time series generated by the MASIG and GFDL ocean models. For the multiple path case, line + noise models, where the noise is modeled as a multivariate autoregressive (AR) process are considered. It is shown that the time necessary to detect the presence of a warming trend in a time series for a single path is reduced by considering time series for multiple paths. [Work sponsored by ARPA.]
1aAO6. Are faster than predicted arrival times seeing Arctic Ocean warming? Peter N. Mikhalevsky (Sci. Appl. Intl. Corp., McLean, VA 22102), Alexander Gavrilov (Andreev Inst. of Acoust., Moscow, Russia), and Arthur B. Baggeroer (MIT, Cambridge, MA 02139)

Arrival times of $M$ sequences transmitted across the Arctic in the spring of 1994 during the trans-Arctic acoustic propagation (TAP) experiment [P. N. Mikhalevsky, J. Acoust. Soc. Am. 95, 2851(A) (1994)] are faster than modeled arrival times using historical climatology. The modal dependence of the travel times appears to be consistent with a warming of the Atlantic intermediate water (AIW) in the Arctic Ocean. Calculations of the effects of this type of climate change signal, as well as ambient variability on the modal arrival times, will be presented. The possibility that the TAP results are consistent with new reports of AIW warming in the Arctic [Carmack et al., Geophys. Res. Lett. (in press) and K. Aagaard and E. C. Carmack, Science 266 (23 December 1994)] will be discussed. [Work supported by ONR, ARPA, and the Ministry of Science, Russian Federation.]

\section{$9: 45$}

1aA07. Long-distance low-frequency modal propagation into the Lincoln Sea. Rich Pawlowicz (Inst. of Ocean Sci., P. O. Box 6000, Sidney, BC V8L 4B2, Canada), David M. Farmer (Inst. of Ocean Sci., Sidney, BC V8L 4B2, Canada), Barbara Sotirin (NCCOSC-RDTE, San Diego, CA 92152-5000), and Siobhan Sozard (Univ. of Victoria, Victoria, BC, Canada)

One-Mm transmissions from the April 1994 trans-Arctic acoustic propagation (TAP) experiment recorded by a vertical line array deployed from an icecamp at the edge of the continental shelf in the Lincoln Sea are analyzed. The received phase is astonishingly stable, and appears to vary mainly with source/receiver motions. Travel times determined from $M$-sequence transmissions are less stable and are not consistent with the phase measurements. Modal decomposition shows that bottom effects strip out the higher-order modes as sound propagates onto the shelf. The amplitude of the surface-trapped first mode is also weaker than predicted by standard loss mechanisms. Some implications of this analysis for the design of future Arctic low-frequency tomography experiments are discussed.

\section{0:00-10:15 Break}

\section{0:15}

1aA08. Use of simplified coupled mode propagation for the prediction of impulse responses of megameter trans-Arctic propagation paths. Herbert A. Freese (Sci. Applic. Int. Corporation, McLean, VA 22102)

In the spring of 1994, a long-range propagation experiment was conducted in the Arctic during which large time-bandwidth product signals ( $M$ sequences) were transmitted to two receiver sites. These data were processed to extract the pulse response of the propagation channel at the vertical arrays at each site and a horizontal array at one of the sites. These data are being used for an assessment of the utility of Arctic acoustic measurements for global warming signature detection and monitoring. A critical part of this measurement is the identification of the observed paths with specific propagating modes. A method has been developed based on coupled normal modes which allows us to estimate pulse responses quickly. The results agree favorably with the experimental data when historical environment data are used as the model inputs. The modeling methods used, comparisons with the measurements and implications, as well as the impact on sound speed accuracy required, will be discussed. 
1aA09. On the feasibility of modal group delay-based tomography in the deep ocean. Michael G. Brown and John Viechnicki (RSMAS-AMP, Univ. of Miami, 4600 Rickenbacker Cswy., Miami, FL 33149)

The modal description of sound propagation in deep ocean environments is considered. Several recently published inversion algorithms have assumed that modal group time delays can be measured. Such a measurement requires that the frequency bandwidth: (1) be sufficiently broad that successive modal arrivals are resolved in time, and (2) be sufficiently narrow that, across the band, the group slownesses of neighboring mode numbers do not overlap. These conflicting requirements can be reconciled only at long range. In the deep ocean, the late arriving, low-order modes are most likely to satisfy condition (2). Unfortunately, recent measurements and numerical simulations suggest that these modes are susceptible to internal wave-induced mode coupling - especially at long range. The following pessimistic conclusion is made: Modal group delay-based inversion schemes can be applied to measurements of acoustic wavefields in the deep ocean only for a carefully selected choice of experimental parameters (range, center frequency, and bandwidth) which may not be experimentally accessible. [Work supported by ONR.]

\section{0:45}

1aA010. Ray identification theory in ocean acoustic tomography. D. Mauuary (Inst. Für Meereskunde, Düstembrooker Weg 20, D-24143 Kiel, Germany)

The identification problem in multipath ocean acoustic tomography is not only one of the more crucial but also one of the more difficult signal processing problems to solve for further inverse studies. It is only recently that a tool, based on the Bayesian decision theory and close to the data association problem in RADAR, has been proposed by Mauuary and Moura. It fundamentally uses prior information ocean variability which transits through the ray acoustic model. It also statistically solves the ray identification problem with a Bayesian strategy. Despite the inherent complexity of resulting algorithms, a first successful attempt has been made on a French tomographic set of data (GASTOM). Some simplifications and further experimental use are now being investigated on the Mediteranean set of data (THETIS). With another approach given by Send, those are the only practical tools, but, they are sufficiently general to solve the identification problem in the most difficult conditions given by unresolved and unstable data. Both solutions are very close to the generalized Kalman filter theory and joint use of these algorithms in data assimilation models can be expected.

\section{1:00}

1aAO11. Frequency interpolation method for increasing speed of long-range broadband parabolic equation (PE) calculation. $K$. D. Heaney and W. A. Kuperman (Marine Phys. Lab., Scripps Inst. of Oceanog., La Jolla, CA 92093-0238)

An interpolation method that increases the speed of broadband rangedependent calculations by an order of magnitude using the parabolic equation has been developed. By decomposing the vertical field into complex modal amplitudes, interpolation across frequency is possible. The method was designed to be used in Monte-Carlo studies of the effects of internal waves. Signal processing algorithms are being developed to extract the deterministic signal from the internal wave noise. The method has been benchmarked against the fully broadband PE for several ocean environments. The results are in good agreement with the PE for 1000-km propagation through internal waves and for $3000-\mathrm{km}$ mildly varying ocean environments. The reproduction of the internal wave effects in the SLICE89 experiment reported by Colosi [Colosi et al., J. Acoust. Soc. Am. 96, 452-68 (1994)] has also been possible. Frequency interpolation predictions of the ATOC Acoustic Engineering Test will be presented and compared with preliminary results from the experiment. In particular, simulation of the statistics of the internal wave effects as measured in the Acoustic Engineering Test is of main concem.
1aA012. Internal wave effects on long-range ocean acoustic tomography. M. A. Wolfson, J. L. Spiesberger (Dept. Meteorol. and ARL, Penn State Univ., 602 Walker Building, University Park, PA 16802), and F. D. Tappert (Univ. of Miami, Miami, FL 33149)

Full-wave numerical simulations of internal wave scattering of lowfrequency sound in the deep ocean are performed with an efficient broadband PE model based on a new parabolic approximation that is secondorder accurate, and an internal wave model based on a new representation of the GM spectrum that allows efficient generation of internal wave fields that evolve in geophysical time. Results are displayed as plots of acoustic travel time versus geotime for various source-receiver separations (up to $8 \mathrm{Mm}$ ), and various center frequencies and bandwidths. Quantitative examination (coherent and incoherent geotime averages) yields information about the temporal coherence and stability of each multipath. It is found that the later near-axial arrivals are unstable and unresolvable, and therefore not useful for tomography, at ranges and frequencies that are consistent with the predictions of Dozier and Tappert [J. Acoust. Soc. Am. 64, 533-547 (1978)] who used a coupled mode model. [Work supported by ONR and ARPA.]

\section{$11: 30$}

1aAO13. Horizontal multipaths caused by mesoscale structure and their effects on global tomography with near-axial paths. F. D. Tappert (Appl. Marine Phys., Univ. Miami, RSMAS, 4600 Rickenbacker Cswy., Miami, FL 33149) and M. A. Wolfson (Penn State Univ., University Park, PA 16802)

The optimistic and questionable assumption that the lowest acoustic normal modes are adiabatic is used. Then, for each low mode a parabolic wave equation in the horizontal plane is derived that contains lateral variability of sound speed near the sound channel axis caused by mesoscale structure that is modeled by homogeneous and isotropic fluctuations having a single scale length, $L \sim 100 \mathrm{~km}$. In the geometrical acoustics limit, horizontal rays are found to be chaotic with the growth rate (Lyapunov exponent) given by $\nu \sim \epsilon^{2 / 3} / L$, where $\epsilon \sim 5 \times 10^{-3}$ is the rms sound speed fluctuation. Horizontal multipaths begin at the focal range, $r_{f} \sim \nu^{-1} \sim 3$ $\mathrm{Mm}$, and thereafter the number of horizontal multipaths per mode increases exponentially at the rate $\nu$ until diffraction effects limit this ray chaos. Full saturation is found to occur at the "log range," $r_{s} \sim r_{f} \ln \left(L^{2} / \lambda r_{f}\right) \sim 14 \mathrm{Mm}$, for the acoustic wavelength $\lambda \sim 30 \mathrm{~m}$. Since mode coupling is neglected, this saturation range is a hard upper bound for doing global ocean acoustic tomography with near-axial paths. [Work supported by ONR and ARPA.]

\section{1:45}

1aA014. Travel time effects of mesoscale structure on rays and waves at global ranges. F. D. Tappert and Xin Tang (Appl. Marine Phys., Univ. Miami, RSMAS, 4600 Rickenbacker Cswy., Miani, FL 33149)

A range-dependent ray trace model and a broadband PE model are used to model sound propagation at fixed bearing through a field of mesoscale baroclinic modes in order to study the effects of mesoscale structure on travel time at long ranges. The ray model exhibits chaos at ranges beyond a few $\mathrm{Mm}$ as manifested by an exponentially increasing number of eigenrays and triplications, especially in the late-time near-axial arrivals. In addition, the ray model predicts a mesoscale travel time bias, in the direction of later time, of the last axial arrival amounting to about 100-200 $\mathrm{ms} / \mathrm{Mm}$. At center frequency $75 \mathrm{~Hz}$ and bandwidth $50 \mathrm{~Hz}$, the full-wave PE model qualitatively confirms the ray trace predictions of chaos, and shows that the later near-axial arrivals are smeared out into a continuum of unresolvable multipaths characterized by saturated (Gaussian) statistics at ranges of a few $\mathrm{Mm}$, and also quantitatively confirms the mesoscale bias of $100-200 \mathrm{~ms} / \mathrm{Mm}$ of the last axial arrival. The steeper, early raylike arrivals are relatively stable in the presence of mesoscale structure and may be useful for long-range tomography. [Work supported by ONR.] 


\title{
Session 1aBV
}

\section{Bioresponse to Vibration and to Ultrasound: Progress in Vibrotaction: From Periphery to Cognition}

\author{
Ronald T. Verrillo, Chair \\ Institute for Sensory Research, Syracuse University, Merrill Lane, Syracuse, New York 13244-5290
}

Chair's Introduction $-8: 30$

Invited Papers

$8: 35$

1aBV1. Biophysical properties of a tactile mechanoreceptor: The Pacinian corpuscle. B. W. Pietras and S. J. Bolanowski (Dept. of Bioeng. and Neurosci., Inst. for Sensory Res., Syracuse Univ., Syracuse, NY 13244)

The Pacinian corpuscle is a tactile mechanoreceptor composed of a neural element surrounded by a multilayered, fluid-filled accessory capsule. Receptor potentials recorded from isolated Pacinian corpuscles in response to sinusoidal vibrations have nonlinear, asymmetric full-wave rectified transfer functions. The frequency response is U-shaped with a best frequency near $300 \mathrm{~Hz}$. The Pacinian corpuscle's accessory capsule was studied experimentally and modeled theoretically to determine its mechanical contribution to the observed physiological response. Measurements of reactive force in response to sinusoidal compression showed that the reactive force is linear, and increases with frequency and amplitude. The compressive force can be described by a first-order linear differential equation of the form $f(x, t)=k x+c d x / d t$. Interlamellar fluid velocities and lamellar displacements were also computed for a range of frequencies. Computational simulations of the interlamellar fluid flow in response to sinusoidal displacement of the surface of the capsule shows that the multilayered structure linearizes the interlamellar fluid velocity, thereby linearizing the interlamellar compressive force. Thus both the mechanical filtering by the accessory capsule and the electrodynamical properties of the neurite contribute to the nonlinear, physiological response profiles seen in the receptor potential. [Work supported by NSF, IBN-9211561.]

\section{9:05}

1aBV2. Tactile perception with haptic displays. Janet $M$. Weisenberger (Dept. of Speech and Hear. Sci., Ohio State Univ., Columbus, $\mathrm{OH}$ 43210)

Tactile sensing of objects and surfaces is typically accomplished via movement of the hand or fingers relative to the object being sensed, a mode known as haptic touch. Despite this fact, most previous work on the tactile response to complex vibratory stimuli has employed stationary displays. Although pattern movement can be simulated on these displays, kinesthetic feedback from active movement of the hand is absent. The present paper describes a series of studies employing a haptic vibratory display for the index fingertip that can be moved across a surface to encounter and scan virtual stimuli, thus preserving kinesthetic feedback. Experiments comparing this haptic scanning mode to stationary display modes showed a significant superiority of haptic scanning. However, most of this advantage was attributable to the ability to scan patterns repeatedly and from multiple directions, and not to haptics per se. Further experiments examined whether reductions in the display's field of view could be compensated by haptic scanning. Dramatic reductions in the field of view showed minimal decreases in performance when haptic scanning was employed. Results are discussed in terms of the role of haptics in tactile sensing and implications for the development of tactile displays. [Work supported by Systems Research Laboratories and ASFOSR.]

9:35-9:45 Break

1aBV3. Cortical plasticity and representation of spatially temporally distributed tactile stimuli. Xiaoqin Wang and Michael M. Merzenich (Coleman Lab. and Keck Ctr. for Integrative Neurosci., Univ. of California at San Francisco, P.O. Box 0732, San Francisco, CA 94143-0732)

An important property of the cerebral cortex is its ability to reorganize itself in response to changing sensory environment throughout life. Representational "maps" of skin surfaces in the primary somatosensory cortex (SI) are remodeled after many forms of input alteration. Two questions were addressed in our recent plasticity experiment: Under what specific conditions are spatially distributed and time-varying sensory inputs (a) integrated, or (b) segregated in their representations by the dynamic mechanisms underlying cortical function? The results showed that the topographic map of skin surfaces in SI was dramatically remodeled in adult owl monkeys trained to discriminate two tactile stimuli applied to distal and proximal segments of their fingers in alternation. Subsequent mapping of area $3 \mathrm{~b}$ revealed neural responses with either distal or proximal multiple-digit receptive fields, reflecting cortical integration of inputs from temporally coincidentally excited skin surfaces. By contrast, the cortical representations of temporally noncoincidentally stimulated skin surfaces were segregated from each other. These findings directly demonstrate that for learning induced plastic changes in cortical topographic maps, afferent input integration and segregation are dependent on stimulus coincidence, and that regularly practiced hand use results in a representation of its sensory surfaces in the brain that reflects that specific use. 
1aBV4. Influences of pain and learning on vibrotaction. Stanley J. Bolanowski, Lisa M. Maxfield, Karen L. Hall, and James C. Makous (Dept. of Bioeng. and Neurosci., Inst. for Sensory Res., Syracuse Univ., Syracuse, NY 13244)

The parallel processing of tactile information by independent channels as defined psychophysically, physiologically and anatomically has been amply described in the literature. For a unified percept to occur, however, information carried by these separate channels must be combined centrally. Furthermore, the unified percepts are affected by submodality interactions, such as the gate theory of pain whereby tactile stimulation suppresses pain, and cognitive factors (e.g., attention). Several series of experiments are presented showing that painfully hot and cold stimuli can substantially diminish vibrotactile sensation, but only when co-localized with the tactile stimuli. The effect occurs regardless of the tactile channel activated. This tough-gate acts oppositely to the pain gate and indicates centrally located touch-pain interactions. The results of additional experiments show that training of observers in an intensity-discrimination task can significantly affect the ability to distinguish differences in vibrotactile intensity. This learning effect appears to be bilateral and affects intensity as well as spatio-temporal perceptions of tactile stimuli. The effect indicates that high-level cognitive functions can influence tactile perceptions; the neural basis for these perceptions are presumably located at the cortical level.

\section{Contributed Papers}

\section{0:45}

1aBV5. Changes in mechanoreceptor-specific vibrotactile thresholds with ongoing occupational exposure to hand-transmitted vibration. A. J. Brammer and J. E. Piercy (Inst. for Microstruct. Sci., Natl. Res. Council Canada, Ottawa, ON Canada), S. Nohara (Asahi Mutual Life Insurance Co., Fukui, Japan), and H. Nakamura (Tokushima Univ., Tokushima, Japan)

The purpose of this paper is to explore the influence of occupational exposure to hand-transmitted vibration on mechanoreceptor acuity, for possible future use as a screening test for early symptoms of the hand-arm vibration syndrome (HAVS). Mechanoreceptor-specific vibrotactile thresholds were determined using the tactometer at frequencies mediated by the SAI receptors $(4 \mathrm{~Hz})$, FAI receptors $(32 \mathrm{~Hz})$, and FAII receptors $(100 \mathrm{~Hz})$ [A. J. Brammer and J. E. Piercy, "Measuring vibrotactile perception thresholds at the fingertips of power-tool operators," Proc. U.K. Informal Group Meeting on Human Response to Vibration, Buxton (1991)]. Thresholds were obtained from 33 seasonal operators of chain saws and brush cutters, who had so worked for up to 39 years. Twenty-three of the workers were judged clinically to suffer from mild to moderate symptoms of HAVS. A progressive reduction in acuity with increasing years of exposure was observed, on average, in this group, for each mechanoreceptor population. A reduction in acuity remained even after correcting for changes in threshold with age.

\section{1:00}

1aBV6. The effects of the intensity and duration of a masking stimulus, $\Delta v$, and signal duration on vibrotactile forward masking. George A. Gescheider (Dept. of Psych., Hamilton College, Clinton, NY 13323 and Inst. for Sensory Res., Syracuse Univ., Syracuse, NY 13244-5290), Kristine Santoro (Hamilton College, Clinton, NY 13323), James C. Makous, and Stanley J. Bolanowski (Syracuse Univ., Syracuse, NY 13244-5290)

Vibrotactile thresholds were found to increase as a function of the duration and intensity of a forward masking stimulus and to decrease as a function of the time interval between the termination of the masking stimulus and the onset of the signal $(\Delta t)$ and signal duration. The effects of the intensity and duration of the masking stimulus were similar for both the $\mathrm{P}$ and NPI channels as indicated by the nearly identical results measured at 250 and $20 \mathrm{~Hz}$. The observed declines in threshold as a function of $\Delta t$ and signal duration were both attributed to an increase in the time interval between the offset of the masking stimulus and the offset of the signal.

\section{$11: 15$}

1aBV7. Nonauditory effects of waterborne sound: Subjective responses during under water hearing conservation studies. $C$. $C$. Steevens and P. F. Smith (Naval Submarine Med. Res. Lab., Groton, CT 06249)

Divers have reported that intense waterbome sound can be felt as well as heard. Subjective reports of nonauditory sensations were obtained from 19 divers during recent underwater hearing conservation studies. The divers were interviewed following each noise exposure trial. The waterbome exposure stimuli were warble tones ( $\pm 5 \%$ center frequency) at center frequencies varying from 125 to $6000 \mathrm{~Hz}$ at sound-pressure levels as high as $145 \mathrm{~dB}$ re: $20 \mu \mathrm{Pa}$. Exposure durations ranged from $43 \mathrm{~s}-15 \mathrm{~min}$ per exposure. Reports of direct effects on divers' bodies, and effects on diver equipment were solicited. Nonauditory sensations were reported for frequencies of $1000 \mathrm{~Hz}$ and below, but none was reported above $1000 \mathrm{~Hz}$. The lower the frequency, the lower the threshold for nonauditory sensations. At $125 \mathrm{~Hz}$, sensations were first reported at a level of $104 \mathrm{~dB}$ re: 20 $\mu \mathrm{Pa}$. Nonauditory sensations reported were primarily vibrations felt at various locations on the divers' bodies. Anatomical localization of sensations varied with frequency, but were not exclusively related to gas filled viscera. There were also several reports of minor effects on diving equipment. No evidence for nonauditory, physiological hazard was found during these limited exposures. [Work supported by U.S. Navy.] 


\title{
Session 1aPA
}

\section{Physical Acoustics: Scattering and Propagation}

\author{
Guillermo C. Gaunaurd, Chair \\ Naval Surface Warfare Center, Code 684, White Oak, Silver Spring, Maryland 20903-5640
}

Chair's Introduction $-8: 30$

\section{Contributed Papers}

\section{8:35}

1aPA1. On local versus global parametrization of short-pulse-excited scattering and spectra: Poisson summation revisited. Leopold B. Felsen (Dept. of Aerospace and Mech. Eng., Boston Univ., 110 Cummington St., Boston, MA 02215)

Poisson summation has conventionally been employed for conversion of local scatterings due to individual elements in an infinite periodic array into global Bragg spectra that characterize the collective phenomena due to the entire array. In a recent generalization, time harmonic and transient local-global phenomena in finite periodic and quasiperiodic arrays have been related via finite Poisson summation, with the global outcome interpreted as radiation from equivalent sources distributed over the finite array aperture [L. B. Felsen and L. Carin, J. Acoust. Soc. Am. 95, 638-649 (1994)]. This analysis is now re-examined in the context of multiple scatter scenarios under short-pulse time-domain excitation. Since short enough pulsed incident fields can time-gate individual scattered field arrivals, the early time response at the observer is necessarily parametrized locally. As multiple interaction develops, one may reparametrize any finite number of these collectively in terms of spectra associated with equivalent sources that are smoothly distributed over the corresponding multipass finite spacetime aperture. This results in global algorithms based partly in the configuration domain and partly in the spectral domain. These concepts are developed and examined rigorously and asymptotically with respect to the time evolution of global spectra from highly resolved early scatters under short-pulse time-domain conditions. Corresponding statistical aspects, when the scattering hierarchy is randomly perturbed, are explored as well. [Work supported by AFOSR and ONR.]

\section{8:50}

1aPA2. Short pulse sound wave scattering by a fluid shell model target near a randomly distorted bottom interface: Wave oriented data processing for target-clutter discrimination. T. T. Hsu, L. Carin (Dept. of Elec. Eng., Polytechnic Univ., Six Metrotech Center, Brooklyn, NY 11201), and L. B. Felsen (Boston Univ., Boston, MA 02215)

A finite difference time-domain (FDTD) code has been used to assemble a comprehensive data base of realizations for short-pulse plane wave scattering by a fluid shell model target submerged in the presence of a randomly distorted bottom interface. The entire problem is two dimensional with the scattered acoustic pressure observed along an elevated track above the target and parallel to the mean bottom boundary. Two bottom topographies are examined: (1) a smoothly deformed interface between the wated column and a fluid bottom; (2) a collection of soft randomly pitched thin flat strips. The database furnished by the ensemble of realizations for the randomly irregular bottom, in which the deterministic target remains unchanged, is subjected to wave-oriented data processing that yields spacewave number and time-frequency phase space distributions extracted via windowed transforms and refined by locally applied high resolution algorithms. The processing is applied to the target alone, the cluster alone, and the target-clutter combination. Target resonances are regarded as a major discriminant which one needs to enhance by input signal shaping and output filtering while simultaneously de-emphasizing the comupting effect of the clutter. Statistical measures pertaining to the clutter are given consideration, as is the effect of system noise added to the data. Results are presented and discussed. [Work supported by ONR and AFOSR.]

\section{9:05}

1aPA3. Acoustic response of interacting gas bubbles. Daniel Goldman, Paul E. Barbone, and Ali Nadim (Dept. of Aerospace and Mech. Eng., Boston Univ., 110 Cummington St., Boston, MA 02215)

Following a method similar to that used to obtain the standard Rayleigh-Plesset equation, equations are derived describing the volume oscillations of two interacting spherical bubbles, whose centers are assumed to be a large distance (relative to the equilibrium radii of the bubbles) apart in an infinite fluid. Although the bubbles are initially assumed to be fixed, these approximate equations also describe the movement of the bubble centers. First, the natural frequencies of the two-bubble system are obtained via linearization. Then, for bubbles with the same equilibrium radii, the nonlinear stability of the symmetric and antisymmetric modes is studied both analytically and numerically. The response of the two-bubble system to a time-periodic pressure field is also studied. Finally, the equations for the two-bubble system are generalized to those describing an $n$-bubble system, and an estimate is obtained for the lowest resonant frequency of the $n$-bubble cloud.

\section{9:20}

1aPA4. Scattering from a fluid-loaded elastic spherical shell in proximity to a rough fluid-elastic interface: Numerical results. Judy Smith and Gamer C. Bishop (Nav. Undersea Warfare Ctr. Div., Newport, RI 02841-5047)

A null field $T$-matrix formalism is developed and used to calculate scattering of a pressure wave from a fluid-loaded elastic spherical shell in proximity to a rough fluid-elastic interface. The Helmholtz-Kirchhoff integral representations of the various scattered pressure and displacement fields are constructed. The surface fields are required to satisfy the elastic boundary conditions and the scattered fields are required to satisfy the extended boundary condition. The free-field $T$ matrices for the elastic shell and the rough fluid-elastic interface are constructed and used in the Helmholtz-Kirchhoff and the null field equations for the shell-interface system. It is shown that the $T$ matrix for the system is simply related to the free-field $T$ matrices for the shell and the rough fluid-elastic interface. A perturbative solution is obtained for scattering from arbitrary roughness, and an "exact" solution is obtained for scattering from periodic roughness. The "exact" solution is used to obtain a variety of numerical results that show some of the effects of the acoustic coupling that occurs between the rough interface and the elastic spherical shell. 
phone may be on the opposite side of the sheet. The understanding and

1aPA5. The effect of turbulence drift on acoustical scattering into a shadow region. Michael R. Stinson (Inst. for Microstruct. Sci., Natl. Res. Council, Ottawa, ON KIA 0R6, Canada)

The turbulence structures responsible for acoustical scattering in the atmosphere are not static and evolve in time. As a first approximation, a uniform drift of the turbulence with the prevailing winds, consistent with Taylor's hypothesis, can be anticipated. This drift can be accommodated through an extension of the Green's function parabolic equation (GF-PE) approach. Normal implementations of the GF-PE compute the sound field assuming a static realization of turbulence. Such calculations can be repeated, though, for successive realizations of a turbulent atmosphere, with each realization corresponding to an additional shift in space of the initial turbulent structure. The resulting series of sound field "snapshots" shows the evolution of the sound field as the turbulence drifts. Recent simulations using the GF-PE have examined scattering by drifting turbulence into an acoustical shadow, formed during upward refracting conditions, and the time evolution of the scattered field will be presented. The nature of these time variations is relevant to the use of beamforming arrays in an acoustic shadow. Some observations about predicted array responses will be discussed.

\section{9:50}

1aPA6. Resonant behavior of two eclipsed shells: Quasiharmonic study. P. Pareige, Elm. Kheddioui, J. M. Conoir, and J. L. Izbicki (Lab. d'Acoust. Ultrason. et d'Electron., U.R.A. C.N.R.S. 1373, Univ. du Havre, place Robert Schuman, 76 610 Le Havre, France)

The purpose of this paper is the presentation of temporal, frequential, and angular experimental studies of the acoustic scattering by two identical air-filled elastic shells immersed in water. They are insonified by a quasiharmonic wave at normal incidence. The distance between the shells is about 1 to 3 diameters of one shell. The frequency range investigated corresponds to the whispering gallery wave resonances $(l=2)$. The configuration considered is the eclipsed one: Emitter and shells are on the same axis. The complex structure of the backscattered temporal signal is analyzed and theoretically justified. According to the part of the temporal signal considered, different backscattering spectra are obtained and analyzed. The results are discussed in term of acoustic rays involving internal and external acoustic waves. In addition, a phenomenon of acoustic interference is clearly shown. At the resonance frequencies of one shell, the angular diagram shapes are justified and compared to those provided by an analytic model. In all cases, the results are compared to the well-known results provided by the study of a single shell.

\section{0:05-10:20 Break}

1aPA7. Pressure impulse response measurements for elastic scatteners in water and regulation of the source spectrum. Philip $L$. Marston and Scott F. Morse (Dept. of Phys., Washington State Univ., Pullman, WA 99164-2814)

A large PVDF sheet adjacent to the scatterer has been used to excite the plane-wave pressure impulse response of elastic shells over a wide spectral range [Kaduchak et al., to be published in J. Acoust. Soc. Am. (1995)]. Since the sheet is transparent to the scattered signal, the receiver hydro- control of the spectrum of the radiated pressure pulse is useful for facilitating the detection of specific target spectral features. An idealized circuit model is examined where the sheet is excited by a step voltage source having a real impedance given by the resistance $R$. Let $C$ denote the sheet capacitance and let the step occur at $t=0$. The radiated pressure for a thin sheet becomes a step followed by an exponential tail: $\exp (-t / R C)$. The magnitude spectrum is proportional to $\left[1+(\omega R C)^{2}\right]^{-1 / 2}$. Increasing $R$ reduces the high-frequency content which may be advantageous for certain measurements. Applications include the impulse excitation of lowfrequency structural resonances of shells in water. Adjacent modes exhibit a prominent beating in the response. [Work supported by the Office of Naval Research.]

\section{0:35}

1aPA8. Sound diffraction by a ground with barriers or impedance discontinuities in a refracting atmosphere. $\mathrm{Y}$. $\mathrm{L}$. $\mathrm{Li}$ (Wave Propagation Lab., Dept. of Elec. and Comput. Eng., Univ. of Illinois at Urbana-Champaign, 332 CSRL, 1308 W. Main Street, Urbana, IL 61801) and Michael J. White (U.S. Army Construction Eng. Res. Lab., Champaign, IL 61826-9005)

The exact solution for the total field is obtained for the sound diffraction due to a time-harmonic line source above a ground in a medium with linear sound-speed profile. The ground may have barriers with arbitrary shapes or impedance discontinuities parallel to the line source. The solution is in terms of an integral equation which is numerically solved by an extension of the method of moments. The effects of scattering in the region around barriers or impedance discontinuities are studied for situations for various values of the refracting strength of the sound-speed profile.

\section{0:50}

1aPA9. Application of the PE method to up-slope sound propagation. Marta Galindo and Karsten Bo Rasmussen (Acoust. Lab., Bldg. 352, Tech. Univ. of Denmark, DK 2800 Lyngby, Denmark)

The wide-angle PE method is applied to the prediction of sound propagation in a range-dependent environment. The finite-difference model treats the sloping ground by a staircase approximation. The method allows impedance and slope angle variations to be taken into account. The implementation of sloping interfaces in one-way PE models introduces a fundamental problem of energy conservation. This is generated at the lower boundary condition. The ground is assumed to be a locally reacting surface where the ground impedance is defined as the ratio of pressure to the normal component of particle velocity at the surface. The staircase PE method approximates the normal at the slope surface with the vertical component at the stair-step surface. A numerical correction can be included. Using a scale model facility [K. B. Rasmussen, J. Acoust. Soc. Am 96, 3617-3620 (1994)], a series of measurements is carried out. The results are compared with the PE calculations. The magnitude of the error due to the interface condition is evaluated. 
Preliminary experiments using Styrofoam indicate that similar trends exist above the low impedance rough surface as did over the high impedance rough surfaces. [Work supported by USDA.]

1aPA10. An experimental investigation of the propagation of sound over a flat rough surface of finite impedance. James $P$. Chambers and James M. Sabatier (Natl. Ctr. for Phys. Acoust., Univ. of Mississippi, University, MS 38677)

Recent work on rough surfaces by Medwin et al. [J. Acoust. Soc. Am. 79, 657-665 (1986)] has indicated the presence of acoustic boundary waves in the fluid directly above rough surfaces with high acoustic impedances. The effect of this boundary wave is that the sound field above the rough surface is louder than it would have been over a similarly smooth surface at close ranges due to multiple coherent scattering. The opposite is true at longer ranges since the scattering becomes incoherent. The ranges of coherent and incoherent scattering depend on the roughness scale and frequency of the sound source. The experimental work described above was performed over surfaces with high acoustic impedances such as wax and steel. The purpose of this present work is to find out if similar trends exist in the propagation of sound over rough surfaces that possess a lower acoustic impedance. Sound signals will be measured above a rough surface and compared to the signal above a smooth surface of the same material.

\section{1:20}

1aPA11. Locally reacting boundary condition over sloping boundaries for the parabolic equation. B. G. Tollison, J. S. Robertson (Dept. of Math. Sci., U.S. Military Academy, West Point, NY 10996-1786), and W. L. Siegmann (Rensselaer Polytech. Inst., Troy, NY 12180-3590)

A boundary condition which models locally reacting boundaries is derived for the generalized parabolic equation. This formulation is valid over nonhorizontal boundary surfaces. The derived condition is discretized using Lagrange polynomials. Particular implementation schemes are discussed for nonhorizontal boundaries. Numerical results compare the accuracy of this approach with other methods that have been used with the PE. Comparison with experimental results may also be presented. [Work supported by ONR and USACECOM.]

TUESDAY MORNING, 30 MAY 1995

CONGRESSIONAL HALL B, 8:30 A.M. TO 12:00 NOON

\title{
Session 1aSC
}

\section{Speech Communication: Speech Processing and Perception/Psychophysics (Poster Session)}

\author{
W. Van Summers, Chair \\ Walter Reed Army Medical Center, Research Section, Army Audiology and Speech Center, Washington, DC 20307
}

\section{Contributed Papers}

\begin{abstract}
All posters will be on display from 8:30 a.m. to 12:00 noon. To allow contributors an opportunity to see other posters, contributors of odd-numbered papers will be at their posters from 8:30 to 10:15 a.m. and contributors of even-numbered papers will be at their posters from 10:15 a.m. to 12:00 noon. To allow for extended viewing time, posters will remain on display until 8:00 p.m.
\end{abstract}

1aSC1. The effects of talker variability across $\mathrm{CV}$ and $\mathrm{VC}$ environments. Lynn M. Farnsworth and John W. Mullennix (Dept. of Psych., Wayne State Univ., 71 W. Warren St., Detroit, MI 48202)

A consonantal speeded classification task was used to assess the effects of talker variability across $\mathrm{CV}$ and VC environments. In addition, conditions were blocked by syllable type ( $\mathrm{CV}$ or $\mathrm{VC}$ ) or consisted of mixed sets of $\mathrm{CV}$ and $\mathrm{VC}$ syllables. Talker variability was manipulated by presenting stimuli spoken by a single talker or by many talkers. The results showed that the magnitude of the effect of talker variability was approximately the same when comparing performance across talker contexts for CV's and VC's. In addition, the talker variability effect was stronger under conditions where syllables were blocked. The results provide further information about the underlying mechanisms involved in processing perceptual variation in the speech signal.

1aSC2. Adult attribution of the infant vocalizations. Yoko Shimura (Dept. of Educ., Saitama Univ., 255 Shimo-Okubo, Urawa-shi, Saitama, 338 Japan) and Satoshi Imaizumi (Univ. of Tokyo, 7-3-1 Hongo, Bunkyo-ku, Tokyo, 113 Japan)

Adult attribution of the infant vocalizations was investigated by perceptual rating experiments for 717 voice samples recorded from seven infants at $2,6,9,12$, and 17 months of age. Perceptual rating was performed using nine vocalization- and emotion-related reference words by 15 normal heating adult listeners. By a principal factor analysis, three factors representing the emotional contrast of crying/frightened/sad versus laughing/pleased/happy, shout/surprising versus secret-talk/calm, and speaking versus singing were studied. Even at 2 months of age, significant individual differences were observed in the extracted factor scores, although three were less variability compared to those obtained from the elder infants' vocalizations. These results suggest that the ability to express emotion through vocalization seems to be developing during the observed months of age, although infants even at 2 months of age can express some aspects of emotion through vocalization.

1aSC3. A longitudinal investigation of children's duration and temporal variability in speech production. Bruce L. Smith, Mary Kay Kennedy, and Sarmad Hussain (Dept. of Commun. Sci. and Disord., Northwestern Univ., 2299 North Campus Dr., Evanston, IL 60208-3570)

A number of cross-sectional studies have observed that children's speech segments tend to decrease in duration and also become less variable from younger to older groups of subjects. Because cross-sectional investigations have certain limitations when they are used to study children's development, it is also important to conduct longitudinal analyses of speech production development. The present study provides data based on a longitudinal analysis of the speech of 12 children. When they were initially seen, the children ranged from 7-11 years of age; they were evaluated again after approximately $1-1 / 2$ years. At both ages, the children were recorded as they produced 25 repetitions of each of several different words and short phrases, from which various segments and syllables were measured. Results indicate that, on average, segment and syllable durations decreased from the initial to the follow-up recordings by approximately $10 \%$, whereas temporal variability was found to decrease by about 
$40 \%$. Although the relative decrease in the children's temporal variability was substantially greater than the decrease in their duration across the year-and-a-half time period, their durations were considerably more adultlike than their variability at both times they were studied.

1aSC4. Speech during simultaneous communication: Temporal characteristics and perceived naturalness. Robert $L$. Whitehead (Comm. Res. Natl. Tech. Inst. for the Deaf, 52 Lomb Memorial Dr., Rochester, NY 14623-5604), Nicholas Schiavetti (State Univ. of New York, Geneseo, NY 14454), Brenda H. Whitehead (Natl. Tech. Inst. for the Deaf, Rochester, NY 14623), and Dale Even Metz (State Univ. of New York, Geneseo, NY 14454)

Simultaneous communication is a process which combines spoken English with various manual representations of English words in the form of signs and fingerspelling. The present study investigated the durational characteristics and perceived naturalness of speech produced during simultaneous communication with increasing fingerspelling complexity. Ten normal-hearing adult females, skilled in the use of simultaneous communication, served as speakers. Experimental words of differing fingerspelling complexity were embedded in sentences which were: (a) spoken and (b) spoken and signed, except for the experimental words which were fingerspelled. Five temporal measures of speech were calculated: (a) sentence duration, (b) experimental word duration, (c) diphthong duration preceding the experimental word, (d) pause duration preceding the experimental word; and (c) pause duration following the experimental word. Speech naturalness was rated using a nine-point naturalness rating scale for all stimulus samples. Results indicated significant differences in durational measures and naturalness ratings between the speech-only and simultaneous communication conditions, and between levels of fingerspelling complexity. A multiple regression analysis was performed to predict speech naturalness from the durational measures used as independent variables. The multiple $R^{2}$ was 0.698 . [Work supported by U.S. Department of Education.]

1aSC5. Speaker normalization for tone. Corinne B. Moore (Dept. of Mod. Lang. and Lit., 203 Morrill Hall, Cornell Univ., Ithaca, NY 14853)

Segments with identical acoustic characteristics may not be perceived identically, because listeners normalize for acoustic variability due to vocal tract differences among speakers. While normalization studies have been done for vowel perception, the present experiment uses Mandarin Chinese to investigate speaker normalization for lexical tones. In this study, two speakers were identified for whom the low tone (tone 3) of the highpitched speaker occurred in the same $F O$ region as the midrising tone (tone 2) of the low-pitched speaker. Synthetic syllables formed three continua used in a series of perception tests. The three continua varied along either $F 0$, timing of the turning point, or both acoustic dimensions. Stimuli were placed after a natural precursor from each of the two speakers. Test items were randomized and presented in a mixed block condition. Results show that category boundaries shift contrastively in the direction predicted; that is, the same stimuli were perceived differently in the two precursor conditions, although only for stimuli varying along the $F 0$ and both $F 0$ and turning point dimension, but not for stimuli varying along the duration dimension (turning point) alone. Further testing is currently being conducted on normalization in the duration dimension. [Work supported by Sigma Xi.]

1aSC6. The effect of stimulus familiarity on magnitude-estimation scaling of different languages. Donald Fucci (School of Hear. \& Speech Sci., Lindley Hall 219, Ohio Univ., Athens, OH 45701), Ramesh Bettagere, Maria D. Gonzales, Mary E. Reynolds (Ohio Univ., Athens, $\mathrm{OH} 45701$ ), and Linda Petrosino (Bowling Green State Univ., Bowling Green, $\mathrm{OH}$ 43403)

The purpose of this study was to examine listener's abilities to judge the subjective parameter of loudness of familiar and unfamiliar spoken languages. Two groups of subjects consisting of 20 adults each, ranging in age from 20-32 years, participated. Group 1 consisted of subjects who were familiar with English and not familiar with Hindi. Group 2 consisted of subjects who were familiar with English as well as Hindi. Two separate magnitude-estimation scaling tasks were performed. For the first task, subjects in both groups were instructed to assign a numerical value that cor- responded to the loudness of each of nine suprathreshold intensity levels of an English sentence. For the second task, subjects in both groups were instructed to assign a numerical value that corresponded to the loudness of each of nine suprathreshold intensity levels of the same sentence spoken in Hindi. Statistical analysis showed no significant difference between the two groups in the scaling of the English and Hindi sentences. Results suggested that magnitude-estimation scaling appears to be an effective measure of loudness of familiar and unfamiliar languages, and that subjects scale the loudness of a familiar language the same way as they scale the loudness of an unfamiliar language.

1aSC7. Talker-based selective adaptation. Gary E. Star and Mark A. Pitt (Dept. of Psych., Ohio State Univ., 1885 Neil Ave., Columbus, $\mathrm{OH}$ 43210-1222)

When during perception does the auditory system differentially process certain classes of sound objects? For example, in some models of speech perception, only phonetic stimuli are processed in the highest levels of analysis. Prior work in this lab [M. A. Pitt, J. Acoust. Soc. Am. 95, 2975(A) (1994)] found that stimuli varying in musical timbre yielded selective adaptation results qualitatively similar to those obtained with phonetic stimuli. The generality of this finding was assessed by conducting comparable experiments on a nonphonetic dimension that originates from the same source as a phonetic percept: talker identity. Two selective adaptation experiments were conducted using a voice continuum. Talkers spoke the same vowel. Endpoint adaptors and different-vowel adaptors were presented ipsilateral and contralateral to the ear of identification. Results are similar to prior findings, and suggest that these classes of objects are processed similarly to a fairly abstract level of analysis. [Research supported by NIH.]

1aSC8. The effects of talker-specific information on immediate memory span. Helena M. Saldaña (Speech Res. Lab., Dept. of Psych., Indiana Univ., Bloomington, IN 47405)

Recent evidence suggests that talker-specific information is retained along with codes for words and phonemes in long-term memory [Palmeri et al., JEP:LMC 19, 309-328 (1993); J. W. Mullenix and D. B. Pisoni, J. Acoust. Soc. Am. 85, 365-378 (1990)]. If this indexical information is retained, one would expect talker-specific information to also effect a listener's performance on short-term memory tasks. However, the predominant trace decay theories of short-term memory do not predict this. For example, in the articulatory loop model, items are stored as memory traces that fade after approximately $2 \mathrm{~s}$ unless revived by an articulatory control process [A. D. Baddeley and G. J. Hitch, Psych. Learn. Motiv. (1974)]. The number of items that can be reactivated within the decay time can be retained indefinitely. Therefore, immediate memory span is defined in terms of time or duration of items. In the present investigation, memory span experiments were conducted in which voice information was held constant or was changed for each item in the list. Initial results indicate that a change in voice information results in a decrease in memory span. Follow-up experiments manipulate speaking rate and presentation level. A reconceptualization of the articulatory loop model is discussed which takes into account the encoding of stimulus variability. [Work supported by NIH.]

1aSC9. The role of experience in the development of category structures. Barbara E. Acker and Richard E. Pastore (Dept. of Psych., SUNY Binghamton, Binghamton, NY 13902)

Recent speech research and music work have found empirical evidence for protolypes or exemplars. Whereas the function of a speech prototype is debatable, experiments using musically trained subjects found better discrimination around major chord prototypes than near less representative members of the category. While the specific function of prototypes is of interest, the development of prototypes is also important in elucidating perceptual processes. Developmental processes in speech and music have been studied by comparing infant and adult dara. Utilizing musically naive adult subjects and musical stimuli, the current work investigates perceptual differences attributable to training and experience, but avoids problems associated with infant subjects. The ability of nonmusically trained subjects to discriminate in-tune (prototype [P] set) and mistuned (nonprototype [NP] set) major chords was evaluated in an AX task. Unlike musicians 
who showed significantly better performance with the $P$ set, the naive subjects showed no differences between the $\mathrm{P}$ and NP sets of stimuli. These results demonstrate the need for experience for the development or activation of prototypes. Whereas the music results may not provide a direct parallel to speech, they do provide a basis for comparison. [Work supported by AFOSR.]

1aSC10. The effects of feedback in auditory looming tasks. A. Paige Wuestefeld and Lawrence D. Rosenblum (Dept. of Psych., Univ. of California, Riverside, CA, 92521)

Previous research [Rosenblum et al., Perception 22, 1467-1482 (1993)] demonstrates that listeners are able to make somewhat accurate anticipatory judgments regarding the time to arrival of a looming sound source and that performance feedback significantly improves these judgments. The present research examines the effects on performance of providing feedback to listeners and subsequently withdrawing it. Some visual research involving the extrapolation of movement suggests that, upon withdrawing feedback, performance will revert back to its original level. Subjects made judgments about the time to arrival of an approaching car based on various portions of the event. Each subject participated in three experimental sessions on consecutive days. Subjects were divided into three groups which received differing feedback schedules. For all groups, the first session contained no performance feedback. The withdrawal group received feedback during the second session, but not the third. One control group received no feedback for the remainder of the experiment. A second group control group received feedback for both the second and third sessions. When feedback was withdrawn, performance between the second and third sessions did not differ significantly. Thus, unlike the previous visual effects, performance did not revert back to its original level.

1aSC11. Age differences in the uptake of acoustic cues in spoken word recognition. Robert Allen Fox and Julie McGory (Dept. of Speech Hear. Sci., Ohio State Univ., 110 Pressey Hall, 1070 Carmack Rd., Columbus, $\mathrm{OH}$ 43210-1002)

This study compares the uptake of acoustic-phonetic information during lexical access in younger (20-30 years) and older ( $55-70$ years) listeners to determine if these groups are equally efficient in utilizing the coarticulatory information in VC transitions. Examined were place contrasts between stops ( $\mathrm{p} / \mathrm{t}$ and $\mathrm{p} / \mathrm{k}$ ) and fricatives ( $\mathrm{s} / \int$ and $\left.\mathrm{z} / \mathrm{z}\right)$, and manner contrasts between stops and nasals $(b / m$ and $d / n)$ using the gating paradigm [Warren and Marslen-Wilson, Percept. Psychophys. 41, 262-275 (1987)]. Stimuli consisted of 21 pairs of $\mathrm{CVC}$ words differing only in terms of the final consonant (e.g., pot versus pop). These words were heard in their natural or cross-spliced forms in which the initial CV of one word was appended to the consonant offset of its match. Gated versions of each word were constructed on the basis of this "splicing" point such that the first gate included the initial $\mathrm{CV}$ up to $100 \mathrm{msec}$ before end of the vowel and then proceeded in 20-ms increments (gates that included portions of voiceless stop closure providing no additional acoustic information were eliminated). Results will be discussed in terms of possible age-related decrements in phonetic processing. [Work supported by NIA.]

1aSC12. The cocktail party effect in infants. Peter W. Jusczyk and Rochelle Newman (Dept. of Psych., SUNY at Buffalo, Buffalo, NY 14260)

Most research on speech. recognition by infants occurs in quiet laboratory rooms with no outside distractions. But the real world may not resemble the laboratory: Much speech directed to infants occurs with competing acoustic signals. To learn language, infants need to follow their care-givers' speech even under less than ideal listening conditions. Infants' abilities to follow a female speaker's voice when a male voice was talking simultaneously were examined. In pilot studies, infants presented with two competing streams of fluent speech showed no evidence of recognizing words spoken by the target voice. In the present study, infants heard a target voice repeating isolated words, while the second voice spoke fluently at either of three different loudness levels. Subsequently, infants heard passages produced by the target voice using either the familiar words, or novel words. Infants listened longer to the familiar words when the target voice was 10 or $5 \mathrm{~dB}$ louder, but not when the distractor was equally loud. This suggests that infants could separate the two voices, provided the target voice was at least $5 \mathrm{~dB}$ louder. Hence, infants have some capacity to extract information from speech even in the face of competing acoustic signals. [Wotk supported by NICHD to PWJ and an NSF Graduate Fellowship to RN.]

1aSC13. The relationship between the discriminability of formant transitions and certain cognitive measures. Thomas Carrell (Barkley Ctr., Univ. of Nebraska, Lincoln, NE 68583-0738), Nina Kraus, Tenry McGee, and Steven Zecker (Northwestern Univ., Evanston, IL 60208)

This work concerns the relationship between two measures of auditory processing and several measures of cognitive abilities. The discriminabilities of formant transitions in synthesized consonant-vowel syllables were collected from over 100 children aged 7-11. Specifically, the discriminabilities of the $F_{3}$ onset frequencies were measured in a set of stimuli which ranged perceptually from $/ \mathrm{da} /$ from $/ \mathrm{ga} /$. Also, the discriminabilities of the onset transition durations of $F_{1}, F_{2}$, and $F_{3}$ were measured in stimuli which ranged perceptually from $/ \mathrm{ba} / \mathrm{to} / \mathrm{wa} /$. A PEST adaptive tracking paradigm was employed to make these measurements. The average just noticeable difference was $101 \mathrm{~Hz}$ for the $F_{3}$ onset frequencies and $6 \mathrm{~ms}$ for the $F_{1}, F_{2}$, and $F_{3}$ frequency ramps. A battery of tests of cognitive ability were administered to the same children. The cognitive ability tests revealed a wide range of scores in the subjects tested. A correlational analysis of these data indicated that better onset frequency discriminability was significantly correlated with higher scores on the reading subtests, whereas better scores on transition duration tasks were not significantly correlated with any of the cognitive ability measures. It was also found that better formant onset frequency discriminability did not predict higher transition duration discriminability. That is, better $/ \mathrm{da} / \mathrm{vs}$ /ga/ discrimination did not predict better /ba/ vs /wa/ discrimination. [Work supported by NIH.]

1aSC14. On the "locus equation" and its relation with the consonant place of articulation. Samir Chernoukh, René Carré (Sig. Dept. ENST, 46 Rue Barrault, 75634 Paris, France), and Björn Lindblom (Univ. of Stockholm, S-10691 Stockholm, Sweden)

The specification of the consonant place of articulation from the locus equation coefficients [Sussman et al., J. Acoust. Soc. Am. 90, 1256-1268 (1991)] is subject to discussion. For instance, Fowler [Percept. Psychophys. 55, 597-610 (1994)] claims that these coefficients can vary significantly within homorganic consonants. In order to study the relation between these coefficients and place of articulation, $\mathrm{V} 1 \mathrm{CV} 2$ utterances were stimulated using the distinctive region model and three different instants of initiating the second vowel: before the acoustic segment of the consonant, at the beginning of that segment, and, in the third case, after it. The results show that linear locus equations can be acceptably fitted to those conditions, but that their coefficients vary as a function of the second vowel onset. It was noted that the locus equations are sensitive to degree of consonant-vowel coarticulation. Also, when y intercepts were plotted against the associated slopes for a given place and across the simulated timing conditions, highly linear relationships were observed. Thus the coefficients of such "second-order" locus equations stay constant for a given place despite variations in temporal and coarticulatory conditions. Hence, for natural speech, such "second-order" parameters might be a more stable place correlate than first-order coefficients.

1aSC15. Influence of the number of syllables and morae on reaction time in Japanese speech production. Shinobu Masaki and Kiyoshi Honda (ATR Human Information Process. Res. Labs., 2-2 Hikaridai Seika-cho, Soraku-gun, Kyoto, 619-02 Japan)

In order to estimate the processing unit of Japanese speech production, the influence of the number of syllables and morae on reaction time (RT) in speech production was analyzed. In experiment 1 , RT was measured for one- to four-mora nonwords consisting only of Japanese CV syllables [ka] or [ta]. Five subjects produced stimulus words as soon as possible after the visual onset signal was presented. RT was defined as the duration from the moment of onset signal presentation to the release of word-initial plosive consonant. Results showed a tendency for RT to increase as the number of $\mathrm{CV}$ syllables increases. In experiment 2, stimulus words consisted of CV syllables and vowels without a syllable-initial consonant, or CV syllables and nasal sounds [N] without a syllable-final vowel. Note that, in Japanese, 
a vowel and nasal sound $[\mathrm{N}]$ can be a mora like a $\mathrm{CV}$ syllable. For these stimuli, the RT was not influenced by the increase of the number of morae. These results suggests a possibility that a mora consisting of a CV syllable is the smallest segment of the processing unit in Japanese speech production. However, morae consisting of a single vowel or nasal sound cannot perform as independent processing units.

1aSC16. Perceptual interaction of $F 1$ and $F 0$. Jose $R$. Benki (Linguist. Dept., Univ. of Massachusetts, Amherst, MA 01003)

While $F 1$ is a primary perceptual correlate of vowel height, other dimensions of vowels, such as $F 0$, covary with $F 1$ in natural speech. This study investigates the perceptual interaction of $F 0$ and $F \mathrm{l}$ in back vowels using the Garner paradigm. Two factors are hypothesized to determine the interaction: the magnitude of $F]-F 0$ and the locations of the harmonics. $F 1$ and $F 0$ are predicted to integrate negatively for vowels in a threshold region (3.0 Bark $<F 1-F 0<3.5$ Bark), whereas subthreshold $(F 1-F 0$ $<3.0$ Bark) and suprathreshold vowels $(F 1-F 0 \gg 3.0$ Bark) are predicted to show no interaction. Previous work [Benki et al., J. Acoust. Soc. Am. 95, 2977(A) (1994)] suggests that $F 1$ and $F 0$ do not interact in the threshold region but integrate positively in the suprathreshold region. The predicted dependence on the locations of the harmonics follows from work by Hughes and Diehl [J. Acoust. Soc. Am. 95, 2978(A) (1994)] showing that $F 1$ discriminability is enhanced when a harmonic is near nominal $F 1$. To assess these effects, CVC stimuli ranging in $F 1$ from $300-600 \mathrm{~Hz}$ and $F 0$ from $90-120 \mathrm{~Hz}$ were presented to listeners in the Garner baseline and correlated classification tasks. A perceptual $F 0 / F 1$ space is inferred using a detection-theory analysis of the accuracy data. [Work supported by NSF and NIH.]

1aSC17. Word frequency effects on the acoustic duration of morphemes. Beth L. Losiewicz (Dept. of Psych., Colorado College, Colorado Springs, CO 80901)

The acoustic duration of the English past tense (ED) morpheme was measured for matched high- and low-frequency verbs (e.g., KNEADED/ NEEDED). The ED on low-frequency verbs was of longer acoustic duration than the ED on matched high-frequency words. The rhyming portion of the matched verbs also showed a lengthening effect for the lowfrequency words; in contrast to previous reports that word frequency does not affect word acoustic duration [Geffen and Luszcz, Mem. Cogn. 11, 13-15; Wright Mem. Cogn. 7, 411-419]. However, this effect was statistically independent of the ED length effect, and the final phonetic segment of a low-frequency monomorphemic verb stem was not longer in acoustic duration than a homophonous segment on a matched high-frequency verb (e.g., the /d/ in KNEAD/NEED). Further, the ED morpheme is of longer acoustic duration than a homophonous segment in a nonverb homophone (e.g., RAPPED/RAPT), as earlier reported for the morpheme /s/ [Walsh and Parker, J. Phon. 11, 201-206]. This set of evidence corroborates a frequency-dependent dual-access processing theory of linguistic morphology: that high-frequency complex words are processed holistically and low-frequency complex words are processed componentially [cf. Bybee, Morphology (1985)]

1aSC18. Adaptation in speech production to transformed auditory feedback. John F. Houde and Michael I. Jordan (Dept. of Brain and Cognit. Sci., MIT, 79 Amherst St., Cambridge, MA 02139)

This study investigated the ability of the speech production system to learn to compensate for changes in auditory feedback. The setup used for this was a DSP system that transformed the immediate feedback a subject received when speaking. This system can analyze a subject's speech into a formantlike representation, possibly alter it, and then use it to resynthesize speech which is fed back to the subject with no noticeable delay (16 ms). The first of the experiments investigated whether subjects would learn to compensate for a change in vowel identity when producing CVC words. It was found that compensatory articulations were indeed learned, and that these persisted even when no auditory feedback was provided. The findings suggest similarities between speech and other sensorimotor tasks, such as reaching, which also show such adaptation. Other experiments characterizing the degree to which this effect generalizes across differing word and vowel environments will also be presented.
1aSC19. Differential masking of individual words within a sentence. Theodore S. Bell (UCLA School of Medicine, Head \& Neck Surgery, 31-24 Rehabilitation Ctr., Los Angeles, CA 90024-1794) and Richard Wright (UCLA, Los Angeles, CA 90024-1543)

Three experiments tested the effectiveness of adjusting the amplitude of a noise masker to selectively alter intelligibility of individual words within sentences. Stimulus sentences contained three key words; all were commonly used and phonetically unique. A speech-shaped noise was presented at $65 \mathrm{~dB}$ SPL for all experiments. The initial study tested 12 listeners under two conditions: the noise level remained flat, or was attenuated $6 \mathrm{~dB}$ under the middle key word. The speech was presented at 54 and $58 \mathrm{~dB}$ SPL. The middle key word improved by as much as $30 \%$ ( $p$ $<0.001$ ), while other key words were unaffected. In the next two experiments, the noise was either increased or decreased by $3 \mathrm{~dB}$ at a key word. In one, the manipulation was at the first key word, and in the other was at the last key word. In both experiments, the speech was presented to ten listeners at $56 \mathrm{~dB}$ SPL. The 3- $\mathrm{dB}$ increment in noise significantly decreased the word's intelligibility, and the 3-dB decrease increased the intelligibility $(p<0.01)$ without affecting other key words in the sentence. This technique has application in reducing variability in sentence intelligibility and greatly reducing perceptual dynamic range. Implications for "all-or-none" contextual recognition in adverse conditions are discussed.

1aSC20. Pitch perception physiology and psychophysics as a basis for the design of pitch detection algorithms. Robert A. Houde (RIT Res. Corp., 75 Highpower Rd., Rochester, NY 14623) and James Hillenbrand (Western Michigan Univ., Kalamazoo, MI 49008)

The generation of high-quality speech with a source-filter vocoder depends to a very great extent on accurate analysis of source parameters. After decades of research, even state-of-the-art pitch detection algorithms tend to make gross errors in the analysis of signals that present no diffculty for the human listener. In this study a review of a broad range of pitch detection algorithms was undertaken, with particular attention to the plausibility of those algorithms in relation to what is currently known about the psychophysics of pitch perception and the neural coding of speech signals. Our principal conclusion from this review is that the most plausible model is a time-domain pitch perception scheme proposed more than 4 decades ago by Licklider [J. C. R. Licklider, Experientia 7, 128-133 (1951)], and extended in more recent studies. The implications of these findings for source-filter vocoders will be discussed, and an implementation of the Licklider model using level-crossing interval histograms will be described.

1aSC21. Contribution of different frequency regions to detection of additive noise in vowels. Muralidhar R. Kudumala (Electr. Eng., Univ. of Oklahoma, Oklahoma City, OK 73170) and Blas Espinoza-Varas (Univ. Oklahoma Health Sciences Ctr., OkJahoma City, OK 73170)

An important issue in audio coding is the detection of quantization noise masked by speech. This investigation examined how different frequency regions contribute to the detection of broadband noise masked by a vowel. The vowel was synthesized by addition of the first 32 harmonics of a $200-\mathrm{Hz}$ fundamental, with amplitudes appropriate to $\mathrm{i} / \mathrm{V}$. Broadband noise was synthesized by random-phase addition of the harmonics of a $10-\mathrm{Hz}$ fundamental that fall within the vowel bandwidth. Noise detection thresholds were measured in a 2IFC task with an adaptive procedure (Levitt, 1971). Detection thresholds were obtained for noise with spectrum envelope parallel to that of the vowel, and for the same noise containing $-6.0-\mathrm{dB}$ spectral notches in either of the following bands: (a) $0.2-0.5$ $\mathrm{kHz}$; (b) $0.2-1.0 \mathrm{kHz}$; (c) $4.2-5.0 \mathrm{kHz}$; and (d) $4.7-5.0 \mathrm{kHz}$. Thresholds obtained in three highly trained listeners ranged from -27 to $-32 \mathrm{~dB}$ (expressed in terms of noise-to-vowel power ratio). Threshold differences between spectral notch conditions were small. The results were compared to predictions of the excitation pattern model proposed by Moore and Glasberg [Hear. Res. 28, 209-225 (1987)]. [Work supported by OCASTHR4-064.] 
1aSC22. Toward understanding the role of formant transitions for distinctions of stops from glides. Sally G. Revoile, Peggy B. Nelson, and Lisa Holden-Pitt (Gallaudet Univ., Ctr. for Auditory and Speech Sci., 800 Florida Ave., N.E., Washington, DC 20002)

Our understanding is incomplete of the properties of vowel formant transitions that contribute to distinctions of voiced stop and glide consonants in speech. Research appears to have established some of the important transition cues for discernment of bilabial synthetic stops versus glides. However, the stop/glide transitions studied have typically been more stylized than those found in natural speech. This investigation examined the importance of transitions to listeners' identification of initial stops and glides in spoken /CVk/ syllables. Performance was assessed for the stops and glides with progressive deletion of segments from the syllables' onsets. Bilabial and velar stops and glides as well as alveolar stops were tested in /Cuk/, /Cak/, /Cæk/ contexts to examine differences in transition use among phoneme environments. Twelve normal-hearing young adults participated as listeners. In general, when the initial stop bursts were deleted, the $F 2$ transition frequency extent was significantly correlated with subjects' consonant identification response patterns. That is, longer $F 2$ frequency extents yielded a higher percentage of glide responses. In addition, shorter $F 2$ frequency extents resulted in a higher proportion of "no initial consonant" responses. Neither $F 2$ transition duration nor $F 1$ transition duration/frequency extent significantly correlated with the subjects' consonant identifications.

1aSC23. The role of formant synchrony in the coherence of vowels. Peter C. Gordon and Erika Manning (Dept. of Psych., Univ. of North Carolina, Chapel Hill, NC 27599-3270)

The coherence of vowels as auditory objects was studied by comparing identification thresholds in noise for synthetic vowel sounds (differing only in the center frequency of a single formant) to identification thresholds for the distinctive formant presented in isolation. The bandwidth of the noise masker was limited so that it only interfered with perception of the distinctive formant. Thresholds for accurately identifying the vowel sounds were lower than those for identifying the isolated formant. This demonstrates that vowel sounds cohere in the sense that unmasked formants reduce the masking of a formant embedded in noise. The advantage of a complete vowel over an isolated formant appears to depend on the temporal alignment of the formants. When the onset of the distinctive formant coincides with the offset of the other formants, then listeners can still identify the vowel sound in modest amounts of noise. However, in this case thresholds are not lower for vowel identifications than for identifications of isolated formants. This indicates that temporal synchrony plays a basic role in the psychoacoustic coherence of vowels.

1aSC24. Dynamic and static properties of imaged speech sounds. Deborah A. Gagnon (Moss Rehab. Res. Inst., 1200 w. Tabor Rd., Philadelphia, PA 19141)

The type of information stored in memory for speech sounds was tested using a primed, speeded classification task. The relationship between prime and target was varied in terms of phoneme constituency, phoneme order, or both. Primes were presented either auditorally or visually, allowing for a contrast between perceptual and imaged speech codes. Two other manipulations were made to assess whether the temporal nature of the stimuli, the stimulus quality, or possibly both, play a role in determining imageability: (1) Stimuli either contained stop (dynamically cucd) or fri cative (relatively statically cued) consonants; and (2) stimuli were either natural or synthetic. Inhibitory effects were found when an auditory prime was presented at a I00-ms ISI, supporting earlier evidence for a positionally specific perceptual speech code (Gagnon and Sawusch, 1992). It was also found that both the manipulation of the type of consonant (stop versus fricative) present in the targel and the quality of the stimulus set (natural versus synthetic) had an effect on imageability, supporting both a temporal nature (Surprenant, 1992) and stimulus quality account of imageability. These results will be discussed within the context of current theories of memory, imagery, and speech perception. [Work supported by NIDCD Grant R01 DCO0219 to SUNY at Buffalo and Mark Diamond Research Fund grant to Deborah A. Gagnon.]
1aSC25. Quantitative measures of envelope cues in speech recognition. Johnny Saade (Dept. of Elec. Eng., Univ. of California, Los Angeles and House Ear Inst., Los Angeles, CA 90057), Fan-Gang Zeng, John J. Wygonski, Robert V. Shannon, Sigfrid D. Soli (House Ear Inst., Los Angeles, CA 90057), and Abeer Alwan (Univ. of California, Los Angeles, CA)

A quantitative procedure is derived to evaluate the relative contribution of envelope cues to speech recognition. Recognition data of 16 consonants in the $/ \mathrm{aCa} /$ form were collected using signal-correlated noise stimuli in seven normal-hearing listeners. Several distance measures were calculated directly from duration and amplitude of the acoustic envelope. One amplitude distance measure was the Euclidean distance which was computed from the squared difference of the sample-by-sample amplitudes. The second measure was the envelope difference index (EDI) [Fortune et al., Ear Hear. 15. 93-95 (1994)] which was computed from the absolute value of the difference of the sample-by-sample amplitudes. A multidimensional scaling analysis was used to convert the perceptual confusion matrix into a distance matrix and to normalize the different distance measures. Correlation coefficients were computed between the different distance measures and the perceptual data. Preliminary analysis of data from six stop consonants showed that the consonant duration alone is sufficient to explain the perceptual data $(r=0.92)$. Although Euclidean distance conveyed less information $(r=0.75)$ than duration, it was a better measure than the EDI $(r=0.31)$. Evaluation of these measures on the full 16 consonant set will be discussed.

1aSc26. Onset-sensitive time-frequency masking and its application to speech recognition. Kiyoaki Aikawa (ATR Human Information Process. Res. Labs., 2-2 Hikaridai, Seika-cho, Soraku-gun, Kyoto, 619-02 Japan)

This paper proposes an onset-sensitive time-frequency masking mechanism in order to improve dynamic feature extraction. Application of the proposed mechanism to Japanese 23-phoneme recognition using hidden Markov models demonstrated that onset-sensitive MASP outperforms time-invariant MASP. Masked Spectrum (MASP) [Aikawa et al., Proc. ICASSP93 II, 668-671 (1993)] is a new spectral representation incorporating time-frequency forward masking and has been reported to provide excellent performance when used for speaker-dependent and speakerindependent speech recognition. The masking pattern production mechanism was previously modeled by a time-invariant time-frequency filter, but the masking level rises at the onsets and offsets in a speech sound [T: Hirahara, J. Acoust. Soc. Jpn. E12 (2), 57-68 (1991); E. Miyasaka, J. Acoust. Soc. Jpn. 39 (9), 614-623 (1983)]. This phenomenon suggests that an adaptive masking mechanism is effective for balancing instantaneous and transitional spectral features depending on vowels or consonants. The masking pattern is calculated as the weighted sum of the smoothed preceding spectra obtained by time-distance-dependent spectral smoothing lifters. The masking level is controlled by the slope of the temporal contour of the instantaneous sound energy. The masked spectrum is obtained by subtracting the masking pattern from the current spectrum. Onsel-offset-sensitive masking models are also examined.

1aSC27. Difference limens for vowel-vowel formant transitions. William A. Ainsworth (Dept. of Commun. and Neurosci., Keele Univ., Keele, Staffordshire ST5 5BG, United Kingdom)

Second-formant transitions in vowel-vowel utterances are not always of the same duracion as those of the first formant and they often begin and end at different instants. In other cases the formant frequencies sometimes first move in a different direction from their final targets. In order to investigate whether these formant movements are perceptually significant, a number of difference limens for formant transitions have been measured for synthesized versions of the vowel pair $/ \mathrm{a} / \mathrm{f} / \mathrm{i} /$. It was found that differences in duration between the first- and second-formant transitions of up to $70 \mathrm{~ms}$ were not perceived. It was also found that delays between the starts and ends of the first and second transitions of up to $50 \mathrm{~ms}$ were not perceived. These results suggest that the differences in durations and delays between the first and second formants found in natural vowel-vowel utterances are unlikely to be of perceptual significance. [Work supported by EC Science Contract SC1-CT92-0786.] 
1aSC28. Female alaryngeal speech enhancement for improved speaker identification using linear predictive synthesis. Renetta $G$. Tull, Janet C. Rutledge, and Jerry J. Mahler (Elec. Eng./Comput. Sci. Dept., Northwestern Univ., Evanston, IL 60208)

Linear predictive synthesis was applied to female alaryngeal speech as an enhancement technique to reduce gender ambiguity during speaker identification. Esophageal speech, a form of alaryngeal speech resulting from the removal of the larynx due to cancer, perturbs the theoretical acoustic models for laryngeal speech. This form of pathological speech also changes the expected spectral characteristics and affects perception. The female esophageal speaker's low fundamental frequency often causes her to be identified as a male speaker over telephone lines. The errors made in speaker identification can be reduced by obtaining vocal tract parameters of the aperiodic, esophageal speech input from a linear predictive coding (LPC) model and resynthesizing the LPC prediction coefficients using an appropriate pitch period that is better suited for female voice. This enhancement technique was implemented using the TMS $320 \mathrm{C} 30$ floating point digital signal processor from Texas Instruments. This implementation supports the potential of a portable processor that enhances the perceptual quality of the female alaryngeal voice and improves the input speech signal used by both human listeners and automatic speech and speaker identification systems.

1aSC29. Vocal tract simulations based on fluid dynamic analysis. G. Richard, M. Liu, D. Sinder, H. Duncan, Q. Lin, J. Flanagan (Rutgers Univ.-CAIP Ctr., Piscataway, NJ 08855-1390), S. Levinson (AT\&T Bell Labs., Murray Hill, NJ 07974), D. Davis, and S. Simon (General Dynamics, Groton, CT 06340)

An alternate approach to speech synthesis based on direct numerical solution of Navier-Stokes (NS) and Reynolds-averaged-Navier-Stokes (RANS) equations is described. Unlike the traditional methods based on linear acoustic theory, the NS and RANS formulations are not limited by the assumptions of linearity, negligible viscous effects, and plane-wave propagation. The expected results are high-quality synthesis and a new parametrization of speech for applications in automatic recognition and low bit-rate coding resulting from a parsimonious modeling of articulatory shapes and dynamics. In the present formulation, the Navier-Stokes equations are discretized and solved using a finite-difference method. An initial application involves a 2-D simulation of flow through ideal channels (straight, dilating, and constricting tubes) with rigid walls and constant boundary conditions (constant flow velocity at inlet, zero pressure at outlet). As expected for these simple geometries, the resonance frequencies correspond to those predicted by linear acoustics. In a second application, the formulation is applied to the geometry of the three cardinal vowels. For these cases, periodic inflow boundary conditions are also used (a train of short pulses to represent vocal cord excitation). Synthetic speech sounds of encouraging quality are obtained for the three vowels. [Research supported by NSF/ARPA IRI-9314946 and ARPA DAST 63-93-C-0064.]

1aSC30. Removal of phase distortion from vowel-like signals. Kenton A. Shipley, ${ }^{\text {a) }}$ Casey R. Winkel, and P. G. Vaidya (Dept. of Mech. and Mater. Eng., Washington State Univ., Pullman, WA 99164)

In this paper, specific types of signals are considered. To generate these signals, we begin with $x(t)=A \cos [w t+\phi(t)]$, where $A$ and $w$ are constants, but $\phi(t)$ is a function of time generated by a random walk. The other step to generate the final signal is to pass $x(t)$ through a nonlinear filter such that, were $\phi(t)$ to be constant the output would be of simple harmonics at $w, 2 w, 3 w$, etc. However, since $x(t)$ is nonstationary, the output signal, say $y(t)$, would also be nonstationary. It would be shown that human vowel signals seem to follow this nonlinear pattern. It is of interest to remove (or modify) the phase distortion due to the randomness of $\phi(t)$. This has been accomplished by using two different methods. One uses the trans-spectral coherence technique [P. G. Vaidya and M. J. Anderson, J. Acoust. Soc. Am. 89, 2370-2378 (1991)] and the other uses the complex FFT components in a manner similar to a rigid body rotation in dynamics. These methods have been used to produce both distortion-free and highly

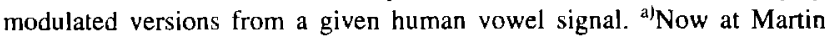
Marietta, Knolls Atomic Power Labs, Schenectady, NY.
1aSC31. Perception of voice-onset-time cued by articulatory information. David R. Williams (Sensimetrics Corp., 26 Landsdowne St., Cambridge, MA 02139)

This paper presents results of a first perceptual assessment of VOT continua synthesized using articulatory rather than acoustic parameters. The research employed Sensimetrics' HLSYN synthesis system, which embodies an articulo-acoustic model of speech production [K. N. Stevens and C. A. Bickley, J. Phon. 19, 161-174 (1991)]. The system permits time-varying control of fundamental frequency, vocal-tract shape, and of glottal, oral, and nasal orifice sizes; calculated aerodynamic quantities provide the basis for estimating KLSYN88 synthesizer source parameters. Six 10-member VOT continua (/ba/-/pa/, /da/-/tal, /ga/-/ka/, /bi/-/pi/, /di/-/ $\mathrm{ti} /, / \mathrm{gi} /-/ \mathrm{ki} /$ ) were constructed by varying the glottal adduction trajectory so that voicing onset occurred $0,15,30, \ldots$, or $135 \mathrm{~ms}$ after oral release. In addition, two peak glottal openings $\left(18,30 \mathrm{~mm}^{2}\right)$ were tested; for $/ \mathrm{a} /$, rate of oral release was also varied (slow, fast). Subjects rated the "goodness" of the CVs as exemplars of the appropriate voiceless stop on a ten-point scale. In general, subjects preferred longer VOTs for velars than for labials. For alveolars, longer VOTs were preferred before /i/t than before /a/. Effects of peak glottal opening and oral release rate on VOT preferences were negligible. The results are discussed relative to traditional findings for acoustically defined VOT continua. [Work supported by NIMH.]

1aSC32. A computationally efficient articulatory synthesizer. Jacob Rael, James Chang, and Abeer Alwan (Dept. of Elec. Eng., 66-147E Engr. IV, UCLA, 405 Hilgard Ave., Los Angeles, CA 90095)

In this study, a computationally efficient articulatory synthesizer that utilizes the popular analog circuit simulator SPICE is developed. The synthesizer uses a transmission-line analog model of the vocal tract. An analog model has many advantages over digital representations: (1) Side branches (needed for modeling nasals and $/ /$ ) can be simulated easily by additional transmission lines in parallel; (2) drive-dependent sources, at any location, could be added; and (3) the number of sections can be varied without changing the sampling rate, as is the case with a digital synthesizer. A computer interface, using MATLAB, is developed such that the input to the synthesizer can be specified in terms of the area function of the vocal tract and the type and location of dependent or independent sources (voltage or current.) By simulating the transfer function of the vocal tract, transient and steady-state responses are generated. Using Fant's vowel area functions (1960), vowels were synthesized with their first four formant frequencies almost identical to those given by Fant. The feasibility of implementing the analog synthesizer using modern ICs, such as the gyratorbased inductance simulator and switched capacitor filter circuits, is assessed.

1aSc33. Identification of vowels resynthesized from $/ \mathrm{hVd}$ utterances: Effects of formant contour. James Hillenbrand (Speech Pathol. and Audiol., Western Michigan Univ., Kalamazoo, MI 49008)

The purpose of this study was to examine the role of formant frequency movements in vowel recognition. Measurements of fundamental frequency and formant contours were taken from a database of acoustic measurements of $1668 \mathrm{hVd}$ utterances spoken by 45 men, 48 women, and 46 children [Hillenbrand et al., J. Acoust. Soc. Am. 97 (in press)]. A total of 300 utterances were selected from this database, representing equal numbers of 12 vowels $(\mathrm{i}, \mathrm{I}, \mathrm{e}, \varepsilon, \mathfrak{x}, \mathrm{a}, \mathrm{\jmath}, \mathrm{O}, \mathrm{U}, \mathrm{u}, \Lambda, \mathfrak{x})$ and approximately equal numbers of tokens produced by men, women, and children. Listeners were asked to identify three versions of each utterance: (1) the original, naturally produced signal, (2) a formant-synthesized version that followed the measured contours of $F 1-F 3$, and (3) a formant-synthesized version with $F 1-F 3$ fixed at their steady-state values. Preliminary results indicate: (1) the original signals are identified with substantially greater accuracy than either of the synthetic versions, and (2) the synthetic signals that follow the original formant contours are identified with greater accuracy than the flat-formant signals. 
1aSC34. Speech synthesis based on an overlap-add sinusoidal model. Michael W. Macon and Mark A. Clements (Dept. of Elec. and Comput. Eng., Georgia Inst. of Tech., Atlanta, GA 30332)

A general framework for waveform synthesis in a concatenation-based text-to-speech system is presented. Natural speech is segmented into subword units and analyzed using an iterative analysis-by-synthesis procedure originally presented in [E. B. George and M. J. T. Smith, J. Audio Eng. Soc. 40, 497-516 (1992)]. Synthesis is then performed by an efficient overlap-add resynthesis and modification method. This method eliminates the need for precise, hand-corrected pitch pulse marking in analysis (as required in some other popular concatenation methods), by incorporating a pitch pulse onset time estimation function based on [R. J. McAulay and T. F. Quatieri, Proc. ICASSP, 1713-1715 (1986)]. The sinusoidal model is capable of natural-sounding prosodic modification of both continuous speech and concatenated segments, making it an ideal candidate for application in a TTS system. Furthermore, the model provides for a computationally tractible and conceptually simple decoupling of various properties of the speech signal, making it an excellent platform for other transformations of the synthesized speech.

1aSC35. Generating gestural scores from articulatory data using temporal decomposition. Michael J. Collins, Stanley C. Ahalt, and Ashok K. Krishnamurthy (Dept. of Elec. Eng., The Ohio State Univ., Columbus, OH 43210)

Through empirical investigations, the automatic generation of gestural scores for articulatory data corresponding to consonant-vowel-consonant (CVC) tokens was studied. The articulatory data consist of the movements of flesh points measured using an $\mathrm{x}$-ray microbeam, and is first "warped" to resemble the vocal tract variables of constriction location and constriction degree. The multichannel warped data are then analyzed using temporal decomposition. The resulting target functions provide candidates for gestures, from which the best candidates are chosen statistically by examining the magnitudes of the elements of their associated reconstruction weights. Onset and durational analysis of the candidate target functions result in the gestural score. Human and Elman Recurrent Neural Network recognition tests are performed to ascertain the accuracy of the generated gestural scores. Comparisons with "correct" gestural scores are also performed. The results of this work should provide a stepping stone for future acoustic and articulatory based recognizers employing the same strategy.

1aSC36. Layered language understander: A speech recognition system using layered abduction. Kevin A. Lenzo (ATR Interpreting Telecommun. Res. Labs., Dept. 3, 2-2 Hikaridai, Seika-cho, Soraku-gun, Kyoto, 619-02 Japan), John Josephson, Osamu Fujimura, and Christopher Bailey-Kellogg (Ohio State Univ., Columbus, OH 43210)

A speech recognition system is under development in which a generalpurpose inference engine for natural language processing is loaded with domain knowledge and a preprocessing periphery that allows for the integration of prosodic information. Processing takes place in well-defined layers, in which results at one level of abstraction become data to be explained by hypotheses in the next, using a layered-abduction inference mechanism that allows for integration of both top-down and bottom-up information [J. R. Josephson and S. G. Josephson, eds., Abductive Inference: Computation, Philosophy, Technology (Cambridge U. P., 1994)]. The hypothesis types and their interactions are based upon the converter/ distributor model of phonetic implementation [ O. Fujimura, "Syllable Timing Computation in the C/D Model," Proceedings of the Third International Conference on Spoken Language Processing (1994)]. Each hypothesis is annotated with a numerical magnitude that is used in computing a "prosodic contour" for the overall utterance, which aids in the generation of expectations, implications, and knowledge of which hypotheses can account for what data. Preprocessing events are explained by the features, which, in turn, are explained by hypotheses at the demisyllabic layer, the syllabic layer, and, finally, by the word-level hypotheses.
1aSC37. Toward automatic segmentation and syllabification of dysarthric speech. Guangsheng Zhang and $\mathrm{H}$. Timothy Bunnell (Univ. of Delaware. Dept. of Linguist., and Speech Process. Lab, A. 1. duPont Inst., 1600 Rockland Rd., Wilmington, DE 19899)

A program has been developed which uses heuristic processing of the output of a neural network to locate syllable boundaries in connected speech. The neural network attempts a broad segmental (8 category) classification. This is followed by a program which locates syllable boundaries using phonological constraints and the pattern of network classifications over time. On test data consisting of 150 sentences (about 1800 syllables) from the TIMIT database, this program correctly identifies between $83 \%$ and $88 \%$ of the syllable boundaries (depending on the error criteria), including syllables bounded by sonorant consonants and syllables containing syllabic consonants. This program is now being applied to the identification of syllable boundaries in speech from talkers with cerebral palsy. In preliminary analyses of 20 sentences from ten dysarthric talkers (140 syllables) only 12 boundary omissions occurred. However, since dysarthric speech often contains hesitations, false starts, and extraneous noises, assessing boundary insertion errors is more difficult; about 50 boundary insertions occurred. Roughly $2 / 3$ of these represented instances in which the talker inserted additional syllabic features into the speech. Further studies will examine methods for reducing the number of these "unintended" boundaries identified by the algorithm. [Work supported by the Nemours Foundation.]

1aSC38. Adding articulatory features to acoustic features for automatic speech recognition. Igor Zlokarnik (Los Alamos Natl. Lab., CIC-3, MS B256, Los Alamos, NM 87545)

A hidden-Markov-model (HMM) based speech recognition system was evaluated that makes use of simultaneously recorded acoustic and articulatory data. The articulatory measurements were gathered by means of electromagnetic articulography and describe the movement of small coils fixed to the speakers' tongue and jaw during the production of German $\mathrm{V}_{1} \mathrm{CV}_{2}$ sequences [P. Hoole and $\mathrm{S}$. Gfoerer, J. Acoust. Soc. Am. Suppl. I 87, S123 (1990)]. Using the coordinates of the coil positions as an articulatory representation, acoustic and articulatory features were combined to make up an acoustic-articulatory feature vector. The discriminant power of this combined representation was evaluated for two subjects on a speaker-dependent isolated word recognition task. When the articulatory measurements were used both for training and testing the HMMs, the articulatory representation was capable of reducing the error rate of comparable acoustic-based HMMs by a relative percentage of more than $60 \%$. In a separate experiment, the articulatory movements during the testing phase were estimated using a multilayer perceptron that performed an acoustic-to-articulatory mapping. Under these more realistic conditions, when articulatory measurements are only available during the training, the error rate could be reduced by a relative percentage of $18 \%$ to $25 \%$.

1aSC39. Vocal tract normalization in speech recognition: Compensating for systematic speaker variability. Jordan Cohen (CCR-IDA, Princeton, NJ), Terri Kamm, and Andreas G. Andreou (Johns Hopkins Univ., Baltimore, MD 21218)

The performance of speech recognition systems is often improved by accounting explicitly for sources of variability in the data In the SWTTCHBOARD corpus, studied during the 1994 CAIP workshop [Frontiers in Speech Processing Workshop II, CAIP (August 1994)], an attempt was made to compensate for the systematic variability due to different vocal tract lengths of various speakers. The method found a maximum probability parameter for each speaker which mapped an acoustic model to the mean of the models taken from a homogeneous speaker population. The underlying acoustic model was that of a straight tube, and the parameter estimation was accomplished by warping the spectrum of each speaker linearly over a $20 \%$ range (actually accomplished by digitally resampling the data), and finding the maximum a posteriori probability of the data given the warp. The technique produces statistically significant improvements in accuracy on a speech transcription task using each of four dif- 
ferent speech recognition systems. The best parametrizations were later found to correlate well with vocal tract estimates computed manually from spectrograms.

1aSC40. Use of TRACTTALK for adaptive voice mimic. Qiguang Lin, Gael Richard, Jingyun Zou, Dan Sinder, and James Flanagan (CAIP Ctr., Rutgers Univ., Piscataway, NJ 08855-1390)

Various speech-processing technologies necessitate parametrization of the speech waveform. Cepstrum coefficients (including their derivatives and variants) are to date commonly used in speech and speaker recognition. This paper seeks more compact parametric description of speech information based on the adaptive voice mimic [Flanagan et al., J. Acoust. Soc. Am. 68, 780-791 (1980)]. The mimic system utilizes an articulatorybased speech synthesizer to generate synthetic speech, which is adapted to arbitrary speech input. The perceptually weighted spectral difference between the input and synthesized speech is next minimized by optimizing the underlying articulatory parameters until the difference is driven below a predetermined level. The resultant representation, adapted moment by moment, provides efficient parametrization of the signal information by which the problems of speech synthesis, speech recognition, and low bitrate speech coding are coalesced into a compact framework. In this paper, an articulatory speech synthesizer, TRACTTALK, is first described. TRACTTALK simulates the vocal tract based on principles of linear acoustics and incorporates features which include interaction between the voice source and the vocal tract. Preliminary results of adaptive mimicking using TRACTTALK are presented and discussed. [Work supported by ARPA Contract \#DAST 63-93-C-0064.]

1aSC41. Effects of syllable duration on syllable-final stop-glide perception by humans and monkeys. Joan $M$. Sinnott (Comparative Hearing Lab, Psych. Dept., Univ. So. Alabama, Mobile, AL 36688), Melissa A. Borneman, and Paul A. Dagenais (Univ. So. Alabama, Mobile, AL 36688)

Humans and monkeys were compared in their perception of phoneme boundary shifts along two synthetic syllable-final stop-glide /bab/-baU/ continua differing in overall syllable duration (150 vs $320 \mathrm{~ms}$ ). Humans were first tested with a written identification procedure and showed a boundary shift to longer transition durations with increased syllable duration, as previously reported in the literature for syllable-initial stop-glide /ba-wa/ data. Humans and monkeys were then tested with a lowuncertainty discrimination procedure but showed little evidence of a sensory-level discontinuity underlying the identified boundaries: Instead sensitivity appeared to follow Weber's law. Finally, both human and monkeys were tested with a go/no-go identification procedure specifically designed for monkeys. Both species clearly showed the shift effect, but quantitative differences emerged between the species that were generally consistent with comparative psychoacoustic measures of temporal discrimination. [Work supported by NIDCD.]

\title{
Session 1pAO
}

\section{Acoustical Oceanography: Ocean Acoustic Tomography: Observing the Ocean in the 1990s II}

\author{
Bruce M. Howe, Cochair \\ Applied Physics Laboratory, University of Washington, 1013 N.E. 40th Street, Seattle, Washington 98105 \\ B. Edward McDonald, Cochair \\ Naval Research Laboratory, Washington, DC 20375
}

Chair's Introduction-1:00

Invited Paper

1:15

1pAO1. What acoustic tomography can tell us about the state of the ocean: Theoretical considerations. Robert N. Miller (College of Oceanic and Atmos. Sci., Oregon State Univ., Corvallis, OR 97330)

Acoustic tomographic data consist of line integrals of sound speed along ray paths. The community has little experience with line integral data, and most of the methods for mapping oceanic properties and for data assimilation (i.e., combining observed data with results from dynamical models) have been designed and tested with point measurements in mind. In this work, the tools of estimation theory were used to investigate the potential for reconstructing time series of oceanic pressure and density fields by assimilation of line integral data into a simple ocean model. Under ideal conditions, with noise-free data and ocean dynamics given exactly by a simple combination of linear waves, time series of integral quantities along the edges and diagonals of a square array are sufficient to determine time series of amplitudes of a large collection of waves, most of whose wavelengths are much shorter than the length of the edge of the array. Simulation experiments were performed to investigate the consequences of imperfect, possibly nonlinear dynamical ocean models and noisy data. 
1pAO2. Testing a regional ocean model using acoustic travel time observations. Bruce D. Comuelle, Peter F. Worcester, David B. Chester (Scripps Inst. of Oceanogr., Univ. of Califomia at San Diego, La Jolla, CA 92093), Brian D. Dushaw, and Bruce M. Howe (Univ. of Washington, Seattle, WA 98105-6698)

The Acoustic Mid-Ocean Dynamics Experiment (AMODE) measured time series of reciprocal acoustic travel time and temperature with a 700 km-diam array of six moorings south of Bermuda during 1991-1992. Time-independent inversions give a series of snapshots of the barotropic and baroclinic eddy field. Observations taken at different times can be combined, using a dynamical ocean model, to generate improved estimates of the evolving eddy field, provided that the model is accurate. The AMODE observations have been used to test a nonlinear, quasigeostrophic, limited-area ocean model by attempting to fit a long-term dataset, and by initializing the model with a 'training' dataset and then predicting forward in time for comparison to data not used in the initialization. Using an mns error measure of misfit between the observations and the predicted data, the model prediction has $40 \%$ less error than persistence over 12 days, and about $20 \%$ less error than persistence over 24 days. The extent to which the data misfit is sensitive to external model parameters, such as the radius of deformation or viscosity, allows the parameters to be optimized to give maximum predictability, and also gives a measure of how well the model has been tested by the dataset. [Work supported by ONR.]

\section{1:55}

1pAO3. Assimilating tomographic travel time data using an open ocean circulation model. Chris G. Walter, Bruce M. Howe, James A. Mercer (Appl. Phys. Lab., Univ. of Washington, Seattle, WA 98105), and Robert N. Miller (Oregon State Univ.)

Observing and predicting the ocean's meso-scale circulation presents a formidable task for oceanographers. Ocean acoustic tomography uses travel times between acoustic sources and receivers to measure the ocean's low wave-number sound speed (temperature) and current fields. Experiments are being performed with simulated ocean data to determine the relative effectiveness of tomographic and point measurements in constraining a numerical circulation model. The primary tool is data assimilation, i.e., an optimized combination of model output and observations. Relative weights of observation and model output are based on a priori estimates of the model and observation error covariance. Results from identical twin simulations using tomographic and point measurements are presented. In the twin experiment, two assimilation schemes are examined, one in which the estimated model error covariance is fixed (optimal interpolation) and another in which a Monte Carlo [G. Evensen, J. Geophys. Res. 99, $10143-10162$ (1994)] method is used to estimate the model error covariance dynamically. Error statistics of the energy and fux fields are used to quantify the performance for each observation and updating scheme. The number of measurements required to achieve a target error level is estimated for each method. [Work supported by ONR and by the Strategic Environmental Research and Development Program through ARPA.]
1pA04. A 4-D view of convection by assimilation of acoustic and classical measurements. F. Gaillard, Y. Desaubies (Lab. de Phys. des Océans, IFREMER, BP 70, 29280 Plouzané, France), U. Send, and F. Schott (IFM, $2300 \mathrm{Kiel}$, Germany)

Acoustic tomography has been applied to observation of winter convection in the Gulf of Lion during the THETIS-1 experiment conducted during the 1991--92 winter by a European group (IFM Kiel, IFREMER Brest, and IACM Heraklion). A global analysis of all types of temperature measurements collected during THETIS 1 will be presented. Data issued from temperature sensors, thermistor chains, and CTD have been merged with the tomographic measurements in order to recover the time evolution of the full 3-D temperature field. The method relies on inversion and Kalman filtering methods. It can be considered as a new step toward "observation systems." Monitoring of the whole area during the "preconditioning" phase shows the influence of surface cooling on the vertical stratification. This effect is inhomogeneous and the situation evolves with time, influenced by horizontal advection. The relative importance of surface forcing and advection is responsible for the intermittent nature of convection which happens with different intensity from year to year. After convection, the behavior of the mixed water chimney and the evolution of the vertical profile toward the initial state is documented.

\section{2:25}

1pAO5. Evolution of the large-scale temperature field in the Greenland Sea during 1988-89 from tomographic measurements. Werner M. L. Morawitz, Peter F. Worcester, Bruce D. Cornuelle (Scripps Inst. of Oceanogr., Univ. of California at San Diego, La Jolla, CA 92093), Philip J. Sutton (New Zealand Oceanogr. Inst., Kilbirnie, Wellington, New Zealand), James F. Lynch (Woods Hole Oceanogr. Inst., Woods Hole, MA 02543), and Richard Pawlowicz (Inst. of Ocean Sci., Sidney, BC, Canada)

Moored thermistor, hydrographic, and tomographic measurements have been combined using least-squares inverse methods to study the evolution of the $40 \mathrm{~km}$ and larger three-dimensional temperature field in the Greenland Sea during winter 1988-89. In February, the sub-surface temperature maximum at around $200-\mathrm{m}$ depth disappears over a large area. Upper waters warm around this time, while intermediate waters cool, consistent with vertical mixing. A chimney structure reaching depths in excess of $1000 \mathrm{~m}$ is observed to the southwest of the gyre center during March. The chimney has a spatial scale of about $50 \mathrm{~km}$, a time scale of about 10 days, and breaks up in about 3-6 days. A one-dimensional vertical heat balance adequately describes changes in total heat content in the chimney region from autumn 1988 until the time of chimney break-up. A simple one-dimensional mixed layer model is successful in reproducing fall to winter bulk temperature and salinity changes, as well as the observed evolution of the mixed layer. The estimated annual deep water production rate in the Greenland Sea for 1988-89 is about 0.1 Sverdrups, comparable to the decreased production rates since 1983 derived from tracer measurements. [Work supported by NSF and ONR.]

\section{2:40-2:55 Break}

\section{Invited Paper}

\section{2:55}

1pAO6. Basin-scale tomography in the western Mediterranean. Uwe Send (Inst. für Meereskunde an der Univ. Kiel, Düsternbrooker Weg 20, 24105 Kiel, Germany)

An international acoustic tomography experiment had been deployed in the western Mediterranean from January to October 1994. Seven transceivers spanned the region between France, Algeria, the Balearic Islands, and Sardinia/Corsica. The basin (maximum range $600 \mathrm{~km}$ ) was "illuminated" by a single HLF-5 source, the remaining sources had a shorter range but could communicate with their neighbors. Extensive hydrographic measurements and analysis of historical data accompanied the observations, including a large-scale 
survey during deployment and recovery and a 2-weekly XBT section along the main transmission path. Some paths went along/through complicated topography, and in addition a shipboard acoustic survey was carried out to study the propagation into shallow water. All instruments remained operational for the entire period, and 6 of the 7 had 100\% return of received data. The receptions show a high $\mathrm{S} / \mathrm{N}$ ratio with well-resolved arrivals that agree well with acoustic propagation predictions. The seasonal variation of the travel times appears to be a good measure of the cycle of the large-scale heat content. The 3-D basin-averaged temperature is estimated to have an accuracy of $3 \%-4 \%$ of the seasonal amplitude. A system like this, installed with shore-cabled instruments, should be a valuable tool for monitoring interannual and climate-scale water mass variability.

\section{Contributed Papers}

$3: 20$

1pA07. Characteristics of signals recorded near shore in the Mediterranean during the THETIS 2 experiment. Y. Desaubies (Lab. de Phys. des Océans, IFREMER, BP 70, 29280 Plouzané, France), T. Terre (LPO/FREMER), B. Faure (CEPHAG, Grenoble), and F. Gaillard (LPO/IFREMER)

Most of the western Mediterranean coast is characterized by a narrow shelf and steep rise. Thus it should be fairly easy to install sources and/or receivers cabled to shore for permanent acoustic monitoring of the basin. The study of the feasibility of this option was one of the goals of the THETIS 2 project. Results of a ship-borne acoustic survey over the continental rise will be presented. Signals were recorded at 26 stations, opening up in range, with an 8-channel vertical array. Several of the receptions implemented a synthetic aperture array concept. Where available, the results will be compared to those obtained at French Navy, fixed hydrophones locations, in particular, a long time series at a single shallow water hydrophone. [Work supported by EC/MAST and IFREMER.]

\section{$3: 35$}

1pAO8. Large-scale circulation and variability in the northwest Atlantic as determined by long-range reciprocal acoustic transmissions. David B. Chester, Peter F. Worcester, Bruce D. Cornuelle (Scripps Inst. of Oceanogr., Univ. of California at San Diego, La Jolla, CA 92093), and Brian D. Dushaw (Univ. of Washington, Seattle, WA 98105)

Reciprocal acoustic transmissions are analyzed to determine the general circulation of the region between Bermuda and Puerto Rico. Six tomographic transceivers were moored from March 1991 to March 1992 in the region centered at $25^{\circ} \mathrm{N}, 66^{\circ} \mathrm{W}$ during the Acoustic Mid-Ocean Dynamics Experiment (AMODE). Large-scale range averages (over 300 to $700 \mathrm{~km}$ sections) of temperature and velocity and large-scale areal averages of relative vorticity have been estimated through inversion of tomographic travel time data. The barotropic and first baroclinic mode are well resolved by the acoustic sampling, with poorer resolution of the second baroclinic mode. The 300-day mean circulation of the region indicates a band of westward flow of a few $\mathrm{cm} / \mathrm{s}$ in the surface and thermocline waters. An active and resolvable mesoscale eddy field is present, with variability typically dominated by motions with spatial scales of $100-200$ $\mathrm{km}$, and with a general westward drift of mesoscale eddy features. The tomographic observations have been combined with a quasi-geostrophic numerical ocean model to further investigate eddy kinematics in the region. The model is used both to evaluate the evolution of the barotropic and baroclinic flow field, and to illustrate the utility of tomographic data for constraining a quasi-geostrophic model. [Work supported by ONR.]

\section{$3: 50$}

1pA09. Acoustic travel time biases caused by mesoscale eddy. E. C. Shang. Y. Y. Wang. and A. G. Voronovich (CIRES. Univ. of Colorado/ NOAA/Environ. Technol. Lab., Boulder, CO 80303)

Smaller scale fluctuations can be smooth-out by taking a long range propagation under linearized assumption. But the nonlinearity of the equations relating ocean perturbations to acoustic travel times leads to a bias if the problem is linearized about a range-independent reference state. The travel time $t_{\mathrm{RD}}$ in the range-dependent channel differs from the travel time $t_{\mathrm{RA}}$ through the range-averaged channel. In ray theoretical regime, a warm bias $\left(t_{\mathrm{RD}}<t_{\mathrm{RA}}\right)$ has been found for mesoscale eddy in general. In this paper, numerical simulation of modal travel time shows that there is a "cold" bias $\left(t_{\mathrm{RD}}>t_{\mathrm{RA}}\right)$ caused by mesoscale eddy in mode theory regime. [Work supported by ONR, ARPA, and NOAA.]

\section{4:05}

1pAO10. Ocean structure inversion by horizontal refraction tomography. A. G. Voronovich and E. C. Shang (CIRES, Univ. of Colorado/NOAA/Environ. Technol. Lab., 325 Broadway, Boulder, CO 80303)

Numerical simulations for the horizontal refraction tomography (HRT) proposed previously [A. G. Voronovich and E. C. Shang, J. Acoust. Soc. Am. 95, 2851(A) (1994)] were conducted. HRT is based on measurements of horizontal refraction angles (HRA) related to different acoustic modes. HRA are measured with the help of two distant ocean interferometers (each one consists of a pair of long mode resolving vertical arrays separated about $10 \mathrm{~km}$ apart). The signal is produced by tonal towed acoustic source. Both model eddy and real oceanic eddy of $100 \mathrm{~km} \times 100 \mathrm{~km}$ scale measured in Canary Basin were used for simulations. The 3-D tomography inversion proceeded in two stages: (1) retrieving the values of propagation constants in the knots of a horizontal rectangular greed (linear 2-D problem) and (2) reconstruction of the sound-speed profile (SSP) in each knot (1-D nonlinear problem). Up to 81 values of propagation constants for each mode were retrieved in both cases. Then SSP were calculated in each knot with the help of a special invariant-imbedding based procedure. With error-free simulated HRA data, SSPs were exactly reconstructed. The effect of errors on tomography inversion and requirements to the accuracy of phase measurements were also estimated. [Work supported by ONR.]

\section{4:20}

1pAO11. Ocean tomography via matched-field processing. A. Tolstoy (Integrated Performance Decisions, 4224 Waialae Ave., Ste. 5-260, Honolulu, HI 96816) and B. Sotirin (NCCOSC-NRaD, San Diego, CA 92152)

The tomographic determination of 4-D ocean sound speeds depends upon the nature of the system design, the analysis technique, and upon the resources used for generating and measuring the acoustic fields sampling the ocean region of interest. Matched-field tomography has recently been proposed as a high-resolution method potentially capable of synoptically imaging ocean regions as large as 1000 by $1000 \mathrm{~km}$ with rms accuracies less than $1 \mathrm{~m} / \mathrm{s}$ [A. Tolstoy, J. Comput. Acoust. 2(1), 1-10 (1994)]. However, all results to date for the determination of deep ocean sound-speed pronles have been simulated. A major drawback to the aciual implementation of the technique has been the requirement for several long $(1000 \mathrm{~m})$ vertical arrays, and these arrays have been neither affordable nor readily available. New technology is on the verge of producing relatively inexpensive, air-deployable, lightweight versions of these arrays suggesting that the MFP method may soon be testable. [Work supported by ONR.] 
1pAO12. Investigation of the layered bottom structure influence on propagation of high-frequency acoustic signals. Margarita S. Fokina and Boris V. Kerzhakov (Inst. of Appl. Phys., Russian Acad. of Sci., 46, Ulyanov St., 603600 Nizhny Novgorod, Russia)

Peculiarities of frequency-angle dependences of Fresnel's reflection coefficients $V(f d, \theta)(f=$ frequency, $d=$ layer thickness, $\theta$ =grazing angle) from a system of elastic layers on the elastic substrate are investigated. The method of calculating the phase and the module of the reflection coefficient from the layered elastic absorbing substrate is numerically realized. The resonant behavior of the reflection coefficient at different grazing angles for a given bottom model is investigated. The influence of the set of parameters of bottom layers and the substrate on the frequency-angle dependence of the reflection coefficient and the behavior resonance of the reflection coefficient is considered. The effects of the layer structure of the elastic absorbing bottom on the space-frequency dependences of acoustic fields in oceanic waveguides in the frequency range $20-1000 \mathrm{~Hz}$ is studied. The relationship between the type of sediments that forms bottom layers and frequency ranges, where the least losses due to propagation are observed, i.e., optimum propagation frequencies, is ascertained. [Work supported by RFFI project N 94-05-1725-a.]

\section{4:50}

1pA013. Numerical investigation of ray path stability in the Mediterranean under the condition of nonstationary signal receiving. Vladimir N. Fokin and Margarita S. Fokina (Inst. of Appl. Phys., Russian Acad. of Sci. 46, Ulyanov St., 603600, Nizhny Novgorod, Russia)

Using the concrete $c(z)$ data ( $c=$ sound speed, $z=$ depth) obtained in the Mediterranean during the THETIS-2 experiment on board the ACADEMICIAN SERGEY VAVILOV research vessel, the possibility of ray path identification using nonstationary receiving are numerically investigated. Sufficient $c(z)$ data were obtained for numerical simulation of the sound propagation along different acoustic routes. Sensitivity of different rays paths to errors caused by the erroneous determination of depth and distance is analyzed. The possibility of using the data obtained from the research vessel for ocean acoustic tomography is discussed.
1pAO14. Determination of the inhomogeneity position by the time structure of a scattered signal. Vladimir N. Fokin and Marganita S. Fokina (Inst. of Appl. Phys., Russian Acad. of Sci., 46, Ulyanov St., 603600, Nizhny Novgorod, Russia)

The method is based on the fact that the mean velocity of sound pulse propagation along the ray trajectory depends on the angle of the ray exit. The efficiency of the method is analyzed numerically for the signal reception at a horizontal array and a single hydrophone. It is shown that the signal of illumination may be suppressed and the scattered signal may be separated by time gating. It is also shown that the depth of the deposition of a space-localized inhomogeneity may be estimated by the width of the scattered signal autocorrelation function. The distance to the inhomogeneity may be defined by the cross-correlation function of the scattered signal at various array receivers. The influence of the depth of the submergence of a scatterer and the distance between a source and a receiver on the scattered signal structure is analyzed. [Work supported by RFFI project $\mathrm{N}$ 94-05-1725-a.]

1pA015. Definition of geoacoustic characteristics of an elastic layered bottom using the spatial dependence of an acoustic field. Margarita S. Fokina, Vladimir N. Fokin, and Boris V. Kerzhakov (Inst. of Appl. Phys., Russian Acad. of Sci., 46, Ulyanov St., 603600, Nizhny Novgorod, Russia)

The effect of the layer structure of the elastic absorbing bottom on the space-frequency dependences of acoustic fields in shallow and deep seas in the frequency' range $20-500 \mathrm{~Hz}$ is studied. The comparison of the experimental losses due to sound propagation in the deep-water part of the Atlantic Ocean at three frequencies and the results of numerical calculations by ray programs has shown that the experimental and calculated losses may agree satisfactory only if the layer bottom structure is taken into account. The numerical study in the frames of the approximation of adiabatic modes has shown that an absorbing layer of the geological bottom structure of the Barents Sea influences significantly the characteristics of sound fields and enriches the field mode spectrum. It is shown that the degree of this effect depends on the seasonal fluctuations of the sound velocity profile structure. The relation between the type of sediments that form boltom layers and frequency ranges, where the least losses due to propagation are observed, i.e., optimum propagation frequencies, is ascertained. [Work supported by RFFI project N 94-05-1725-a.] 


\title{
Session 1pEA
}

\section{Engineering Acoustics and Physical Acoustics: Optical Vibration and Pressure Sensors and Acoustic Measurements and Instrumentation}

\author{
David A. Brown, Cochair \\ Department of Physics, U.S. Naval Postgraduate School, Monterey, CA 93943 \\ Stephen C. Butler, Cochair \\ Analysis and Technology, Inc., 258 Bank Street, New London, Connecticut 06320
}

Contributed Papers

\section{2:30}

1pEA1. Design of a miniature fiber optic acoustic sensor for an acoustic viscometer. José D. Guevara (Dept. of Mech. Eng., The Cooper Union, 51 Astor Pl., New York, NY 10003) and Daniel R. Raichel (The Cooper Union and the Graduate Ctr. of the City Univ. of New York)

An acoustic fiber optic sensor was designed for the miniature version of an acoustic viscometer under development at The Cooper Union Acoustic Research Center. The sensor consists of a single mode fiber oplic cable embedded in the center of a $50-\mathrm{mm} \times 50-\mathrm{mm} \times 5 \mathrm{~mm}$ compliant polymer layer. It was noted that the geometrical versatility of the sensor could be minimized further, allowing for shorter lengths of sensitized fiber without sacrificing acoustical sensitivity. A Mach-Zender interferometric system is employed to detect the phase shift of the modulated optical signal in the sensitized fiber. A flat frequency response in the $200 \mathrm{~Hz}$ to $20 \mathrm{kHz}$ was predicted. The length of the fiber determines the minimum value of detectable pressure. In addition to immersion in water the sensor has the polential to also withstand immersion in mildly acidic and alkaline solutions.

12:45

1pEA2. A fiber optic dual-beam laser Doppler vibrometer for measurement of electrostrictive and piezoactive response of thin films. F. Guillot, J. Jarzynski (Georgia Inst. of Technol. Atlanta, GA 30332), and E. Balizer (NSWC, Silver Spring, MD 20903)

A two-beam laser Doppler vibrometer (LDV) is described, designed to measure the $d_{33}$ electromechanical coupling coefficient for electrostrictive and piezoactive thin films. Optical fiber couplers are used to split the light into multiple beams and optical fibers are used to simultaneously illuminate the same spot on both sides of the film. The LDV measures the normal displacement on each side and the change in thickness of the film is determined from the algebraic sum of the two LDV signals. It is found that large bending motion of the film can lead to a significant error in the measured $d_{33}$ coefficient. This is illustrated with data for $\mathrm{PVF}_{2}$ films and sources of this error are identified. The application of the LDV system to measure the large electrostrictive effect in polyurethane films is discussed. [Work supported by the Office of Naval Research.]

\section{1:00}

1pEA3. Target visibility measurements: Three-dimensional laser vibrometer. Joseph Vignola, Otis G. Zehl (SFA, Landover, MD 20785). and Brian H. Houston (Naval Res. Lab., Washington, DC 20375-5000)

A three-dimensional laser vibrometer has been constructed, tested, and used for simultaneous measurements of three components of induced transient motion of target surfaces. Such target surfaces need to satisfy a wider range of optical scatter requirements than those for one-dimensional mo- tion measurement. This presentation outlines some of these requirements for high signal visibility and describes several interesting target surfaces under test, including stainless steel, paints, polymer, and retroreflecting preparations. Data presented for these surfaces include signal strength (visibility), repeatability, stability, and effects of surface roughening and a water environment. Results are interpreted in terms of simple physical models where possible to better understand potential specialized target fabrication for improving future vibrometer measurement system performance.

\section{1:15}

1pEA4. Detection of strain-induced wavelength shifts in an optical fiber Bragg grating using an over-coupled coupler. Q. Zhang, D. A. Brown (Phys. Dept., Naval Postgraduate School, Monterey, CA 93943 and Brown Univ., Providence, RI 02912), H. Kung, J. Townsend, L. Reinhart, and T. F. Morse (Brown Univ., Providence, RI 02912)

A highly over-coupled fused biconical tapered fiber coupler has been used to interrogate the strain-induced wavelength shifts from an optical fiber Bragg grating. Both the $95 \%$ reflective fiber gratings and the overcoupled couplers were fabricated in our laboratory. The coupler has a spectral period of approximately $4 \mathrm{~nm}$ at the optical interrogation wavelength of $1550 \mathrm{~nm}$. The outputs are processed using a software-based demodulator programmed in Labview. Results of static and dynamic induced strains and sensor prototypes will be presented. [Work supported in part by NPS and NSF.]

\section{1:30}

1pEA5. Simple intensity type fiber optic vibration sensors and sensor systems. Edward F. Carome (Dept. of Phys., John Carroll Univ., Cleveland, $\mathrm{OH}$ 44118) and Vilnis E. Kubulins (Edjewise Sensor Products, Inc., Cleveland, $\mathrm{OH}$ 44122)

Research is continuing on the development of simple intensity type fiber optic vibration sensors suitable for use in hostile environments, such as automotive and aircraft engine cavities. The transduction element consists of a single or a pair of optical fibers mounted on a cantilever reed. The intensity of the optical output signal is directly proportional to the reed displacement and, therefore, to acceleration at frequencies well below the reed fundamental. Using readily available materials an operating temperature range of $-40^{\circ}$ to $+225^{\circ} \mathrm{C}$ has been achieved. In addition, by employing simple multiplexing techniques, small groups of these sensors may be addressed with a single optical source and/or detector. The basic design of the sensors and the results of extensive laboratory tests are presented in detail. [Work supported in part by NASA.] 
1pEA6. Fiber-optic microphone based on combination of Fabry-Perot interferometry and intensity modulation. Chonghua Zhou, Stephen V. Letcher (Dept. of Phys., Univ. of Rhode Island. Kingston, RI 02881), and Arun Shukla (Univ. of Rhode Island, Kingston, RI 02881)

A fiber-optic microphone based on a combination of Fabry-Perot interferometry and intensity modulation is described. The combination technique eliminates fringe counting ambiguity and provides a wide range measurement of optical phase change without any complicated electromechanical devices. The experimental data are compared with the result of a theoretical analysis. The microphone has a flat frequency response from 20 $\mathrm{Hz}$ to $10 \mathrm{kHz}$ with a high sensitivity of $1.6 \mathrm{rad} / \mathrm{Pa}$. The dynamic range is more than $65 \mathrm{~dB}$. The frequency response and sensitivity can be modulated by changing the size and surface tension of the membrane. The microphone can be easily implemented and can be made very small. [Work supported by NSF]

\section{2:00}

1pEA7. Field tests of a high-temperature fiber optic lever microphone. Allan J. Zuckerwar (MS 236, NASA Langley Res. Ctr., Hampton, VA 23681) and Frank W. Cuomo (F. W. Cuomo Consulting Services, East Providence, RI 02914)

The operating principle of a high-temperature fiber optic lever microphone (FOLM), rated at $538^{\circ} \mathrm{C}\left(1000^{\circ} \mathrm{F}\right)$ continuous service, is based on intensity modulation of light reflected from a vibrating membrane. The frequency response of the microphone, calibrated in the laboratory at ambient temperature and $538^{\circ} \mathrm{C}$ by the electrostatic actuator method, is typjcally $60-70 \mathrm{kHz}$. Applications to high-temperature field testing include the thermal acoustic fatigue apparatus at Langley Research Center, an advanced short takeoff and vertical landing model at Lewis Research Center, the Mach 22 helium tunnel at Langley (ambient temperature only), interior mixer-ejector surfaces at GE Aircraft Engines, Cincinnati, and a high temperature acoustic impedance tube at Langley. In most of these applications the FOLM made possible high-temperature, high-frequency acoustic data to be taken for the first time. Typical test results will be discussed.

\section{2:15}

1pEA8. An innovative probe microphone design for use in high-frequency sound field measurements. Sean $M$. Connelly and Linda P. Franzoni (North Carolina State Univ., Ctr. for Sound and Vib., Dept. of Mech. and Aerosp. Eng., P. O. Box 7910, Raleigh, NC 27695-7910)

There is a need for nonintrusive measurement devices. When the wavelength of the sound wave is physically the same scale as that of the microphone, its sound pressure cannot be measured accurately. Acousticians have worked around this difficulty by using smaller microphones and, when necessary, using probe tubes with even smaller diameters attached to small microphones. This need for very small diameter devices is especially critical at high frequency. The use of probe tubes introduces another level of complexity to acoustic measurements. The resonant dynamics of the tube itself must be taken into account. The lube supports standing waves that cause the microphone frequency response to be very irregular. Typically, damping material, such as foam or steel wool is introduced into the probe tube to attenuate standing waves. However, even when damping is successfully applied, the response remains irregular and the damping material results in a significant reduction of signal-to-noise ratio. The new concept is designed to eliminate standing waves without intmoducing damping in the signal path between the microphone and the end of the tube. Therefore, the signal-to-noise ratio will not suffer significant degradation, and behavior will be consistent from probe to probe.
1pEA9. Use of a background noise correction. Michael A. Staiano (Staiano Eng., Inc., 1923 Stanley Ave., Rockville, MD 20851)

A correction scheme has been proposed which consists of repetitive measurements of the source-signal-with-background and background noise alone than the computation of a signal estimate and prediction interval. The procedure assumes that both the source of interest and background noise are: uncorrelated, normally distributed, random processes which are stationary over the duration of the measurements. For useful results, the numbers of measurement must be selected to provide for a calculated confidence interval which acceptably contains the prediction errors. These requirements are strongly influenced by the variability of the measured parameters. For relatively low background noise situations, the technique is useful primarily for quantifying expected measurement confidence bounds. Application of the procedure to a field measurement situation with mean source band sound levels ranging approximately from 2 to $15 \mathrm{dBA}$ re: mean background band sound levels gave a $99.5 \%$-confidence upperbound margin of $0.3-1.3 \mathrm{~dB}$ with $17-19$ measurements per band.

\section{2:45-3:00 Break}

\section{3:00}

1pEA10. Precision of reference sound source hemianechoic sound power calibration using a fixed microphone array: Relation to ISO 6926 revision. Angelo J. Campanella (Campanella Assoc. \& ACCULAB, 3201 Ridgewood Dr., Columbus, OH 43026)

Reference sound source calibration by the hemianechoic ISO 3745 method allowed a course array of 10 measurement microphone positions on a hemisphere. Draft revision of ISO 6296 provides for a difficult meridional arc constant vertical velocity microphone scan. An improved array of multiple fixed microphone positions is proposed as an alternate to the meridional scan method. Bias of the improved array was simulated with Mathcad 4.0. Agreement was predicted to be within $0.2 \mathrm{~dB}$ up to $4 \mathrm{kHz}$ for 10 points and up to $8 \mathrm{kHz}$ for 20 points. Experimental results for three microphone position arrays were compared to reverberation room results over the limited frequency range of 125 to $8000 \mathrm{~Hz}$. The standard deviation of the difference was 0.84 to $0.89 \mathrm{~dB}(0.81 \mathrm{~dB}$ predicted) for the old ISO 3745 array. For an array having 10 fixed height difference positions from $0.15 R$ to $0.95 R$, the standard deviation was $0.62 \mathrm{~dB}(0.61 \mathrm{~dB}$ predicted). For 20 fixed heights from $0.025 R$ through $0.975 R$ the standard deviation was found to be 0.63 ( 0.18 predicted). Calculated results and experimental test results, compared with reverberation room results, will be presented.

\section{3:15}

1pEA11. Underwater linear horn array simulator. Stephen C. Butler and W. Jack Hughes (Appl. Res. Lab., Penn State Univ., P. O. Box 30, State College, PA 16804)

Sonar transducers with a radiating face smaller than one half wavelength tend to have a low radiation impedance load and consequently a narrow transmitting bandwidth. Thus, when the transducer is tested alone, the measured results yield a poor representation of its intended array performance. The objective of this research was to develop a fluid means to " $\rho c$ " load a single sonar transducer element by imposing a condition similar to its intended use in an array. A new method which accomplishes this and attains " $\rho c$ " loading utilizes a linear endfired waveguide, which forces a single transducer to behave as if it were in an array under " $\rho c$ " loading conditions. This is accomplished by an "underwater linear hom array simulator" which is composed of a rigid-walled cylindrical tube with slots of equal area equally spaced along the length of the tube. Acoustical circuit theory shows that $85 \% \rho c$ loading over an octave bandwidth would be achieved. Measurements on a physical model confirm the prediction. Measured mechanical $Q$ 's of the sonar transducer without the horn is 7.5 , and with horn is 5 , showing that the loading effects of the horn have been achieved. [Work supported by ONR and NUWC, New London, CT.] 
mathematically identical and produce similar experimental results. Comparison of the impedance tube and free-field measurements suggest that the latter approach produces more reliable results. However, due to practical limitations of the source/receiver geometry free-field measurements at low frequencies are more complicated in that the plane wave assumption is not valid.

\section{$4: 15$}

IpEA16. Panel response to jet noise under near sonic conditions. Charles C. Fenno, Ir. (NASA Langley Res. Ctr., Hampton, VA 23681), Alvin Bayliss (Northwestern Univ., Evanston, IL 60208), and Lucio Maestrello (NASA Langley Res. Cir., Hampton, VA 23681)

The problem of the response of an array of flexible aircraft-type panels excited by noise from a jet under near sonic conditions from a converging nozzle is numerically studied. The problem is computed by solving the Euler equations for the unsteady field in the jet, fully coupled to equations describing the panel motion. Computations of the far-field sound, panel response, and panel radiation are presented. The effect of nozzle geometry on the radiated sound and jet instabilities is studied. In addition, the relationship between panel location, relative to the jet exit, and panel response is determined. The computation simulates the development of large amplitude, slowly propagating instability waves in the jet which act as additional sources of sound. Thus the computation allows for direct computation of the natural sources of jet noise, as well as the propagation of the resulting jet noise. The results demonstrate large disturbances in the vicinity of the converging nozzle which act as additional sources of sound. The panel response is concentrated in progressively lower frequencies as distance from the jet exit increases.

\section{$4: 30$}

1pEA17. Acoustic detection of jet aircraft. Brian G. Ferguson (Defence Sci. and Technol. Organisation, P. O. Box 44, Pyrmont, Australia 2009)

A passive broadband acoustic technique is described that detects the transit of a subsonic jet aircraft. The outputs from a pair of spatially separated microphones are cross-correlated in three overlapping frequency bands. The time lag that corresponds to the maximum value of the crosscorrelogram is extracted for each of the three bands every second. Coincidence detection of this time lag information results in the detection of the jet transit with a negligible false alarm rate. The detection range is extended by applying the technique to the beamformed outputs from a pair of microphone arrays. The results of applying the technique to real data from 25 jet transits are presented.

\section{$4: 45$}

1pEA18. Measurement of the distortion level of a loudspeaker using an adaptive filter algorithm in a reverberation and noisy environment. Chun-Duck Kim (Dept. of Elec. Eng., Natl. Fisheries Univ. of Pusan, Pusan, Korea), and Suk-Kun Jee (Kunsan Natl. Univ., Kunsan, Korea), and Abe Masato (Tohoku Univ., Sendai, Japan)

This paper describes a method to measure the distortion level of a loudspeaker and an amplifier using a LMS (least-mean square) adaptive filter. The acoustic apparatus checks the distortion level and the residual noise using a bandpass filter having a sharp cut-off frequency characteristic and a sinusoidal oscillator. The above bandpass filter, however, is expensive and has a long response characteristic. It is impossible to check each hamnonic distortion. Also, to check the harmonic distortion of a loudspeaker in a room with ambient noise, the experiment room needs to be an anechoic chamber. On the other hand, the proposed method offers an easy way to measure the specified harmonic distortion level with a small amount of hardware. Moreover, this method is not affected by ambient noise which has no correlation with the test signal. The measurement can be carried out in a noisy environment. The system consists of a 32-bit DSP96002, 2ch of 16-bit analog to digital converter, and a Sth Chebyshev filter with a $50 \mathrm{kHz}$ sampling frequency. The effectiveness of the proposed method confirmed by experiments using a loudspeaker carried out in a room with ambient noise. 


\title{
Session 1pNS
}

\section{Noise: Active Noise Control and Duet Acoustics}

\author{
Susan K. Numrich, Chair \\ Naval Research Laboratory, Code 5580, Washington, DC 20375-5000
}

Contributed Papers

1pNS1. Active control of broadhand random noise using energy density control. Young C. Park and Scott D. Sommerfeldt (Appl. Res. Lab. and Graduate Program in Acoust., Penn State Univ., P.O. Box 30, State College, PA 16804)

Previous work on active noise control in enclosures has indicated that one can often achieve improved global attenuation of deterministic signals by minimizing the acoustic energy density, rather than the squared pressure. The purpose of this work is to present numerical results that compare the global attenuation of broadband noise achieved by minimizing energy density with the attenuation achieved by minimizing squared pressure in a one-dimensional enclosure. A theoretical approach based on the frequency domain enables one to establish the basic physical limitations of active noise control systems. However, this approach cannot necessarily be applied when controlling a broadband random noise, since it often yields optimal control solutions that are noncausal in the time domain, even though such a frequency domain approach is entirely satisfactory for deterministic signals. Analyses reported here are undertaken in the time domain in a manner that yields the form of the causally constrained optimal controller. Numerical results are presented to predict the performance of the active noise control system designed to control a broadband noise, and to indicate the improved global attenuation of broadband noise that can be achieved by minimizing energy density, rather than squared pressure.

\section{$1: 15$}

1pNS2. Multichannel block adaptive filters for real-time, nonparametric identification of time-varying plants. William $\mathrm{C}$. Nowlin, Gary S. Guthart, and Gregory K. Toth (Appl. Control \& Signal Process. Group, SRI International, Menlo Park, CA 94025)

Many real-world applications of active noise control are characterized by transfer functions that vary significantly and unpredictably. The controller's transfer function models must adapt to these variations. Presented here is a class of adaptive filters that accomplish quasiperiodic system identification updates for feedforward control by using blocks of inputoutput histories. The algorithms form a one-dimensional family linking nomalized LMS adaptive filters and optimal Wiener filters, and are termed "block projection" algorithms. The system identification proceeds noninvasively, producing nonparametric (FIR) impulse responses. The multichannel generalization and application of these algorithms to system identification, as presented here, is novel. Considerations are described that arise from the algorithms' implementation in the context of system identification; in particular, the proper weighting of input and output data pairs is discussed. The resulting multichannel control algorithrns have been implemented successfully for quieling of a compact distributed source in an anechoic environment, and for local quieting of a diffuse field in a reverberant room. In both cases, error microphones could be moved about, providing a "mobile quiet zone," and performance was obtained for bandwidths exceeding a decade.
1pNS3. Active control of sound transmission through a thin metal plate. Xiaoqi Bao, Vasundara V. Varadan, and Vijay K. Varadan (Dept. of Eng. Sci. and Mech., Penn State Univ., University Park, PA 16802)

The performance of active sound isolation by using a piezoelectric sensor and an actuator on a thin plate is investigated experimentally. In the experiment, the aluminum plate covers an opening of an acoustic enclosure, where a sound source is located. The outside acoustic field is measured by an 11-element microphone array, which scans on a surface of a hemisphere. The isolation performance of the original plate is poor at its resonance frequencies. The sound transmission from inside to outside of the enclosure is actively controlled by a one-sensor one-actuator control system which minimizes the output of the on-plate sensor. Global reductions of 15-20 dB are achieved at the first three resonance frequencies.

\section{$1: 45$}

1pNS4. Near-field acoustic holography for enclosures with unknown Green's functions. Kelly Q. Kay (Virginia Polytech. Inst. \& State Univ.-Virginia Consortium of Eng. and Sci. Universities, 2713-D Magruder Blvd., Hampton, VA 23666), Chris R. Fuller (Virginia Polytech. Inst. and State Univ., Blacksburg, VA 24061), and Karen H. Lyle (U.S. Army Vehicle Structures Directorate, NASA Langley Res. Ctr. Hampton, VA 23681)

A method for predicting the acoustic field in acoustically complex cavities is developed based upon current near-field acoustic holography (NAH) techniques. Current NAH methods make use of analytical Green's functions to generate predictions of global acoustic fields. For this reason, NAH is easily applied to generate predictions in free fields, and in cavities with simple geometries and uniform boundary impedances. For acoustically complex cavities, however, the absence of a known analytical Green's function, renders current NAH methods either not solvable, or in cases where approximate Green's functions are used, not accurate. The method requires no prior knowledge of the acoustically complex cavity's Green's function. As well, no approximations about the boundary conditions are required. Instead, the method takes advantage of the discrete nature of NAH predictions, in conjunction with an initial measurement of a cavities acoustic pressure field, to generate a cavity specific discrete Green's function. Computational and experimental results will be presented along with a detailed explanation of the method. [Work supported by NASA Langley Research Center.]

\section{2:00}

1pNSS. Principal component analysis applied to the active control of turbofan inlet noise. Ran Cabell (Virginia Polytech. Inst. and State Univ.-Virginia Consortium of Eng. and Sci. Universities, 2713-D Magruder Blvd., Hampton, VA 23666) and Chris Fuller (Virginia Polytech. Inst. and State Univ., Blacksburg, VA 24061)

An analytical study of the active control of fan noise in a turbofan engine with wall-mounted acoustic sources is described. The transfer functions between the acoustic sources and microphones outside the duct are decomposed into principal components (PCs). The active control problem 
is then solved using a subset of the principal components. The subset consists of PCs that are statistically significant for accurately modeling the primary radiated noise. The resulting elimination of certain PCs is a mathematically efficient way to optimally reduce the number of channels of control. In certain cases it can be shown that the PCs describe the radiating modes of the control system. This provides substantial insight into the mechanisms of control, and can be used in the controller to derive specific modes to the exclusion of all other modes. [Work supported by NASA Langley Research Center.]

$$
\text { 2:15 }
$$

1pNS6. Optimal design of enclosures for active noise control and isolation. Vasundara V. Varadan, Jaehwan Kim, and Vijay K. Varadan (Res. Ctr. for the Eng. of Electron. and Acoust. Mater., Dept. of Eng. Sci. \& Mech., Penn State Univ., University Park, PA 16802)

An optimal design technique is used to find the optimal conditions for several parameters of an enclosure to reduce the noise radiated from an internal source. Design variables in the optimization procedure are the size and thickness of the enclosure, size and thickness of actuators, the location of actuators, and the applied voltage on the actuators. The objective function in optimal design is the total radiated sound power at far field. The practical limits of the design variables are taken as constraints. The acoustic field acts as a distributed load on the walls of the enclosure. A 3-D finite element model is used to model the walls and the PZT actuators. With an applied voltage on the actuators and with acoustic pressure loading, the velocity distribution on the outside of the walls can be found by solving the finite element equations. These are used in the Helmholtz integral representation to compute the radiated sound field to the exterior of the enclosure. The design is performed at a single frequency which may represent the dominant frequency in the noise spectrum. By comparing the initial design and the optimal design results, a noise reduction of $12-18 \mathrm{~dB}$ has been achieved.

$$
2: 30
$$

1pNS7. Attenuation of broadband noise in a reverberant room using a multichannel active noise control system. Gary S. Guthart, William C. Nowlin, and Gregory K. Toth (Appl. Control \& Signal Process. Group, SRI International, Menlo Park, CA 94025)

Active noise control (ANC) is often discussed in the context of reducing noise in vehicles. The acoustic reverberation of typical vehicle cabins makes the application of ANC to quieting interior noise a stiff challenge. While some ANC approaches take advantage of the modal structure of reverberant enclosures to provide reduction throughout the enclosure, these approaches become infeasible for many frequency bands of practical interest because of increasing modal density with increasing frequency. For high-bandwidth, diffuse fields, noise reduction is achieved locally by specifying a quiet zone within the enclosure. Experimental results for broadband, local quieting in a diffuse field using a multichannel ANC system are presented. The reverberant enclosure is an ordinary room, measuring approximately $1.4 \mathrm{~m} \times 2.4 \mathrm{~m} \times 2.4 \mathrm{~m}$ and containing a seated operator with six microphones defining the quiet zone near the operator's ears. The control system uses a single reference signal and two error channels to drive four control speakers. An average of $20-\mathrm{dB}$ reduction in sound pressure level is found at the quiet-zone microphones for the frequency range 50 to $1000 \mathrm{~Hz}$. These results demonstrate the viability of real-time, multichannel ANC for locally attenuating random noise in diffuse acoustic fields.

\section{2:45-3:00 Break}

3:00

1pNS8. Experimental investigation of low-frequency noise from jet engine test cells. Robert G. Gibson (Wyle Labs., 2001 Jefferson Davis Hwy., Ste. 701, Arlington, VA 22202)

Low-frequency noise emission from facilities designed for the ground runup testing of jet engines can be a source of annoyance to communities near military air bases. Noise measurements, using arrays of microphones and accelerometers at a number of U.S. Air Force hush houses, provide information regarding the source characteristics. Comparisons are made between noise from a single aircraft both inside and outside a hush house. Source location calculations indicate that low-frequency noise originates from a region near the trailing edge of the jet exhaust deflector. Coherence between selected near-field and far-field sensors is found to be sufficiently high to, warrant experimental investigation of the active reduction of noise from a full scale hush house, using feedforward control techniques, following on from the small scale hush house experiments described by J. P. Smith et al. [J. Acoust. Soc. Am. 96, 3332(A) (1994)]. [Work sponsored by the U.S. Air Force.]

\section{$3: 15$}

1pNS9. Active control of acoustic intensity in a duct. Karl $\mathrm{M}$. Reichard, David C. Swanson, and Scott M. Hirsch (Appl. Res. Lab., Penn State Univ., P.O. Box 30, State College, PA 16804)

A frequency-domain implementation of the filtered- $x$ algorithm is used to actively control acoustic intensity in a duct. The frequency-domain filtered- $x$ algorithm uses FFT's of the necessary signals to compute the transfer function of the optimal control filter. A version of the algorithm has been adapted to use acoustic intensity, computed from the cross spectrum from two pressure microphones, as the error signal. The results of experiments conducted in a duct are presented which demonstrate the effects of measurement error and control source configuration on controller performance.

\section{$3: 30$}

1pNS10. Insertion loss studies of a baffle-simple expansion chamber system. P. Xiao and M. G. Prasad (Noise and Vibration Control Lab., Dept. of Mech. Eng., Stevens Inst. of Technol., Castle Point on the Hudson, Hoboken, NJ 07030)

Insertion loss (IL) is the difference of sound pressure levels measured at a reference point without and with a muffer in a duct system. Thus, IL describes the acoustical performance of the whole system which includes source, muffler, and tail pipe. IL studies of a simple expansion chamber has been reported in the literature. However, there are not many IL studies of complicated geometries in a muffler from a system design point of view. This study reports both experimental and analytical work on the influence of a baffle on IL of a simple expansion chamber. The hole diameter and location of the baffler are the parameters of this study. An Altec electroacoustic driver is used as a source for experimental work conducted in an anechoic chamber. The analytical work is carricd out using the boundary element method. The results indicate a significant influence (due to the interaction of source and load) on the insertion loss.

\section{3:45}

1pNS11. Reflection of higher-order modes at the end of rectangular ducts. Ralph T. Muehleisen and David C. Swanson (Appl. Res. Lab., Penn State Univ., University Park, PA 16802)

Reflection coefficients for higher-order modes impinging on the ends of finite rectangular ducts were predicted and measured. The results show coupling between propagating and evanescent modes. Expressions for the reflection coefficients in terms of modal coefficients and modal impedances have been developed. Radiation patterns were predicted from the knowledge of the coupling coefficients. It was found that higher-order modes radiate most of their energy off towards the sides and less straight ahead than the plane wave mode. 


\section{4:00}

1pNS12. Acoustical studies of a duct system using a power flow model. Woo-sung Kim (HDD Design Eng., Samsung Electronics Co., Ld., 416, Maetan-3Dong, Paldal-Gu, Suwon City, Kyungki-Do, Korea) and M. G. Prasad (Stevens Inst. of Technol., Hoboken, NJ 07030)

Any duct system from an acoustical point of view can be modeled as a source-load system. The power flow analysis in a source-load system is important for a system's acoustical design. The present work describes the development of a power flow model utilizing the source and load impedances as parameters. Also, the source is described in terms of its power rather than the pressure or the volume velocity. The study includes both analytical and experimental work. The analytical work deals with the development of expressions for power flow in the system. The experimental work is carried out using an Altec electroacoustic driver as the source and various ducts as the loads. The system performance is evaluated using the power flow model for various loads. The study has yielded good results validating the power flow model for duct systems.

\section{4:15}

1pNS13. A multi-mode acoustic filter hased on Bragg resonance. Muhammad A. Hawwa, Chris R. Fuller, and Ricardo A. Burdisso (Vib. and Acoust. Labs., Dept. of Mech. Eng. Virginia Polytech. Inst. and State Univ., Blacksburg, VA 24061-0238)

A unified method is proposed to suppress duct acoustic waves. According to this method, a circular cylindrical duct is designed to have a geometric periodicity at its wall in order to act as a wave filter. The filter action of the periodic rigid wall is based on the mode coupling occurring under the Bragg condition resulting in a strong stopband interaction. The problem is attacked using the perturbation method of multiple scales, leading to the derivation of the coupled-mode equations. The performance of the duct as a wave attenuator is presented in terms of the reflection coefficient or the transmission iuss factor. Due to the nature of the dispersion curves of the cylindrical duct, many modes could share the same stopband. Hence, a multi-mode acoustic filter could be designed over a certain frequency band. This theoretical study might provide a basis for design concepts of acoustic silencers.

\section{$4: 30$}

1pNS14. Studies of source-load interaction in a duct system using acoustic noise synthesis. Anilkumar Bhate and M. G. Prasad (Noise and Vib. Control Lab., Dept. of Mech. Eng., Stevens Inst. of Technol., Castle Point on the Hudson, Hoboken, NJ 07030)

Acoustic noise synthesis (ANS) for duct systems was first developed by the authors (M. G. Prasad and T. V. Ananthapadmanabha, INTERNOISE 89 Proceedings) based on the principles of speech synthesis using a source-filter model. The ANS allows the designer of a duct system to simulate the perceptual quality of the acoustical output of the system. The ANS is based on the transfer function which is evaluated using the known geometry of the duct system. The present work describes the application of ANS to study the interaction between source and load on the perceptual quality of a duct system acoustic output. An electroacoustic driver as a source and ducts of various lengths are used as loads. Good results are obtained which validate the application of acoustic noise synthesis in design of duct systems with emphasis on the perceptual quality and subjective evaluation.

TUESDAY AFTERNOON, 30 MAY 1995

RENAISSANCE WEST B, 1:00 TO 5:00 P.M.

\title{
Session 1pPA
}

\section{Physical Acoustics and Noise: Sonic Boom}

\author{
David T. Blackstock, Cochair
}

Applied Research Laboratories and Mechanical Engineering Department, University of Texas at Austin, P.O. Box 8029, Austin, Texas 78713-8029

Allan D. Pierce, Cochair

Department of Aerospace and Mechanical Engineering, Boston University, 110 Cummington Street, Boston, Massachusetts 02215

Invited Papers

1pPA1. Current strategies in sonic-boom research. Christine M. Darden (NASA Langley Res. Ctr., Hampton, VA 23665)

During NASA's high-speed research program, a renewed emphasis has been placed on understanding and reducing the sonic boom generated by an airplane during flight at speeds faster than the speed of sound. When the current program was initiated in 1990, a three-pronged approach was instituted: (1) human acceptabilities studies; (2) atmospheric propagation studies; and (3) airplane design and operation studies. Original goals were to use special design and operation techniques to reduce the sonic boom to levels which would be acceptable for either unrestricted overland flight or overland flight through low-populated corridors. Recent human response studies have indicated that sonic booms are much more disturbing than continuous noise sources such as engines around airports; thus any commercial overland supersonic flight is highly unlikely within the near future. These conclusions have resulted in a redirection of the sonic-boom program. This talk will discuss current efforts at sonic-boom softening (small design changes), continuing efforts to understand the impact of the atmosphere on the rise time of the sonic-boom signature, and strategies to understand what impact, if any, the sonic boom has on marine life. 
1pPA2. Studies of sonic booms with seismic networks. Bradford Sturtevant, Joseph E. Cates (Graduate Aeronaut. Labs., California Inst. of Technol., Caltech 301-46, Pasadena, CA 91125), and Hiroo Kanamori (California Inst. of Technol., Pasadena, CA 91125)

Seismographs are sufficiently sensitive to detect ground motions induced by atmospheric pressure waves, so seismic networks have the potential to monitor sonic booms over large areas of the United States. They are especially well suited for the analysis of long-range sonic-boom propagation. Ground motion or displacement data provide accurate arrival times and useful estimates of wave amplitude and waveform. The instrumentation is most sensitive to the disturbance produced by the arrival of sonic booms at the measuring station, thus serving as sonic-boom event recorders, but seismographs have also detected seismic waves remotely generated by anomalous coupling of sonic boom into soil. Direct and indirect sonic booms from aircraft operations are routinely detected by the Southern California Seismic Network which consists of 250 seismic stations covering $50000 \mathrm{sq} \mathrm{km}$. Indirect booms from space shuttle landings has been observed at ranges of hundreds of kilometers from the flight path. Data from the network identified "mystery booms" heard in 1992-93 to be long-range indirect sonic booms from offshore operations. Sonic booms generated by space shuttle reentry at Mach 20 and by meteoritic entries into the atmosphere have been detected by seismic networks in the Northwest and Canada.

\section{$1: 40$}

1pPA3. The prediction of sonic-boom distortion using a scattering center based calculation. Richard Raspet and Henry E Bass (Dept. of Phys. and Astron., Natl. Ctr. for Phys. Acoust., Univ. of Mississippi, University, MS 38677)

A numerical scattering technique has been developed to calculate the effect of turbulence on sound propagation. This technique resolves the atmosphere into discrete "turbules," calculates the scattered field from each turbule using the Born approximation, then sums all the contributions to form the scattered waveform. The effect of single "turbules" of different scales will be discussed. This technique has been applied to model the atmosphere and the propagation of sonic booms through the planetary boundary layer during the JAPE-2 tests at White Sands. Good agreement was achieved. The limitations and planned improvements will be described. [Work supported by NASA Langley Research Center.]

1pPA4. Effects of atmospheric stratification on sonic-boom propagation. Robin O. Cleveland (Appl. Res. Labs., P.O. Box 8029, Austin, TX 78713-8029)

Sonic-boom propagation is affected by stratification, geometrical spreading, nonlinear distortion, absorption and dispersion, and turbulence. Stressed in this paper is stratification, in particular its indirect effect on distortion and absorption. The stratification of the density and sound speed leads to refraction and impedance variation, which play a major role in determining the amplitude of the waveform on the ground. Stratification, and associated spreading, can also control the amount of nonlinear distortion a sonic boom suffers. In extreme cases the amount of nonlinear distortion is finite-a phenomenon known as waveform freezing. Analysis shows that for aircraft in the lower $20 \mathrm{~km}$ of the atmosphere waveform freezing does not occur. Through their dependence on temperature, pressure, and humidity, absorption and dispersion are also stratified. A new time domain algorithm, based on a Burgers-type equation, was developed to analyze the effect of the stratification of absorption [J. Acoust. Soc. Am. 96, 3275 (A) (1994)]. The code is used to predict sonic-boom waveforms on the ground. It is demonstrated that sonic-boom shocks are affected not just by local absorption but also by absorption along path for the past $5 \mathrm{~km}$ or so. [Work supported by NASA.]

\section{2:20}

1pPA5. USAF flight test investigation of focused sonic booms. Micah Downing (USAF Armstrong Lab., 2610 Seventh St., Wright-Patterson AFB, OH 45433) and Noel Zamot (USAF TPS, Edwards AFB, CA 93524)

In April 1994, the USAF Armstrong Laboratory in cooperation with USAF Test Pilot School conducted a measurement study of controlled focus booms generated by supersonic maneuvers. The objective of this study was to collect focus and post-focus booms and to assess the ability of aircrews to control the placement of the focal region during basic maneuvers. Forty-nine flights were performed and included level linear acceleration, level turn, accelerating dives, and climbout/pushover maneuvers. Sonic booms were collected by up to 25 boom event analyzer recorders (BEARs) placed in a 13000 - $\mathrm{ft}$ linear array. The BEAR units were spaced 500 to $2000 \mathrm{ft}$ apart with the denser spacing at the expected focal region. This spacing was chosen to evaluate the thickness of both the focal and post-focal regions. Of the 49 flights, a focus boom was successfully placed within the array 35 times which demonstrated the ability to place controlled focus booms. This ability of the aircrew can be employed to avoid collateral damage to noise sensitive receptors. Along with capturing focus $U$ waves, complex $N-U$ signatures were recorded at distances away from the foci. Turbulent conditions had a defocusing effect, resulting in smaller maximum overpressures.

\section{$2: 40$}

1pPA6. The theoretical and computational basis of focused sonic booms. Kenneth J. Plotkin (Wyle Labs., 2001 Jefferson Davis Hwy., Suite 701, Arlington, VA 22202)

For the past 20 years sonic-boom signatures in focus "superboom" regions have been computed using a method based on a scaling law by Guiraud [J. Mech. 4, 215-267 (1965)] and a numeric solution by Gill [Ph.D. thesis, Cornell University (1974)]. The method recognizes that the smooth caustic case treated by Guiraud and Gill is by far the most common focusing situation, and that smooth caustics caused by aircraft accelerations and turns and by atmospheric gradients are topologically similar. Gill's numeric result for a shock may thus be treated as a canonical result, and scaled to arbitrary situations by Guiraud's similitude. The process has been 
incorporated into an existing sonic-boom program. When a focus is detected, the program fits the solution by (a) computing ray and caustic curvatures required by the theory, (b) determining a matching position consistent with Gill's boundary conditions, and (c) applying the scaled Gill solution to each shock. The computational requirements of this process are reviewed, together with empirical support for the results.

\section{3:00}

1pPA7. Effects of sonic booms on animals: Past history and future directions. Ann E. Bowles (Hubbs-Sea World Res. Inst., 2595 South Shores Rd., San Diego, CA 92109)

Models developed to describe and mitigate damage to human hearing after sonic-boom exposure can be applied to a wide range of species. If humans and laboratory animals are poor models for a species of concern, or if intense focused sonic booms are anticipated, experimental assessments will be needed. Although attenuation across interfaces (air-water and air-soil) will protect many aquatic and fossorial species, attenuation may not protect individuals near the interface if boundary phenomena like evanescent energy are found to be important. Panic reactions are a likelier hazard for animals, at least in air. Damage in panics is predicted best by species-typical avoidance behaviors (e.g., panic flight), sound exposure level (after appropriate weighting), and previous experience with impulsive man-made noise. Dose-effect models have not been developed because controlled experimental studies with booms at levels up to $150 \mathrm{~dB}$ (peak flat SPL) have failed to elicit panics. However, widespread concern continues because there are circumstantial accounts of catastrophic damages, notably the "Sooty Tern Incident." Controlled studies of habituation using initially naive animals are needed to develop dose-effect models for damaging responses. Effects of impulse-induced frights on (1) gametogenesis, conception, and early embryonic development, (2) parental care, (3) activity, and (4) habitat use should be investigated as well.

$$
\text { 3:20-3:30 Break }
$$

\section{Contributed Papers}

$$
\text { 3:30 }
$$

1pPA8. Sonic boom from the Titan IV space launch vehicle launched from Vandenberg Air Force Base. Jon K. Francine and Brent $S$. Stewart (Hubbs-Sea World Res. Inst., 2595 Ingraham St., San Diego, CA 92109)

The sonic boom generated by a Titan IV space launch vehicle launched on 7 November 1991 was recorded at two locations on San Miguel Island, California. A Bruel \& Kjaer microphone carrier system recorded the overpressure near a haul-out site for about $\mathbf{4 0 0 0 0}$ pinnipeds (seals and sea lions); a boom event analysis recorder (BEAR) was placed under the predicted focal region. The boom reached the pinniped site $4 \mathrm{~min}$ and $38 \mathrm{~s}$ after launch. It was neither an $N$ nor a $U$ wave at either site; rather, it had several distinct positive peaks, which indicates that the focal region was uprange of the recording sites. The first and fourth positive peaks were 853 ms apart. At the pinniped site, most of the boom's energy was at low frequencies $(\mathrm{ca}<500 \mathrm{~Hz}$ ), as indicated by the $46-\mathrm{dB}$ difference between the 124-dB unweighted sound exposure level (SEL) and the $78 \mathrm{~dB}$ A-weighted SEL. The peak overpressure recorded near the pinnipeds was 64.1 Pa (1.34 psf; $130 \mathrm{~dB}$ peak SPL). The BEAR system recorded a peak overpressure of $90.5 \mathrm{~Pa}$ (1.89 psf; $133 \mathrm{~dB}$ peak SPL). Empirical tests of predictive models for focal regions and descriptions of the frequency characteristics of focused booms are still needed to evaluate the potential risks to wildlife from these booms.

\section{3:45}

1pPA9. Sonic-boom noise penetration into the ocean: 1995 update. Victor W. Sparrow (Grad. Prog. Acoust., Penn State Univ., 157 Hammond Bldg., University Park, PA 16802)

This talk is an update to a previously gircn prusentation [J. Acoust. Soc. Am. 94, 1850-1851 (A) (1993)] in which a first-pass model for the penetration of sonic boom noise into the ocean was described. Since that presentation was given, a number of refinements to the theory have been identified, the primary of which is the effect of aircraft Mach number on the duration of the sonic boom. The general trend for aircraft flying at a fixed altitude is that the evanescent acoustic wave underwater due to a sonic boom penetrates more deeply for increased aircraft speeds [J. Acoust. Soc. Am. 97, 159-162 (1995)]. Other refinements currently under development include appropriate initial models for sound level decay as a function of depth and for focusing and defocusing of the penetrating sonic boom noise by "frozen" sinusoidal and trochoidal ocean surface waves. [Work supported by NASA Research Grant NAG-1-1638.]
4:00

1pPA10. Seasonal acoustic propagation effect. Wayne R. Lundberg (3312 Braddock St., Kettering, OH 45420)

Seasonal variations in wind and temperature gradients are known to influence the propagation of aircraft noise and sonic booms. This paper examines predictive techniques in current use for potential refinement and application to seasonal environmental impact statements. The effects of seasonally averaged refractive atmospheres were studied using sonic-boom propagation models which include refractive effects. The atmospheric environment was chosen to be that near Edwards Air Force Base where sonic booms are known to affect the public. Sonic-boom carpet predictions for a representative flight of an F-111 at Mach 1.3 and $10000 \mathrm{ft}$ above mean sea level were documented. The effect of such atmospheres on predicted noise exposure contours due to a real-world mix of supersonic flights was calculated using appropriate adjustments to the CORBOOM model. Two distinct atmospheric averaging techniques were applied for seasonal and annual comparisons. The resulting single-event and sonic-boom exposure predictions were compared to each other and to predictions obtained using the U.S. Standard Day Atmosphere and the Annual Reference Atmosphere for Edwards AFB. The results show the potential benefit of conducting seasonal acoustic predictions in forecasting the effects which low-altitude aircraft flight operations have on the public.

\section{4:15}

1pPA11. Application of model experiment data to test sonic-boom propagation models. Bart Lipkens (MacroSonix, 1054 Technology Park Dr., Glen Allen, VA 23060)

Model experiments are successful in simulating the propagation of sonic booms through the atmosphenc turbulent boundary layer. In the model experiment spark-produced $N$ waves propagate across the turbulent flow field of a plane jet. The $N$ waves are measured by a wide band condenser microphone and the turbulence is measured by hot-wire anemometry. The results from the model experiment, pressure waveforms and turbulence characteristics, are used to test various sonic-boom models for propagation through turbulence. Both waveform distortion models and rise time prediction models are tested. Only Pierce's model [A. D. Pierce, J. Acoust. Soc. Am. 49, 906-924 (1971)] based on the wavefront folding mechanism at a caustic yields a fairly accurate prediction for the rise time of the mean waveform after propagation through the turbulence. Results from other models are not confirmed by the model experiment data. [Work supported by NASA Langley.] 
1pPA12. On simulation of sonic-boom propagation with realistic modeling of the atmospheric turbulence. Allan D. Pierce (Dept. of Aerosp. and Mech. Eng., Boston Univ., 110 Cummington St., Boston, MA 02215)

The propagation of sonic booms is from high above the ground to ground level, and over the path the dominant acoustic length scales in the signatures fall within what is known as the inertial subrange, for which there is a considerable body of basic theoretical knowledge. Any model of boom-turbulence interaction that requires a value for an outer scale has very weak theoretical basis. However, the range of turbulence scales within the inertial subrange is huge and different scales have different effects. The present paper argues that the turbulence can be split in a well-defined and logical manner, with the resulting scale at which the split occurs serving as an outer scale with respect to the turbulence that affects the rise times of sonic booms. Although molecular relaxation accounts for a substantial fraction of the rise times, the bulk of the thickening during daytime overflights is associated with turbulence. A theory for such thickening that simultaneously took both nonlinear steepening and turbulence into account had been proposed in the early 1970 s by Plotkin and George, but had been criticized for its dependence on the choice of an outer scale. The proposed partitioning removes this objection and combines the salient ideas of Plotkin and George with others proposed by the author during the same epoch for the effects of inertial-subrange-scale turbulence on rise times.
1pPA13. Comparison of computer codes for propagation of sonic booms through the atmosphere. James P. Chambers, Henry E. Bass, Richard Raspet (Natl. Ctr. for Phys. Acoust., Univ. of Mississippi, University, MS 38677), Robin O. Cleveland, Mark F. Hamilton, and David T. Blackstock (Univ. of Texas, Austin, TX 78713-8029)

NASA initiated an exereise to compare sonic-boom propagation codes. Three different codes developed at our two universities are compared here: SHOCKN (Mississippi), THOR (Texas), and ZEPHYRUS (Texas; developed by L. D. Robinson). Each code includes nonlinear steepening, absorption and dispersion (relaxational and thermoviscous), geometric spreading, and atmospheric stratification. All three codes calculate steepening in the time domain. The main difference is in the calculation of absorption and dispersion. SHOCKN and ZEPHYRUS use the frequency domain; the FFT is used to transfer back and forth between the time and frequency domains. ZEPHYRUS employs weak shock theory to reduce the number of FFT operations. All of THOR's calculations are done in the time domain. Code accuracy and effectiveness is judged by comparing ground level predicted waveforms (input waveforms are specified by NASA). The codes are capable of dealing with arbitrary atmospheric data. Results are presented here for uniform and isothermal atmospheres, with and without the effects of molecular relaxation. Early comparisons revealed discrepancies, which led to improvement of codes. Current comparisons show excellent agreement. Ground waveforms show surprising sensitivity to input stratification data. Some comparisons of code running time have been made. The FFT operations apparently make SHOCKN and ZEPHYRUS run more slowly than THOR. [Work supported by NASA.]

\title{
Session 1pSC
}

\section{Speech Communication: Psychophysics of Speech Perception: What Critical Data are Needed to Improve Low-Bit-Rate Coding and Automatic Recognition}

\author{
Oded Ghitza, Chair \\ Acoustics Research Department, AT\&T Bell Laboratories, 600 Mountain Avenue, Murray Hill, New Jersey 07974
}

Invited Papers

$1: 30$

1pSC1. Psychophysics of speech perception: What critical data are needed to improve low bit-rate-coding and automatic recognition? Oded Ghitza (Acoust. Res. Dept., AT\&T Bell Labs., 600 Mountain Ave., Murray Hill, NJ 07974)

The purpose of this special session is to call the attention of the hearing science community to the need for new knowledge on how speech segments of durations of 50-150 ms long (e.g., phonemes, diphones), are being represented in the auditory system. In this session, the need for such knowledge will be addressed in the context of two specific speech-technology applications-low bit-rate coding and automatic recognition (ASR) - which rely on processing speech information of a segment-length duration. Schemes for low bit-rate coding rely on signal manipulations that spread over durations of several tens of ms, and schemes for speech recognition rely on phonemic/articulatory information that extends over similar time intervals. Current research efforts are focused on the psychophysics of stationary acoustic inputs, for example, aspects of masking, pitch perception, and sound segregation. Research efforts also exist on the cognitive aspects of speech perception, for example, lexical access. In contrast, research on the psychophysical aspects of speech dynamics, as manifested in the acoustic properties over durations of tens of $\mathrm{ms}$, still is in its infancy.

1:35

1pSC2. Psychophysics of speech and speech-like stimuli. Tammo Houtgast (TNO Human Factors Res. Inst., Kampweg 5, 3769 De Soesterberg, The Netherlands)

The starting point is the representation of speech in the spectro-temporal domain. From this representation, a number of interesting physical characteristics can be derived on the dynamic properties of speech. These include the temporal and spectral envelopes, correlation patterns, and synchrony. The perceptual relevance of these characteristics will be illustrated by reviewing a number of psychophysical data from experiments including speech-like sounds or specific laboratory-made dynamic stimuli. Within the context of low bit-rate coding and automatic recognition, the ultimate goal of this comparison between speech characteristics and psychophysics is to help identify the cues in the speech signal which are perceptually significant. 
1pSC3. Speech perception, memory, and cognition: Implications for automatic speech recognition systems. Patricia Kuhl (Dept. of Speech and Hear. Sci., Univ. of Washington, WJ-10, Seattle, WA 98195)

Recent experiments suggest that people form perceptual representations for speech as a result of experience with a specific language. These representations are argued to take the form of perceptual maps stored in memory that specify distances between speech stimuli. This point will be illustrated with data from cross-language experiments on American and Japanese adults using the segments $h /$ and $/ /$. The results show that the underlying perceplual space encompassing $/ \mathbf{r} /$ and $/ /$ varies greatly in American and Japanese listeners- their perceptual maps are dramatically different. Cross-cultural developmental studies show that language-specific speech representations are present early in life and differ in infants reared in different linguistic environments. Further work suggests that the perceptual maps resulting from speech experience not only influence auditory speech perception, but influence auditory-visual speech perception in adults and speech production in infants. Implications for automatic speech recognition systems will be discussed. [Work supported by NIH.]

2:25

1pSC4. Automatic speech recognition using signal processing based on auditory physiology and perception. Richard Stern (Dept. of Elec. and Comput. Eng., School of Comput. Sci., and Biomed. Eng. Program, Carnegie Mellon Univ., Pittsburgh, PA 15213-3890)

Signal processing for automatic speech recognition has traditionally been inspired more by models of speech production than by models of auditory perception. While some aspects of human auditory processing have been implicit in traditional signal analysis for speech recognition, there is growing interest in the development of more computationally demanding signal processing strategies that are directly motivaled by knowledge of auditory physiology and perception. The use of physiologically motivated signal processing has been shown to improve the accuracy of some automatic speech recognition systems, particularly in difficult acoustical environments. This talk will review and discuss the role that knowledge of human hearing has played in the design of speech recognition systems. The common features of auditory models that have emerged from various laboratories as well as their differences will be discussed. The recognition accuracy obtained using auditory models will be compared with the accuracy obtained using conventional signal analysis techniques, as well as the accuracy obtained using other approaches to robust recognition that are not physiologically based. Finally, an attempt will be made to identify aspects of monaural and binaural auditory processing that appear to be most helpful for robust recognition. [Work supported by ARPA.]

\section{2:50-3:05 Break}

\section{3:05}

IpSC5. Achieving high quality speech at low bit rates: Fundamental issues in psychophysics. Bishnu Atal (Speech Res. Dept., AT\&T Bell Labs., Rm. 2D-535, Murray Hill, NJ 07974)

Excellent progress has been made in recent years in synthesizing high quality natural sounding speech at bit rates as low as $8 \mathrm{~kb} / \mathrm{s}$. Rapidly increasing demand for speech transmission over digital radio channels requires that the bit rates be decreased even further-to $4 \mathrm{~kb} / \mathrm{s}$ or lower. But, one is not able to achieve high quality speech at these low bit rates. Why? Low bit rate speech coders exploit auditory masking to mask the quantizing noise in the coded signal. The current models of auditory masking are valid only for relatively stationary segments, such as vowels, in the speech signal. Much of the distortions we heard in low bit rate coders are introduced during transitional segments, resulting from lack of psychophysical data on perception of such distortions. A human listener is able to delect such distortions in the coded version without comparing the coded version with the original. Why is it so? How does our hearing system know that the coded signal is distorted? Such questions must be answered before progress can be made in achieving high quality speech at low bit rates.

1pSC6. On the perceptual distance between speech segments. Oded Ghitza and M. Mohan Sondi (Acoust. Res. Dept., AT\&T Bell Labs., Rm. 2D-536, Murray Hill, NJ 07974)

For many tasks in speech signal processing it is of interest to develop an objective measure that correlates well with the perceptual distance between speech segments. (Speech segments means pieces of a speech signal, of duration 50-150 ms.) Such a distance metric would be useful for speech coders at low bit rates because perturbations introduced by such coders typically last for several tens of milliseconds. It would also be useful for automatic speech recognition in adverse conditions. Since human beings perform well in spitc of gross distortions of the signal (e.g., due to reverberation, noisy environments, etc.) it is justifiable to assume that mimicking human behavior will improve recognition performance. In this talk, attempts at defining such a metric will be described. The problem is approached in the framework of the Diagnostic Rhyme Test [DRT]. The errors made by subjects were measured when judiciously chosen time-frequency "tiles" were interchanged between the words in each pair of the DRT test [Ghitza, J. Acoust. Soc. Am. 94, 2507-2515 (1993)]. Next the same task is performed with an array of automatic speech recognizers [Ghitza and Sondhi, Comp. Speech Lang. 7(2), 101-120 (1993)], using a parametrized distance metric. Finally, the parameters of the distance metric are optimized so as to minimize the difference between the error patterns of the human listeners and those of the automatic speech recognizers. 


\title{
Session 1eID
}

\section{Interdisciplinary: Tutorial on Flow-Induced Sound}

\author{
Kenneth J. Plotkin, Chair \\ Wyle Laboratories, 2001 Jefferson Davis Highway, Suite 701, Arlington, Virginia 22202 \\ Chair's Introduction-7:00 \\ Invited Paper \\ $7: 05$
}

1eID1. Flow-induced sound. Alan Powell (Dept. of Mech. Eng., Univ. of Houston, Houston, TX 77204-4792)

After a brief historical account introducing some lasting concepts, the origins and main features of aerodynamically generated sound are described in terms of the melding of some pertinent elements of fluid dynamics and of acoustics. These are, for example, flow separation, flow instability, and vortices on the one hand, coupled with the hydrodynamic flows of acoustic monopoles, dipoles, and quadrupoles on the other. With emphasis on the vortex theory of aerodynamic sound, various theoretical approaches are generally described in physical terms and are illustrated by a variety of sound-generating flows. These may be classified as free flows with no solid surfaces present (spinning vortices, turbulent jet noise, supersonic jet screech), flows over rigid surfaces (boundary layer noise, whistling telephone wires, edge tones, pipe tones, vocal fricatives, and whistling, organ pipes), interaction with steadily moving surfaces (helicopter blade slap, fan blade interaction, but not including the rotational noise of fans and propellers), flow with excited surfaces (interior aircraft boundary layer noise, aeolian tones, reed and lip-driven musical instruments, vocal chord vibration, snoring and raspberries). Some of these involved no resonance at all, while others have flow resonance, acoustic resonance or mechanical vibration or resonance.

\section{Session 2aAA}

\section{Architectural Acoustics and Noise: The Americans with Disabilities Act and Acoustical Criteria for the Disabled}

\author{
David Lubman, Cochair \\ David Lubman and Associates, 14301 Middletown Lane, Westminster, California 92683 \\ John Erdreich, Cochair \\ Ostergaard Acoustical Associates, 100 Executive Drive, West Orange, New Jersey 07052
}

Chair's Introduction-8:30

Invited Papers

8:35

2aAA1. Meeting the challenges of the Americans with Disabilities Act: Providing access for people with communication disabilities. Io Williams (Professional Practices Dept. American Speech-Language-Hearing Association, 10801 Rockville Pike, Rockville, MD 20852)

The American with Disabilities Act (ADA) is significant not only as landmark civil rights legislation, but also because it is a turning point in public policy regarding the importance of communication and communication access. In the past, accessibility policy has focused on physical/mobility concerns and largely ignored needs related to sensory/cognitive impairments. More than 34 million Americans of all ages-approximately one of every ten people-have hearing, speech, and/or cognitive language impairments that 
affect their ability to work, learn in school, and participate in everyday activities. ADA regulations address communication access in employment, community programs and businesses, transportation, and telecommunications. Strategies for communication access and employment accommodations vary with the individual/disability and the type of activity/business. Most individuals need strategies that optimize auditory and visual information exchange, including improvements in environmental conditions (such as reduced ambient noise levels). New approaches and rethinking are needed regarding (1) assessing communication (dis)ability and needs, (2) the concept of accessibility, and (3) the role of professionals, including interdisciplinary collaborative efforts, in ADA implementation.

9:00

2aAA2. Room acoustics needs of people with hearing impairment. Anna K. Nabelek and Igor V. Nabelek (Univ. of Tennessee, Knoxville, TN 37996-0740)

Speech perception of people with hearing impairment is negatively affected to a greater degree by background noise and reverberation than the speech perception of people with normal hearing. As a consequence, rooms which have adequate acoustics for normal-hearing people might be too noisy or too reverberant for hearing-impaired users. Causes of perceptual difficulties of hearingimpaired listeners and data comparing effects of noise and reverberation on speech perception of normal-hearing and hearing-impaired listeners will be presented. The number of people with hearing loss grows with age. Two major causes are noise exposure and aging processes. People with hearing loss can obtain improvement in speech communication with hearing aids but poor room acoustics can limit hearing benefits and cause hearing aid rejection. Advantages of acoustical modifications, preferential sitting, and use of amplification in small rooms will be discussed. Listening systems such as induction loops, FM, and infrared will be considered as alternatives or additions to acoustical modifications of large rooms. The listening systems can be utilized by hearing aid users and by people who do not use hearing aids, even those with normal hearing.

9:25

2aAA3. The effects of room acoustics on normal-hearing children: Implications for intervention. Carl C. Crandell (Dept. of Commun. Processes \& Disorders, Univ. of Florida, 461 Dauer Hall, Gainseville, FL 32611) and Joseph Smaldino (Univ. of Northern Iowa, Cedar Falls, IA 50614)

It is well recognized that the acoustical environment in a classroom is an important variable in the psycho educational achievement of hearing-impaired children. To date, however, there remains a paucity of information concerning the importance of classroom acoustics for populations of children with normal-hearing sensitivity. The present discussion will examine: (1) commonly reported levels of classroom noise and reverberation; (2) the potential effects of classroom acoustics on the speech recognition of several populations of pediatric listeners (young children, children with language/articulation disorders, non-native English children, children with minimal hearing loss, developmentally disordered children, children with central auditory processing disorder); (3) intervention strategies, such as sound field amplification systems, to benefit normal-hearing children in the classroom setting; and (4) implications of appropriate classroom acoustics to the American with Disabilities Act (ADA).

9:50

2aAA4. Proposed guidelines for quiet areas in restaurants for hearing-impaired individuals. Ron Moulder (Empire Acoustical Systems, 89 Park Ave. W, Mansfield, OH 44902) and David Lubman (D. Lubman and Assoc., Westminster, CA 92683)

The predominant problem of the hearing-impaired individual is speech communication. In noisy and reverberant restaurants where speech communication is difficult to impossible, these individuals cannot enjoy the experience of dining out. This fact was conveyed to the US government during hearings for the Americans with Disabilities Act. A program was funded by the federal government to develop recommendations for improving the acoustical quality of restaurants so that hearing-impaired individuals could enjoy the experience of dining out. A three-phase program consisting of a literature search, the evaluation of the acoustical environment in selected restaurants, and the development of proposed recommendations to improve speech communication by hearing-impaired individuals in restaurants was carried out by Battelle. An advisory panel of professional and lay individuals was established to review the work of this program. The proposed recommendations developed focused on reducing reverberation times and noise levels in restaurants. Several recommendations are presented for achieving these goals.

10:15-10:25 Break

Contributed Papers

$10: 25$

2aAA5. Evaluation of accessibility needs in a home for the aged. $M$. Kathleen Pichora-Fuller (School of Audiol. and Speech Sci., Univ. of British Columbia, 5804 Fairview Ave., Vancouver, BC V6T 123, Canada)

Audiological rehabilitation has traditionally focused on the hard-ofhearing individual. In contrast, the needs of groups who perform specific activities in specific locations must be evaluated in planning facility-based accessibility programs. Such evaluations are necessary when planners design programs where solutions may be achieved by a combination of strategies including modification of the environment, provision of personal or institutional assistive technology, or communication training. The present study demonstrates how accessibility needs were evaluated in a home for the aged. For a group of 30 residents, scope of participation in activities and quality of communication during activities were measured four times over two years, twice before and twice after program implementation. In a pilot study, groups of residents and staff identified situations at the home where they felt that hearing was important. The evaluation of communication function was keyed to these situations. The patterns of scope and quality of communication were used to formulate an accessibility plane. Baseline and outcome data regarding the extent to which the program accomplished accessibility will be presented. For example, the program rendered meetings and chapel services accessible to most residents whereas situations such as dining in the dining hall remained inaccessible. [Work supported by NHRDP.] 
increases the capability for auditory communication by: (1) lowering interfering reverberant noise levels generated by people and trains, and (2) increasing the clarity of speech from people and audio systems providing railway information.

\section{1:10}

2aAA8. Influence of impulse noise on speech transmission index. Miomir Mijić (Faculty of Elec. Eng., P.O. Box 816, 11000 Belgrade, Yugoslavia) and Miodrag Popović (INTELSAT, Washington, DC 20008)

This paper analyzes the influence of impulse noise impairments in a telecommunication channel on the speech transmission index (STI) value, which was measured using RASTI method. So far STI has not been applied in telecommunications as a speech transmission quality measure Impulse noise components were formed as IEEE standard impulse streams. Impulse noise amplitude level relative to the level of reference (RASTI) signal as well as the duration between successive impulse events were varied in a channel. Consequently, STI value was determined as a function of these two parameters. In the same channel PB-Word Score evaluation was also performed. As a result, the correlation between PB-Word Score and STI value for a telephone channel with impulse noise impairments present was established in this paper.

2aAA7. Acoustical design of the New York railway stations with improved accessibility for hearing and visually impaired persons. Robert Nichols (Nichols Design Assoc., Inc., P.O. Box 11251, Washington, DC 20008) and Gerald Henning (Miller Henning Assoc., Inc., McLean, VA 22101)

11:25-11:30 Break

An acoustical design of the New York railway stations is proposed to improve the acoustical accessibility for persons with hearing and visual impairments. The acoustical design includes methods and materials for reducing reverberation times in the stations. Reducing reverberation times

\title{
11:30-12:00 PANEL DISCUSSION
}

WEDNESDAY MORNING, 31 MAY 1995 GRAND BALLROOM CENTRAL, 8:00 A.M. TO 12:45 P.M.

\section{Session 2aAO}

\section{Acoustical Oceanography: Ocean Acoustic Tomography: Observing the Ocean in the 1990s III}

\author{
Yves Desaubies, Chair \\ Laboratoire de Physique des Oceans, IFREMER, BP 70, 29280 Plouzane, France
}

Chair's Introduction-8;00

Contributed Papers

\section{8:05}

2aAO1. The internal tide of the western North Atlantic observed using long-range reciprocal acoustic transmissions. Brian D. Dushaw (A.P.L., Univ. of Washington, 1013 NE 40th St., Seattle, WA 98105-6698), Peter F. Worcester, Bruce D. Comuelle (Univ. of California, La Jolla, CA 92093-0213), and Bruce M. Howe (Univ. of Washington, Seattle, WA 98105-6698)

Time series of ray travel times were obtained at 350-, 410-, and 670-km ranges in the western North Atlantic during the 1991-2 Acoustic Mid-Ocean Dynamics Experiment (AMODE). Transmissions were recorded for approximately 300 days between six transceivers in a pentagonal array. Sound-speed variability is separated from current variability by calculating the sum of reciprocal travel times. Both phase-locked and narrow-band internal-tide sound-speed variability, caused by the internaltide isotach displacement, are observed by the sum travel times. The description of the acoustic array as a high-directivity antenna for the incident internal tide will be given. Beamforming the internal tide $M_{2}$ and $K_{1}$ harmonic constants determined on each of the 15 acoustic paths suggests two origins of significant internal tide generation are the Blake Escarpment and the continental shelf north of Puerto Rico. The nontidal, highfrequency variability ( $>1 \mathrm{cpd}$ ) is due to internal-wave sound-speed and current variability.

\section{$8: 20$}

2aAO2. Tomographic inversion of fish parameters in shallow water. Orest Diachok. (SACLANT Undersea Res. Ctr., La Spezia, Italy)

Simulations of Weston's measurements of the temporal variability of anomalously large attenuation due to fish, suggest the possibility of tomographic inversion of pelagic fish parameters over large spatial dimensions (nominally $50 \mathrm{~km}$ radius) in shallow water. These measurements reveal resonances which are a function of fish bladder size and depth. Computations suggest that the latter may be inferred from modal attenua- 
tion measurements [Diachok, European Conference on Underwater Acoustics (1994)], hypothetically permitting unambiguous estimation of bladder size. In the horizontal dimension pelagic fish concentrate into "clusters" of schools at scales, which are driven by the "patchiness" of plankton, which in tutn is hypothetically controlled by atmospheric and bottom conditions, and by oceanic mesoscale phenomena such as eddies, fronts, and upwelling events. A fixed, large-scale tomographic array consisting of several widely spaced transmitters and receivers, designed to invert ocean parameters at low frequencies and fish parameters over a broad range of higher frequencies, together with quasisynoptic measurements of plankton parameters (probably from ships), would permit investigation of fish adaptation(s) to the temporally evolving mesoscale environment. Criteria for the design of such a bioacoustic observatory, and its advantages and disadvantages compared to shipborne methods and fixed long-range active monitoring, will be discussed.

\section{$8: 35$}

2aA03. Scattering of acoustic tomographic signals in shallow water by internal waves. James F. Lynch (Woods Hole Oceanogr. Inst., Woods Hole, MA 02543), Guoliang Jin (Academia Sinica, People's Republic of China), Peter Traykovski (MIT/WHOI Joint Program in Oceanography and Oceanographic Engineering. Woods Hole, MA 02543), Ching-sang Chiu, and James H. Miller (Naval Postgraduate School, Monterey, CA 93943)

This talk examines how internal waves in shallow water cause travel time fluctuations in acoustic tomographic transmissions. Ray and mode theory expressions are developed and calculations are compared to travel time fluctuation data taken from the 1992 Barents Sea Polar Front experiment. While there is basic agreement, there is still much room for improvement of the basic theory and experiments. Directions of further research in both theory and experiment are discussed. [Work supported by ONR.]

\section{$8: 50$}

2aA04. Internal-tide inversions from a 1000-km Pacific acoustic propagation experiment in the Pacific. Charles Bracher and Stanley M. Flatte (Phys. Dept., Univ. of California, Santa Cruz, CA 95064)

The Slice89 experiment sent sound pulses through the Pacific over a $1000-\mathrm{km}$ path. Different identified wavefronts showed clear M2 internaltidal signals in their arrival-time series. we have examined internal-tide models composed of the first three vertical modes in order to fit the Slice89 data. The horizontal directions of the three modes were allowed to be different. The horizontal structure of the models varied from plane waves to waves that are localized along the direction of acoustic propagation. It has been found that the best-fit internal-tide model within the parametrization, and the resulting fit is reasonable. These results along with those of other experiments in the same geographical area have been compared.

\section{9:05}

2aA05. Numerical simulation of the sensitivity for the temperature remote acoustics control in Fram Strait environmental conditions. Konstantin A. Naugolnykh (Environmental Tech. Lab., NOAA. Boulder. CO 80303), Ola M. Johannessen (Nansen Environmental and Remote Sensing Ctr., Bergen N5037. Norway), Igor B. Esipov, Oleg B. Ovchinnikov, Yury I. Tujilkin, and Viktor V. Zosimov (N. Andreyev Acoust. Inst., Moscow 117036, Russia)

An important role of the general problem of global climate changes is the heat inflow from the Atlantic Ocean to the Arctic basin control. The effective tool to perform it is the acoustic methods application. Some aspects of this problem connected with the average water temperature measurements in some cross section of the Fram Strait are considered. It is supposed that the temperature change takes place in the upper 200-m layer of the ocean which is typical for the winter Fram Strait environmental conditions. Variations of the amplitude and the arrival time of the acoustic signals during their propagation in the Fram Strait cross section with variable upper layer mean temperature were considered on the base of the ray theory approximation. The results of computer simulation of the process are presented.

2aA06. A full field approach to acoustic tomography of oceanic currents. Oleg A. Godin (NOAA/Atlantic Oceanogr. and Meteorol. Lab., 4301 Rickenbacker Cswy., Miami, FL 33149) and Dmitry Yu. Mikhin (P. P. Shirshov Oceanogr. Inst. of the Russian Acad. Sci., Moscow 117851, Russia)

Traditionally it is nonreciprocity in travel times along identified pairs of spatially close eigenrays that serves as input data for a current's velocity inversion. In shallow water many of the rays are bottom-interacting and cannot be resolved or identified. Hence the traditional approach results in a very poor or nonexistent resolution in the vertical plane [D. S. Ko et al., J. Geophys. Res. 94, 6197-6211 (1989)]. To avoid these problems it is suggested that one match nonreciprocity of acoustic continuous waves measured at a set of points, e.g., at a vertical array. Analytic studies indicate that nonreciprocity of the phase of a cw signal is sensitive to the flow velocity but does not depend, to first order, on fluctuations in sound speed or on uncertainties in transceiver location and, therefore, can be used as input data for currents inversion. Respective matched-phase nonreciprocity tomography (MPNT) is simulated numerically, including effects of acoustic SNR, systematic and random environmental mismatches, number of transceivers in the array, uncertainty in their positions, etc. and compared to other possible inversion schemes. It is concluded that MPNT could be a practical tool for monitoring ocean dynamics in shallow water. [Work supported by NRC and RBRF.]

\section{9:35}

2aA07. 1994 moving ship tomography experiment in the western Mediterranean. Dmitry Yu. Mikhin. Dmitry L. Aleynik, Sergey V. Burenkov, Yury A. Chepurin, Viktor G. Selivanov, Sergey Ya. Molchanov (P. P. Shirshov Oceanogr. Inst. of the Russian Acad. Sci., Moscow 11785I, Russia), and Oleg A. Godin ${ }^{\text {a) }}$ (NOAA/Atlantic Oceanographic and Meteorological Lab., Miami, FL 33149)

A tomographic network of seven moored transceivers was deployed in the western Mediterranean basin in early 1994 by IfM (Kiel, Germany). IFREMER (Brest, France), and WHOI (Woods Hole, USA) in the framework of the THETIS-2 project. Signals emitted by the transceivers were also utilized in the 8-week moving ship tomography experiment, MOST. Acoustic measurements were made with a single hydrophone and an eightelement vertical array deployed from a drifting vessel and were complemented by CTD casts. The set of observation points was chosen to provide detailed CTD and acoustic data along existing propagation paths of the THETIS-2 network for comparison of traditional and moving ship tomography under well-controlled environmental conditions as well as to create numerous additional propagation paths to improve resolution in the horizontal plane. By precise compensating the Doppler shift due to vessel drift, the data processing technique used made it possible to resolve eigenray arrivals up to $400-600 \mathrm{~km}$ range for different sources. Preliminary results of the MOST data processing, analysis, and interpretation will be presented at the meeting. [Work supported by ISF and INTAS.] ${ }^{\text {a) }}$ On leave from P. P. Shirshov Oceanography Institute, Moscow.

\section{9:50}

2aA08. Acoustic footprints of an interthermocline eddy: Field experiment and numerical simulations. Dmitry Yu. Mikhin. Dmitry L. Aleynik, Sergey V. Burenkov, Yury A. Chepurin, Viktor G. Selivanov, Sergey Ya. Molchanov (P. P. Shirshov Oceanogr. Inst. of the Russian Acad. Sci., Moscow 117851, Russia), and Oleg A. Godin ${ }^{\text {a) }}$ (NOAA/ Atlantic Oceanogr. and Meteorol. Lab., Miami, FL 33149)

A moving ship tomography experiment (MOST) was carried out in the western Mediterranean basin in 1994. Six moored transceivers deployed in the area by IfM (Kiel, Germany), IFREMER (Brest, France), and WHOI 
(Woods Hole, USA), in the framework of the THETIS-2 project, were utilized as wide-band sound sources for the MOST. An interthermocline eddy with cold core was found during the MOST. Detailed CTD surveys were performed along several sections of the eddy. In the horizontal, it was close to an ellipse with axes of 25 and $40 \mathrm{~nm}$. The sound-speed difference at given depth inside and outside the eddy was up to $2 \mathrm{~m} / \mathrm{s}$. Within the eddy core the sound-speed gradient was an order of magnitude less than in surrounding waters. Acoustic signals from the transceivers were recorded at 16 points within and around the eddy in order to study feasibility of reconstruction of mesoscale inhomogeneities of this type by acoustic tomography means. A technique of coherent signal processing used made it possible to resolve multiple eigenray arrivals from four transceivers at each point of observations. Results of mathematical modeling of the acoustic effects of the eddy and their comparison with the experimental data will be

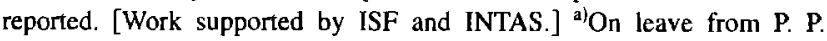
Shirshov Oceanography Institute, Moscow.

\section{0:05-10:20 Break}

\section{0:20}

2aA09. On acoustic tomography of internal waves. John A. Colosi (Scripps Inst. of Oceanogr., IGPP-0225, UCSD, La Jolla, CA 92093) and Stanley M. Flatte (Univ. of California, Santa Cruz, CA 95064)

With the planned acoustic instrumentation of several of the worlds ocean basins, the possibility exists for measuring the strength of the internal wave field as a function of depth, time, and geographical position. The variability of internal-wave energies and spectra over these parameters will provide information on the sources and sinks of internal waves. In the early eighties Flatte formulated an analytic, ray-based framework for internalwave tomography. Analysis of the SLICE89 experiment combined with supercomputer numerical simulations have recently shown that internal waves have even stronger effects on signals transmitted over $1000 \mathrm{~km}$ than were expected from the early ray-based theory plus the Garrett-Munk (GM) spectrum. It was found that the ray-based theory is adequate for identifiable wavefronts that turn at depths below $100 \mathrm{~m}$; energy that reaches very shallow depths and energy that stays near the sound-channel axis do not behave so simply. The numerical simulations establish an internal-wave strength below $100 \mathrm{~m}$ of $0.5 \pm 0.25$ the reference $\mathrm{GM}$ level, and point to known limitations of the GM spectrum in the upper ocean. Simulations to $3000 \mathrm{~km}$ have quantified the range dependence of internalwave effects, and extensions of ray-based theory attempt to explain these effects.

\section{$10: 35$}

2aA010. Internal wave effects on shallow water acoustic tomography. Xin Tang and F. D. Tappert (Appl. Marine Phys., Univ. Miami, RSMAS, 4600 Rickenbacker Cswy., Miami, FL 33149)

Full-wave numerical simulations of internal wave scattering of sound in shallow water are performed with an efficient broadband PE model that also includes scattering by bathymetric variations. Bathymetric scattering by itself gives an exponentially large number of unresolvable multipaths as predicted by ray chaos theory. When internal waves that evolve dynamically in geophysical time are included, the unresolvable multipaths are found to be temporally unstable with coherence times less than a few minutes at ranges greater than a few tens of water depths in shallow water, except possibly for rare paths that are shielded from internal waves such as within strong surface ducts. Since traditional travel-time ocean acoustic tomography requires many stable, resolvable and identifiable paths, conditions that are not satisfied in shallow water at longer ranges, some other inversion method will have to be developed for remote sensing of shallow water environments. [Work supported by ONR.]

\section{0:50}

2aAO11. Decadal variability in acoustic thermometers interpreted with ocean models. John Spiesberger, Mark Keller (Dept. of Meteorol. and the Appl. Res. Lab., 512 Walker Bldg., Penn State Univ., University Park, PA 16802), Mark Johnson (Univ. Alaska-Fairbanks, Fairbanks, AK 99775-1080), Harley Hurlburt (Naval Res. Lab., Stennis Space Center, Bay St. Louis, MS 39522), and James O'Brien (Florida State Univ., Tallahassee, FL 32306)

Travel times of acoustic signals were measured between the bottommounted Kaneohe source near Oahu and seven SOSUS stations at 30004000-km distance during 1983-1989. The Naval Research Laboratory hydrodynamic eddy resolving model yields changes in travel time whose standard deviations are consistent with the data. The model predicts that between 1981-1993, Rossby waves modify travel times by one second. Mesoscale eddies modify travel times little compared to Rossby waves. The largest Rossby waves are descendants of El Nino. Travel times changes are sensitive indicators of predictable features in the Naval Research Laboratory model. [Work supported by the Strategic Environmental Research and Development Program, managed by the Advanced Research Projects Agency.]

\section{1:05}

2aA012. Surface suspended acoustic receiver for acoustic tomography. John S. Spiesberger, Carter Ackerman, and Bruce Einfalt (Dept. of Meteorol. and the Appl. Res. Lab., 512 Walker Bldg., Penn State Univ., University Park, PA 16802)

A surface suspended acoustic receiver (SSAR) is shown capable of processing and transmitting acoustic tomography data through ARGOS to laboratories in near-real time. Ray theory is shown adequate for validating the data from the SSAR. [Work supported by the Strategic Environmental Research and Development Program, managed by the Advanced Research Projects Agency.]

\section{1:20}

2aAO13. ATOC-FACT arrival-time differences. David Palmer (NOAA/Atlantic Oceanogr. and Meteorol. Lab., 4301 Rickenbacker Cswy., Miami, FL 33173), Leon Krige (Inst. for Maritime Technol., Simon's Town, 7995 South Africa), Geoff Brundrit (Univ. of Cape Town, Rondebosch, 7700 South Africa), and Kurt Metzger (Univ. of Michigan, Ann Arbor, MI 48109-2122)

The acoustic thermometry of ocean climate-feasibility ascension Cape Town (ATOC-FACT) propagation experiment took place during November and December of 1992. Explosive charges were detonated near the oceanic sound-channel axis at five locations in the South Atlantic off Cape Town. The resulting signals were recorded on bottom-mounted hydrophones at Ascension, Navy horizontal arrays off Bermuda, bottom-mounted hydrophones off Kaneohe and Wake and from a hydrophone array deployed from a research ship off Southern Tasmania. The primary goal of the experiment was to determine the extent that the acoustic path between Ascension and Cape Town is blocked by Bonaparte's Seamount and other bathymetric features. Secondary goals were to determine the horizontal arrival structure at Bermuda and the temporal arrival structure at Ascension, Hawaii, and off Tasmania. After giving an overview of the experiment, we use recorded data to discuss the question of how precisely arival-time differences of explosive signals can be measured. Detonations separated both temporally and spatially are considered. It is hoped this work will contribute to an understanding of the role explosive signals can play in the design of a global ATOC network. 


\title{
$11: 35$
}

2aAO14. Observing the ocean in the 1990s: Where do we stand now? Carl Wunsch (Dept. of Earth, Atmospheric and Planetary Sciences, Room 54-1524, MIT, Cambridge, MA 02139)

Thirteen years ago, a paper with almost the same name as this present session was published by Munk and Wunsch. Ways in which oceanographers were likely to be observing the ocean 10 years later was speculated upon. The special focus was climate-scale problems with acoustic tomography and satellite altimetry as the natural complementary observational technologies. At the time, the tomographic idea was only about 4 years old, and now that it is half-way through the 1990s, it is interesting and perhaps useful to look back to understand the extent to which the vision of the role of tomography in oceanography has come to pass. A number of problems will be considered, from the technical to the sociological and attempt to make yet another forecast of where tomography in oceanography might be in another 10 years.

12:00-12:45

PANEL DISCUSSION

\section{Session 2aEA}

\section{Engineering Acoustics and Noise: Active Control of Noise and Vibration I}

\author{
Sung H. Ko, Cochair \\ Naval Undersea Warfare Center, New London Detachment, New London, Connecticut 06320 \\ Elizabeth Â. McLaughlin, Cochair \\ Naval Undersea Warfare Center, New London Detachment, New London, Connecticut 06320
}

Chair's Introduction-8:00

Invited Papers

8:05

2aEA1. Active control of noise and vibration: Overview and current applications. Chris R. Fuller (Vib. and Acoust. Lab., Dept. of Mech. Eng., Virginia Polytech. Inst. and State Univ., Blacksburg, VA 24061)

In this introductory presentation the principles and components of active control systems will be briefly overviewed. Current research topics and areas of application will be discussed including duct noise, machinery isolation, wave control in beams, interior noise in aircraft, and structurally radiated sound. The use of adaptive materials in active noise and vibration control will also be touched upon. The presentation will finish with a discussion on the present limitations of active control and future promising directions.

\section{8:35}

2aEA2. Active control: New paradigms for structural acoustics and dynamics. Albert J. Tucker (Office of Naval Res., $800 \mathrm{~N}$. Quincy St., Arlington, VA 22311-5660)

Originally the concept of active control conveyed the simple notion of creating "antinoise" or "antivibration;" these ideas implied that simple antiphase motions could be propagated to cancel unwanted noise. However, recent advances in signal processing, coupled with gigaflop digital signal processors, augur new ways to understand and control complex dynamic systems. Among these are shifting wave numbers from radiating to nonradiating components and modifying structural impedances to create large dynamic masses, stiffnesses, and/or damping. Hence one electronically modifies the dynamics of complex systems to achieve acoustic radiation reduction, or to have a physically lightweight structure emulate the dynamics of a heavier, stiffer structure. This fundamental scientific understanding offers new opportunities for development of new technology for control algorithms, sensors, and actuators. As a result, active control is emerging as viable addition to the engineering toolkit for noise and vibration reduction. 
2aEA3. Silencers: The impact of active control. L. J. Eriksson (DIGISONIX, Inc., 8401 Murphy Dr, Middleton, WI 53562)

Over the past decade, powerful new adaptive techniques have been developed for the active control of sound and vibration. One of the areas that has received the most attention is the application of active control to enhance the performance of silencers used on ducts or pipes. Active silencing is often combined with passive silencing to create a new form of hybrid silencer. These silencers can be used as intake or discharge silencers for heating, ventilating, and air conditioning (HVAC) fans, industrial fans, rotary blowers, compressors, internal combustion engines, gas turbines, pumps, and related equipment. This paper will review the use of active control for these applications with particular emphasis on the different reasons generating the interest in active control for each application. Among the potential advantages of active control for silencers are improved low frequency performance, reduced flow restriction, and decreased size and cost. The degree to which these potential advantages can be realized in specific applications can vary considerably as well as the ultimate impact of the improvement for the end user. These issues will be discussed using a number of actual examples and implications for the future use of active control will be suggested.

2aEA4. Active control of aircraft cabin noise. Gopal P. Mathur (McDonnell Douglas Aerospace, 1510 Hughes Way, Mail Code 71-35, Long Beach, CA 90810-1870)

Aircraft cabin noise control in the past has relief heavily on improving sidewall attenuation by passive "add-on" treatments. The conventional passive methods, such as adding mass, damping, or acoustic absorption, etc., not only impose a stiff weight penalty, they are also ineffective in improving the low-frequency sound transmission loss of an aircraft fuselage sidewall. Active control of sound inside aircraft cabins has been the focus of research in recent years and has shown considerable promise. Laboratory and in-flight tests of prototype active control systems for tonal noise reduction using secondary speakers have demonstrated the feasibility of active noise control (ANC) in aircraft cabins. In recent years active structural acoustic control (ASAC) has also been applied to aircraft fuselage structures in controlling low- to mid-frequency structural sound radiation. In the ASAC technique, control forces are applied directly to the vibrating structure by actuators (such as piezoelectric transducers) instead of using loudspeakers to minimize the radiated sound field. The ASAC approach is also important in the design of "smart structures," which incorporate both sensors and actuators in the structure for noise and vibration control. This paper presents results of investigations (conducted at McDonnell Douglas) of application of both ANC and ASAC techniques to a full scale aircraft fuselage. Significant sound pressure reductions were achieved throughout the cabin for multiple tonal frequencies of excitation. The performance of the ASAC method is compared with that of the ANC system using speakers. The fight test results with a prototype ANC system in an MD-80 aircraft will also be presented.

\section{9:50}

2aEA5. Feedback-stabilized artificial bedrock: A practical implementation of a passive/active vibration isolation system. A. M. Beard, D. W. Schubert (Barry Controls, 40 Guest St., Brighton, MA 02135), and A. H. von Flotow (Hood Technol. Corp., Hood River, OR 97031)

Active control in the presence of resonant structural dynamics is challenging since such dynamics are both uncertain and easy to destabilize. This is particularly true when broadband control is required. Active vibration isolation is plagued by such difficulties. In spite of much development work, few systems are in service and these remain very sensitive to structural resonances. For each such system, the control logic is fine-tuned during installation. When the system's environment changes (sometimes only slightly), unstable behavior is common. This presentation describes basic options in passive/active vibration isolation and highlights mechanical architectures which make robust active control possible. Simple theoretical arguments are supported with experimental evidence. The presentation concludes with a detailed description of a recently developed system which is based upon one such mechanical architecture; Barry Controls' STACIS-2000. This system uses seismic motion sensors (geophones) and piezo-ceramic stack actuators to create feedback-stabilized "artificial bedrock." Vibration-sensitive payloads are mounted to these stiff, quiet points. The system delivers the isolation performance of a well-damped $0.3-\mathrm{Hz}$ passive mount and the stiffness (to inertial space) of a $25-\mathrm{Hz}$ mount. The system is robust to the dynamics of both the vibration source and the receiver.

\section{0:15-10:30 Break}

\section{Contributed Papers}

\section{0:30}

2aEA6. Smart foam design and applications in active structural acoustic control. Cassandra A. Gentry, Cathy Guigou, and Chris R Fuller (Vib. and Acoust. Lab., Dept. of Mech. Eng., Virginia Polytech. Inst. and State Univ., Blacksburg, VA 24061-0238)

Acoustic foams possess inherent high-frequency sound absorbing capabilities for radiation and reflection control of structures. Comparatively, active noise control systems generally do not perform well at high frequencies due to the increasing complexity of the physical model and the necessary high speed digital signal processing. The "smart foam" concept and design originates from the combination of these two control strategies formulating a passive/active noise suppression device that can efficiently operate over a broad range of frequencies. In this research, the experimental device is comprised of a layer of piezoelectric material embedded in a partially reticulated polyeurethane foam. Depending on the specific application, the distributed actuator alters the surface impedance of the passive absorber such that an incident acoustic wave is totally reflected or absorbed. Error sensor configurations include microphones or piezoelectric sensors integrated directly into the noise suppression device creating a compact passive/active system. Experimental results are presented for piston radiation control. An analytical model of smart foam is described and simulations are presented to illustrate the potential of the device and the interaction between the passive and active components. 
2aEA7. Adaptive control of sound reflection. Samson Beyene and Ricardo Burdisso (Vib. and Acoust. Lab., Dept. of Mech. Eng., Virginia Tech., 203 Randolph Hall, Blacksburg, VA 24060)

The presence of an air cavity behind a layer of absorptive material creates an impedance-release boundary condition at the interface between the material and the air cavity. This boundary condition results in high absorption coefficients over relatively narrow frequency bands. These high absorption values depend on the depth of the air cavity which in tum is a function of frequency. Therefore, the impedance-release boundary condition occurs only at a few discrete frequencies. The active control system uses an adaptive feed-forward filtered- $x$ LMS control algorithm to maintain the impedance-release boundary condition over a broad frequency range. This is done by separating the incident and reflected vector intensity within the air cavity using two microphones and a wave deconvolution circuit. A speaker inside the cavity is used as an actuator to minimize the reflected intensity vector. The result is optimum absorption over a broad frequency range, including low frequencies. Very good agreement is found between numerical simulation and experimental results. The experimental results show high absorption coefficient $(0.8-1.0)$ over a wide frequency band $(100-2000 \mathrm{~Hz})$.

\section{$11: 00$}

2aEA8. Optimum design for feedforward active structural acoustic control of complex structures. Héctor $M$. Rodríguez, Ricardo A. Burdisso, and Chris R. Fuller (Vib. and Acoust. Lab., Dept. of Mech. Eng., Virginia Polytech. Inst. and State Univ., Blacksburg, VA 24061-0238)

An efficient design formulation for feedforward active structural acoustic control systems for complex structures and disturbances is presented. The approach consists in a multilevel optimization procedure. The upper level part is carried out in the modal domain where the optimum modal control forces and modal error sensor components which minimize the total radiated power are obtained. These optimum model parameters are then used in the lower level optimization to obtain the physical characteristics of the transducers to be implemented. This separation between the modal domain and the physical domain during the design process allows the designer to investigate different control system configurations in the upper level part with minimum computational effort. The formulation is capable to handle the design of multiple-inputs, multiple-outputs control systems and multiple frequency excitations. The proposed approach is demonstrated for the case of a simply supported plate excited by a periodic point force. The first eight modes are included in the analysis. Several different control system configurations are investigated. The results show that the optimum configurations yield reductions in the total radiated power of up to $26 \mathrm{DB}$, depending in the number of control channels and the amount of allowed control effort. [Work supported by the Office of Naval Research.]
2aEA9. Implementation of fast transversal filters for active structural acoustic control. Marcus J. Bronzel and Christopher R. Fuller (Vib. and Acoust. Lab., Virginia Tech., Blacksburg, VA 24061-0238)

The design of an optimal feedforward controller in active noise control applications for controlling the sound radiation from a vibrating structure can be seen as a system identification problem. Most control implementations use a stochastic gradient search algorithm to adaptively identify the impulse response of the optimal control filter. The convergence properties of the widely used filtered- $x$ LMS algorithm depend on the signal properties of the filtered reference signals. Sensing the reference signals from the vibrating structure is inevitable in most real applications and will effectively result in structural filtering of the unknown noise source. The filtered reference signals from a structural sensor generally exhibit a large eigenvalue spread resulting in poor convergence properties of the adaptive controller. A new extended-filtered- $x$ approach has been developed which makes the implementation of fast RLS type algorithms possible. These algorithms are based on an orthogonal projection of the predictor error into the subspace of the filtered reference signals and therefore converge independent from the underlying signal statistics. Experiments for active control of noise from a vibrating plate have been conducted which demonstrate the superior control performance of fast transversal filters.

\section{1:30}

2aEA10. Normalized stochastic gradient method for nonstationary environment. K. Lee and Jung G. Shin (Dept. of Elec. Eng. and Comput. Sci., Stevens Inst. of Technol., Castle Point on the Hudson, Hoboken, NJ 07030)

The normalized least-mean-squares (NLMS) method has been adopted for a wide range of adaptive filter applications due to its simple structure and low complexity of computation. However, under certain circumstances, the convergence rate may not be satisfactory. The performance may even be poorer under certain nonstationary situations. A new normalized stochastic gradient (NSG) method is suggested to overcome the drawbacks of the NLMS method. The main features of the NSG method are (1) the time varying convergence parameter and (2) the onset detection. While in the NLMS method the convergence parameter is fixed, it is a function of time in the NSG method. It is selected to minimize the conditional variance of the filter coefficients at the next step given a set of the previous coefficients. The time-varying sequence of the convergence parameter makes the NSG method converge fast. The new NSG method has the capability of the onset detection. It detects the statistical changes of the desired input and by resetting the adaptive procedure it keeps tracking the changed signal. The simulations show that the new NSG method outperforms the NLMS method under both stationary and nonstationary environments. [Work supported by Daewoo Motot Company, Seoul, Korea.] 


\title{
Session 2aMU
}

\author{
Musical Acoustics: The Bowed String \\ Roger J. Hanson, Chair \\ Physics Department, University of Northern Iowa, Cedar Falls, Iowa 50614
}

Chair's Introduction-8:25

Invited Papers

8:30

2aMU1. Musical oscillator simulations: From curiosity-driven science to commercial product. Robert T. Schumacher (Dept. of Phys., Carnegie Mellon Univ., Pittsburgh, PA 15213)

The first computer simulations of bowed string oscillations were done by McIntyre and Woodhouse in 1977. Generalizations to wind instruments followed within five years. The first commercial product using the principles of these simulations appeared in 1994. This talk will give a brief history of these developments, and will then concentrate on recent work on computer simulation of bowed string motion, much of which is presented in detail by other papers in this and an associated session. the relative success of bowed string simulations compared to simulations of wind instrument oscillations requires some explanation, which will be provided. The talk will conclude with a summary of the recent work by the author with James Woodhouse using fast computation to explore the enormous parameter space of the string instrumentalists' world.

\section{9:00}

2aMU2. Evaluation of transients through observations and simulations of bowed strings. Xavier Boutillon (Lab. d'Acoust. Musicale, C.N.R.S.-Université Paris 6, Case 161, 4 place Jussieu, 75252 Paris Cedex 05, France)

Despite their well-established importance on the perception of sound and their crucial role in music, transients are far less studied than steady-state regimes in musical instruments. How long a transient lasts is certainly a major question, among others: Is pitch variation noticeable? How different are transients from note to note, from one instrument to another? In order to investigate these questions, direct and reciprocal observations will be presented as well as various partial simulations: fully numerical simulations, tests with a computer-controlled bowing machine, simulations based on transfer functions. This variety of approaches is aimed at sorting out what could be attributed to the player, the bow, the string and the body of the instrument. The question of adequate signal processing or representation will be addressed and the relationship between the transient length and the quality of the instrument will be outlined. [Work supported in part by the French Ministry of Cullure.]

\section{9:30}

2aMU3. Some aspects of bow resonances-Conditions for spectral influence on the bowed string. Knut Guettler (Norwegian State Acad. of Music, Post Box 5190, Majorstua, 0302 Oslo, Norway)

The resonant bow seems to some extent capable of modifying the power spectrum of the bowed string, even during a steady-state Helmholtz motion, where only one slipping interval occurs during a fundamental period. Longitudinal resonances in the bow hair, strongly coupled to the transverse vibrations of the stick, are being excited by the changes of the frictional force which occur during each individual period. In general, these velocity fluctuations-superimposed on the steady bowing velocity-show small amplitudes compared to those of the string under the bow. In certain frequency regions however, dependent on the bow/bridge distance, impedances, etc., their energy content is sometimes great enough to cause noticeable modification of the string velocity. Such spectral changes would at any rate be small, but may still bear some acoustic significance. The present analysis is based on measurements on a violin bowed by a computer-controlled bowing machine, and supported by computer simulations. [Thanks are extended to Dr. Anders Askenfelt for sharing his experience in measuring bows, and to The Swedish Royal Institute of Technology for the use of their bowing machine and other laboratory facilities.]

\section{0:00}

2aMU4. Effects of brow-hair compliance on bowed-string motion. Roland Pitteroff and James Woodhouse (Cambridge Univ.. Eng. Dept., Trumpington St., Cambridge, U.K.)

Bow-hair compliance is likely to have a strong effect on bow-string interaction. The first line of evidence comes from analyzing experimental results presented by Cremer [L. Cremer, The Physics of the Violin (MIT, Cambridge, MA, 1985)] of the reflection and transmission behavior of transverse waves on a string incident on a bow at rest. The second line of evidence comes from simulations of the bowed string taking into account the finite width of the bow. The nature of the "differential slipping" which may arise due to the kinematical incompatibility of uniform bow velocity across the width of the bow and the string velocity in standard bowed-string motion (Helmholtz motion) is strongly dependent on bow-hair compliance. Simulations also demonstrate that suitable tilting of the bow can reduce the extent to which "differential slipping" is detrimental to the establishment of the desired string motion. Preliminary results of an experimental investigation of finite bow-width effects will be discussed. 


\section{Contributed Papers}

10:35

2aMU5. Mode frequency and damping changes due to chemical treatments of the violin bridge. Machele Bailey and George Bissinger (Phys. Dept., East Carolina Univ., Greenville, NC 27858)

Hammer-impact modal analysis using a microphone as a no-load vibration transducer was performed on violin bridge blanks chemically treated two different ways to extract free-free vibrational mode frequencies, dampings and shapes for comparison with untreated bridges. Data were taken for both in-plane (IP) and out-of-plane (OP) vibrations over a $0-20 \mathrm{kHz}$ range. Average effects of these chemical treatments on mode frequency and damping were determined from IP and OP single-point excitation on six Formalization (FA), six Resorcin/formaldehyde (RE) bridges, and eight untreated (UN) maple violin bridge blanks. All the bridges exhibited three well-defined IP modes and six OP modes. Few significant changes were observed between the frequency and damping parameters of the IP and OP mode shapes, although the RE treated bridge exhibited a possibly unique doublet at $\sim 9 \mathrm{kHz}$. Since no mode frequency variations $>1.5 \%$ were found, even though the RE treatment increased bridge masses $9.1 \%$, it was concluded that the combined mass/stiffness changes of the wood were related to the acoustic effects observed with these bridges [H. Yano and K. Minato, J. Acoust. Soc. Am. 92, 1222-1227 (1992)].

\section{Invited Papers}

2aMU6. Anomalous low-pitched tones from a bowed violin string. Roger J. Hanson (Phys. Dept., Univ. of Northem Iowa, Cedar Falls, IA 50614), Frederick W. Halgedahl (Univ. of Northem Iowa, Cedar Falls, IA 50614), and Knut Guettler (Norwegian State Acad. of Music, P.B. 5190, Majorstua, 0302 Oslo, Norway)

With a bow force greater than the Schelleng maximum and careful control, it will be demonstrated that it is possible to produce sounds on a violin of definite pitch ranging from approximately a musical third to a twelfth or more below the normal pitch. The lowered pitch is in agreement with the fundamental frequency of the observed harmonic series. The fundamental itself is very weak if the sounds are produced on the open G string. Mari Kimura has utilized the effect in performances [New York Times, 21 April 1994, p. B3, and Strings, Sept./Oct. 1994, 60-66]. These anomalous low frequencies (ALF) occur when the bow force is great enough to prevent the Helmholtz kink from triggering the normal release of the string from the bow hair. As a result of pronounced bow-nut and bow-bridge reflections there is at the bow a very complex string waveform, some portion of which regularly triggers the slipping of the string. ALF can also be produced on a bowed string mounted on a steel beam, where the motion is detected optically. Computer simulation is used to show how a string can be forced to vibrate at frequencies lower than the natural fundamental frequency of the string.

2aMU7. "Subharmonics:" An extended technique for the violin. Mari Kimura (Dept. of Music and Performing Arts Professions, School of Education, New York Univ., 35 West 4th Street, Suite 777, New York, NY 10012)

It has been found that unusual ways of drawing the bow on the violin could produce pitches that are as low as one octave below the fundamentals. The technique was developed from a bowing exercise meant to improve sound production by scratching harder on the string. After much practicing, all the audible transient noises were eliminated including the fundarnentals, so that only those lower notes could be heard. Then the sounds were polished into steady, clear, and loud "subharmonic" pitches, suitable for musical purposes. It was not until April 1994, that these "subharmonics" were publicly introduced as a musical element at Kimura's New York debut recital in the third movement of ALT for solo violin by Kimura, which will be performed during this presentation. In ALT, "subharmonics" were incorporated into the music, requiring precise control of the bow in order to freely switch between the new technique and the usual bowing. The musical effect was noted as a "revolutionary bowing technique... astonishing effect" [E. Rothstein, The New York Times (21 April 1994)]. It was also found that seconds and thirds below the fundamentals can be produced in a similar manner [R. Neuwirth, Strings, Sept./Oct. (No. 44, 1994)]. 


\title{
Session 2aPP
}

\section{Psychological and Physiological Acoustics: Auditory Perception and Physiology (Poster Session)}

\author{
Donna L. Neff, Chair \\ Boys Town National Research Hospital, 555 North 30th Street, Omaha, Nebraska 68131
}

\section{Contributed Papers}

\begin{abstract}
All posters will be on display from 7:45 to 11:45 a.m. To allow contributors an opportunity to see other posters, contributors of odd-numbered papers will be at their posters from 7:45 to 9:45 a.m. and contributors of even-numbered papers will be at their posters from 9:45 to 11:45 a.m.
\end{abstract}

2aPP1. The effect of extraneous stimuli on sample discrimination for frequency. Donna L. Neff and Christina J. Kessler (Boys Town Natl. Res. Hosp., 555 N. 30th St., Omaha, NE 68131)

Four listeners completed 2JFC sample-discrimination tasks for frequency (SDF), in which they judged which of two tones or tone pairs was drawn from the higher of two frequency distributions. The distributions were separated in mean frequency by $200 \mathrm{~Hz}(100-\mathrm{Hz}$ s.d.). The main conditions examined SDF at $2100 / 2300 \mathrm{~Hz}$, in isolation or with randomfrequency tones (Gaussian distributed, $100 \mathrm{~Hz}$ s.d.), fixed-frequency tones, or noise bands (600-Hz wide) added on both sides of the SDF region, or a single random-frequency tone added on the high or low side. Stimuli were equated in total power. Frequency position of the extraneous stimuli relative to the SDF region was varied. Flanking random-frequency tones produced the largest decrements in performance, with most listeners showing little recovery even at maximum distance from the SDF region. A single random-frequency tone below the SDF region degraded performance more than one presented above. Flanking fixed-frequency tones also produced large performance decrements that were relatively independent of distance. Noise bands produced little interference unless they overlapped the SDF region. Thus SDF can be affected by distant extraneous tonal stimuli, particularly those sharing similar patterns of variation. [Work supported by NIDCD.]

2aPP2. Informational masking in the identification of simple auditory patterns. Gerald Kidd, Jr., Tanya L. Rohtla, and Christine R. Mason (Dept. of Commun. Disorders, Boston Univ., 635 Commonwealth Ave., Boston, MA 02215)

Listeners identified six auditory patterns comprised of eight sequential tone bursts [cf. Kidd et al., J. Acoust. Soc. Am. 95, 2916(A) (1994)]. Several factors affecting performance were examined including center frequency, level and the size of the frequency steps forming the patterns. In masked conditions, a multitone masker was played synchronously with each pattern element. The components of the multitone masker were chosen randomly on every burst within a presentation interval. Masker components were excluded from a region of $20 \%$ of the pattern center frequency to minimize "energetic masking." The results in quiet indicated that identification performance was nearly perfect for pattern frequency steps as small as $1 \%$ and at levels as low as $20 \mathrm{~dB}$ SL. Considerable "informational masking" was produced by the random multitone masker bursts. The masked identification-level functions were shallow, rising on average about $2 \%$ per $\mathrm{dB}$. Several signal and masker presentation schemes were examined that were intended to reduce the observed masking. To date, the most effective means for improving signal pattern identification has been to present the signal to one car and identical maskers to both ears with the masker leading in time $(8 \mathrm{~ms})$ in the ear contralateral to the signal. [Work supported by NIH.]

2aPP3. The influence of pattern-based frequency expectations on resolving power for components of multitone sequences. Gary $\mathbf{R}$. Kidd (Dept. of Speech and Hear. Sci., Indiana Univ., Bloomington, IN 47405)

The effect of deviations from frequency expectations on resolving power for components of tonal sequences was studied with a method similar to that used earlier to determine the effect of temporal deviations [G. R. Kidd, J. Acoust. Soc. Am. 95, 2966(A) (1994)]. In a same-different task, listeners were asked to compare the last two of four twelve-tone patterns presented on each trial. The first two patterns were identical and served to establish expectations from which the third and fourth pattems could deviate. In the third pattern, the frequency of a single "target" tone was lowered by either 3 or 7 semitones, or left unchanged. The frequency change to be detected was added to the target component in the fourth pattern on half the trials. Thresholds for this frequency increment were estimated for each deviation condition (as defined by the target-tone frequency used in the third pattern). Just as with deviations from temporal expectations, deviations from frequency expectations produced threshold increments that increased with the size of the deviation. These findings suggest that the superior discriminability typically observed with farmiliar patterns is due to the appropriate targeting of attention in frequency and in time during pattern presentation. [Work supported by NIDCD.]

2aPP4. Modeling the perceived urgency of multitone signals. Ellen C. Haas (Human Research and Engineering Directorate, U.S. Army Research Lab., Bldg. 520, Aberdeen Proving Ground, MD 21005) and Judy Edworthy (Univ. of Plymouth, Plymouth, United Kingdom)

In some environments, there is a serious mismatch between the perceived (psychoacoustic) urgency of a warning and its situational urgency (the urgency associated with the situation or condition that the signal represents). Several parameters which were believed to affect the perceived urgency of multitone auditory warning signals were investigated in a factorial experiment. These parameters were fundamental frequency $(200,500$, and $800 \mathrm{~Hz})$, time between signals $(0,300$, and $500 \mathrm{~ms})$, and signal level $(5,25$, and $40 \mathrm{~dB}$ Lin SPL above the ambient noise level of a sound-treated chamber). The stimuli were 27 auditory signals, each signal consisting of a train of four repetitions. Free-modulus magnitude estimation quantified the relationship between auditory signal parameters and changes in perceived urgency. The effect of the three variables on per- 
ceived urgency will be discussed. The results of this experiment will be used in the development of a mathematical model in which the relationship between signal parameters and perceived urgency can be defined.

2aPP5. IWAIF model predictions of a profile analysis task. Mark A. Ericson, Lawrence L. Feth (Dept. of Speech and Hear. Sci., Ohio State Univ., Columbus, $\mathrm{OH} 43210$ ), and Ashok K. Krishnamurthy (Ohio State Univ., Columbus, $\mathrm{OH} 43210$ )

A profile analysis task was chosen to test the predictions of a multichannel IWAIF model. A 2Q, 2AFC three up-one down, adaptive staircase procedure was incorporated with a $20-\mathrm{dB}$ roving signal level to determine the detection threshold of a $1-\mathrm{kHz}$ target tone in each profile. The masker profiles included 11 adjacent of the 21 possible components ranging from 200 to $5012 \mathrm{~Hz}$. The components of the profile were spaced logarithmically at frequency ratios of $1: 1.18$ so that no two components fell into any one critical band. The fixed frequency target tone was placed at either the second lowest, middle, or second highest component in each 11 component profile. The IWAIF model predictions are compared with channel theory predictions [Durlach et al., J. Acoust. Soc. Am. 80, 63-72 (1986)] for listener performance in the profile analysis task. [Work supported by AFOSR.]

2aPP6. Identification of the incremented component in profile stimuli. Hedwig Gockel and Hans Colonius (Graduate College "Psychoacoustics," FB 5, Dept. of Cagn. Psychol., Carl-von-Ossietzky Univ. of Oldenburg, D-26111 Oldenburg, Germany)

The ability to identify the frequency of the incremented component in a multitone complex was assessed in two conditions, both using the same 11-tone complexes (fixed frequency, equilog spaced, random phases). All components had equal amplitude, except one of the inner 9 , which was incremented. The overall level was randomly varied on each presentation. In condition $\mathrm{A}$ the tone complex, with the increment position randomized, was followed by a sinusoid either at the increment frequency or at that of an adjacent component (below or above). In condition B the same complex with flat power spectrum was additionally presented on each trial. The sequence of flat and incremented profile stimuli was randomized over the first two presentation intervals, followed by the sinusoid. In both conditions subjects had to indicate whether the frequency of the incremented component was same or different from that of the sinusoid. Thresholds for these two conditions were estimated by the use of an adaptive procedure and compared to the thresholds of the same subjects in discriminating between the flat and an incremented profile (with signal uncertainty). Results indicate that identification ability is at least as good as discrimination. [Work supported by DFG.]

2aPP7. Discrimination of static versus dynamic, and log versus harmonic profiles. Charles S. Watson and Ward R. Drennan (Dept. of Speech and Hear. Sci., Indiana Univ., Bloomington, IN 47405)

"Profile" stimuli consisting of multiple simultaneous fixed-frequency sinusoidal components are more representative of naturally occurring sounds than the spectrally simpler waveforms more often used in psychoacoustic experiments. However, most naturally occurring sounds are characterized by dynamic rather than static spectra, and by harmonically spaced rather than the log-spaced components used in most profile experiments. In preparing to study a variety of dynamic profiles, the discriminability of static and frequency-glide profiles was determined, using both log- and harmonically spaced components. Discriminations were based on the detection of an intensity increment added to the mid-frequency component of 11-component, 400-ms profiles. Each profile had a starting frequency range of 200 to $2000 \mathrm{~Hz}$. Dynamic profiles increased in frequency continuously over their 400 -ms durations. Each subject was run under four stimulus conditions (static-log, static-harmonic, dynamic-log, dynamicharmonic) generally for a minimum of 2000 trials per condition, in an adaptive-tracking procedure. Mean differences between asymptotic thresholds for the stimulus conditions were small compared to differences among the subjects. Harmonic yielded somewhat lower thresholds than the log spaced components, while very modest differences, if any, were found between static and dynamic profiles. [Work supported by grants from NIH/ NIDCD and AFOSR.]
2aPP8. Pitch perception based on a temporal energy rate model. Henning Reetz (Allgemeine Sprachwissenschaften, Univ. of Konstanz, Postfach 5560 (D186), D-78 464 Konstanz, Germany)

Theories about the mechanisms of human's pitch perception either are based an the temporal firing rate of the haircells in the basilar membrane or on the place of these cells in the membrane in a sort of spectral representation. The insensitivity to the phase of individual components of a complex signal and the ability to perceive pitch for binaural presented harmonics. has given experimental evidence for place-based pitch perception models. This paper explains theses findings with an alternative temporal rate model. The proposed explanation is based on a model of the inner ear that computes the energy of an acoustic signal in the time domain, leading to a temporal energy distribution of the signal. This temporal energy distribution is converted by a simple decision logic to a pitch value. The model will be theoretically discussed, reanalyses of reported experimental findings are presented, new experimental evidence for the proposed model will be given, and a computer program to determine the pitch of a speech signal based on the model's principles will be evaluated.

2aPP9. Effects of interaction of low- and high-frequency tone complexes on periodicity pitch perception. Sridhar Krishnamurti and John W. Hawks (School of Speech Pathol. and Audiol., Kent State Univ., Kent, OH 44242)

The effects of interaction of low-frequency tone complexes and highfrequency tone complexes on the thresholds of discrimination (DLs) for a change in periodicity pitch in one of the two frequency regions were studied. Stimuli were digitally constructed by summing sine waves spaced at $100-$ or $200-\mathrm{Hz}$ intervals. The amplitude of components of one of the interacting complexes was fixed while the periodicity pitch of the other complex was obtained by decrementing amplitude of components in 1-dB steps. DLs were obtained for (1) low-frequency complexes only, (2) highfrequency complexes only, (3) low-frequency complexes in the presence of high-frequency complexes, and (4) high-frequency complexes in the presence of low-frequency complexes. Preliminary results indicate elevated DLs for high-frequency complexes in condition 2 and elevated DLs (significant interference effects) in conditions 3 and 4 . Interference effects were greater for condition 4 relative to condition 3 . Implications in terms of temporal fine structure and envelope cues are discussed.

2aPP10. Difference limens for roved glides. John P. Madden (Dept. of Commun. Disorders, Univ. of North Dakota, University Station, Grand Forks, ND 58202-8040)

The study determined glide difference limens (GDLs) for up-glides in several conditions and estimated how well a level-detection model of the auditory periphery could account for the data. Signal durations were 50 , 100 , and $200 \mathrm{~ms}$, and the average stimulus center frequencies were 1 and $4 \mathrm{kHz}$. To avoid the confounding effect of between-stimuli static pitch cues, the center frequencies of the standard and target stimuli were "roved" about the center frequencies [Neill and Feth, J. Acoust. Soc. Am. Suppl. $187, S 23$ (1990)]. The standard signals increased in frequency by 0,200 , and $400 \mathrm{~Hz}$. The subject's task was to distinguish between the standard and a target signal with a greater increase in frequency in a 2 -alternative, forced-choice task. The increase in the target signal was varied adaptively until discrimination threshold was obtained. The results for the $0-\mathrm{Hz}$ standard were consistent with previous findings that the change in $\mathrm{Hz}$ at threshold is constant across duration in a glide-detection task. The results for the 200 and $400 \mathrm{~Hz}$ standards extend this finding to a glide-discrimination task. The modeling results suggest that the auditory system tracks gliding signals by monitoring the change in level of the output of the auditory filters. [Work supported by NIDCD.]

2aPP11. Pitch-level functions for pure tones in the presence of partial masking. Edward M. Burns (JG-15, Univ. of Washington, Seattle, WA 98185)

The purpose of this experiment was to determine if the pitch changes of pure tones with changes in level are dependent on the spread of excitation with increasing level. Pitch-level functions were obtained in quiet, and in the presence of band-reject masking noise, for frequencies ranging from 250 to $8000 \mathrm{~Hz}$. Both the spectrum level of the masking noise and the width of the the band-reject region surrounding the pure tones were para- 
metrically varied. In addition, estimates of the amount of partial masking for each condition were obtained from loudness matches. Preliminary results suggest that, for levels above $60 \mathrm{~dB}$ SPL, the pitch shifts associated with partial masking usually reduce or eliminate pitch changes with level. [Work supported by NIDCD and the Virginia Merrill Bloedel Hearing Research Center.]

2aPP12. Frequency discrimination in noise by untrained listeners. Lynne A. Werner (Dept. Speech \& Hear. Sci., JG-15, Univ. of Washington, Seattle, WA 98195)

The frequency discrimination performance of adults, with no prior experience as listeners in psychoacoustic experiments, was examined for tones of 1000 and $4000 \mathrm{~Hz}$ presented in a background of broadband noise. The tones were presented at a signal-to-noise ratio expected to produce $80 \%$ correct detection. The level of the stimuli was either 45 or $60 \mathrm{~dB}$ SPL. The subjects were tested in a procedure similar to one used in infant psychoacoustics [Olsho et al., Devel. Psychol. 23, 627-640 (1987)]. Threshold values of $\Delta f$ were found to be more similar to those of welltrained listeners at $4000 \mathrm{~Hz}$ than at $1000 \mathrm{~Hz}$. The effects of stimulus level were similar to those reported by Dye and Hafter [J. Acoust. Soc. Am. 67, 1748-53 (1980)] for well-trained listeners: Threshold $\Delta f$ increased with increasing level at $4000 \mathrm{~Hz}$, but not at $1000 \mathrm{~Hz}$. This result suggests that the effects of level on frequency discrimination in noise are robust enough to be replicated in subjects with little listening experience. Further, the effects of level on human infants' frequency discrimination in noise may provide important information about why their frequency discrimination in quiet is more adultlike at high frequencies. [Work supported by NIDCD DC00396.]

2aPP13. On the perceptual asymmetry in frequency modulation discrimination. Tao Zhang, Lawrence L. Feth, and Ashok K. Krishnamurthy (Dept. of Speech and Hear. Sci. \& Elec. Eng., Ohio State Univ., Columbus, OH 43210)

Several researchers have observed perceptual asymmetry in frequency modulation discrimination experiments. Porter et al. (1991) found that when listening to a formant transition followed by a frequency plateau, listeners detected the onset frequency much better for the falling transition than for the rising transition. Demany and McAnally (1994) discovered that the frequency difference limen measured at frequency maxima of a $5-\mathrm{Hz}$ cosine frequency modulation is significantly smaller than at frequency minima. Heil et al. (1992) also observed asymmetry in the FM response in chick auditory cortex. They proposed that this effect was due to the asymmetrical lateral inhibition in the unit. Preliminary results in this lab indicated that this perceptual asymmetry also exists when listeners were asked to discriminate between a sinusoidal plus linear ramp frequency modulation (SLRFM) and a linear ramp frequency modulation (LRFM) (Zhang et al., 1994). In this presentation, perceptual asymmetry is systematically studied for both rising and falling LRFMs. The effect of starting phase of a SLRFM signal is also examined. The experimental results are discussed in terms of existing modulation models and physiological explanations. [Work supported by AFOSR.]

2aPP14, Stimulus-driven, time-varying weights for comodulation masking release. Søren Buus (Commun. and Digital Signal Processing Center, Dept. of Elec. and Comput. Eng., 409 DA, Northeastern Univ., 360 Huntington Ave., Boston, MA 02115-5096), Lei Ji Zhang, and Mary Florentine (Northeastern Univ., Boston, MA 02115)

This study tests the hypothesis that CMR is mediated by "listening in the valleys" [S. Buus, J. Acoust. Soc. Am. 78, 1958-1965 (1985)]. Detectability was measured for signals consisting of 6 consecutive $25-\mathrm{ms}$, $1-\mathrm{kHz}$ tone bursts presented in a $50-\mathrm{Hz}$ wide masker or in maskers consisting of seven $50-\mathrm{Hz}$ wide noises, one critical band apart, with either correlated or uncorrelated envelopes. Each burst varied randomly around masked threshold according to Gaussian distributions with 3- or 6- $\mathrm{dB}$ standard deviations. For each listener and condition, the responses from 5000 trials were sorted to construct conditional psychometric functions for $d^{\prime}$ as a function of burst energy for 10 ranges of short-term level of the on-frequency masker band during the burst. The slopes of these functions for three normal listeners decrease markedly with increasing short-term masker level for the correlated multiband masker, but are largely constant for the other maskers. This indicates that the weight applied to the signal channel is high when the masker level is low and vice versa for the correlated masker, but is approximately constant for single-band and uncorrelated mutliband maskers. This finding provides direct evidence that CMR is mediated by "listening in the valleys." [Work supported by NIHNIDCD R01DC00187.]

2aPP15. Temporal resolution and CMR can depend upon frequency, Sid P. Bacon and Jungmee Lee (Psychoacoust. Lab., Dept. of Speech and Hear. Sci., Arizona State Univ., Tempe, AZ 85287-1908)

An estimate of temporal resolution can be obtained by subtracting the signal threshold obtained in the presence of a modulated masker from that obtained in the presence of an unmodulated masker (yielding the modulated-unmodulated difference, or MUD). An estimate of comodulation masking release (CMR) can be obtained by measuring the MUD with both a broadband and critical-band masker. The present study obtained MUD and CMR for signal frequencies from 250 to $4000 \mathrm{~Hz}$. The masker was unmodulated or sinusoidally amplitude modulated at rates from 2 to $16 \mathrm{~Hz}$. For all rates and both masker bandwidths, the MUD increased monotonically with signal frequency; it was larger for the broadband masker, indicating an across-channel CMR. The CMR increased from a few dB at $250 \mathrm{~Hz}$ to $14 \mathrm{~dB}$ at $4000 \mathrm{~Hz}$. The frequency effect (for MUD and $\mathrm{CMR}$ ) was considerably reduced when the modulation depth $(\mathrm{m})$ of the masker was decreased from 1.0 to 0.9 , and was essentially eliminated at a depth of 0.5 . The results of a forward-masking experiment using the unmodulated masker at both masker bandwidths suggest that the frequency effect is due to differences in the recovery of forward masking, and that suppression can influence CMR (and MUD) at very large modulation depths. [Work supported by NIDCD.]

2aPP16. Modeling modulation perception: Modulation low-pass filter or modulation filter bank? T. Dau, B. Kollmeier (Graduate College 'Psychoacoustics', FB 8, Postbox 2503, University of Oldenburg, 26111 Oldenburg, Germany), and A. Kohlrausch (IPO, Eindhoven, The Netherlands)

In current models of modulation perception, the stimuli are first filtered and nonlinearly transformed (mostly half-wave rectified). In order to model the low-pass characteristic of measured modulation transfer functions, the next stage in the models is a first-order low-pass filter with a typical cutoff frequency of 50 to $60 \mathrm{~Hz}$. From physiological studies in mammals it is known that many neurons in, e.g., the inferior colliculus, show a bandpass characteristic in their sensitivity to amplitude modulation. Results from psychophysical studies of modulation masking also suggest some kind of bandpass analysis of modulation frequencies. Results of two experiments on modulation detection that allow discrimination between models incorporating a low-pass filter and those using a modulation filterbank are presented. In the first experiment, modulation detection thresholds were measured for noise carriers of bandwidths between 3 and $6000 \mathrm{~Hz}$. In the second experiment, modulation detection for a sinusoidal carrier was measured in the presence of interfering modulation components with a bandpass characteristic in the modulation spectrum. The results from these experiments could not be simulated by a model including a modulation low-pass filter, but were successfully simulated by a model using a modulation filterbank.

2aPP17. Gating effects in CMR. J. W. Hall, III, J. H. Grose, and D. R. Hatch (Div. Otolaryngol./Head \& Neck Surgery, Univ. North Carolina at Chapel Hill, Chapel Hill, NC 27599-7070)

CMR is sometimes smaller for gated than continuous maskers. Our current work on CMR for multiple, comodulated narrow noise bands indicates that the gating effect is minimized when the number of comodulated bands is large. The present study extends this work to the CMR paradigm where pure tone signal thresholds are obtained as function of modulated and unmodulated noise bandwidth. Results indicate that in unmodulated noise, gated and continuous thresholds do not differ, regardless of noise bandwidth. In modulated noise, thresholds are often higher for gated noise than continuous noise when noise bandwidth is narrow, but not when the noise bandwidth is considerably wider than the auditory filter bandwidth. These results may suggest that gating effects on CMR are 
generally small when comodulation information is available across a large number of auditory channels. Results will be discussed in terms of competing cues for auditory grouping. [Research supported by AFOSR.]

2aPP18. Multiband detection of energy fluctuations. J. H. Grose and J. W. Hall, III (Div. Otolaryngol./Head \& Neck Surgery, Univ. North Carolina at Chapel Hill, Chapel Hill, NC 27599-7070)

The filtering properties of the ear permit the auditory periphery output to be viewed as a bank of parallel quasi-independent channels. The purpose of this study was to examine how information contained among the independent channels is combined. Four related experiments were undertaken in which the detection of an energy change within a channel was measured as a function of the number of channels carrying that change. Each experiment examined the detection of a different signal type: (1) The detection of multitone signals masked by narrow bands of noise; (2) the detection of intensity increments (intensity discrimination) for multiple noisebands; (3) the detection of intensity decrements for the same stimuli as (2); and (4) the detection of temporal gaps in multiple noisebands. Eight normal-hearing listeners participated and performance was measured for a 1-, 2-, 4-, and 8-channel stimulus. The center frequencies for the 8 channels were 356, 494, 663, 870, 1125, 1442, 1838, and $2338 \mathrm{~Hz}$. For each signal type, performance improved with increasing number of channels carrying the signal. The comparative patterns of improvement will be discussed in terms of multiband detection models. [Work supported by the NIDCD R01-DC01507.]

2aPP19. Synthesis of common-envelope signal pairs. Jayanth Anantharaman, Ashok K. Krishnamurthy, and Lawrence L. Feth (Depts. of Elec. Eng. \& Speech and Hear. Sci., 2015 Neil Ave., Ohio State Univ., Columbus, $\mathrm{OH} 43210$ )

A large class of sounds can be described in terms of rapid pressure fluctuations known as the "fine structure" and relatively slower amplitude changes called the "envelope." Signals having the same envelope and occupying the same range of frequencies, but differing in their fine structure, are important to the study of fine structure perception. Voelcker's [Proc. IEEE 54, 340-353, 735-755 (1966)] complementary two-tone complex pair is one of the simplest signals in this class, and has been used in a number of pitch related experiments. As an extension of these ideas, a computational algorithm is described to generate a common-envelope pair occupying the same range of frequencies of any bandlimited signal. Several properties of common-envelope signals are presented and methods of combining these signals to create other common-envelope signals are given. A few examples of multicomponent common-envelope signals are spectrally reversed linear-spaced complexes, spectral ramps, and multiple Voelcker two-tone pairs. Applications of these signals to psychoacoustic experiments are discussed. [Work supported by AFOSR.]

2aPP20. Roughness and the critical bandwidth at low frequency. Jian-Yu Lin and William Morris Hartmann (Dept. of Phys., Michigan State Univ., East Lansing, MI 48824)

A plausible model represents the sensation of roughness as the product of three factors, a speed factor that increases with increasing modulation rate, a temporal modulation transfer function (TMTF), and a spectral window that is determined by auditory filter widths. At high signal frequency, where critical bands are broad, the TMTF is the controlling factor. At low signal frequency the auditory filters dominate. Therefore, roughness experiments can serve to measure the critical bandwidth at low frequency. To implement this idea, listeners adjusted both the modulation rate for $\mathrm{AM}$ signals and the beat rate of beating sine pairs in order to achieve maximum roughness. The center frequency varied from 2000 to $70 \mathrm{~Hz}$, and SPLs were 60 and $80 \mathrm{~dB}$. The data could be fitted with a model in which roughness is summed over all auditory filters. The data require the critical bandwidth parameter to continue to decrease with decreasing frequency below $500 \mathrm{~Hz}$ so that it becomes considerably narrower than critical bands from the bark scale. [Work supported by the NIDCD.]
2aPP21. Concurrent amplitude modulation detection with two carriers. Stanley Sheft and William A. Yost (Parmly Hearing Inst., Loyola Univ., 6525 N. Sheridan Rd., Chicago, IL 60626)

The ability to detect amplitude modulation (AM) of two concurrent tonal carriers was measured in a 4 AFC task; each carrier was independently modulated during one of two observation intervals. Carrier frequencies were 700 and $2350 \mathrm{~Hz}$ and AM rates were 4, 16, and $200 \mathrm{~Hz}$. In the concurrent detection task, the modulation depths were either 1.0 or set for a detection $d^{\prime}$ of 0.7 or 2.0 with modulation of just one carrier. Results showed large performance decrements in the concurrent detection procedure that were greater than typically obtained in other two-channel listening tasks. Partitioning of the $4 \times 4$ response matrix indicated interaction between carrier channels with performance generally better when both tones were simultaneously modulated and for most subjects at the greater modulation depths when both responses were correct. Cross-channel estimates of correlation derived from the bivariate normal distribution were highly dependent on the subject's response strategy. When both tones were modulated at the same rate, results are consistent with past work indicating difficulty associating modulation with its appropriate carrier. Overall results indicate limited ability to independently process two concurrent sources of modulation information. [Work supported by NIH.]

2aPP22. Identification of flat-spectrum vowels on the basis of amplitude modulation. Brian C. J. Moore and Joseph I. Alcántara (Dept. of Exp. Psychol., Univ. of Cambridge, Downing St., Cambridge CB2 3EB, England)

These experiments investigate whether amplitude modulation of "formants" allows vowel sounds to be identified. The periodic complex sounds had a fundamental frequency of $100 \mathrm{~Hz}$ and a level of $85 \mathrm{~dB}$ SPL. Harmonics were added either in cosine or random phase. Harmonics with frequencies below $3000 \mathrm{~Hz}$ were equal in mean amplitude. Three pairs of successive harmonics, located at the first, second, and third formant frequency values of six possible vowels, were sinusoidally amplitude modulated. For a $10-\mathrm{Hz}$ modulation rate, vowel identification of cosine-phase stimuli improved with increasing modulation index, $m$, up to $m=0.4$, after which it reached an asymptote of about $80 \%$. For the random-phase stimuli, performance was essentially at chance for all modulation depths, despite the fact that the amplitudes of the "formant" harmonics were greater than those of "background" harmonics at certain points of the modulation cycle. For a $2-\mathrm{Hz}$ modulation rate, performance improved for the random-phase stimuli, but remained below that for the cosine-phase stimuli. Modulating the "formants" at different rates or modulator phases made performance worse for random-phase stimuli but had little effect for cosine-phase stimuli. The results are interpreted in terms of the cochlearfiltered waveforms and in terms of perceptual grouping mechanisms.

2aPP23. Effects of amplitude envelope expansion and compression on nonsense sentence recognition. Richard L. Freyman, Debra Speicher, and G. Patrick Nerbonne (Dept. of Commun. Disorders, Univ. of Massachusetts, 6 Amold House, Amherst, MA 01003)

When spectral information in speech is obscured, listeners presumably fall back on secondary cues such as those containted within the gross waveform envelope. This study examined the extent to which listeners' ability to use such cues is resistant to distortions of the envelope. Signal correlated noise samples, created individually to follow the amplitude envelopes of 200 nonsense sentences, were used as maskers of those sentences, with signal-to-noise ratios of $-4,-2,0$, and $+2 \mathrm{~dB}$. The resulting waveforms preserved the original wideband envelopes, but spectral information was degraded to a degree determined by the S-N ratio. The sentences were presented monaurally to nornal hearing subjects at $70 \mathrm{~dB}$ SPL. Recognition of key words in these sentences was compared to that obtained for waveforms that had been processed with two different levels 
of amplitude expansion and two levels of compression. The results indicated that amplitude expansion severely reduced recognition scores, while compression had little effect. [Work supported by NIH \#DC01533.]

2aPP24. Defining features of steady-state timbres. Michael D. Hall (Dept. of Speech and Hear. Sci. W1-10, Univ. of Washington, Seattle, WA 98195) and Richard E. Pastore (Dept. of Psychol., State Univ. of New York at Binghamton, Binghamton, NY 13902-6000)

While several dimensions contribute to voice and instrument timbre, perceptual interactions between dimensions have not been adequately assessed. As a result, the information used in timbre identification is unclear. Three experiments sought to define steady-state timbral features. First, Gamer classification reflected the perceptual integrality of spectral slope and formant structure (a source and filter property, respectively); classification speed along either dimension depended upon variability along the other dimension. Feature detection then was evaluated within arrays of distractor pitches with homogeneous timbre. When targets had /á/ vowel formants with shallow spectral slopes, search time increased as a log function of array size consistent with parallel processing. Parallel search was not obtained for targets with /// formants and steep slopes, suggesting a feature coded as the presence or absence of $/ a$ formants with shallow slopes. A final search experiment using heterogeneous distractor timbres showed that this feature was separable from pitch. Search times for conjunctions of pitch and timbre linearly increased with array size, implying serial processing. Thus attention was needed to conjoin attributes to perceive tonal objects. Implications are discussed for the nature of attention and the perception of properties relevant to communication. [Work supported by AFOSR.]

2aPP25. Individual differences in speech and nonspeech processing among normal-hearing subjects. Aimee M. Surprenant (Dept. of Psychol. Sci., Purdue Univ., 1364 Psychol. Sci. Bldg., West Lafayette, IN 47907-1364) and Charles S. Watson (Indiana Univ., Bloomington, IN 47405)

While a large portion of the variance among listeners in auditory speech processing is associated with the audibility of components of the speech waveform, it is not possible to predict individual differences in speech perception strictly from the audiogram. Psychoacoustic measures of spectral-temporal acuity with nonspeech stimuli also have been shown to correlate only weakly (or not at all) with speech processing. In a replication and extension of an earlier study (Watson et al., J. Acoust. Soc. Am. Suppl. I 71, S73) 100 normal-hearing college students were tested on speech perception tasks (nonsense syllables, words, sentences in a noise background) and on 6 spectral-temporal discrimination tasks using simple and complex nonspeech sounds. Factor analysis showed that the abilities that explain performance on the nonspeech tasks are quite distinct from those that account for performance on the four speech tasks. Performance was significantly correlated among speech tasks, and among nonspeech tasks. Either, (a) auditory spectral-temporal acuity for nonspeech sounds is orthogonal to speech processing abilities, OR (b) we have yet to identify the appropriate task or types of nonspeech stimuli that exercise the abilities required for speech recognition. [Work supported grants from NIH and AFOSR to Indiana University.]

2aPP26. Shrinking illusion of speech duration caused by noise/gap replacement. Minoru Tsuzaki and Hiroaki Kato (ATR Human Information Processing Res. Labs., 2-2 Hikaridai, Seika-cho, Soraku-gun, Kyoto, 619-02 Japan)

To investigate the process of perceptual resynthesis of an "auditory object," the apparent duration of a noise-replaced speech signal was measured. Subjects adjusted the duration of synthesized speech to subjectively match that of the target speech. When a portion of the target speech was replaced by a noise burst, the duration was perceived to be shorter than when no replacement was made. Besides this effect, the apparent duration of speech segments was found to be affected by their deviation from the internal template. The greater the physical duration of the target deviated from the "naturally" spoken token, the greater the degree of underestimation became. Interestingly, these two effects were independent of each other. This independence was replicated in the second experiment which tested wider range of durational deviations. Again, the apparent duration of noise-replaced speech was shorter than when no replacement was made. The apparent duration of gap-replaced speech was also measured in this experiment. The degree of underestimation was far greater for the latter case. These findings suggest that a shrinkage of duration occurs in the process of resynthesizing an "auditory object" where the process is assumed to be at a different stage than that utilizing speech-specific schema.

2aPP27. The effects of incremental change in temporal envelope correlation on auditory fusion. John R. Carter, Patrick W. Rappold (Dept. of Speech Pathol. and Audiol., Univ. of South Alabama, Mobile, AL 36688), and Madhuri S. Muelkar (Univ. of South Alabama, Mobile, AL 36688)

The effects of incremental change in degree of temporal envelope correlation on the auditory fusion of narrow-band noise (NBN) pairs was examined by placing fusion in competition with sequential streaming for one component of a NBN pair. Seven levels of envelope correlation were represented $(r=1.0,0.75,0.50,0.25,0.00,-0.25,-0.35)$ by three sets of NBN pairs, for a total of 21 NBN pairs. A NBN pair consisted of a target-band spectrally centered at $2000 \mathrm{~Hz}$, and a flanker-band centered at $1500 \mathrm{~Hz}$. A NBN pair was played alternately with the target-band alone (captor-band) at an initial interstimulus-interval (ISI) of $500 \mathrm{~ms}$. The ISI decreased automatically in 5- or 2-ms increments until a subject exited the program when judging the perceptual prominence of sequential streaming as greater than that of fusion. The ISI at exit was used to infer strength of fusion for the NBN pair. Shorter ISIs suggested stronger fusion; longer ISIs suggested weaker fusion. Results showed a trend for ISI to increase with a decrease in the degree of envelope correlation, which suggested a gradual decline in fusion strength as envelope correlation was incremented downward from perfect correlation.

2aPP28. Attention and grouping in vowel perception. R. W. Hukin and C. J. Darwin (Lab. of Exp. Psychol., Univ. of Sussex, Falmer, Brighton BN1 9QG, UK)

The effective contribution of a $500-\mathrm{Hz}$ component of a steady-state vowel was measured by the position of the vowel boundary on a $/ \mathrm{I} /-/ \mathrm{e} /$ continuum varying only in first formant $(F 1)$ frequency. Shifts in the vowel boundary were calibrated against those produced by level changes in the $500-\mathrm{Hz}$ component. When the $500-\mathrm{Hz}$ component was given an ITD of $666 \mu$ s and the remaining components an ITD of $-666 \mu \mathrm{s}$, there was a small but significant reduction in the $500-\mathrm{Hz}$ component's contribution to phonetic quality. When the vowel was embedded in a sequence of six $500-\mathrm{Hz}$ tones, each having the same ITD as the $500-\mathrm{Hz}$ component of the vowel, there was a significant reduction its contribution compared to when all components had the same ITD. When the sequence conditions were presented in the same experimental block as the previous ITD conditions, then the latter conditions did show an effect of grouping by common ITD. These results show that listeners can group sounds on the basis of ITD information, but their ability to do so is greatly enhanced if the position and frequency have been previously cued. [Work supported by UK MRC.]

2aPP29. A comparison of two procedures for studying auditory image formation. Richard A. Roberts, Patrick W. Rappold, Michael P. Cannito (Dept. of Speech Pathol. and Audiol., Univ. of South Alabama, Mobile, AL 36688), and Madhuri S. Muelkar (Univ. of South Alabama, Mobile, AL 36688)

The results from an off-line procedure (scaling) were compared to those from an on-line procedure ("rate adjustment") for effectiveness in examining the fusion strength of partials within two, three-tone complexes. In both procedures fusion strength was inferred by the ease in which a tone presented in isolation (captor tone), and alternately with a complex, was able to capture a member (target tone) of the complex into a sequential stream. In the rate adjustment procedure the interstimulus-interval (ISI) between captor and target was decreased until the listener judged the prominence of streaming to be greater than that of fusion. In the scaling procedure the ISI was held constant. On a visual analog scale, having its end points labeled "fast" and "slow," subjects judged the prominence of the rate at which they heard the pitch of the captor tone. Stimulus factors were tone relationship (octave/inoctave) and tone position (low, middle, high). More of the total variation was explained by the rate adjustment procedure, but the coefficients of variation for each tone position were 
smaller for the scaling procedure. Neither procedure showed an effect of tone relationship (octave/inoctave), and both procedures showed the same effect of tone position.

2aPP30. The effects of narrow-band noise maskers on overshont. Peter Marvit and Virginia M. Richards (Dept. of Psychol., Univ. of Pennsylvania, 3815 Walnut St., Philadelphia, PA 19104)

The elevation of detection thresholds for a short signal added just after the onset of a masker versus when it is added later during the masker has been called "overshoot." Previous work with broadband maskers suggests masker frequency component near, but not at, the signal frequency produce overshoot. The relative contribution of different frequency regions to the overshoot effect was investigated here with narrow-band noise maskers of different center frequencies. The 350-ms maskers were $\frac{1}{2}$ octave wide with center frequencies ranging from 1400 to $8500 \mathrm{~Hz}$. The 10 -ms signal was $2500-\mathrm{Hz}$ tone added 4 or $325 \mathrm{~ms}$ after the onset of the masker. Masker spectrum levels were $35 \mathrm{~dB}$ SPL. The three subjects generally showed little or no overshoot when the masker spectrally overlapped the signal, but showed a maximum overshoot of $8-12 \mathrm{~dB}$ for masker bands centered $\frac{1}{2}$ to 1 octave above and below the signal frequency: increased signal-masker frequency difference diminished but did not abolish the overshoot effect. Detection thresholds were maximal when the masker band spectrally overlapped the signal and fell as signal-masker frequency separation grew. A similar pattem of results was obtained using low-pass, high-pass, bandpass, and notched noise maskers bounding on a $750-\mathrm{Hz}$-wide region around the $2500-\mathrm{Hz}$ signal frequency. [Work supported by NIH.]

2aPP31. Forward-masked intensity discrimination: Duration effects. Robert S. Schlauch and Nicole Lanthier (Dept. of Commun. Disorders, 115 Shevlin Hall, Univ. of Minnesota, Minneapolis, MN 55455)

Forward-masked intensity discrimination shows elevated justnoticeable differences (jnd's), for mid-level standards, a finding argued to be consistent with sensory adaptation [F. Zeng and $\mathrm{C}$. W. Turner, J. Acoust. Soc. Am. 92, 782-787 (1992)]. Accordingly, the size of jnd's should increase with increases in masker duration. We tested this notion by measuring forward-masked jnd's as a function of level for $1000-\mathrm{Hz}$ tones in four listeners with normal hearing. Jnd's were measured for 10 -ms standards in quiet and in the presence of a $1000-\mathrm{Hz}$ masker with either a $10-\mathrm{ms}$ or a $250-\mathrm{ms}$ duration. The masker level was fixed at $90 \mathrm{~dB}$ SPL and the interval between masker offset and standard onset was $100 \mathrm{~ms}$. Forcedchoice procedures were employed. The results show significantly less masking for the longer duration masker $(250 \mathrm{~ms})$ than for the short one (10 $\mathrm{ms})$. These results are contrary to the results reported for forward-masked detection. Listening bands revealed by measuring jnd's as a function of masker frequency suggest that off-frequency listening may be responsible for this finding [Work supported by the Bryng Bryngelson Fund.]

2aPP32. Mechanisms underlying nonmonotonic Weber functions in forward-masked intensity discrimination. Bart R. Clement and Robert S. Schlauch (Dept. of Commun. Disorders, Univ. of Minnesota, 164 Pillsbury Dr. S.E., Minneapolis, MN 55455)

Nonmonotonic growth in threshold for intensity discrimination has been noted for pure tones preceded by intense sinusoidal maskers; thresholds are highest for 40-60 dB SPL standards, producing what has often been described as a "midlevel hump." Both physiologic and cognitive factors have ben suggested as mechanisms underlying this phenomenon. Three subjects received four conditions designed to examine this issue. A 2IFC paradigm assessed intensity discrimination of $1000-\mathrm{Hz}, 100-\mathrm{ms}$ sinusoids. A no-masker condition provided a baseline. Three forwardmasked conditions were implemented: An ipsilateral condition that typically produces a mid-level hump; a contralateral condition in which the hump is typically absent or greally reduced; and a binaural condition presenting these two maskers simultaneously (contralateral stimulation was $13 \mathrm{~dB}$ greater than ipsilateral stimulation; listeners perceived only the contralateral masker). It was argued that if this condition produced a hump despite a perceptually absent ipsilateral masker, a sensory explanation would be suggested. Conversely, data mirroring the no-masker or contralateral results would be consistent with a cognitive hypothesis. Results suggest a strong sensory component, with only a small portion of the effect having a possible cognitive origin. Data will be compared to those of related literature. [Work supported by Bryng Bringelson Foundation.]

2aPP33. Referential coding and the "severe departure" from Weber's law. Christopher J. Plack (Dept. of Exp. Psychol., Univ. of Sussex, Brighton BN1 9QG, UK)

The Weber fraction for intensity discrimination for a $30-\mathrm{ms}, 6.5-\mathrm{kHz}$ sinusoidal pedestal is greater at medium pedestal levels that a low or high pedestal levels, particularly when the pedestal is gated with notched noise [R. P. Carlyon and B. C. J. Moore, J. Acoust. Soc. Am. 76, 1369-1376 (1984)]. In the present experiments, Weber fractions were measured for a 30-ms, 6-kHz pedestal, at a range of levels between 40 and $70 \mathrm{~dB}$ SPL. Measurements were made in quiet and in the presence of a $110-\mathrm{ms}$ notched noise with an onset $40 \mathrm{~ms}$ before the onset of the pedestal. The bandstop portion of the noise was $1-\mathrm{kHz}$ wide, centered on $6 \mathrm{kHz}$, and the noise had a fixed spectrum level of $20 \mathrm{~dB}$. In contrast to the earlier results, at pedestal levels of around $50 \mathrm{~dB}$ SPL the notched noise caused a reduction in the Weber fraction. It is suggested that the noise might improve performance by providing a proximal reference for coding the intensity of the pedestal. [Work supported by The Royal Society.]

2aPP34. Short-term temporal integration at high-frequencies: Evidence for the influence of peripheral nonlinearity? Andrew $J$. Oxenham and Brian C. J. Moore (Dept. of Exp. Psychol., Univ. of Cambridge, Downing St., Cambridge CB2 3EB, UK)

Temporal integration in the auditory system can be modeled by a compressive nonlinearity followed by a temporal integrator. For a fixed integrator, the more compressive the nonlinearity, the greater the effective integration. Compressive nonlinearity on the basilar membrane is marked at medium levels, but is reduced at low and high levels. To assess whether this influences temporal integration, thresholds of a $6.5-\mathrm{kHz}$ sinusoid were measured in the presence of a long-duration broadband-noise masker as function of signal duration, using three different noise spectrum levels, $-10,20$, and $50 \mathrm{~dB}$ (re: $20 \mu \mathrm{Pa}$ ). Signal level $\mathrm{dB}$ was plotted against $\log ($ time) for each level. For signal durations between 20 and $200 \mathrm{~ms}$, there was no significant difference in the slopes of the three functions. However. between 1 and $10 \mathrm{~ms}$ the slope of the function for the mid-level masker was steeper by a factor of between 1.5 and 2 . This is consistent with the greater mid-level compression on the basilar membrane, if one assumes a fixed short-term temporal integrator. Performance in other psychoacoustic tasks, including differences between normal-hearing and hearing-impaired listeners, is also discussed in terms of peripheral processing. [Work supported by the MRC, EPSRC, and Meridian Audio (UK).]

2aPP35. Temporal integration of loudness as a function of level. Mary Florentine (Commun. Res. Lab., Dept. of Speech-Language Pathol. and Audiol., 133FR, Northeastern Univ., Boston, MA 02115), S6ren Buus (Northeastern Univ., Boston, MA 02115), and Torben Poulsen (Tech. Univ. of Denmark, DK-2800 Lyngby, Denmark)

This study compared temporal integration of loudness for 1-kHz tones and broadband noises. Absolute thresholds and levels required to produce equal loudness were measured for 5-, 30-, and 200-ms stimuli using adaptive, two-interval, two-alternative forced-choice procedures. Levels ranged from 5 to $80 \mathrm{~dB}$ SL for noises and from 5 to $90 \mathrm{~dB}$ SL for tones. Results for 6 listeners with normal hearing show that the amount of temporal integration, defined as the level difference between equally loud 5- and 200 -ms stimuli, varies nonmonotonically with level. The average amount of temporal integration varies from about 10-12 $\mathrm{dB}$ near threshold, to a peak of 18-19 dB when the 5-ms tone is about $56 \mathrm{~dB}$ and the 5-ms noise is about $76 \mathrm{~dB}$ SPL; at higher levels, the amount of temporal integration decreases to $10 \mathrm{~dB}$ for tones and $13 \mathrm{~dB}$ for noises with levels around 100 $\mathrm{dB}$ SPL. These results indicate that modified power functions cannot account for the growth of loudness for tones at all durations. They also indicate that the growth of loudness may, at least in part, be consistent with the nonlinear input/output function of the basilar membrane. [Work supported by NIH-NIDCD R01DC02241.] 
2aPP36. Modeling loudness growth and loudness summation in hearing-impaired listeners. S. Launer, V. Hohmann, and B. Kollmeier (AG Med. Phys., FB8, Univ. Oldenburg, Postbox 2503, 26111 Oldenburg, Germany)

The goal of this study is to model the effect of sensorineural hearing impairment on loudness perception for stationary stimuli of variable bandwidth. Loudness growth functions were obtained employing a categorical scaling technique with 10 categories. Loudness scaling was performed with 9 normal-hearing and 14 sensorineural hearing-impaired subjects employing bandfiltered noises with bandwidths between 1-6 critical bands. For normal-hearing listeners, categorical scaling revealed similar differences across stimulus conditions as with loudness balancing. The loudness functions of the hearing-impaired listeners show both, a steeper increase (recruitment) and reduced loudness summation. Both aspects were successfully modeled by Zwicker's loudness model with three extensions to take account of hearing impairment. Raised audiometric threshold is modeled by a frequency-dependent attenuation after calculation of excitation patterns. Increasing the exponent in calculating the specific loudness yields recruitment. Reduced frequency selectivity is accounted for by the normal dependence of filter bandwidth on level in the excitation patterns. The extended model describes well the measured individual loudness growth functions of hearing-impaired subjects for stimuli of differing bandwidth.

2aPP37. Efficiency of selective listening by normal-hearing and hearing-impaired listeners. Karen A. Doherty (Commun. Sci. and Disorders, Syracuse Univ., 805 S. Crouse Ave., NY 13244) and Robert A. Lutfi (Univ. of Wisconsin, Madison, WI 53705)

A COSS analysis procedure [B. G. J. Berg, J. Acoust. Soc. Am. 88, $149-158$ (1990)] was used to estimate the efficiency with which 15 normal-hearing and 15 hearing-impaired listeners detected a level increment in a target tone embedded in a multitone complex. The complex was comprised of 6 oct frequencies from $250-8000 \mathrm{~Hz}$ and the target tone was selected as either the $250-, 1000-$, or $4000-\mathrm{Hz}$ tone. The hearing-impaired listeners had moderate, sloping, high-frequency sensorineural hearing impairments. Two efficiency measures were used to account for the listeners' less than ideal performance on the task; weighting efficiency $\left(\eta_{\text {wgt }}\right)$, which measures how well the listener attends to or weighs the target, and noise efficiency $\left(\eta_{\text {noise }}\right)$, which accounts for all other factors unrelated to weights. Weighting efficiencies were significantly higher for hearing-impaired listeners than normal-hearing listeners for the $4000-\mathrm{Hz}$ target, which was in the region of hearing loss. No significant $\eta_{\text {wg1 }}$ differences between normalhearing and hearing-impaired listeners were observed for the other two target tones. Even though hearing-impaired listeners sometimes weighted information more efficiently than normal-hearing listeners their overall performance on the task was significantly poorer as was reflected by smaller values of $\eta_{\text {noive }}$

2aPP38. On measuring psychometric functions. Huanping Dai (Psychoacoust. Lab., Dept. of Psychol., Univ, of Florida, Gainesville, FL 32611)

Psychometric functions of simulated observers were obtained using the conventional constant-stimulus method and an adaptive up-down method through a post-hoc analysis of the track. The form of the psychometric functions was assumed to be $P C=\Psi\left(d^{\prime} / 2^{1 / 2}\right)$, where $\Psi$ is the cumulative Gaussian probability function. The detectability $d^{\prime}$, is related to the signal level $x$ by $d^{\prime}=a x^{k}$, where $a$ and $k$ are the two parameters to be estimated. Among the factors considered in the simulation were step size and number of trials. For small number of trials $(n \leqslant 120)$, and particularly when the step size was also small, the slope values $(k)$ estimated using the adaptive method were systematically greater than the true slopes. Such biases were smaller with the constant-stimulus method. When the number of trials was reasonably large $(n>300)$, the constant-stimulus method showed no clear advantage over the adaptive method. The only a priori information required in the adaptive method is the starting stimulus level, whereas the constant-stimulus method requires pilot measurements in order to set all the stimulus levels properly. Thus the adaptive method is reasonably efficient for measuring psychometric functions. [Work supported by NIH.]
2aPP39. The effects of criterion variability on relative operating characteristics. William S. Brown (Brookhaven Natl. Lab., Bldg. 130, Upton, NY 11973-5000) and David S. Emmerich (State Univ. of New York at Stony Brook, Stony Brook, NY 11974)

The form of the relative operating characteristic (ROC) describing auditory detection typically differs from that expected based on the assumption that the observer's responses reflect underlying distributions that are normal and of equal variance [e.g., Green and Swets, Signal Detection Theory and Psychophysics]. Specifically, it is found that binormal ROCs often have slopes less than one, and are not strictly linear [Watson et al., J. Acoust. Soc. Am. 36, 283-288 (1964)]. It has been suggested that nonlinear binormal ROCs may be the result of extreme criteria being more variable than those more centrally positioned [Emmerich and Binder, J. Acoust. Soc. Am. Suppl. 1 65, S59 (1979)]. The forms of rating ROCs obtained in an experiment designed to reveal the effects of such variability were consistent with the proposition that criteria associated with "no" responses are more variable than those associated with "yes" responses. Criterion operating characteristics [Wickelgren, J. Math. Psychol. 5, 102122], which reflect the relative locations and variances of the boundaries of confidence categories, favored the same interpretation. It is concluded that the variability of criteria defining categories of rated confidence is sizable and not necessarily constant across criteria, and that such variability significantly influences the forms of empirical ROCs determined using confidence ratings in auditory signal detection.

2aPP40. Application of confidence intervals and joint confidence regions to the estimation of psychometric functions. Monica $\mathrm{L}$. Hawley and H. Steven Colburn (Dept. of Biomed. Eng., Boston Univ., 44 Cummington St., Boston, MA 02215)

A mathematical description of a psychometric function with two free parameters is fit to fixed-increment data by a nonlinear gradient search technique that incorporates a weighted least squares algorithm. The statistical confidence in the parameter estimates is considered by comparing results from standard confidence interval analysis with those from joint confidence region analysis. Confidence intervals estimate the variability of each parameter alone, ignoring the interaction between the parameters, whereas the joint confidence region gives the confidence in the joint estimation of both parameters together. In our study, both analyses were applied to fixed-increment data from a variety of tests (interaural time and intensity discrimination and $N_{0} S_{\pi}$ binaural detection) and subjects for two-interval forced choice experiments. Results show that the parameter estimates are affected primarily by the data collected near the midpoint which is not surprising since the parameters in the model are the slope and translation at the midpoint. However, the joint confidence region analysis shows that the statistical confidence of the parameter estimates are greatly affected by data collected at levels that give performance near chance and near perfect. [Work supported by NIDCD (Grant DC00100).]

2aPP41. Temporal symmetry in auditory perception and the auditory system. Toshio Irino (NTT Basic Research Lab., 3-1, Morinosato Wakamiya, Atsugi-shi, Kanagawa, 243-01, Japan) and Roy D. Patterson (MRC Applied Psychology Unit, Cambridge CB2 2EF, UK)

When a repeating damped exponential is used to modulate a sinusoid or noise, the carrier component of the perception is suppressed. When these "damped" sounds are reversed in time, producing "ramped" sounds, the carrier is restored. [R. D. Patterson, J. Acoust. Soc. Am. 96, 1419-1428 (1994); M. A. Akeroyd and R. D. Patterson, J. Acoust. Soc. Am. 95, 2941(A) (1994)]. We report matching experiments designed to measure the perceptual asymmetry between damped and ramped sounds directly. Listeners compared the relative strengths of the transient and continuous components in pairs of damped and ramped sounds, and the damped half life was varied to produce a match in the relative strengths of the transient and continuous components of the two sounds. For sinusoidal and noise carriers, the half life of the damped sound has to be, respectively, 5 and 2.5 times that of the corresponding ramped sound to produce the match. A "delta-gamma" theory of intensity enhancement around acoustic transients [T. Irino and R. D. Patterson, J. Acoust. Soc. Am. 95, 2943(A) (1994)] is used to explain the perceptual asymmetry. The theory is also used to compare the asymmetry produced by different auditory models. 
2aPP42. Temporally directed attending in the discrimination of tempo: Further support for an entrainment model. J. Devin McAuley (Dept. of Comput. Sci., Cognitive Science Program, Indiana Univ., Bloomington, N 47405) and Gary R. Kidd (Dept. of Speech and Hear. Sci., Indiana Univ., Bloomington, IN 47405)

The effect of deviations from temporal expectations on tempo discrimination was investigated using four-tone isochronous sequences. On each trial, a standard sequence was followed by a comparison sequence that was slightly faster or slower than the standard. Listeners judged which sequence was faster. Temporal deviations consisted of advancing or delaying the onset of the comparison pattern in relation to an onset predicted by an extension of the periodicity of the standard (i.e., an "expected" onset, based on an entrainment model's predictions). The interonset-interval in the standard sequence was always $400 \mathrm{~ms}$, and the onset of the comparison sequence was manipulated in relation to an "expected" interval of $800 \mathrm{~ms}$ between the onset of the last tone of the standard sequence and the onset of the comparison sequence. Discrimination thresholds were determined for conditions in which the comparison pattern onset was early, late, or at the expected temporal location. Thresholds for "early" conditions were found to be significantly higher than for the "expected" condition, but this was not the case for "late" conditions. These data will be compared with the quantitative predictions of an entrainment model [McAuley and Kidd, J. Acoust. Soc. Am. 95, 3257 (1994)]. [Work supported by NIMH and NIDCD.]

2aPP43. The effect of different component means and variances on the discrimination of temporal patterns. Toktam Sadralodabai and Robert D. Sorkin (Dept. of Psychol., Univ. of Florida, Gainesville, FL 32611)

Listeners were presented with two successive 9-tone sequences. The task was to discriminate between the temporal patterns defined by the intertone times in each sequence (tone duration $=25 \mathrm{~ms}$, tone frequency $=1000 \mathrm{~Hz}$ ). The listener had to indicate whether the two pattems had the same or different (partially correlated) temporal envelopes. A technique suggested by Lutfi [R. Lutfi, J. Acoust. Soc. Am. 97, 1339 (1995)], was used to determine the importance of each temporal position on the listener's decision. In the first experiment, one of the intertone times was assigned a different (either higher or lower) mean duration than the others. This intertone time occurted either at an early temporal position (2nd) or at a late position (6th). Results indicated that two positions, the first temporal position and the position with the different mean, had more influence on the listener's decision than other positions. In the second experiment, the first 4 intertone times were assigned a different variance than the Jast occurring 4 intertone times. Initial results suggest that listeners give higher weights to the temporal positions with the lower variance. [Work supported by AFSOR.]

2aPP44. Detection and recognition of familiar sounds in noise. Mark A. Stellmack and Robert A. Lutfi (Waisman Ctr., Univ. of Wisconsin-Madison, Madison, WI 53706)

Three experiments compared listeners' detection and recognition in broadband noise of five familiar and five unfamiliar sounds with identical long-term spectra. The familiar sounds were ice in a glass, zipper, crashing wood blocks, creaking door, and gas station bell. The unfamiliar sounds were these same five sounds played backwards. Thresholds were obtained using a single-interval, yes-no procedure. In the first two experiments, detection thresholds were measured for each sound by playing the same sound within each block of trials (fixed signal), and by playing one of the ten sounds with equal probability on each signal trial (randorn signal). In the third experiment, both detection and recognition thresholds were obtained for three of the familiar sounds played forward and backward. On each trial, listeners were asked first to make a yes/no detection judgment and then to identify which signal was played regardless of their first judgment. In all three experiments, there were no significant differences between thresholds obtained for the sounds played forward and thresholds for corresponding sounds played backwards. There was a small $(1 \mathrm{~dB})$ but statistically significant difference between thresholds for fixed and random signals. These results suggest that naturally occurring, familiar sounds have no special status among auditory stimuli in simple detection and recognition tasks [Research supported by NIDCD.]
2aPP45. Binaural delection with reproducible narrow-band maskers. Scott K. Isabelle and H. Steven Colburn (Dept. of Biomed. Eng., Boston Univ., 44 Cummington St., Boston, MA 02215)

Responses of five subjects for the binaural detection of a $500-\mathrm{Hz}$, 300 -ms, interaurally out-of-phase tone masked by statistically independent samples of narrow-band, interaurally identical noise are reported. A set of 30 noise samples were taken from a noise process with a power spectrum that is $115 \mathrm{~Hz}$ wide, centered at $500 \mathrm{~Hz}$. The rms noise pressure was $75 \mathrm{~dB}$ SPL. For each subject, the responses show good self-consistency and a strong dependence on individual noise samples. (For example intrasubject correlation coefficients for subsets of the results were typically 0.85 and always higher than 0.72 ). However, there are significant differences between subjects in the pattern of responses across the set of masker samples. (Intersubject conrelation coefficients were lower than 0.7). Subjects' performance values for individual noise samples are compared to the predictions of psychophysical models for binaural detection. Sample-level predictions of models for which the decision variable is dominated by the energy in the particular noise sample have no significant correlation with the patterns of responses across noise samples for any subject. In contrast, models that are based on variability of the interaural differences have predictions that are statistically significantly correlated with most subjects' responses (although no correlation coefficients were larger than 0.7). [Work supported by NIDCD (Grant DC00100).]

2aPP46. Infinite-Impulse-Response filter models of the head-related transfer function. Abhijit Kulkami and H. Steven Colburn (Dept. of Biomed. Eng., Boston Univ., 44 Cummington St., Boston, MA 02215)

Directional-transfer-functions (DTFs) derived from head-related transfer functions (HRTFs) measured from human subjects in an anechoic environment were modeled using auto-regressive (AR) and auto-regressive moving-average (ARMA) modeling techniques. The HRTFs, which were provided by Dr. Fred Wightman (Wightman and Kistler, 1989), were decomposed into a directional-transfer-function (DTF) and a mean-function (computed as the mean of all measured HRTFs for that subject). Model reconstructions of the HRTFs was done under the assumption that HRTFs are minimum-phase functions. The AR estimator which is derived from the theory of linear prediction, corresponds to an all-pole model of the DTF. The ARMA model extends the all-pole model to a pole-zero model and is derived by posing a weighted-least-squares formulation of the modifiedleast-squares problem proposed by Kalman (1958). The validity of each model was assessed psychophysically using a $4 I, 2 \mathrm{AFC}$ paradigm. Using a 80-ms white noise stimulus and testing a different position on each trial, for a 25-pole all-pole model and a 6-pole 6-zero pole-zero model, subjects performed close to chance for most of the 13 representative positions tested. [Work supported by NIDCD (Grant DC00100).]

2aPP47. Efficient finite-impulse-response filter models of the head-related transfer function. Abhijit Kulkarni and $\mathrm{H}$. Steven Colburn (Dept. of Biomed. Eng., Boston Univ., 44 Cummington St., Boston, MA 02215)

The head-related transfer function (HRTF) is empirically measured as a finite-impulse-response (FIR) filter e.g., Wightman and Kistler, 1989. In this study we explore reduced-order approximations of measured HRTFs for use in virtual acoustical displays. The HRTFs tested were measured from human subjects and provided by Dr. Fred Wightman. In all model reconstructions it is assumed that HRTFs can be approximated as minimum-phase functions. Theoretical results are derived which allow for Iwo model-order reduction strategies with optimal criteria. First, we demonstrate that the partial energy contained in the first $n$ taps of a minimumphase FIR filter is optimal in the Parseval sense. Secondly, we demonstrate that the HRTF expressed by its cepstral coefficients (Oppenheim and Schafer, 1979) constitutes a Fourier series. A partial sum from this Fourier series then provides the best mean-square approximation to the logmagnitude function of the HRTF. The validity of the reconstructed HRTFs was assessed psychophysically using a 4I-2AFC procedure. Using a 80-ms white noise stimulus and testing a new position on each trial, for a 64-tap HRTF reconstruction by both methods, subjects performed close to chance for most of the 13 representative positions tested. [Work supported by NIDCD (Grant DC00100).] 
2aPP48. Effects of reverberation cues on distance identification in virtual audio displays. Douglas S. Brungart (AL/CFBE, 2800 Q Street, WPAFB, $O H$ 45433-7901) and William D'Angelo (AL/CFBA, WPAFB, OH 45433-7901)

Although reverberation is known to play an important role in the perception of distance, it is not possible to isolate the effects of early and late reflections in a real environment. A virtual audio display, however, can be used to examine the effects of early and late reflections on distance identification. In this experiment, a virtual audio display simulates distant sound sources under 4 conditions: Without reflections, with an early reflection (floor reflection) only, with late reflections (reverberation) only, and with both early and late reflections. In each trial, the subjects identify the distance of the simulated sound source from one of 5 possible distances: $2,4,8,16$, and $32 \mathrm{ft}$. Numbered targets are placed at the locations of the simulated sound sources, allowing the subjects to visualize the distances involved. White noise and speech are used as stimuli in the experiment, and their amplitudes are randomized over $24 \mathrm{~dB}$ to prevent identification based on intensity alone. The results will be analyzed to measure the percentage of correct responses at each distance and the information transfer in each of the 4 conditions.

2aPP49. Measurement of localization ability using virtual audio cues and visual target acquisition. William R. D'Angelo (AL/CFBA, 2610 Seventh St., WPAFB, OH 45433-2901), Richard L. McKinley (AL/ CFBA, WPAFB, OH 45433), David R. Perrott (California State Univ., Los Angeles, CA), and Larry Hettinger (Logicon Technical Services, Inc., WPAFB, OH 45433)

Visual target detection was used to demonstrate the ability of subjects to localize with a virtual audio display. The testing facility consisted of audio presented over headphones with and without localization cues and a projection screen bearing the visual scene. In each trial the visual target appeared as one pixel at a random location of the screen. The target grew in size until found by the subject. The sound cue came on at threshold level and became progressively louder until the target was found. The audio cue was pink noise while the visual targets consisted of aircraft icons. Subject reaction time was measured from target onset to target detection. As indicated by the preliminary data, the localized audio condition faithfully provided the location of the target resulting in reaction times significantly better than with the nonlocalized audio cue

2aPP50. Aurally aided visual search under static and moving conditions. Debra L. Ferguson and David R. Perrott (Dept. of Psychol., California State Univ., 5151 University Dr., Los Angeles, CA 90032)

Auditory localization of static and dynamic targets in the presence or absence of an (broadband noise) auditory cue was examined in a twoalternative, forced-choice paradigm. The location of the target varied relative to the subject's initial fixation point. The right and left hemispheres were tested at 45,90 , and 135 deg azimuth on the zero degree elevation plane. Also tested were zero and $180 \mathrm{deg}$ locations. The range of target velocities was $0,10,20$, or 40 deg per second, with direction of travel and velocity randomized in each trial. Each main effect was significant as well as two-way interactions between condition $\times$ location and condition $X$ velocity. These results suggest the utilization of spatially correlated auditory cues under static and moving conditions, particularly in the posterior field and at higher velocities. [Work supported by NSF.]

2aPP51. The role of auditory cues in assisting pursuit motor functions. Renato G. Villacorte, David R. Perrott, and Debra L. Ferguson (Psychoacoust. Lab., California State Univ., Los Angeles, CA 90032)

Eight experienced subjects were used in a two-alternative, forcedchoice visual search paradigm in order to investigate the auditory system's contribution in maintaining pursuit eye movements after the cue that indicated the target's location, velocity, and direction of travel was removed. Subjects were instructed to use the target motion information provided by the cue to anticipate the location of the target onset after the delay following the cue's offset. Predictably, conditions that included visual target information produced faster RT's than a cue consisting of only auditory information. Performance also declined when the velocity of the target or the duration of the delay was increased. These results may suggest the importance of information retention in maintaining pursuit tasks when target information is removed. The importance of the auditory system in processing spatial information for pursuit movement tasks will be discussed. [Work supported by NSF.]

2aPP52. Evaluation of three-dimensional (3D) auditory beacons for navigation of remote-controlled vehicles. Tuyen V. Tran, Tomasz R. Letowski, and Kim S. Abouchacra (US Army Res. Lab., AMSRL-HR-SD, Bldg. 520, Aberdeen Proving Ground, MD 21005-5425)

A series of experiments was conducted to evaluate 3D auditory beacons to be used by' drivers of remote-controlled vehicles. The auditory beacons were generated from an external sound source, conditioned using the Convolvotron ${ }^{\mathrm{TM}}$ and then presented through Sennheiser HD-580 earphones. In addition, a pink noise masker was presented through an overhead loudspeaker at a level of $80 \mathrm{dBA}$ measured under the earphones. Ten listeners with normal hearing were asked to (1) judge sound quality of nine auditory beacons, and (2) move a beacon from a predetermined starting location to a position directly in front of the listener in the 3D display. The beacons differed regarding type of sound as well as rate and mode (continuous versus noncontinuous and single versus oscillating sound source) presentation. Results of the experiments indicate that listeners preferred (1) continuous versus interrupted presentation of the beacons, (2) nonspeech versus speech beacons, and (3) a rate of 1.1 repetitions per second over 0.7 or 2.5 repetitions per second. Mode of presentation did not affect the overall localization accuracy or the number of front versus back confusions made by the listeners. Results of the experiments will assist in the selection of beacons for auditory displays used for operation of remote-controlled vehicles.

2aPP53. A cognitive perspective on audio delay tolerance in telecommunications. Larry Marturano (Northwestern Univ., Evanston, IL. 00208)

A set of tests are described in which the effects of echo-free audio delay on PCM-encoded speech are evaluated. In these tests, a conversation-type subjective speech assessment technique is used, which is augmented with several objective measures of conversational performance. At the same time, a measure of mental workload is studied to assess the cognitive effects of audio delay on the human end user. The workload measure that is used is a secondary task involving short term, or working, memory. In describing these experiments, relevant background information is provided on audio delay, which illustrates the difficulties in measuring user acceptability of audio delay. Information on human memory models and key experimental findings are reviewed, which motivate the choice of workload measure used in this study. The test methodology and results are then reviewed in detail. User tolerance of audio delay is found to decrease with increasing audio delay, in accordance with other researchers findings. In addition, added cognitive load is demonstrated in the presence of long delays via decreased working memory performance.

2aPP54. The relationship between tone frequency and perceived elevation under headphone listening conditions. Alan D. Musicant (Dept. of Psychol., Middle Tennessee St. Univ., Box X063, Murfreesboro, TN 37132)

The relationship between pure tone frequency and perceived in head elevation was investigated. Stimuli were pure tones ranging from 1000 to $1200 \mathrm{~Hz}$ in $1000 \mathrm{~Hz}$ steps. Insert earphones were utilized for presentation. Subjects were instructed to indicate, on two separate diagrams, the image location of the stimulus after presentation of a brief tone. Dimensions were left-right, front-back and elevation. All stimuli were presented diotically, that is with equal intensity to the two ears. Tones were presented at approximately 70 phons with a variation of $\pm 3 \mathrm{~dB}$. Perceived in head location was found to vary systematically in the elevation dimension. Tones with lower frequencies were perceived as lower in elevation than tones of higher frequency up to about $9 \mathrm{kHz}$. Stimuli with frequencies of $10 \mathrm{kHz}$ and higher were perceived as lower in elevation relative to the perceived elevation of the 8- and 9-kHz stimuli. Results of this experiment were compared to results from free field experiments and found to be in general 
agreement with those reported by Butler [Conference on Binaural and Spatial Hearing, 1993]. Results of this experiment are compared with results obtained in the more typical lateralization paradigms.

2aPP55. Perception of multiple echoes. Daniel D. McCall, Brian $\mathbf{L}$. Costantino, Rachel K. Clifton (Dept. of Psychol., Univ. of Massachusetts, Amherst, MA 01003), and Richard L. Freyman (Univ. of Massachusetts, Amherst, MA 01003)

Since Ebata et al. [J. Acoust. Soc. Am. 44, 537-541 (1968)], little experimental or theoretical work has been done on the perception of multiple echoes. In the current experiment, listeners seated in an anechoic chamber were presented with 4-ms noise bursts in a lead-lag-lag configuration. The lead burst was presented from a loudspeaker at $45^{\circ}$ left of midline and one lag burst ( $\operatorname{lag} A$ ) was presented at midline. Listeners discriminated the location of a second lag burst (lag B) presented from a loudspeaker at either $35^{\circ}$ or $55^{\circ}$ right of midline. Keeping the delay between lead and lag B constant, a range of delays between lead and lag A was presented to determine whether changing the delay of lag $A$ had an effect upon the perception of $\operatorname{lag} B$. Presentation of lag A interfered with the perception of lag $B$ over a wide range of lag A delays, such that subjects' discrimination of the location of lag $B$ was poorer when compared to the discrimination of lag B when lag A was absent. The data suggest that the introduction of an earlier echo serves to suppress directional information from a subsequent echo. [Work supported by NIH grant DC01625.]

2aPP56. Localization suppression and echo suppression aspects of the precedence effect. Xuefeng Yang and D. Wesley Grantham (Bill Wilkerson Ctr. and Div. of Hear. and Speech Sci., Vanderbilt Univ. School of Medicine, 1114 19th Ave. South, Nashville, TN 37212)

The precedence effect is a phenomenon that may occur when a sound from one direction (the lead) is followed within a few milliseconds by the same or a similar sound from another direction (the lag, or the echo). Typically the lag sound is not heard as a separate event and the lag sound cannot be localized. Traditionally these two aspects of precedence (echo suppression and localizalion suppression) have been assumed to represent the operation of a single mechanism. The hypothesis is proposed in this study that localization suppression and echo suppression are at least partially independent phenomena. A series of experiments was conducted to test this hypothesis as well as to investigate the acoustic parameters that affect the magnitude of localization suppression and echo suppression. In the first experiment subjects showed a significantly greater degree of echo suppression than of localization suppression when presented with an extended train of lead-lag noise burst pairs. In other experiments it was shown that the magnitude of localization suppression is affected primarily by the degree of spectral overlap of lead and lag sounds, and to a lesser extent by the relative "localization strength" of the two sounds [P. L. Divenyi, J. Acoust. Soc. Am. 91, 1078-1084 (1992)]. [Work supported by NIDCD.]

2aPP57. The effect of background noise on the precedence effect. Yuan-Chuan Chiang and Richard L. Freyman (Dept. of Commun. Disorders, 6 Arnold House, Univ. of Massachusetts, Amherst, MA 01003)

The current experiment examined the influence of noise on the precedence effect. focusing on the importance of noise source location. Listeners seated in an anechoic chamber judged whether the image produced by a lead-lag pair of 4-ms noise bursts (2-ms delay) was to the left or right of midline. The lag loudspeaker was fixed at $45^{\circ}$ to the left or right, while the lead loudspeaker was positioned at a variable number of degrees to the opposite side. The angle of the lead loudspeaker producing $50 \%$ judgements favoring the lead was used to estimate its perceptual weighting relative to the lag. This weighting, which was quantified using the $c$ metric developed by Shinn-Cunningham et al. [J. Acoust. Soc. Am. 93, 29232932 (1993)], strongly favored the lead when the sounds were presented in quiet. The value of $c$ was reduced markedly when background broadband noise was introduced from $0^{\circ}$ or $180^{\circ}$ angles, supporting previous findings of a weakened precedence effect in noise. When the background noise source was off-midline, or lead-lag pairs of noise sources were used, the effects of noise on $c$ were complex and asymmetric. [Work supported by NIH \#DC01625.]
2aPP58. Interference with interaural time difference and intensity discrimination: Empirical and modeling study of effects of component level, spacing, and perceptual grouping. William S. Woods (Univ. Oldenburg, FB8/Medizinische Physik, D-26111 Oldenburg, Germany)

The discrimination of the ITD of a $600-\mathrm{Hz}$ tone was measured in the presence of other tones, with levels of 75 and $45 \mathrm{~dB}$ SPL/tone, spacings of 200 and $400 \mathrm{~Hz}$, and in the presence of noisebands. Noiseband levels were such that excitation patterns with tonal and noise interferers were equal in a region around the target. Tonal interferers were presented with simultaneous onsets and offsets, 250-ms onset asynchrony, or continuously. Noisebands were presented continuously. At the lowest presentation level with the widest spacing and continuous tonal interferers, jnds showed no increase over the target-alone jnd, i.e., no interference. Decreased spacing or increased level caused increased jnds (i.e., interference). Lesserexperienced subjects showed more interference in the asynchronous-onset condition than in the continuous-tone condition. Measurements of intensity jnds in a subset of conditions yielded similar results. These results are consistent with interference caused by spread of excitation and pulsing of interferers being as significant as interference expected from auditory grouping. A filter-based cross-correlation model for ITD processing is shown to be consistent with past and present ITD jnd results. [Work supported by NIDCD (Grant DC00100).]

2aPP59. Binaural profile analysis: On the comparison of interaural time difference and interaural correlation acrass frequency. William S. Woods (Univ. Oldenburg, FB8/Medizinische Physik, D-26111 Oldenburg, Germany), Andrew R. Brughera, and H. Steven Colburn (Boston Univ., Boston, MA 02215)

Experiments are reported on that werè., aimed at determining if a "profile-analysis" process is performed in" the binaural domain, specifically with interaural time difference (ITD) and interaural correlation (IC). Sensitivity to variations in these parameters across frequency was investigated by roving the parameters across intervals and requesting subjects to choose the interval containing an incrementally different parameter value in a target band. The size of the increment needed to identify the target interval was measured for several bandwidths of the total stimulus. In the ITD experiments, tone complexes were used and the target was a tone at $500 \mathrm{~Hz}$. For harmonic complexes (100- $\mathrm{Hz}$ spacing), the addition of reference tones (out to $4 \mathrm{kHz}$ ) does not aid detection of the target interval but usually degrades performance. For logarithmic complexes (spacing factor l.3) performance improved at the widest ( $4 \mathrm{kHz}$ ) bandwidth. In the IC experiment, spectrally continuous noise was used, with a $115-\mathrm{Hz}$ wide target band at $500 \mathrm{~Hz}$. Adding fringe bands increased thresholds significantly for a narrow stimulus bandwidth $(354 \mathrm{~Hz}$ ), and performance retumed to that measured for the target alone for wide bandwidths. These results are discussed in terms of possible binaural contributions to auditory grouping. [Work supported by NIDCD (Grant DC00100).]

2aPP60. Interaural phase locking as a function of interaural level. G. G. R. Green, A. Rees (Dept. of Physiol. Sci., The Medical School, Newcastle-upon-Tyne NE2 4HH, UK), and G. B. Henning (Oxford Univ., Oxford OX1 3UD, UK)

Sinusoidal phase modulation in a sinusoidal carrier was monaurally presented to observers' left ears in standard two-interval forced-choice experiments: The carrier was phase modulated in one of two randomly chosen observation intervals and the observers were required to indicated the interval having phase modulation. In the dichotic condition, a pure tone with the same frequency $(500 \mathrm{~Hz})$ and level $(70 \mathrm{~dB}$ SPL) as the carrier was presented to the right ear in both observation intervals; in the monaural condition, the pure tone was attenuated by $71 \mathrm{~dB}$. The 1.5 -s stimuli were gated simultaneously with 20 -ms Gaussian rise/fall times. At low modulation rates, the depth of phase modulation corresponding to $75 \%$ correct responses in the dichotic condition was an order of magnitude smaller than in the corresponding monaural condition consistent with the results of $G$. G. R. Green, J. S. Heffer, and D. A. Ross [J. Physiol. (London) 260, 49P-50P (1976)]. The dichotic advantage is scarcely affected by two orders of magnitude reduction in the level of the pure tone in the right ear. The results are related to the precision of phase locking in the auditory system. [Work supported by the Royal Society.] 
2aPP61. Across-frequency interaural pattern discrimination. Jennifer J. Lentz (Dept. of Bioeng., Univ. of Pennsylvania, Philadelphia, PA 19103) and Virginia M. Richards (Univ. of Pennsylvania, Philadelphia, PA 19103)

The ability of observers to detect changes in interaural intensity difference across frequencies was tested using multitone complexes. The tonal components ranged in frequency from 200 to $5000 \mathrm{~Hz}$, with amplitudes randomly drawn from a Gaussian distribution having a mean of 60 $\mathrm{dB}$ SPL and a given variance. For the standard stimulus, variance was zero, providing equal-amplitude tones. The signal stimulus had a nonzero variance. Because independently drawn signal stimuli were presented to the left and right ears, interaural level differences varied randomly across frequency. Percent correct signal detections were measured as a function of the variance of the signal stimulus. To encourage discrimination based on the pattern of interaural level differences across frequency rather than the change from diotic (standard) to dichotic (standard+signal), the overall levels of the stimuli presented to each ear were independently chosen from a 7-dB range. Because standard and standard+signal power spectra differed, monaural cues to the presence of the signal were available. Thus psychometric functions were separately obtained using the same signal stimulus at both ears. Results indicate that the detectability of changes in interaural level differences across frequency is nearly the same as the detectability of changes in power spectra for diotic presentations. [Work funded by NIH.]

2aPP62. Distortion product otoacoustic emissions created through the interaction of a spontaneous otoacoustic emission and an externally generated tone. Linda W. Norrix and Theodore J. Glattke (Dept. of Speech \& Hear. Sci., Univ. of Arizona, Tucson, AZ 85721)

An SOAE $\left(f_{2}\right)$ and one XT $\left(f_{1}\right)$ were used to create $2 f_{1}-f_{2}$ DPOAEs. External tones were applied to the ear canal at $\mathrm{SOAE} / f_{1}$ ratios between 1.08 and $1.22 \mathrm{XT} / \mathrm{SOAE}$ intensity differences varied from 0 to approximately $50 \mathrm{~dB}$ SPL. DPOAE amplitude and SOAE suppression characteristics were variable across subjects. However, the following trends were noted: (1) At larger frequency ratios, DPOAE generation and SOAE suppression were associated with greater XT SPLs; (2) DPOAE growth functions were characterized by slopes $<1 \mathrm{~dB} / \mathrm{dB}$, a maximum, rollover and disappearance into the noise floor; (3) maximum DPOAEs were observed at frequencies approximately $\frac{1}{2}$ oct below the SOAEs; (4) DPOAE maximums were associated with a significant amount of SOAE suppression. The results are consistent with DPOAEs created using two low-level tones [Brown and Gaskill, in Mechanics and Biophysics of Hearing (SpringerVerlag. Berlin, 1990)] and can be interpreted by considering suppression in a nonlinear cochlear model [Zwicker, J. Acoust. Soc. Am. 80, 163-176 (1986).] [Work supported by NIH-NIDCD grant 1 P60 DC-01409 and Univ. of AZ Graduate Student Development Fund.]

2aPP63. Masking and aging: I. Gerbil auditory brain-stem response thresholds. Flint A. Boettcher, John H. Mills, and Barbara N. Schmiedt (Dept. of Otolaryngol. and Commun. Sci., Medical Univ. of South Carolina, 17l Ashley Ave., Charleston, SC 29425-2242)

Masked thresholds estimated from auditory brain-stem responses (ABR) of aging gerbils are $20-30 \mathrm{~dB}$ higher than the masked thresholds of young gerbils when quiet thresholds are equal. In this presentation, we will discuss data on young ( 6 month) gerbils with normal hearing and aged (36 month) gerbils with a range of hearing levels. ABR thresholds were measured at $1,2,4,8$, and $16 \mathrm{kHz}$ in quiet and in the presence of a low-pass filtered noise ( $1 \mathrm{kHz}$ upper bound) at levels of 50,60 , and $70 \mathrm{~dB}$ SPL. Young subjects had linear growth of masking of signals within the passband of the noise. At higher frequencies, only minimal masking was observed. In contrast, aged subjects showed much higher masked thresholds at 2 and $4 \mathrm{kHz}$ compared to young subjects. This occurred for both aged subjects with minimal age-related threshold elevation and those with large hearing losses. In accordance with previous studies, masked ABR thresholds in aging gerbils are much higher than those in young gerbils. This phenomenon is examined in human subjects with behavioral and ABR techniques in the companion presentations. [Work supported by NIDCD.]
2aPP64. Masking and aging: II. Human behavioral and auditory brain-stem response thresholds. Judy R. Dubno, John H. Mills, Jayne B. Ahlstrom, and Lois J. Matthews (Dept. of Otolarygol. and Commun. Sci., Medical Univ. of South Carolina, 171 Ashley Ave., Charleston, SC 29425-2242)

Masked thresholds measured psychophysically are equivalent for young and aged human subjects with equal quiet thresholds. However, higher masked thresholds derived from auditory brain-stem responses (ABR) are observed for aged than for young gerbils, independent of quiet thresholds. The purpose of this exeriment was to characterize further agerelated changes in masking by comparing masked thresholds measured electrophysiologically and psychophysically for young and aged humans with normal hearing. Signals were Gaussian 1.8-ms tone pips at 1.0, 2.0, and $4.0 \mathrm{kHz}$; the masker was a $1.0 \mathrm{kHz}$, low-pass-filtered noise. Using identical signals, $A B R$ and behavioral thresholds were measured in quiet and in maskers with overall levels of 74,82 , and $90 \mathrm{~dB}$ SPL. Thresholds measured psychophysically are equivalent for young and aged subjects in both on- and off-frequency masking conditions, consistent with previous findings. However, quiet and masked ABR thresholds are higher for aged than for young subjects, and masking functions are shallower. Thus, whereas ABR thresholds of aged humans are higher than their behavioral thresholds, they are consistent with elevated ABR thresholds observed in aged gerbils. More direct comparisons of the characteristics of human and gerbil ABRs are contained in a companion poster. [Work supported by NIDCD.]

2aPP65. Masking and aging: III. Human and gerbil neural input/output functions. John $\mathrm{H}$. Mills, Lois J. Matthews, Richard A. Schmiedt, Flint A. Boettcher, Judy R. Dubno, and Jayne B. Ahlstrom (Dept. of Otolaryngol. and Commun. Sci., Medical Univ. of South Carolina, 171 Ashley Ave., Charleston, SC 29425-2242)

To account for higher masked ABR thresholds, input-output (VOO) functions of the ABR were examined. For both aging humans and gerbils, amplitudes of the ABR were smaller and slopes of ABR VO functions were less steep than those of young subjects. Smaller amplitudes could indicate abnormal function of the brainstem or an altered input to the brain stem from the periphery. Analysis of $\mathrm{I} / \mathrm{O}$ functions of the compound action potential (CAP) of the auditory nerve of aging gerbils also showed a decrease in slope. Decreases in the slope of the CAP can be attributed to a loss of $10 \%-30 \%$ of spiral ganglion cells, to a reduction in synchronous neural activity of the remaining nerve fibers, and to a reduction of the endocochlear potential. Thus, our hypothesis is that the unusual amounts of masking observed in the ABR of both aging humans and gerbils can be accounted for by age-related alterations in the auditory periphery. It is also our hypothesis that behavioral measurements of normal quiet and masked thresholds in aging humans do not necessarily reflect an intact auditory periphery. [Work supported by NIDCD.]

2aPP66. Neural encoding of level in the auditory nerve and anteroventral cochlear nucleus: $A$ study of neural models and physiological responses. M. A. Burock and L. H. Carney (Dept. of Biomed. Eng., 44 Cummington St., Boston Univ., Boston, MA 02215)

Models for level discrimination based on neural counts require "pooling" of auditory-nerve (AN) fiber responses to explain human performance. Neither the mechanism nor site of this "pooling" is known. This study concerns the transformation of AN responses in the anteroventral cochlear nucleus (AVCN). An AN model with statistics consistent with physiological data [mean-to-variance ratio $(\alpha) \approx 2$ ] provided inputs to coincidence-detecting models for AVCN cells. The $\alpha$ of coincidencedetecting cell models is lower than the $\alpha$ of AN fibers. Responses of most gerbil AVCN neurons to (frozen) wideband noise had $\alpha$ less than 2, consistent with a coincidence-detection mechanism. The lower $\alpha$ implies these cells would not be as effective as AN fibers for encoding level in terms of rate. To optimally "pool" these responses would require either summation of a greater number of elements, or steeper rate-level curves (to compensate for lower $\alpha$ ) and thus more elements to span a wide dynamic range. Neural coincidence detection may contribute to processing spectral information (monaurally) and localization information (binaurally). This 
mechanism does not provide an advantage, and perhaps introduces a disadvantage, for processing intensity information in the form of discharge rate. [Work supported by NIH, Whitaker Foundation.]

2aPP67. Responses of cochlear nucleus units in the chinchilla to best frequency tones amplitude modulated by low-frequency, two-tone complexes. W. P. Shofner, S. Sheft, and S. Guzman (Parmly Hear. Inst., Loyola Univ. Chicago, 6525 N. Sheridan Rd., Chicago, IL 60626)

A tone that is amplitude modulated by two tones $\left(f_{\bmod 1}\right.$ and $\left.f_{\bmod 2}\right)$ contains no spectral energy at the envelope beat frequency $\left(f_{\bmod 2}-f_{\bmod 1}\right)$ for either the stimulus waveform or the half-wave rectified waveform. Preliminary data showed that Fourier analysis of post-stimulus time histograms of chinchilla $\mathrm{CN}$ units recorded in response to $\mathrm{BF}$ tones that were amplitude modulated by two tones $\left(f_{\text {mod } 1}, f_{\bmod 2}<64 \mathrm{~Hz}\right)$ yields spectral peaks at $f_{\operatorname{mod2} 2}-f_{\text {modl }}$ in addition to the peaks at $f_{\operatorname{mod1}}$ and $f_{\bmod 2}$ [Shofner and Sheft, J. Acoust. Soc. Am. 93, 2410(A) (1993)]. The magnitudes of these spectral peaks are measures of synchrony at each frequency component. For all units, synchrony at $f_{\bmod 1}$ and $f_{\text {mod2 } 2}$ is greater than the synchrony at $f_{\text {mod2 }}-f_{\text {mod1 }}$. For a given unit, synchrony at $f_{\text {modl }}$ and $f_{\text {mods }}$ remains relatively constant as a function of overall level, whereas synchrony at $f_{\bmod 2}-f_{\bmod 2}$ decreases as the level increases. Synchrony at $f_{\bmod 1}$ and $f_{\bmod }$ is largest in chopper units, intermediate in primarylike units, and smallest in onset units. This hierarchy differs from that described in the literature for $\mathrm{CN}$ units in response to $\mathrm{BF}$ tones modulated at best modulation frequencies. No hierarchy appears to exist for synchrony at $f_{\text {mod2 } 2}-f_{\text {mod1 }}$. [Work supported by a PPG from NIDCD.]

2aPP68. A comparison of the efficiency and accuracy of medial superior olive cell simulations. Trevor M. Shackleton (Dept. of Exp. Psychol., Univ. of Sussex, Brighton, E. Sussex BN1 9QG, UK)

Binaural processing of interaural time differences (ITDs) is widely believed to be performed by a cross-correlation mechanism. Cells have been found in the medial-superior olive (MSO) and inferior colliculus which respond most strongly when presented with stimuli with a characteristic ITD (EE cells). These cells are believed to form the basis for such a mechanism. In this study we compare the performance of cells based on simple cross-correlation, coincidence detector cells based on a novel deterministic method, and coincidence detectors based on Monte-Carlo simulation. The deterministic model is based upon the requirement that the probability of a given number of inputs from the auditory nerves of either ear must exceed a threshold amount within' a finite time (coincidence window) before the modeled cell can fire. Wé examine the firing rate and vector strength as a function of ITD, overall level, and interaural level difference (ILD) and compare our results with the data from cells in the MSO. We find that a simple cross-correlation cell is inadequate to describe the data. However, the deterministic cell and Monte-Carlo simulation, both with a coincidence window width of $0.2 \mathrm{~ms}$, describe the dala very well. [Work supported by The Wellcome Trust, UK.]
2aPP69. Rate of rise sensitivity of medial geniculate neurons in the squirrel monkey. John F. Olsen (Lab. Neurophysiol., NIMH, NIH, Bldg. 110, P.O. Box 608, Poolesville, MD 20837)

In this study neural sensitivity to rate of rise was measured. The responses of 32 medial geniculate neurons to linear increments of a broadband noise from a defined baseline level were recorded extracellularly. Step rise time (3-96 ms), step amplitude (6-24 dB), and baseline level (0-73 dB SPL) were varied independently. A typical response to the step consisted of a transient burst of 1-8 spikes. Response probability increased as a function of the step's rate of rise (amplitude/rise time), such that increases in rise time could be traded for proportional increases in step amplitude to obtain a criterion response. Increases in baseline level decreased the slope of the probability versus rate of rise function and increased the threshold rate of rise. Response probability was independent of peak level (=baseline level+step amplitude). The results show that the response of a common type of medial geniculate neuron is determined by the rate at which stimulus amplitude increases from a defined background level, indicating a possible role for these neurons in the perceptual segregation of auditory events [A. S. Bregman, P. A. Ahad, and J. Kim, J. Acoust. Soc. Am. 96, 2694-2703 (1994)].

2aPP70. Neural processes of auditory scene analysis observed by magnetencephalography. S. Imaizumi, K. Mori, S. Kiritani (Res. Inst. of Logopedics and Phoniatrics, Faculty of Medicine, Univ. of Tokyo, 7-3-1 Hongo, Bunkyo-ku, Tokyo, 113 Japan), and M. Yumoto (Univ. of Tokyo, 7-3-1 Hongo, Bunkyo-ku, Tokyo, 113 Japan)

Auditory-evoked magnetic field elicited by three sound stimuli consisting of a spoken word, a click, and their overlapping version, was measured using BTi 37ch SQUID MEG system from the contra lateral hemisphere of the stimulated ears of seven normal hearing subjects. Three stimuli were presented in a random order with a random interstimulus interval between 1 and $2 \mathrm{~s}$ at the most comfortable hearing level. Two tasks were imposed: One is to count the click, and the other is to count the spoken word. Significant differences in the response were observed between the tasks. For both the tasks, a neural activity in the left and right temporal auditory cortices was observed for all the stimuli. When counting the click, a wide spread activity was elicited by the overlapping click suggesting possible contribution of a frontal neural activity in the right hemisphere. The results suggest that plural neural mechanisms may concern the auditory scene analysis by which the overlapping sound objects are distinctively perceived. The temporal-lobe activity may reflect automatic memory trace construction, and the frontal-lobe activity may concern attentional selection of a memory trace. 


\title{
Session 2aSA
}

\section{Structural Acoustics and Vibration: Scattering}

\author{
Hanson Huang, Chair \\ Naval Surface Warfare Center, Indian Head Division, White Oak, Silver Spring, Maryland 20903-5640
}

\section{Contributed Papers}

8:30

2aSA1. Phase-space processing of scattering from a submerged elastic layer. Raymond J. Nagem, Leopold B. Felsen, and Brian J. Collins (Dept. of Aerospace and Mech. Eng.. Boston Univ., 110 Cummington St., Boston, MA 02215)

In scattering from an insonified submerged elastic layer, whose surface is perturbed with periodic or quasiperiodic variations, the resulting Bragg modes are expected to couple to the leaky modes that can be radiated by the unperturbed smooth layer. If these two distinct but coupled phenomenologies can be separately identified and extracted from acoustic scattering data by "wave-oriented" processing, one may gain discriminants for imaging the properties of the elastic layer and of its surface topology. A simple two dimensional model-a finite array of filamentary scatterers on the insonified face of a fluid layer-has been investigated previously [T. Hsu, L. B. Felsen, and L. Carin, to appear in IEEE Trans. Antennas Propag.]. It has been shown that windowed-transform processing of data observed along a track parallel to the layer extracts space-wave-number phase space distributions in which the leaky mode and Bragg mode footprints are clearly separated; these features are poorly resolved in the unprocessed data. The present paper explores these phenomenologies and the corresponding phase-space processing when the fluid layer is replaced by an elastic layer with its far richer variety of layer-guided modes. Parametric studies show how the space-wave-number distributions are affected by the processing window size, the physical parameters of the layer, and the size and spacing of the filament array.

\section{8:45}

2aSA2. Variable phase coupling coefticient for leaky waves from resonance scattering theory and physical aspects of background contributions. Philip L. Marston (Dept. of Phys., Washington State Univ., Pullman, WA 99164-2814)

Approximations are examined for the coupling coefficient $G_{I}$ for the launching and detachment of leaky waves having application to situations where thin shell theory is not applicable. The approach is based on a comparison of ray and resonance scattering theory (RST) formulations which includes effects on the phase of $G_{l}$ of higher-order Debye approximations of Hankel functions and derivatives [P. L. Marston, Wave Motion (accepted for publication)]. The comparison includes a frequency dependent transition (between soft and rigid limits) for the RST background factor in the product expansion of the $S$ function. The transition is formulated such as to not affect $\left|G_{l}\right|$. The high frequency phase of $G_{\text {, for limiting }}$ background cases previously examined [P. L. Marston and K. L. Williams, J. Acoust. Soc. Am. Suppl. 1 83, S94 (1988)] S94 (1988)] is recovered. A variable phase term depending on the background transition function corresponds to a term given by the convolution formulation of variable curvature scattering [P. L. Marston, J. Acoust. Soc. Am. 97, 34-4I (1995)] when applied to thin circular cylindrical shells. Other correlations with shell theory are evident. Diffractive and inertial aspects of the background contribution are also examined. [Work supported by the Office of Naval Research.]

\section{9:00}

2aSA3. The exact sonar cross section of an elastic spherical shel] near the sea surface. H. Huang (Naval Surface Warfare Center, Indian Head Div., Silver Spring. MD 20903-5640) and G. C. Gaunaurd (Naval Surface Warfare Ctr., Carderock Div,, White Oak, Silver Spring, MD 20903-5640)

The acoustic scattering by a submerged spherical elastic shell near a free surface and insonified by plane waves at arbitrary angles of incidence is analyzed in an exact fashion by the classical separation of variables method. To satisfy the boundary conditions at the free sea surface, as well as on the surfaces of the elastic shell, the mathematical problem is formulated using the method of images. The scattered wave fields are expanded in terms of spherical wave functions using the addition theorems. The final results come out in terms of Wigner 3-j symbols or Clehsch-Gordan coefficients. Quite similar to the problem of scattering by multiple spheres, the computation of the scattered pressure field involves the solution of an ill-conditioned complex matrix system, of size that depends on the number of terms in the modal series required for convergence. This, in turn, depends on the value of the frequency and on the proximity of the steel shell to the free surface. The matrix equation is solved by the Gauss-Seidel method. Many backscattered echoes from the shell are computed, and its form function is displayed for various depths and in broad frequency intervals. The plots show that the large-amplitude, low-frequency resonance features in the sonar cross-sections shift upward as the shell approaches the free surface. This can be attributed to the decrease of added mass for the shell vibration. [Work partially supported by the Independent Research Program of the CDNSWC.]

\section{9:15}

2aSA4. A theory for predicting the scattered signals from elastic spheroidal shells near smooth absorbing interfaces. M. F. Werby (Naval Res. Lab., Stennis Space Center, MS 39529) and N. A. Sidorovskaia (Univ. of New Orleans, New Orleans, LA 70148)

A new formulation that allows one to describe backscattered echoes from elastic shells near absorbing plane interfaces is presented. This formulation is always consistent even at low frequencies and large distances from the interfaces and allows the rapid reproduction of backscattered echoes over frequency ranges of practical interest. This novel formulation is developed and is shown to be consistent with that reflecting interfaces. The method has been implemented and numerical results for both pulse and continuous wave signals are carried out. [Work sponsored by NRL and the Office of Naval Research.]

\section{9:30}

2aSA5. Animations of pulse signals scattering from submerged resonating structures. M. F. Werby (Naval Res. Lab., Stennis Space Center, MS 39529) and N. A. Sidorovskaia (Univ. of New Orleans, New Orleans, LA 70148)

A formulation that allows one to describe scattering from objects in a waveguide has been developed. The method is based on coupling the free-field $T$-matrix to a waveguide using the normal mode code SWAMP 
and the $T$-matrix codes developed at NRL. This formulation allows for the rapid reproduction of solutions and allows one to replicate pulse scattering from resonating objects in a waveguide. Animations of signals scattering from resonating objects is presented. The results are shown to enhance insight into the physics of such scattering processes.

\section{$9: 45$}

2aSA6. Elastic scattering of a spherical acoustic wave by an infinite fluid-filled cylindrical shell. Stirling S. Dodd and Charles M. Loeffler (Advanced Sonar Group, Appl. Res. Lab., Univ. of Texas at Austin, P.O. Box 8029, Austin, TX 78713-8029)

A method of describing spherical acoustic waves in cylindrical coordinates is applied to the problem of point source scattering by an elastic infinite fluid-filled cylindrical shell. Léon et al. developed the scattering matrix for plane-wave scattering by an infinite hollow cylindrical shell [Léon et al., J. Acoust. Soc. Am. 91, 1388-1397 (1992)]. By using a method of expressing a spherical wave in cylindrical coordinates in a form similar to that of a plane wave in cylindrical coordinates [ $\mathrm{Li}$ and $\mathrm{M}$. Ueda, J. Acoust. Soc. Am. 87, 1871-1879 (1990)], the result of Leon et al. is manipulated into an exact solution for the spherical wave, fluid-filled scattering problem. The exact solution is in an integral form that is treated numerically. It is applied to distributed source and receiver arrays by treating them as sets of point sources and point receivers and then integrating over their spatial extent.

\section{0:00-10:15 Break}

\section{0:15}

2aSA7. Scattering from a fluid-loaded cylindrical shell with axially periodic circumferential constraints using analytical numerical matching. Rickard C. Loftman and Donald B. Bliss (Mech. Eng. and Mater. Sci., Duke Univ., Box 90300, Durham, NC 27708)

Acoustic scattering from an infinitely long, thin elastic cylindrical shell with axially periodic circumferential constraints leads to the excitation of flexural, longitudinal, and shear waves on the shell. Of interest is the interaction of these structural waves with the discontinuous constraints, and the effect of this interaction on the resultant scattered sound field. This three-dimensional, fully coupled, structural acoustic problem is solved with the aid of a new method called analytical/numerical matching (ANM). The method combines high-resolution local analytical solutions and low-resolution global numerical solutions to more efficiently mode] structural discontinuities. ANM local solutions have been developed to efficiently capture the rapid variation in system response across the discontinuous constraint. The local solutions are combined with a smooth global solution, modeled by modal methods, to form an accurate, uniformly valid composite solution. The ANM composite solution is more accurate and converges more rapidly than the traditional modal approach. An important observation is that this scattering problem exhibits considerable sensitivity to modeling accuracy and convergence of structural response in the region of the discontinuities. Errors in these regions have an overall effect on the structure and the associated scattered field. [Work supported by ONR.]

\section{$10: 30$}

2aSA8. Diffraction analyses with and without flow: $A$ unifying spectral approach. R. Martinez (Cambridge Acoust. Assoc., Inc., 200 Boston Ave., Ste. 2500, Medford, MA 02155)

This paper discusses sample diffraction problems from the fields of structural acoustics in a still medium and of aeroacoustics at high subsonic Mach numbers. Respectively and specifically: Diffraction by a tandem arrangement of thin circular plates of finite radius and inertia, and scattering of high-frequency rotor/stator noise by the nacelle of a modem aeroengine. The presence of a freestream endows the elemental diffraction loads in the second class of problems with the capacity to inject vorticity into the medium. The need to account for it has classically prompled the development of a new mathematical interpretation of scattering, called "unsteady aerodynamics," so that flow and flowless diffraction communities have tended to work in mutual isolation. A side objective of this paper is to speak to both groups by presenting a completely formal complexcontour representation of the fundamental lift kemel for the flow case. That spectral formulation displays the role of vorticity explicitly through an additional singularity that shifts away to complex infinity as the flow is turned off. The remaining spectrum is the Fourier transform of the hypersingular diffraction kernel of ordinary acoustics.

\section{0:45}

2aSA9. Generalized holography for imaging elastic structures. Robert P. Porter, Daniel Rouseff (Appl. Phys. Lab., Univ. of Washington, Seattle, WA 98105), and David Bennink (Appl. Measurement Systems, Bremerton, WA)

Generalized holography is a technique for determining the properties of acoustical sources or scatterers [R. P. Porter, Progress in Optics 27, 1989]. In its most general form, the radiating or scattered field and its normal derivative are measured on a recording surface of arbitrary shape. The resulting measurements can be forward propagated into the far field to calculate the radiation pattern. The field can also be backpropagated toward the original source location to generate an image. For the special case of a closed recording surface surrounding the source, the image is independent of the shape of the hologram. Moreover, the image will be independent of evanescent (subsonic) components of the field even when the measurements are made in the near field. This is an important consideration for locating sources of radiation on an object. In this talk, generalized holography is applied to simple elastic structures to determine the modes of vibration. Numerical results will be presented for spherical shells excited by localized surface pressure or by an incident pressure wave.

\section{1:00}

2aSA10. Acoustical scattering by a penetrable wedge. Anthony M. J. Davis (Dept. of Math., Univ, of Alabama, Tuscaloosa, AL 35487-0350)

Consider the two-dimensional scattering of a time-harmonic sound wave generated by a line source and incident upon a penetrable wedge. The wave speeds in the interior and exterior of the wedge are distinct and the radiation condition of only outgoing waves at infinity is applied in all directions. At the boundary of the wedge there is a pair of transmission conditions which ensure continuity of the acoustic pressure and normal velocity. By using suitably modified Green's functions and considering separately the symmetric and antisymmetric parts of the pressure field with respect to the center plane of the wedge, a pair of disjoint integral equations of the first kind can be obtained for the two parts of the normal velocity on just one face of the wedge. Transformation to equations of the second kind is then achieved by using a technique for solving integral equations with Hankel function kernels [D. Porter, IMA, J. Appl. Math. 33, 211-228 (1983)]. The new kemels are bounded but defined on the interval $(0, \infty)$. A numerical solution must describe the far field which, at the wedge boundary, will exhibit some mixture of the two wave speeds.

\section{1:15}

2aSA11. Acoustic and flexural wave scattering from a three member junction. Douglas A. Rebinsky and Andrew N. Norris (Dept. of Mech. and Aerospace Eng., Rutgers Univ., Piscataway, NJ 08855-0909)

A three member junction is formed by a pair of semi-infinite plates in contact with fluid on one side and a mechanical structure on the other. The latter is described by an impedance matrix and the plates are modeled by the classical theory of flexure. The scattering problem for oblique incidence is solved using the impedance matrix for the plate junction without the attachment. This is accomplished using the Wiener-Hopf technique to solve the dual integral equations for the unknown pressure on the plates. Explicit formulae are obtained for the pressure transform, and correspond- 
ing explicit and relatively simple expressions are given for the various diffraction coefficients associated with the fluid/structure interaction. $\mathrm{Nu}$ merical results for the redistribution of energy from flexural to fexural plus acoustic at a thickness discontinuity will be discussed in detail with respect to frequency and angle of incidence including the effect of various critical angles. It is verified that the general solution reduces to that for a pair of plates with clamped and welded junction conditions as limiting cases when the frame impedance is zero and infinite. [Work supported by ONR.]

WEDNESDAY MORNING, 31 MAY 1995

GRAND BALLROOM SOUTH, 7:45 TO 11:45 P.M.

\title{
Session 2aSC
}

\section{Speech Communication: Bi-modal Speech Perception (Poster Session)}

\author{
Stephen A. Zahorian, Chair \\ Electrical and Computer Engineering Department, Old Dominion University, Norfolk, Virginia 23529
}

\section{Contributed Papers}

\begin{abstract}
All posters will be on display from 7:45 to 11:45 a.m. To allow contributors an opportunity to see other posters, contributors of odd-numbered papers will be at their posters from 7:45 to 9:45 a.m. and contributors of even-numbered papers will be at their posters from 9:45 to 11:45 a.m.
\end{abstract}

2aSC1. Effects of modality on subjective estimates of frequency and recency of spoken and printed words. Daniel E. Gaygen and Paul A. Luce (Language Perception Lab., Dept. of Psychology. University at Buffalo, Buffalo, NY 14260)

Previous research (Pisoni and Garber, 1990; Garber and Pisoni, 1991) has demonstrated that subjective familiarity judgments for words are not differentially affected by the modality (visual or auditory) in which the words are presented, suggesting that subjects base their judgments on fairly abstract, modality-independent representations in memory. However, in a recent large scale study in Japanese (Amano et al., in press), markedly modality effects on familiarity ratings were observed. The current research further examines possible modality differences in subjective ratings and their implications for word recognition. Specially selected words were presented to subjects for frequency and recency judgments. In particular, subjects were asked how frequently (or recently) they READ, WROTE, HEARD, or SAID a given spoken or printed word. These ratings were then used to predict accuracy and processing times in auditory and visual lexical decision and naming tasks. Our results suggest modality dependence for some lexical representations, primarily for words that occur fairly rarely in the language. [Work supported by NIDCD.]

2aSc2. Speechreading with and without auditory $F 0$ supplementation: Effects of lexical and sentential context. Robin $S$. Waldstein and Arthur Boothroyd (Ctr. for Res. in Speech and Hear. Sci., Graduate School and Univ. Center, City University of New York, 33 W. 42 St., New York. NY 10036)

This study examined the effects of lexical and sentential context on speechreading with and without supplementation by an auditory indicator of voice fundamental frequency $(F 0)$ in 12 adults with normal hearing. Lexical context effects were assessed by measuring phonetic segment and syllable recognition in CVC words and nonwords. Sentential context effects were assessed by measuring word recognition in normal sentences, in syntactically correct but semantically anomalous sentences, and in isolation (i.e., no context). For the lexical contexts, improved performance was seen by both speechreading plus $F 0$ and speechreading alone in CVC words relative to CVC nonwords. $F 0$ supplementation contributed to improved consonant recognition, presumably due to the provision of cues to voicing presence or absence. For the sentence contexts, $F 0$ supplementation resulted in best recognition of words in normal sentences, while recognition of words in anomalous sentences was comparable to that for words in isolation. By contrast, results by speechreading alone revealed similar recognition of words in normal sentences and words in isolation, and poorer recognition of words in anomalous sentences. The study showed that $F 0$ supplementation significantly enhanced the visual perception of both segments and word and phrase boundaries, and interacted with context. [Work supported by NIH Program Project Grant \#5PO5DC00178.]

2aSC3. Age-related differences in performance measures for a cross-modal auditory Stroop task. John W. Hawks, Anthony J. Caruso, and Wojtek W. Chodzko-Zajko (School of Speech Pathol. and Audiol., Kent State Univ., Kent, OH 44242)

Physiologic and task performance measures are compared for young and elderly subjects on a simple speeded task, an auditory Stroop task and a cross-modal auditoryvisual Stroop task using verbal responses. The results suggested that all three tasks are perceived as physiologically stressful by both subject groups and that older subjects were more physiologically reactive to visual stimuli than younger subjects. Differences in reaction times suggested a longer overall processing time for older subjects, as well as greater interference in processing incongruent stimuli. However, analysis of response errors indicated significantly better accuracy on difficult tasks for the older subjects. The implications of these findings will be discussed in terms of task strategic differences and resource conservation.

2aSC4. The role of visual information in speaker normalization of fricatives. Elizabeth A. Strand (Dept. of Linguist., Ohio State Univ., 222 Oxley Hall, 1712 Neil Ave., Columbus, OH 43210-1298)

This study examines the role of visual information in speaker normalization of fricatives by comparing listeners' responses to audilory stimuli only with responses to audiovisual (McGurk-type) stimuli to determine the effect that visual cues have in speaker normalization. Voices judged by listeners as auditorily ambiguous and unambiguous with respect to gender were used to construct four continua of tokens from /sad/ "sod" to / / $\mathrm{ad} /$ "shod"; the continua were synchronized with videos of visually unambiguous male and female faces saying the same words. The audiovisual stimuli, as well as the auditory signals only, were then presented to listeners for identification as either "sod" or "shod." Preliminary results suggest that visually disambiguating information affects the perception of auditorily ambiguous voices. This paper will present the results of additional tests on a larger pool of listeners. [Work supported by NIH.] 
2aSC5. Effects of listener expectation on eye movement behavior during audiovisual perception. Inge-Marie Eigsti (ATR Human Information Processing Labs, 2-2 Hikaridai, Seika-cho, Soraku-gun, Kyoto 619-02, Japan), Eric Vatikiotis-Bateson, Sumio Yano, and Kevin G. Munhall (Queens Univ., Canada)

In previous work [Vatikiotis-Bateson et al., Proc. ICSLP-94 2, 527 530 (1994)], listener eye movements were examined during audiovisual perception. Clear effects of masking noise level were observed in the stability and location of the listener's gaze. At higher noise levels, listeners fixated primarily on the mouth and very little on the eyes. Listeners were implicitly directed to focus on phonetic detail in the stimuli, by means of questions used to determine intelligibility of the stimulus; however, attention to phonetic detail is only part of what goes on during audiovisual communication. In the current study, different patterns of eye movement were elicited from 10 English listeners by biasing the listener's focus of attention. Two listener biases were tested-one toward phonetic discriminations, as previously studied, and the other toward practic information, e.g., having to do with perceived characteristics of the speaker. If visual cues during audiovisual perception are only partly phonetic, then differences in the listener's interpretation of the experimental goals may evince different patterns of eye movement. For example, the perceived goal of the experiment may affect the relative proportion of gaze fixations on the eyes and the mouth.

2aSC6. The effect of face and lip inversion on audiovisual speech integration. Deborah A. Yakel, Lawrence D. Rosenblum (Dept. of Psychol., Univ. of California, Riverside, Riverside, CA 92521). Kerry P. Green (Univ. of Arizona), Chantel L. Bosley, and Rebecca A. Vasquez (Univ. of California, Riverside, Riverside, CA 92521)

Seeing a speaking face can influence observers' auditory perception of syllables [McGurk and McDonald, Nature 264, 746-748 (1976)]. This effect decreases when the speaker's face is inverted [e.g., Green, J. Acoust. Soc. Am. 95, 3014 (1994)]. Face recognition is also inhibited with inverted faces [e.g., Rock, Sci. Am. 230, 78-85 (1974)] suggesting a similar underlying process. To further explore the link between face and audiovisual speech perception, a speech experiment was designed to replicate another face perception effect. In this effect, an inverted face and an inverted face containing upright lips are perceived as looking normal, but an upright face with inverted lips looks grotesque [Thompson, Perception 9, 438-484 (1980)]. An audiovisual speech experiment tested four presentation conditions: Upright face-upright mouth, upright face-inverted mouth, inverted face-inverted mouth, inverted face-upright mouth. Various discrepant audiovisual syllables were tested in each condition. Visual influences occurred in all but the upright face-inverted mouth condition for some of the syllable combinations thereby mimicking the face perception effect. However, other syllable combinations revealed visual influences in all four conditions. Results are interpreted in terms of articulatory dynamics and the vertical symmetry of the visual stimuli.
2aSC7. The McGurk effect in infants. Jennifer A. Johnson, Lawrence D. Rosenblum (Dept. of Psychol., Univ. of California, Riverside, Riverside, CA 92521), and Mark A. Schmuckler (Univ. of Toronto, Scarborough Campus, Toronto, Canada)

In the McGurk effect, auditory speech syllables are influenced by simultaneous presentation of discrepant visible speech syllables [McGurk and McDonald, Nature 264, 746-748 (1976)]. The effect is known to be stronger for native English than native Japanese-speaking subjects [Sekiyama and Tokhura, J. Acoust. Soc. Am. 90 (1991)]. It is unknown whether the strength of the effect is something lost or gained through developmental exposure to a given language. To explore this issue, two experiments tested for the McGurk effect in 5-month-old English-exposed infants. Infants were first gaze-habituated to an audiovisual $/ v a /$. They were then presented three different audiovisual combinations: audio /va/-visual $/ \mathrm{va} /$; audio $/ \mathrm{ba} /$-visual $/ \mathrm{va}$ (perceived by adults as $/ \mathrm{va} /$ ); and audio $/ \mathrm{da} /-$ visual $/ \mathrm{va} /$ (perceived by adults as $/ \mathrm{da} /$ ). The infants habituated to the tokens with audio $/ \mathrm{ba} /$ and $/ \mathrm{va} /$ components at the same rate, but habituated more slowly to the token with audio /dal. Further tests with a neutral static face and changing auditory syllables revealed that these habituation differences were not due to the auditory similarity of $/ \mathrm{ha} /$ to $/ \mathrm{va} /$ relative to /dad. This suggests that the infants were visually influenced in the same way as English-speaking adults.

2aSC8. Auditory localization and audiovisual speech perception. J. A. Jones and K. G. Munhall (Dept. of Psychol., Queen's Univ., Kingston, ON K7L 3N6, Canada)

The influence of the spatial position of the acoustic signal in audiovisual speech perception was investigated in a series of experiments using the McGurk effect. Subjects viewed video disk recordings of faces producing visual VCV nonsense syllables while simultaneous acoustic VCV stimuli were presented from one of 7 different speakers locations. The speakers were positioned in a semicircular array in front of the subject. In separate studies subjects were required to name the intervocalic consonant, indicate the location of the sound source using a computerized pentiometer system, or perform both tasks. Preliminary results suggest that the subjects' ability to localize the position of the auditory stimulus was influenced by the presence of a visual stimulus. Specifically, subjects tended to localize the sound closer to the position of the monitor showing the visual stimulus. However the strength of the McGurk effect was not influenced by the spatial position of the sound source. Subjects perceived the visual /g/ and the auditory $/ \mathrm{b} /$ combination as $/ \mathrm{d} /$ equally often at all sound locations. The independence of the ventriloquist effect and audiovisual integration in the perception of the McGurk effect will be discussed in terms of spatial constraints on the cross-modal perception of speech. 


\title{
Session 2aSP
}

\section{Signal Processing in Acoustics: Wavelet Analysis Techniques and Applications I}

\author{
Randy L. Smith, Chair \\ Applied Research Laboratories, University of Texas at Austin, P.O. Box 8029, Austin, Texas 78713-8029
}

Chair's Introduction $-8: 25$

Invited Papers

8:30

2aSP1. Overview of wavelets and applications. Joseph D. Lakey (Dept. of Mathematics, Texas A\&M Univ., College Station, TX 77843-3368)

Construction of orthogonal and biorthogonal wavelets as well as redundant wavelet frames will be reviewed. Fast computational wavelet-based algorithms will be outlines and their performance will be compared with Fourier-based algorithms. Various applications of wavelet analysis in speech compression and speech recognition, as well as broader applications in acoustic signal processing including denoising and transient analysis, will be discussed.

9:00

2aSP2. Wavelet packets as a tool for sound processing. Ronald Coifman (Dept. of Mathematics, Yale Univ., 10 Hillhouse Ave., New Haven, CT 06520)

Wavelet packet analysis of sound corresponds to an optimized musical transcription in which the sound is organized in different structures (orchestration), each of which is described by a "musical score." This representation is a superposition of wavelet packets (notes) of different duration, pitch, location in time, and amplitude. Fast algorithms permit real-time transcriptions with a minimal number of notes. In this talk we will give a precise definition of these statements as well as applications to audio compression, denoising, recognition, and modeling.

\section{Contributed Papers}

\section{$9: 30$}

2aSP3. Composite wavelet transform and frames. Wade Trappe and Joseph D. Lakey (Appl. Res. Labs, Univ. of Texas, P.O. Box 8029, Austin, TX 78713-8029 and Dept. of Math., Univ. of Texas, P.O. Box 8029, Austin, TX 78712 and Texas A\&M Univ., College Station, TX $77843)$

Motivated by the human auditory system, a new signal transform is presented which models the way humans hear. Cochlear processing acts like a constant bandwidth bank of filters in the low frequency range but is of proportional bandwidth at higher frequencies. This new transform, which we call the composite wavelet transform, is better able to model this process than standard signal processing techniques such as the short-time Fourier transform (STFT) and the continuous wavelet transform (CWT). The composite wavelet transform in fact provides a signal analysis tool that is able to examine signals with competing signal structures whereas the STFT and the CWT do not. In order to insure stable signal recovery, the theory of frames is examined. An overview of the theory of frames will be given and wavelet and Gabor frames will be discussed. Recent work on frames for the composite wavelet transform will also be presented. Finally, some applications of frames, especially to speech signal processing will be considered.

\section{9:45}

2aSP4. One-dimensional blood flow measurement methods: Narrow-band versus wideband/wavelet transform techniques. Lora G. Weiss (Appl. Res. Lab., Penn State Univ., State College, PA 16804)

The goal of blood flow velocimetry is to obtain an accurate measurement of the velocity of the blood as it flows through a blood vessel. If the measurement technique underestimates the blood flow velocity, then the measurement may appear normal and an occlusion may go undetected. To prevent an incorrect diagnosis, new methods for blood flow velocimetry are needed. This paper presents a technique for blood flow velocimetry using wavelet transforms, and it compares this technique to the conventional narrow-band Doppler methods often applied. Ultrasonic signals with high fractional bandwidths and/or large time-bandwidth products are processed with wavelet transforms. By applying wavelet transforms, many narrow-band assumptions typically invoked when measuring blood flow may be removed. With the wavelet method presented, instead of measuring the Doppler shift associated with the reflection of a signal, the time-scaling of the signal is obtained along with the round trip travel time. This time scaling more accurately reflects the effects of motion on the signals than does a Doppler shift since a Doppler shift is an approximation to time scaling. The continuous wavelet transform is then used to obtain the axial velocity of scatterers.

\section{0:00}

2aSP5. Signal and echo arrival-time estimation using wavelets. Phillip L. Ainsleigh (Naval Res. Lab., Underwater Sound Reference Detachment, Orlando, FL 32856-8337)

A wavelet-based method is introduced for estimating the arrival times of signals and echoes observed in noisy multipath environments. This problem arises when trying to model any signal containing overlapping segments of direct and reflected components. The method presented is more general than cepstrum-based techniques since it is not limited to echoes that are scaled replicas of the direct signal. The statistics of the wavelet coefficients are derived for pyramid-algorithm wavelet decompositions, and these are employed in a statistical detection algorithm for obtaining the arrival-time estimates. The method will be used to estimate signal and echo arrival times in data observed during underwater transducer calibration experiments conducted in small pressurized tanks. 
2aSP6. A wavelet method for estimating frequency-dependent damping in structures. Gary R. Wilson (Appl. Res. Labs., Univ. of Texas, P.O. Box 8029, Austin, TX 78713-8029)

Estimation of damping irom the measurement of the vibrations or acoustic radiation of structures provides some insight into energy storage and dissipation mechanisms in the structure. Because damping is typically proportional to frequency, a method of estimating damping that scales with frequency is suggested. A wavelet approach has been taken to exploit the natural scaling properties of wavelets to estimate frequency-dependent damping. A wavelet transform, the damped complex exponential (DCE) transform, has been designed specifically for measuring damping [R. D. Priebe and G. R. Wilson, IEEE ICASSP 3, 205-208 (1994)]. This transform has been applied to stimulate impulse response frequencies to determine its accuracy and sensitivity to mismatch between the assumed damping/frequency ratio used by the wavelet transform and the actual damping/frequency ratio of the impulse response. It has also been applied to the measured impulse response of a fluid loaded thin elastic shell. Results of these measurements show reasonable agreement with theoretical predictions of damping.

\section{0:30-10:45 Break}

\section{0:45}

2aSP7. Use of wavelet template analysis for condition monitoring of rotational machine elements. David C. Swanson, Karl M. Reichard, and Michael B. Van Dyke (Appl. Res. Lab., Penn State Univ., P. O. Box 30, State College, PA 16804)

Rotational elements of machinery, such as bearings and gears, have been shown to exhibit specific vibration signal spectral patterns over various stages of failure, associated with certain defect frequencies characteristic of the elements and their components. It has been demonstrated that during advancing stages of wear, spectral peaks at defect frequencies are accompanied by increasing numbers of sidebands of other defect frequencies. In this paper, the use of wavelet template analysis to characterize vibration signals of defective rotational elements is presented as a method for identifying and quantifying element wear. The premise of this approach is that the dynamics of a worn component are characterized by an increasing degree of variation around its rotational frequency as the component degrades at its points of contact with other components, resulting in a vibration signal analogous to a frequency-modulated signal.

\section{1:00}

2aSP8. Detection and characterization of blade-vortex interaction noise with wavelet analysis. Wyatt $O$. Davis (Dept. of Mech. and Mater. Eng., Washington State Univ., Pullman, WA 99164-2920), Charles Pezeshki (Washington State Univ., Pullman, WA 99164-2920), and Marianne Mosher (NASA Ames Res. Ctr., Moffett Field, CA 94035-1000)

A discrete implementation of the wavelet transform (WT) was used to analyze blade-vortex interaction (BVI) noise in acoustic helicopter noise signals. A BVI detection algorithm was developed which takes advantage of the prominence of BVI noise in certain subbands. Isolated-BVI signals were constructed by detecting impulsive BVI events and extracting them from the subbands. Four metries were tested for suitability as BVI estimators. Two of these, the rms levels of the isolated-BVI signals and a metric computed from the A-weighted frequency spectra of the isolated-BVI signals, were seen to characterize BVI noise severity, but were computationally expensive. The other two metrics were computed from the amplitudes of the BVI events in the subbands. The first, based on the exponential behavior of the amplitudes across subbands, failed to characterize BVI noise severity for certain signals and was highly sensitive to noise. The second, computed from the amplitudes of BVI events in a single subband, was an effective BVI estimator and was relatively insensitive to noise. This estimator is especially convenient because it is easily used as a decision threshold in the detection algorithm, thereby making possible simultaneous BVI detection and characterization.
2aSP9. Time-frequency analysis techniques exploiting the harmonic relationships of outside sound source emissions, for the purpose of detection and classification. John C. Mossing and Mohammad A. Karim (Dept. of Elec. Eng., Univ. of Dayton, 300 College Park, Dayton, OH 45469)

Acoustic sensors can be used to passively detect, track, and identify non-line-of-sight sound sources. The sound sources discussed in this paper contain strong harmonic components and range from being "pseudo" stationary to transient in nature. This research project investigates the use of various time-frequency analysis techniques for the purpose of selecting features to be extracted from the acoustic signatures of motorized sound sources. Acoustic data were filtered and digitized using a commercially available analog-digital convertor. The short time Fourier transform (STFT) was used as the initial identifier, followed by narrow-band peak detection algorithms used to select peaks above a user defined SNR. When sufficient "nonsmeared" peaks exist, these peaks are used to generate a set of harmonically related features which are input to a bayesian quadratic classifier. When the signal became more "nonstationary" and the use of the STFT resulted in excessive "smearing," wavelet and Wigner-Ville analysis technique were employed. Results are presented highlighting the trade-offs of these time-frequency analysis techniques. Additional results are presented discussing the harmonic relationship algorithm employed to normalize the frequency shifts (due to rotor/engine RPM fluctuations) which exist in this type of signature data.

\section{1:30}

2aSP10. Improved sound localization employing modified wavefront reconstruction. Steven D. Trautmann (Center for Comput. Res. in Music and Acoust., P.O. Box 9675, Stanford, CA 94309)

Audio wavefront reconstruction is similar to visual holography in which optical wavefronts are reconstructed by reproducing phases and amplitudes to create the impression of an object being present when it is not there. In audio there are far fewer speakers than necessary to exactly reproduce a wavefront this way. In order to construct the closet possible approximation to a desired wavefront, information about the listening environment such as the number, placement and qualities of the loudspeakers and the nature of local reverberation can be used. The perceived quality of this approximation can be improved by using psychoacoustical properties of sound localization, such as treating amplitude as more vital than phase, ear, and head filtering, precedence effects and masking. Thus despite limited numbers of loudspeakers, improvements can be made in sound localization for quadraphonic and stereo systems, especially at low frequencies. This is done by using signal processing techniques including partial inverse filtering of the acoustical environment to cancel out unwanted room reverberation, and a criterion applied to a multidimensional system in order to minimize the maximum difference relative to the desired perception anywhere a listener might be located.

\section{1:45}

2aSP11. Audio compression based on sine-crossing representations of signals. Michael L. Hilton and Prasanjit Panda (Dept. of Comput. Sci., The Univ. of South Carolina, Columbia, SC 29208)

The sampling theorem of Bar-David [J. Bar-David, Info. Control 24, 36-44 (1974)] provides an implicit representation of bandlimited signals using their crossings with a sine function. This sine function is chosen in a way that guarantees a unique representation of the signal. On a finite signal, this leads to a multiplicative representation involving a Riesz product whose roots form the representation of the original signal. These roots can be encoded to provide high-quality, near lossless audio compression. The sine crossing representation can also be combined with the wavelet transform to provide further compression. [Work supported by ONR.] 


\title{
Session 2aUW
}

\section{Underwater Acoustics: Signal Processing (Poster Session)}

\author{
Jeffrey L. Krolik, Chair \\ Department of Electrical Engineering, Duke University, Box 90291, Durham, North Carolina 27708-029I
}

\section{Contributed Papers}

\begin{abstract}
All posters will be on display from 8:30 a.m. to 12:00 noon. To allow contributors an opportunity to see other posters, contributors of odd-numbered papers will be at their posters from 8:30 to 10:15 a.m. and contributors of even-numbered papers will be at their posters from 10:15 a.m. to 12:00 noon.
\end{abstract}

2aUW1. Unambiguous noise and reverberation measurements from a dual line horizontal array. Bruce K. Newhall (Johns Hopkins Univ., Appl. Phys. Lab., Laurel, MD 20723), Walter S. Allensworth (Appl. Hydro-Acoustics Res., Inc., Rockville, MD 20850), and Iman W. Schurman (Johns Hopkins Univ, Laurel, MD 20723)

Single horizontal line arrays are often utilized to resolve the azimuthal character of the low-frequency noise dominated by long-range shipping sources. However, such arrays are subject to an azimuthal ambiguity on conjugate bearings. Coherent beamforming of an array compased of two parallel horizontal lines was recently employed to eliminate this left/right ambiguity using a minimum variance adaptive algorithm with single-point main response constraints under an assumed horizontally isotropic noise field. The ability of this array and beamformer to resolve the directional characteristics of the acoustic noise field was demonstrated in the Atlantic and the Mediterranean. Median left/right rejection of $20 \mathrm{~dB}$ or more was observed over a wide frequency and azimuthal band. The measured beam noise exhibits temporal and spatial variability that could not have been quantified with a single-line system. Median noise gain improvements of up to $7 \mathrm{~dB}$ above that of a single line were measured. Distant lowfrequency reverberation ambiguity resolution was also measured using the reverberation from signals underwater sound (SUS) explosive charges deployed in the Allantic. Reverberation left/right rejection of $20 \mathrm{~dB}$ was achieved despite imperfect, nonparallel line tow conditions.

2aUW2. Reverberation characterization and suppression in the presence of channel spreading. Geoffrey $S$. Edelson (Lockheed Sanders, Inc., MAN6-2000, P.O. Box 868, Nashua, NH 03061-0868) and Ivars P. Kirsteins (SACLANT Undersea Research Centre, Viale San Bartolomeo, 400, 1-19138 La Spezia, Italy)

In active sonar, the reverberation plus target time series can be modeled as the joint convolution of the signal with the channel and scatterer impulse responses. Especially in shallow water, the channel response cannot be adequately modeled as propagation over discrete paths. We propose a maximum likelihood type approach for estimating the arival times of signals which have propagated via a continuum of paths, i.e., temporally spread channels. The channel spreading is included in the model by using a discrete prolate spheroidal sequence (DPSS) to represent the channel impulse response of given duration, but unknown shape. Thus the unknown parameters are the arrival times and the scale factors of the DPSS expansion. The parameters are estimated using an iterative methodology which decomposes the original data into its constituent components and then estimates the parameters of the individual components through a sequence of one-dimensional searches. Experimental data and computer simulation examples indicate that the method performs well.
2aUW3. Appearance of elastic wave contributions in high résolution acoustic images. Gregory Kaduchak, Chad M. Wassmuth, and Charles M. Loeffler (Appl. Res. Labs., Univ. of Texas, P.O. Box 8029, Austin, TX 787i3-8029)

Studies involving plane wave scattering by finite cylindrical shells at oblique incidence display monostatic echo contributions from surface elastic waves. These waves follow helical paths along the shell's surface and are guided back in the source direction after reflection from the cylinder truncation [X.-L. Bao, J. Acoust. Soc. Am. 94, 1461-1465 (1993)]. The present research examines such effects viewed in the time record of a high resolution sonar system. The target consists of a finite cylindrical shell with a radius to thickness ratio of $5 \%$. The shell is immersed in water and is subject to both exterior and interior fluid loading. It is ensonified with short tone bursts of narrow beam width over a range of incidence angles $90 \geqslant \phi \geqslant 50$, where $\phi$ is measured relative to the cylinder axis. The backscattered echo signature exhibits prominent artifacts as a result of scattering processes involving the $s_{0}$ and $a_{0}$ Lamb waves interacting with the cylinder truncation. The echo contributions are extremely sensitive to the angle of incidence and interior fluid loading which may assist in target classification schemes.

2aUW4. An approach to matched field processing in correlated multipath environments. Robert Zeskind (The MITRE Corp., 7525 Colshire Dr., McLean, VA 22102-3481) and Mark Owen (PRESEARCH, Inc., Fairfax, VA 22031)

Matched field processing (MFP) is based on some type of comparison of actual received signal across the elements of an array to predicted array element response based on complicated propagation models for postulated source locations. It is well known that these techniques are very sensitive to model mismatch. Investigators have proposed modified MFP techniques of varying complexity to be more robust to mismatch. A simplified approach to MFP in a correlated multipath environment is to estimate the parameters of the path arrivals at the array based on an assumed wavefront model for each path. The parameters to be estimated are arrival angles, relative received level for each path, and the correlation coefficient between pair of paths. For spherical wavefronts, the distance traveled along each path is also estimated. It is assumed that there are only a few dominant parhs and their number is known. The parameter estimation problem is posed as a well-known optimization problem. The solution to the optimization problem not only acts as a detector, but provides the set of estimated parameters to be used in a raytrace propagation model to back propagate the multipath to localize the detected target. An example is presented to illustrate the method. 
2aUW5. Multiple-frequency robust adaptive matched-field processing (MFP) in shallow water. Yung P. Lee (Science Applications International, Inc., 1710 Goodridge Dr., MS T1-3-5, McLean, VA)

Application of robust adaptive algorithms to multiple-frequency MFP in shallow water has been studied and the minimum-detectable-level (MDL) has been examined via simulations. The multiple-frequency MFP output is obtained by incoherently averaging the MFP outputs of the discrete narrow-band-components. Performance of three different averaging techniques (arithmetic-mean, geometric-mean, and harmonic-mean) have been compared. The arithmetic-mean averages the amplitudes, the geometric-mean averages the $\mathrm{dBs}$, and the harmonic-mean averages the reciprocals of the amplitudes. These algorithms have been applied to shallow water data from the Hudson Canyon experiment and have demonstrated excellent source localization results. simulations show a 10 - to 20-dB improvement in the MDL against the simulated near or distant surface noise.

2aUW6. Fisher information for fluctuating intensity and the efficiency of logarithmic measures. Nicholas C. Makris (Naval Res. Lab., Washington, DC 20375)

The optimal resolution of a set of parameters to be estimated from a set of measurements of fluctuating intensity can be determined by computing the respective Fisher information matrix. This matrix is dependent upon the conditional distribution of the fluctuating intensity measurements, given the parameters to be estimated. It is assumed that fluctuating intensity is measured from circular complex Gaussian random (CCGR) fields. Such fields are commonly measured in acoustics, optics, and radar. For example, CCGR fields typically arise in scattering from fluctuating targets and surfaces, random sources, and ocean-acoustic propagation scintillation. Distributions for intensity, log-intensity, and acoustic flow (analogous to optical flow) are derived as a function of the measurement-system averaging time and the temporal coherence of the fluctuating field under the CCGR field assumption. (This advances previous work in ocean-acoustic propagation scintillation that was limited to instantaneous measurements.) It is shown that the Fisher information for a measurement of 'fluctuating intensity is expressed in terms of parameter variations over the expectation value of a logarithmic measure of intensity. This gives a mathematical justification for the engineering intuition that fluctuating intensity should be measured in logarithmic units to efficiently convey information. A generalized Fisher information matrix is derived for the estimation of parameters from intensity images.

2aUW7. Parameter estimation bounds for acoustic intensity images. Nicholas C. Makris (Naval Res. Lab., Washington, DC 20375)

A generalized Cramer-Rao lower bound is derived for the estimation of parameters from intensity images. It is assumed that the intensity assigned to each pixel value in an image is measured from a circular complex Gaussian random (CCGR) field. Such fields are commonly measured in acoustics, optics, and radar. However, intensity images obtained from acoustic measurements generally have lower SNR than in optics or microwave radar, making parameter estimation more difficult. This is because acoustic coherence time scales are generally much larger than those in optics or microwave radar relative to the respective stationary time scales. Therefore, it is especially important to determine the practicality of the parameter estimate by determining the optimal resolution attainable. Various analytic bounds are derived including those for estimation of scattering strength, transmission loss, the orientation of a Lambertian surface by stereoscopic analysis, the location and recognition of an object, the resolution of blurred features in a set of images, and the motion or acoustic flow (analogous to optical flow) of an object over a series of blurred images. The applications presented are for underwater imaging systems, but the formulation is generally applicable to arbitrary beamformed imaging of CCGR fields. This work is directly applicable to pattern recognition.

2aUW8. A rapid forward model for matched-field inversion. R. J. Cederberg and Michael D. Collins (Naval Res. Lab., Washington, DC 20375)

The forward model is an important component of a matched-field inversion algorithm. The forward model must be efficient because it is often necessary to compute a large number of replica fields during the parameter search. An efficient forward model will be described for an experimental configuration involving a source and a vertical array separated by up to several tens of wavelengths in range. The operators of the self-starter and the split-step Padé solution are combined into a single operator that acts on a delta function to give the field on the array. On a parallel-processing computer, this approach provides the replica field in the computation time it takes to solve a single tridiagonal system in a conventional parabolic equation algorithm. This efficiency gain is not achieved by sacrificing accuracy. The approach typically involves the solution of about ten tridiagonal systems. Finite-difference based separation of variables solutions typically require the solution of hundreds or thousands of similar tridiagonal systems. The efficiency of the rapid forward model will be demonstrated for the matched-field inversion problem of estimating the parameters of the ocean bottom.

2aUW9. Localizing a source buried in noise in an uncertain environment. Laurie T. Fialkowski and Michael D. Collins (Naval Res. Lab., Washington, DC 20375)

A source in an uncertain environment may be localized using focalization, which involves a parameter space that includes both source and environmental parameters. A source buried in noise may be localized using noise-canceling techniques, which involve matching both signal and noise, or eigen-processing techniques, which are capable of extracting signals from noisy data. These techniques have been combined to solve localization problems involving a source buried in noise in an uncertain environment. Noise-canceling techniques requires knowledge of the noise. Since it may not be practical to model the noise as part of the parameter search, it is assumed that the noise covariance matrix is obtained from data taken when the source is not present (mismatch associated with temporal environmental variability is included in the simulations). Eigen-processing techniques are less effective than noise-canceling techniques at suppressing noise and require source motion when multiple sources are present. However, they have the advantage of not requiring a priori knowledge of the noise.

2aUW10. Fast inversion of the ill-conditioned shallow water propagation operator for pulsed signals. Jacob Roginsky (Acoust. Div., Naval Res. Lab., Overlook Ave., S.W., Washington, DC) and G. W. Stewart (Univ. of Maryland, College Park, MD)

Time series deconvolution in underwater acoustics is a difficult problem due to the practically singular nature of the propagation operator. The situation is even more complicated in the presence of a multipath environment. In particular, when the time window is shorter than the propagation time, the process requires the solution of a large triangular Toeplitz system that is quite ill conditioned. Satisfactory results can be obtained by truncating the singular value decomposition (SVD), but only at the cost of ignoring the Toeplitz structure. The object of this work was to investigate the alternative of using Tichonov-Phillips regularization to lessen the effects of the ill conditioning. An algorithm of Lars Eldén [SIAM J. Sci. Stat. Comput. 5, 229-236 (1984)] that takes advantage of the Toeplitz structure can be adapted to this purpose. The results are as good as those obtained from the SVD, but the cost is $O\left(n^{2}\right)$ in the matrix size as opposed to $O\left(n^{3}\right)$ for the SVD. For the problems considered here, this amounts to a speedup of two orders of magnitude.

2aUW11. Matched-field replica model optimization and bottom property inversion using multitone signals in shallow water. Paul A. Baxley (Ocean and Atmospheric Sciences Div., Acoust. Branch, NCCOSC RDTE Div. Code 541, 53560 Hull St., San Diego, CA 92152-5001) and Newell O. Booth (NCCOSC RDTE Div., San Diego, CA 92152-5001)

Matched-field replica models based on an inaccurate knowledge of geoacoustic parameters such as bottom antenuation, shear, and interfacial sound-speed discontinuities, can predict an incorrect number of propagating modes for a shallow-water channel. The resulting degradation in the matched-field ambiguity surface can be substantially reduced by optimizing over the modal-sum-limit used in the replica model. The use of this technique for multitone $(70,95,145$, and $195 \mathrm{~Hz})$ source-tow data recorded near San Diego during the first shallow water evaluation cell experiment (SWellEX-1) significantly increased matched-field correlation 
levels for all narrow-band tones (and their average), with the maximum peak more frequently coinciding the correct source location than that observed with a previous nonoptimized model [Schey and Ryan, J. Acoust. Soc. Am. 95, 2981 A (1994)]. The predicted number of propagating modes was also reduced substantially. An inversion for bottom properties (attenuation, interfacial sound-speed discontinuities, no shear) provided sediment attemuation estimates which agree well with Hamilton's models and were an order of magnitude greater than that used in the nonoptimized model, which accounts for the reduction in the number of modes. A simulated model decomposition using the new attenuation verifies the number of modes predicted by the modal-sum-limit optimization.

2aUW12. Broadband matched-field processing of low level signals in shallow water. Newell Booth and Phil Schey (Ocean and Atmos. Sci. Div., NCCOSC RDTE Div. Code 541, San Diego, CA 92152-5001)

Matched-field processing analysis is presented for multitone signals from the shallow water evaluation cell experiment $\# 3$ (SWellEx-3) which was carried out in July 1994 west of Point Loma in $200 \mathrm{~m}$ water of complex bathymetry. The multitone test signal was transmitted from an acoustic source towed at various depths over tracks designed to test matched-field processing replica generation over complex propagation paths. The multitone signal consisted of 5 sets of 10 tones, each set spanned $50-200 \mathrm{~Hz}$ and was transmitted at various levels with 22-dB dynamic range. Using noise level estimales obtained at frequencies between the tones, the input signal + noise-to-noise ratio is estimated. Bartlett and minimum variance distortionless response (MVDR) adaptive matched-field processing results are presented from this data set over various paths for input signal to noise ratios spanning +12 to $-10 \mathrm{~dB}$.

2aUW13. Effects of ship trim and dynamic attitude on the relative noise level measured by a hull-mounted multibeam bathymetric sonar. C. de Moustier (Marine Physical Lab., Scripps Inst. of Oceanogr., La Jolla, CA 92093-0205)

Acoustic data recorded in various sea conditions with the hull-mounted multibeam swath bathymetry system SeaBeam 2000 aboard R. V. MELVILLE have been analyzed to quantify the relationship between the ship's attitude and the relative noise levels observable in the data. The data consist of concurrent time series of echo magnitude and direction of arrival for each transmission cycle, and of the three attitude components roll, pitch, and heave. Although higher noise levels are usually associated with pitch, in these data heave has the greatest impact on noise level, presumably because of bubble syphoning from the areated surface layer. In addition, a slight change in the trim of the ship from horizontal to bow up results in an average relative noise increase of about $12 \mathrm{~dB}$. [Research supported by ONR.]

2aUW14. Gradient-based receiver structures for detecting sound directions. Terry L. Henderson and Terry J. Brudner (Appl. Research Labs., The Univ. of Texas, Austin, TX 78713-8029)

The use of a pressure gradient microphone for detecting the source direction of an incident plane wave is well known. Furthermore, any receiving aperture that applies a specified apodization can be regarded as extracting a point-sample of a spatially filtered version of the incident wave. Our result is that when the gradient method is applied to the spatially filtered wave (which is also a plane wave), the answer can be expressed in terms modified apodizations, formed from the originally specified apodization function and its vector gradient, integrated over the original aperture and/or its boundary, together with simple temporal filters. Fortunately, this seemingly complicated structure collapses to a much simpler one in many cases of interest, and it is even possible to extend the method to the higher order case in which two or more wave directions are detected simultaneously. Altematively, one wave direction can be measured while steering a broadband null in another specified direction, and the steered-null direction can be altered dynamically even after beamforming. Examples are given. Our results are especially applicable to transient sounds of arbitrary bandwidth. [Work supported by ARL:UT IR\&D.]
2aUW15. Broadband matched-field source localization with a horizontal line array in shallow water. W. S. Hodgkiss, J. J. Murray, K. H. Kim, and G. L. D'Spain (Marine Physical Lab., Scripps Inst. of Oceanogr., La Jolla, CA 92093-0701)

SWellEx-3 (shallow water evaluation cell experiment \#3) was carried out in July 1994 west of Point Loma in approximately $200 \mathrm{~m}$ water. A MPL. 64-element vertical array was deployed from the R/P FLIP and the NRaD SWSS (shallow water sensor string) horizontal line array was codeployed $500 \mathrm{~m}$ to the east. The focus of this paper is on broadband $(70-370 \mathrm{~Hz})$ matched-field source localization with the SWSS horizontal line array. Examples of processing results from a CPA event near endfire using a $\mathbf{3 0 0}$ m subaperture of the HLA will be presented. In order to better understand these results, a comparison will be made with the results from similar processing of the data collecled by the VLA over the same period of time. In addition, simulations of the SWellEx-3 environment will be presented showing the effect on the matched-field ambiguity surface of increasing the HLA aperture. [Work supported by ONR, Code 321.]

2aUW16. Minimum variance matched-field source localization with SACLANT Mediterranean sea trial data. Jeffrey L. Krolik (Dept. of Elec. Eng., Duke Univ., Box 90291, Durham, NC 27708)

The minimum variance (MV) adaptive beamformer has been widely proposed for matched-field processing because it provides a means of suppressing ambiguous beampattern "sidelobes." In this paper, the performance of three robust MV methods is evaluated using vertical array data from the Mediterranean Sea collected by the NATO SACLANT Centre north of Elba [D. F. Gingras and P. Gerstoft, J. Acoust. Soc. Am. 96, 3234 (1994)]. The three MV methods considered are (1) the reduced MV method (RMV) [C. L. Byme et al., J. Acoust. Soc. Am. 87, 2493-2502 (1990)], (2) the MV method with neighborhood location constraints (MVNLC) [H. Schmidt et al., J. Acoust. Soc. Am. 88, 1851-62 (1990)], and (3) the MV method with environmental perturbation constraints (MV-EPC) [J. Krolik, J. Acoust. Soc. Am. 92, 1408-19 (1992)]. Real data results indicate that each approach has different merits. Projecting the data onto the reduced space defined by the modal eigenfunctions as in the RMV is necessary to avoid instabilities caused by highly correlated noise. Using neighboring location constraints as in the MV-NLC reduces the need to finely sample the ambiguity surface. And using constraints derived from the second-order statistics of the signal wavefront averaged over an ensemble of perturbed environmental parameters as in the MV-EPC increases robustness to environmental mismatch. [Work supported by ONR.]

2aUW17. Mirages in shallow water matched-field processing. G. L. D’Spain, J. J. Murray, W. S. Hodgkiss (Marine Physical Lab., Scripps Inst. of Oceanogr., San Diego, CA 92152-6400), and N. O. Booth (NCCOSC RTDE Div., San Diego, CA 92152-5001)

Broadband (50-200 $\mathrm{Hz}$ ) matched field processing was performed on vertical line array data from a recent shallow water experiment. Although the actual water depth steadily decreased from 200 to $100 \mathrm{~m}$ over the source tow track, the replica vectors were calculated assuming a rangeindependent environment of $200 \mathrm{~m}$ depth. Rather than breaking up due to the increasingly severe environmental mismatch, the broadband matched field output peak in range and depth behaved in a consistent way; both the predicted range and depth of the source became increasingly greater than its true range and depth as the true water depth decreased. A simple analytical model was developed to predict the behavior of these MFP mirages. The good agreement between model predictions and actual results suggests that the model can be used to quantify the impact of unknown water depth changes (e.g., ocean swell on synthetic aperture studies) and to "calibrate" water depth in a region using the sinking mirages of surface ships of opportunity. By use of the concept of "effective depth" developed by D. E. Weston et al, the model also can be applied to studies of ocean bottom geoacoustic parameter mismatch. [Work supported by ONR, code 321.]

2aUW18. Robustness and resolution of a ray travel time inversion scheme. I-Tai Lu (Polytechnic Univ., Route 110, Farmingdale, NY 11735)

A ray travel time inversion scheme has been developed not only for localizing the source and calibrating the array position, but also for characterizing environmental parameters such as sound-speed profiles in both 
water column and sediment [I-Tai Lu, J. Acoust. Soc. Am. 95, 2983A (1994)]. Here, we will present the detailed studies of robustness and resolution of this method.

2aUW19. Broadband hydrophone calibration below 1 MHz. Gerald R. Harris, Jeffrey M. Porter, Panl M. Gammell, and Christopher A. Patton (FDA/CDRH, 12721 Twinbrook Pkwy., HFZ-132, Rockville, MD 20852)

Recent calibration efforts for miniature hydrophones used to measure medical diagnostic ultrasound fields have been devoted to increasing the upper frequency range of calibration (>10-15 MHz). However, a bandwidth extending to at least 10 times below the diagnostic pulse center frequency is needed for accurate (error $\approx 5 \%$ ) measurement of the peak rarefactional pressure and mechanical index, both important quantities. Since at present no commercial hydrophones for medical ultrasound use provide sensitivity information below $1 \mathrm{MHz}$, a study was undertaken to determine these low frequency sensitivities. The technique uses broadband, plane-wave pressure pulses generated by electrical shock excitation of a thick piezoceramic disk. The hydrophone response is calculated from measurements of the source transducer and hydrophone voltage waveforms. The frequency responses of both needle and membrane polymer hydrophones were measured using this technique. The membrane hydrophones studied had bandwidths extending below $\approx 0.2 \mathrm{MHz}$, but one of the needle probes began rolling off above $0.5 \mathrm{MHz}$. Therefore, given the above criterion regarding diagnostic pulse center frequency, sensitivity to $0.1-0.2$ $\mathrm{MHz}$ should be established for diagnostic use hydrophones, because a uniform response below $1 \mathrm{MHz}$ cannot be assumed.

2aUW20. Broadband guide source helps localization in shallow water. Martin Siderius, Darrell Jackson, and Robert Porter (Appl. Phys. Lab., Univ. of Washington, Seattle, WA 98105)

Acoustic measurements from a broadband guide source are used in a highly variable shallow ocean to augment sparse environmental information. The concept of using a broadband guide source is inspired by the astronomical technique in which light from a star is used to correct atmospheric aberration of a nearby (in the angular sense) object. The acoustic technique investigated here is similar to holography [P. D. Mourad et al., J. Acoust. Soc. Am. 92, 1031-1039 (1992)] in which a vertical array of continuous wave (cw) reference sources is used to localize unknown targets by reconstructing the wavefront at the location of the reference source array, removing much of the distortion caused by the ocean. Mourad et al. showed that this method is effective where the adiabatic approximation is valid. The present technique differs by using a single broadband guide source in shallow water where mode coupling can occur. In this work, we develop a method similar to holography valid for strong range-dependent environments, together with single hydrophone matched field methods to solve the source localization problem. Numerical simulations are also given for a variety of environments to demonstrate the effectiveness of the method. [Work supported by Office of Naval Research.]

2aUW21. Source depth dependence of mismatch-induced range and depth localization errors in conventional matched-field processing. George B. Smith (Naval Res. Lab., Code 7173, Stennis Space Center, MS 39529-5004), Murty A. Akundi (Xavier Univ. of Louisiana, New Orleans, LA 70124), and Nichalos D. Gardner (Mississippi State Univ., Mississippi State, MS 39762)

In previous work [Smith and Akundi, J. Acoust. Soc. Am. 95, 2980(A) (1994)] analytic expressions for mismatch-induced range localization error and signal gain degradation for a linear correlator matched-field processor were obtained. Those results agreed with previously obtained results [Shang and Wang, J. Acoust. Soc. Am. 89, 2285-2290 (1991)]. In this work the problems of mismatch-induced depth localization error and the source depth dependence of both range and depth localization errors are addressed. Computer simulations show that the analytic predictions obtained here are not perfect but are close enough to indicate that this line of research is on the right track. [This work was supported by the Office of Naval Research, Program Element 61153N, with technical management provided by the Naval Research Laboratory, Stennis Space Center, Mississippi, The NAVY/ASEE summer faculty research program, and by the MAMP program.]
2aUW22. Nonambiguous beamforming for a high resolution twin-line array. Jean P. Feuillet (Space and Naval Warfare Systems Command, Washington, DC 20999), Walter S. Allensworth (Appl. Hydro-acoustic Res., Inc., Rockville, MD 20850), and Bruce K. Newhall (Johns Hopkins Univ., Laurel, MD 20723)

Technology of towed line arrays and associated signal processing has progressed in recent years to the point that wideband multiline array coherent signal processing can now be performed in real time. Earlier implementation (1993) of a multiline array beamformer centered around the ability to convolve a conventional line array beam response with that of an orthogonal dipole. Performance of this implementation provided a unique nonambiguous right/left beam with roughly $-20-\mathrm{dB}$ energy rejection in the null steered direction over a significant range of vertical arrival angles. However, performance suffered significantly with other than straight and parallel towed lines. Recent (1994) implementation of a beamformer for a twin-line towed array has shown the ability to form deep backlobe nulls $(-37 \mathrm{~dB})$. Implementation of this bearnformer is similar to the MVDR method except an isotropic noise field is assumed in the horizontal plane. This technique allows for uniform suppression of sidelobes at all off-MRA azimuth directions while maintaining backlobe rejection and MRA shape. Spatial weights are adaptively calculated based on the estimate of the twin-line array shape. This method has been shown to be robust for nonparallel and dissimilar shapes.

2aUW23. Shallow water active sonar Issues. William Carey (ARPA, 3701 North Fairfax Dr., Arlington, VA 22203) and Peter G. Cable (BBN Systems Technol., New London, CT 06320)

The central questions in sonar system design concern the limits of coherent processing and are: What is the optimum frequency range and bandwidth?; How large of a receiving aperture is required?; What is the achievable detection range? These questions are determined by the propagation and scattering of sound. In the shallow water channel, under downward refraction, the bottom is the determinant factor. A parametric sonar equation under reverberation limited conditions shows two factors are important, the effective bottom back scatter strength and the coherent signal gain. A parametric analysis is presented that focuses on the frequencydependent characteristics of the bottom backscattering strength and the effect of coherence on receiving array aperture. Several recent reverberation and signal coherence measurements are presented and the needs for future studies are delineated. The backscattering strength is shown to be strongly frequency dependent and to depend on the depositional sediment layer. The coherence is also found to depend on frequency and range as determined by signal gain measurements.

2aUW24. Acoustic calibration of shallow underwater explosive charges. Peter G. Cable and William J. Marshall (BBN Systems and Technol., Union Station, New London, CT 06320-6147)

Broadband underwater acoustic measurements are often performed using explosive charges as sources. For moderate weight charges detonated at shallow depths the bubble pulse oscillation period can exceed the separation between direct and surface reflected arrivals for any source-toreceiver geometry. Because of the overlap of multipath arrivals with the time signature of the charge, a direct determination of free-field source levels and spectral characteristics for such a situation can be problematical. In order to perform acoustic calibrations of explosive charges in shallow water, a method has been developed that uses the multiple arrivals on a vertical receiving array of hydrophones separated from the source detonation by one or two water depths. The technique involves averaging the time series from the vertical array phones after time aligning the direct shock arrivals on all the phones. This focused beamforming method has been used to obtain source calibrations from data obtained during area characterization test II (ACT II) in water 50 fathoms deep using $4 \mathrm{lb}$ Comp B charges detonated at $\mathbf{2 5}$ fathoms. High levels of consistency and repeatability were obtained from several sources detonated at different ranges from the array. The calibration technique will be described and illustrated, and the achievable precision of the method discussed. [Work supported by ARPA under the Adverse Environments Program.] 
2aUW25. Pressure effects on the dynamic and static properties of perforated elastomers used in underwater acoustics. Stephane Beretti (DCN Ingenierie Sud, Dept. of Underwater Warfare, BP 30, 83800 Toulon Naval, France), Christian Audoly (DCN Ing. Sud., Toulon, France), and Philippe Guillaussier (PONS, 13872 Aubagne Cedex, France)

The microinhomogeneous materials made with air-filled cavities in viscoelastic matrices, have many possible uses in underwater acoustics including anechoic coatings, acoustic decoupling, or reflectors for arrays. Theoretical models are necessary to predict the acoustic properties of these materials by evaluating the properties of the equivalent homogeneous material, as a function of frequency, pressure, and temperature. During recent works an abnormal effect of the applied pressure on these materials was noted: The plot of the longitudinal sound speed and of the bulk modulus, versus static pressure, had the lowest value for 10 to 20 bars of applied pressure, instead of a regular increasing plot predicted by the models [G. Gaunaurd et al., J. Acoust. Soc. Am. 76, 173-177 (1984)]. Different results, including test panel measurement in a tank, or measurement of the static and dynamic bulk modulus agree with this consideration. Theoretical models to predict the static and dynamic effective material properties as a function of the pressure were compared with these results.

2aUW26. Nonlinear transformations for spatial matched filtering (detect-on-track). Yung P. Lee (Science Applications International Corp., 1710 Goodridge Dr., McLean, VA 22102) and Haw-Jye Shyu (Naval Res. Lab., Washington, DC 20375)

A detect-on-track algorithm based on the Hough transform has been applied to acoustic broadband correlograms for passive detection and localization. The Hough transform integrates (sums) the amplitudes along a set of delay curves of interest. The delay curves are calculated over a range of closest point of approach (CPA), speed, and heading of the targets. When normalized by the number of points, the Hough transform computes the arithmetic-mean along the track. This process is referred to as an arithmetic-sum (AS) transform. This AS transform optimally reduces the variance of the noise, but can also generate significant ambiguous sidelobes. To reduce the sidelobe, two nonlinear transforms are proposed: The logarithmic-sum (LS) transform and the harmonic-sum (HS) transform. The LS-transform sums dB's while the HS-transform sums the reciprocal of the amplitudes along the track. When normalized by the number of points, the LS transform computes the geometric-mean and the HS transform computes the harmonic-mean along the track. Simulations show that the nonlinear transforms perform the same as the AS transform in noiselimited environments but outperform the AS transform in sidelobe-limited environments.

2aUW27. Shallow water source track localization using matched multipath correlations. D. P. Knobles, Evan K. Westwood, and K. C. Focke (Appl. Res. Labs., The Univ. of Texas at Austin, Austin, TX 78713)

A method for localizing a broadband moving source using the signals received on a horizontal planar array is presented. The method involves finding the constant-velocity, straight-line source track that generates the least error between measured and modeled correlation traces over a period of time. Measured correlation traces are obtained by cross-correlating multiple pairs of received time series. Simulated correlation time delays are obtained using a ray model that includes the effects of refraction on the ray arrival times. A nonlinear optimization routine is used to obtain the best match in measured and simulated time delays over time and receiver pair. The main problem in shallow water is to identify which multipath pairs produce the correlation traces observed in the measured data. An approach for overcoming this problem that involves testing multiple hypotheses concerning the multipath pairs will be presented. An example application of the method to a shallow water dataset with three bottom-mounted receivers in a triangular configuration is presented. In order to validate the localization, the measured correlagram is compared with a correlagram produced by a broadband normal mode model using the derived source track. [Work supported by the Office of Naval Research (Code 322).]

2aUW28. Autonomous environmental measurement system. J. M. Stevenson, J. R. Olson, and B. J. Sotirin (Acoust. Branch, Code 54I, Naval Command, Control and Ocean Surveillance Center, RDT\&E Div., San Diego, CA 92152-6435)

For experiment planning and optimized array deployment, experiment planners and anray designers often seek a quick, affordable means to help determine best placement of a underwater acoustic measurement system prior to deployment. Array design geometry relative to ambient noise levels and variability, seafloor morphology, currents, and water column structure (e.g., ducts) is aided by a priori knowledge of the setting. It is desirable to determine some of these unknowns before undertaking the time and effort of a system deployment. Likewise, experiment planners and array designers would like to be able to explore deployment schemes that are locally optimized for the environment in order to reduce sensor sparseness in critical areas. This presentation describes an expendable, inexpensive sensor suite installed approximately one week prior to the deployment of an underwater acoustic measurement system. The instrument collects environmental data and provides advanced information to the experiment planners prior to a final deployment, facilitating optimized placement of the measurement system. Parameters sampled include: Variability and average level of ambient noise, SVPs, seafloor type and sediment thickness, surface wave heights, tidal measurements, and water currents. Characteristics of the system described include its small size, low weight, low power, affordability, and simple, low-bandwidth data collection scheme based on slack line array technology. [Work supported by ONR, Code 321.]

2aUW29. Target range and depth estimation from surface multipath. James A. Doutt (Dept. of AOPE, Woods Hole Oceanographic Inst., Woods Hole, MA 02543) and Edward H. Hug (Old Ironsides, Inc., New London, CT 06320)

The range and depth of a target are determined and compared with the known depth and the range as derived from differential GPS navigation. The data consists of a single return from a monostatic sonar configuration. The method uses a cost function defined as the sum of the squares of the differences between the arrival times measured from the data and those predicted by ray trace for an assumed target depth and range. The depth and range are then varied so as to minimize the cost function. Only the direct and surface-reflected paths have been used. However, the method is easily generalized to use more arrivals if the bottom depth is known and bottom-interacting arrivals can be identified.

2aUW30. Results on the sequential classification of spherical objects in shallow water. Roger F. Dwyer (Naval Undersea Warfare Center, New London, CT 06320)

Based on the theoretical results reported in [J. Acoust. Soc. Am. 93, 1460 (1993)] and the simulated results reported in [J. Acoust. Soc. Am. 96, 3312(A) (1994)] an experiment to determine the performance of sequential classifying spherical objects in shallow water has been carried out. The shallow water data were obtained from experiments off the East Coast of the United States in 1993. Five spherical objects of different composition were embedded in shallow water data and the performance of a sequential classifier to correctly identify the objects was obtained. The performance results of these tests using standard measures of effectiveness will be reported.

2aUW31. Efficient acoustic-field computations for matched-field inversion for geoacoustic properties. S. E. Dosso, N. R. Chapman (Defence Research Establishment Pacific, FMO Victoria, BC V0S $1 \mathrm{~B} 0$, Canada), R. G. Racca, and N. G. Henson (Jasco Research Ltd., Brentwood Bay, BC V8M 1P7, Canada)

Matched-field inversion for source location, environmental properties, or a combination of the two (focalization) is based on comparing measured acoustic fields to replica fields predicted for a variety of possible environmental parameters and/or source positions using a numerical propagation model. Although this approach is conceptually straightforward and widely 
applicable, it requires a large number of replica-field computations and can be impractically slow if the propagation modeling is not carried out in an efficient manner. This is particularly true for environmental inversion and focalization where the expanded parameter search space generally requires a very large number of replica field computations, even if a relatively efficient optimization algorithm such as simulated annealing (SA) is employed. This paper describes matched-field inversion for geoacoustic properties using SA and rapid acoustic-field modeling. The replica-field modeling is based on the normal mode solution and makes use of multivariate interpolation of precomputed "look-up" tables of modal quantities stored as a function of the geoacoustic parameters. The optimum grid-element size for the modal tables is considered, and two interpolation schemes, linear and Shepard's method (locally quadratic), are investigated. An example is given of geoacoustic inversion in a North Pacific environment.

2aUW32. Sonar array self-noise analysis using hydrophone cross-correlation functions. Christian Audoly (DCN Ingénierie Sud, Dept. LSM, B.P. 30, 83800 Toulon Naval, France)

The performances of acoustic arrays for passive sonar systems are limited by ambient noise and by self-noise phenomena, such as turbulent flow-induced noise, machinery noise propagating along the hull, or propellor noise. To predict the performances of the system and to improve the design of the arrays, it is important to determine which self-noise component is dominant on a given ship at different speeds. This paper will present the behavior of the typical coherence functions between the hydrophones of a flank array, for the different noise components. As the evolution along sensor separation and/or frequency for each component is characteristic, the analysis of the coherence in a real experiment can be used to determine the dominant contribution. An example on a submarine will be given.

2aUW33. Large-array acoustic signal processing in random deep-water channels: Fffects of coherence. Alexander I. Malekhanov and Alexander G. Sazontov (Inst. of Appl. Phys., Russian Acad. of Sci., 46 Ul'yanov St., 603600 Nizhny Novgorod, Russia)

Approximate analytical solution of the matrix transport equation for the mutual coherence function (MCF) of an acoustic field in terms of normal modes was derived. The proposed approach is capable of including stochastically rough surface as well as volume fluctuations in the index of refraction, and allowed one to reduce the problem of the MCF calculation in a refractive channel to the analogous problem in a free space. These results have been then employed to simulate the statistical effects of longrange sound propagation in realistic deep-water environments from the North-West Pacific on the horizontal array detection performance for several beamforming techniques. The most attention was paid to the gain degradation for the sound frequency of $250 \mathrm{~Hz}$ over megameter ranges under the basic assumption that internal waves or surface wind waves are, respectively, the main source of acoustic signal fluctuations. Rough surface scattering has been shown to cause the most significant effects. In particular, for the array length of order 100 acoustic wavelengths and wind speed of $15 \mathrm{~m} / \mathrm{s}$ the coherence loss was of about $6 \mathrm{~dB}$ even for optimal quadratic processor and, in comparison, of about $12 \mathrm{~dB}$ for conventional plane-wave beamformer. [Work supported by Russian Basic Research Foundation, Grant No. 94-02-04544a.]

Session 2pAA

\title{
Architectural Acoustics: Health and Legal Aspects Regarding the Use of Porous Materials
}

\author{
Richard H. Talaske, Cochair \\ The Talaske Group, Inc., 137 North Oak Park Avenue, Oak Park, Illinois 60301
}

Angelo J. Campanella, Cochair

Campanella Associates, 3201 Ridgewood Drive, Columbus, Ohio 43026

Chair's Introduction-1:30

Invited Papers

1:35

2pAA1. Fiberglass health and safety update: Moving heyond the science. Rick Versen (Schuller International, Inc., P.O. Box 625005, Littleton, CO 80162)

Fiberglass has provided numerous acoustical and insulation benefits for over 50 years. Today, engineers, architects, and specifiers musl weigh the benefits derived from various products against the potential risks that may be involved. During the past several years significant efforts have been undertaken to evaluate the potential health hazards and risks associated with exposure to fiberglass materials to facilitate meaningful benefit/risk analyses. Large studies of workers and various animal tests have been conducted and reviewed by numerous groups and agencies. While many scientists believe that the research indicates that fiberglass is not very hazardous, if at all, to humans, others contend that it may be just as harmful as asbestos. While disagreement among scientists is nothing new, the confusion and controversy created in the minds of nontoxicologists and epidemiologists observing the debates is unfortunate. Comfort should be taken in the extensive weight of the scientific evidence that is available on this important material. Most important, we should understand the limitations of science and focus on the handling and use recommendations which can assure the safe use of fiberglass products. 
2pAA2. Weight of scientific evidence approach shows fibrous glass presents little public health risk. Charles W. Axten (Health, Safety, and Environmental Affairs, North America Insulation Manufacturers Assoc., 44 Canal Ctr. Plaza, Ste. 310, Alexandria, VA 22314)

To date, several scientific research studies have been published discussing the health effects of exposure to fibrous glass. Although the majority of these studies have failed to demonstrate any causal association between exposure to fibrous glass and adverse health effects, the material has nevertheless been classified/labeled as a "possible carcinogen." This paper discusses the basis for this classification/abel as well as a more realistic "weight of the evidence" approach to evaluating the scientific data on fiberglass. Using such an approach and integrating the substantial toxicological, epiderniological, exposure, and chemical data accumulated on fibrous glass over the past 50 years, one is led to the conclusion that the material poses little, if any, risk to the public health under normal conditions of use.

\section{2:15}

2pAA3. Porous insulation in HVAC systems. Philip R. Morey (Clayton Environmental Consultants, Inc., 1729 Christopher Ln., Norristown, PA 19403)

Porous insulation used to line the air stream surfaces of HVAC equipment provides a locus for the accumulation of dirt and debris. Dirt and debris are hydrophilic and the insulation on the air stream surfaces of mechanical cooling systems thus provides a niche for mold growth. The mold growing on porous insulation unlike moldy debris on a hard surface such as sheetmetal cannot be removed by duct cleaning. Actions for proactively preventing biocontamination of HVAC insulation include the following. (1) Porous insulation shall not be used to line the air stream surfaces of HVAC plenums where wetting is likely such as in the vicinity of cooling coils, water spray systems, humidifiers, and other sources of water. Porous insulation may, however, be used in these HVAC components provided that the insulation is separated from moisture sources by a barrier that is both air and water tight. (2) Because of possible mold growth, the use of porous insulation should be minimized on the air stream surfaces in mechanical ventilation systems where the relative humidity consistently exceeds $65 \%$.

\section{$2: 35$}

2pAA4. ASHRAE sound and vibration technical committee position statement on the use of fiberglass in HVAC systems. Russell A. Cooper (Jaffe Holden Scarbrough Acoustics, Inc., 114A Washington St., Norwalk, CT 06854)

Fiberglass duct liner continues to be the most cost-effective solutions to noise control in most HVAC air duct systems. There has been a recent increase in the number of institutional, educational, and medical projects for which the use of fiberglass has been banned or severely limited. The decisions are based on concerns that the fibers may be carcinogenic and that the products may promote microbial growth. This paper will present the ASHRAE TC2.6 (Sound \& Vibration) technical committee's response to these developments, describe the state of the medical research and briefly outline alternatives and the consequences of duct lining removal from HVAC systems.

\section{2:55}

\section{2pAA5. Measurement of shredded mineral fibers. Per V. Brüel (Brüel Acoust. ApS, Gl. Holtevej 97, Denmark)}

Throughout the world there has been a desire to test mineral wool for the shredding of mineral fibers. There is a general concern, that sound absorption materials, consisting of fibers made of rock or glass, are damaging even in small amounts. Until now a standard method for the testing of acoustical absorbers has not been found. This paper shows a test chamber and describes a method for the objective measurement of shredded fibers. This method is simple and fast to carty out and shows a level of accuracy, which seems better than earlier attempts.

\section{3:15}

2pAA6. Nonfibrous air and gas flow silencers and sound absorbers. Martin Hirschorn (Industrial Acoust. Co., 1160 Commerce Ave., Bronx, NY 10462) and Uno Ingard (MIT, Cambridge, MA)

Post-WWII jet aviation, air conditioning, quieter working and living conditions, and OSHA legislation necessitated new noise control products largely based on fibrous sound absorptive materials. In June 1994 the U.S. Départment of Health and Human Services announced that fiberglass is a material "reasonably anticipated to be a carcinogen." Nonfibrous silencers, noise suppressors, and sound absorbers have already been developed, for reasons unrelated to this announcement, for over 40 years-longer when automobile mufflers, Maxim silencers, and Helmholtz resonators are considered. Nonfibrous aircraft ground jet engine noise suppressors provide superior acoustic performance and durability. Nonfibrous HVAC silencers and sound absorbers are used in medical operating rooms and others requiring a risk-free environment. The design of nonfibrous silencers, rooms, sound absorbers, and encapsulated fiber designs for various acoustical requirements will be discussed. Most sound absorptive materials lend themselves well to mathematical modeling. Absorption characteristics of a variety of future absorber configurations, including their nonlinear behavior in intense sound fields, will be presented. Such designs are often perfected by means of laboratory models tests, with airflow if applicable, before finalization. For certain nonfibrous duct liners, the possibility of negative insertion loss must be considered. 


\title{
Session 2pAO
}

\section{Acoustical Oceanography: Acoustical Determination of Ocean Processes}

\author{
Michael D. Richardson, Chair \\ Naval Research Laboratory, Stennis Space Center, Bay Saint Louis, Mississippi 39529-5004
}

Chair's Introduction-2:00

\section{Contributed Papers}

\section{2:05}

2pAO1. Effects of salinity on the acoustics of bubble plumes. Gregory J. Orris and Michael Nicholas (Naval Res. Lab., Washington. DC 20375)

A previous paper [J. Acoust. Soc. Am. 95, 2826(A) (1993)] presented acoustic data collected near bubble plumes of various void fractions and sizes produced by varying the flow velocity of a water jet impinging normally on a water surface. Subsequent analysis has revealed that the data's spectral content varies considerably between the two phases of the experiment - one involving fresh water in Lake Washington and the other involving salt water in Puget Sound. In the fresh-water portion of the experiment the spectra are completely dominated by modes corresponding roughly to the gross dimensions of the plume. In the salt-water portion of the experiment, the salts and other contaminants (which act as surfactants and lower the surface tension) dramatically reduce the mean bubble size with respect to that of fresh water. The acoustic data from the salt-water experiment are drastically different from that of the fresh water, and show little or no modal structure. For this particular physical situation it is suggested that the dominant acoustic generation process is likely to be turbulence. Furthermore, differences in the acoustic signatures of the experiment could be exploited to determine, amongst other things, the concentration of the salts and contaminants.

2pAO2. Direct numerical simulation of the acoustic behavior of bubble clouds. M. Watanabe and A. Prosperetti (Dept. of Mech. Eng., Johns Hopkins Univ., Baltimore, MD 21218)

The description of a bubble assembly, or cloud, by means of an "effective medium" approximation has found widespread application in the underwater acoustics community. In this approach the bubbly liquid is regarded as a continuum endowed with properties different from those of the pure liquid. In this study the effective medium results are compared with those of a direct numerical simulation in which the effects of the mutual interaction of the bubbles are simulared. A spherical assembly of bubbles immersed in an incompressible liquid is studied. The potential problem is solved numerically with a singularity expansion method including both volume pulsations and translational oscillations of the bubbles. Numerical results for both single realizations and ensemble averages are presented and discussed. [Work supported by the Ofnce of Naval Research.]

\section{2:35}

2pA03. A theoretical study of natural frequencies of bubbles in sediments. Andrea Prosperetti and Hasan N. Oguz (Dept. of Mech. Eng., Johns Hopkins Univ., Baltimore, MD 21218)

Bubbles that are highly efficient scatterers have dramatic effects on the acoustic characteristics of sediments. The incorporation of such effects in continuum models requires the knowledge of individual bubble frequency and response to acoustic waves. For bubbles that are smaller than the acoustic wavelength but large enough that the liquid viscosity is insignificant, the potential flow approximation is reasonable. The natural frequency of bubbles surrounded by sediment grains of comparable size are computed by a boundary integral formulation allowing in detail for bubble deformation and the presence of rigid boundaries. Even though the geometry is restricted to axisymmetric cases, main features of this process are adequately captured by simulating a number of cases. Several configurations are tested and, in many instances, substantial differences between the current calculations and the commonly used formula have been found. [Work supported by the Office of Naval Research.]

\section{2:50}

2pA04. Bubble size distribution measurement using an inverse acoustic scattering technique: Theory and experiments. Ramani Duraiswami, S. Prabhukumar, and Georges L. Chahine (Dynaflow, Inc., 7210 Pindell School Rd., Fulton, MD 20759)

Determination of the bubble population in liquids is an important problem in many fields. Acoustic methods have advantages over other methods of measurement in that bubbles are very responsive to sound, that reasonably large volumes may be sampled, and that the method can be relatively inexpensive. However, previous attenpts at using acoustical means of measurement have not led to well-established measurement techniques due to the ill-posed nature of the problem. In the present work, a multiphase model for sound propagation through bubbly liquids is used to obtain two integral equations of the first kind for determining the bubble distribution from measured phase-velocity and attenuation data. New algorithms for regularization of the ill-posed problem by imposing a number of physical constraints in a direct minimization setting are developed. These algorithms have been tested on analytical data with varying artificial noise added, and found to be successful. Based on this technique a new bubble measurement device- the acoustic bubble spectrometer (ABS) is being developed. The device is being compared in a series of tests with other methods of bubble distribution measurement such as venturi cavitation susceptibility meters, and microphotography. Results from these experiments will be presented. [Work supported by NSF.]

\section{3:05}

2pAO5. A semianalytical method for the noise emission of finite size objects in shallow water. Hasan N. Oguz (Dept. of Mech. Eng., Johns Hopkins Univ., Baltimore, MD 21218)

Point sources are commonly used in underwater ambient noise computations to model wave breaking noise. A semianalytical solution to sound emission by a finite size object in shallow water is developed. The coefficients of the normal modes obtained by this technique are compared with the coefficients given by the point source approximation for the case of a hemispherical bubble cloud. The comparison is only good when the size of the bubble cloud is much smaller than the acoustic wavelength. Substantial differences occur when the radiation pattern near the bubble 
cloud deviates from a circular shape associated with the point dipole emission. Accurate normal modes coefficients given by the current method could be coupled with shallow water propagation models used in ambient noise calculations. [Work supported by the Office of Naval Research.]

\section{3:20}

2pA06. A model for underwater ambient noise at low wind speeds. Hasan N. Oguz (Dept. of Mech. Eng., Johns Hopkins Univ., Baltimore, MD 21218)

When the wind speed is low enough, wave breaking does not lead to bubble clouds of substantial size. The collective mode of oscillations that is dominant at high wind speeds may be neglected. In this case, wave breaking that is still the major source of noise is approximated by a finite area of the sea surface over which single bubbles are entrained and emit individual acoustic pulses. Each breaking wave has a certain emission characteristics depending on bubble size distribution, densily, wave size, rate of entrainment, etc. An empirical correlation between the whitecap coverage ratio and the wind speed is employed to infer the size of the bubble entrainment area. The contribution of all waves are summed and the ambient noise levels are computed as a function of the wind speed. The results are in good agreement with field measurements. [Work supported by the Office of Naval Research.

\section{$3: 35$}

2pA07. Depth-dependent gas fractions in acoustic models of marine sediments. Frank A. Boyle and Nicholas P. Chotiros (Appl. Res. Labs., Univ. of Texas, P.O. Box 8029, Austin, TX 78713-8029)

Models have recently been developed for acoustic scattering from trapped gas bubbles in sandy and silty marine sediments [F. A. Boyle and N. P. Chotiros, J. Acoust. Soc. Am. 96, 3219 (A) (1994) and F. A. Boyle and N. P. Chotiros, J. Acoust. Soc. Am. 94, 1766 (A) (1993)]. These preliminary models may be somewhat limited in applicability due to an assumption that the bubbles are distributed homogeneously throughout the half-space below the sediment interface. Observations suggest that actual sediments are horizontally stratified, and that gas fractions have strong depth dependence. The models have therefore been modified to allow specification of a depth-dependent gas fraction. Studies suggest that the backscattering strength is significantly affected, with considerable influence on its grazing angle dependence. [Work supported by Naval Research Laboratory, Stennis Space Center under the MCM Tactical Environmental Data System (MTEDS) project.]

\section{3:50-4:00 Break}

\section{$4: 00$}

2pA08. Recent developments in the in-situ measurement of sediment geoacoustic properties. Michael D. Richardson (Naval Res. Lab., SSC, MS 39529-5004) and Sean R. Griffin (Omni Technol., Inc., Metairie, LA 70001)

The latest version of the in-situ sediment acoustic measurement system (ISSAMS) allows direct, in-situ measurement of sediment geoacoustic properties. Geoacoustic probes, which are mounted on a rigid frame, are inserted into the sediment hydraulically. The entire operation is controlled and monitored in real-time from the surface. Data is collected and processed by a wet-side computer and transmitted to the surface for waveform display and analysis. Video cameras both monitor insertion of probes and provide preliminary indication of sediment type. A seabird CTD is used to measure bottom water temperature, salinity, and sound speed. Sediment compressional wave velocity and attenuation are measured over pathlengths of 40 to $110 \mathrm{~cm}$ and at depths of between 0 to $50 \mathrm{~cm}$ below the sediment-water interface. Pulsed sine waves are transmitted at $58-\mathrm{kHz}$ and time delays and voltages are used to determine values of velocity and attenuation between identical radial-poled ceramic cylinders (two transmit and two receive). Shear wave velocity and attenuation are measured using a similar pulse technique. Bimorph bender elements mounted in flexible silicone rubber mounts and driven at 100 to $2000 \mathrm{~Hz}$ to generate shear waves. Values of sediment geoacoustic properties from muddy sediments of Eckernförde Bay, Baltic Sea and carbonate sediments of the Florida Keys presented used to illustrate the operation and utility of the new system.

\section{$4: 15$}

2pA09. Using shear waves to predict the liquefaction potential of seafloor sediments. James Pyrah and Angela Davis (School of Ocean Sci., Univ. College of North Wales, Menai Bridge, Gwynedd LL59 5EY, UK)

There is growing evidence to support the use of the seismic shear wave velocity as a predictor of seafloor sediment liquefaction potential. At the University College of North Wales laboratory experiments are being performed to determine the steady-state characteristics of a variety of noncohesive sediments. The conventional laboratory approach involves an investigation of void ratio/effective stress relationships, and provides an indication of the sediment's likely behavior-contractive or dilativewhen subjected to cyclic shear stresses such as those imposed by earthquake or water wave loading. In the tests currently being carried out at UCNW, the shear wave velocity is also being monitored to establish velocity/void ratio/effective stress relations which offers the possibility of extrapolating the laboratory findings to the field situation. Thus given a knowledge of the in situ shear wave velocity, grain characteristics, and static stress regime, it becomes possible to provide an estimate of the sediment's stability under cyclic load conditions.

\section{4:30}

2pA010. Determination of vertical correlation lengths in a channel using SWELLEX-1 thermistor data. T. Barnard and $M$. J. Beran (Dept. of Elect. Eng., The Catholic Univ. of America, Cardinal Station, Washington, DC 20064)

In order to properly determine the volume scattering in a channel, it is necessary to know the characteristic vertical and horizontal correlation lengths associated with the random index-of-refraction fluctuations. The results we have obtained for characteristic vertical correlation lengths using SWELLEX-I vertical-array thermistor data are discussed. The data have been analyzed for day-time and night-time observations. In addition, the results are dependent upon averaging times and this effect is discussed. Graphs are given for the standard deviation and normalized cross correlations of the fluctuations, as a function of depth. [The vertical temperature data analyzed in this presentation were produced by NRL Code 7120 and NRAD Code 541 under ONR sponsorship. The data were supplied to us by B. Pasewark of NRL Code 7120. Our analysis was supported by ONR.]

\section{$4: 45$}

2pA011. Using Rytov acoustic wave modeling to test ocean variability models. Terry E. Ewart and Daniel Rouseff (Appl. Phys. Lab. and School of Oceanogr., Univ. of Washington, Seattle, WA 98105)

Stochastic acoustic models of acoustic phase propagation were used to validate ocean internal wave models in Ewart and Reynolds [SPIE Press, PM09 (April 1993)]. In that work Rytov modeling of the phase variability provided very accurate agreement with a linear ocean internal wave model. Such tests require extreme accuracy in the phase measurements and hence very accurate position stability in the acoustic arrays. In the AIWEX acoustic transmission experiment the phase variability was so small that millimeters of motion of the array were significant in the phase variance. When the propagation is in the Rytov regime for both the phase and $\log$ amplitude variability, the amplitude should be equally usable to test sto- 
chastic ocean models. In this work we develop the Rytov model for the case of variability in the sound velocity profile and a nonconstant Vaisala frequency profile where the scintillation index of the acoustic fluctuations indicates that Rytov models hold. Simulated linear internal wave fields and PE propagation are used to generate the complex wave fields. The stochastic inverse for the phase and log amplitude is compared with the input transverse spectrum of internal waves. The regimes of applicability of these methods, the directions of further research, and the incorporation of these techniques in the conduct of traditional oceanographic measurements to enhance our ability to interpret the internal wave environment will be discussed.

\section{$5: 00$}

2pA012. Mode coupling effect of Kuroshio front in Taiwan's Northeast Sea. Chifang Chen (Dept. of Naval Architecture and Ocean Eng., National Taiwan Univ., 73 Chou-Shan Road, Taipei, Taiwan, Republic of China)

The Kuroshio current turns toward the northeast while it passes the Okinawa Trough in Taiwan's Northeast Sea. In this region, there are different waters mixing, namely, water from the Taiwan Strait, water of the East China Sea, and the Kuroshio current. During the year, except in the summer months, the Kuroshio current is characterized by its distinct temperature difference from other waters, i.e., the Kuroshio front is a predominant feature in this region. The bottom topography in this region is composed of shelf and slope features. The acoustics propagation is studied numerically using the FOR3D code. Severe mode coupling is realized while the sound is transmitted through the Kuroshio front. [Work supported by National Science Council of Republic of China.]

\section{$5: 15$}

2pAO13. Three-dimensional ultrasonic imaging of temperature distributions. V. I. Mirgorodsky, V. V. Gerasimov, and S. V. Peshin (Inst. of Radioeng. and Electron. of RAS, Fryazino, 141120 Moscow Region, Russia)

The investigation of the new opportunity of an acoustothermometer's space resolution realization is described. The method is based on the correlation processing of signals received by the multi-element piezotransducer. It is convenient to illustrate the method by the example of a reception of the signals irradiated by a point noise source by means of the two spaced transducers. The mutual correlation function of these signals has a maximum at the delay that is proportional to the path's difference between the signal's source and piezotransducers. Space resolution that is determined by the correlation maximum's width is about a coherence length of the received signals. At the easily achieved frequency band of the received signals $(\sim 1 \mathrm{MHz})$ it may be of a relatively small value $(\sim 0.1 \mathrm{~cm})$. The analytical solution of the problem of space distribution of noncoherent radiation's sources by use of correlation processing of signals received by various elements of the transducers is presented. The opportunity of realization of a space resolution less than $1 \mathrm{~cm}$ at a distance of about $7 \mathrm{~cm}$ is shown by the computer simulation. It is essentially better using a known method based on the frequency's analysis. Results of preliminary experiments are presented.

\title{
Session 2pEA
}

\section{Engineering Acoustics: Transducers, Arrays, and Modeling}

\author{
Harold C. Robinson, Chair \\ Naval Undersea Warfare Center, New London, Connecticut 06320
}

\section{Contributed Papers}

1:00

2pEA1. Applications of the combustive sound source. Preston $S$. Wilson, Thomas G. Muir, John A. Behrens (Appl. Res. Labs. Univ. Of Texas, P.O. Box 8029, Austin, TX 78713-8029), and Janet L. Elizey (Univ. of Texas, Austin, TX 78712)

The combustive sound source (CSS) can be used to generate highintensity, low-frequency acoustic energy in a variety of mediums by using deployment configurations suitable to each medium. The basic principle of operation remains configuration independent. A gaseous fuel/oxidizer mixture is introduced into a combustion chamber and ignited with a spark. The ensuing combustion produces a bubble of expanding gas which, in turn produces high-intensity, low-frequency acoustic pulses. Three different deployment configuration experiments will be discussed, including water column, ocean-bottom seismic, and earth-surface seismic. In-situ measured energy levels and spectra produced by CSS are compared to other sources typical to each medium, including explosive and inertial. It is shown that CSS is capable of producing bulk waves in both water and earth mediums, as well as seismic interface waves for both air/earth and water/sea floor cases. It is demonstrated that CSS is a truly versatile acoustic energy source with a low cost deployment configuration which can be tailored easily to different environments. [Work supported by the U.S. Navy Office of Naval Research.]
1:15

2pEA2. An acoustical model of the pulse combustion process. Jeffrey A. Zimmer (BBN Syst. and Technol., 70 Fawcett St., Cambridge, MA 02138)

Pulse combustors derive many of their advantages as thermal devices from the large acoustic oscillations that are characteristic of the pulse combustion process. There have been numerous attempts to model this process and predict the frequency and amplitude of the oscillations. Many of these modeling efforts have relied heavily upon computation while providing little in the way of explanation of the underlying physical mechanism. The present model treats the pulse combustor as an acoustical system with feedback. The dependence of the operating frequency on various system parameters is studied through stability analysis. The dynamics of the combustion event itself are characterized as a time delay. The presenice of this delay in the feedback loop causes the operating frequency to differ significantly from the frequency one would calculate by treating the combustor as a Helmholtz resonator at resonance. Preliminary experimental verification of the theory is presented, as follows. Both the operating frequency and combustion time delay are measured for a pulse combustion boiler, as are temperatures in the unit to establish sound speeds. When the measured temperatures and delay are inserted into the model, it predicts the operating frequency to within $4 \%$. [Work supported by The Gas Research Institute.] 
problem is presented to model a future PCTSCM transducer. Linear excitation of a PCTSCM transducer is accomplished through the usual application of a potential difference across the two faces of a thin film realiza-

2pEA3. Noise reduction of an experimental pulse combustion work station. Jeffrey A. Zimmer (BBN Syst. and Technol., 70 Fawcett St., Cambridge, MA 02138) and Robert D. Collier (49 Eagle Ridge Dr, Lebanon, NH 03766)

Pulse combustors are pulsating acoustical systems which radiate highintensity radiated noise. The Forbes energy engineering experimental pulse combustor was operated in a "high pressure mode" at a fundamental frequency of $50 \mathrm{~Hz}$ and an output between 330000 and $390000 \mathrm{BTU} / \mathrm{h}$. The noise levels of the baseline configuration, measured around the exhaust outlet, had an SPL over $110 \mathrm{~dB}$ at $50 \mathrm{~Hz}$ and a series of high intensity narrow-band components up to $2 \mathrm{kHz}$. The A-weighted broadband SPL in the range of $100 \mathrm{~dB}(\mathrm{~A})$ exceeded damage/risk criterion by as much as 25 $\mathrm{dB}$. The noise reduction goals corresponded to NC50 and SIL of $50 \mathrm{~dB}$. The approach consisted of three parts: (1) A cowl silencer installed at the outlet vent for control of mid to high frequency broadband noise; (2) a custom designed tuned low-frequency muffler installed as part of the exhaust decoupler; and (3) thermoacoustic cladding applied to the structure. The silencer provided a reduction of $20 \mathrm{~dB}(\mathrm{~A})$ in the mid-frequency range and the muffler resulted in narrow-band reductions of 25 to $40 \mathrm{~dB}$ at the lowest frequencies and 5 to $15 \mathrm{~dB}$ up to $2 \mathrm{kHz}$. The results, including sound intensity mapping, are discussed in terms of the goals, design modifications, and operational factors. [Work supported by The Gas Research Institute.]

\section{$1: 45$}

2pEA4. The effects of multilayering piezoelectric plates with implication to 1-3 piezocomposite materials. David J. Powell (Dept. of Ocean Eng., Florida Atlantic Univ., Boca Raton, FL 33431), Thomas R. Howarth, and Robert Y. Ting (Naval Res. Lab., Orlando, FL 32856-8337)

This paper discusses the considerations of stacking piezoclectric plates mechanically in series (on top of each other) and electrically in parallel or series. Benefits include increased displacement for projectors and increased signal-to-noise ratio (SNR) for hydrophones (because of selfshielding and decreased electrical impedance). The presentation will show why these enhancements are realized by using a mathematical solution of the one-dimensional (1-D) wave equation to describe how each layer is related to that in adjacent layers via continuity of particle displacement and force. The resultant set of simultaneous equations is solved using matrix manipulation algorithms over a specified frequency range in terms of the free-field voltage sensitivity (FFVS), transmitting voltage response (TVR), and electrical immitance characteristics. A temporal transient response is obtained through the inverse fast Fourier transform (IFFT). This computational efficient model is capable of piezoceramic or $1-3$ piezocomposite transducer layers, as well as inactive layers. The $1-3$ piezocomposites are input by using the Smith expressions for representing the piezocomposite as a homogeneous material. Application of 1-3 piezocomposites for multiple layering will be introduced and discussed. [Work sponsored by the Office of Naval Research.]

\section{2:00}

2pEA5. Axial shear wave transducer constructed of a piezoelectric continuously twisted structurally chiral medium. Steven F. Nagle and Akhlesh Lakhtakia (Dept. of Eng. Sci. and Mech., Penn State Univ., University Park, PA 16802-1401)

The material properties of a piezoelectric continuously twisted structurally chiral medium (PCTSCM) vary helicoidally along the axial direction. The quasielectrostatic approximation was implemented for axial propagation modes in PCTSCMs [S. F. Nagle and A. Lakhtakia, J. Acoust. Soc. Am. 96, 3226(A) (1994)]. The following conclusions were drawn: Axial propagation of shear modes is affected by piezoelectric stiffening. Axial propagation displays selective attenuation and the frequency response was classified into three zones: A zone of no attenuation and two handednesses (zone I); a zone of selective attenuation (zone II); and a zone of no attenuation and one handedness (zone III). Now, a boundary value tion. It is shown that such a transducer would launch linearly polarized shear waves with controlled polarization. Within the transducer, the polarization does not, in general, follow the material inhomogeneity. Shear wave characteristics are dependent on the specific frequency response zone (see above) in which the transducer is operated. [Work supported by NSF Graduate Fellowship.]

\section{2:15}

2pEA6. A transduction model of lead magnesium niobate-titanate. Jean C. Piquette and S. E. Forsythe (Underwater Sound Reference Detachment, Naval Res. Lab., P.O. Box 568337, Orlando, FL 32856-8337)

Lead magnesium niobate (PMN) and lead magnesium niobate-lead titanate (PMNPT) are electrostrictive ceramics that exhibit high strains when a sample is subjected to a high voltage. The strain as a function of voltage is an almost perfect square-law over a large range of voltages, neglecting saturation and hysteresis. Unlike piezoelectric ceramics, these materials will not accept a significant permanent polarization, so the behavior cannot be linearized in this way. A transduction model of these materials based on an analogy to the Hunt electrostatic transducer is presented. The Hunt model is generalized for radiation loading and for a passive dielectric material between the plates. The dielectric model includes saturation but excludes hysteresis. The model is inverted analytically to determine a driving-voltage waveform which permits a PMN(PT)based transducer to be driven at high voltage in a harmonic- and transientfree manner. Successful experimental applications of the model to measurements obtained from PMN(PT) samples are presented. The results include a description of a successful attempt to drive a $\mathrm{PMN}(\mathrm{PT})$ sample in a harmonic- and transient-suppressed manner at high voltage.

$$
\text { 2:30 }
$$

2pEA7. Low-frequency underwater transducer modeling using the direct global stiffness method. J. Robert Fricke and Mark A. Hayner (Dept. of Ocean Eng., MIT, Cambridge, MA 02139)

The direct global stiffness matrix method (DGSM), described by Fricke and Hayner [direct global stiffness matrix method for 3-D Truss Dynamics, submitted to the ASME 15th Biennial Conference on Mechanical Vibration and Noise, 17-21 September 1995], provides an efficient method for analyzing two-dimensional and axisymmetric, low-frequency, underwater, transducer geometries. The DGSM method is a wave-type solution based on the Euler-Bernoulli beam element. The mechanical motion of the transducer element is modeled using a collection of beam elements welded together at joints. By imposing dynamic equilibrium conditions at these joints, a banded symmetric stiffness matrix is formed. The matrix is then solved using Gaussian elimination to find joint displacements and rotations with beam energies calculated on a post-processing basis. The radiation load due to the surrounding water is included using a compact source assumption. Thus fluid loading interaction effects, important for low quality factor transducers, are properly addressed. Analysis of a class IV flextensional transducer is offered as an example of the usefulness of this approach.

\section{2:45-3:00 Break}

\section{3:00}

2pEA8. In-water modal analysis using a combined finite element/boundary element method. Clementina $M$. Siders and $M . D$. McCollum (NRL, Orlando, 3909 Summerlin Ave., Orlando, FL 32856-8337)

A combined finite element/boundary element modeling technique can be used to analyze fluid-loaded transducers to compute the displacements of the structure at specified frequencies. The problem with this technique is that there is no way of knowing a priori the in-water resonance frequency corresponding to a given in-air mode. A common approach is to "guess" 
a frequency range that will encompass the in-water resonance frequency by using a coarse frequency step size. A fluid-loaded analysis is then performed in this frequency range. By examining the resulting displacements versus frequency, it can be determined whether the resonance has occurred in the frequency range. If the in-water resonance frequency is located, a finer frequency step can be used over a smaller frequency range to determine the resonance to a better resolution. This paper describes a method to determine the in-water resonance frequency of a finite element/boundary element model using an iterative eigenvalue solution, thus avoiding the lengthy convergence process of a harmonic solution. The time savings between this new in-water modal solution and the traditional harmonic solution can be phenomenal. Several example problems are also presented.

\section{$3: 15$}

2pEA9. Near-field acoustic solution of a rotating point source in frequency domain. Hongbin Ju, Menyu Sheng (Div. of Fluid Mech., Dept. of Eng. Mech., Tsinghua Univ., Beijing 100084, People's Republic of China), and Fangyuan Zhong (Shanghai Jiaotong Univ., Shanghai 200030, People's Republic of China)

To do research on the aeroacoustic mechanism of turbomachinery or any other rotating machines, it is important to study the acoustic characteristics of the rotating sources. Based on the frequency domain solution of moving sound sources, an approximate acoustic solution of a rotating point source in the near field, which is the superposition of a far-field solution and three near-field modification iterns, is given if the rotating radius is acoustical compact when compared with the field sound wavelength. Accordingly, the near-field Green's function in free space is obtained. Then, the directionality characteristics of the sound field induced by rotation is discussed, and the influence of source position, source frequency, and rotating frequency is studied in detail. The results reveal that the distribution of near-field modifications is discrete around the viewing angle, and the influence on near-field modification by the rotating radius happens mostly around the rotating plane, while the influence of the axial position of the source happens mostly near the rotating axis.

\section{3:30}

2pEA10. Variational model for the radiation impedance of class IV transducers II. Harold C. Robinson and Elizabeth A. McLaughlin (Naval Undersea Warfare Ctr., New London Detachment, New London, CT 06320)

In a previous paper [H. C. Robinson and E. A. McLaughlin, J. Acoust. Soc. Am. 96, 3318(A) (1994)], the variational method of determining modal radiation impedances was applied to a pair of class IV transducers. The self- and mutual radiation impedances for the quadrupole, octopole, and monopole modes were presented. This paper extends the analysis to include the effects of other asymmetric modes of the class IV. The in-vacuo mode description of the transducer's surface velocity will be improved by using three-dimensional mode shapes. The trial functions describing the surface pressure are modified as well. Changes in the impedance characteristics for the symmetric modes due to this improved transducer description will be shown. The characteristics of the nonsymmetric modal impedances are compared to those of the symmetric modes as a function of dimensionless major axis $k a$ and dimensionless separation distance $k d$. Suitable methods for combining these modal impedances into a total radiation impedance for the transducer will be presented. The effect of relative orientation of the transducers on the radiation impedance is investigated. The results will be compared to equivalent circuit models and experimental data. [This work was sponsored by the Office of Naval Research.]

\section{3:45}

2pEA11. Comparison of using extended-line projectors versus single-element projectors in a water-filled traveling-wave tube. $M$. K. Beason and L. D. Luker (Underwater Sound Reference Detachment, Naval Res. Lab., P.O. Box 568337, OrIando, FL 32856-8337)

This presentation considers the effect of projector design on reducing pressure-field deviations within the long-line hydrophone calibrator (LLHC). The LLHC is a water-filled traveling-wave tube which simulates a plane-wave pressure field arriving from any bearing. This is accomplished by measuring the electroacoustic transfer matrix between hydrophones and projectors that are placed within a steel pipe with an inner diameter of approximately $30 \mathrm{~cm}$. This transfer matrix is then used to compute projector drives suitable for creating the desired field. Two types of projectors were investigated for use in the LLHC: (1) single-element projectors using a hollow PZT-4 sphere with an outer diameter of $38.1 \mathrm{~mm}$ and a wall thickness of $4.76 \mathrm{~mm}$, and (2) projector lines constructed of 10 capped cylinders of PZT-4 with $12.7 \mathrm{~mm}$ length, $12.7 \mathrm{~mm}$ diameter, and $0.79 \mathrm{~mm}$ wall thickness. Model simulations and measured data will be presented indicating that the extended-source projectors result in lower deviations from the desired pressure field than single-element projectors at the same spacing. [Work supported by NAVSEA.]

\section{4:00}

2pEA12. Estimation of the uncorrelated and correlated spatial components of the self-noise in towed arrays. Brian G. Ferguson, Gary C. Speechley (Defence Sci. and Technol. Organisation, P.O. Box 44, Pyrmont, Australia 2009), and David V. Wyllie (Defence Sci. and Technol. Organisation, APW2-316, Canberra, Australia 9600)

The self-noise in towed arrays can be divided into two components on the basis of its spatial correlation properties. In this paper, optimal estimates of the uncorrelated and correlated self-noise components are obtained using an adaptive frequency-wave-number analysis technique. The results of applying the technique to real data from various towed-array types are presented. The spatially uncorrelated contribution to the total self-noise is often ignored in the presence of the correlated structure-borne vibrations that travel in both axial directions along the array with a phase speed that is less than the speed-of-sound propagation in the underwater medium. The increasing importance of the uncorrelated component at higher tow speeds is discussed and, under some circumstances, the uncorrelated component is shown to dominate.

\section{$4: 15$}

2pEA13. Acoustic coupling in directional loudspeaker arrays. Hahn Vu-Maesto, Ananda Chakravarty, Vineet Mehta, and Charles Thompson (Ctr. for Adv. Comput. and Telecommun., Dept. of Elec. Eng., Univ. of Massachusetts, Lowell, MA 01854)

The performance of directional loudspeaker and microphone arrays is of interest with regard to conferencing applications. Transducer arrays can be steered to enhance transmission and or reception at a prescribed location. In reverberant environments, directional microphone arrays have shown promise in enhancing speech intelligibility in hand-free applications. Steerable loudspeaker arrays, on the other hand, have met with limited success. The differentiating feature is the input impedance of the loudspeaker. In this presentation the role of the acoustic coupling between adjacent transducers in a loudspeaker array will be examined. The acoustical interaction between transducer elements is modeled. The impact of this interaction on beamforming is examined.

\section{4:30}

2pEA14. Variational principles derived for discontinuous electromagnetic fields. G. Aşkar Altay (Boğaziçi Univ., Istanbul, Turkey) and M. Cengiz Dökmeci (Istanbul Tech. Universit-Teknik Univ., P.K.9, Taksim, 80191 Istanbul, Turkey)

A unified procedure based on a general principle of physics (e.g., Hamilton's principle) together with Legendre's (or Friedrichs's) transformation is proposed to systematically derive certain variational principles 
for discontinuous electromagnetic fields which are useful to treat electromagnetic waves and vibrations in dielectrics. The integral and differential types of variational principles generate Maxwell's equations and the associated natural boundary and jump conditions as well as the initial conditions, as their Euler-Lagrange equations, for a regular finite and bounded dielectric region with or without a fixed, internal surface of discontinuity. Special cases of the variational principles, including a reciprocal one, are recorded which have those for time-harmonic motions and a dielectric region within a vacuum or a perfect conductor, and they are shown to agree with and to recover some of earlier variational principles [e.g., M. C. Dökmeci, IEEE Trans. UFFC UFFC-35, 775-787 (1988); UFFC-37, 369-385 (1990) and G. A. Aşkar, J. Acoust. Soc. Am. 95, 3007 (1994) and references therein]. [Work supported in part by The Scientific and Technical Research Council of Turkey.]

\title{
Session 2pED
}

\section{Education in Acoustics: Demonstrations in Acoustics}

\author{
Sally G. Revoile, Chair \\ Center for Auditory and Speech Sciences, Gallaudet University, Washington, DC 20002
}

Chair's Introduction-1:00

Invited Papers

1:05

2pED1. How musical instruments make sound. Thomas D. Rossing (Phys. Dept., Northern Illinois Univ., DeKalb, IL 60115)

Simple demonstration experiments help us understand how bars, membranes, plates, and air columns vibrate and how they produce musical sound. They also help us understand the physics of vibrations and waves.

$1: 20$

2pED2. Analysis and synthesis of speech. Tom Tremain (U.S. Dept. of Defense, 9800 Savage Rd., Fort George G. Meade, MD 20755-6000)

Speech produced by individual audience participants will be stored on a computer. Speech waveforms in the time and frequency domains will then be displayed by the computer. The speech will be played back in its natural form, and also after it has been analyzed and synthesized. Various transforms of the speech will be implemented and played back. For example, the speech will be speeded up and slowed down. The gender of the voice will be changed by altering the pitch periods. The speech will be made to seem as if the talker had a cold by changing the voicing of the consonant sounds.

\section{$1: 35$}

2pED3. A dynamic absorber and a side-branch resonator for vibration and noise control. M. G. Prasad, E. Zanone, and S. Abbattista (Noise and Vibr. Control Lab., Dept. of Mech. Eng., Stevens Inst. of Technol., Castle Point on the Hudson, Hoboken, NJ 07030)

This paper presents two demonstrations. The first one deals with control of vibrations of a motor under unbalance excitation. A dynamic absorber is used to reduce vibrations of the motor. The resonance phenomenon and mode shapes in vibrating systems will be shown. The second system is a finite length duct with an acoustic source (speaker) at one end. A Helmholtz resonator as a side-branch device is used to reduce the noise radiating from the open-end of the duct. Audible effects due to acoustic resonances in the duct and side-branch resonator system will be presented.

2pED4. A lecture demonstration of the cochlea. Robert M. Keolian (Naval Postgraduate School, Dept. of Phys,, Code PH/Kn, Monterey, CA 93943)

Part of the mystery of the cochlea, the organ of hearing in mammals, is how it distinguishes different frequencies from one another so well. Even though the cochlea is small, filled with fluid, and made out of tissue, its frequency selectivity corresponds to a quality factor $Q$ which is quite high-about 300 . Part of the answer lies with an unusual traveling wave that deposits the energy of different frequencies at different positions. This will be shown with a lecture demonstration that displays the physics of this wave. 
2pED5. Demonstrations of acoustic resonant systems. James M. Sabatier, Richard Raspet, and Bruce Denardo (Natl. Ctr. for Physical Acoust. and Dept. of Phys. and Astron., Univ. of Mississippi, University, MS 38677)

The phenomena of "sonic wind," viscous drag, and high " $Q$ " in resonant acoustic systems is demonstrated. The first two are the well-known Christmas tree balls and rotating cup demonstration. The third is a large heavy tuning fork which rings for a very long time after being struck.

\section{2:20-2:35 Break}

\section{$2: 35$}

2pED6. Demonstration of room acoustic parameters using a digital multieffects processor. Steven M. Haas (Jaffe Holden Scarbrough Acoust. Inc., 114A Washington St., Norwalk, CT 06854)

Digital multieffects processors is a useful tool to study and demonstrate room acoustic parameters. Live and prerecorded speech and audio program will be processed through a multieffects device and reproduced through a pair of loudspeakers. The listeners will be allowed to hear the effect of varying a number of acoustic parameters that occur in natural spaces. These include: reverberation time, echoes, diffusion of sound, high frequency decay rate, initial time delay gap, spaciousness, and depth. A brief explanation of each parameter will be given before its audible demonstration. The means in which an architectural acoustician designs an acoustic space with these parameters in mind will also be discussed.

2pED7. 15 minutes of chaos. Murray S. Korman (Dept. of Phys., U.S. Naval Acad., Annapolis, MD 21402) and Lawrence A. Crum (Applied Physics Lab., Univ. of Washington, Seattle, WA 98105)

Apparatus (both mechanical and electrical) will be presented to illustrate the complexities of a certain nonlinear behavior termed as chaos. In this demonstration, you will see a "chaos man" (a store bought sticklike compound pendulum) oscillate with a very complex motion. A simple pendulum will be made to oscillate (inverted) with the pivot at the bottom and the mass at the top. An electric circuit (modeling a nonlinear mechanical system) will be used to demonstrate chaotic behavior-which occurs when the system is driven with sufficient amplitude at certain frequencies.

\section{3:05}

2pED8. How a wind instrument makes a sound. Peter L. Hoekje (Phys. Dept., Univ. of Northern Iowa, Cedar Falls, IA 50614-0150)

Two demonstrations illustrate the mechanism of sound production in musical wind instruments. First, a "water trumpet" inspired by $\mathrm{A}$. H. Benade produces waves in a water-filled trough. The wave action is generated at one end the trough by a feedback valve which regulates the inlet flow of water according to the height of the water waves at that same end of the trough. This is analogous to the generation of sound in lip reed brass instruments and cane reed woodwinds in which the flow of air through the reed valve is affected by the pressure inside the instrument mouthpiece. The second demonstration again mimics the feedback mechanism of the reed with a simple electronic device which allows a wind instrument to sound itself. The air in the mouthpiece is excited by a small speaker which is driven by an amplified signal from a microphone sensing the pressure in the mouthpiece. The playing frequency depends on the resonances of the instrument and corresponds to a normal note of the instrument. Thus the demonstrator can finger a scale on the instrument and the instrument will play along.

2pED9. Reverberant sound in one-, two-, and three-dimensional spaces. William J. Strong and David C. Copley (Dept. of Phys. and Astron., Brigham Young Univ., Provo, UT 84602)

The method of images was used to calculate impulse responses for a large $(30 \mathrm{~m} \times 23 \mathrm{~m} \times 18 \mathrm{~m})$ three-dimensional space, a large (30 $\mathrm{m} \times 23 \mathrm{~m}$ ) two-dimensional space, and a large $(30 \mathrm{~m})$ one-dimensional space. The reverberation time for these spaces was set to approximately $1.5 \mathrm{~s}$ and all sound absorption took place at the walls. Similar impulse responses were calculated for small rooms whose dimensions were one-tenth those of the large rooms and whose reverberation times were smaller. A short (10-s) sample of singing was recorded in an anechoic chamber and then convolved with the various impulse responses. The resulting six reverberated samples of singing were recorded binaurally on audio tape. Graphs of the impulse responses and the taped examples will be presented. (Although the taped examples will be presented via loudspeakers, they are best heard via earphones.)

3:35-4:00

\section{Audience Participation with Exhibits}




\title{
Session 2pNS
}

\section{Noise: Advances in Aircraft Noise Modeling and Monitoring}

\author{
Sanford Fidell, Cochair \\ BBN Systems and Technologies, 21120 Vanowen Street, Canoga Park, California 91303 \\ Nancy S. Timmerman, Cochair \\ Massport, 600 Control Tower, East Boston, Massachusetts 02128
}

Chair's Introduction-1:00

Invited Papers

1:05

2pNS1. Measuring aircraft noise impact in a low signal-to-noise environment. Nathan B. Higbie (Larson Davis Systems, 131 Middlesex Trpk., Burlington, MA 01803)

The agreements negotiated for the new Denver Airport present an interesting example of how legal considerations can govern how noise measurements are made. The agreements stipulate certain noise limits on communities surrounding the airport. These limits are expressed in aircraft Leq(24), and are placed at 102 points, some over 15 miles away. There are financial penalties if any values are exceeded for a year. A signal-to-noise measurement problem resulted since modeled values of the aircraft Leq(24) were lower than measured Leq(24) community noise. The problems that needed solving were detection and quantification of aircraft noise in low signal-to-noise, and assignment of each noise event to its source. Arrays and other spatial techniques were proposed, but were too costly and would not meet Type 1 measurement requirements. A floating threshold was implemented so that noise events could be detected for any ambient condition. To date, all airport monitoring systems have used a fixed threshold since signal-to-noise is not a problem. The events are then correlated with the flight track data using a statistical pattern recognition algorithm whose parameters are optimized for each monitor location.

\section{$1: 25$}

2pNS2. Integrated noise model (INM) 5.0. Jake A. Plante (Analysis and Evaluation Branch, Office of Environment and Energy, Federal Aviation Administration, Washington, DC 20590)

The ability to accurately assess and predict noise exposure is an increasingly important factor in the design and implementation of airport and airspace improvements. To meet this challenge, the Federal Aviation Administration (FAA) Office of Environment and Energy (AEE) is undertaking a series of operations research initiatives to improve the modeling and other quantitative methods used to evaluate aviation noise impacts. A major effort in recent years has been the redesign of the integrated noise model (INM 5.0) in Windows. While rewritten in a different computer language, the noise calculation algorithms in 5.0 remain essentially the same as V4.11, based on the SAE-AIR-1845 methodology. There are, however, many technical enhancements to the computer code which provide faster run times and more accurate noise predictions. Among the enhancements are (1) a noise calculation module that is between 1.5 and 6 times faster depending on the study case, (2) a new exposure fractioning algorithm for improved accuracy, (3) additions to the aircraft database, (4) new metrics (e.g., $L_{\max }, L_{\text {eq(n) }}$, user-defined), (5) user-defined profile generation for the study of noise abatement landings and approaches, (6) a lateral track dispersion methodology, and (7) GIS analysis with Census, CAD, NFDC, elevation, and ARTS radar data capabilities.

$1: 45$

2pNS3. Statistical methods for automated INM path derivation. David A. Flynn (Larson Davis Labs., $1681 \mathrm{~W} 820$ N, Provo, UT 84601)

Flight paths at an airport can be viewed as samples of a statistical distribution. An individual sample path $x_{i}$, is described by the position as a function of distance from the airport. The mean paths of distribution $\left\langle x_{m}\right\rangle$ minimize $\Sigma x_{i}-\left(x_{\text {closes }}\right)$, where the operator "- " is an approximation of the difference in ground noise projection of two paths, and " $m$ " is the desired number of mean paths. Cluster analysis methods can be used to find this set of mean paths: they will by definition be the optimal $m$ paths for use in the INM in place of the original sample paths. These methods can reduce hundreds of thousands of radar monitored paths into a concise set of INM tracks. Moreover, they do not suffer measurement anomalies that gate penetration analysis suffers.

2pNS4. Modeling aircraft departure profile using ARTS radar data. Paul Dunholter (Mestre Greve Assoc., 280 Newport Ctr. Dr., Newport Beach, CA 92660)

The current method for the modeling of aircraft departure profiles is to use the standard departure profiles contained in the INM noise model. The INM model accounts for the different departure gradients based upon the fight distance to the aircraft's destination. Aircraft flying a longer distance are assumed to be heavier and as a result, would climb at a lower gradient. New techniques involve using the actual flight profiles from the FAA's ARTS radar data. State-of-the-art airport noise monitoring systems collect flight track 
data [Aircraft identification, Type of operation, and aircraft position ( $X, Y$ and $Z$ )] from the ARTS data. This information can be processed to determine the actual flight profiles for each aircaft type as well as a range of profiles for a specific aircraft. Using this technique, the departure profiles used in the model account for not only the departure distance, but also pilot techniques, the actual aircraft load as well as meteorological conditions such as altitude, density, and wind speed. The result is that the modeling of aircraft departure flight profiles are based upon actual data specific to the flights and conditions at the airport under study.

\section{$2: 25$}

2pNS5. Use of flight track information to resolve individual and community-wide aircraft noise concerns. Karen $L$. Robertson (Dallas/Fort Worth Int'l. Airport, P.O. Drawer 619428, DFW Airport, TX 75261-9428)

Technological advances in airport noise monitoring systems have provided the "missing link" of data needed to manage an airport noise office effectively. The addition of aircraft flight track and altitude information has given airport proprietors the necessary tools to understand, if not alleviate, certain community concerns regarding aircraft noise and overflights. Airport neighbors often contact the local airport or FAA office when an aircraft operation is annoying. The annoyance may be a result of one, or many, concerns ranging from noise to aircraft flight patterns to overall safety. Once concerns are voiced, airport neighbors want immediate information regarding the annoying event, followed by the ever-allusive "corrective action." Specific examples of how the new generation airport noise monitoring and flight tracking systems can respond to community or individual concerns will be demonstrated in an interactive format, followed by innovative, intriguing uses for the system. Information from these systems can surprise even the most knowledgeable airspace analyst or airport noise official.

2:45

2pNS6. Wide area modeling of en route aircraft noise. Nicolaas Reddingius (BBN Syst. and Technol., 21120 Van Owen St., Canoga Park, CA 91304) and Michael Ebersole (National Park Service)

Most aircraft noise modeling methods have been so carefully focused on aiport-based policies and analyses that they are awkward to use for other purposes. For example, standard noise modeling software is of limited utility for characterizing en route noise exposure or assessing the potential impacts on nonurban populations. The National Park Service has developed alternative methods for modeling aircraft noise and its impacts in outdoor recreational settings. Software created to support Public Law 100-91 analyses permits users to perform observer-based (rather than source-based) aircraft noise analyses; to consider the masking provided by localized sources when modeling noise impacts over areas of tens of thousands of square kilometers; to take terrain relief into consideration; and to present results in the form of map layers for a geographic information system.

$$
\text { 3:05-3:15 Break }
$$

\section{Contributed Papers}

\section{$3: 15$}

2pNS7. Applications of Boston-Logan's noise monitoring system. Nancy S. Timmerman (Massport, 600 Control Tower, E. Boston, MA 02128-2042)

Boston's Logan International Airport has an advanced noise monitoring system consisting of noise, aircraft track, weather, and land use information. The system has been used to support INM 5.0 validation efforts, to improve INM track definitions, to assist in assessing aircraft departure procedures, and to isolate the types of aireraft affecting various communities. Examples of the newer applications pursued will be presented.

$$
\text { 3:30 }
$$

2pNS8. A comparison of different aircraft noise metrics for large, medium, and small airports. Melissa Burn, Eric Stusnick, and Gary Ehrlich (Wyle Labs., 2001 Jefferson Davis Hwy., Ste. 701, Arlington, VA 22202)

A wide variety of metrics are available to represent and evaluate noise impacts from aircraft operations. Two of these have gained wide acceptance and are used extensively in noise studies and mitigation programs throughout the United States: Day night average sound level (DNL) and sound exposure level (SEL). A third metric, time above (TA), is not often used but is very often requested by community members who believe it will represent their noise problem more convincingly than the others. Each metric differs significantly from the others both in the way it represents noise impact and in way the measure is best employed. For example, since DNL accounts for the loudness of individual events and the number of operations, an equivalent DNL value can result from a few very loud overflights or a large number of quieter ones. DNL is considered useful in predicting the average response of communities but not of individuals. The authors evaluated the noise impact for different sized airports (based on number of operations) by examining each of the three metrics DNL, SEL, and TA. The noise impacts are compared and the best use of the information is discussed.

\section{$3: 45$}

2pNS9. Classification of noise events as aircraft overflights by means of one-third octave band algorithms. Matthew Sneddon (BBN Syst. and Technol., 21120 Van Owen St., Canoga Park, CA 91304)

A set of algorithms for real time classification of noise events as low altitude military aircraft overflights was developed for use in a personal noise monitoring system. The classification algorithms, which are applied to polynomial curve fits to time series of sound pressure level measurements rather than to raw data, operate primarily on the temporal symmetry and relative rates of increase and decrease of sound levels in different one-third octave bands. Correct classification performance and misclassification errors vary with disjunctive decision rules applied to the outputs of the algorithms. [Work supported in part by the U.S. Air Force NSBIT program.]

\section{4:00}

2pNS10. Effects of military aircraft noise on residential property sale prices near Langley Air Force Base. Sanford Fidell (BBN Syst. and Technol., 21120 Van Owen St., Canoga Park, CA 91303), Barbara Tabachnick (California State Univ., Northridge), Laura Silvati (BBN Syst. and Technol.), and Brenda Cook (U.S. Air Force Air Combat Command)

An empirical, market-based method was developed to assess whether military aircraft noise exposure has affected residential property values in the vicinity of Langley Air Force Base over the last two decades. Multiple regression models that accounted for the bulk of the variance in property sale prices were developed and validated for independent samples drawn 
from areas of Hampton, VA exposed to levels of military aircraft noise lower than $\mathrm{DNL}=60 \mathrm{~dB}$. The predictive performance of these models was indistinguishable in areas of Hampton exposed to military aircraft noise in excess of $\mathrm{DNL}=65 \mathrm{~dB}$. Maps constructed by geo-coding property sale locations permitted spatial comparisons of property values with aircraft noise exposure contours. These maps confirmed the independence of the spatial distributions of property values and aircraft noise contours in the vicinity of Langley Air Force Base.

4:15

2pNS11. Six months of continuous measurement of one-hour average sound levels, one mile sidewise from NAS Miramar, San Diego. Robert W. Young (1696 Los Altos Rd., San Diego, CA 92109-1321)

From 18 July 1994 to 17 December 1994, 1-h average sound level was measured continuously by Computer Engineering Ltd. model 493 integrating sound level meter feeding model $238 \mathrm{~A}$ secondary processor. The timemean-square average level printed automatically on the hour, and recorded in an RS232C memory for later analysis, is that during the hour preceeding the hour printed. The site of noise monitoring is the roof 7084 Miramar Road, San Diego, about a mile sidewise north of Runway 6L-24R, NAS Miramar. After adjustments to remove steady ventilating and compressor noise, aircraft noise was tabulated for each day as community noise equivalent level (CNEL), the day-evening-night weighted 24-h average level specified for California airports. The 148-day average CNEL was 69 dB. In one week CNEL varied from $58 \mathrm{~dB}$ on Sunday to $75 \mathrm{~dB}$ on Tuesday. Such noise variability is being investigated in relation to future movement of Navy aircraft from Miramar to NAS Lemoore, and Marine aircraft from MCAS El Toro to Miramar. Also being here reported for 7084 Miramar Road are the A-weighted sound exposure levels (ASEL) in excess of 100 $\mathrm{dB}$; the greatest thus far is $116 \mathrm{~dB}$

\section{$4: 30$}

2pNS12. The SEL noise reduction provided by barriers to residences located near runway ends. Marcio Avillez (Wyle Labs., 2001 Jefferson Davis Hwy., Ste. 701, Arlington, VA 22202)

The T.F. Green State Airport in Warwick, Rhode Island, has a noise barrier intended to reduce noise at residences on one side of Runway 05 , near the start of the takeoff roll. Measurements were conducted to evaluate the attenuation provided by the barrier. This was accomplished by selecting pairs of measurement positions that were located on opposite sides of the runway and equidistant from the centerline of the runway. The measurement results indicate that barriers can significantly reduce SEL of departing aircraft, and consequently the DNL, at residences located near the end of a runway.

\title{
Session 2pSA
}

\section{Structural Acoustics and Vibration: Wayne Reader Memorial Session}

\author{
Walter M. Madigosky, Cochair \\ Carderock Division, Naval Surface Warfare Center, 10901 New Hampshire Avenue, White Oak, Silver Spring, \\ Maryland 20903
}

Gerald Castelluci, Cochair

Vector Research Company, Inc., 2101 East Jefferson Street, Suite 701, Rockville, Maryland 20852

\author{
Introduction-1:00 by Maurice Sevik \\ Carderock Division Naval Surface Warfare Center, Bethesda, Maryland 20084
}

Invited Papers

1:05

2pSA1. The measurement of target strength of complex underwater structures-The early years. Jan M. Niemiec (Naval Surface Warfare Ctr., Carderock Div., Bethesda, MD 20084-5000)

By the early 1970's, few measurements of target strength of large scale underwater structures had been made. Novel measurement techniques to control the range, depth, and aspect angle of the underwater target had to be developed. Unique instrumentation had to be devised to measure the incident sound level at the target and to reliably record the scattered sound wave on a ping by ping basis without interrupting the rotating target. Dr. Wayne Reader lead a group of scientists and engineers who successfully overcame many of these difficulties and measured the target strength of a large scale model of a complex structure known as KAMLOOPS. In addition to providing the leadership for the resolution of many technical problems, Wayne Reader developed a unique method to calibrate the scattering measurements by using the doubly curved surface of the KAMLOOPS dome as a standard target. Many of the measurement techniques pioneered by Wayne are still in use today. This talk outlines the aspects of a model target strength measurement and illustrares some of the many contributions that Wayne made to this field of science.

\section{1:25}

2pSA2. Sonar baffles. Ronald P. Radlinski (Naval Undersea Warfare Ctr., Detachment New London, New London, CT 06320)

Acoustic decoupling baffles are often used to minimize noise contamination al hydrophone and transducer arrays. To maintain sensitivity near the nominally pressure release surface of the baffle, hydrophones can be placed at an odd multiple of a quarter wavelength from the baffle or near a heavy signal conditioning plate inserted between the hydrophones and the baffie. In either case, coherent interference between the incident wave and the wave reflected from the baffle limits the bandwidth of high sensitivity. Wayne Reader reasoned that by inserting a broadband absorber between the hydrophones and the decoupling baffle, a smoother hydrophone 
response could be attained of potentially less weight with relatively small loss in sensitivity. The talk describes the use of gradual transition absorbers developed by Wayne for sonar applications and his interactions with the author to combine these materials with broadband decouplers such as arrays of compliant tubes. His careful and thoughtful approach to the theoretical and experimental aspects of acoustic and material research was inspirational and we miss his insight, advice, and encouragement.

\section{1:45}

2pSA3. Wayne T. Reader: The Vector Research Company years, 1988-1994. C. Warren Vest (Vector Res. Co., Inc., Ste. 700, 2101 E. Jefferson St, Rockville, MD 20852)

After 30 years of distinguished government service Dr. Wayne T. Reader retired in 1988 and joined Vector Research Co., Inc. as principal research scientist. He continued his Navy-sponsored anechoic materials research program. He recruited a talented research and development team and established a state-of-the-art dynamic material evaluation laboratory. He developed unique experimental procedures for evaluating static and dynamic properties of viscoelastic materials, an extensive material properties database, and analytical computer programs to predict acoustic performance of layered media including anechoic lining systems. He continued to seek economical solutions to acoustic quieting and won a competitive Navy procurement to design and install an innovative lining system to a large anechoic test tank. While continuing his work with passive voided polymers, he began exploring the advantages of composites containing discrete and distributed piezoelectric particles in a polyurethane medium. In addition to his technical work, Wayne Reader established a working environment which met not only his interests but also the company's and the Navy's as well. He attracted and mentored several young scientists who continue to work in this field.

\section{2:05}

2pSA4. The development of 1-3 piezocomposite materials for large-area actuator applications. Robert Y. Ting (Naval Res. Lab., Underwater Sound Reference Detachment, P.O. Box 568337, Orlando, FL 32856-8337)

One of the technical subjects to which the late Dr. Wayne Reader made a significant contribution in recent years was the development of 1-3 piezoelectric composite materials, which consist of thin ceramic rods aligned and embedded in a polymeric matrix. The combination of good piezoelectric properties and the potential of low-cost manufacturing for large area coverage makes this new class of composite materials attractive for application as large area actuators in vibration control. Some of Dr. Reader's work will be reviewed, and the most recent development of new 1-3 piezocomposites will be discussed. Special new fabrication methods and the resulting material will be introduced. Acoustical properties of these new samples have been evaluated as a function of pressure and temperature and some of the results will be reported in this presentation.

2pSA5. History and status of working group S2-79 on the characterization of the dynamic properties of viscoelastic polymers. Bruce Hartmann (Polymer Sci. Group, Naval Surface Warfare Ctr., 10901 New Hampshire Ave., Silver Spring, MD 20903-5640)

Recognizing a long-standing need for standard materials to calibrate acoustic measurement equipment, Wayne Reader initiated a Working Group with the title "Characterization of the Dynamic Properties of Viscoelastic Polymers." The Working Group was organized under Standards Committee S2 of the Acoustical Society of America and was given the designation S2-79. The desirable qualities for the materials were: Ready availability, wide range of acoustic properties, stable with time, reproducible from lot to lot, and thermorheologically simple. He drew Group members from government, private industry, and universities, both in this country and abroad. Under his leadership, the Working Group progressed to the point that its work is nearly complete. This presentation will give an overview of the results from various laboratories on the most promising candidates. Emphasis will be given to a high loss polyurethane, for which the most data is available. In contrast, data for a low loss polyurethane will be presented to illustrate the reasons it was eliminated as a standard.

2:45

2pSA6. A technique for measuring the reflectivity of panels at low frequencies and high oblique angles. J. J. Dlubac and R. J. Deigan (Code 721, Signatures Directorate, Carderock Div. of the Naval Surface Warfare Ctr., Bethesda, MD 20084-5000)

Conventional short-pulse techniques for measuring the reflectivity of acoustic panels require that the reflected and incident waves be separated in time so that their ratio can be established. This technique is limited to moderate angles of incidence, since at high angles the direct and reflected waves overlap. An interference method is investigated as a way of making reflectivity measurements at high oblique angles. The total field (incident and reflected) near an oblique panel is measured relative to the incident field without the panel. The total field, which depends on frequency and angle of incidence as well as the panel material, is then examined and manipulated to extract information on the reflected wave. The technique is demonstrated with data reduced on steel plates. 
2pSA7. The design and development of an anechoic lining system for the acoustic test facility at NUWC Newport N. Scott Emery, Lois Peña, Shanti Satyapal, Kin Ng, and David Sauter (Vector Res. Co., lnc., Ste. 700, 2101 E. Jefferson St., Rockville, MD 20852)

During his years at Vector Research Company, Inc., Dr. Wayne T. Reader applied his extensive knowledge and experience in absorptive and decoupling coatings to the design and development of anechoic lining systems for large acoustic test tanks. The result of Dr. Reader's efforts was the award of a competitive Navy procurement 10 Vector to design and install a broad frequency ( 1 to $100 \mathrm{kHz}$ ) anechoic lining system in the acoustic test facility (ATF) and NUWC Newport. Based on Dr. Reader's designs and concepts, a prototype anechoic lining system for the ATF and NUWC Newport was tested successfully and the full system will be installed in the Spring of 1995. Background on the design and development of the lining system and data from prototype testing will be presented.

\section{3:35}

2pSA8. Basic model for acoustic reflection and absorption from multilayered underwater, sound-attenuating composites. Donald Brill (U.S. Naval Acad., Phys. Dept., Annapolis, MD 21402), Armando Santiago (Naval Surface Warfare Ctr., Annapolis, MD 21402), and Guillermo Gaunaurd (Naval Surface Warfare Ctr., White Oak, MD 20903-5640)

To reduce backscattered acoustic echoes from submerged elastic structures, these are routinely covered with viscoelastic (i.e, sound-absorbing) composite multilayers. This old and well-studied area is revisited and a basic, simple, analytical model to predict how these composites behave when tested is presented. The multilayer can have $N$ layers of homogeneous (or inhomogeneous) materials with different elastic/viscoelastic properties, all bonded to each other, and the whole, to a backing (metal) plate. The structure is fluid-loaded and extemally excited with plane, monochromatic sound waves. We have developed a "propagator matrix" approach that yields the reflected, transmitted, and absorbed waves from the structure as a product of the $N$ transfer matrices of the layers. The approach is computerized and used to evaluate and plot the reflectivity and/or absorptivity of the structure as a function of frequency for pertinent (often complex) values of the layers' material parameters and thicknesses. The predictions are experimentally verified for selected test cases (i.e., $N=2,3$ ), and the enhancement of the echo-reduction plots at the resonance frequencies of the layers is noted. Additional complexities can be handled in a straightforward fashion. [Work partially supported by the IL-IR Program of the CDNSWC.]

\section{3:50}

2pSA9. Dynamic mechanical analysis ASTM/ISO. James S. Peraro (Himont USA, 912 Appleton Rd, Elkton, MD 21921)

Dynamic mechanical analysis (DMA) is used to characterize the behavior of a variety of materials. The development of the methodology for conducting these tests is the responsibility of ASTM D20.10.15 on dynamic mechanical properties. Similar activities are conducted in ISO under the jurisdiction of subcommitte \#2. The test methods being developed cover a broad range of responses (temperalure, frequency, and time) as well as stress states (i.e., tensile, bending, shear, and compression). Instruments have been developed which have greatly simplified the measurement of dynamic mechanical properties. The use of advanced computers and microprocessors and improved computer programs, to properly digest this information, have increased the demand for more sophisticated test results. The ease of developing this test data has resulted in DMA being a requirement in material characterization and specifications. The demand of the global market for this information has increased the interest of both ASTM and ISO members to develop standardized test methods for conducting and reporting DMA test data.
2pSA10. The loss factor of beams with thick constrained layers. Joel Garrelick (Cambridge Acoust. Assoc., Inc., 200 Boston Ave., Medford, MA 02155)

A constrained layer beam, consisting of a base beam, viscoelastic layer, and cover plate, is analyzed with each layer modeled using the twodimensional equations of viscoelasticity. The (three) layers are "cascaded," enforcing appropriale equations of continuity. A shape factor is invoked to account for the effect of the beam's finite width on the dilatational wave speed of the constrained layer. Composite loss factors are first defined solely for the freely propagating (modified) flexural wave-number component of the response and then in terms of the overall drive point response of the beam to a mid-span load. At low frequencies results are compared to values obtained from Kerwin's [1959] classic thin layer model. At the other end, high frequency asymptotic limits are obtained by viewing the constrained layer as being semi-infinite and/or "fuzzy." Finally, predictions are compared to measurements performed on a steelNitrile-steel beam [Junger et al., 1986].

\section{4:20}

2pSA11. Results of recent development to measure and analyze the dynamic properties of materials. Ahid Nashif (Roush Anatrol, 935 Benecia Ave., Sunnyvale, CA 94086)

Although the vibrating beam technique has been widely used and demonstrated to be an effective technique for measuring the dynamic properties of materials, it has its own limitations. This is because the present analysis uses the fourth-order beam equations which do not adequately handle the boundary conditions of the test specimens especially for the fundamental mode of vibration. Because of that, measurements on the first and sometimes the second modes of vibration have been omitted which limits the frequency range where measurements could be made. To overcome such limitations, a new approach using finite element analysis has been developed to compute the dynamic properties of materials for several modes of vibration, including the lower ones. This analysis is suited for either extensional or shear deformation, depending on the geometry of the specimen. Results of using this analysis to compute the dynamic properties of the various proposed materials for the S2WG79 standard will be presented and discussed.

\section{4:35}

2pSA12. Flow-induced self-noise on a spherical sensor. Andrew $\mathbf{R}$. Jones and Gerald C. Lauchle (Graduate Program in Acoust. and Appl. Res. Lab., Penn State Univ., P.O. Box 30, State College, PA 16804)

An experimental effort to characterize the low Reynolds number flowinduced self-noise on a spherically shaped inertial sensor operating underwater is described. The transducer is a small geophone encased in a sphere, $76.2 \mathrm{~mm}$ in diameter. The sphere itself is cast from a 4:1 by volume of polystyrene microballons and epoxy resin, which yields a slightly negatively buoyant bluff body that is capable of sensing weak fluctuations in velocity such as those resulting from acoustic disturbances. When such a sensor is used in ocean environments, a spurious self-noise signal oftentimes exists due to low velocity currents. The research described in this paper quantifies this flow-induced noise signal as a function of the sphere diameter Reynolds number $\left(0<\operatorname{Re}_{d}<20000\right)$ and it is found that the broadband ( 10 to $1000 \mathrm{~Hz}$ ) flow-induced signal is due to the turbulent flow structures shed by the sphere. Virtually no broadband noise is produced when the Reynolds number is subcritical and the shed vortices are laminar. [Work supported by Naval Air Warfare Center, Warminster, PA under ONR contract.] 


\title{
Session 2pSC
}

\section{Speech Communication: Cross-Modal Integration and Speech Perception}

\author{
Lynne E. Bernstein, Cochair \\ Center for Auditory and Speech Sciences, Gallaudet University, Washington, DC 20002 \\ Kenneth W. Grant, Cochair \\ Walter Reed Army Medical Center, Army Audiology and Speech Center, Washington, DC 20307-5001
}

Chair's Introduction-1:15

Invited Papers

$1: 25$

2pSC1. Speechreading aids based on automatic speech recognition: Prospects for the automatic generation of cued speech. L. D. Braida, R. M. Uchanski, and L. A. Delhome (Res. Lab. of Electron., MIT, Cambridge, MA 02139)

Current progress in the development of automatic speech recognition (ASR) systems may soon permit discrete symbolic speechreading supplements to be derived from the speech signal. Such supplements could be similar to those used in manual cued speech, in which the talker uses discrete hand positions and shapes to provide distinctions between constants and vowels that are often confused in speechreading. Highly trained receivers of manual cued speech can achieve nearly perfect reception of everyday connected speech materials at normal speaking rates through the visual sense alone. To understand the accuracy that might be achieved with automatically generated cues, we measured how well trained spectrogram readers and an automatic speech recognizer could assign cues for various cue systems. A model of audiovisual integration was then applied to these measurements and data on human recognition of consonant and vowel segments via speechreading was published. This analysis suggests that with cues derived from current recognizers, consonant and vowel segments can be received with accuracies in excess of $80 \%$, roughly equivalent to the segment reception accuracy required to account for observed levels of manual cued speech reception. To provide guidance for the development of automatic cueing systems, we describe techniques for determining optimum cue groups for a given recognizer and speechreader, and estimate the cueing performance that might be achieved if the performance of current recognizers were improved.

\section{$1: 55$}

2pSC2. Measures of auditory-visual integration. Ken W. Grant, John L. Clay, and Brian E. Walden (Walter Reed Army Med. Ctr., Army Audiol. and Speech Ctr., Washington, DC 20307-5001)

The easiest and most effective way to improve speech recognition for hearing-impaired individuals, or for normal-hearing individuals listening in noisy or reverberant environments, is to have them watch the talker's face. Auditory-visual (AV) speech recognition has been shown consistently to be better than either hearing alone or speechreading alone for all but the most profoundly hearing-impaired individuals. When AV recognition is less than perfect, several factors need to be considered. The most obvious of these are poor auditory (A) and poor visual (V) speech recognition skills. However, even when differences in unimodal skill levels are taken into account, differences among individual AV recognition scores persist. At least part of these individual differences may be attributable to differing abilities to integrate $A$ and $V$ cues. Unfortunately, there is no widely accepted measure of AV integration ability. Recent models of AV integration offer a quantitative means for estimating individual integration abilities for phoneme recognition. In this study, we compare several possible integration measures, along with model predictions, using both congnuent and discrepant AV phoneme and sentence recognition tasks. The focus of this talk will address the need for independent measures of AV integration for individual subjects. [Work supported by NIH Grant DC00792.]

2:15

2pSC3. Perceiving talking faces. Dominic W. Massaro and Michael M. Cohen (Dept. of Psychol., Univ. of California, Santa Cruz, Santa Cruz, CA 95064)

Speech perception has been studied extensively in the last decades. It has been learned that people use many sources of information in perceiving and understanding speech. This talk focuses on the important contribution of visible information given in the talker's face in face-to-face communication. This visible speech is particularly helpful when the auditory speech is degraded due to noise, bandwidth filtering, or hearing impairment. Although the influence of visible speech is substantial when auditory speech is degraded, visible speech also contributes to performance even when paired with intelligible speech sounds. The importance of visible speech is most directly observed when conflicting visible speech is presented with intelligible auditory speech. These studies use a synthetic talking face to achieve control over the visible speech and to study those visible aspects that are informative. The talking head can be heard, communicates paralinguistic as well as linguistic information, and is controlled by a text-to-speech system. A fuzzy logical model of perception (FLMP) has been shown to provide an accurate description of how people evaluate and integrate audible and visible speech in a broad range of experimental studies. Demonstrations of the talking head and various psychological phenomena will be provided. [Work supported by NIDCD.] 
2pSC4. A neural basis for the synthesis of sound, sight, and touch. Barry E. Stein (Dept. Neurobiol. \& Anat., Bowman Gray School of Medicine, Wake Forest Univ., Winston-Salem, NC 27157-1010)

That visual cues affect speech perception has been known for some time, but is only one part of the much broader phenomenon of cross-modal integration. This fundamental characteristic of the CNS has a profound influence on perception and behavior and is evident not only in speech perception, but in the facilitated detection, identification, and reaction to combinations of concordant cues from different modalities, and in the striking perceptual anomalies that can occur when these cues are discordant. Understanding the mechanisms by which these functions are achieved requires a fundamental knowledge of the neural bases of multisensory integration; specifically, the circuits involved and the principles by which such "multisensory" neurons synthesize their convergent sensory information. Perhaps the best known site of multisensory convergence is the superior colliculus, a midbrain structure involved in attentive and orientation functions. The spatial, temporal, and receptive field characteristics of its constituent multisensory neurons serve as an excellent model for understanding the neural principles of multisensory integration throughout the CNS and for predicting overt behavior. It will be these multisensory circuits, the neural principles by which they affect multisensory integration, and the impact of this integration on overt behavior which will be emphasized in this discussion.

2pSC5. Crass-modal perception: Similarities and communalities. Lawrence E. Marks (John B. Pierce Lab. and Yale Univ., 290 Congress Ave., New Haven, CT 06519)

Cross-modal perception encompasses two distinct domains. One domain consists of those objects, events, or environmental conditions (e.g., phonemes, shapes of objects, locations in space or time) about which different sensory modalities can provide common information; the other domain consists of dimensions or qualities of perceptual experience in different modalities that bear a perceptual similarity that itself need not reflect any communality in the environment (e.g., between greater auditory pitch and greater visual brightness). Cross-modal relations pervade perception, and they are readily determined through several psychophysical paradigms, including measures of perceptual equivalence between stimuli presented to different modalities (cross-modal matches); measures of cross-modal similarity, assessed by multidimensional scaling; and measures of cross-modal interactions in response speed and accuracy during stimulus classification. Although many cross-modal relations are grounded in perceptual similarities or environmental communalities, interactions in the processing of stimuli presented to different senses can readily be modulated by language, and can also depend on semantic recoding. [Work supported by NIH Grant DC00271.]

4:05

2pSC6. Unisensory and multisensory convergence in a phonetotopic register. Robert E. Remez (Dept. of Psychol., Barnard College, 3009 Broadway, New York, NY 10027)

Descriptive studies have noted that speech perception is ineluctably multisensory if the listener can hear and see the talker. In such cases, the resolution of linguistic attributes occurs by combining auditory and visual inflow rather than by phonetic analyses in each modality before convergence. Because subjects readily acquire unanticipated proficiency in haptic and auditory integration, some researchers have conceptualized speech perception as a multisensory means of detecting a talker's phonetic production, independent of memorized sensory prototypes. These findings have motivated a definition of the problem of sensory convergence in terms that are literally multisensory: How do auditory and visual and haptic afferents coalesce? Recent studies of perceptual organization in a speech mode present a contrasting view, based on a phenomenon of unimodal convergence in speech perception. In this research employing tonal analogs of speech signals, phonetic perception occurs if auditory constituents cohere. Failure of convergence in this empirical paradigm is equal to failure of phonetic perception. Together, these studies suggest that integration of sensory attributes in speech perception is equivalent across changes in domain, from uni- to multisensory, and warrants an account of convergence that is general over changes in the scale of the sensory projection. [Work supported by NIDCD.]

4:35-5:00

\section{DISCUSSION}




\title{
Session 2pSP
}

\section{Signal Processing in Acoustics: Wavelet Analysis Techniques and Applications II}

\author{
David I. Havelock, Chair \\ National Research Council, IMS/ASP, M36 Montreal Road, Ottawa, Ontario KIA OR6, Canada
}

\section{Invited Papers}

\author{
1:15
}

\begin{abstract}
2pSP1. A wavelet auditory model and noise suppression. Anthony Teolis (Naval Res. Lab., 4555 Overlook Ave., S.W., Bldg. 210, Washington, DC 20375-5000)

Based on a wavelet auditory model (WAM) of the mammalian auditory system, discrete time-scale representations of acoustic signals which are inherently robust to "noise" emerge. The model consists of the two main processes of (i) a "continuous" wavelet transform with a causal analyzing wavelet determined by a "shark-fin"-shaped frequency response (cochlear filter), and (ii) a specific and signal-dependent sampling of the wavelet transform. In general the pattern of samples in the wavelet domain is irregular and determined at lower frequencies by activity at higher frequencies. By analyzing the model in terms of the theory of mathematical frames, it is shown that acoustic signals may be fully recovered from their WAM representation through iterative reconstruction algorithms. Noise is thought of as that portion of an acoustic signal which is "incoherent" with respect to the underlying WAM frame functions. Because coherent energy is highly localized and incoherent (noise) energy is necessarily scattered in the time-scale plane by the WAM frame representation, wavelet shrinkage techniques provide powerful algorithms for noise suppression. Several numerical examples of the noise suppression abilities of these algorithms are presented in the paper.
\end{abstract}

1:45

2pSP2. Adaptive wavelet collocation methods and wave propagation. Wei Cai (Dept. of Math., Univ. of California, Santa Barbara, CA 93106)

An adaptive wavelet collocation method for the initial value boundary problem of nonlinear PDE's is studied. The collocation method is based on a cubic spline wavelet decomposition for the Sobolev space $H_{0}^{2}(I)$, where $I$ is a bounded interval. Based on a special "point-wise orthogonality" of the wavelet basis functions, a fast discrete wavelet transform (DWT) is constructed. This DWT transform will map discrete samples of a function to its wavelet expansion coefficients in $O(N \log N)$ operations. The issue of efficient data structure for the wavelet collocation methods will also be discussed. Numerical results for various PDE's including wave propagation will be presented.

\section{Contributed Papers}

\section{2:15}

2pSP3. Exploratory wavelet transform analysis of seismoacoustic data. Randall W. Smith, Steven A. Stotes, and Robert A. Koch (Appl. Res. Labs., Univ. of Texas, P.O. Box 8029, Austin, TX 78713-8029)

The one-dimensional continuous wavelet transform is applied to the analysis of seismoacoustic data. The data consist of received signals, due to an impulsive source, measured by a triaxial seismometer. The wavelet transform provides a time-frequency decomposition of the signal, which in turn yields information about dispersion and arrival times of the various propagation modes. The primary goal of the analysis is to explore methods for extracting information from the time-frequency representation. The use of the wavelet transform will be complemented by modeling efforts. In particular, the different modes of propagation, estimating geoacoustic parameters, and localizing the source will be identified and analyzed.

\section{2:30}

2pSP4. Wavelet applications to bottom sediment classification. Russell Priebe, Nicholas P. Chotiros (Appl. Res. Labs., Univ. of Texas, Austin, TX 78713-8029), Donald J. Walter, and Douglas N. Lambert (Naval Res. Lab., Stennis Space Center, MS 39529)

Wavelet signal decomposition techniques applied to fathometer echoes were used for seafloor classification. Fathometer echoes were deconvolved with their source signals to yield transfer functions representative of the seafloor. These transfer functions were then expanded onto damped complex exponential wavelet bases. In this case a discrete implementation of the continuous wavelet transform was used. This technique allowed the signals be decomposed into distinct modes by localizing signal component energies in time and frequency. Modal features were then analyzed for clues to the physical makeup of the seafloor. Techniques were developed for extracting modal features from the wavelet signal expansions. These modal features include modal densities, center frequencies, bandwidths, duration, and most importantly, decay rates. The modal features were analyzed for clues to the physical makeup of the sea bottom. Available information was exploited to invert for internal sediment structure, particularly sediment density and the presence of trapped bubbles. Feature-based bottom maps were generated allowing classification of the seafloor. [Work supported by NRLSSC under the MTEDS program.]

\section{2:45}

2pSP5. Signal denoising using matching pursuits. Wade Trappe (Appl. Res. Labs. and Dept. of Math., Univ. of Texas, P.O. Box 8029, Austin, TX 78713-8029)

This paper will examine in brevity the matching pursuit algorithm proposed by Mallat and Zhang which yields an adaptive signal decomposition that may be used to derive a phase plane representation of a signal. Matching pursuits is closely related to wavelet analysis and may be considered a superset of wavelet multiresolution analysis. A methodology for 
coherent feature extraction, or denoising, using matching pursuits will be developed and explained. Acoustic signals derived from speech and music will be examined. Synthetic white noise and acoustic noise will be injected and matching pursuits will be used to attempt to remove noise from these signals.

\section{$3: 00$}

2pSP6. Noise isolation and reduction using spline wavelets. Phillip L. Ainsleigh (Naval Res. Lab., Underwater Sound Reference Detachment, Orlando, FL 32856-8337) and Charles K. Chui (Texas A \& M Univ., College Station, TX 77843-3368)

A technique is introduced for removing unwanted components from data using $B$-spline-based multiresolution ( $B$-wavelet) analysis. The time and frequency localization capabilities of wavelets allow two advantages over standard filtering methods: (1) better noise detection arising from the ability to isolate noisy components in wavelet bands where the signal energy is small relative to the noise energy, and (2) less signal loss by selectively filtering only those segments within wavelet bands containing unwanted noise. The procedure is used to remove impulsive noise from time series, and to eliminate multipath interference from frequency domain data.

\section{$3: 15$}

2pSP7. A method of analysis of acoustic emission signals due to leakage by wavelet transform. Q. M. Chen, Y. S. Ho, and T. P. Leung (Dept. of Mech. and Marine Eng., The Hong Kong Polytechnic Univ., Hung Hom, Kowloon, Hong Kong)

In the test of continual acoustic emission due to leakage, the rms was used to evaluate the energy of AE signals. However, the value of rms strongly relates to measuring conditions, including sensitivity of sensor, mounting pressure, setting amplification, etc. Detecting points of sharp variation of $\mathrm{AE}$ signals by wavelet transform to evaluate leakage is explored in this paper. The parameter evaluating leakage is sample zerocrossing counts of wavelet transform at each scale. Based on the sharp variation of $\mathrm{AE}$ signals instead of amplitude, this method is robust to measuring conditions. The experimental results obtained under different test conditions demonstrates the applicability of the method.

\section{3:30-3:45 Break}

\section{$3: 45$}

2pSP8. Design of $M$-sequences for a shallow water transmission experiment. S. E. Forsythe (Underwater Sound Reference Detachment, Naval Res. Lab., P.O. Box 568337, Orlando, FL 32856-8337) and Mohsen Badiey (Office of Naval Res., Arlington, VA 22217-5660)

Narrow-band signals centered at frequencies from $600 \mathrm{~Hz}$ to $7 \mathrm{kHz}$ were transmitted from a fixed projector to a fixed hydrophone array located $200 \mathrm{~m}$ away as part of the shallow water acoustic/geologic experiments at the New Jersey Atlantic Generating Station Site. Both the projector and hydrophone array were fixed $2 \mathrm{~m}$ off the ocean floor in roughly $15 \mathrm{~m}$ of water. The signals were Gaussian pulses $1 \mathrm{~m}$ in width modulated by weighted binary maximal length sequences ( $M$-sequences). These $\boldsymbol{M}$-sequences were designed to increase effective signal-to-noise ratio while retaining the time/space discrimination of the original Gaussian pulse. The pulse width was narrow enough to allow separation of component arrivals (e.g., direct and bottom reflection). A design method is presented that allows simultaneous control of frequency- and time-domain content of the transmitted signal. Examples of recovery of the transmitted signals and their multipath information are presented.
2pSP9. Equal-likelihood decision making with limited information. Richard Pitre (Naval Res. Lab., Washington, DC 20375-5350)

Performance measures are examined for a detector-estimator based on an equal-likelihood signal probability model [N. R. Davis and R. Pitre, J. Acoust. Soc. Am. 97, 978-992 and 993-1005 (1995)]. With limited integration time and SNR, detection and parameter estimation analysis based solely on estimation of cumulants is incomplete. Conventional performance measures like pfa and uncertainty measures such as cost uncertainty and decision information loss are examined as a function of integration time and estimation resolution. [Work sponsored by ONR(NRL).]

\section{$4: 15$}

2pSP10. Effect of ergodicity failure on detection performance prediction. George E. Ioup (Dept. of Phys., Univ. of New Orleans, New Orleans, LA 70148), Lisa A. Pflug (Naval Res. Lab., Stennis Space Center, MS 39529), and Juliette W. Ioup (Univ. of New Orleans, New Orleans, LA 70148)

Formulas based on the noise variance and the first four signal moments to predict the performance at the minimum detectable level of ordinary and higher order correlation central ordinate threshold detectors have been derived. The passive prediction formulas have been reported and will be published. The active prediction results have also been presented. These prediction formulas agree remarkably well with computer-based Monte Carlo simulations. A breakdown in the agreement has been observed for simulations containing a small number of sample points-in the observation window for the passive case and in the signal for the active case. This breakdown can be traced to the inapplicability of the ergodicity assumption for various time averages over a small number of sample points, since key steps in the derivations contain the interchange of expectations and summations for nonlinear terms. Small sample number time averages lead to correlation central ordinate value probability density functions which are non-Gaussian. Prediction errors in the formulas as great as $2 \mathrm{~dB}$ are observed for simulations with 32 sample points. These errors decrease as the number of sample points is increased. Both passive and active examples are given. [Research supported by ONR/NRL-SSC.]

\section{$4: 30$}

2pSP11. Frequency normalized leakage correction technique. Casey R. Winkel, Kenton A. Shipley, ${ }^{\text {) }}$ P. G. Vaidya, and Tim A. Doughty ${ }^{()}$ (Dept. of Mech. and Mater. Eng., Washington State Univ., Pullman, WA 99164)

Leakage is a problem in nearly all aspects of signal processing, especially when the FFT is involved. Leakage takes the form of wide frequency spikes due to a "spilling over" of information across frequency bins and occurs most often when the sampling frequency is not an integer multiple of the base frequency. The leakage correction method presented here substantially reduces this problem. The method resamples the original time domain data within specified limits. A frequency error measurement is taken for each sampling rate within the defined limits. The minimum frequency enror is recorded along with the fundamental frequency of the record. This method resamples the entire signal one record at a time with a linear interpolator. Nyquist criteria must be satisfied at all times to validate the use of the linear interpolation. Nyquist criteria is satisfied if the sampling rate is at least twice as large as the highest frequency in the signal. The resampled data must satisfy three conditions to eliminate leakage. (1) $2^{n}$ points per period. (2) Integer number of periods per record. (3) Satisfy Nyquist criteria. The method here satisfies these conditions and

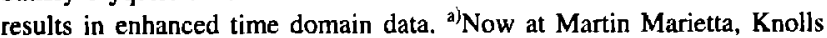
Atomic Power Labs, Schenectady, NY. ${ }^{b}$ Now at Dept. of Mech. Eng., Purdue Univ., West Lafayette, IN. 

and to the length scales much smaller than $\lambda$ as the microscales. A complexity frequently encountered in scattering problems is the one characterized by a microscale heterogeneity that occupies domains in space measured on the macroscale. A new formulation, tuned to govern the macroscale response component, has been presented in several recent papers. This formulation is sufficient for estimating the radiation of a sound field into the far-field of a surrounding fluid. In this presentation we concentrate on the formulation's implications. The questions addressed are: What type of microscale heterogeneity has no footprint in macroscale response measures? What classes of microscale heterogeneity have an identical footprint in macroscale response measures?

WEDNESDAY AFTERNOON, 31 MAY 1995

CONGRESSIONAL HALL B, 1:15 TO 4:15 P.M.

\title{
Session 2pUW
}

\section{Underwater Acoustics: Propagation (Poster Session)}

\author{
Richard B. Evans, Chair \\ Science Applications International Corporation, \#2 Shaw's Cove, Suite 203, New London, Connecticut 06320
}

\section{Contributed Papers}

\begin{abstract}
All posters will be on display from 1:15 to 4:15 p.m. To allow contributors an opportunity to see other posters, contributors of odd-numbered papers will be at their posters from 1:15 to 2:45 p.m. and contributors of even-numbered papers will be at their posters from 2:45 to 4:15 p.m.
\end{abstract}

\begin{abstract}
2pUW1. The status of Naval underwater acoustic modeling. Paul $\mathrm{C}$. Etter (P.O. Box 5622, Rockville, MD 20855-5622)
\end{abstract}

Results from a survey of recent developments in Naval underwater acoustic modeling are compared with those of two previous surveys conducted at 8-year intervals [P. C. Etter and R. S. Flum, Sr., J. Acoust. Soc. Am. Suppl. 1 65, S42 (1979); P. C. Etter, I. Acoust. Soc. Am. Suppl. 182 , S102 (1987)]. All surveys cover basic acoustic models and sonar performance models. Basic acoustic models include propagation, noise, and reverberation models. Sonar performance models integrate basic acoustic models, signal processing models and supporting databases into cohesive operating systems organized to solve the active or passive sonar equations [P. C. Etter, Underwater Acoustic Modeling (Elsevier, Amsterdam, 1991); 2nd ed. (Chapman \& Hall, 1995)]. Summary tables identify and highlight noteworthy characteristics of 83 propagation models, 15 noise models, 14 reverberation models, and 14 sonar performance models. These surveys provide a retrospective view of modeling developments over the past two decades and document a $160 \%$ increase in the number of models that are now available for applications in sonar technology. This updated baseline also suggests future directions for Naval modeling in an era of defense conversion including new applications in oceanographic research.

2pUW2. Combined volume and surface scattering in a channel using a modal formulation. Shimshon Frankenthal and M. J. Beran (Dept. of Elec. Eng., Catholic Univ. of America, Cardinal Station, Washington, DC 20064)

In previous work, a modal approach was used to study random volume scattering in a shallow channel [M. J. Beran and S. Frankenthal, J. AcousI. Soc. Am. 91, 3203-3211 (1992)]. Here, the way to include the effects of a rough channel surface in the formulation is shown. To include the effects of a rough surface, the modes are taken to be dependent on the range and transverse coordinates in addition to the depth coordinate. The propagation is studied in terms of the ensemble-averaged two-point coherence function and the equation governing the coherence function is derived. In order to insure energy conservation when the generalized modal field equations are simplified, the parabolic approximation is replaced by a method which includes both forward and backward propagating fields. The two-point coherence function is expressed as the sum over both self-modal and crossmodal coherence functions. The difference between the equations governing the self-modal coherence functions and the cross-modal coherence functions is considered. A numerical example which uses typical shallow water parameters is presented. Figures portray how the mode energies are transferred between the modes as the acoustical field propagates. [This work was partially supported by ONR.]

2pUW3. Investigation of three-dimensional bathymetric refraction using laboratory scale modeling. Stewart A. L. Glegg, Anthony LaVigne, and Joseph M. Riley (Ctr. For Acoust. \& Vib., Florida Atlantic Univ., Dept. of Ocean Eng., Boca Raton, FL 33431)

Experimental measurements have been carried out to identify the effect of bathymetry on three-dimensional underwater sound propagation. The measurements have been conducted over a 1/10 000th scale model of the Santa Lucia Escarpment off the coast of California. Experiments measured the bearing error obtained in locating a source with a horizontal line array using a phase-wave beamformer. The results of this experiment demonstrated significant variations in the measured bearing error. Differences in the detected bearing of the variations beamformer and the actual source location were as large as $18 \mathrm{deg}$, with the magnitude of these errors being a function of bearing line and source frequency. An analytical solution of the acoustic field in a shear supporting wedge using the method of images has been used to approximate the results of the bearing error experiment. Comparison of experiment and theory shows the same effects with bearing errors of the same magnitude. [Work supported by ONR.] 
2pUW4. Modeling range-dependent propagation using trapped and leaky wedge modes. John A. Fawcett (SACLANT Undersea Res. Ctr., Viale San Bartolomeo, 400, 19038 La Spezia, Italy), Evan K. Westwood (Appl. Res. Labs., Univ. of Texas, Austin, TX 78713-8029), and Christopher T. Tindle (Univ. of Auckland, Auckland, New Zealand)

The use of coupled wedge modes in modeling range-dependent underwater acoustic propagation has been previously described by Primack and Gilbert [H. Primack and K. E. Gilbert, J. Acoust. Soc. Am. 90, 3254-3262 (1991)]. Their approach has been extended by using leaky modes, in addition to the trapped ones, in wedge mode computations. Trapped modes decay exponentially in the basement and correspond, in terms of rays, to angles of propagation which are totally internally reflected above the basement interface; leaky modes are not well behaved in the basement, but provide an excellent discrete approximation to the continuous spectrum contribution in the water column. Because leaky modes are included in the computations, it is possible to model accurately shallow water environments where trapped/leaky mode conversions are important. Within a single wedge domain, adiabatic wedge mode theory is used for computations; at wedge junctures, a least-squares mode coupling scheme is employed. Numerical examples where the results of the coupled wedge mode method are compared to parabolic equation and standard adiabatic mode computations are presented.

2pUW5. Accuracy of parabolic approximations for travel time. F. D. Tappert and M. G. Brown (Appl. Marine Phys., Univ. Miami, RSMAS, 4600 Rickenbacker Cswy., Miami, FL 33149)

Many different full-wave parabolic approximations are analyzed with regard to the accuracy of their travel time predictions by considering the geometrical acoustic (ray tracing) limit. The two small parameters are $\epsilon=\left(1-n^{2}\right) / 2$ and $\mu=p^{2} / 2$, where $n$ is the depth and range-dependent index of refraction and $p$ is a scaled ray grazing angle. By expanding the exact and approximate Hamiltonian and Langrangian functions in powers of $\epsilon$ and $\mu$, which tend to have the same order of magnitude, it is found that among the class of parabolic approximations that can be implemented with the efficient "split-step Fourier" algorithm only the recently developed $c_{0}$-insensitive approximation [Tappert et al., J. Acoust. Soc. Am., to be published] has full second-order accuracy. The highly touted "modified log" parabolic approximation of Berman et al. [J. Acoust. Soc. Am. 86, 228-233 (1989)] has only first-order accuracy, the same as the "standard" parabolic approximation, and furthermore it has a second-order bias toward times that are too early. Numerical calculations confirm and quantify these results. [Work supported by ONR.]

2pUW6. Acoustic interaction with a propagating internal soliton wave packet in shallow water. Michael K. Broadhead (Code 7173, Naval Res. Lab., Stennis Space Center, MS 39529-5004)

One-way ocean acoustic propagation through shallow water internal waves was numerically simulated, where the oceanographic model consisted of a two-layer density/temperature stratified fluid overlying flat bathymetry. For sufficiently small amplitudes in a lossless medium, shallow water internal waves are governed by the Korteweg-de Vries (KdV) equation, which possesses soliton solutions. A finite difference scheme was used to numerically time evolve initial conditions, the details of which determine the number and properties of the soliton events that emerge. The internal waves (in a center-of-mass frame) were introduced through the index of refraction in the acoustic wave equation. Range-dependent normal mode and PE models were used to compute transaction-loss for various frequencies ( $100 \mathrm{~Hz}-10 \mathrm{kHz}$ ), for different times during the soliton wave packet evolution, and for different initial conditions. For lower frequencies, refraction effects caused an increase in the mean TL level due to increased bottom interaction, while for higher frequencies, the predominant effect was spatial/temporal fluctualions and the enhancement of surface duct propagation. Dispersive terms exist for all but special initial conditions, but their amplitudes were generally too low to be significant. [Supported by Office of Naval Research and Naval Research Laboratory.]
2pUW7. Fluctuations of high-frequency acoustic pulses in shallow water. Marcia A. Wilson (Naval Res. Lab., Code 7174, Bldg. 1005, Stennis Space Center, MS 39529)

High-frequency acoustic propagation and scattering experiments were conducted near Panama City, FL, in August of 1991 and 1993, and in Eckernforde Bay, Germany in May 1993. The Panama City site has a hard sand bottom, but the bottom of Eckernforde Bay is soft mud. Environmental measurements were made in conjunction with acoustic measurements. The water depth at both sites was approximately $30 \mathrm{~m}$. The sources and the receiver arrays were mounted 6 to $8 \mathrm{~m}$ from the bottom and were separated by about $90 \mathrm{~m}$. A bottom reflected signal was detected in Panama City, and in some cases, in Germany. The soft mud bottom of Eckernforde Bay, however, absorbed most of the energy directed toward it. Data from pulses $1.0 \mathrm{~ms}$ in length, al 1-s intervals, will be shown for frequencies from 20 to $180 \mathrm{kHz}$. Means, standard deviations, and coefficients of variation of 100 or 150 pulses for each frequency characterize two scales of temporal variability in the data. Data from several of the receiving hydrophones are compared. Sparial variability is shown and related to modeled and measured changes in the sound velocity profile. [Work supported by the Office of Naval Research.]

2pUW8. Jovian acoustics: Short-range observation and long-range prediction. Michael D. Collins, B. Edward McDonald (Naval Res. Lab., Washington, DC 20375), W. A. Kuperman (Scripps Inst. of Oceanogr., La Jolla, CA 92093), and William L. Siegmann (Rensselaer Polytechnic Inst., Troy, NY 12180)

Waves were observed propagating in the stratosphere of Jupiter near the impact sites of several of the fragments of Comet Shoemaker-Levy 9. Concentric rings appearing in Hubble Space Telescope images have tentatively been identified as gravity and acoustic waves based on their horizontal group speeds of 450 and $900 \mathrm{~m} / \mathrm{s}$. Interpreting the images is complicated by the fact that the outer ring is located amidst the debris of the collapsed fireball. Since the fireball rose back through the obliquely oriented path of the incoming fragment, the debris field is asymmetric and offset from the impact site. It is therefore unlikely that the outer ring is part of the debris. If waves are observed far from the impact sites, they might provide new information about the Jovian atmosphere. A threedimensional adiabatic mode solution predicts that the Jovian zonal winds cause horizontal caustics to form to the east and west of the impact sites about $10 \mathrm{~h}$ after impact. An adiabatic mode solution has also been derived for gravity waves, which exhibil similar horizontal caustics. Although the acoustic and gravity wave equations are drastically different, the horizontal wave equations for the mode coefficients are identical

2pUW9. Three-dimensional acoustic propagation in a waveguide of variable thickness. Robert A. Coury, William L. Siegmann (Rensselaer Polytechnic Inst., Troy, NY 12180-3590), and Michael D. Collins (Naval Res. Lab., Washington, DC 20375)

The parabolic equation (PE) method has proven to be an efficient method for solving range-dependent ocean acoustics problems. Recently an extension has been made to handle two-dimensional waveguides of varying thickness. This type of modeling is necessary when the upper boundary of the waveguide is allowed to change, as for example in propagation up a beach or in an ocean with a gradually undulating surface. The adiabatic mode $\mathrm{PE}$ is another extension that has been developed to solve global scale, three-dimensional propagation problems. In this paper, the three-dimensional adiabatic mode PE is extended to handle problems involving a gradual range dependence in the overall thickness of the waveguide. The modified adiabatic PE has several applications. On relatively small scales, it may be applied to solve three-dimensional beach acoustics problems or to model diffraction by an island. It may also be applied to solve global-scale seismoacoustic problems in which topography on the continents plays a significant role. [Work supported by ONR.] 
2pUW10. Global-scale acoustic modeling in an ocean overlying elastic bathymetry. Gregory J. Orris, Michael D. Collins (U.S. Naval Res. Lab., Washington, DC 20375), Grant Deane (Scripps Inst. of Oceanogr., Univ. of California, San Diego, La Jolla, CA 92093), and Michael B. Porter (New Jersey Inst. of Technol., University Heights, Newark, NJ 07102)

A three-dimensional propagation model is presented that takes into account the elastic properties of the ocean bottom and is applicable to problems of long range and low frequencies. This method allows globalscale calculations appropriate for ocean monitoring programs to be performed with environments for which solution techniques have been heretofore unavailable. The model is an extension of the adiabatic parabolicequation method, which uses the normal-mode solutions in the waveguide for the depth dependence of the acoustic field. A modified two-dimensional parabolic equation model is used to determine the range and azimuth dependence of the acoustic field, thus providing a three-dimensional solution. It is shown that the inclusion of elastic material, with both compressional and shear waves, in bathymetric features causes larger loss and larger shadow zones behind sea mounts and islands due to the effective softening of the material. The method is compared with exact solutions and experimental results and is found to be in excellent agreement for regimes for which the adiabatic approximation is valid (i.e., for regions of slowly varying bathymetry and sound-speed profiles).

2pUW11. An accurate, efficient rough surface parabolic equation program. Allan P. Rosenberg and Sleven F. Magruder (Johns Hopkins Univ., Appl. Phys. Lab., 7-334, Laurel, MD 20723-6099)

The parabolic equation program Fepe has been extended to handle a rough air-water interface treated as a series of stair steps. Because much finer vertical spacing may be needed to resolve the interface than to propagate an acoustic wave accurately in the rest of the domain, an option to use one vertical spacing near the surface and a coarser one in the rest of the ocean has been implemented. The necessary Galerkin approximations of derivatives on an unequally spaced grid have been worked out using the symbolic manipulation program Mathematica. A test case with a realistic surface is provided to demonstrate the accuracy of the new program Fepe rsfc. The code is efficient enough to allow thousands of runs for realistic sized problems, for example to simulate the effect of a moving surface. [Work supported by the office of the Chief of Naval Operations.]

2pUW12. Modeling a directional source in an underwater acoustic waveguide. Richard B. Evans (Sci. Appl. Intl. Corp., \#2 Shaw's Cove, Ste. 203, New London, CT 06320)

Techniques for modeling a single frequency directional source in an underwater acoustic waveguide are applied and tested. The techniques are evaluated, with regard to accuracy and efficiency, for use in prediction of source perfornance. Existing discussions of the topic often focus on one technique. Here, the goal is to consider a larger class of methods. They range from mode matching in the far-field, of the source, to the direct application of a free-space beam pattern at range zero. The techniques are applied to problems that arise due to sparseness of calibration data and the inappropriateness of a free-space beam pattern for waveguide applications. [Work supported by ARA.]

2pUW13. Comparison of theory and experiment of acoustic bottom penetration in a shallow water site. Nicholas P. Chotiros, Morris Stem, Adrienne Mautner (Appl, Res. Labs., Univ. of Texas, P.O. Box 8029, Austin, TX 78713-8029), Ảge Kristensen, and Enzo Michelozzi (SACLANT Undersea Res. Ctr., 19026 La Spezia, Italy)

Signals from a spark source in water and received by acoustic sensors buried in a sandy sediment in a site off La Spezia, Italy, are compared to theoretical model predictions from a fast field model. The model is a modification of OASES 1.6 that includes Biot's poroelastic wave propagation model. The received acoustic pulses are compared to determine the transfer function from the in-water source to the buried acoustic sensors. From a theoretical point of view, the sediment is treated as a poroelastic solid governed by Biot's theory of acoustic propagation. It is predicted to support two acoustic waves. Comparisons are made between theory and experiment. [Work supported by Office of Naval Research, Ocean Acoustics Program, code 321 OA, under the initiative for basic research in the physics of moderate to high frequency acoustics.]

2pUW14. Propagation characteristics from the trans-Arctic propagation source as measured at a receiver in the Lincoln Sea. Thomas N. Lawrence, Ilene McCool, and Nancy R. Bedford (Appl. Res. Labs., Univ. of Texas, P.O. Box 8029, Austin, TX 78713-8029)

The trans-Arclic propagation (TAP) source was a Russian device deployed north of Svalbard transmitting at about $20 \mathrm{~Hz}$. Transmissions received in the Lincoln Sea north of Ellesmere Island were recorded on a 20-element vertical array located on the continental shelf. The propagation path between the source and receiver is over the Arctic Mid Ocean Ridge, then skirting the Morris Jesup Plateau, and finally propagating up the continental slope. The ice cover along this path of propagation is highly varied, ranging from central Arctic roughness levels (usually about 1-2.5 m standard deviation) to the higher roughness (about $4 \mathrm{~m}$ s.d.) of the Canadian Archipelago. Amplitude and phase fluctuations of signal and environmental noise over varied time periods, and the results for the statistical character of the water column will be presented. Comparisons will be made with modeled results to help determine the effects from this complex environment and to predict the impact of variation in ice conditions along the propagation path.

2pUW15. Mode vector parabolic equation. Ahmad T. Abawi, W. A. Kuperman (Marine Phys. Lab., Scripps Inst. of Oceanogr., Univ. of California, San Diego, La Jolla, CA 92093-0238), Michael D. Collins (Naval Res. Lab., Washington, DC 20375), and Michael B. Porter (New Jersey Inst. of Technol., Newark, NJ 07102)

A mode vector parabolic equation (MVPE) is derived for the propagation of normal modes in the ocean waveguide. This model, which includes mode coupling, is a generalization of the adiabatic mode PE [M. D. Collins, J. Acoust. Soc. Am. 94, 2269 (1993)]. The main features of this model are: (I) It is based on an initial value differential equation which propagates normal modes rather than a method that involves matching solutions at interfaces. (2) The solution vector whose components are the amplitude of the modes is not derived from a procedure involving a reference wave number and thus each vector component is locally accurate around each mode. (3) It agrees with previous benchmark solutions. (4) The model is tractable in three dimensions. MVPE has three parts: Spatial derivative of the mode amplitudes, eigenvalue, and coupling coefficients marrices. The latter two are precomputed; the coupling matrix is based on McDonald's formulation in terms of environmental parameters which is summarized in [McDonald et al., J. Acoust. Soc. Am. 96, 2357 (1994)]. The system of equations can either be solved by matrix inversion or iteration. To illustrate the method, the solution to the problem of propagation of waves in a two-dimensional wedge is presented in detail.

2pUW16. Application of the mode vector parabolic equation to the 3-dimensional wedge problem. Ahmad T. Abawi and W. A. Kuperman (Marine Phys. Lab., Scripps Inst. of Oceanogr., Univ. of California, San Diego, La Jolla, CA 92093-0238)

The MVPE [Abawi et al., this session] is applied to the problem of propagation of waves in a 3-dimensional coastal wedge and the results are compared with those when horizontal coupling is neglected and with the results obtained from conventional 3-dimensional methods. The components of the solution vector are the amplitudes of the vertical modes which are coupled in both range and azimuth. The coupling matrices for both range and azimuth are precomputed by the method summarized in [McDonald et al., J. Acoust. Soc. Am. 96, 2357 (1994)]. In the absence of mode coupling, the solution reduces to the solution of the adiabatic mode parabolic equation [M. D. Collins, J. Acoust. Soc. Am. 94, 2269 (1993)]. The system of equations are numerically solved by the Crank-Nicholson finite difference method. For a single vertical mode, this system of equations closely resembles a system of discretized parabolic equation in range and azimuth. For multiple modes, the matrices involved are band diagonal with the coupling matrices appearing in their diagonal. The size of these matrices are thus equal to the number of points in azimuth times the number of vertical modes. When the physics of the problem is contained in 
a subset of coupled modes, this method offers additional advantages in numerical computation over the conventional 3-dimensional methods.

2pUW17. Semiclassical techniques for long-range, low-frequency propagation. Frank S. Henyey, Stephen A. Reynolds, and Terry E. Ewart (Appl. Phys. Lab., Univ. of Washington, 1013 NE 40th St., Seattle, WA 98105)

The Chester, Friedman, and Ursell (CFU) uniform asymptotic expansion near caustics has been implemented as an adjunct to a ray-tracing code. The method is applied to the propagation of $400 \mathrm{~Hz}$ sound from a 200-m deep source in a Munk sound-speed profile through a range of 300 $\mathrm{km}$. Making the parabolic approximation in the ray trace, the validity of "semiclassical" wave functions with the CFU expansion near caustics can be determined by comparison with a parabolic equation code. Good results for the semiclassical wave function are found. If the CFU expansion is not used, the field is badly inaccurate over hundreds of meters in the vertical. A video of the propagation comparing the Wigner function with the ray depth-angle plot is shown. [Work supported by ONR.]

2pUW18. Probabilistic range dependence in a numerical average underwater acoustic propagation method. Roger M. Oba (Naval Res. Lab., Stennis Space Center, MS 39529-5004)

A computational method for forward propagation of the Helmholtz equation has been developed for numerically computing average complex pressure field in a single pass. It used sound-speed profiles be piecewise probabilistically independent in finite sized range steps to calculate transmission loss and phase for the average solutions in the continuous wave case. This required that the uncertainty is probabilistically modeled within range intervals as ensembles of sound speeds which were independent from one range step to the next. This method can be modified to represent certain cases for which there is statistical dependence in range. This can be achieved by nonlocal averaging over several range steps. The inter-relation of range sound speed and statistical dependence will be considered, and results from numerical experiment will be shown. Similar calculations allow the calculation of growth of standard deviation over range. This modification can also be considered in the limit of small intervals of uncertainty. [Work supported by Naval Research Laboratory and the Office of Naval Research.]

2pUW19. Characterization of oceanographic/acoustic variability in littoral regions: Examples from two diverse test sites. Hassan B. Ali (Code 7173, Naval Res. Lab., Stennis Space Center, MS 39529-5004)

As a result of complex oceanographic and atmospheric processes in littoral regions, environmental parameters are characterized by considerable spatial and temporal variability. The preceding imposes severe constraints on signal processing schemes, thereby adversely influencing systems based on acoustical, optical, magnetic, and oceanographic parameters. Acoustic signals are degraded by extensive interaction with the surface and bottom boundaries, the passage of internal waves, tidal changes, ambient noise, the influx of fresh water from rivers and marshes, etc. It is unlikely that these diverse phenomena will all be present in any single geographic area. Rather, the number and significance of the phenomena will vary from site to site. As a result, it is important to identify and characterize the germane ocean processes and environmental features at particular sites of interest, in order to isolate and assess their acoustic effects. The objectives of this task are to ascertain the minimum number of oceanographic parameters controlling the acoustic propagation, and their requisite spatial and temporal resolutions. The results of such investigations for two diverse sites off the U.S. and U.K. coasts are provided in this paper.

2pUW20. Solution of the acoustic wave equation in underwater environments using an improved finite difference method. R. A. Zingarelli (Naval Res. Lab., Acoust. Simulation Section, Stennis Space Center, MS 39529)

At the fall meeting, a finite difference solution to the acoustic wave equation for real pressure fields, its implementation, and several applications were presented. An improved differencing scheme developed by Cole et al. has subsequently been incorporated into this numerical model. Using this method runtimes and memory usage for $\mathrm{cw}$ problems have been re- duced by factors of 10 and 5, respectively, with no loss of accuracy or computational parallelism, making its computational requirements similar to other solution techniques. Because the weightings of various grid elements in this method are based on the source frequency, its usefulness in TD calculations with pulsed sources is limited. Despite this, some lowfrequency TD calculations are possible. Applications and limitations of the higher-order method will be presented. [Work supported by ONR.]

2pUW21. A method for computing adiabatic modal wavefronts. Evan K. Westwood (Appl. Res. Lab., P.O. Box 8029, The Univ. of Texas at Austin, Austin, TX 78713-8029)

An approach has been developed for determining the modal wavefronts that allow normal modes to be propagated adiabatically in smoothly rangedependent ocean waveguides. The method is motivated by the fact that propagation in an isospeed penetrable wedge is accurately modeled by "adiabatic wedge modes," which are identical to the usual "vertical modes" except that the wavefront curvature induced by the sloping bottom is taken into account. For the wedge, the wavefronts are assumed to be circular arcs centered on the wedge apex. In order to generalize the notion of wedge modes to other types of range dependence, wavefronts are constructed numerically such that the derivative of each mode in the direction normal to its wavefront, $\partial \phi / \partial s$, is zero. The result is that the first-order modal coupling coefficients, which involve $\partial \phi / \partial s$ integrated over depth, are zero, and the adiabatic algorithm can be used. The quantities $\partial \phi / \partial z$ and $\partial \phi / \partial r$ versus depth are used to obtain the directions normal to the wavefront. For the isospeed penetrable wedge, wavefronts computed in this manner do indeed correspond closely to the expected circular arcs. [Work supported by the ARL Internal Research and Development Program.]

2pUW22. A comparison of broadband and narrow-hand transmission loss in shallow water. Karen D. Frech (Bolt Beranek and Newman, 1300 North 17th St., Arlington, VA 22209)

Calibrated acoustic measurements were made at the Hudson Canyon Experiment site [L. Maiocco and W. Carey, J. Acoust. Soc. Am. Suppl. 1 86, S8 (1989)] during area characterization test $\amalg(A C T$ II) conducted in September 1993. This site was chosen because it is a surveyed area with known geophysical and geoacoustic properties. Transmission loss was measured along the previously run radials from bore hole 6010 using calibrated small broadband explosive charge sources and a narrow-band continuous wave (cw) towed acoustic projector. These sources were, for the purposes considered here, co-located in range because of low test ship speed (under $5 \mathrm{kn}$ ). The receiving sensor was a 25 element stationary vertical line array spanning the majority of the water column $(73 \mathrm{~m})$, with 19 elements extending off the bottom $(60 \mathrm{~m})$ and 6 elements along the bottom $(30 \mathrm{~m})$. Using the principle of reciprocity, the hydrophone elements at each of the source depths enabled comparisons of the transmission loss measurements for broadband and $\mathrm{cw}$ sources to be made over a range interval from $4-22 \mathrm{~km}$ at two frequencies $(200$ and $900 \mathrm{~Hz})$. The comparisons were performed by means of linear regression of the data from the two sources. The results of the comparison will be presented and will include a detailed discussion of the precision of the results and of possible sources of experimental error. Finally, the cw transmission loss from ACT II will be compared with the previous measurements of Maiocco and Carey.

2pUW23. The effect of roughness on acoustic penetration of the seafloor as given by a fluid-fluid perturbation model and comparison with recent sediment penetration experiments. John $E$. Moe, Eric I. Thorsos, Darrell R. Jackson, and Kevin L. Williams (Appl. Phys. Lab., Univ. of Washington, 1013 NE 40th St., Seattle, WA 98195)

Recent experimental results [F. E. Boyle and N. P. Chotiros, J. Acoust. Soc. Am. 91, 2615-2619 (1992)] reveal acoustic penetration from water into sandy sediments at grazing angles below the compressional critical angle in relation to the mean surface. These authors interpret the results to indicate the excitation of a biot slow wave in the sediment. An additional mechanism for subcritical penetration will be discussed, based on assuming a small level of roughness at the water-sediment interface. Computer simulations of these experiments using theoretical calculations based on Rayleigh-Rice perturbation theory for 2-D surfaces reproduce experimental results, indicating that the acoustic penetration of the surface may be 
due to scattering (diffraction) from low levels of roughness. The accuracy of perturbation theory for the level of roughness being considered is verified using comparisons with exact calculations in the special case of 1-D surfaces. [Work supported by ONR.]

2pUW24. Marching wave-number-integration approach to range-dependent, two-way seismoacoustic propagation modeling. Henrik Schmidt (Dept. of Ocean Eng., MIT, Cambridge, MA 02139)

An approximate solution to two-way seismoacoustic propagation problems can be obtained by recursive use of wave-number-integration in a stepwise range-dependent environment. The approach is conceptually simple and straightforward. In the range-independent sector containing the source, an "exact" integral representation of the field can be obtained versus range and depth using, e.g., the SAFARI/OASES code [H. Schmidt and F. B. Jensen, J. Acoust. Soc. Am. 77, 813-825 (1985)]. At the vertical boundary of the next sector we then solve the reflection-transmission problem locally for each plane-wave component, assuming vertical homogeneity of the field. The resulting particle motions now act as virtual sources, the wave-number representation of which can be stated explicitly using the seismic source representation theorem. The resulting transmitted and reflected fields can then be computed at any depth and range within the range-dependent sectors. The procedure is repeated at any vertical cut in a marching scheme. A single-scatter approximation to the backscattered field is subsequently obtained by a backward marching scheme, similarly to the approach used in the two-way elastic PE [M. D. Collins, J. Acoust. Soc. Am. 93, 1815-1824 (1993)]. A special version of OASES incorporating this approach has been developed, and its performance is demonstrated by solutions to canonical problems such as the ASA benchmark, and seismic problems previously solved using the two-way elastic PE.

2pUW25. Comparison of exact and approximate solutions of multiple scattering between interfaces in rough waveguides. Kevin D. LePage (Bolt Beranek and Newman, Inc., 70 Fawcett St., Cambridge, MA 02138)

Modeling propagation in rough waveguides introduces the interesting question of how to treat multiply scattered waves. One approach is to treat the rough surface scattering at the waveguide boundaries under an extended Born approximation [W. Kuperman and H. Schmidt, J. Acoust. Soc. Am. 86 (1989)]. Under this approach multiple scatter effects are incorporated into the mean field through a second-order correction in surface roughness, and the scattering amplitudes at each interface are assumed to be driven by the corrected mean field alone. Coupled integral equations which the total field in a rough waveguide must satisfy at the source depth are available in the literature [D. Berman, J. Acoust. Soc. Am. 92 (1992)] making it possible to evaluate the approximate results in a consistent way. Here the results obtained by solving the integral equations are compared to those obtained by adding the scattered field to either a corrected or an uncorrected mean field. Conclusions regarding the bounding behavior of the approximate solutions are drawn for various roughness and waveguide parameters in the low frequency regime.

2pUW26. Sediment layer structure and composition effects on pulse propagation at the Atlantic generating station (AGS) site. Kevin P. Bongiovanni (Rensselaer Polytechnic Inst., Troy, NY 12180-3590), Mohsen Badiey (Univ. of Delaware, Newark, DE 19716), and William L. Siegmann (Rensselaer Polytechnic Inst., Troy, NY 12180-3590)

An investigation of broadband pulse propagation in a shallow water environment is performed using high-order frequency- and time-domain parabolic equation models. High resolution geoacoustical profiles are constructed from cores obtained at the extensively sampled AGS site which lies 5 miles offshore southern New Jersey. These, along with CTD and detailed bathymetric data, are used to model the environment. Acoustic experiments necently conducted at this site [M. Badiey et al., J. Acoust. Soc. Am. 96, 3593-3604 (1994)] provide broadband acoustic data for comparisons with the modeling predictions. Simulations of transmission loss versus frequency show very good agreement with experimental data over several different propagation tracks. Time-domain simulations are compared with time series of pressure variations generated by the impulsive experimental source along corresponding tracks. Strongly rangedependent mechanisms are observed over short ranges with both models and can be correlated with specific sediment features. A discussion of the modeling results in connection with both environmental and acoustic data sets will be given.

2pUW27. Three-dimensional acoustic/geoacoustic propagation modeling of the New Jersey Atlantic generating station site. Mohsen Badiey (Ocean Acoust. Prog., Office of Naval Res., Arlington, VA 22217). George Botseas, Ding Lee (Naval Undersea Warfare Ctr., New London, CT 06320), and William L. Siegmann (Rensselaer Polytechnic Inst., Troy, NY 12180)

Propagation effects of 3-D geological features in shallow water sediment layers at the Atlantic generating station (AGS) continental shelf site are investigated. Profiles of compressional and shear sound speed, attenuation, and density have been generated from 23 available geological cores at the AGS site and discussed in connection with a recent acoustic experiment conducted there [Badicy et al., J. Acoust. Soc. Am. 96, 3593-3604 (1994)]. A 3-D geoacoustic data set is constructed from these profiles using interpolation schemes based on Akima splines and on krieging. Correlation properties of the sound speeds and density fields clearly show the existence of sublayer geological features. The geoacoustic fields, along with detailed bathymetry and water sound speed, are used for input a 3-D frequency domain PE propagation model. Both 3-D and N $\times 2-\mathrm{D}$ simulations are performed over a circular region corresponding to the experimental configuration. Relative influences of sediment sound-speed structure and water-sediment interface topography on transmission loss are determined for frequencies up to $1 \mathrm{kHz}$. Simulation results are compared with spectra obtained from the broadband experimental transmission loss.

2pUW28. Use of empirical orthogonal functions to estimate wave number variance in a stochastic shallow-water channel. Michael $\mathbf{J}$. Longfritz, William L. Siegmann, Melvin J. Jacobson (Rensselaer Polytechnic Inst., Troy, NY 12180-3590), and Mohsen Badiey (Ocean Acoust. Lab., University of Delaware, Newark, DE 19716)

The variances of horizontal acoustic wave numbers in stochastic shallow-water environments are estimated using empirical orthogonal functions (EOFs). Using perturbation methods and an adiabatic normalmode propagation model, the wave number fluctuations are related to range variations in the ocean and/or sediment sound-speed profiles, which can be conveniently and efficiently represented by EOFs. This relationship can be used to estimate both the wave number deviations arising from selected realizations of the stochastic ensemble and the overall wave number variance. The procedure is illustrated by application to a shallow-water waveguide with a multilayered sediment bottom. Both the layer depths and the intralayer sound speeds are modeled as randorn variables. The accuracy of the estimation procedure is investigated for various choices of the layer depth and sound-speed statistics by comparing with results from computational simulations. A particular environment examined is the New Jersey Shelf Atlantic Generating Site, where relatively extensive geoacoustic profiles are available [M. Badiey et al., J. Acoust. Soc. Am. 96, 3593-3604 (1994)]. Comparisons are made between results from the estimation procedure and from the KRAKEN and FEPE propagation codes.

2pUW29. Multiple ocean layering models and mode trapping in sublayers for high frequency propagation. N. A. Sidorovskaia (Dept. of Phys., Univ. of New Orleans, New Orleans, LA 70148) and M. F. Werby (Naval Res. Lab., Stennis Space Center, MS 39529)

It is possible for sublayers in a layered ocean to have pronounced effects on the propagating modes in the water column. For example, atypical effects occur for sound speeds less than the overlaying water column or some overlying sublayers. In some cases one predicts the concentration of a large energy flux in that layer even at model eigenvalues that prohibit propagation in the water column while supporting propagating modes in the sublayer. Since some data suggest the possibility of underlying gaseous regions that support low sound speeds, this is a practical problem. This allows for the possibility that for a layer thick enough to absorb energy before arriving in a higher speed sublayer, the number of propagation modes in the water column can be reduced with increasing frequency. Since this is a frequency-dependent effect [effective (absorbing) thickness of layers is inversely proportional to wavelength or directly proportional to frequency], absorption into the overlying sublayers becomes more pro- 
nounced with increased frequency thus reducing the possibility of "tunnelling" which leads to a reduction in propagating modes in the water column. This effect is the converse of the usual case for ocean bottom layering and can be directly measured. Some interesting effects are studied and compared with available data using the high frequency mode model SWAMP. [Work sponsored by the Office of Naval Research.]

2pUW30. Analysis of spatial-time characteristics of broadband pulse propagation through ocean sound channels by using shallow water acoustical mode propagation model. Nataly A. Sidorovskaia (Dept. of Phys., Univ. of New Orleans, New Orleans, LA 70148) and Michael F. Werby (Naval Res. Lab., Stennis Space Center, MS 39529)

Broadband pulse propagation through an oceanic sound channel is investigated to characterize some important oceanic properties, namely, the vertical sound profile, bottom structure, and also source localization. Examples of waveguides with surface and bottom ducting are considered. The new fast normal mode method (SWAMP) based on a new expansion method allows one to study the high frequency range in which large numbers of modes can be excited. In this situation the complicated structure of the wavefront that is the result of the interference of the low-order trapped refractive modes and the high-order reflected modes is observed in the waveguides. To understand the structure of this informatively rich wavefront, two techniques are presented: The static spatial-time maps of the sound field intensity distribution in a waveguide and video movies permitting one to observe the time evolution of a pulse through the fixed spatial region. The later technique gives much additional information on the process and can offer additional insight into the nature of pulse evolution along a waveguide. [Work sponsored by the Office of Naval Research.]

2pUW31. Scholte-Stoneley waves on a solid layered ocean floor. Louis Hargenrader and Herbert Überall (Dept. of Phys., Catholic Univ., Washington, DC 20064)

The existence of a Scholte-Stoneley interface wave has been pointed out for the two-layer propagation model of Pekeris, as generalized to a shear-supporting ocean bottom. Further, the occurrence of apparent resonances in a solid sediment covering the ocean floor has been noted [S. J. Hughes et al., J. Acoust. Soc. Am. 88, 1283 (1990)]. By finding the poles in the complex frequency plane corresponding to the various interfacewave (Scholte-Stoneley) and trapped-wave modes (including Love waves), a detailed study is carried out of the various wave modes that exist in the elastic layered problem taking note of their identities and of transitions between mode types, and the resonance behavior associated with them is investigated.

2pUW32. Measurement of upper-sediment geoacoustic properties in the Canadian high Arctic. S. E. Dosso (Defence Res. Establishment Pacific, FMO Victoria, BC v0S 1BO, Canada) and G. H. Brooke (Numerical Decisions Group, Victoria, BC V8Z 3K3, Canada)

Geoacoustic properties of the ocean bottom can significantly affect acoustic propagation in shallow water. However, to date, few measurements of geoacoustic properties have been reported for the high Arctic. A seismoacoustic survey was carried out to determine a geoacoustic model for the upper sediments on the continental shelf of the Lincoln Sea, north of Ellesmere Island, Canada. The experiment involved deploying an ocean-bottom seismometer through $5 \mathrm{~m}$ of polar pack ice, and recording signals from seafloor sources at ranges of 35-900 m. Subsequently, a layered compressional-speed model of the upper sediments was determined from a travel-time analysis of refracted compressional waves (head waves), and a shear-speed model was determined by matching the observed dispersion characteristics of shear and seismic-interface (Scholte) waves. Frequency-dependent compressional and shear attenuation coefficients were estimated from an analysis of the decay of signal amplitude with range: An approximately linear dependence of attenuation on frequency was observed for both wave types. The sensitivity of acoustic propagation in the ocean to the various geoacoustic parameters was investigated using a numerical propagation model.

2pUW33. The relationship between rays and modes in a waveguide with large-scale inhomogeneities. Anatoly L. Virovlyansky (Inst. of Appl. Phys., 46 Ulyanov St., 603600 Nizhny Novgorod, Russia)

The well-known results of Tindle and Guthrie, Felsen, and other authors on studying the relationship between rays and normal modes in a range-independent waveguide are generalized for a waveguide with largescale inhomogeneities of refractive index. It is shown that in complete analogy with the range-independent case, the raylike behavior of a cluster of normal modes takes place. The notion of Fresnel zones for normal modes is introduced, which is analogous to the usual Fresnel zones introduced for rays. Using Fresnel zones for modes one can simplify the analysis of mode scattering at large-scale inhomogeneities (such as an opaque screen or an inhomogeneity of refractive index) in a waveguide. Simple and statistical averaging-suited formulas are deduced to calculate mode amplitude variations under scattering at inhomogeneities of refractive index, which are "mode analogues" of the formulas of geometrical optics and those of the Rytov method.

2pUW34. Some general properties of ray arrival sequences in an underwater sound channel. Anatoly L. Virovlyansky (Inst. of Appl. Phys., 46 Ulyanov St., 603600 Nizhny Novgorod, Russia)

Some properties of ray arrival sequences valid in a waveguide with an arbitrary sound-speed profile are ascertained using the simple approximate formula connecting differences in ray travel times and adiabatic invariants of their trajectories. It is noted that the following conservation law takes place: The mean difference in travel times of rays having their launch angles inside the fixed narrow angular interval does not depend upon the distance between the source and the receiver. This law holds true not only in the range-independent waveguide but in the adiabatically varying waveguide as well. Some general conclusions concerning the relationship between travel times and launch angles of rays reaching the receiver are made. 
Session 3aAA

\title{
Architectural Acoustics: Concert Hall and Opera House Acoustics
}

\author{
George E. Winzer, Chair \\ Winzer Associates, 17721 Mill Creek Drive, Rockville, Maryland 20855
}

Chair's Introduction-8:30

Contributed Papers

8:35

3aAA1. Computer model versus reality in a nonshoebox orchestra hall. Part I. Richard H. Campbell (ECE Dept., Worcester Polytech. Inst., 100 Institute Rd., Worcester, MA 01609)

The Portland (Maine) City Hall Auditorium was the venue for acquisition of occupied and unoccupied acoustical data on 4 October 1994, using the MLSSA system. During intermission, an omnidirectional loudspeaker was positioned at center stage in the plane of the proscenium arch and occupied data were collected with nearly a full audience. Three volunteers equipped with eyeglass microphone-pairs and DAT machines occupied six preselected seats in $3.5 \mathrm{~min}$ to acquire 18 binaural 64-K impulse responses. The same seat locations were used earlier in the day to obtain the unoccupied data. An ODEON (c) computer model of this hall, created by Kirkegaard and Associates, is used to estimate acoustical parameters from the same source and receiver locations as used in the live audience acquisition. Particular attention is given to response features due to the design of this hall, which is a double-balcony horseshoe-shaped space with curved ceiling. [Work supported by the Concert Hall Research Group.]

\section{8:50}

3aAA2. Computer model versus reality in a nonshoebox orchestra hall. Part II. David Griesinger (Lexicon, Inc., 100 Beaver St., Waltham, MA 02154)

The Portland (Maine) City Hall Auditorium was the venue for acquisition of occupied and unoccupied acoustical data on 4 October 1994, using the MLSSA system. An ODEON (c) computer model of this hall, created by Kirkegaard and Associates, is used to estimate acoustical parameters from the same source and receiver locations as used in the live audience acquisition. The data collected in the hall are analyzed for musical intelligibility and reverberance using new and modified standard measures. Comparisons are made to the informal impressions of the listeners at the concert. The relevant usefulness of the data estimated from the computer model is discussed. The role of computer model surface definitions with respect to coefficients of absorption and diffusion is investigated. [Work supported by the Concert Hall Research Group.]

\section{9:05}

3aAA3. A room acoustics measurement system round robin. J. $S$. Bradley (IRC, Natl. Res. Council, Montreal Rd., Ottawa, ON KIA 0R6, Canada)

This paper reports preliminary results of a room acoustics measurement system round robin. The round robin uses a commercial digital reverberator to represent three standard rooms that can be sent from one measurement group to another. Thus the tests compare basic calculation procedures and do not include the effects of variations due to types and placement of transducers. Each participant measures octave band values of monaural measures such as decay times, early/late ratios, and RASTI values on three different reverberator settings. Where possible, participants can also measure interaural cross correlations. Initial comparisons include both commercially available measurement systems and the measurement systems of individual research groups. The initial results will compare measurements from groups in North America, Japan, and Europe. Subsequent work will seek to explain the cause of the observed differences.

\section{$9: 20$}

3aAA4. Technical matters relating to the measurement of interaural cross-correlation (IACC) coefficients in concert halls and opera houses. Takayuki Hidaka and Toshiyuki Okano (Takenaka Res. \& Dev. Inst., 5, 1-chome, Ohtsuka, Inzai-machi, Inba-gun, Chiba 270-13, Japan)

The interaural cross-correlation coefficient (IACC) has been shown to be an important measure, along with others, of the quality of acoustics in concert halls and opera houses [Schroeder et al., J. Acoust. Soc. Am. 56, 1199 (1974); Ando, Concert Hall Acoustics (Springer-Verlag, Berlin, 1985)]. When measured in auditoriums with binaural heads (real or dummy) using an impulse source, means must be adopted to achieve accuracy and precision, and to select the type of impulse and processing methods to make IACC most useful in the prediction of acoustical quality. Subjects covered include: (1) type of impulse, (2) convolution signal, (3) integration limits, (4) directivity and positions of source in the hall, (5) parameters of head, (6) comparisons of measurements made with real and dummy heads, (7) value of torso, (8) measuring positions in hall, (9) comparison of measurements of IACC in halls with upholstered and nonupholstered seats, (10) comparison with IACC measurements made by other laboratories, and (11) some preliminary data on differences among IACCs measured in occupied and unoccupied halls.

\section{9:35}

3aAA5. Determination of categories of acoustical quality in concert halls using the questionnaire and interview method. Leo L. Beranek (975 Memorial Dr., Ste. 804, Cambridge, MA 02138-5755)

There are three common alternative techniques for judging subjective acoustical quality in concert halls and opera houses: (1) the binaural comparison method; (2) the expert-group round-robin listening method; and (3) the questionnaire and interview method. The questionnaire and interview method was utilized by me in rank-ordering 55 concert halls in 22 countries. Conductors, musicians, and music critics were interviewed and each was asked to rank-order the halls he/she knew well. In addition, to cover all halls, opinions were solicited from those who listen often to symphonic music in many halls, which included acoustical consultants and selected listeners who travel extensively and attend symphonic concerts. I have attended symphonic concerts in all but six. The halls were then separated into six categories from $\mathrm{A}+$ (super), $\mathrm{A}$ (excellent), $\mathrm{B}+$ (good to excellent), $\mathrm{B}$ (good), $\mathrm{C}+$ (fair to good), and $\mathrm{C}$ (fair). No rank-orderings were attempted within each category. It is probable that some halls might be ranked one category higher or one category lower than its present assignment if a different group if interviewees were selected. A shift of two categories is highly unlikely. The results for the 55 concert halls are presented and discussed. 
3aAA6. Interaural cross-correlation (IACC), lateral fraction (LF), and low- and high-frequency sound levels (G) as measures of acoustical quality in concert halls. Takayuki Hidaka (Takenaka Res. \& Dev. Inst., 5, 1-chome, Ohtsuka, Inzai-machi, Inba-gun. Chiba 270-13, Japan), Leo L. Beranek (975 Memorial Dr., Ste. 804, Cambridge, MA 02138-5755), and Toshiyuki Okano (Takenaka Res. \& Dev. Inst., Chiba 270-13, Japan)

Interaural cross-correlation coefficient (IACC) and lateral efficiency (LF) as correlates with subjective ratings (see companion paper, L. Beranek, "Determination of categories of acoustical quality in concert halls" (this session)] were analyzed. To make IACC sensitive to quality ratings, a multi-octave-band average was developed, based on Blauert $e t$ al. [Acustica 59, 292] and on a subjectively derived set of equal ASW contours that showed the $0.5,1.0$, and 2.0 octave bands to be of equal importance. This $\mathrm{IACC}_{3}$ was divided into $\mathrm{IACC}_{\mathrm{E} 3}$ integrated over first $0.08 \mathrm{~s}$ after arrival of the direct sound, and $\mathrm{IACC}_{\mathrm{L}, 3}$ same, 0.08 to $1 \mathrm{~s}$. Subjective judgments were also made to determine the effects of increased sound levels of symphonic music at frequencies above and below $355 \mathrm{~Hz}$ on apparent source width ASW. $\mathrm{IACC}_{\mathrm{E} 3}$ and GL both appear important for determining ASW. The measured quantity $\left[1-\mathrm{IACC}_{\mathrm{E} 3}\right.$ ] alone was found to separate the 17 concert halls of this study for which IACC data were available into the same three category groups as those determined from the interviews. Lateral fraction (LF) was found to extend over a very small range and there were so many overlaps among halls when separation into three rating groups was attempted that LF was judged not to be suitable for rating acoustical quality.

\section{0:05-10:15 Break}

\section{0:15}

3aAA7. Correlations between categories of acoustical quality for forty concert halls and measured physical parameters for them: RT, EDT, IACC, LF, G, ITDG, BR, C(80) and diffusion. Leo L. Beranek (975 Memorial Dr., Ste. 804, Cambridge, MA 02138-5755)

Through the cooperation of many acoustical laboratories, architects, and hall managers, physical measurements of reverberation time (RT), early decay time (EDT), early interaural cross-correlation coefficient [IACC(E)], lateral fraction (LF), sound-pressure level (G), initial-timedelay gap (ITDG), bass ratio (BR), early/reverberant energy ratio [C(80)], and diffusion [IACC $(\mathrm{L})]$ have been collected in whole or in part for 66 concert halls and 10 opera houses. RTs are for both occupied and unoccupied conditions; others are for unoccupied states. The parameters IACC or LF, or both, are available for only 40 of the concert halls. The above acoustical parameters for these halls are individually correlated with the rating categories given in the companion paper [Beranek, "Determination of Categories of Acoustical Quality in Concert Halls"]. It is shown that six of these quantities seem necessary to correlate physical data with the subjective ratings, namely, RT (or EDT), IACC(E), G, ITDG, BR, and some measure of diffusion not yet developed.

\section{0:30}

3aAA8. Toward an overall computational scheme embodying the modern acoustical measures and correlation of the scheme with the categories of acoustical quality of concert halls as determined from the questionnaire and interview method. Leo L. Beranek $(975$ Memorial Dr., Ste. 804, Cambridge, MA 02138-5755)

An overall computational scheme for estimating from modern acoustical measurements the acoustical quality of concert halls is explored in this study. The method showing promise is based on Ando [Concert Hall Acoustics (Springer-Verlag, Berlin, 1985)]. Identified are the most important acoustical attributes to the rating scheme: RT, G, ITDG, IACC(E), and BR. Preferred values for each of these acoustical attributes is estimated from the results presented in a companion paper [Beranek, "Correlations between categories of acoustical quality and modern physical quantities"]. Formulas are borrowed from the Ando study and constants are chosen that express the relative importance, and the degree of falloff in contribution to quality with deviations from optimum, of each of these acoustical attributes. By process of adjustment of the constants, the best fit between the measured physical quantities and the subjective determination of acoustical quality of concert halls is determined. Excellent correlation between the final result and the rank-orderings is obtained. It must be emphasized, however, that other factors are important in concert hall design which are not included in this rating scheme, for example, the degree to which musicians can hear each other and hear themselves on stage and balance among the sections of an orchestra. There may be other unknown acoustical factors that contribute to acoustical quality.

\section{0:45}

3aAA9. The use of the Sabine and Eyring reverberation time equations to churches. António Pedro $O$. Carvalho (Acoust. Lab., Dept. Civil Eng., Faculty of Eng., Univ. of Porto, R. Bragas, P-4099 Porto Codex, Portugal)

Reverberation time (RT) measurements were taken at several source/ receiver locations in 41 Catholic churches in Portugal built in the last 14 centuries, using the impulse-response method. The use of the Sabine and Eyring reverberation time equations was tested to estimate the measured RTs in this sample of churches. The effect of coupled spaces was analyzed, and a new algorithm for the application of the Sabine equation in churches was developed producing an average of $16 \%$ in the differences between the predicted and measured RTs compared to $71 \%$ using the standard Sabine equation. Coupled spaces were found to act as windows with a characteristic absorption coefficient depending on their dimensions. The recesses in churches were grouped in three types: main altar area, chapels, and lateral aisles, each having a particular acoustical behavior. It was found that those recesses only acted as coupled spaces if their length/opening_width $>0.6$ or if the aisle_width/opening_height $>0.4$ in lateral aisles. The remaining differences found between the RTs measured and predicted with this new algorithm were hypothesized to be related to what was called a reverberant ceiling effect, which is presumed to be due to a two-dimensional reverberant sound field that builds up near a very tall ceiling. [Work supported by JNICT/Ministry of Planning and Univ. of Porto, Portugal.]

\section{1:00}

3aAA10. Evaluating acoustical qualities of four rooms using questionnaires. Gary W. Siebein, Martin A. Gold, Mitchell Spolan, and Christopher Herr (Arch. Tech. Res. Ctr., 231 ARCH, Univ. of Florida, P.O. Box 115702, Gainesville, FL 32611-5702)

A large group of students listened to anechoic recordings of music and speech samples at several locations in four different performance rooms: a large 2000-seat multipurpose performing arts center; a black box theater; a 250-seat lecture hall; and an open field. A brief piece by Mozart played by a string quartet, a portion of a symphony by Bruckner, a soliloquy from Hamlet, and standard speech intelligibility word lists were played through a single loudspeaker located at the center front of the performing area of the room. Students evaluated qualities of sound including clarity, intimacy, envelopment, balance, reverberance, loudness, and overall impression on a bi-polar semantic rating scale. They also took speech intelligibility tests. The overall impression of the pieces of music in the black box theater and the lecture hall were scored higher than the other two rooms. There were significant differences in the ratings of the different musical pieces in all rooms. The ratings in the outdoor tests were consistently less than those in all of the indoor locations by over two scale points illustrating the importance of room effects. The ratings in the performing arts center for the recorded music were significantly lower than ratings obtained at the same locations by a group of students evaluating live orchestral performances in the room. [Work supported by NSF.] 
3aAA11. Audibility of individual reflections in a complete sound field. II. Søren Bech (Bang and Olufsen Ltd., Peter Bangs Vej 15, Struer DK-7600, Denmark)

This is a continuation of paper $I$, presented at the Wallace Clement Sabine Centennial Symposium in Boston 1994. The threshold of detection (TD) and just noticeable difference (jnd) for an increase in level was measured for six individual reflections in a complete sound field. The subjects were instructed to use changes in timbre as the criteria for detection. The sound produced by a single loudspeaker in a normal size listening room was simulated using an electroacoustic setup. The simulated loudspeaker had directivity characteristics according to the measured characteristics of a real loudspeaker and the room surfaces had frequencydependent absorption characteristics according to measurements. Values of TD and jnd were measured using four subjects for noise and speech. The results confirmed that the first-order floor reflection contributes individually to the timbre of the sound field. The results have further shown that energy in the frequency range $500-2 \mathrm{kHz}$ is important for the TD level. The noise and speech stimuli have been found to result in significantly different TD and jnd values for all reflections, and is contrary to the previous reported results. [Work financed by Eureka project EU105.]
3aAA12. A preliminary analysis of image shifting in concert halls. Gilbert A. Soulodre (Dept. of Psych., Carleton Univ., 1125 Colonel By Dr., Ottawa, ON K1S 5B6, Canada)

Under typical conditions, the early reflections in a concert hall are attenuated relative to the direct sound and are thus integrated with the direct sound. However, there are instances where, due to focusing effects, the level of a reflection can be greater than or equal to the level of the direct sound. This can result in an apparent shifting of the image away from the true source and toward the source of the reflection. This paper reports on a series of subjective tests designed to provide an initial look at the parameters of the reflected sound which influence image shifting. Tests were conducted using two methods: binaural simulation and multiple loudspeakers in an anechoic chamber. Image shifting was examined as a function of the level, delay, angle of arrival, and spectral content of the reflected sound relative to the direct sound. The results were examined in the context of the Orpheum Theatre in Vancouver where pronounced image shifting occurs at certain locations in the hall due to several large concave surfaces in the ceiling.

THURSDAY MORNING, 1 JUNE 1995

MEETING ROOM 3, 7:45 TO 11:45 A.M.

\title{
Session 3aEA
}

\section{Engineering Acoustics and Noise: Electromagnetic Compatibility of Acoustical Instruments}

\author{
Victor Nedzelnitsky, Cochair \\ National Institute for Standards and Technology, Sound Building 233, Room A147, Gaithersburg, Maryland 20899-0001
}

\author{
Richard J. Peppin, Cochair \\ Scantek, 916 Gist Avenue, Silver Spring, Maryland 20910
}

Chair's Introduction-7:45

Invited Papers

7:50

3aEA1. Electromagnetic interference (EMI) concerns and electromagnetic compatibility (EMC) modeling for acoustic instrumentation. David S. Dixon (Electromagnetic Compatibility Branch, Nav. Undersea Warfare Ctr., New London Detachment, New London, CT 06320)

Traditionally, the electromagnetic interference (EMI) concerns of submarines have been dominated by the susceptibility of low-frequency subsystems associated with acoustic, control, and electromagnetic systems. The uniqueness of the efforts to ensure the nondegraded operation of these systems is the fact that the frequency range of the susceptible systems and the typical EMI sources overlap. Therefore, many low-frequency, low-amplitude signals can be degraded by low-frequency conducted and radiated EMI created by the power generation and distribution system. Power line rectification and switching harmonics are radiated magnetically as well as conducted along cables, shields, and ground plane components. In addition, the use of modern wideband digital systems coupled with the use of lightweight, low-cost, nonmetallic enclosures has produced an additional concern caused by the rapidly increasing radiated electric field environment. In summary, the electromagnetic environmental $\left(E^{3}\right)$ concerns associated with acoustic instrumentation in very similar to the $\mathrm{E}^{3}$ concerns that are required for shipboard systems. This paper will discuss the typical EMI coupling mechanisms that will degrade the expected performance of low-frequency systems, such as audiometers, acoustic instrumentation, telephones, cabling, etc. In addition, it will discuss the EMI models that have been developed for components as well as the latest analysis and predictive EMC design techniques that have been developed to achieve cabinet and compartmental (room) compatibility.

8:15

3aEA2. Electromagnetic compatibility standards for acoustical instruments. Ted Kuemmel (Quest Tech., $510 \mathrm{~S}$. Worthington St., Oconomowoc, WI 53066)

There is an increasing awareness of the problems caused by interference from electromagnetic fields on electronic devices. The rapid expansion of the use of personal radio communication devices suggests a growing problem. The sophisticated electronic technology now commonly used in acoustical instruments is more susceptible to electromagnetic interference than previous technolo- 
gies. In 1991, the ANSI Committee S1, Acoustics, formed working group 21 to develop a new standard to address the radio frequency electromagnetic susceptibility of acoustical instrumentation. Now in its eighth draft, the standard provides general specifications for the characteristics of acoustical instruments when used in the presence of radiated electromagnetic fields. The standard provides limits on allowable deviation from specified acoustical performance based on the accuracy class of the instrument. The standard also presents minimum testing requirements and sample test methods to demonstrate conformance to the specifications. Intemationally, the IEC Technical Committee 29, Electroacoustics, is also working on this problem. The current status of these efforts and the issues driving them are presented and compared.

\section{$8: 40$}

3aEA3. Electromagnetic susceptibility of sound level meters. Hans-O. Finke (Physikalisch-Technische Bundesanstalt, Bundesallee 100, D-38116 Braunschweig, Germany)

Tests of electromagnetic susceptibility (EMS) are part of the type of approval procedure for sound level meters in the field of legal metrology. Starting in January 1996 every electronic device on the European market has to have a label that indicates a certification of the device, which also includes requirements on electromagnetic susceptibility. The EMS test of sound level meters at the Physikalisch-Technische Bundesanstalt (PTB) are performed in a radio frequency anechoic chamber. The electric field strength is 6 $\mathrm{V} / \mathrm{m}$, the frequency range is $26-1000 \mathrm{MHz}$. The frequency is varied in steps of $4 \%$ and the signal is modulated with $1000 \mathrm{~Hz}$ $(m=80 \%)$. An acoustical signal is applied to the sound level meter and the difference of the displayed levels with and without the electromagnetic field has to be within specified tolerances $(0.5$ to $2 \mathrm{~dB})$. At the moment not all types of sound level meters comply with these tolerances; with instruments under test deviations up to $18 \mathrm{~dB}$ had been observed. The present test procedure will be improved for future regulations and standards (higher strength of field, wider frequency range, influence of accessories). Methods and results will be reported.

\section{9:05}

3aEA4. Electromagnetic susceptibility of audiometers. Utz Richter (Physikalisch-Technische Bundesanstalt, Lab. 1.51, Bundesallee 100, D-38116 Braunschweig, Germany)

As early as 1991 the Physikalisch-Technische Bundesanstalt (PTB) in Germany decided that tests of electromagnetic susceptibility have to be performed, in general, with all measuring instruments submitted to PTB for pattern evaluation, among them also pure-tone and speech audiometers. A large shielded room is available where electromagnetic fields $(10 \mathrm{~V} / \mathrm{m}, 10-935 \mathrm{MHz}, 1-\mathrm{kHz}$ tone $\mathrm{AM}$ modulation) can be generated. No suitable test method and no limits were found in any known standard for audiometers including IEC 645-1, and no available acoustic measuring equipment did pass the EMC test by itself. So a new test method had to be developed based on loudness balance tests between the $1-\mathrm{kHz}$-interference tone caused by the electromagnetic field and the ordinary $1-\mathrm{kHz}$ test tone of the audiometer. The tests are performed in the shielded room at fixed carrier frequencies. Taking into account the uncertainty of this subjective test method, an upper limit of $60 \mathrm{~dB}$ HL for the interference level was established. Of the first 25 types tested, $92 \%$ audiometers did fail the EM susceptibility test in the first run. All of them passed the test after reconstruction.

9:30

3aEA5. OIML standards on performance requirements for acoustical instruments. Samuel E. Chappell (Office of Standards Services, NIST, Gaithersburg, MD 20899)

The International Organization of Legal Metrology (OIML) is a treaty organization, having currently 53 (voting) member and 40 (non-voting) member nations, most of the latter nations being characterized as developing. OIML's objective is to harmonize national regulations for the performance requirements of legal measuring instruments among member nations in order to facilitate international commerce of measuring instruments and affected products, to ensure public health and safety, and to protect the environment. This effort intends to avoid or minimize technical barriers to international trade. An OIML technical committee or subcommittee develops OIML recommendations (standards or model performance regulations) to address particular types or classes of instruments. If approved, member nations have a moral obligation to implement a recommendation in their national regulations for the instrument addressed. The obligation applies both to new regulation and to harmonizing any existing regulations. This presentation addresses OIML work projects for acoustical instruments and the potential impact of relevant OIML recommendations on instrument manufacturers. The basis for requirements including electromagnetic compatibility and the means by which the United States participates in the work are covered.

\section{9:55}

3aEA6. Complicated cases and shielded rooms: Audiometric booths shielded to attenuate electromagnetic interference (EMI). Victor Nedzelnitsky (NIST, Sound Bldg. 233, Rm. Al47, Gaithersburg, MD 20899-0001)

Electromagnetic compatibility (EMC) issues involving acoustical instruments, especially their susceptibility and immunity to electromagnetic interference (EMI), are increasing in importance. This increase is driving domestic and international standards development. Of particular significance is the impact on commercial practice and international trade of two Councils of the European Communities directives regarding generic apparatus (Council Directive 89/336/EEC, 3 May 1989) and medical devices (Council Directive 93/42/EEC, 14 June 1993). Directive 93/42/EEC is being interpreted to include hearing aids and audiometric instruments. Numerous voluntary International Electrotechnical Commission (IEC) electroacoustical performance standards are available for some of these instruments. However, extension of these complicated standards to include all pertinent EMC issues is difficult. Development of standards for other instruments, such as those for measuring auditory brain-stem response, would be even more difficult. In some 
circumstances, the use of shielded rooms to attenuate radiated EMI is practical and cost-effective. In the U.S.A., at least, existing standards for measuring such shielded-room performance were not specifically developed for audiometric booths. Methods in some of these standards have been adapted and applied to that purpose. Selected standards, methods, and some considerations regarding their application to measuring the shielding performance of audiometric booths in hospital/clinical environments are discussed.

\title{
Contributed Papers
}

10:20

3aEA7. Effect of electromagnetic interference on a continuous wave ultrasonic field. David L. Cosnowski and Mardi C. Hastings (Ohio State Univ., Dept. of Mech. Eng., 206 W 18th Ave., Columbus, OH 43210)

Continuous-wave ultrasound for imaging and other measurement applications requires the use of a separate source and receiver transducer. When this system is used in air or water, the receiver transducer often detects a signal at the ultrasonic frequency even in the absence of a target. At ultrasonic frequencies, significant electromagnetic coupling between the transducers can occur due to their own and extraneous electromagnetic fields. This study examines the effect of this interference on the 3-dB sample volume defined by the crossed beams. The sample volume is determined mathematically by modeling the source and receiver transducers as plane piston radiators [J. Zemanek, J. Acoust. Soc. Am. 49, 181-191 (1971)]. The effect of interference is obtained by adding a constant term to the sample volume matrix, which, in general, is complex. Experimental measurements using an ultrasonic source and receiver in air at several frequencies from 40 to $100 \mathrm{kHz}$ correlate well with model predictions. The electromagnetic interference was found to have a significant effect on the observed field. [Work supported by NSF Grant No. MSS 9058607.]

10:35

3aEA8. New requirement for electromagnetic compatibility of acoustical instrumentation. Ernst Schonthal and Helge Larsen (Bruel \& Kjaer Instruments, Inc., Denmark)

Most electronic equipment is sensitive to electromagnetic signals. Often electronic equipment also radiates electromagnetic signals causing in-

THURSDAY MORNING, 1 JUNE 1995 terference to other electronic equipment. To insure that the electrical equipment can work without influence from the electromagnetic environment and also can operate without disturbing the environment, all electric equipment including acoustical measuring instruments sold within the European Community must fulfill the requirements per EU50082-1 and -2 , starting 1 January 1996. To achieve this goal a new special test laboratory has been established at Bruel \& Kjaer in Denmark. Here, all existing and new measuring instrumentation are tested for conformance to EU50082-1 and -2. The results have shown that a number of the existent instrumentation will have to be modified or redesigned. The new Bruel \& Kjaer Falcon ${ }^{\mathrm{TM}}$ range of measuring microphones, preamplifiers, and power supplies has been designed in accordance with the new requirements of EU50082-1 and -2. This paper will describe measurement results and give examples of experience gained through this work.
11:00-11:45

PANÉL DISCUSSION

MEETING ROOM 2, 8:30 A.M. TO 12:00 NOON

\section{Session 3aNS}

\section{Noise: State of the Art-Noise Effects and Control}

\author{
Henning E. von Gierke, Cochair \\ Medical Research Laboratory, Armstrong Aerospace, AL/CF 2610 Seventh Street, Wright Patterson Air Force Base, \\ Ohio 45433-7901
}

Daniel L. Johnson, Cochair

Biophysical Operations, EG\&G Special Projects, P.O. Box 9100, Albuquerque, New Mexico 87119

Chair's Introduction-8:30

\section{Invited Papers}

8:35

3aNS1. Noise environments outdoors and the effects of community exposure. Edgar A. G. Shaw (Inst. for Microstructural Sci., Natl. Res. Council. Ottawa, ON K1A 0R6, Canada)

Man-made noise, especially transportation noise, creates acoustical environments that are vastly different from those associated with pristine habitats. During the past 25 years, the day-night average sound level, $L_{\mathrm{dn}}$, has gained substantial acceptance as a valid measure of the magnitude of any given pattern of community noise, taking into account its complex temporal and spectral characteristics. During the same period, the effects of various levels of noise exposure on human activities and well-being have been widely studied. Data from many social surveys have been brought together and analyzed to clarify the relationship between noise level and the prevalence of annoyance which provides a broad indication of the impact of intrusive noise on human communities. Criteria based 
on such studies are now widely used in urban planning. In recent years, there have been impressive reductions in the noise emissions from commercial aircraft and notable improvements in the control of airport noise, while highway barriers now provide some relief from motor vehicle noise. Finally, the potential effects of noise on wildlife, especially endangered species, now come under close scrutiny when major projects are planned in remote areas.

3aNS2. Occupational noise exposures: Where do we stand today? Alice H. Suter (Alice Suter and Assoc., 575 Dogwood Way, Ashland, OR 97520) and Daniel L. Johnson (EG\&G Special Projects, P.O. Box 9100, Albuquerque, NM 87119)

There have been several major improvements in the field of occupational noise exposure over the last 25 years. ISO 1999 has been revised and now provides an accepted method of relating hearing loss to noise exposure. Hearing protection devices have been improved considerably, impulse noise is being assessed more effectively, and there has been a shift among U.S. agencies toward using the equal-energy rule in noise exposure standards. Perhaps the most important advancement has occurred with the promulgation of OSHA's hearing conservation amendment to its noise standard. The future, however, poses many challenges: OSHA's noise standard needs revision, more emphasis on enforcement is needed, engineering controls should be re-emphasized after many years of inattention, and total reliance on hearing protection devices should be de-emphasized. In addition, the benefits of the hearing conservation amendment need to be extended to currently underserved workers, such as miners and workers in construction, agriculture, and the oil and gas industries. Perhaps the greatest challenge is the need to investigate the effectiveness of hearing conservation programs on a national scale to determine the extent to which they are actually preventing hearing loss.

3aNS3. Noise environments and their effects. Recreational exposures. Alf Axelsson (Hear. Res. Lab., Lindholmen Dev., Box 8714, S-402 75, Goteborg, Sweden)

Many efforts have been made lately to combat occupational noise exposure, and the situation in many industries conceming noise preventive measures seems promising. Conversely, with regard to recreational activities the situation seems less promising. There are reasons to raise concerns about noisy activities such as personal cassette players (PCPs), pop/rock concerts, car stereos, motor sport, shooting activities, etc. For most of the musically oriented activities the situation is probably generally satisfactory; even if the sound levels frequently are very high, the exposure time and number of exposures/year is limited. For impulsive noisy activities the situation is more serious. As is well known, the impulsive noise in connection with the use of weapons, fire-crackers, and toy-cap guns without hearing protection may lead to a permanent noise induced hearing loss under accidental conditions. The general attitude concerning recreational noisy activities should obviously be to strive for lower sound levels, limited exposure, and better use of hearing protection.

\section{9:35}

3aNS4. Aircraft noise control. David G. Stephens (Fluid Mech. and Acoust. Div., NASA-Langley Res. Ctr., Hampton, VA 23681-0001)

The purpose of this paper is to describe the research program in aircraft acoustics being conducted by the NASA-Langley Research Center. The program includes research to understand, predict, and reduce the sources of aircraft noise; to quantify the transmission of noise through the atmosphere and/or aircraft structures; and to define the effects of noise on people and structures. The research is focused on a broad range of aircraft with emphasis on advanced subsonic transports including conventional and tiltrotor rotorcraft, supersonic transports, high-performance military vehicles and hypersonic configurations. Specialty areas of research include computational aeroacoustics, jet noise, rotating blade acoustics, and structural and subjective acoustics. The resulting technology is used to design and operate aircraft to reduce community noise impact and/or to minimize the effects of noise on structural integrity and interior noise.

\section{9:55}

3aNS5. Advances in noise control for products other than aircraft. William W. Lang (Phys. Dept., Box 539, Vassar College, Poughkeepsie, NY 12601)

How much progress has been made in advancing the technology of noise control over the past quarter century? Different techniques of quieting have been applied to the many products that are grouped in the following broad categories: stationary noise sources, moving noise sources (other than aircraft), and specialized industrial machinery and equipment. For all products, the control of noise by engineering design is the goal. To arrive at estimates of progress, two subsidiary questions must be addressed. How is progress to be measured in quantitative terms? Recognizing that trade secrets and patented innovations may be involved, how is it possible to learn what progress has been made for the different classes of products? A key factor is that changes in functional technology may reduce the noise emissions as a desirable by-product; for example, ink-jet and laser printers are much quieter than impact printers. The estimates of progress in product quieting in terms of the reductions of A-weighted sound power levels range from $0 \mathrm{~dB}$ for leafblowers up to $50 \mathrm{~dB}$ for devices which 25 years ago required cooling fans for their safe and efficient operation, but today require none. 
3aNS6. Twenty-five years of progress in noise standardization. Paul D. Schomer (U.S. Army Construction Eng. Res. Lab., Champaign, IL 61826-9005)

This paper documents the more than ten-fold growth in the number of Standards and the program of work carried out by the ASA Standards directorate for the American National Standards Institute (ANSI) and for related international work with the International Organization for Standardization (ISO) and the International Electro-technical Commission (IEC). Not only has there been a huge increase in the work effort, but the emphasis has changed greatly. Now we work to ensure that ISO Standards are fair to all players and to ensure that ANSI Standards are compatible with ISO. While major U.S. industries are participating in this effort, more participation is needed by industry and government. Case histories are used to show how our efforts in the standards process are currently helping some U.S. manufacturers to market their products worldwide. Major industries with some significant involvement include automotive, construction and agricultural equipment, computer and business machines, hearing conservation equipment, and audiological testing, and sirens and alarms. Many other industries such as aircraft, mining, rotating electrical machinery, and manufacturers of acoustical materials, attenuators, mufflers, and soundproof enclosures currently lack significant involvement in the ISO process. Thus greater involvement is required by significant sectors of U.S. industry.

\section{0:45}

3aNS7. Federal regulations and government activities in noise control. R. L. Miller (Harris, Miller, Miller \& Hanson, Inc., 15 New England Exec. Park, Burlington, MA 01803)

Since the demise of the EPA's Office of Noise Abatement and Control at the hands of former President Reagan, coordinated regulatory action for the control of noise at the Federal level has essentially disappeared. Instead, governmental agencies are left to deal with their noise problems on their own, usually meaning that they respond only to specific legislation, political pressures, or litigation. Agency coordination, when it does occur, is imposed by Executive Order or law. This paper briefly reviews existing Federal regulations controlling the world of transportation noise, but focuses to a greater extent on the on-going efforts of our government that will influence our noise environment into the next century. The paper summarizes the actions of the Federal Aviation Administration, the National Aeronautics and Space Administration, and the Department of Defense as they confront issues of aircraft noise and airspace management; the National Park Service as it struggles to resolve conflicting mandates to preserve natural quiet and solitude while providing access to and public enjoyment of National Parks and Forests; the Federal Railroad Administration and the Federal Transit Administration in their endeavors to control noise from transit, high-speed rail, and mag-lev systems; and the Federal Highway Administration as it develops better tools to assess and control the noise impact of traffic. The paper concludes, speculating on the effects that reduced Federal spending will have on these efforts.

\section{1:05}

3aNS8. A global vision for the noise control marketplace. Bennett M. Brooks (Brooks Acoust. Corp., 27 Hartford Tnpke., Vernon, CT 06066), T. James DuBois (Acentech, Canoga Park, CA 91303), Robert M. Hoover (Hoover and Keith, Inc., Houston, TX 77082), George C. Maling (Empire State Software Systems, Ltd., Poughkeepsie, NY 12603), and Louis C. Sutherland (Rancho Palos Verdes, CA 90274)

Much progress has been made over the past 25 years in the United States to define the dangers of excessive noise exposure, and to develop the means to control it. A significant portion of this progress was stimulated by government actions, to regulate the industries which produce noise, establish community and workplace noise standards, and to promote reseanch and development in noise control. Today, the pressures of competition in the global marketplace, which did not exist 25 years ago, are rapidly increasing the motivation for noise control in the private sector. Those industries which seek to sell their products worldwide, particularly in Europe or Japan, find that they must meet stricter standards than now exist in the U.S. Further, foreign companies which already live with stricter standards are producing quiet products that compete for sales in the U.S. These challenges present both a threat and an opportunity to the U.S. economy. Several actions which can be immediately taken to address these issues are suggested.

$$
\text { 11:25-11:55 }
$$

\section{PANEL DISCUSSION}

11:55-12:00 CONCLUSION 


\title{
Session 3aPA
}

\section{Physical Acoustics: Ultrasonic Techniques}

\author{
Inder Raj S. Makin, Chair \\ Department of Engineering, Swarthmore College, Swarthmore, Pennsylvania 19081-1397
}

Chair's Introduction-8:00

Contributed Papers

\section{8:05}

3aPA1. Acoustic radiation pressure in an infinite lossy medium. Zhong-Yue Jiang and James F. Greenleaf (Dept. of Physiol. and Biophys., Mayo Clinic and Foundation, Rochester, MN 55905)

Theories on acoustic radiation pressure associated with plane acoustic waves and lossless media have been examined extensively. The radiation pressure produced by a non-plane wave in a lossy unbounded medium was analyzed. The distinction between Lagrangian and Eulerian pressure was used to clarify the understanding of radiation pressure. The radiation pressure exerted on a perfectly absorbing pointwise target by an arbitrary acoustic wave in an unbounded lossy medium was then considered. By using an indirect approach developed in Chu and Apfel [B.-T. Chu and R. E. Apfel, J. Acoust. Soc. Am. 72, 1673-1687 (1982)], the radiation pressure was analyzed without appealing to the detailed solutions of the goveming fluid dynamic equations. It was shown that even in lossy media with arbitrary waves, the radiation pressure experienced by a pointwise target is equal to the Langevin pressure which is the time average energy density at that point. The radiation pressure exerted on a specific target by an arbitrary acoustic wave in an infinite lossy medium was studied and a concise theoretical result was obtained. [The work was supported by grants CA 43920 and HL 41046 from the National Institutes of Health.]

\section{8:20}

3aPA2. Simulation of ultrasonic propagation through abdominal wall. T. Douglas Mast, Laura M. Hinkelman, Robert C. Waag (Dept. of Elec. Eng., Univ. of Rochester, Rochester, NY 14627), and Victor W. Sparrow (Penn State Univ., University Park, PA 16802)

Ultrasonic pulse propagation through the human abdominal wall is simulated using a model for two-dimensional propagation through anatomically realistic tissue cross sections. The time-domain wave equation for a medium of variable sound speed and density is discretized and solved as a set of coupled finite-difference equations. The finite-difference algorithm used is a two-step MacCormack scheme that is fourth-order accurate in space and second-order accurate in time. The inhomogeneous tissue of the abdominal wall is represented by two-dimensional matrices of sound speed and density values. These values are determined by mapping scanned images of abdominal wall cross sections that have been stained to identify connective tissue, muscle, and fat, each of which is assumed to have a constant sound speed and density. The computational configuration is chosen to simulate the experimental wave-front distortion measurements of Hinkelman et al. [J. Acoust. Soc. Am. 95, 530-541 (1994)]. Qualitative agreement is found between those measurements and the results of the present computations. Visualization of calculated sound fields is used to illustrate the salient characteristics of ultrasonic wave-front distortion in vivo.

\section{8:35}

3aPA3. The use of high-intensity focused ultrasound for noninvasive surgery. Lawrence A. Crum (Appl. Phys. Lab., 1013 NE 40th St., Univ. of Washington, Seattle, WA 98105) and Wing Law (Focus Surgery, Fremont, CA 94539)

High-intensity focused ultrasound (HIFU) can be used to destroy tissue in vivo; recent applications of this modality to the treatment of benign prostatic hypertrophy (BPH) have met with significant success. The lesions formed by focused ultrasound can be induced by both thermal and mechanical (cavitation) mechanisms. However, the relative contributions of these two mechanisms, under varied HIFU parameters, are often difficult to ascertain. Descriptions are presented of some simple experiments designed to elucidate the relative roles of cavitation and thermal effects in specific tissues; these tests were undertaken in turkey breast tissues, but seem to correlate well with previous in vivo experiments in dogs and humans. The experiments indicate that cavitation, once induced, tends to shorten and broaden the thermally induced lesions, suggesting that the presence of free gas generated by the cavitation blocks passage of the ultrasound.

\section{8:50}

3aPA4. Measurements of finite-amplitude acoustic pulses radiated from focused piston sources in water. Michalakis A. Averkiou, Lawrence A. Crum (Appl. Phys. Lab., Univ. of Washington, Seattle, WA 98105), and Mark F. Hamilton (Univ. of Texas at Austin, Austin, TX 78712-1063)

Measurements of finite-amplitude acoustic pulses radiated by a focused piston source in water are reported. Pulses with center frequency of 2.25 $\mathrm{MHz}$, durations ranging from two to ten cycles, and peak sound pressures up to $3 \mathrm{MPa}$ were measured with a wideband $(1-20 \mathrm{MHz})$ membrane hydrophone. The combined effects of focusing, diffraction, and finiteamplitude distortion on short pulses are demonstrated. Propagation curves for the peak positive and peak negative pressure along the axis of the source are shown. The peak negative pressure, which is an important factor in cavitation dynamics, is maximized in the prefocal region and shifts closer to the source with increasing source amplitude, as predicted earlier [Averkiou $t i$ al., J. Acoust. Soc. Am. 96, 3306(A) (1993)]. Tissue images during treatment with a therapeutic ultrasound device that show increased cavitation activity in the prefocal region are presented. The measurements are compared with theoretical predictions based on the $\mathrm{KZK}$ equation and good agreement is found. [Work supported by the National Institutes of Health.] 
3aPA5. Magnetic field studies of the superconducting transition in $\left(\mathrm{La}_{1-r} \mathrm{Sr}_{\mathbf{r}}\right)_{2} \mathrm{CuO}_{4}$ single crystal by ultrasonic measurements. $\mathrm{M}$. J. McKenna, Hong Zhang, Bimal K. Sarma, Moises Levy (Dept. of Phys., Univ. of Wisconsin-Milwankee, Milwankee WI 53201), T. Kimura, K. Kishio, and K. Kitazawa (Univ. of Tokyo, Tokyo, Japan)

The temperature dependence of the ultrasonic attenuation and the relative sound velocity were measured in a single crystal of $\left(\mathrm{La}_{1-x} \mathrm{Sr}_{x}\right)_{2} \mathrm{CuO}_{4}$ $\left(x=0.075\right.$, nominal) with dimensions of about $4 \times 4 \times 4 \mathrm{~mm}^{3}$, in a temperature range of 4.2 to $50 \mathrm{~K}$. The superconducting transition of this sample measured by ac susceptibility is at $36.6 \mathrm{~K}$. The frequency of longitudinal sound waves propagating along the $c$ axis was extended up to $305 \mathrm{MHz}$. The attenuation shows two peaks, one at about $27 \mathrm{~K}$ and the other at around $36.7 \mathrm{~K}$, while the sound velocity exhibits softenings of about 20 and $80 \mathrm{ppm}$ at the corresponding temperatures, respectively. Magnetic fields of $11 \mathrm{KOe}$ were applied in the $a-b$ plane to study the effects of the field on both the temperatures and the heights of these features in attenuation and velocity. The results, combined with the frequency dependences of these features, suggest that the transition at $36.7 \mathrm{~K}$ may possibly be produced by both a structural transition and the superconducting transition. In addition, results on the temperature dependence of the attenuation upon cooling in magnetic fields will be presented. [Work supported by the Office of Naval Research.]

\section{9:20}

3aPA6. Pressure and cavitation measurements of an intra-arterial angioplasty device. Inder Raj S. Makin and E. Carr Everbach (Dept. of Eng., Swarthmore College, Swarthmore, PA 19081-1397)

Variation of the acoustic field radiated from a low-frequency (22.5 $\mathrm{kHz})$ catheterlike angioplasty device in a relatively large $(600 \mathrm{~L})$ tank of water was presented earlier [I. R. S. Makin and E. C. Everbach, J. Acoust. Soc. Am. 95, 2855(A) (1994)]. The normal volume of the human torso or the human limb into which the ultrasonic wire is inserted during therapy cannot be treated, however, as an infinite medium for the $66.6-\mathrm{mm}$ wavelength (calculated for water) due to the wire source. Therefore the sound field of the device was investigated in a liquid-filled Plexiglas ${ }^{\circledR}$ cylinder (200-mm diameter, 350-mm height) to simulate anthropometric dimensions. Measurement of pressures along the axis of the cylinder for the wire source positioned axially at one end of the cylinder shows a marked standing wave pattern even up to an axial range of $250 \mathrm{~mm}$. Such strong standing waves could potentially exist far away from the probe tip in vivo. In addition, the occurrence of cavitation at the tip of the wire was confirmed using a $20-\mathrm{MHz}$ passive cavitation detection technique.

\section{9:35}

3aPA7. Correction of errors in Doppler peak velocity measurements made with commercial ultrasound systems. Andrew $J$. Winkler and Junru Wu (Dept. of Phys., Univ. of Vermont, Burlington, VT 05405)

Commercial Duplex ultrasound systems primarily measure peak velocity of blood flow to provide information in diagnosing vascular disease. However, due to errors caused by intrinsic spectral broadening (ISB), the accuracy decreases as Doppler angle increases. In general, vascular technologists keep the Doppler angle under $60^{\circ}$. Since most vessels in the body run almost parallel with the skin surface, any criteria requiring a Doppler angle of say $45^{\circ}$ or less would be difficult to achieve. Using the transverse Doppler equation [Newhouse et al., J. Acoust. Soc. Am. 95, 2091-2098 (1984)] as a correction for ISB, in cojunction with the classical Doppler equation, much more accurate peak velocity measurements were obtained at Doppler angle as high as $80^{\circ}$.

\section{0:05}

3aPA8. Temperature rise generated by diagnostic ultrasound in a transcranial phantom. Junru Wu, Frances Cubberley, Gerard Gormley (Dept. of Phys., Univ. of Vermont, Burlington, VT 05405), and Thomas L. Szabo (Hewlett-Packard Imaging Systems, Andover, MA 01810)

Temperature rises generated by diagnostic ultrasound from a commercial system (Sonos 1000 Hewlett-Packard) in a transcranial phantom that consists of human temporal bone and tissue-mimicking material are measured. Significant temperature rises were found at the external and internal temporal bone surfaces. The experimental resuits are compared with Cranial Thermal Indices (TIC) developed by American Institute of Ultrasound in Medicine and National Electrical Manufacturers association in 1992 for various modes. For all the modes compared, TIC underestimated temperature rise at the external temporal bone surface. The differences between the data and temperature rises predicted by TIC can be attributed to transducer surface heating. [Work supported by Hewlett-Packard Co.]

\section{0:20}

3aPA9. Numerical anomalies in the Rayleigh-Ritz method for calculating the normal mode vibrations of arbitrarily shaped elastic solids. P. S. Spoor and J. D. Maynard (Dept. of Phys., Penn State Univ., University Park, PA 16802)

A matrix implementation of the Rayleigh-Ritz variational method was used by Demarest [H. H. Demarest, J. Acoust. Soc. Am. 57, 768] to make the first successful calculation of the eigenfrequencies and eigenmodes of an isotropic elastic cube, and was later generalized by Ohno [I. Ohno, J. Phys. Earth 57, 355] and Visscher [W. M. Visscher et al., J. Acoust. Soc. Am. 90, 2154-2162 (1991)] to apply to solids of arbitrary shape and elastic symmetry. This became the core of a new method of elastic constant determination based on measurements of the normal mode spectra of elastic parallelepipeds and spheres, called resonant ultrasound spectroscopy (RUS). In theory, one should be able to use Rayleigh-Ritz to determine the effect of imperfect parallelepipeds (due to small errors in sample preparation) on the accuracy of RUS. It has been discovered, however, that the method may not converge properly for many relevant cases, producing spurious results. A variety of examples, including comparisons with loworder approximations, will be discussed. [Work supported by NSF Grant DMR- 9000549 and by the Office of Naval Research.]

\section{0:35}

3aPA10. Ultrasonic characterization of the quality of an epoxy resin. B. Faiz, A. Moudden (Lab. d'Instrum. et de Mesures, Faculté des Sci. d'Agadir, Univ. Ibnou Zohr, Agadir, Morocco), D. Décultot, and G. Maze (Université du Havre, Le Havre, France)

A pulse ultrasonic technique has been developed to study the quality of the liquid/solid transformation of an epoxy resin made up of two components (resin and hardener). The epoxy resin is in a tank with parallel faces. A reflection technique allows the measurement of the phase velocity, the attenuation, and the acoustical impedance. The first and second parameters give an average state of the resin hardening in the thickness. The temporal evolution of the last parameter allows an evaluation of the state of the resin at the interface resin/tank wall. These experiments have been achieved for different proportions of the hardener. The velocity and the attenuation increase at first and then stabilize after a time which is as short as the hardener concentration is high. The experiment shows that $10 \%$ of the hardener is the oplimum value and that $20 \%$ of it produces a vitreous transition. The experimental results also show that the acoustical impedance stabilizes after a time longer than the one observed for the velocity and the altenuation. The velocity and the attenuation are significant parameters of the optimum value of the hardener concentration. The acoustical impedance is the significant parameter of the bond quality. 
3aPA11. Polarimetric fiber-optic ultrasonic sensor for the nondestructive evaluation of materials. Chonghua Zhou, Stephen V. Letcher (Dept. of Phys., Univ. of Rhode Island, Kingston, RI 02881), and N. Narendran (MTI, Latham, NY 12110)

A fiber-optic polarimetric interferometer has been used as an ultrasonic sensor in a feasibility study of flaw detection and nondestructive evaluation of materials. The sensor is based on acoustic modulation of the birefringence of a polarization-maintaining fiber. The small size of the fiber and the ability to embed it in materials provide significant advantages over conventional transducers. When the fiber and sample are both immersed in water, sample thickness and flaw location, size, and orientation have been evaluated. The fiber has also been embedded in a Plexiglas specimen and has shown the potential to locate internal flaws. [Work supported, in part, by NSF.]

\section{1:05}

3aPA12. Concentration, fractionation, and separation of small particles in liquid by ultrasound. Kenji Yasuda, Shin-ichiro Umemura (Advanced Res. Lab., Hitachi Ltd., 2520 Akanuma, Hatoyama-chou, Hiki-Gun, Saitama 350-03, Japan), and Kazuo Takeda (Central Research Lab., Hitachi Ltd., Tokyo 185, Japan)

The efficacy of using an ultrasonic standing plane wave to concentrate small particles in a liquid was theoretically estimated and compared with experimental results. Acoustic energy density was measured by ultrasonic absorption, and particle distribution was observed by darkfield microscopy. The theory predicts that the effect of diffusion is negligible in concentrating polystyrene spheres larger than $5 \mu \mathrm{m}$ in diameter when they are subjected to $4 \mathrm{~J} / \mathrm{m}^{3}$ ultrasound at $500 \mathrm{kHz}$. The half-width of the steady-state particle distribution in the experiment was of the same order of magnitude as that of the theory. This concentrating technique was applied to the fractionation of polystyrene spheres $10 \mu \mathrm{m}$ in diameter by using a capillary tube. More than $90 \%$ of the particles in a laminar flow were successfully collected. A separation technique based on the competition between the acoustic radiation force and the electrostatic force was also tested. A mixture of polystyrene spheres with two different radii, which lined up on a pressure node of a stationary standing wave, was successfully separated according to their radii by applying a uniform electrostatic force parallel to the acoustic radiation force.

\section{1:20}

3aPA13. Collinear ultrasonic four-wave mixing mediated by a suspension. Christopher S. Kwiatkowski and Philip L. Marston (Dept. of Phys., Washington State Univ., Pullman, WA 99164-2814)

In previous work, ultrasonic four-wave mixing mediated by a suspension was observed by oblique Bragg reflection of a high-frequency probe wave from a periodic grating [H. J. Simpson and P. L. Marston, J. Acoust. Soc. Am. 90, 2244(A) (1991)]. The grating in the number density of suspended polymer microspheres was induced by the radiation presşure of a standing pump wave. In the present investigation, a different geometry is of mediated interactions of sound with sound. The new geometry uses a high-frequency probe beam that is collinear with the axis of the pump standing wave. The Bragg signal for this geometry is amenable to analysis with transfer matrices. The interaction investigated has potential applications to the acoustical probing of suspensions. [Work supported by the Office of Naval Research.]

\section{1:35}

3aPA14. Greenspan acoustic viscometer: Recent results. Keith $A$. Gillis, Michael R. Moldover (NIST, Thermophys. Div., Gaithersburg, MD 20899), and James B. Mehl (Univ. of Delaware, Newark, DE 19716-2570)

Acoustic resonators consisting of a duct coupling two cavities (Greenspan viscometers) have a low-frequency mode whose response is a sensitive function of the viscous diffusivity of the enclosed gas. The response function is determined experimentally by measuring the acoustic pressure in one cavity as a function of the frequency of a source in the other cavity. A theoretical response function, based on a model which includes viscous and thermal boundary layer phenomena in both the duct and the cavities, as well as inertial and resistive duct-end corrections, is fit to the data to determine the viscous diffusivity. Recent measurements in argon, helium, and propane near room temperature span a range of viscous diffusivity from $7 \times 10^{-7}$ to $2 \times 10^{-4} \mathrm{~m} / \mathrm{s}^{2}$. Experimental values determined with three different viscometers differ from reference values by average values of $2.7 \%$ (viscometer 0 ), $0.8 \%$ (viscometer 1 ), and $1.8 \%$ (viscometer 2). [Work supported by the Office of Naval Research.]

\section{1:50}

3aPA15. Numerical investigations of double-Helmholtz resonators and duct-end effects. James B. Mehl (Dept. of Phys. and Astron., Univ. of Delaware, Newark, DE 19716-2570 and NIST Gaithersburg, MD 20899)

The acoustic fields and eigenfrequencies of double Helmholtz resonators (Greenspan viscometers) have been investigated using a boundaryintegral-equation technique. The resonator geometry consists of a circular duct connected at both ends to concentric cylindrical cavities. Comparisons of the numerically determined fields with analytic expressions based on a Green's function expansion establish the high accuracy of the method. The acoustic flow near the duct-cavity orifices has the expected singular behavior at the edge of the duct orifice. The associated duct-end inertance corresponds to a length correction of approximately 0.82 times the duct radii, slightly smaller than the classical formulas of Rayleigh and Ingard [J. Acoust. Soc. Am. 25, 1037-1061 (1953)], which are based on an assumed uniform (piston) flow within the orifice. Numerical integrations of the square of the tangential particle velocity over the walls of the duct and the cavity wall surrounding the orifice have been used to estimate the orifice resistance. This quantity was found to correspond to a resistance effective length of approximately 0.79 times the viscous penetration length, a result of importance in the modeling of Greenspan viscometers. [Work supported by the Office of Naval Research.] 


\title{
Session 3aPP
}

\section{Psychological and Physiological Acoustics: Pitch and Temporal Perception; Auditory Detection}

\author{
William A. Yost, Chair \\ Parmly Hearing Institute, Loyola University, 6525 North Sheridan Drive, Chicago, Illinois 60626
}

\section{Contributed Papers}

$7: 45$

3aPP1. Pitch and pitch strength of iterated rippled noise. William A. Yost, Sandra J. Guzman, and Stanley Sheft (Parmly Hear. Inst., Loyola Univ. Chicago, 6525 N. Sheridan Rd., Chicago, IL 60626)

A cascade of add, delay $(d \mathrm{~ms})$, and attenuate $(-1 \leqslant g \leqslant 1)$ circuit excited with noise produces iterated rippled noise (IRN) stimuli. The matched pitch and discriminability between pairs of IRN stimuli were studied as a function of $g, d$, and the number of circuit iterations $(n)$. For $g>0$, the pitch of all IRN stimuli equals $1 / d$. For $g<0$, pitch depends on $n$ : For small $n$, there were two pitches in the region of $1 / d$, while for large $n$ there was a single pitch equal to $1 / 2 d$. Peaks in the autocorrelation function of IRN stimuli accounted for all of the results. Peaks in the autocorrelation functions for IRN stimuli indicate the number of intervals in the waveform with durations $p d(p=1,2, \ldots, n)$, and for $g<0$ intervals related to peaks near $1 / m d$ ( $m=$ odd integers) caused by assumed auditory filtering. The number of intervals (i.e., the heights of the autocorrelation peaks) determines the discriminability between IRN stimuli, while the reciprocal of the interval duration determines the matched pitch. These results support a temporal rather than a spectral account of the pitch of IRN stimuli. [Work supported by NIH.]

\section{8:00}

3aPP2. Extracting the F0's of two overlapping groups of harmonics. Robert P. Carlyon (MRC Appl. Psych. Unit, 15 Chaucer Rd., Cambridge CB2 2EF, England)

Two concurrent harmonic sounds were mixed and passed through a bandpass filter, with cutoffs of either $20-1420 \mathrm{~Hz}$ ("low condition;" components resolved by auditory system) or $3900-5400 \mathrm{~Hz}$ ("high condition;" components unresolved). One complex (the "masker") had an $F 0$ of 210 $\mathrm{Hz}$ in both intervals of each 2IFC trial. Sensitivity $\left(d^{\prime}\right)$ was measured to $F 0$ differences (" $\triangle F 0$ 's") in the other ("target") complex between the two intervals of each trial. In the low condition, listeners could hear two clear pitches in each interval, and performance on the discrimination task was reasonably good ( $d^{\prime}$ approximately 0.75 for $\Delta F 0=4 \%$ ) regardless of whether the masker was gated synchronously with the target or started 150 ms before and ended $150 \mathrm{~ms}$ after it. In contrast, the mixture in the high condition was perceived as a single, aperiodic sound, similar to a crackle, and discrimination in the presence of an asynchronous masker was close to chance, even for $\Delta F 0=16 \%$. This, together with other findings, suggests that listeners are poor at extracting the $F 0$ 's of two groups of overlapping unresolved harmonics. Performance in the high condition was better with a synchronous than with an asynchronous masker. and experiments will be presented which test alternative explanations for this difference.

\section{8:15}

3aPP3. Trade-off between depth and phase of modulation in the perception of pitch of complex amplitude-modulated tones. Pierre L. Divenyi (Speech and Hear. Res., V.A. Medical Center, Martinez, CA 94553), Steven Greenberg (Univ. of California, Berkeley, CA 94720), and Alain P. Algazi (V.A. Medical Ctr., Martinez, CA 94553)

Complex amplitude-modulated (CAM) tones were generated through amplitude modulating a high-frequency carrier $f_{\text {carH }}(3-10 \mathrm{kHz})$ by an $\mathrm{AM}$ tone having a modulating frequency and a carricr $f_{\mathrm{carl}}$ about $10 \%$ of $f_{\mathrm{carH}}$ modulated at a rate $f_{0}$ in the $100-\mathrm{Hz}$ range. CAM sounds have been shown to have a low pitch corresponding to $f_{0}$, whose discriminability decreases as the modulation of $f_{\text {car }}$ is shifted from AM to QFM phase [S. Greenberg and P. L. Divenyi, Proc. 18th Midwinter Mtg. ARO, p. 55 (1995)]. In the present experiments, pitch discrimination was measured for pairs of CAM tones having fixed $f_{0}$ differences but either variable depth (1\%-200\%) or variable phase $(1-1.5 \pi)$ of modulation in the $f_{\text {carl }}$ complex. Results of the experiments show a pattem of modulation depth-phase trade-off over a range is constant above, and much reduced below $f_{\text {carH }}=4 \mathrm{kHz}$. These findings portray time-based pitch extraction as being a process in which phase spectrum plays a role consistent with the one proposed by W. $H$. Huggins [J. Acoust. Soc. Am. 24, 582-589 (1952)] in his theory. [Work supported by NIH and the VA Medical Research.]

8:30

3aPP4. A bridge between place and time. I. The peripheral/central origins of frequency analysis. Steven Greenberg (Int. Comput. Sci. Inst., 1947 Center St., Berkeley, CA 94704)

Auditory theory has traditionally pitted "place" (the tonotopically organized spatial pattern of excitation) versus "time" (the temporal pattern of discharge) with respect to the neural representation underlying specific attributes of acoustic sensation. This long-standing controversy has been of particular significance for models of pitch and frequency analysis, but casts its theoretical shadow over the discipline as a whole. A potential resolution of this historical opposition is proposed, in which place and time are viewed as flip sides of a complex representational matrix of neural activity, bound together through the mechanics of the cochlear traveling wave and its interaction with central loci of coincidence detection and inhibition. Frequency analysis is viewed as possessing two components. One is excitatory, based on spatially circumscribed patterns of temporally coherent peripheral activity and processed by central coincidence-sensitive neural elements. The other involves central inhibitory elements driven by nonsynchronous activity distributed over a broad tonotopic domain. Together, these two components can account for the preservation of frequency selectivity across a wide range of frequencies and sound-pressure levels, despite dramatic changes in the average-rate-based profile of neural activity.

\section{8:45}

3aPP5. A bridge between place and time. II. Its significance for theories of pitch. Steven Greenberg (Int. Comput. Sci. Inst., 1947 Center St., Berkeley, CA 94704)

The opposition between "place" and "time" perspectives in auditory function finds its fullest theoretical expression in the long-standing controversy over the neural basis of pitch. Traditionally, the two perspectives have been viewed as mutually exclusive, with pitch derived from either place or temporal cues, but not both. The controversy persists, in large part, because neither perspective, by itself, can fully account for the full range of perceptual experience associated with the pitch of complex signals [E. F. Evans, Audiology 17, 369-420 (1978)]. It is proposed that pitch stems from the interplay of place and time information, bound together into a coherent representation through the operation of central coincidencesensitive neural populations. This framework provides a principled basis 
for understanding the neural mechanisms underlying the major properties of pitch (such as the "dominance" and "existence" regions, the first and second effects of the pitch shift, phase-insensitivity and dichotically mediated pitch) and illustrates the manner in which both frequency selectivity and synchrony are required for computing a signal's periodicity with precision and reliability.

9:00

3aPP6. The effects on comodulation masking release (CMR) of systematic variations in on- and off-frequency masker modulation patterns. Emily Buss and Virginia M. Richards (Dept. of Psych., Univ. of Pennsylvania, Philadelphia, PA 19104)

Detection thresholds were obtained for a $500-\mathrm{Hz}$ tone signal added to a masker comprised of an amplitude modulated tone centered at the signal frequency (on-frequency masker) and an array of amplitude modulated tones centered at $300,700,800,900,1000$, and $1100 \mathrm{~Hz}$ (off-frequency maskers). The on-frequency masker was a sinusoidally amplitude modulated tone, and the off-frequency maskers were square-wave modulated tones. On- and off-frequency modulators were either in-phase or of random phase relative to one another, and different conditions used square wave modulators with different duty cycles $(0 \%-100 \%)$. The difference between thresholds obtained in the in-phase and random phase conditions (CMRs) were as large as $12 \mathrm{~dB}$, comparable to the CMRs found with perfectly matched modulators. Thresholds in the random phase condition did not depend on duty cycle, but for the in-phase condition the plot of thresholds as a function of duty cycle is " $U$ " shaped. The data are in rough agreement with "cued-listening" models of CMR in which the detection of the added tone is enhanced by the cue provided by the off-frequency masker minima. [Work supported by NIH.]

\section{9:15}

3aPP7. Mixed modulation masking: AM masking FM and FM masking AM. Brent W. Edwards and Neal F. Viemeister (Dept. of Psych., Univ. of Minnesota, Minneapolis, MN 55455)

In a series of experiments, the masking of AM by FM and the masking of FM by AM was investigated. All modulation was sinusoidal, and the carrier was a $1 \mathrm{kHz}$ tone. The masking of $16-\mathrm{Hz} \mathrm{AM}$ by $\mathrm{FM}$ as a function of the modulation frequency of the FM showed the same broad tuning seen with AM modulation masking [Bacon and Grantham, J. Acoust. Soc. Am. 85. 2575-2580 (1989)]. This is consistent with FM being primarily encoded as AM for small modulation depths. Masking of 16- $\mathrm{Hz}$ FM by AM showed broader tuning; this can be attributed to the "second cue" for FM hypothesized by Edwards and Viemeister [J. Acoust. Soc. Am. 96, 733740 (1994)]. AM detection thresholds also were obtained as a function of the modulation index, $\beta$, of the FM masker. Thresholds approached $100 \%$ modulation depth for large $\beta$, indicating an inability to process energy fluctuations that occur in both time and frequency. Finally, thresholds were obtained as a function of the relative phase between the masker and signal modulations. These phase results are discussed with respect to current modulation encoding theories. [Work supported by DC00683.]

\section{9:30}

3aPP8. Detection of sinusoidal amplitude modulation having unexpected rates. Beverly A. Wright (Keck Ctr., Box 0732, Univ. of Calif., San Francisco, CA 94143-0732) and Huanping Dai (Univ. of Florida, Gainesville. FL 32611)

The detectability of sinusoidal amplitude modulation having unexpected rates was assessed using a probe-signal method. With this method, three listeners were led to expect a target modulation rate $(4,32$, or 256 $\mathrm{Hz}$ ) by presenting the signal most often at that rate, and sensitivity to modulation at six other unexpected rates between 4 and $256 \mathrm{~Hz}$ was measured via occasionally presented probe modulation rates. The modulation phase was random on each 2IFC trial and the overall level of the 500-ms wideband carrier was randomly varied between 55 and $75 \mathrm{~dB}$ SPL across intervals. The modulation depth at each rate was set so that the modulation was detected on about $90 \%$ of the trials when only that rate was presented.
Performance at the unexpected rates depended upon the target rate. For the 4-Hz target, modulation at all rates was detected on about $80 \%$ of the trials. For the 32- and 256- $\mathrm{Hz}$ targets, unexpected modulation rates of $16 \mathrm{~Hz}$ and above were detected on $80 \%-90 \%$ of the trials, but modulation rates below $16 \mathrm{~Hz}$ were detected nearly at chance. Thus, contrary to the predictions of several popular models, the ability to detect modulation was clearly influenced by expectation of the modulation rate. [Work supported by NIH.]

9:45-10:00 Break

\section{0:00}

3aPP9. Detection of decrements and increments in sinusoids at high overall levels. Brian C. J. Moore (Dept. of Exp. Psych., Univ. of Cambridge, Downing St.. Cambridge CB2 3EB, England), Robert W. Peters (Univ. of North Carolina, Chapel Hill, NC 27599-7190), and Brian R. Glasberg (Univ. of Cambridge, Cambridge, England)

Thresholds for the detection of decrements in level of sinusoidal signals were measured as a function of duration $(2,4,6,10$, and $14 \mathrm{~ms})$, level $(70,80$, and $90 \mathrm{~dB}$ SPL) and frequency $(250,500,1000,2000$, and 4000 $\mathrm{Hz}$ ). Seven normally hearing listeners were tested at each frequency. Thresholds for detecting a 10-ms increment in level were also measured. The sinusoids were presented in a background noise intended to mask spectral splatter associated with the decrement or increment. Performance improved with increasing frequency for all decrement and increment durations. Performance also tended to improve with increasing level at $\mathbf{2 0 0 0}$ and $4000 \mathrm{~Hz}$. The results were analyzed using a four-stage model consisting of an auditory filter, a nonlinearity, a sliding temporal integrator, and a decision mechanism. The analysis indicated that the improved performance with increasing frequency and increasing level can be attributed mainly to changes in the detection process following the temporal integrator rather than changes in the equivalent rectangular duration (ERD) of the temporal integrator. The ERD varied little with changes in frequency and level. At higher frequencies and levels a smaller change is required at the output of the integrator for threshold to be reached.

\section{0:15}

3aPP10. Gap defection thresholds measured for symmetric and asymmetric marker frequency combinations surrounding a silent temporal gap. Craig Formby (Dept. of Surgery, Univ. of Maryland School of Medicine, 419 W. Redwood St., Baltimore, MD), Sheldon $\mathrm{Li}^{\mathrm{a})}$ (Johns Hopkins Univ., Baltimore, MD 21218), and LaGuinn Sherlock (Univ. of Maryland School of Medicine, Baltimore, MD)

Temporal gap detection (TGD) thresholds were measured between sinusoids that varied in frequency from 2000 to $3100 \mathrm{~Hz}$ for combinations of 2,3 , or 4 markers (e.g., combinations of one or two pre-gap markers with one or two post-gap markers). Sinusoidal frequencies $F_{1}$ and $F_{4}$ were used as pre-gap markers, while $F_{2}$ and $F_{3}$ served as post-gap markers. TGD thresholds were measured from three normal-hearing adults who tracked $70.7 \%$ correct detection thresholds adaptively across blocks of 50 2AFC trials. For symmetric marker conditions, where a pair or pairs of pre- and post-gap markers are equivalent in frequency on both sides of the silent gap (e.g., $F_{1}=F_{2}$ or $F_{1}=F_{2}$ and $F_{3}=F_{4}$ ), TGD thresholds are small (i.e., $<10 \mathrm{~ms})$. However, for asymmetric marker conditions, where only three markers are presented on a trial (e.g., $F_{1}=F_{2}, F_{2} \neq F_{3}$, no $F_{4}$ ), performance is highly variable. TGD thresholds for asymmetric marker conditions may be very large (e.g., $20-80 \mathrm{~ms}$ ), and tend to increase as the 1requency separation is increased belween markers $F_{3}$ or $F_{4}$ and markers $F_{1}=F_{2}$. These patterns of TGD results are inconsistent with a masking process and probably reflect mechanisms that are important in grouping of auditory images. [Research supported by NIH and The Center for Lan-

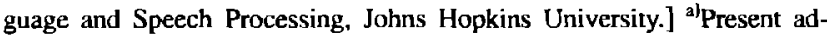
dress: AT\&T Bell Laboratories, 200 Laurel Ave., Middletown. NJ 07748. 
3aPP11. The relationship between auditory filter bandwidth and temporal resolution. Elizabeth A. Strickland (Dept. of Psych., Univ. of Florida, P.O. Box 112250, Gainesville, FL 32611-2250)

Temporal resolution was examined in the context of an envelope detector model. This model incorporates two limits to temporal resolution: filtering by the peripheral auditory system, and a central temporal window. It predicts that temporal resolution should increase with the bandwidth of the peripheral filter up to a certain filter width, beyond which it should be determined by the central limitations. To determine whether the predicted relationship between peripheral filtering and temporal resolution could be observed, temporal resolution and auditory filter widths were measured as a function of frequency region and level. Because auditory filters widen with level and with center frequency, the relationship between temporal resolution and filter width could thus be tested both within and across frequency regions. Temporal modulation tranifer functions (TMTFs) were measured for frequency regions from 500 to $4000 \mathrm{~Hz}$ using narrow-band noise. Auditory filter functions were measured in the same frequency regions, using the notched-noise method with probe level fixed. Both the TMTFs and filter functions were measured at two levels in each frequency region. As predicted, time constants were correlated with filter width for narrow filters, and were constant for wider filters. [Work partially supported by a post-doctoral fellowship from NIH.]

\section{0:45}

3aPP12. The effect of temporal placement on gap detectability. Karen B. Snell (Dept. of Audiol., Rochester Inst. of Technol., 52 Lomb Memorial Dr., Rochester, NY 14623-5604) and Hue-Lu Hu (Smith College, Northampton, MA 01060)

The detectability of a masked sinusoid increases as its onset approaches the temporal center of a masker. This study was designed to deternine whether a similar change in detectability would occur for a silent gap as its onset was parametrically displaced from the onset of a noise burst. Gap thresholds were obtained for four subjects. Signals were 150-ms, 6-kHz low-passed noise bursts presented at an overall level of 75 $\mathrm{dB}$ SPL. A 10-ms cosine-squared envelope shaped the onset and offset of the noise burst. Gaps were shaped by a 1-ms cosine-squared envelope. Gaps onsets were displaced by $0,1,3,5,10,30,50,100,110$, and $120 \mathrm{~ms}$. The displacement was measured from the first point of full amplitude following the 10-ms shaped onset. Gap thresholds were determined in a 3IFC procedure using 75-trial runs. Subjects completed five replications without prior practice. The mean gap threshold was largest for the noise burst closest to signal onset $(5.67 \mathrm{~ms})$ and smallest for the gap offset by 60 $\mathrm{ms}$ from the signal onset $(2.01 \mathrm{~ms})$. For all subjects, gap thresholds decreased as the gap approached the noise burst center.

\section{1:00}

3aPP13. Shared attention in detection and identification. Ervin $R$. Hafter and Anne-Marie Bonnel (Dept. of Psych., Univ. of California, Berkeley, CA 94720)

Brief, incremental $[S(+)]$ or decremental $[S(-)]$ signals were added to an ongoing pedestal. For detection, correct responses were either "yes" to both $S(+)$ and $S(-)$ or "no" to nonsignal trials. For identification, a signal was presented on every trial and responses were eirher " $S(+)$ " or " $S(-)$." Subjects monitored either a single channel or, in the "dual task," two simultaneous channels with independent stimuli. Expanding on previous work with two visual stimuli, here there were one visual stimulus (on a CRT) and one auditory (pure tone). As before, dual-task performance in detection was better than in identification. In addition, detection was as good on each of two channels as on one alone, indicating parallel processing, but identification declined, suggesting shared attention. Testing the idea that pedestal-to-signal transients account for these differences, pedestals were turned off before and after the signal intervals, removing transients as sources of information. This did not affect identification, but detection was now worse than identification and declined in the dual task, suggestive of shared attention.
3aPP14. Peak detection for auditory sound discrimination. Julius L. Goldstein (Central Inst. for the Deaf, $818 \mathrm{~S}$. Euclid, St. Louis, MO 63110-1594) and Joseph L. Hall (AT\&T Bell Labs.; Murray Hill, NJ 07974-0636)

Auditory detection of envelope maxima in temporal responses of cochlear frequency-analyzing filters has been hypothesized to account for phase effects in psychophysical discrimination [J. L. Goldstein, J. Acoust. Soc. Am. 41, 458-479 (1967)]. Re-examination of this hypothesis in the context of asymmetry of masking [R. Hellman, Percept. Psychophys. 11. 241-246 (1972)] reveals that it also provides an adequate explanation for this phenomenon. Peak discrimination between a tone and tone masker plus narrow-band-noise probe is more sensitive to probe energy than is the inverse discrimination between noise and noise masker plus tone probe, in agreement with psychophysics. Simulations of this model indicate that asymmetry of masking is a function of the product of noise bandwidth and temporal duration. Psychophysical experiments on masking asymmetry were performed with both masker and probe bandwidth ranging from pure tone to supracritical band. The experimental design included both fixed and roving levels, with random phases fixed throughout each experiment. A standard detection-theory model was fit to the data, assuming that ratios of peaks or of normalized peaks are measured by an optimal noisy discriminator. Predictions based upon stimulus envelopes are in good agreement with the data.

\section{1:30}

3aPP15. Auditory detection of changes in mass density and elasticity of a tuning fork. Robert A. Lutfi and Eunmi Oh (Dept. of Commun. Disord. and Psych., Univ. of Wisconsin, Madison. WI 53706)

The principles of theoretical acoustics were applied to reconstruct the sound-pressure waveform at the ear as would be generated by an idealized tuning fork (struck, clamped bar). The result is an inharmonic sum of damped sinusoids whose individual acoustic parameters (frequency, intensity, and decay modulus) are determined by the mass density and elasticity of the material comprising the bar. In the standard 2IFC procedure, listeners were asked, in effect, to detect changes in the material composition of the bar based on their perception of the acoustic waveform. Listener strategies for detecting such changes were estimated by perturbing slightly the individual acoustic parameters from trial to trial and computing correlations with the listener's response [cf. R. A. Lutfi and E. Oh, J. Acoust. Soc. Am. 95, 2963(A) (1994)]. In general, the correlations revealed that listeners fail to make optimal use of the information in the acoustic waveform by tending to give undue weight, for a given material change, on one or more parameters of the acoustic waveform. The results are discussed in terms of their implications for ecological notions regarding the perception of "higher-order" variables in the determination of natural resonant sources. [Research supported by NIDCD.]

\section{1:45}

3aPP16. Estimating perceptual distributions for endpoint stimuli M. E. H. Schouten and A. J. van Hessen (Res. Inst. for Lang. and Speech, Utrecht Univ., Trans 10, 3512 JK Utrecht, The Netherlands)

Rather than to assume that all stimuli on a continuum give rise to perceptual distributions that are Gaussian and equal, it is sometimes desirable to determine these distributions more directly, for example when the stimuli are speech sounds.' This can be done by means of (absolute) identification or (non-numerical) magnitude estimation. A problem with such methods is that the apparent distributions obtained with them become skewed as the stimuli approach either end of the continuum. An attempt was made to-recover the underlying distributions by extending the response range by three stimulus steps beyond the continuum endpoints, both for intensity and for stop consonants. The resulting distributions were fitted by means of Gaussian functions, so that a direct measure of $d^{\prime}$ in these tasks was obtained. It is assumed that in tasks "other than identification or magnitude estimation, the widths of the distributions change, but that their means are unaffected. 


\title{
Session 3aSA
}

\section{Structural Acoustics and Vibration: Structural Intensity I}

\author{
Sabih I. Hayek, Cochair \\ Department of Engineering Science and Mechanics, Pennsylvania State University, University Park, Pennsylvania 16802 \\ Scott D. Sommerfeldt, Cochair \\ Graduate Program in Acoustics, Pennsylvania State University, University Park, Pennsylvania 16802
}

Chair's Introduction-8:30

Invited Papers

8:35

3aSA1. The equation of diffusion applied to energy density of vibrating beams. Myriam Djimadoum (L.V.A., I.N.S.A. Bât 303, Villeurbanne, France) and Jean Louis Guyader (Institut National des Sciences Appliquées, 69621 Villeurbanne Cedex, France)

Inspired by precedent studies on the possibility of writing simple differential equations governing the evolution of vibratory energy density [D. J. Nefske and S. H. Sung, Trans. ASME 111, 94-100 (1989)], and equation of diffusion and its related conditions at discontinuities have been developed for an energetic quantity $\Omega$ in the case of flexural waves propagating in beams, where $\Omega$ represents the space-averaged far-field part of the displacement autospectrum, is proportional to energy density, but does not need extra time averaging. Several assumptions based on frequency and space averaging allow writing energetic conditions at discontinuities and defining a complete formalism for monodimensional problems. The procedure is applied to two coupled Euler Bemoulli beams: $\Omega$ is numerically in very good agreement with "exact" space and frequency-averaged far-field results and so the conditions at discontinuities are validated. Knowing that $\Omega$ allows for the ability to obtain an approximation of the energy flow in the beams and so gives an idea of how the energy propagates in the structure. These good results are encouraging, but the generalization of the procedure to plates is not trivial and requires further assumptions.

\section{9:00}

3aSA2. Measurement of the flexural wave power transmission and reflection coefficients of joints using wave-number domain methods. Robert J. Unglenieks and Robert J. Bernhard (School of Mech. Eng., Purdue Univ., 1077 Ray W. Herrick Lab., West Lafayette, IN 47907-1077)

Recently, a number of investigators have proposed so-called energy flow analysis techniques to allow engineers to perform high-frequency structural vibration studies of coupled structural elements. One key parameter required for this analysis is the power transmission and reflection coefficients of the joints that couple structural elements. A wave-number domain technique has been developed which uses a spatial fast Fourier transform to transform velocity data obtained in the spatial/frequency domain into the wave-number/frequency domain. The resulting wave-number domain spectrum allows estimation of energy flow segregated into right and left traveling components. Using the segregated energy flows for all of the structural elements coupled at a joint, it is possible to estimate the power transmission and reflection coefficients of the joint. A ladder frame structural system is analyzed to determine the flexural wave power transmission and reflection coefficients of ell- and tee-joints that comprise the structure. The structure is forced so that only flexural and longitudinal waves are excited. A scanning laser Doppler vibrometer is used to collect the flexural wave velocity data. The estimates of the joint coefficients are presented and compared to the analytical values for rigid joints.

3aSA3. Active control of structural intensity in an elastic plate. S. I. Hayek, M.-Y. Nam (Dept. of Eng. Sci. and Mech., Penn State Univ., University Park, PA 16801), and S. Sommerfeldt (Penn State Univ, University Park, PA 16801)

Active control of structural intensity (SI) in a finite elastic plate coupled to a point damper is achieved through the use of a judiciously located actuator. An algorithm was developed using the magnitude of the structural intensity vector to achieve local or global reduction of SI. The control algorithm showed that controlling each component of the intensity vector $\left(I_{x}\right.$ or $\left.I_{y}\right)$ generates a parabolic surface, which may or may not intersect the null plane. Under certain conditions based on the locations of the primary mechanical source and the error sensors, as well as the dynamic parameters of the plate, the surface intersects the null plane. If the surface intersects with the null plane, then the intersection generates a circle of possible actuator magnitudes and phases that will make either $I_{x}=0$ or $I_{y}=0$. If the circles exist for both $I_{x}=0$ and $I_{y}=0$, then inequalities were derived that will indicate when and if these circles will intersect. The intersection of these circles allows only two possible control solutions that will make the magnitude of the vector SI at the error sensor vanish. When the intersections do not occur, then minimization rather than extinction is achieved. These control strategies are explored and applied to an elastic simply supported plate coupled to a point damper and excited to vibration by a point force. The role of structural damping on control strategies is fully explored. 
3aSA4. Structural and acoustic intensity analysis for a fluid-loaded elastic plate with a distributed inhomogeneity. J. M. Cuschieri (Ctr. for Acoust. and Vib., Dept. of Ocean Eng., Florida Atlantic Univ., Boca Raton, FL 33431) and D. Feit (David Taylor Res. Ctr., Bethesda, MD 20084)

The solutions developed for the response and scattered pressure from a fluid-loaded plate with a distributed mass inhomogeneity, which were presented at the ASA 128th meeting in Austin, Texas, are extended to included distributed stiffness inhomogeneities. The same four types of distributions, with varying degrees of "smoothness" are considered for the stiffness distribution. The scattering characteristics between the mass and stiffness inhomogeneities are different, and while the scattering is dipolelike for the mass inhomogeneity, it is quadrupolelike for the stiffness inhomogeneity. The results for this presentation are presented in the form of structural and acoustic intensity maps, both as vector plots and streamline plots. For frequencies lower than the critical frequency of the fluid-loaded plate, the results show the constant exchange of power between the plate and the fluid. The streamline plots for the reactive intensity show a band very close to the plate surface. For higher frequencies, the characteristics of the intensity maps become dependent on the relative length and "smoothness" of the distributed inhomogeneity.

\section{0:15}

3aSA5. Vorticity characteristies of the vibration intensity field and the structure-borne sound. Nobuo Tanaka (Vib. and Sound Div., Mech. Eng. Lab., 1-2 Namiki, Tsukuba Science City, Ibaraka 305, Japan)

This paper deals with the characteristics of real vibrational power flow in a simply supported rectangular panel under the action of feedforward vibration control. It is found that the path of the power flow is a combination of translations and rotations, the rotations induced by the interference of two modes which products a "vortex generating block." Particular emphasis is placed on the vortex power flow in the vibration intensity field. A qualitative formula for predicting the number of power flow vortices, as well as the discussion of the vortex period, is put forward. Then, by introducing an energy stream function, the generation mechanism of the vortex power flow is quantitatively discussed. The fundamental characteristics of the vortex power flow are further clarified by vortex function derived from the intensity field. A novel method to induce a vortex at an arbitrary location of the plate is also shown, which may have practical applications in controlling the path of vibrational power flow in systems of large extent. Moreover, the influence of the induced vortex power flow on the plate onto the acoustic intensity distribution is investigated, showing that the rotational direction of the vortex on the plate is not always the same with that of the acoustic intensity in the nearfield.

\section{0:40-10:50 Break}

\section{Contributed Papers}

\section{0:50}

3aSA6. Vibration studies of joints in structures. S. K. Kim and M. G. Prasad (Noise and Vib. Control Lab., Dept. of Mech. Eng., Stevens Inst. of Technol., Castle Point on the Hudson, Hoboken, NJ 07030)

Joints are used extensively in connected structures which are part of machine systems. Joints introduce discontinuity in structures which not only disturb the energy flow but are also possible locations of failure. Thus it is necessary to characterize the joints to determine their rigidity. A joint can be characterized in terms of the energy reflection coefficient and impedance. This study presents both analytical and experimental work on vibration characterization of joints. The analytical work is based on both classical and finite-element methods. The experimental work is based on the two sensor method for impedance measurements. The vibration behavior of a joint is studied in terms of reflection coefficient and impedance. The joints considered are bolted joints in specimen structures such as cantilever beams. Good results are obtained which supports the application of such studies in design and diagnostics of structural joints. [Work supported by Daewoo Motor Company, Seoul, Korea.]

\section{1:05}

3aSA7. Vibrational power flow through adhesively bonded beams. Qunli Wu, Yiren Hong, and M. K. Lim (School of Mech. and Prod. Eng., Nanyang Technol. Univ., Singapore 2263, Republic of Singapore)

A theoretical and experimental study of power flow through adhesive lap-joint beams was carried out in this paper. The mathematical model of power flow in the beams was developed by treating the joint as a sandwich structure. The power flow analysis for different joint parameters was performed. The relationship between the joint parameters and the power flow was investigated. The power flow measurement system was calibrated in a stepped beam with known reflection coefficient of the step, and then the measurements were carried out on aluminum beams with epoxy bonded joints. The measured results agreed well with the theoretical prediction. The termination effects on the power flow measurement are discussed.

\section{1:20}

3aSA8. Structural intensity of acoustically excited waves in a flud-loaded elastic plate. Steven L. Means and Ralph R. Goodman (Appl. Res. Lab., P.O. Box 30, Penn State Univ., State College, PA 16804)

When an acoustic plane wave is incident on a elastic plate, various wave types are excited in the plate for different angles of incidence. The present study determines the structural intensity as a function of incident angle at different depths along the cross section. It is found that for near normal incidence the direction of the intensity parallel to the plane of the plate is opposite the intensity in the fluid and for other angles of incidence. Previously, a similar analysis for a semi-infinite medium by Bunney and Goodman [J. Acoust. Soc. Am. 53, 1658-1662 (1973)] did not yield such a result. To gain physical insight into this phenomenon, a study was performed to determine which wave modes are excited at these angles of incidence and to calculate their group velocities. 


\title{
Session 3aSC
}

\section{Speech Communication: Contacting Linguistic Structure from Perception}

\author{
John C. Kingston, Chair \\ University of Massachusetts, Box 37130, Amherst, Massachusetts 01003-7130
}

Chair's Introduction-8:20

Invited Papers

8:25

3aSCl. Access to phonological structure in listening to speech. Carol A. Fowler (Haskins Labs., 270 Crown St., New Haven, CT 06511)

In a direct-realist theory of speech perception, listeners are in immediate (in the sense of unmediated) contact with the phonological units of their language when they use structure in acoustic speech signals as information for its causal source-phonological gestures of the vocal tract. In the theory, phonological categories include, minimally, the sets of motor-equivalent articulatory movements producible by a synergy of the vocal-tract, each set, thereby, counting as a token of the same phonological gesture for producer/ perceivers of speech. Maximally, categories include a set of similar gestures that members of a language community do not distinguish. Categories, thus, are defined gesturally, not acoustically, as for example, research on prototypes in speech have been interpreted as suggesting. Striking behaviors of listeners that index their extraction of information about phonetic gestures from the acoustic speech signal is their parsing of acoustic signals. A literature review suggests that listeners do not hear such unitary acoustic dimensions as fundamental frequency or duration as unitary. Rather, they parse each dimension into its distinct, converging gestural cause. Complementarily, listeners use as information for a phonological unit the constellation of diverse acoustic consequences of the units gestural realization. [Work supported by NICHD.]

8:50-9:05 Commentary by Patricia K. Kuhl

Department of Speech and Hearing Science, University of Washington, WJ-10, Seattle, Washington 98195

9:05 9:15 Discussion

9:15

3aSC2. Undoing place assimilation. Aditi Lahiri (Fachgruppe Sprachwissenschaft, Post Fach 5560, Univ. Konstanz, D-7750 Konstanz, Germany)

Phonological variation in the form of different phonetic shapes of words pose a challenge for theories of speech perception and language comprehension. Postlexical phonological processes like assimilation and deletion frequently cause the phonetic shape of a word to change in a given phonological context. Such processes can easily lead to the creation of nonwords. In a sentence like "I detest green bananas," the word green may well be pronounced as [grim] in the context of a following [b]. Listeners, however, apparently have no problems in parsing and identifying the word correctly in ongoing speech. How is the linguistic system organized such that it can accept the sequence [grim] as the word green? In this talk, competing theoretical accounts dealing with such variation will be presented. An account which assumes a highly abstract lexical representation will be presented. This account assumes that the central component of the linguistic system is the lexicon which contains, among other things, a diclionary of uninflected words with their phonological forms, semantic properties, and syntactic properties of various sorts. The phonological form is assumed to have a highly abstract representation on the basis of which the listener accepts or rejects phonetic variance. In addition, a recent hypothesis will be argued against that assumes an on-line process of phonological inference and an abstract representation will be presented to deal with phonetic variation. The data to support the hypothesis comes from German assimilation processes.

9:40-9:55 Commentary by Paul A. Luce

Psychology Department, State University of New York, Buffalo, New York 14260

9:55-10:05 Discussion

10:05-10:20 Break

10:20

3aSC3. On the internal perceptual structure of phonological features: The [voice] distinction. Randy L. Diehl, Wendy A. Castleman (Dept. of Psych., Univ. of Texas, Austin, TX 78712), and John Kingston (Univ. of Massachusetts, Amherst, MA 01003)

Apart from phonological features and their individual phonetic correlates, an intermediate level of structure apparently exists in which subsets of phonetic properties form perceptually coherent units, referred to here as "integrated perceptual properties." The mapping between each successive level of structure is arguably many-to-one, elevating both redundancy and distinctiveness at the level of phonological features. For the distinctive feature [voice], a main integrated perceptual property conresponding to the [+voice] value 
is the presence of low-frequency energy during or near the consonant, which may be further analyzed into at least three phonetically distinct subproperties: voicing during the consonant constriction, a low $F 1$ near the constriction, and a low $F 0$ in the same region. Two predictions follow if these three subproperties contribute to a single integrated perceptual property. One is that the effects on [voice] judgments of varying either a low $F 1$ or $F 0$ should pattern in similar ways for a given utterance position and stress pattern; this prediction was confirmed. The second prediction is that two stimuli in which separate subproperties of the low-frequency property are positively correlated (i.e., they are either both present or both absent) will be more distinguishable than two stimuli in which the subproperties are negatively correlated; though not yet confirmed for the pairing of $F \mathbf{l}$ with $F 0$, this prediction was confirmed for pairings of $F 1$ or $F 0$ with constriction voicing.

10:45-11:00 Commentary by Burt Schouten

Research Institute of Language and Speech Transmission, University of Utrecht, $103512 \mathrm{JK}$, Utrecht, The Netherlands

\section{1:00-11:10 Discussion}

11:10

3aSC4. Speech perception as a pattern recognition. Terrance M. Nearey (Dept. of Linguist., Univ. of Alberta, Edmonton, AB T6G 2E7, Canada)

Phonetics involves three domains of events: (1) acoustic/auditory, (2) articulatory/gestural, and (3) symbolic/phonological. Theories of perception hold different views about the relationships among these domains. Auditorists (e.g., Kingston and Diehl) emphasize a strong (i.e., simple, direct) relation between (1) and (3). Gesturalists (e.g., Liberman and Mattingly) propose a strong relation between (2) and (3). Advocates of double-strong theory (e.g., Blumstein and Stevens) propose strong relations of both (1) and (2) to (3). Difficulties with all these theories will be discussed. An alternative, "double-weak" theory will be described and evidence supporting it will be presented. This approach views speech production and perception as distinct but cooperative systems. This view accepts the gesturalist contention that the acoustic mapping if phonological units is modified by context in ways that are important to perception. However, it also contends that the accommodation of contextual effects in perception is limited and highly stylized. Assuming a concomitant stylization of gestural patterns in production, relatively simple pattern-recognition strategies in perception may suffice for successful communication.

11:35-11:50 Commentary by Keith R. Kluender

Psychology Department, University of Wisconsin, Madison, Wisconsin 53706

11:50-12:00 Discussion

THURSDAY MORNING, 1 JUNE 1995

GRAND BALLROOM SOUTH, 7:45 A.M. TO 12:00 NOON

Session 3aUW

Underwater Acoustics and Acoustical Oceanography: Seismoacoustic Determination of Sea Ice Processes

Peter J. Stein, Chair

Scientific Solutions, Inc., 18 Clinton Drive, Hollis, New Hampshire 03049-6576

Chair's Introduction-7:45

Invited Papers

7:50

3aUW1. Determination of sea ice processes using geophone arrays. Peter J. Stein and Steven E. Euerle (Scientific Solutions, Inc., 18 Clinton Dr., Hollis, NH 03049-6576)

Similar to land-based geophysics, seismoacoustic techniques are necessary to study sea ice mechanics. Most ice fracturing can only be detected via the elastic/acoustic waves which radiate. One of the best means of determining the spatially averaged mechanical properties is through probing the ice with elastic waves. Here the results are described from two separate experiments in which large (order 1-km aperture) arrays of triaxial geophones were deployed to study the sea ice mechanical properties via the elastic waves which propagate in the ice. The first test was conducted on "clean" first-year shore-fast ice off the coast of Resolute Bay Canada in Spring 1992. The second was a winter-over system deployed on multiyear ice during the Fall 1993 SIMI field operation in the Beaufort Sea 
(subsequently recovered in March 1994). Additionally, during SIMI an automated "hammer blow" source was deployed which impacted the ice every $7 \mathrm{~h}$. Various signal processing and data analysis techniques for studying sea ice processes such as ice growth and thermal fracturing will be described. Results will be presented along with comparisons between first-year and multiyear ice. [Work supported by ONR.]

\section{8:10}

3aUW2. Seismoacoustic observations as related to thermally induced stresses and fracturing in pack ice. James $\mathrm{K}$. Lewis (Ocean Phys. Res. \& Dev., 207 S. Seashore Ave., Long Beach, MS 39560)

On-ice geophone data and under-ice noise data contain a significant amount of information about the stress state of pack ice and how ice fractures in response to the stress. This information can be used to study the rheology of sea ice and the related mechanics of fracturing. A review is presented of the current understanding of ice mechanics related to thermal fracturing based on seismoacoustic observations. A rheology is presented and discussed based on a thermodynamic model and an associated stress/strain relationship. The rheology has an enhancement which prescribes how the extent of existing cracks in the ice affect (1) the stress state of the ice, (2) the number of fractures when the ice exceeds its yield strength, and (3) the stress relief after fracturing occurs. This allows us to simulate first-year and multiyear ice using the same model by differentiating the two ice types based on the extent of existing cracks. Examples are shown of how under-ice acoustic data can be used to estimate the vertical distribution of the fractional area of existing cracks in multiyear ice.

\section{8:30}

3aUW3. Seismo-acoustic remote sensing and inversion of sea ice fracture events. H. Schmidt, Y. Dudko (MIT, Cambridge, MA 02139), K. von der Heydt, and E. K. Scheer (Woods Hole Oceanogr. Inst., Woods Hole, MA 02543)

A new, layered seismo-acoustic remote sensing concept was developed and applied during the SIMI field experiments in the Beaufort Sea in the Fall of 1993 and the Spring of 1994. A large-aperture, 32-element, horizontal hydrophone array was used to record the acoustic emission from ice events. Using real-time array processing, maps of the seismic activity of the ice cover out to a range of 2 to $3 \mathrm{~km}$ were continuously generated. Once an active zone was detected, clusters of five 3-axis geophones and a single hydrophone were deployed in the active zone for near-field recording of the seismo-acoustic emission. The data were transmitted back to the main camp via a wireless local area network, and recorded on tape. During the Spring experiment data were recorded continuously on the hydrophone array for 4 weeks, and several deployments of the geophone clusters were performed in the vicinity of active ice mechanical processes such as ridge building, finger rafting, and floe fracturing. In addition, the clusters were deployed on specimens used for artificial fracturing experiments. The layered remote sensing concept is described, and examples are given of the seismoacoustic emission produced by the different types of ice events. Finally, the matched-field fracture plane analysis of the seismic data is described.

\section{$8: 50$}

3aUW4. Acoustic and seismic measurement of ice processes. David M. Farmer and Yunbo Xie (Inst. of Ocean Sci., P.O. Box 6000 , Sidney, BC V8L 4B2, Canada)

As sea ice responds to environmental forcing, it deforms leading to the storage of strain energy. When mechanical failure occurs, most of this energy is dissipated through fracturing, but a small portion radiates as seismic and acoustic waves. These waves provide useful signals for sensing the failure process in the ice. In a recent ice mechanics experiment conducted north of Prudhoe Bay (SIMI '94), a large number of ice failure events were observed using geophone and hydrophone arrays. Preliminary results are presented, including a large-scale tensile fracture test and naturally occurring sounds near a closing lead. For the artificial fracture, the acoustic signals allow determination of cracking rate, fracture advance, and crack propagation velocities. The overall crack propagation speed is estimated to be of order $50 \mathrm{~m} \mathrm{~s}^{-1}$; maximum cracking activities occur prior to peak loading. In the second data set, the naturally occurring stick-slip process was observed as two ice sheets moved against'each other in a closing lead. A wide range of frequencies can occur simultaneously representing different components of the sliding and slipping mechanisms.

\section{9:10}

3aUW5. A study of the evolution of under-ice fresh water layer during summer melt phase in the Arctic. Subramaniam D. Rajan, Henry Laible (Woods Hole Oceanogr. Inst., Woods Hole, MA), and Walter B. Tucker, III (Cold Regions Res. and Eng. Lab., Hanover, NH)

In the Arctic, the entire snow cover and a significant part of the ice melt during the summer months. The melt water, which is at about $0{ }^{\circ} \mathrm{C}$, reaches the underside of the ice where it overlies much colder and denser saline water and forms under-ice melt ponds. It has been suggested that these under-ice melt ponds can play a significant role in heat flux through the ice. The changes in acoustic propagation in a region very close to the bottom of the ice using data collected during a multiseason cross-hole tomography experiment conducted in the Arctic are presented. Since acoustic propagation is dependent on the properties of the medium, the changes in acoustic propagation to the changes in the characteristics of the medium are related. Further an investigation is made as to whether these changes correspond to those observed by Martin and Kauffman [J. Fluid Mech. (1974)] in a laboratory experiment conducted to study the development of under-ice melt ponds. [Work supported by ONR.]

\section{9:30}

3aUW6. Seismoacoustic ultrasonic modeling characterization of sea ice processes. Jacques R. Chamuel (Sonoquest Adv. Ultrason. Res., P.O. Box 81153, Wellesley Hills, MA 02181-0001)

Sea ice processes affect the elastic properties, structure, and boundaries of the ice cover, and change the composition and acoustic properties of the top water layer underneath the ice. Upward refraction in the water creates substantial interaction between underwater acoustic waves and the ice cover influencing long-range low-frequency propagation. In order to develop a basic understanding of sea 
ice processes, it is essential to characterize seismoacoustic phenomena associated with sea ice growth, melting, refreezing, cracking, ridging, and rafting. Controlled laboratory experiments were conducted to study diverse transient liquid/solid seismoacoustic wave phenomena. Examples from scaled ultrasonic modeling results are presented characterizing near-grazing Scholte wave backscattering from trenches at liquid/solid interfaces, effective attenuation of flexural waves from horizontal refraction, seismic profiling from $45^{\circ}$ oblique boreholes, viscous waves in ice suspensions, variability of shear wave speed in sea ice cores, edge wave propagation along truncated and range-dependent apex angle wedges, focused multiple scattering, and finger-rafting. The findings provide physical insight into high-latitude seismoacoustic oceanography and contribute to the interpretation of Arctic field data. [Work supported by ONR.]

\section{9:50-10:00 Break}

\section{Contributed Papers}

\section{0:00}

3aUW7. Ice activities during the AREA 88 experiment. I. A pictorial and video description. T. C. Yang (Naval Res. Lab., Washington, DC 20375)

During the AREA 88 experiment (Spring of 1988), an ice camp was deployed on an ice floe in the general neighborhood of $78 \mathrm{~N}$ and $55 \mathrm{~W}$ in the central Arctic. The ice floe became active on and off for a period of days. The ice activities were clearly audible in the air (a kind of lowfrequency roar). One can unmistakably determine the direction of the noise by stepping outside the tent. Open and refrozen leads were found between the floes. One of them quickly closed up in a matter of minutes swallowing up the geophones just deployed on the refrozen lead. Immediately following this lead closing, the floe next to us started to move laterally at a speed approximately $3 \mathrm{ft} / \mathrm{min}$. During a peak ice activity period, the ice at the edge of our ice floe started to pile up into a form called ice ridges, apparently due to forces between the ice floes which broke the ice and pushed them into a pile of irregular shapes. This ice ridging was recorded on a video tape. Tens to hundreds of blocks of ice of about 5- to 10-ft thickness were also found neatly lying on top of adjacent ice floes, a scene which looked like an act of God.

\section{0:15}

3aUW8. Ice activities during the AREA 88 experiment. II. Noise events recorded on geophones. T. C. Yang (Naval Res. Lab., Washington, DC 20375)

During the AREA 88 experiment (Spring of 1988), an ice camp was deployed on an ice floe in the general neighborhood of $78 \mathrm{~N}$ and $55 \mathrm{~W}$ in the central Arctic. The ice floe became active on and off for a period of days. Many of the noise events were recorded on a planar array of geophones. The response of three-axis geophones were used to analyze the forcing mechanism of the noise events by comparing the geophone response to the ice activities with that generated by man, i.e., hammer blows. Specifically, frequency-wave-number analysis was used to sort out the longitudinal, shear, and flexural waves in the geophone data. While the examples shown here indicate that floe bumping/rubbing was the likely cause for the very-low-frequency noise signals analyzed, long-term accumulative geophone data indicate vertical forcing was the dominant source for ice generated noise.

\section{0:30}

3aUW9. Acoustic scattering in an elastic medium as it relates to sea ice and the determination of effective moduli. Henry $A$. Laible (Woods Hole Oceanogr. Inst. and MIT, Woods Hole. MA 02543) and Subramaniam D. Rajan (Woods Hole Oceanogr. Inst., Woods Hole, MA 02543)

The scattering of sound in ice is a complex problem which depends upon the material properties of the ice as well as the frequency of the sound source. Because of the complexity of the problem, a simplified approach to acoustic wave scattering is taken. The ice is modeled as a two-phase medium in which fluid-filled cylindrical cavities (brine channels) are embedded in an attenuating elastic matrix. An analytical solution is obtained for the acoustic scattering of longitudinal waves from an infi- nitely long cylinder. The solution is extended for low-ka wave propagation in order to determine effective moduli (Lamé parameters and density) and effective wave speeds. An experiment is conducted in the lab to determine the scattering cross section versus angle for a single cylindrical scatterer for frequencies of 160 and $200 \mathrm{kHz}$. In addition, effective moduli are determined as a function of cavity concentration. The experimental and theoretical results are presented. [Work supported by ONR.]

\section{0:45}

3aUW10. Temporal evolution of compressional wave speed in sea ice. Subramaniam D. Rajan (Woods Hole Oceanogr. Inst., Woods Hole, MุA 02543)

A year-long cross-hole tomography experiment was conducted in sea ice during 1992 to 1993 . The analysis of the data is presented and the variations in the compressional wave speed structure in sea ice with season are investigated and related to the changes that are predicted by thermodynamic model. [Work supported by ONR.]

\section{1:00}

3aUW11. A model of nonlinear behavior of ice cracks. Lev A. Ostrovsky (NOAA/ERL/ETL/CIRES, 325 Broadway, R/E/ET-1, Boulder, CO B0303), Alexander E. Ekimov, Andrey V. Lebedev, and Alexander M. Sutin (Inst. of Appl. Phys. of Russian Acad. Sci., Nizhni Novgorod 603600 , Russia)

A work presented at the previous ASA meeting has demonstrated that the cracks may provide anomalously strong vibroacoustic nonlinearity in ice. Experimental data were obtained from the field experiments on a fresh-water lake, where a strong subharmonic signal was, in particular, registered. Here, a theoretical model is suggested for the description of the effects observed and possible use of them for characterizing the crack parameters. The model is based on consideration of nonlinear flexural oscillations of the ice plate with a crack which may be "opened" from its upper or lower part due to flexural oscillations. Together with the added mass of the surrounding area of the plate, a nonlinear oscillator may be formed which admits both higher harmonic and subharmonic generation (a parametric effect). The oscillator has a strong nonlinearity and relatively low $Q$ factor which may provide a possibility of parametric excitation if there is a rather wide frequency range. The model considered seems to be applicable for real sea conditions.

\section{1:15}

3aUW12. Acoustic measurements of ice cover variations in the Arctic Ocean. Victor V. Artel'nyi and Mikhail A. Raevsky (Inst. of Appl. Phys., 46, Ulyanov St., 603600 Nizhny Novgorod, Russia)

Climatic monitoring of the Arctic Ocean implies long-duration observations of the ice cover and water temperature variations by measuring the characteristics of low-frequency acoustical signals traveled along stationary paths. The sensitivity of sounding signal parameters to the variations of the ice cover characteristics is one of the important problems in climatic monitoring. The time structure of the pulse signal in the Arctic waveguide is investigated. The relative influence of the ice cover thickness, density, 
shear, and compressional sound speeds on the variations of the phase and time delay of the normal modes is studied. It is found that, in the frequency range $f \leq 30 \mathrm{~Hz}$, the variations of the ice cover density and thickness are more efficient than sound-speed variations. Taking the desalination of the subsurface layer into account decreases ice thickness influence. These effects are compared with signal fluctuations in the Arctic Ocean. The fluctuations of the cw signal of the frequency $f=20 \mathrm{~Hz}$ in the Arctic waveguide are investigated. The internal waves, the submesoscale eddies, and the ice cover drift are considered as signal fluctuations sources. According to the data of numerical simulation, the influence of submesoscale eddies is primary.
$11: 30-12: 00$

PANEL DISCUSSION

THURSDAY MORNING, 1 JUNE 1995

MEETING ROOM 17, ROOM, 9:00 A.M.

\title{
Meeting of Accredited Standards Committee S2 on Mechanical Vibration and Shock
}

to be held jointly with the

\section{U.S. Technical Advisory Group (TAG) Meeting for ISO/TC 108 Mechanical Vibration and Shock}

\author{
D. J. Evans, Chair S2
}

National Institute of Standards and Technology (NIST), Building 233, Room A 147, Gaithersburg, Maryland 20899

D. F. Muster, Chair, U.S. Technical Advisory Group (TAG) for ISO/TC 108, Mechanical Vibration and Shock 4615 O'Meara Drive, Houston, Texas 77035

\begin{abstract}
Standards Committee S2 on Mechanical Vibration and Shock. Working group chairs will present reports of their recent progress on writing and processing various shock and vibration standards. There will be a report on the interface of S2 activities with those of ISO/TC 108 (the Technical Advisory Group for ISO/TC 108 consists of members of S2, S3, and other persons not necessarily members of those committees) including a report on the activities of ISO/TC 108, including plans for its September 1996 meeting in Sydney, Australia.
\end{abstract}

Scope of S2: Standards, specifications, methods of measurement and test, and terminology in the fields of mechanical vibration and shock, and condition monitoring and diagnostics of machines, but excluding those aspects which pertain to biological safety, tolerance, and comfort.

THURSDAY AFTERNOON, 1 JUNE 1995

MEETING ROOM 16, 12:45 TO 2:20 P.M.

\section{Session 3pAA}

\section{Architectural Acoustics: Room Acoustics-Measurements}

Angelo J. Campanella, Chair

Campanella Associates, 3201 Ridgewood Drive, Columbus, Ohio 43026

Chair's Introduction-12:45

\section{Contributed Papers}

$12: 50$

3pAA1. Preliminary values of clarity and intelligibility for small auditoria, meeting, and teleconference rooms. Angelo J. Campanella (Campanella Assoc. \& ACCULAB, 3201 Ridgewood Dr., Columbus, OH 43026)

Clarity (C80) and intelligibility (D50), normally measured for large auditoria, provide an alternative to reverberation time (RT60) to evaluate the acoustical performance of many critical smaller rooms. A Larson Davis
2900 real time analyzer and sound level meter was used to record a rapid sequence of octave band spectra from impulsive sound. A PC program was written to extract this data from the LD2900 and process it into C80, D50, and TCT values. Experimental values were obtained in ten different rooms including auditorium, church, theater, lab, chapel, gym, music, rehearsal, teleconference, and living rooms. Room qualities varied from good through those in need of correction for the intended room use. Measured quantities were compared with opinions on existing room performance to provide preliminary desirable ranges of C80, D50, and TCT according to room use. 
upon the amount of absorption, the center frequency wavelength and/or the dimensions of the absorber are used to determine the size of nonuniform

3pAA2. Spatial selectivity of differential microphone arrays in rectangular enclosures. Ea-Ee Jan and James L. Flanagan (CAIP Ctr., Rutgers Univ., Piscataway, NJ 08855-1390)

Omni-directional microphones have been employed in delay-and-sum beamformers to mitigate room reverberation and ambient noise. A onedimensional beamformer has spatial selectivity in only one dimension. Therefore, higher-dimensional arrays are used to improve spatial selectivity. In comparison, a single differential microphone provides fixed spatial selectivity. This report discusses the performance of arrays of differential microphones. To compute sound behavior in the enclosure, calculation of the incident angle for every arriving signal is required. An image model using the ray-tracing method has been developed to calculate the multipath in an enclosure up to a prescribed order of images. Nonuniform wall reflectivities can be included. For the special case of rectangular enclosures, the algorithm efficiently calculates the incident angle for each arriving signal and all of its associated reflection points. Additionally, it is noted that the number of images for a given order more nearly resembles an arithmetic series instead of a geometric series. [Research supported by NSF Grant No. MIP-9121541 and NSF Subcontract 397-26740.]

3pAA3. An infinite impulse response model for sound travel in a small conference room. Paul S. Kovitz (2877 S. Buchanan St., \#A2, Arlington, VA 22206)

A new method for predicting an infinite impulse response (IIR) from a source position to a receiver position in a small rectangular room is propased. The method predicts the IIR for all frequencies below an arbitrary cutoff. The IIR is stated in terms of a $z$-transform polynomial; the order of the $z$-transform polynomial follows directly from the room dimensions and the cutoff frequency. The effectiveness of this model is demonstrated from measurements.

\section{1:35}

3pAA4. Effects of absorption placement on interior noise levels-A theoretical study. Danielle S. Labrozzi and Linda P. Franzoni (North Carolina State Univ., Ctr. for Sound and Vib., Dept. of Mech. and Aerosp. Eng., P.O. Box 7910, Raleigh, NC 27695-7910)

The effect of the spatial distribution of absorbing surfaces on the sound field in enclosures is investigated. For a given overall level of absorption, the difference between distributing the absorption uniformly or localizing it in a single region is studied. Attention is focused on the high-frequency range having wavelengths small compared to the enclosure dimensions, but either large or small compared to the absorptive region. The distribution of mean-square pressure in a bandwidth is studied to ascertain the degree of uniformity throughout the enclosure, and particularly in the vicinity of the absorbing region. Of special interest is the effect of absorption on intensification zones, which are the regions near boundaries where there is a high degree of spatial correlation between modes. Depending regions in the enclosure. The study is conducted using classical modal analysis. The results provide physical insight into the behavior of acoustic fields in enclosures.

\section{1:50}

3pAA5. Visualization of acoustic scattering in a three-dimensional enclosure. Sylvia K. Isler, Vardhani Harpanahalli, and Charles Thompson (Ctr. for Advan. Comput., Univ. of Massachusetts, One University Ave., Lowell, MA 01854)

Frequently the results of time-varying computer simulations of acoustic behavior in built environments are difficult to analyze due the multidimensional nature of the numerical results. Graphical displays of acoustic data have been effective in remedying this problem. A technique for visualizing the time evolution of a sound pressure field within an enclosure due to a source localed in the enclosure is presented. The numerical technique for computing the pressure distribution within the enclosure employs the method of images. Padé approximants are used to take into consideration the effects of sound scattering under high-contrast conditions. The graphical representation of the enclosure is created as follows. The walls of the enclosure and objects contained therein are rendered with the standard radiosity method. The graphical display of the interaction of the pressure field with the enclosure and its contents was accomplished by rendering each volume element in the field according to the pressure magnitude. The presentation will include a sequence of images depicting the time evolution of the pressure field in the enclosure.

\section{2:05}

3pAA6. Design and construction of an academic acoustics laboratory at CCNY on a limited budget. Samuel Musora (Dept. of Mech. Eng., The Cooper Union, 51 Astor Pl., New York, NY 10003). Daniel R. Raichel (The Cooper Union and the Graduate Ctr. of City Univ. of New York), Latif Jiji, and Karim Abdulla-Altaii (CCNY)

Establishing an acoustics laboratory from the start on limited funds $(\$ 50 \mathrm{~K}$ ) for the purpose of training engineering students constitutes a formidable task that requires careful planning and design. Cost considerations limit the size of an anechoic chamber, but fortunately a commercially ready unit was found available for the price of $\$ 15 \mathrm{~K}$ and with a cutoff frequency of $125 \mathrm{~Hz}$. A reverberation chamber could be constructed in-house with the incorporation of a reflective door which is also available commercially. The preferred minimum room volume of $7062 \mathrm{ft}^{3}$ results in a cut-off frequency of $125 \mathrm{~Hz}$. In the selection of instrumentation, advantage was taken of the ready availability of task-specific circuit boards which can be inserted into microcomputers which can then serve as FFT analyzers and signals generators. Other equipment include a Class 1 sound level meter kit, a number of extremely low-priced SLMs, audio amplifiers, monitor speakers, measurement microphones, and an $X-Y$ plotter. Also, the available space was reconfigured to minimize outside interference and to maximize the ease of facility usage. 


\title{
Session 3pEA
}

\section{Engineering Acoustics and Noise: Active Control of Noise and Vibration II}

\author{
Kenneth A Cunefare, Chair \\ School of Mechanical Engineering, Georgia Institute of Technology, Atlanta, Georgia 30332-0405
}

\section{Contributed Papers}

\section{$12: 30$}

3pEA1. An adaptive feedback technique for disturbance rejection: Experimental results. Daniel H. Miller and William T. Baumann (Dept. of Elec. Eng., Virginia Polytechnic Inst. and State Univ., Blacksburg, VA 24061)

The paper presents experimental results of a hybrid control technique that blends traditional fixed feedback control with adaptive feedback techniques. The objective is to provide damping for transient disturbances via the fixed part of the controller and rejection of steady disturbances via the adaptive part of the controller. This technique differs from the other hybrid techniques in that it does not require a measurement of an external signal coherent with the disturbance, nor does it require knowledge of how the disturbance enters the system. In addition, it provides damping to the system, unlike neutralization-loop techniques. As with all feedback techniques, stability is an issue. The adaptive controller is implemented in such a way as to minimize stability problems and in the case of perfect system identification it is guaranteed to be stable. The experimental results are from a cantilever beam with piezoceramic actuators and sensors. The results for the fixed feedback controller will be compared to those for the adaptive feedback control technique. The comparison will include results from harmonic, narrow-band, and broadband disturbances. [Work supported in part by ONR contract N00014-92-J-1170.]

\section{2:45}

3pEA2. Acoustic absorptivity of direct acoustic rate feedback control. Daniel G. Cole (Adaptive Technologies, Inc., 620 N. Main St., Ste. 306, Blacksburg, VA 24060) and William R. Saunders (Virginia Polytechnic Inst. and State Univ., Blacksburg, VA 24061-0238)

The fixed-gain feedback methods often provide the only means of limiting transient noise and excessive sound pressures due to unmeasurable, incoherent disturbances. The use of direct acoustic rate feedback (DARFB) to control an enclosure's reverberant energy has the benefit over other feedback methods of larger stability margins and improved stability robustness. It also provides a means for achieving acceptable sound pressure levels in locations and environments which are not suited to surface treatments of sound absorbing materials. The effect of DARFB on the growth and decay of sound in enclosures is investigated and the change in the reverberation time of a sound field is discussed for active absorption using single and multiple controllers. Various models for the dynamics of sound growth are used and are shown to provide equivalent or similar results. Effective Sabine absorption coefficients are shown for active absorption and are compared with sound absorbing materials. The effectiveness of transducer placement is also discussed with respect to active absorption and the closed-loop reverberation time. Numerical examples of acoustic absorption are given for a rectangular enclosure.

$1: 00$

3pEA3. A novel active control approach for aircraft interior noise. Brian H. Houston and Martin H. Marcus (Naval Res. Lab.. Washington. DC 20375-5350)

The results of numerical experiments carried out to study the broadband structural acoustics of an aircraft-like shell under point excitation have been previously reported. The dominant mechanisms that lead to the enhancement of interior acoustic levels are resonances in the structure (both in the endcap and cylinder wall) forcing high interior acoustic levels as well as structural-borne energy coupling into "rigid wall" cavity resonances. Wave-number domain analysis reveals the evidence of free waves on the cylinder modified by finite length effects, flexural Bloch waves, and coupling to the cavity resonances. In this paper, results are presented of active control simulations that employ novel control approaches that are wave-number-domain based. These results also show that global access to low-order interior modes is obtained through simple actuation schemes and that minimization of some of the more dominant interior modes is achieved by the application of simple constraints to the structure.

\section{$1: 15$}

3pEA4. Active control of acoustic impedance with a multi-element system. Brian H. Houston, Douglas M. Pholiadis, J. A. Bucaro, Robert D. Corsaro (Naval Res. Lab., Washington, DC 20375-5350), and Larry A. Kraus (SFA, Inc., Landover, MD 20785)

The use of active control of acoustic impedance is of general interest due to a variety of potential defense and civilian applications. These include control systems to minimize aircraft and rocket payload section interior acoustic levels. Control of impedance at a boundary is one of the most challenging in active conirol due to the collocation of sensors and actuators (implicit in this is feedback control). Some of the technical issues include-the selection of the appropriate physical control law, the degree of inter-connectivity (local versus global control), device linearity, component and processor delays, system identification, nonminimal phase-zero constraints the coupling matrix, and performance versus robustness tradeoffs. Recently, new active boundary control (ABC) experiments were carried out at NRL's Laboratory for Structural Acoustics on a 15-tile array system. The results of these experiments will be discussed with a focus on the application of $H_{\infty}$ control engineering techniques and the physics involved.

3pEA5. The impact of sampling location on the minimization of noise in a cavity with flexible walls. Kenneth A. Cunefare, Van Biesel (George W. Woodruff School of Mech. Eng., Georgia Inst. of Technol., Atlanta, GA 30332-0405), and Steven Engelstad (Lockheed Aeronaut. Systems Co., Marietta, GA 30063)

Many transportation systems, e.g., aircraft and automobiles, have significant interior noise levels. There is substantial interest to reduce such interior noise levels, while minimizing weight penalties. Since the noise field inside such vehicles cannot, in general, be determined analytically, numerical techniques are commonly used to model the structural response of the cavity walls and the accompanying coupling to the interior space. These approaches may then be coupled to an optimization algorithm to determine how the walls should be designed so as to minimize the interior noise. However, a significant feature of such an approach is the need to sample the interior field at a number of points within the volume. This presentation will evaluate how the distribution of the sampling points within a volume may influence the efficiency of an optimization algorithm. 
The relative merits of sampling points distributed throughout the volume will be compared to points restricted to a layer near the cavity boundaries. The subject cavity will be a simple model of an aircraft fuselage.

\section{$1: 45$}

3pEA6. On the use of quadratic boundary elements for sensitivity analyses. Kenneth A. Cunefare (George W. Woodruff School of Mech. Eng., Georgia Inst. of Technol., Atlanta, GA 30332-0405)

The use of the acoustic boundary element method is gaining widespread acceptance. As the BEM matures, new uses for it are being developed, including its incorporation into design optimization and active noise control algorithms. The use of quadratic shape functions in the BEM formulation provides cerain benefits with regard to modeling and surface representation. However, these popular elements do pose challenges when acoustic sensitivity information is desired of the formulation, such as is used in optimization and active noise control. The difficulty arises from the implications of a positive change in a nodal value (e.g., surface normal velocity), and the net change over the balance of the element. Corner nodes will yield a net negative change, while mid-side nodes will yield a net positive change, both for positive nodal change. This presentation demonstrates that the use of quadratic elements requires careful treatment as a consequence of this behavior, and that element-based, rather than nodebased, sensitivities are most appropriate with this element.

\section{2:00}

3pEA7. Simultaneous active/passive control of extensional and flexural vibrations in infinite thin beams. Florence $L$. Deneufve and Chris R. Fuller (Vib. and Acoust. Labs., Dept. of Mech. Eng., Virginia Polytechnic Inst. and State Univ., Blacksburg, VA 24061-0238)

Passive control techniques implemented using added damping, through mass discontinuities or material discontinuities in order to minimize structural vibrations, are limited concerning the amount of attenuation obtained especially in the low-frequency domain. Active control methods are effective for optimally reducing structural vibrations, but may require significant control energy, especially for large disturbance inputs. The combination of passive with active control has much polential for completely reducing vibrations propagating in structures as the control energy required for the active part is found to be reduced and the frequency ranges of operations are complementary. In this research, passive and active techniques are combined to control simultaneously extensional and flexural motions in an infinite thin beam. The passive control is implemented with a hard rubber discontinuity of various lengths and the active control is achieved with two co-located independent piezoelectric actuators bonded on each side of the rubber section. The disturbance is a harmonic point force applied at the origin at an angle in order to excite both extensional and flexural vibrations. The optimal control voltages are calculated to reduce both the extensional and flexural wave amplitudes to zero downstream of the rubber discontinuity. Results of a simulation on an infinite thin beam are presented and discussed.

\section{2:15}

3pEA8. The use of neural networks for optimum actuator grouping in time domain active control applications. G. Clark Smith, Chris R. Fuller (Vib. and Acoust. Labs., Dept. of Mech. Eng.. Virginia Polytechnic Inst. and State Univ., Blacksburg. VA 24061-0238), and Richard J. Silcox (NASA Langley Res. Ctr., Hampton, VA 23665-0001)

Previous work has demonstrated the benefit of grouping actuators to decrease the number of degrees of freedom in an active control system. In this work, a time-domain cost function was developed for on-line actuator grouping and active structural acoustic control (ASAC) of a simplysupported beam excited with a broadband distumance. Actuators are considered grouped when their compensators are equal. Therefore, the cost function presented here incorporales a mean-square error term related to the structure-borne noise and an additional nonquadratic term which penalizes the controller for differences between respective compensator coefficients. The backpropagation neural network algorithm provides the proper procedure to determine the minimum of this cost function. The main disadvantage of using such a stochastic gradient technique while searching the prescribed control surface is converging to local minima. A resolution to this problem is discussed which incorporates using a variety of initialization conditions. Two scenarios are considered here: grouping actuators based upon weights determined by converging the filtered- $x$ LMS algorithm and simultaneously grouping and controlling with the compensator weights started at zero. Computer simulations demonstrate the ability of this new form of the cost function to simultaneously group actuators and control the structure-borne noise with either initial conditions.

3pEA9. Vibration signature monitoring using analytical models of slotted beams. XiuTing C. Man and Robert D. Finch (Mech. Eng. Dept., Univ. of Houston, 4800 Calhoun Rd., Houston, TX 77204-4792)

Vibration signatures may be used in monitoring structural integrity. A procedure was established in which the experimental data were processed using analytical models of the physical structures. Steal beams were used as examples. Saw-cut slots of different sizes and locations were the "damage" to be detected. An analytical model based on a perturbation method was developed, which leads to direct relationships between the modal properties and the slot parameters. The modal frequencies were estimated experimentally using modal analysis techniques. The frequency reductions were computed as the difference between the experimentally estimated modal frequencies and the values theoretically calculated from a uniform beam of identical conditions. The first three modal frequency contours were calculated from the perturbation model and used to quantify the slot depths and slot locations. The slot locations were determined with great accuracy, although the slot depths were estimated lower than the actual values. The reason was that the perturbation model predicted the modal frequency shifts smaller than the actual values, and when used inversely, it tended to give a slot depth of higher value.

\section{2:45}

3pEA10. Active control of vibration of a magnetic levitation platform. Jiangxiong $\mathrm{Li}$, Jiaqiang Pan, and Qiang Hu (Dept. of Mech. Eng., Thejiang Univ., Hangzhou 310027, People's Republic of China)

A study on active control of vibration isolation of a magnetic levitation platform used for the calibration of precision inertia instruments is presented in this paper. Nonlinear and linearized dynamic models of the system in state-space were deduced by using the theory of an electromagnetic field. The stability of both the open- and closed-loop system was investigated in the cases of different configuration of the current coils. A LQG optimal control strategy was employed in the control syntheses of the magnetic levitation system, since the system input perturbance is a stochastic excition acting on the fixed base from the ground. The transfer functions from the exciting current or the control current or the base displacement to the vibratory displacement of the levitation platform were verified experimentally. Open- and closed-loop vibration responses were calculated. The results show that compared to the base displacement, the vibration of the magnetic levitation platfonn can be attenuated more than $20 \mathrm{~dB}$ by using active control, over frequencies ranging from 0.1 to $10 \mathrm{~Hz}$, which cover the main peaks of the ground perturbance.

\section{3:00}

3pEA11. Method of prognostic noise and vibration from industrial setups. Alexander E. Ekimov, Vladimir A. Tyutin (Inst. of Appl. Phys., Russian Acad. of Sci., 46 Ulyanov str., Nizhny Novgorod, 603600, Russia), Rostislav A. Dudnik, and Andrei B. Kolpakov (Inst. Arch. \& Civil Eng., Nizhny Novgorod, 603600, Russia)

This paper considers a method to determine the contribution of vibroactive industrial setups (prior to their mounting on the basement) to outside noises and vibrations. The technique offered is based on the experimental determination of transfer coefficients of noises and vibrations from outside 
sources (acoustic generators and vibrators) into environmental points of interest. The contribution of a setup is determined by multiplying vibration and noise characteristics (measured using an operating analogue setup) and experimentally measured transfer coefficients mentioned above. The technique was used to estimate the levels of vibrations and air noises at the shop of microelectronics at the Communication Equipment Plant in Nizhny Novgorod. The vibrations and noises in question were caused by an electrocompressor located in a separate building near the shop. A vibrator generating a force from 500 to $6000 \mathrm{~N}$ in the frequency range 20-60 $\mathrm{Hz}$ was used to determine vibration transfer coefficients. The experimental estimations showed that electrocompressor noises and vibrations will not affect the operation of precision devices in the shop. The prognosis was fully verified by measurements taken after electrocompressor mounting and the noises and vibrations did not exceed background ones.

THURSDAY AFTERNOON, 1 JUNE 1995

GRAND BALLROOM SOUTH AND CENTRAL, 2:15 TO 3:20 P.M.

\title{
Session 3pED
}

\author{
Education in Acoustics: Internet and the World Wide Web \\ Lawrence R. Rabiner, Chair \\ AT\&T Bell Laboratories, Room 2D-538, Murray Hill, New Jersey 07974
}

Chair's Introduction-2:15

Invited Paper

2:20

3pED1. The evolving Internet. Pat Parseghian (AT\&T Bell Labs., Room 2C-472, 600 Mountain Ave., P.O. Box 636, Murray Hill, NJ 07974-0636)

The World Wide Web and point-and-click browsers have revolutionized the way one looks at the Internet. The Internet is not just for computer scientists anymore-it links elementary schools, businesses, and homes around the globe. Addresses for resources on the World Wide Web appear in national magazines, local newspapers, and are televised on the evening news. Following a brief overview of the Internet and traditional text-based Internet services, this presentation will focus on emerging multimedia uses of the Internet. Multimedia extensions for electronic mail allow us to share images and sounds. The MBone (Multicast Backbone) supports collaborative tools for audio and video conferencing, as well as a shared whiteboard. On the World Wide Web, one can look at the weather report, read colleagues' technical papers, go shopping, and check the traffic conditions, all before leaving home for the office! While the Internet is straining to accommodate its escalating load, advances in networking technology promise a bright future. 


\title{
Session 3pID
}

\section{Interdisciplinary: Hot Topics in Acoustics}

\author{
Yves H. Berthelot, Chair \\ School of Mechanical Engineering, Georgia Institute of Technology, Atlanta, Georgia 30332
}

Chair's Introduction-1:00

A special session on "Hot Topics in Acoustics" is presented at each meeting of the Society. A member is chosen from each of three or four of the Society's technical committees or specialty groups to present a tutorial paper on topics of current special interest. The presentations are intended to help acousticians become familiar with issues and achievements that are not within their own primary fields of interest.

\section{Invited Papers}

\section{1:05}

3pID1. Hot topics in structural acoustics. Andrew N. Norris (Dept. of Mech. Eng., Rutgers Univ., Piscataway, NJ 08855-0909)

This talk surveys some key developments in modeling the acoustic response of fluid-loaded structures. Recent experimental and computational results indicate that wave interaction effects are very significant for structures with even a sinall degree of complexity. The complexity can be as simple as a few ribs or stiffeners which convert supersonic membrane-type wave motion into subsonic flexural energy, and vice versa A greal deal of effort continues to be put into developing efficient numerical methods in order to simulate greater complexity. At the same time, recent analytical findings have increased our understanding of the dynamic interaction at ribs and plate junctions, in terms of wave-like diffraction processes. Ray methods have been developed to handle the multi-wave nature of structural energy flow on nonseparable shapes, and provide a fast numerical method for dealing with high-frequency sirnulation. In general, the complexity of the substructures within the relatively well-defined "master" structure presents the most difficult challenge for modelers. Techniques are only now being developed to distinguish these effects in a logical manner. One approach of great interest is "fuzzy" structural acoustics. The idea is to replace the detailed dynamics of the internal by a smeared out effect, which still contains some of the physics of the internal such as the total mass, and the modal density per unit bandwidth. Fuzzies are also "hot" in that they are guaranteed to generate thermal energy among ASA participants concerned about energy effects.

$1: 25$

3pID2. Hot topics in speech communication. D. H. Whalen (Haskins Labs., 270 Crown St., New Haven, CT 06511)

In speech processing, mapping from acoustics to articulation is becoming more useful. While acoustically based recognizers have achieved impressive success rates, their limitations do not seem to be yielding to further acoustic manipulations. Recovering the articulation underlying the speech offers a way of decoding the complex acoustic manifestations of simple articulatory events. These results also offer benefits in bit-rate reduction and speech synthesis. In speech production, nonlinear dynamic models of speech articulation are beginning to bear fruit. The transition from stasis to vibration of the larynx (the voicing source in speech and singing) can be viewed as a switch from a point attractor (damped oscillator) to a limit cycle (self-sustained oscillation). The tools of nonlinear mathematics and chaos theory allow us to explore such topics as voice breaks, yodeling, and certain voice disorders, as well as normal phonation, Nonlinear techniques have also provided important insights into the production mechanisms of fricative consonants. In speech perception, one of the most interesting new developments is the patterns of brain activity (seen in blood flow) shown by PET scans and by functional MRI. Researchers have found that passive listening to speech activates the temporal lobe, but making judgments on that same speech also involves Broca's area. Reading print also activates Broca's area, indicating a crucial link between speech and reading. Another finding is that males rely mostly on the left premotor cortex in performing phonetic tasks presented in print, while females are not strongly lateralized. As acquisition time declines, fMRI promises to yield even more specific information on speech perception.

3pID3. Hot topics in underwater acoustics. W. A. Kuperman (Marine Phys. Lab., Scripps Inst. of Oceanogr., Univ. of California, San Diego, La Jolla, CA 92093-0238)

Underwater acoustics is in a period of renaissance with researchers actively pursing studies in many areas from short-range high-frequency shallow water acoustics to global low-frequency acoustics. There has been renewed interest in inhomogeneous sediment acoustics as well as propagation in very shallow water bounded by these sediments. The recent Office of Naval Research "SRP" program studying deep water boundary reverberation has not only been fruitful, but has also laid the foundation for innovative 
research in the area of boundary scattering in coastal water acoustics. On the other scale, the Heard Island Feasibility Test as described by 18 papers in the October 1994 issue of JASA has re-awakened interest in the myriad of possibilities of monitoring all scales of the ocean using acoustic methods. In an expanding number of efforts, underwater acoustics is distinguishing itself as developing the most physically based signal processing schemes presently being considered in any field. These and other hot topics will be discussed.

THURSDAY AFTERNOON, 1 JUNE 1995

MEETING ROOM 2, 1:00 TO 3:15 P.M.

\title{
Session 3pNS
}

\author{
Noise: Noise and Hearing \\ Linda L. Pierson, Chair \\ Visual and Auditory Processes Branch, Human Research and Engineering Directorate, U.S. Army Research Laboratory, \\ Aberdeen Proving Ground, Maryland 21005-5425
}

\section{Contributed Papers}

\section{1:00}

3pNS1. Investigation of the impact of changes in regulatory criteria on the daily noise dose (DND) of longwall coal mine workers. J. Alton Burks and Roy C. Bartholomae (U.S. Bureau of Mines, Pittsburgh Res. Ctr., Pittsburgh, PA 15236)

The authors previously examined the impact of anticipated changes in noise regulations on the coal mining industry [R. C. Bartholomae and J. A. Burks, Noise-Con 94, 1017-1022 (1994)]. That analysis focused primarily on assessing the effect on the daily noise dose (DND) resulting from changing the current threshold level from 90 to $80 \mathrm{dBA}$. To more fully explore the effect of other regulatory parameters, as well as the threshold level, 59 sets of full-shift time-resolved dosimeter data were acquired on four occupations associated with longwall coal mining. After these data were downloaded to a computer, realistic combinations (a total of 12) of criterion level $(80,85,90 \mathrm{dBA})$, threshold level $(80,85,90 \mathrm{dBA})$, and tradeoff rate $(3,5 \mathrm{dBA})$ were applied to these data to compute DND's. The results of this "what if" type analysis are presented. In general it was concluded that the magnitude of the computed DND resulting from changes in either the tradeoff rate or the threshold level is unpredictable; instead, it depends on the specific distribution of noise levels associated with a given worker's noise exposure.

1:15

3pNS2. The effects of reverberant blast waves on hearing: Energy and spectral considerations. William A. Ahroon, Sheau-Fang Lei, and Roger P. Hamernik (Auditory Res. Lab., State Univ. of New York, Plattsburgh, NY 12901)

Chinchillas were exposed to 1,10 , or 100 reverberant impulses at 150 , 155 , or $160 \mathrm{~dB}$ peak SPL. The impulses were generated by one of two different shock tubes each producing blast waves having a different spectral composition; one emphasizing low frequencies $(<0.5 \mathrm{kHz})$, the other mid-frequencies $(2-4 \mathrm{kHz})$. Impulses were presented at the rate of $1 / \mathrm{s}$. This parametric paradigm yielded 18 exposure conditions; 15 animals/ condition. Hearing thresholds were measured using auditory evoked potentials and the sensory epithelium was evaluated with the surface preparation. In general, trauma increased as the total energy of the exposure, determined by the peak SPL and number of presentations, increased. The dependent variables (permanent threshold shift and sensory cell loss) varied in an orderly fashion across frequency as the peak and number of presentations was increased for both blast wave sources. There were, however, consistent differences between the effects of the low- and highfrequency energy "content" blast waves. Correlations between the dependent variables and the energy of exposure were highest for P- or A-weighted energies [Patterson et al., J. Acoust. Soc. Am. 93, 2860-2869 (1993)]. [Work supported by U.S. Army Medical Research and Development Command.]

$$
\text { 1:30 }
$$

3pNS3. Validating a mathematical model of noise hazard with varying numbers of rounds and peak pressures produced by a rifle. G. Richard Price, Linda L. Pierson, Joel T. Kalb, and Pam Mundis (Human Res. and Eng. Directorate, Army Res. Lab., Aberdeen Proving Ground, MD 21005)

A mathematical model of noise hazard [G. R. Price and J. T. Kalb, J. Acoust. Soc. Am. 90, 219-227 (1991)] has correlated very highly with hearing loss to 50 Friedlander impulses from two sources at 5 different peak pressures (135 to $145 \mathrm{~dB}$ ). In order to extend the model's range four additional noise exposures were tested: two single impulse exposures (157and $169-\mathrm{dB}$ peak) and 6 or 12 impulses at $143-\mathrm{dB}$ peak pressure. These specific conditions were also chosen to contrast the model's predictions with the "CHABA" damage risk criterion (DRC). Four groups of ten anesthetized cats were exposed to each condition and ABR thresholds at 1 , $2,4,8$, and $16 \mathrm{kHz}$ were monitored to establish hearing loss immediately and 2 months later. In each case the model's predictions were upheld and rankings of hazard by the CHABA DRC were not. Furthermore, no simple linear frequency weighting would have predicted the losses. The model's hazard rating correlated well with the group hearing losses (coefficient near 0.8 ) and refinement of the model's annular ligament and the intracochlear hazard formula is expected to improve this correlation.

\section{$1: 45$}

3pNS4. Effect of "conditioning" on hearing loss from military noise exposures. Lynn W. Henselman (Army Audiol. and Speech Ctr., Walter Reed Army Med. Ctr., Washington, DC 20307-5001), Donald Henderson, Malini Subramaniam, Philip Hofstetter (SUNY, Buffalo, NY 14214), and Vincenzo Sallustio (Centro Di Audiologica, Universita Di Bari, Bari, Italy)

It has been shown with chinchillas as subjects that prophylactic ("conditioning") exposures to an interrupted OBN centered at $0.5 \mathrm{kHz}$ for 10 days provide protection against permanent threshold shift (PTS) from high level impulse noise in chinchillas [L. W. Henselman et al.; Hear. Res. 78, 
1-10 (1994)]. The present investigation studied the effect of "conditioning" with helicopter noise on PTS resulting from high level impulse noise. Thirty-eight chinchillas were randomly assigned to one of four experimental groups (helicopter noise exposure prior to impulse noise exposure) or a control group (impulse noise exposure only). It was shown that (a) interrupted exposures over a 10-day period to helicopter noise presented at 112 $\mathrm{dB}$ SPL for $1.5 \mathrm{~h} /$ day caused TTSs to decrease as exposure days continued at the test frequencies of 0.5 to $8 \mathrm{kHz}$, and (b) after 4 weeks of recovery, subjects were protected from PTS after "conditioning" with helicopter noise (except the group with prolonged "conditioning"). Histological results were consistent with audiological findings and revealed significantly less hair cell loss in the experimental groups that were protected from PTS. The results are discussed in terms of possible application to hearing conservation programs. [Work supported by the U.S. Army Medical Command.]

\section{2:00}

3pNS5. Comparison of impulse noise effects generated by two rifle muzzles. Linda L. Pierson, G. Richard Price, Joel T. Kalb, and Pamela A. Mundis (Visual and Auditory Processes Branch, Human Res. and Eng. Directorate, U.S. Army Res. Lab., Aberdeen Proving Ground, MD 21005-5425)

The current impulse noise standard for US military uses a combination of peak sound pressure level and envelope duration to rate hearing hazard. There is general agreement that this procedure tends to overestimate the hazard from impulses that are broadly peaked at the low frequencies. One possible correction would be to frequency-weight the energy, giving less emphasis to the low frequencies. The current study, using the cat as a model, compares the physiological effect (auditory brainstem response threshold) of one rifle impulse generated either with the standard or an experimental muzzle device. The pressure from the standard muzzle device $(N=9)\left(157.1 \mathrm{~dB}\right.$ Peak, $9.62 \mathrm{~ms}$ B-duration, $\left.0.9 \mathrm{~J} / \mathrm{M}^{2}\right)$ has a peak $13 \mathrm{~dB}$ "below" the pressure generated by the experimental muzzle device $(N$ $=10$ ) $\left(169.9 \mathrm{~dB}\right.$ peak, $6.88 \mathrm{~ms}$ B-duration, $\left.9 \mathrm{~J} / \mathrm{M}^{2}\right)$. It would be expected that the animals in the normal muzzle group would incur substantially less threshold shift than animals in the experimental muzzle group. However, group mean threshold were not significantly different. This finding challenges the current criterion. Furthermore, although both impulses contain essentially the same spectrum, because the energy composition of the impulses was very different, frequency weighting alone does not solve the problem.

\section{2:15}

3pNS6. The effect of the level of impact noise on hearing loss. Stephanie Levine, Donald Henderson, and Phillip Hofstetter (Hear. Res. Lab., Univ. of Buffalo, Buffalo, NY 14214)

Impulse and impact noise found in industry may be particularly dangerous for workers' hearing. This study examines the effect of the level of impact noise on hearing loss. Twenty four adult chinchillas were exposed to impact noise for $7.5 \mathrm{~h}$. The subjects were divided into four groups and exposed to impulses delivered at $1 / \mathrm{s}$ at one of four different intensities: $113,119,125$, and $131 \mathrm{~dB}$. As the level of exposure increased, the permanent hearing loss of the animals increased. At lower levels, 113-125 dB hearing loss increased at approximately $1.8 \mathrm{~dB}$ for each $\mathrm{dB}$ of noise. These findings are consistent with studies using continuous noise which report a 1.7- $\mathrm{dB}$ increase in asymptotic threshold shift for every $\mathrm{dB}$ increase in exposure. However, as the level of the impact increases beyond a critical level, $(125 \mathrm{~dB})$ the resulting threshold shift increases dramatically i.e., 6 $\mathrm{dB}$ for each $\mathrm{dB}$ increase in exposure level. It has been shown in prior research [Hamernik et al., Hear. Res. 13, 229-247 (1984) and Henderson et al., Hear. Res. 76, 101-117 (1994)] that the anatomical damage to the cochlea at this critical level changes from metabolic to mechanical. [Research supported by NIOSH Grant No. 15034470.]

\section{2:30}

3pNS7. Mathematical formulas and summary measures for the audiometric database analysis procedures (ANSI S12.13-1991). Tilahun Adera (Dept of Preventive Medicine, Medical College of Virginia, 1008 East Clay St., Box 980212, Richmond, VA 23298)

The concept of an audiometric database analysis (ADBA) method for evaluating the effectiveness of hearing conservation programs has been appearing in the scientific literature for more than 15 years. This concept was recently developed into a series of procedures and is currently being considered for acceptance as a U.S. National Standard (ANSI S12.131991). Although the method presents the various analytical procedures in a narrative fashion, mathematical formulas expressing the essential principles have not been provided. In addition, there currently is no method for determining the overall effectiveness of a hearing conservation program (HCP) following application of ADBA procedures to a set of audiometric data. This paper provides the mathernatical formulas for four ADBA procedures including the percent worse sequential, percent better or worse sequential, standard deviation applied to single test frequencies, and standard deviation applied to averages of test frequencies. In addition, the paper introduces and illustrates two methods of summarizing results from ADBA procedures, which includes the standard score estimator and the score designator. Each of these methods consists of at least a median and a mean-based procedure. The strengths and limitations of these procedures in determining the overall effectiveness of an HCP are discussed.

\section{2:45}

3pNS8. A comparative study of occupational and nonoccupational noise regulations in South American countries. Jorge P. Arenas (Inst. of Acoust., Facultad de Ciencias de la Ingenieria, Univ. Austral de Chile, Casilla 567, Valdivia, Chile) and Martin Gutierrez V. (Inst. of Acoust., Univ. Austral de Chile, Casilla 1130, Valdivia, Chile)

The final aim of this work is to show a comparative study about the most important aspects of the noise regulations in some South American countries. It includes Argentina, Brazil, Chile, Peru, and Uruguay. Where national standards exist in a particular country, the area of application, and other details of the procedures, should be in accordance with the standards of that country. The differences between national standards are extremely significant for some types of noise. The most important parameters for nonoccupational noise considered when assessing the acceptability of a given noise exposure are: the time of the day (day time, evening, night time), the type of neighborhood, the use to which an area of land or building is put, and sometimes the indoor standards. The basic criteria is not to alter the character of an entire area and dominate its noise climate. In some countries the occupational regulations are combinations of ISO and OSHA standards. Differences were noted in some dose criterias and the trade-off between noise level and permitted exposure time is still the subject of some divergence of opinion. Some standards allows $3 \mathrm{~dB}$ (or 5 $\mathrm{dB}$ ) increase in noise level per halving of exposure duration.

\section{3:00}

3pNS9. Modified method for determination of heightened human sensitivity to noise. Alexander A. Menshov and Vladimir V. Lipovoy (Inst. for Occupational Health, 252033, Kiev, Ukraine)

Occupational hardness of hearing occurs first of all in workers with heightened sensitivity to noise. Along with the objective method for determining higher excitability of the central structures of the auditory analyzer by means of the awaked brain potentials, the heightened sensitivity to noise can be determined by a simpler method [A. Peyser, Acta Otolaryngol. (Stockh.) 28, 443-444 (1940)]. The experimental data have shown that a higher increase in TTS is observed at a tone of $2000 \mathrm{~Hz}$ instead of $1000 \mathrm{~Hz}$ according to A. Peyser. Such a modified method was approved at 42 young weavers. After 3 min of $100-\mathrm{dB}$ noise load at the tone of $2000 \mathrm{~Hz}$ in $13 \%$ of weavers TTS was more than $12 \mathrm{~dB}$ (hightened sensitivity). After work for a year under the conditions of 98-100 dBA, in 
the weavers with hightened sensitivity, TTS at the tone of $2000 \mathrm{~Hz}$ was $11.9 \mathrm{~dB}$, at the tone of $1000 \mathrm{~Hz}$ was $9.4 \mathrm{~dB}$, and at the tone of $4000 \mathrm{~Hz}$ was 9.6 dB. In the weavers insensitive to noise, TTS at the tone of $1000 \mathrm{~Hz}$ was $7.0 \mathrm{~dB}$, at the tone of $2000 \mathrm{~Hz}$ was $7.5 \mathrm{~dB}$, and at the tone of $4000 \mathrm{~Hz}$ was $7.8 \mathrm{~dB}$. In the sensitive to noise weavers, PTS at $1000 \mathrm{~Hz}$ was $2.3 \mathrm{~dB}$, at
$2000 \mathrm{~Hz}$ was $4.3 \mathrm{~dB}$, and at $4000 \mathrm{~Hz}$ was $1.7 \mathrm{~dB}$. In the insensitive to noise weavers, PTS at $1000 \mathrm{~Hz}$ was $1.1 \mathrm{~dB}$, at $2000 \mathrm{~Hz}$ was $1.7 \mathrm{~dB}$, and at 4000 $\mathrm{Hz}$ was $1.3 \mathrm{~dB}$. The determination of human sensitivity to industrial noise by audiometry in this modification enables one to discover workers with heightened auditory sensitivity and predict the PTS auditory level.

\title{
Session 3pPP
}

\section{Psychological and Physiological Acoustics: Hearing Impairment, Cochlear Implants and Hearing Aids}

\author{
Fan-Gang Zeng, Chair \\ House Ear Institute, 2100 West Third Street, Los Angeles, California 90057
}

Contributed Papers

12:40

3pPP1. Simulation of sensorineural hearing loss: Use of linear spectral smearing to reduce frequency resolution. Arthur Boothroyd, Bethany Mulhearn, Juan Gong, and Jodi Ostroff (City Univ. of New York, 33 W. 42 St., New York, NY 10036)

Phoneme and word recognition, was measured in seven hearing adults under various conditions of spectral smearing-produced by multiplying the speech waveform by low-pass-filtered noise. Phoneme recognition fell from $97 \%$ for no smearing to $13 \%$ for complete smearing ( $\pm 10 \mathrm{kHz}$ ). Smearing by $\pm 600 \mathrm{~Hz}$ reduced group mean phoneme recognition to $50 \%$ - a score typical of hearing-impaired subjects with hearing losses around $80 \mathrm{~dB}$, listening to amplified speech. In a second experiment, noise was mixed with the speech before spectral smearing was introduced. Noise susceptibility was measured as the $\mathrm{S} / \mathrm{N}$ ratio required for a phoneme recognition score that was $50 \%$ of the score in quiet. Smearing at $\pm 1 \mathrm{kHz}$ increased noise susceptibility by $20 \mathrm{~dB}$, an amount similar to that found in hearing-impaired subjects with losses in excess of $90 \mathrm{~dB}$. As with hearingimpaired subjects, spectral smearing diminished the perception of consonant place more than the perception of consonant manner and voicing. Unlike with hearing-impaired subjects, however, smearing affected vowel perception more than consonant perception. This last finding may be attributed to the use smearing over a fixed bandwidth-producing a greater percentage smearing in the lower frequencies. [Work supported by NIDCD Grant No. 10078.]

\section{$12: 55$}

3pPP2. Loudness functions in normal-hearing and hearing-impaired listeners. Lidia W. Lee (Dept. of Commun. Disorders, Northem Illinois Univ., DeKalb, IL 60115) and Larry E. Humes (Indiana Univ., Bloomington, IN 47405)

This paper examined whether an excitation-pattem model of loudness could adequately describe the growth of loudness for complex stimuli presented to normal-hearing and hearing-impaired listeners in quiet and in noise. The loudness-growth functions were obtained for three synthesized steady-state vowels $(/ a, \mathrm{i}, \mathrm{u} /$ ), each with two talkers (male: $F 0=120 \mathrm{~Hz}$; female: $F 0=200 \mathrm{~Hz}$ ), and for several pure tones $(125-4000 \mathrm{~Hz})$. All stimuli were presented, at random, from 2 to $90 \mathrm{~dB}$ SPL (in 4-dB steps) in quiet and in broadband noise. Magnitude estimation were used to measure the loudness of each stimulus. These data are used to evaluate the predictions of an excitation-pattern model [modified power-law model, L. Humes and W. Jesteadt, J. Acoust. Soc. Am. 85, 1285-1294 (1989)]. [Work supported by NIA.]
1:10

3pPP3. Relationships between simple reaction time and auditory stimulus intensity in people with sensorineural hearing losses. Philip F. Seitz (Ctr. for Auditory and Speech Sci., Gallaudet Univ., Washington, DC 20002-3695) and Brad Rakerd (Michigan State Univ., East Lansing, MI 48824-1212)

Individual differences in reaction time (RT) to subjectively comfortable, loud, and soft tones were assessed for ten young adult subjects with longstanding, less-than-profound sensorineural hearing losses and ten normal hearing controls. To support estimation of the sensory, central, and motor components of RT, subjects were also tested using an equivalent protocol with bright and dim visual stimuli. Subjects with normal hearing listened to $500-\mathrm{Hz}$ tones at fixed intensities that pilot testing established as corresponding to comfortable, loud, and soft levels. Prior to RT testing, subjects with hearing losses individually adjusted tone intensity to "most comfortable," "very loud but not painful," and "very soft but definitely audible" levels. Results show larger modality-dependent and leveldependent individual differences in RT than have been previously noted in the literature, pointing up a need for individual subject baselines in cognitive experiments that use RT as a dependent variable. Results also indicate that the typically small range between threshold and pain in listeners with hearing losses is, with respect to RT, functionally equivalent to larger ranges in normal hearing listeners in some individuals but not in others. [Work supported by NIH-NIDCD.]

3pPP4. The relationship between aided articulation indices, performance, and subjective satisfaction with programmable and conventional hearing aids. Gabrielle $H$. Saunders and Kathleen $M$. Cienkowski (Audiology (126), Veterans Admin. Med. Ctr., East Orange, NJ 07019)

The Articulation Index (AI) was developed as a method for predicting speech recognition performance from objective measurements. It should therefore be an efficient method for predicting and comparing potential user benefit from different hearing aids. In this study subjects wore six different hearing aid fittings for 3 months each. Performance on a number of speech tests was measured at the start and end of each 3-month period and self-rated satisfaction with each hearing aid was assessed. AIs for each hearing aid fitting were calculated from real ear aided gains. The relationship between the AIs, performance, and satisfaction will be presented and the efficacy of using the $\mathrm{AI}$ as a predictor of performance and satisfaction will be discussed. 


\section{1:40-1:50 Break}

\section{1:50}

3pPP5. Intensity discrimination of amplitude modulated stimuli in electric hearing. Fan-Gang Zeng and Robert V. Shannon (House Ear Inst., 2100 W. 3rd St., Los Angeles, CA 90057)

In cochlear implants loudness is a power function of electrical amplitude at low frequencies $(<300 \mathrm{~Hz})$ and an exponential function for higher frequencies [Zeng and Shannon, Science 264, 564-566 (1994)]. In addition, the just-noticeable-difference (jnd) in intensity is inversely proportional to the slope of the loudness function. Implant speech processors generally use low-frequency modulation of a high-frequency carrier. Because the loudness functions are different for the modulator and carrier frequencies, this raises the question: Do the loudness and jnd of a modulated stimulus follow the modulator or the carrier? Intensity discrimination was measured for a $100-\mathrm{Hz}$ sinusoidally modulated $1000-\mathrm{Hz}$ sinusoid or pulse train in implant listeners. The jnd was measured either as an increment in the carrier level for a fixed modulation depth or as an increment in the modulation depth for a fixed carrier level. The results showed that the jnd function of the modulated stimuli is similar to the jnd function of the high-frequency carrier. At high sensation levels, implant listeners can discriminate extremely small changes in modulation depth $(1 \%-2 \%)$. This implies that modulated stimuli can produce more jnd steps across the dynamic range than either the modulator or the carrier alone.

\section{2:05}

3pPP6. Preliminary evaluations of cochlear implantees using a wearable CIS processor. W. M. Rabinowitz, D. K. Eddington, J. Tierney, and L. A. Delhorne (Res. Lab. of Electron., MIT, 50 Vassar St., Rm. 36-789, Cambridge, MA 02139)

Each channel of a continuous-interleaved-stimulation (CIS) sound processor uses the compressed envelope of its bandpass-filtered output to modulate biphasic current pulses that are delivered to an intracochlear electrode. Pulses are interleaved across channels to avoid simultaneous field interactions, and pulse rates are high $(-2000 \mathrm{pps} / \mathrm{channel})$ to preserve temporal waveform cues. Using up to six monopolar electrodes directly accessible with the Ineraid implant, this strategy has shown considerable promise in acute evaluations conducted in the laboratory [Wilson et al., Nature 352, 236-238 (1991)]. In collaboration with a group in Innsbruck, a prototype portable real-time system has been developed (based on a DSP56001) that can realize some CIS implementations. Two subjects with 9 years of experience using the Ineraid analog sound processor are now wearing the CIS system on a full-time basis. After several weeks, one subject prefers the CIS strategy; however, objective measures of speech reception show no gains (re: the Ineraid processor). The second subject shows large gains with CIS and some evidence of continuing improvements. Without lipreading, he scores near perfect on relatively difficult (IEEE/Harvard) sentences in quiet; for speech reception in noise, he shows a deficit of $7 \mathrm{~dB}$ re: normal-hearing listeners. [Work supported by NIH.]

$$
\text { 2:20 }
$$

3pPP7. Field trials of a portable prototype digital hearing aid. Donna J. Gelnett, Jean A. Sullivan, Michael J. Nilssọn, and Sigfrid D. Soli (House Ear Inst., 2100 W. 3rd St., Los Angeles, CA 90057)

A battery-operated digital processor connected to microphones and receivers located in left and right ear modules was built and used in a hearing aid field trial. Eight hearing impaired individuals with moderate to moderately severe hearing losses served as subjects. All subjects had symmetric hearing losses and were experienced binaural hearing aid users. Four binaural hearing aid algorithms were programmed into the processor for evaluation in the fieid trial. The algorithms all equalized the magnitude and phase insertion effects of the ear modules, but differed in their gain prescription. Two prescriptions based on the Articulation Index (AI), one on NAL-R, and a control prescription were evaluated in the two week field trial. Subjects rated each algorithm in seven categories. Objective measures of speech intelligibility in noise, including measures of binaural directional hearing, were taken before and after the field trial. Intelligibility and directional hearing was best with the AI prescriptions, although these prescriptions did not receive the highest subjective ratings. Details of the binaural algorithms and fittings, as well as objective and subjective measures of their benefit will be reported.

\section{2:35}

3pPP8. Combining ratings and paired comparisons in hearing aid evaluation. Harry Levitt, Arlene C. Neuman, and Christopher Oden (Ctr. for Res. in Speech and Hear. Sci., Graduate School and Univ. Ctr., City Univ. of New York, 33 W. 42nd St., New York, NY 10036)

The method of paired comparisons is a rapid and efficient technique which has proven to be useful in hearing aid evaluation. In the conventional application of the technique, the subject is limited to a binary decision. Greater efficiency can be obtained if, in addition, a confidence rating is obtained. A method for combining confidence ratings with pairedcomparison data is provided. Data are presented in which a set of hearing aids differing in compression characteristics (compression ratio, release time) was evaluated by the traditional paired-comparison technique and the paired-comparison plus rating technique. Data will also be presented on the relative precision and efficiency of the two techniques.

\section{2:50}

3pPP9. Effects of hearing aids on binaural directional hearing in hearing-impaired individuals. Donna J. Gelnett, Michael J. Nilsson, and Sigfrid D. Soli (House Ear Inst., 2100 W. 3rd St., Los Angeles, CA 90057)

Binaural directional hearing, the ability of a listener to "tune out" noise from one direction and listen to a signal from another direction, improves speech intelligibility in noise. This ability is present in the hearing-impaired individual, but may be reduced as a result of hearing impairment. The present research examined the relationship between aided and unaided directional hearing, and directional hearing capacity-as measured under headphones with simulated head-related transfer functions and idealized amplification. Reception thresholds for sentences (RTSs) were measured with and without spatial separation of the speech and a spectrally matched noise for 25 hearing-impaired binaural hearing aid users. Directional hearing capacity for these individuals often fell within the normal range. Unaided RTSs were elevated $3-6 \mathrm{~dB}$ on average over the capacity measures. Aided RTSs were also elevated 2-3 dB over average scores for directional hearing capacity, suggesting that the interaural cues for binaural directional hearing are either inaudible or absent from the hearing aid output. Detailed analyses will be reported with respect to the type of hearing aid, hearing aid transfer function, and degree of hearing loss.

\section{3:05}

3pPP10. Sound localization in the median sagittal plane by hearing impaired listeners. Brad Rakerd, Timothy J. Vander Velde (Dept. of Audiol. and Speech Sci., Michigan State Univ., East Lansing, MI 48824), and William Morris Hartmann (Michigan State Univ., East Lansing, MI 48824)

Previously, it was reported that listeners with substantial highfrequency hearing loss have difficulty localizing sounds in the median sagittal plane [Vander Velde et al., J. Acoust. Soc. Am. 94, 1812(A) (1993)]. When asked to localize broadband noise, they performed near chance level on an elevation task and somewhat better, but far below normal, on a task that required that they distinguish between sources to the front, overhead, and rear. In the present study, these experiments were repeated with new subjects $(n=16)$, and with the following variations, each introduced to encourage improved performance. (1) Low-frequency 
components of the noise stimulus were filtered out to preclude the masking of more informative high-frequency components. (2) The difficulty level of the elevation task was eased. (3) Subjects were tested while wearing their hearing aids. Each of these changes helped a few individuals, typically in small ways, but none produced statistically significant improvement for the group overall $(p>0.05)$. Together, these results point up a significant localization problem for individuals with high-frequency hearing loss of the sort that commonly accompanies aging. The results also support the theory that spectral cues are of the greatest importance for median plane localization. [Work supported by NIDCD.]

THURSDAY AFTERNOON, I JUNE 1995

MEETING ROOMS 12, 13, 14, 1:00 TO 3:10 P.M.

\title{
Session 3pSA
}

\section{Structural Acoustics and Vibration: Structural Intensity II}

\author{
Sabih I. Hayek, Cochair \\ Department of Engineering Science and Mechanics, Pennsylvania State University, University Park, Pennsylvania 16802 \\ Jean Louis Guyader, Cochair \\ Laboratoire Vibrations-Acoustique, INSA-Lyon, 69621 Villeurbanne, Cedex, France
}

Invited Papers

$1: 00$

3pSA1. On the use of acoustical holography for the determination of intensity in structures. Anthony J. Romano, ${ }^{\text {a) }}$ Earl G. Williams, and Charles G. DelVecchio ${ }^{\text {a) }}$ (Naval Res. Lab., Code 7132, Washington, DC 20375-5000)

An overview of the methods for determining structural intensity from information obtained using near-field acoustical holography (NAH) is presented. Toward these ends, a presentation of the theoretical basis and practical methodologies involved in NAH is given, as well as a brief restatement of the structural intensity formulation appropriate for thin shells and plates. A method for obtaining the unknown displacement and stress components from a knowledge of surface pressure and normal velocity is discussed, and these methods are applied to experimental data obtained for the particular cases of a point-driven, fluid-loaded rectangular plate and cylindrical shell. A movie will be presented which displays the instantaneous structural intensity corresponding to the situations previously outlined. a) Also at Sachs/Freeman Associates, Inc., 1401 McCormick Dr., Landover, MD 20785.

3pSA2. Broadband control of multi-mode power flow in beams using wave vector sensors. G. P. Gibbs, M. Bronzel, C. R. Fuller, J. D. Blotter, D. E. Montgomery, and R. L. West (Dept. of Mech. Eng., Virginia Polytechnic Inst. and State Univ., Blacksburg, VA 24061-0238)

Recent work has demonstrated the use of wave filtering techniques to estimate traveling waves in the time domain. These techniques use PVDF surface mounted sensors in conjunction with a digital filter network to estimate the traveling flexural and extensional waves in beams for band-limited spectra. The traveling wave estimates are proportional to the power flow in the beam, and thus can be used as the cost function of an active control system. In this paper, experimental results are presented which demonstrate the broad band active control of flexural and extensional power flow in beams using surface mounted piezoceramic control actuators, surface mounted PVDF wave vector filters, and a TMS320C30 based adaptive controller. The results demonstrate flexural and extensional power flow attenuations of 5 to $20 \mathrm{~dB}$ for band-limited excitation. Independent verification of the total beam response before and after control (three dimensional) is found via a technique called experimental spatial dynamics modeling (ESDM). Velocity measurements from a scanning laser vibrometer are processed using the ESDM technique to produce a full 3-D velocity response field. These data can be further reduced using a method termed experimental spatial structural intensity (ESSI) to determine the complex intensity over the beam. Contour plots are presented showing flexural and extensional power flow in the beam for both uncontrolled and controlled cases. [Work supported by NASA Langley Research Center.]

\section{$1: 50$}

3pSA3. Laser interferometry for structural acoustics. Yves H. Berthelot, Jacek Jarzynski, Hyun-Gwon Kil, Lance Wills, and Ming Yang (School of Mech. Eng., Georgia Inst. of Technol., Atlanta, GA 30332-0405)

Laser interferometry offers the possibility of measuring in real time instantaneous surface velocities in the $\mu \mathrm{m} / \mathrm{s}$ range by noninvasive measurements. It can also be used to measure the three components of the surface vector velocity at several points simultaneously and, therefore, it is a promising way of measuring structural intensity and power flow in arbitrarily complex structures under laboratory conditions. Three different laser interferometric probes are currently being used at Georgia Tech for research in structural acoustics. The advantages and disadvantages of each system will be discussed within the context of structural intensity measurements. [Work supported by the Office of Naval Research, Code 332 SM.] 
3pSA4. Measurement of structural intensity. Gunnar Rasmussen (G.R.A.S. Sound \& Vibration aps, Skelstedet 10B, 2950 Vedbaek, Denmark)

The modeling of structural behavior leads to simplified models of very often complex structures. To get a better understanding of the energy flow inside and out of the structures, the development of measurement methods is very important. Dynamic movements are associated with vibratory and acoustic energy dissipation. If the surface area is large compared to an acoustic wavelength, acoustic intensity is a good indicator of dynamic activity. If the area is small compared to a wavelength at the frequencies in question, surface measurements using the vibratory motion on the structure surface may be a good indicator. By transfer of energy between structural parts, point power measurements should be applied. Measurements may be carried out using acoustical techniques for determination of structure borne energy. The acoustical transducers used must be able to measure correctly in very reactive environments. A proper calibrated intensity probe will offer a noncontact measurement of an area, which may be small or large depending on wavelength for measurement of vectors caused by dispersed waves. The use of two accelerometers or an accelerometer and a force transducer may also be useful for detection of energy flow in a structure. Acoustic calibration of intensity probes has been improved to meet standard requirements. Calibration of accelerometers and force transducers is carried out by substitution measurements against a third transducer. Measurements on structures has found many practical applications.

\section{Contributed Paper}

$$
\text { 2:40 }
$$

3pSA5. Numerical investigation of error mechanisms in near-field acoustic holography (NAH). Gerard P. Carroll (Structural and Hydroacoust. Res. Branch, Carderock Division, Naval Surface Warfare Ctr. (CDNSWC), Bethesda, MD 20084-5000)

Near-field acoustic holography (NAH) has been validated in precisely controlled laboratory conditions for small scale models. The practical validity of the approach in nonlaboratory settings for larger scale models, where increased background noise, inexact sensor positioning, and other environmental contaminants are likely, needs to be established. In this paper, the effect of these error mechanisms on the accuracy of cylindrical near-field acoustic holography are investigated numerically. This is accomplished using simulated hologram plane pressure data obtained from surface pressure and velocity results. The surface pressure and velocity are obtained using FEM/BEM calculations (SARA) for a ribbed cylindrical model. The simulated hologram plane data are obtained from the SARA results using a numerical implementation (Gaussian quadrature) of the Helmholtz integral. The errors in the holographic reconstructions associated with background noise, sensor placement, and other error mechanisms are revealed by comparing the holographically reconstructed results with the original SARA results. Since the ribbed cylindrical model under investigation is quite detailed, this approach is a good test of the ability of NAH to appropriately reproduce the complex surface behavior. [Work supported by ONR.]
3pSA6. Energy partitioning in a truss structure. Kai-Ulrich Machens and Ira Dyer (Dept. of Ocean Eng., MIT, Cambridge, MA 02139)

MIT is investigating both theoretically and experimentally wave propagation in truss structures. Experiments were conducted to measure energy partitioning in flexural, longitudinal, and torsional vibrations of specific truss members. The truss consists of 109 aluminum struts assembled via 35 joints to form a regular structure of 11 cells connected in series. A complete numerical analysis [Y. P. Guo, J. Acoust. Soc. Am. 96, 3291(A) (1994)] finds that energy equilibria among different wave types can be expected, the ratios of which are dependent upon the distinct classes of struts making up the truss. This was tested by simultaneous measurement of the transfer functions at various locations along the axis of each strut considered. The truss was excited by broadband white noise $(5-\mathrm{kHz}$ baseband) at one end of the truss. Wave number processing extracts two complex wave amplitudes, each associated with traveling waves propagating in opposite directions. Thus the spectral energy content of the strut is known and is compared to others in the same class. In addition, struts of other classes are measured to discuss issues related to equipartition of energy, pass/stop bands and natural damping of complex branched structures. 
GRAND BALLROOM SOUTH AND CENTRAL, 3:30 TO 5:30 P.M.

\title{
Plenary Session, Business Meeting, and Awards Ceremony
}

\author{
Jiri Tichy, Chair \\ President, Acoustical Society of America \\ Business Meeting \\ Presentation of certificates to new Fellows
}

Presentation of Awards

R. Bruce Lindsay Award to Beverly A. Wright

Gold Medal to Kenneth N. Stevens

Distinguished Speaker

Gerald T. Garvey

Assistant Director, Physical Sciences and Engineering, Executive Office of the President, Office of Science and Technology Policy, Washington, DC 20500

THURSDAY AFTERNOON, 1 JUNE 1995

GRAND BALLROOM SOUTH AND CENTRAL, 5:30 TO 6:30 P.M. Film Presentation

The Caroling of Atoms: The life's work of Harvey Fletcher

Harvey Fletcher is both "The father of stereophonic sound" and the "father of psychoacoustics." He was the first one to show during his Ph.D. work (with Robert Millikan) that the electron had a fixed quantized charge, and the first to accurately measure the threshold of hearing. He provided T. A. Edison with a shoe-box sized electronic hearing aid, manufactured by Western Electric, and personally demonstrated stereo to A. G. Bell. He produced the first electronic hearing aid, the first electronic (2-A) audiometer, discovered the critical bands of the cochlea, and is well known for "the Fletcher-Munson curves." This film presents some amazing footage from the 1950 celebration of the 25th Anniversary of the Acoustical Society in New York City that might bring tears to your eyes (54 mins, 16-mm color film). 


\title{
Session 4aAA
}

\section{Architectural Acoustics: Orchestra Shell and Stage Acoustics Design}

\author{
Peter D'Antonio, Chair \\ RPG Diffusor Systems, Inc., 65I-C Commerce Drive, Upper Marlboro, Maryland 20772
}

Chair's Introduction-8:15

Invited Papers

$8: 20$

4aAA1. An introduction to performance enclosure acoustics. Dennis A. Paoletti (Paoletti Associates, Inc., 40 Gold St., San Francisco, CA 94133)

This paper will provide an introduction and overview of the acoustical parameters typically established by acoustical consultants in the design of "enclosures" surrounding musicians performing on stage in theaters, concert halls, and multipurpose auditoria. A broad view of enclosure design must include facility design, musical instrument acoustics, engineering, theater consulting, and architectural design. Size, shape, configuration, and materials selection are all important re: the acoustical performance of the enclosure. However, storage for enclosure components, structural considerations (in the engineering design of the enclosure, as well as the engineering of the stage/backstage area that will house the enclosure), and operational and maintenance characteristics must also be considered. As complex as all of these issues are to balance during the design of a performing arts complex, not to mention budget, the success of the enclosure is ultimately in the hands, and ears, of the performing musicians, which leads to further considerations involving psychoacoustics, visual aesthetics, fables, environmental conditions, and even potential conditions surrounding a performance faculty and its performing arts organizations.

\section{8:40}

4aAA2. Measured effects of orchestra shells. J. S. Bradley (IRC, Natl. Res. Council, Montreal Rd., Ottawa, ON K1A 0R6, Canada)

The effects of adding orchestra shells or modifying orchestra shells were measured in six different concert halls varying in volume from 3000 to $32000 \mathrm{~m}^{3}$. Early, late, and total sound levels were seen to increase with the addition of an orchestra shell. Effective orchestra shells were found to increase total sound levels by up to $2 \mathrm{~dB}$ at mid-frequencies in audience areas of halls. Average changes to measured clarity could be larger. The measured increases in sound levels varied with location in the halls, and in several cases sound level increases were greatest towards the rear of halls. In one case, adding an orchestra shell was found to decrease low-frequency levels due to a shift of the seat dip attenuation. On-stage, larger effects were measured. Support values increased by as much as $5 \mathrm{~dB}$ with the addition of an orchestra shell. Partial changes to shells generally produced much smaller effects. In audience seating areas the effects of partial changes and adjustments to orchestra shells were usually less than estimated just noticeable differences in the measured quantities.

\section{9:00}

4aAA3. Stage house coupled shells. Christopher Jaffe (Jaffe Holden Acarbrough Acoustics, Inc., 114 A Washington St., Norwalk, CT 06854)

In the early sixties, the American Symphony Orchestra League conducted a survey of American orchestras to determine their preference of material for demountable symphonic enclosures. Oddly enough, Fiberglass reinforced polyester shells manufactured by Stagecraft Corporation received the number one rating in the country. The Stagecraft shells, constructed of 0.0625- to 0.125-in. Fiberglass sheet, and edge framed in aluminum, were coupled to stage house volumes through openings between the ceiling sections. In the low frequencies some coupling was achieved through the panels themselves, in view of their low transmission loss at the bottom end of the spectrum. This paper will document the success of these enclosures, and explain why these shells worked so well for orchestras performing in the sixties and seventies. Basic changes in multi-use theater design in the eighties reduced the effectiveness of these designs. However, several practitioners have developed new design solutions that have returned stage house coupled shells to the forefront once again.

4aAA4. Stage acoustics measurements with major orchestras. Dana Kirkegaard (Kirkegaard Acoustics, 4927 Wallbank Ave., Downers Grove, IL 60515)

This paper will address objective and subjective measures relating to the author's work onstage with major orchestras. Based on this research, some of the design challenges and potential solutions, including the use of QRDs, will be diagrammed. A formal paper will be available for distribution. 
4aAA5. Active and passive orchestra shells and stage acoustics. W. C. J. M. Prinssen and B. H. M. Kok (System for Improved Acoustic Performance, B. V. Uden, The Netherlands)

This spring the largest theater building of The Netherlands, the Chasse Theatre at Breda, was completed. Of the two main auditoria, which have similar stage houses, one has electronic variable acoustics by means of a SIAP system, including a stage system, and the other has mechanical variable acoustics which includes a large orchestra shell. It will be shown through measurements that active (electronic) stage acoustics can be at least as effective as passive (physical) stage acoustics. It is particularly interesting that loudness, reverberation time, and reflection patterns can be influenced independently with active systems. This flexibility is an advantage for meeting the needs of performers. Furthermore, active control of stage acoustics can also provide a significantly improved foldback to actors and singers for drama, opera performance, etc. when technical stage facilities and scenery make physical acoustic means difficult to apply. Since acoustic coupling of the stage to the auditorium main volume is an important factor, results will also be presented of the achievements of the SIAP system to correct auditoria which have spaces with (extremely) poor acoustic coupling such as balcony and under balcony areas. The orchestra pits, which are of different size and shape, but have the same passive acoustic treatment, will be compared.

\section{0:00-10:10 Break}

\section{0:10}

4aAA6. Adaptable acoustical shells incorporating modular reflective, absorptive, and diffusive surfaces. Peter D'Antonio (RPG Diffusor Systems, Inc., 651-C Commerce Dr., Upper Marlboro, MD 20772)

The optimum percentage and orientation of reflecting and diffusing surfaces in an acoustical shell was determined from objective and subjective measurements using conservatory students at the Cleveland Institute of Music comprising two string quartets, a brass quintet, and a hom duo. Objective impulse measurements were made with balloon bursts and five different microphone systems were used to obtain simultaneous DAT recordings. The Head Acoustics mannequin was placed within the group to determine ensemble blend without self-masking. Etymotic Research probe microphones were inserted into the ear canals of musicians to determine ensemble blend with self-masking, headband microphones were used to monitor ensemble blend with self-masking, an omnidirectional microphone was placed within the group as a monophonic control, and spaced omnidirectional microphones were placed in the front of the house to measure the projected sound quality. Analysis of these and other data led to the development of a variable acoustics modular performance shell utilizing reflecting, absorbing, and/or diffusing modules, which allows both variability and flexibility for different stage uses as well as sectional and personal acoustic environments for the performers. This approach was subjectively evaluated with the full Baltimore Symphony Orchestra with David Zinman conducting.

\section{Contributed Papers}

\section{0:30}

4aAA7. Supplemental partial height concert shell incorporating quadratic diffusor panels. Dana S. Hougland (David L. Adams Associates, Inc., 1701 Boulder St., Denver, CO 80211)

A recently completed project to modify the acoustical performance of Boettcher Concert Hall included the addition of several modifications to improve cross-stage hearing of the musicians. Boettcher Concert Hall utilizes an in-the-round seating arrangement, therefore, the enclosure design was restricted to partial height. Custom designed quadratic diffusors were incorporated into the concert shell design. Tests were conducted both on an individual panel section in an anechoic chamber and in situ. The results of the tests are presented.

\section{0:45}

4aAA8. Orchestra shell design and manufacture: The Wenger way. Ron Probst and Todd Benjamin (Wenger Corp., 555 Park Dr., Owatonna, MN 55060)

Wenger Corporation has manufactured orchestra shells for educational and professional spaces for over 30 years. This paper will present the design philosophy behind the Wenger line of acoustic shells. Pros and cons of standardized equipment versus custom design and manufacture will be discussed. Also, the methodology behind the design and development of the Diva Acoustic Shell and many of its innovative features will be examined.

$$
\text { 11:00-12:15 }
$$

PANEL DISCUSSION: Technical Aspects of Products 


\title{
Session 4aAB
}

\section{Animal Bioacoustics: Passive Acoustic Tracking of Animals: Methods and Applications}

\author{
Khosrow Lashkari, Chair \\ MBARI, 160 Central Avenue, Monterey, Califormia 93950 \\ Chair's Introduction-8:30 \\ Invited Papers

4aAB1. Effects of low-frequency sound (ATOC) on marine mammals: An experimental approach. Daniel P. Costa, Dawn Goley, Danielle Waples, Don Croll, Bumey Le Boeuf (Univ. of California-Santa Cruz), John Calambokidus Cascadia (Research Collective), and James Harvey (Moss Landing Marine Laboratory)

This presentation will outline the research program currently in place to study the effect of the California ATOC experiment on marine mammals. There are three phases to this research: (1) a preliminary baseline data collection period prior to any ATOC transmissions, (2) a pilot study (when ATOC transmissions would be manipulated to assess effects on marine mammals), and (3) a monitoring period (when transmissions are optimized for climate study, ATOC feasibility operations). During the pilot study ATOC transmissions would be 4 days on, 7 days off and changes in the animals distribution, abundance, and general behavior measured. This experimental pattern would be replicated to provide statistical power. Although the objectives are different for each period, the research methodology remains the same. Methods used will be aerial and shipboard surveys to assess distribution and abundance, shipboard observations to assess behavior, a towed array to assess acoustic behavior, photo-identification to examine long-term movements and stock identity, and very high frequency (VHF) radio tags and time depth recorders (TDR) to assess dive behavior and movements. Data collected during the preliminary period are being used to predict sample sizes necessary for studies that would be carried out during the pilot study.

\section{9:00}

4aAB2. Constraints on the sound production mechanism of blue whales. David S. Clark (Naval Command, Control and Ocean Surveillance Ctr., RDT\&E Div. 721, San Diego, CA 92152-5000), Mark A. McDonald, John A. Hildebrand, and Spahr C. Webb (Scripps Inst. of Oceanogr., UCSD, La Jolla, CA 92093-0205)

Broadband, high-fidelity recordings of blue whale calls are used to model parameters of the sound generation mechanism. Arrays of sonobuoys have been used to characterize and localize calling whales, providing broadband $(10-2400 \mathrm{~Hz})$, high dynamic range (90 $\mathrm{dB}$ ) recordings. Blue whales typically produce $15-$ to $20-\mathrm{Hz}$ sounds of up to $30-\mathrm{s}$ duration at levels of $185 \mathrm{~dB}$ re: $1 \mu \mathrm{Pa} @ 1 \mathrm{~m}$. The observed sound levels, frequencies, and harmonic content have implications for the sound generation parameters. Source levels provide constraints on volume displacements. Frequencies and harmonic content relate to resonant structures and their means of excitation. The data show harmonic series with selected overtones emphasized. The relative amplitudes of the overtones vary between different parts of the call.

4aAB3. Acoustic tracking of whales using hydrophone arrays: Implications for behavioral studies and population estimates. Christopher W. Clark (Comell Lab. of Omithol., Bioacoust. Res. Program, 159 Sapsucker Woods Rd., Ithaca, NY 14850)

Understanding how free-ranging whales use sound to communicate, navigate, and find food has been seriously hampered by the inability to consistently identify, locate, and track the animal producing the sound. Only in the most tractable situations (e.g., coastal species, shallow water, breeding/calving season) has it been possible to describe acoustic repertoires and prescribe biological functions. The potential use of such acoustic techniques, in combination with traditional visual observation methods, for population census efforts has been clearly demonstrated in the case of bowhead whales. Recent application of large aperture arrays and beamforming techniques has demonstrated the feasibility of using such systems for locating and tracking vocally active pelagic species. There is every reason to believe that a combination of acoustic tracking and visual observation techniques can be successfully extended for use as a census tool to produce meaningful estimates about relative abundance on blue, finback, and minke whales. Passive acoustic tracking methods, (towed arrays and fixed horizontal arrays) have now been successfully applied to follow individually vocalizing whales for many days, acoustically observe herds of animals, and describe species specific seasonal changes in distribution and relative abundance.

9:50

4aAB4. Localization of animal calls via hyperbolic methods. Steve Mitchell and John Bower (Bioacoust. Res. Program, Comell Univ., 159 Sapsucker Woods Rd., Ithaca, NY 14850)

For over a decade the Comell Bioacoustics Research Program has been using passive sparse acoustic arrays to locate calling marine mammals in the near field in shallow water, as well as birds and other animals in air. The techniques used have been cross correlation followed by all-pairs hyperbolic geometric positioning, using the simplest propagation model, and iterative nonlinear least-squares 
error minimization. This talk describes some of our experiences with this process, including the results of calibrated field tests. Applications to whale population censusing and research into the countersinging behavior of song sparrows will be discussed. Many bioacoustic passive array experiments depend on accurate locations at the extremes of the near field, where range is difficult to determine. Analysis techniques for passive near-field arrays have appeared in the literature over the past 50 years. Recently there has been much work devoted to using detailed propagation modeling to improve the accuracy of undersea localization of marine vessels. It is argued that, in the case of animal calls, there are also accuracy improvements to be had from improved signal modeling.

\section{0:15-10:30 Break}

\section{Contributed Papers}

\section{0:30}

4aAB5. Using combined acoustic tracking and visual observation techniques to study humpback whales. Adam S. Frankel and Christopher W. Clark (Cornell Biacoust. Res. Program, 159 Sapsucker Woods Rd., Ithaca, NY 14850)

The technique of acoustic location was combined with visual tracking and behavioral observation to study humpback whales on the winter mating grounds in Hawaii. The behavior and movements of nonvocalizing whales and singers could be studied simultaneously. Social interactions that had not been previously described were observed. These included affiliations of singers and nonsingers while the singer continued to sing. Visual tracking also allowed the comparison of visual and acoustic locations to verify the accuracy of the acoustic locations. Acoustic location allowed the simultaneous tracking of several humpback singers, and their relative distribution and orientations were examined. Results indicate that song mediates inter-singer separation and that $4 \mathrm{~km}$ was the preferred minimum spacing between singers off Hawaii. Humpbacks have been described as being distributed within the 100 fathom contour while on the mating grounds. However, singers were acoustically located up to $13 \mathrm{~km}$ offshore in water as deep at 305 fathoms. While singers are typically described as stationary, combined tracking techniques revealed singer speeds of up to $3.6 \mathrm{~km} / \mathrm{h}$. In conclusion, the combination of acoustic and visual tracking provided a more comprehensive view of humpback whale behavior and distribution than either technique separately.

\section{0:45}

4aAB6. The use of passive towed arrays for surveying marine mammals. Jeffrey Norris, William Evans, Troy Sparks (Texas A\&M Univ., P.O. Box 675, Galveston, TX 77553), and Robert Benson (Texas A\&M Univ., College Station, TX 77843-3367)

The first marine mammal acoustic survey using a towed passive hydrophore array was recently completed. Other cetacean surveys have used static, how-frequency arrays; the array used is a broadband, mobile system, receiving both baleen and toothed whale signals. For acoustic censusing and tracking (ACT) to become an accepted technique, it's biases and assumptions must be described. ACT has a number of advantages, including a permanent record of all effort, compared to visual methods which are instantaneous samples, with no permanent record. This record permits measurement of perception and availability biases. A dynamic strip width can be calculated by continuously recording SPL. An area of $154621 \mathrm{~km}^{2}$ was surveyed in the north central Gulf of Mexico during seven cruises, recording 1431 tapes. Recording effort occurred over $11997 \mathrm{~km}$ of a total transect distance of $12580 \mathrm{~km}$, corresponding to a $95.4 \%$ level of effort; a concurrent visual survey yielded a $48.5 \%$ level of effort. A total of 157 contacts from 13 cetacean species were recorded, including 67 sperm whale (Physeter macrocephalus), 22 pantropical spotted dolphin (Stenella attenuata), one baleen whale, 327 unidentified dolphin, and 30 unidentified cetacean contacts. Three-dimensional distributions were determined for sperm whale and pantropical spotted dolphin. Estimates of cetacean populations were computed based on this effort.

\section{1:00}

4aAB7. Three-dimensional localization of a blue whale using broadband matched-field processing for range and depth, and plane-wave adaptive beamforming for azimuth. G. L. D'Spain, W. A. Kuperman, W. S. Hodgkiss, and L. F. Berger (Marine Phys. Lab., Scripps Inst. of Oceanogr., San Diego, CA 92152-6400)

During a recent shallow water experiment conducted $10-15 \mathrm{~km}$ west of San Diego, blue whale calls were recorded simultaneously on two underwater hydrophone arrays, an 18-element, 205-m-aperture horizontal line array, and a 64-element, 115-m-aperture vertical line array. These data are used to localize the calling whale in 3-D space and then to make estimates of its source level. First, adaptive plane-wave beamforming is used with the horizontal line array data to obtain finely resolved bearing estimates. Then, the bottom bathymetry profiles along these bearing estimates are used as input to a 2-D full-wave-field normal mode code (written by Mike Porter) for generating "replica vectors" (i.e., predictions of the acoustic field) received by the vertical line array. A search in range and depth for the best match between the replica vectors and the actual field measured by the vertical array ("matched-field processing") then is performed to obtain the best-fit position of the whale in 3-D space. Finally, estimates of the source level of the whale calls are made. [Work partially supported by ONR, Code 321.]

\section{$11: 15$}

4aAB8. Simultaneous source panging and bottom geoacoustic inversion using shallow water, broadband dispersion of fin whale calls. G. L. D'Spain, W. A. Kuperman (Marine Phys. Lab., Scripps Inst. of Oceanogr., San Diego, CA 92152-6400), C. W. Clark, and D. K. Mellinger (Cornell Univ., Ithaca, NY 14850)

Beamformed time series of fin whale calls from the North Atlantic recently have been obtained. Spectrograms of these recordings have an unusual appearance-three to four downsweeping clicks occur in rapid succession, almost as if the whale was stuttering. These arrivals can be explained in terms of the differences in group velocity between the lowestorder modes that propagate in the shallow water (about $350 \mathrm{~m}$ ) waveguide. By matching predicted spectrograms calculated from a shallow water waveguide model with actual spectrograms, estimates of both the range of the whale and the compressional wave velocity in the ocean bottom are obtained. The approach makes various assumptions, including that the whale emits a signal with a downsweep rate of $25 \mathrm{~Hz}$ per second. However, known deviations from the assumptions can be incorporated into the modeling. The results have several implications such as that whale signals may provide loud, broadband, tomographic sources of opportunity for determining oceanographic/geophysical properties, and that some differences in whale call recordings from different populations of the same species may be caused by propagation effects. Might whales take advantage of propagation-induced distortions in order to learn something about the ocean medium? [Work partially supported by ONR.] 
4aAB9. The MAP tag: A new marine mammal tracking system. Cyrus Bazeghi (Dept of Comput. Eng., Univ. of California, Santa Cruz. CA 95064) and Guy W. Oliver (Univ. of California, Santa Cruz, CA 95064)

Development of the movement and position (MAP) tracking system was motivated by limitations of currently available tags for marine mammals. The MAP tag employs a global positioning system (GPS) receiver to accurately fix an animal's position when it surfaces and a tri-axial magnetometer and velocity-time-depth recorder to track in three dimensions underwater movements between surfacings. The tag is microprocessor controlled allowing flexibility and user control of sampling regimes. Data are stored in a high capacity datalogger. To minimize the energy budget the GPS receiver is only switched on when the animal nears the surface and switched off when a fix is obtained. The unit is battery-powered, selfcontained and capable of deployment on animals descending to $1500 \mathrm{~m}$. By attaching a pinger to the animal it can also be tracked acoustically, allowing calibration of passive acoustic tracking systems. In its current configuration, the MAP tag is designed for deployment on elephant seals, but with relatively minor reconfiguration of the package it could be deployed on many large marine vertebrates. It is anticipated that the capability of the MAP tag will dramatically enhance future studies of marine mammal behavior, navigation, orientation, and foraging. [Work supported by ONR.]
4aAB10. The study of acoustic interactions of territorial song sparrows, Melospiza melodia, using passive acoustic tracking. John L. Bower (Bioacoust. Res. Program, Comell Lab. of Ornithol., 159 Sapsucker Woods Rd., Ithaca, NY 14850)

A passive sparse acoustic array of eight microphones was used to characterize the patterns of acoustic interactions of territorial song sparrows. The rectangular array was placed in a four hectare open field (near Ithaca, New York) that contained 11 song sparrow territories. Recordings were made approximately every 3 days from early April, when male song sparrows arrived back from their wintering grounds, through July, when the breeding season ended. Computer analysis of field recordings showed that the array was able to capture vocalizations of all 11 territorial males in the field, and that these vocalizing males could be accurately located. Data were taken during three critical periods of the breeding season: (1) territory establishment, (2) settlement of territories by females, and (3) nesting. For each period, the timing of song, inter-song intervals, and song-type matching were discemed for males. Patterns of acoustic interactions recorded during periods of visually observed male-male conflict were also investigated. These patterns were used to evaluate several hypotheses that seek to explain the function of acoustic interactions in song sparrows.

FRIDAY MORNING, 2 JUNE 1995

AUDITORIUM, 8:55 TO 11:30 A.M.

\title{
Session 4aMU
}

\section{Musical Acoustics: Guitar Acoustics/Performance}

\author{
Uwe J. Hansen, Chair \\ Department of Physics, Indiana State University, Terre Haute, Indiana 47809
}

\author{
Chair's Introduction-8:55
}

Invited Papers

9:00

\begin{abstract}
4aMU1. Acousties of guitars. Thomas D. Rossing (Phys. Dept., Northern Illinois Univ., DeKalb, IL 60115)
The guitar can be considered to be a system of coupled oscillators. The plucked strings excite the top plate, which in turn transmits vibrational energy to the air cavity and back plate. Sound is radiated quite effectively by the vibrating plates and through the sound hole. The considerable amount of research on the guitar during the past 20 years, especially as it reflects on further development of this popular instrument, will be reviewed.
\end{abstract}

4aMU2. Acoustical design criteria for the guitar. Bernard E. Richardson (Dept. of Phys. and Astron., Univ. of Wales College of Cardiff, P.O. Box 913, Cardiff CF2 3YB, UK)

The acoustical function of the guitar is now reasonably well understood. The various modes of the vibrating string couple to structural and air-cavity modes of the body. These in turn interact with the surrounding air thus enhancing the radiation of energy from the string to the listener. The modal properties of the instrument are thus of central importance in determining its sound quality. It is of great interest to the maker to know how these modal properties might be adjusted to produce instruments of pre-determined sound quality. This paper will give a resume of experimental and theoretical work undertaken at Cardiff to determine formal relationships between physical properties of the instrument (construction and materials) and psychoacoustical evaluation of guitar tones. The aim of this work is not to define parameters of an idealized "perfect" instrument—-that would be a futile goal. Instead, the aim is to identify those features of construction which have a perceptible effect on the guitar's sound and to define acceptable limits for modal properties. The latter will be discussed theoretically, but taped demonstrations will be presented to emphasize the points being made. 
4aMU3. The guitar: Air modes and radiated sound fields. Ingolf Bork, Ulrich Heise (Physikalisch-Technische Bundesanstalt, Braunschweig, Germany), and Uwe J. Hansen (Indiana State Univ., Terre Haute, IN 47809)

The experimental technique of modal analysis is generally used to display normal modes of structural vibrations. It provides a phase sensitive method of representing a slow motion animated display of the standing waves supported by the structure. The technique also allows exaggerated displacement representation on the computer screen for ease of observation. An essential element in the technique is associated with its ability to retain spatial phase information for a predetermined geometrical point distribution on the structure in relation to the point of excitation. The work discussed in this paper utilizes this ability to retain phase information and extends the technique to represent the radiated sound field. The grid of observation points is removed from the structure and is replaced by a grid covering the space where the sound field is to be modeled. The excitation point is retained on the structure. This will be illustrated by observing air resonances in a guitar in computer animation, and by representing the radiated sound feld of that guitar at various frequencies.

10:30

4aMU4. Designing and testing of new guitars by criteria of applied physics and psychoacoustics. Michael Kasha (Ctr. for Music Res. and Inst. of Molecular Biophys., Florida State Univ., Tallahassee, FL 32306-3015)

This program represents the interaction of the authors as designer of new guitars, with skilled luthiers as developers, and musicians as evaluators. The ultimate goal is the development of the full brilliance and power which the instrument should be capable of, using models derived from the elementary laws of vibration mechanics. The aim is to enhance the response of the four-octave range of the classical six-string guitar by introducing structural asymmetry, borrowing the principle inherent in the violin family of instruments. As a guide, the standard formulation of the boundary conditions, and the frequency dependence of mechanical impedance, are incorporated-all this within the construction limitations imposed by the luthier, the materials, and the aesthetics of the instrument. The Backhaus criterion of brilliance is adopted, wherein the harmonic spectral intensity distribution is targeted in which the fundamental is dominant, and with decreasing intensity of higher harmonics. A specially designed anechoic chamber has been constructed for testing real-time harmonic spectra of string instrument pairs: conventional versus new designs (taking into account variations of player technique). Measurements will be made to compare with the Backhaus criterion. Ultimately, the judgement of musicians and of the general spectrum of musical auditors will decide the success of this program.

\section{1:00}

4aMU5. Adapting intuitive ideas and applied mechanics to the improvement of guitar acoustics: The luthier's view. Thomas Humphrey, Richard Schneider, and Michael Kasha (Ctr. for Music Res. and Inst. of Molecular Biophys., Florida State Univ., Tallahassee, FL 32306-3015)

The mechanical structure of the guitar underwent a sudden transformation about 150 years ago in the hands of Antonio de Torres Jurado, under the demand on the part of Julian Arcas, a virtuoso guitar performer of the day, for greater acoustical power and brilliance in the guitar. The Torres design features three revolutionary changes, (a) the transverse structural bars of the existing Mediterranean instrument were replaced by longitudinal functional bars, coupling the bridge action to the soundboard, (b) the body dimensions were greatly increased, lowering the body and cavity resonance frequencies, (c) the string length was greatly increased, leading to higher string tension requirements, thereby further increasing acoustical power. Each luthier will present his approach to understanding the mechanical action of the torres design, and its modifications for the acoustical requirements of modern musical performances. The presentation will be followed by live demonstrations of various guitars by musicians. 


\title{
Session 4aNS
}

\section{Noise: Progress Report and Discussion on the Continuing Activity of ASA's Role in Noise and Its Control}

\author{
Robert M. Hoover, Chair
}

Hoover and Keith, Inc., 11381 Meadowglen, Houston, Texas 77082

Invited Paper

8:30

4aNS1. Progress report and discussion on the continuing activity on ASA's role in noise and its control. Robert H. Hoover (Hoover and Keith, Inc., 11381 Meadowglen, Houston, TX 77082)

A discussion meeting is being sponsored by the Technical Committee on Noise to review progress made to date on the actions initiated at the Denver, 1993 meeting to increase the role of the ASA in noise and noise control. Much of this action evolved out of an all-day workshop held at the Ottawa, 1993 ASA meeting. Members of the steering committee and others involved in related supporting activity will review specific progress made in the areas of education, increasing public awareness about noise and noise control, and encouraging joint activity in this area with other professional organizations. Included will be a report on a trial presentation on acoustics and noise given to 6 high school classes. Discussion about, and review of opportunities for involvement with, these activities will be encouraged.

\section{Session 4aPP}

\section{Psychological and Physiological Acoustics: Harvey Fletcher Memorial Session I}

\author{
Jont B. Allen, Cochair
}

AT\&T Bell Laboratories, Room 2D-553, 600 Mountain Avenue, P.O. Box 636, Murray Hill, New Jersey 07974-0636

Mohan M. Sondhi, Cochair

AT\&T Bell Laboratories, Room 2D-553, 600 Mountain Avenue, P.O. Box 636, Murray Hill, New Jersey 07974-0636

\author{
Chair's Introduction-8:00
}

Invited Papers

8:15

4aPP1. Harvey Fletcher: A son's reflections. Stephen Harvey Fletcher (3046 Comanche Ln., Provo, UT 84604)

Harvey Fletcher was born on 11 September 1884, in Provo, Utah, of pioneer parents. He received a B.S. degree from Brigham Young University. He then entered the University of Chicago, where he worked with Robert Millikan on the determination of " $e$ ", and earned his Ph.D. in physics, summa cum laude, in 1911. He was made director of acoustical research at Bell Laboratories in 1928, and the following year, co-founded the Acoustical Society of America. By 1935 he was made director of physical research at Bell Laboratories. As such, he supervised the work of Shockley, Brattain, and Bardeen in the development of the transistor; Dean Wooldridge, James Fiske, Charles Towne, and many other outstanding physicists. Upon retirement from Bell Laboratories, Harvey Fletcher accepted a position as professor of electrical engineering at Columbia University, a position he held for 3 years. In 1952, he returned to BYU as director of research, and from 1960 until his death at the age of 96 , he actively pursued the study of musical tones. 
4aPP2. Harvey Fletcher: Friend and scientific critic. Leo L. Beranek (975 Memorial Dr., Ste. 804, Cambridge, MA 02138-5755)

Harvey Fletcher was active in World War II both in the early days of defense research under the auspicies of the National Defense Research Committee and later as Chief of Section 17.3 of the Office of Scientific Research and Development. He was a member of the National Research Council committee that started and oversaw the research of the Electro-Acoustic Laboratory at Harvard University of which I was named Director. Within a year or so Dr. Fletcher's OSRD Section 17.3 took over the supervision. In the latter part of World War II, I was named Director of the Systems Research Laboratory under the Office of Field Research. Dr. Fletcher was appointed as one of a group of five to oversee the activities of this Laboratory. This paper is a collection of anecdotes and remembrances of Fletcher in the fast-moving and important military research and development period from 1940 until 1945 when I reported often to him. Personal contacts with him continued after the war, frequently at first, and sporadically for the next three decades. "Uncle Harvey," as I came to call him, was both a friendly guide and a demanding scientific critic.

\section{9:00}

4aPP3. Harvey Fletcher: Some personal recollections. Cyril M. Harris (Dept. of Elect. Eng. and Graduate School of Architecture, Columbia Univ., New York, NY 10027)

The author of this paper describes his first visit with Harvey Fletcher in 1940 at the Acoustical Research Department of Bell Telephone Laboratories, then located on West Street in New York City. Five years later, he was invited by Dr. Fletcher to join the Laboratories. A brief description is given of some of the work carried out in Acoustical Research at that time. Upon his mandatory retirement at age 65, "Uncle Harvey" accepted the position of Visiting Professor in the Department of Electrical Engineering at Columbia University. In 1951 the author left Bell Labs for a teaching position in Europe; on his return to the U.S. a year and a half later, he again sought Dr. Fletcher's counsel and it was suggested he take Fletcher's place at Columbia University. This made it possible for Dr. Fletcher to return to Utah, where he devoted the rest of his distinguished career to research in musical acoustics at Brigham Young University.

\section{9:15}

4aPP4. Harvey Fletcher and auditory theory. Nelson Yuan-sheng Kiang (MIT, Harvard Medical School, Massachusetts Eye and Ear Infirmary, 243 Charles St., Boston, MA 02114, and Massachusetts General Hospital)

Harvey Fletcher was an original thinker whose career is worth examining for future scientists. Trained as a physicist, he developed a pragmatic approach to psychophysics that virtually defined the engineering approach to acoustic communication. Although his practical achievements were considerable, he also tried to integrate all that was known about hearing in the 1953 version of his "space-time pattern theory of hearing." It is instructive to compare that formulation with the ideas of today after more than four decades of intense research. Many of the issues that concemed Fletcher have been resolved; many others remain uncertain. For example, the observation that low-frequency stimuli can mask high-frequency stimuli whereas high-frequency stimuli cannot mask low-frequency stimuli was accorded great significance by Fletcher. It is now known that a key factor lies in the asymmetry of tuning curves for basal and apical turn auditory-nerve fibers. On the other hand, the full implications of these phenomena, especially for central processing mechanisms, is virtually unexplored. What is clear is that a major effort to produce a unified theoretical framework for research in hearing is long overdue and a modem Fletcher would be welcome today.

\section{9:30}

\section{4aPP5. Fletcher and pitch. John R. Pierce (Dept. of Music, Stanford Univ., Stanford, CA 94305)}

Preparing remarks on Fletcher's work on pitch changed drastically. My view of the development of our present understanding. In 1940 Jan Schouten showed that canceling out of the fundamental of a pitched tone did not change pitch. Schouten called the surviving pitch residue pitch. This inspired a spate of papers by people ignorant of earlier work. I had known Fletcher [Phys. Rev. 23(3), 427-437 (1924)] had shown that filtering out the fundamental and lower harmonics of a variety of musical sounds did not change the pitch, and, through synthetic sounds, that mere equal frequency spacing of tones did not give a pitch equal to the spacing. Thus, the pitch heard was evoked by harmonic partials. Unhappily, Fletcher proposed an unsound explanation in terms of production of the fundamental in the ear by nonlinearities. In a latter report. Fletcher [J. Acoust. Soc. Am. 1, 311-343 (1930)] gave an incomplete analysis of the functioning of the cochlea. Among his valid conclusions he said "the pitch of a tone is determined both by the position of its maximum stimulation on the basilar membrane and also by the time pattern sent to the brain. The former is probably more important for high tones and the latter for low tones." That is our present understanding. Fletcher [J. Acoust. Soc. Am. 23, 637-645 (1951)] subsequently gave a "correct" analysis of the functions of the cochlea, agreeing with the experimental work of von Bekesy, and going a little beyond papers a year earlier by Peterson and Bogert and by Zwislocki.

\section{9:45}

4aPP6. From Fletcher to Knudsen. Harry Levitt (Ctr. for Res. in Speech and Hear. Sci., City Univ. of New York, Graduate School and University Center. 33 W. 42nd St., New York, NY 10036)

Harvey Fletcher was not only an outstanding researcher and director of one of the world's leading research laboratories, but was also an inspiring teacher and mentor. Vern Knudsen was one of many outstanding researchers who were deeply influenced by Fletcher. Knudsen's contributions in architectural acoustics, psychoacoustics, audiometry, hearing aids, and tactile stimulation were of groundbreaking importance and reflected Fletcher's remarkable influence. As a close disciple of Fletcher, Knudsen was also in a position to learn about many of Fletcher's other accomplishments which were substantial, but unknown to the outside world. 
4aPP7. Prediction of articulation scores. Christine M. Rankovic (Dept. Speech-Language Pathol. and Audiol., Northeastern Univ., 360 Huntington Ave., Boston, MA 02115)

Harvey Fletcher developed articulation theory to enable the prediction of the speech transmission capacity of imperfect communication systems, thereby eliminating the need for the time-consuming speech recognition testing that is otherwise required to differentiate among systems. Two assumptions underlie the theory: (1) a speech signal in each of a number of contiguous frequency bands comprising the audible spectrum contributes independently to the articulation score (percent-correct for lists of nonsense syllables); and (2) the separate contributions are additive. The approach of Fletcher and his colleagues was to define the articulation index, $A$, an entity for which these assumptions hold true. The equations for calculating $A$ contain variables representing hearing acuity. critical bandwidth, masking, loudness, perception, and frequency importance weights so that the communication system's frequency response and gain are translated into perceptual terms. In addition to the derivation of the theory. Fletcher's 1953 book provides a method for calculating $A$ using charts and tabled data, as well as an extensive validation study that included a variety of suboptimal systems. Fletcher's presentation of articulation theory is far more comprehensive than two popular versions [ANSI S3.5-1969; N. R. French and J. C. Steinberg, J. Acoust. Soc. Am. 19, 90-119 (1947)]. Differences among these versions of the $A$ calculation and consequences for predicting articulation scores will be discussed. [Work supported by NIH.]

\section{0:45}

4aPP8. Speech recognition at higher than normal speech and noise levels. Gerald A. Studebaker and Robert L. Sherbecoe (Memphis Speech and Univ. of Memphis Hear. Center, 807 Jefferson Ave., Memphis, TN 38105)

One finding of the early investigators at the Bell Telephone Laboratories was that the articulation index (AI) decreased when speech was amplified above normal conversational levels (French and Steinberg, 1947; Fletcher and Gaht, 1950). Recent efforts to predict the performance of hearing-impaired subjects using hearing aids under real world conditions has produced a renewed interest in this phenomenon. A study will be described that attempts to quantify the effects of higher than normal speech and noise levels. Data for normal-hearing subjects revealed that raising the speech level from 65 to $100 \mathrm{~dB}$ SPL reduced the AI by about 0.003 units per $\mathrm{dB}$ and that this slope remained fairly constant for $\mathrm{S} / \mathrm{N}$ ratios between -2 and $+19 \mathrm{~dB}$. Data on hearing-impaired subjects are currently being collected. Results for both subject groups will be compared with those obtained by Fletcher and his colleagues.

\section{1:00}

4aPP9. Probability theory and speech perception. Arthur Boothroyd (City Univ. of New York, 33 W. 42 St., New York, NY 10036)

Fletcher recognized the value of probability theory in developing a quantitative approach to speech perception-hence the articulation index (AI). He also recognized the need to allow for violations of the independence assumptions that underly basic probability theory. For example, in nonsense syllables, the probability $\left(p_{w}\right)$ of recognizing a word equals $p_{p}^{n}$, where $p_{p}$ is the probability of recognizing the constituent phonemes and $n$ is the number of phonemes per word. In real words, however, $p_{w}=p_{p}^{i}$. where $n \geqslant j \geqslant 1$. Similarly, extending the methods of AI, the probability of recognition of words in sentences $\left({ }^{s} p_{w}\right)$ can be shown to be related to the probability of recognition in isolation $\left({ }^{\prime} p_{k}\right)$ by the equation ${ }^{s} p_{w}=1-\left(1-{ }^{i} p_{w}\right)^{k}$, where $k$ is an exponent reflecting the contribution of the sentence context. From these two basic equations one can derive relationships among many measures of speech perception, ranging from the phonetic level to the sentence level. The empirical values of the exponents $j$ and $k$ can be used both to quantify the effects of various structural and contextual constraints and to assess an individual's use of those constraints. [Work supported by NIDCD Grant No. 10078.]

\section{1:15}

4aPP10. Harvey Fletcher and modern compression hearing aids. Edgar Villchur (Foundation for Hear. Aid Res., P.O. Box 306, Woodstock, NY 12498)

Harvey Fletcher described the effect of a transmission channel on speech recognition in terms of the channel's "articulation" plotted against channel gain. For normal listeners, articulation increases with gain to a maximum and then levels off or decreases. For hearing-impaired listeners, however, Fletcher noted: "... the gain $\alpha_{1}$ corresponding to the tolerable level is reached before it is large enough (to bring articulation to a maximum)." This occurs in cochlear impairment because the dynamic range of hearing is reduced by recruitment. It is the problem to which the design of modern compression hearing aids is addressed. Compression aids compensate for recruitment by providing increased relative gain for weak speech sounds; thus they allow the articulation to reach higher values before tolerance levels are exceeded. An analysis of compression/equalization signal processing is presented. The essential elements of this processing are: (1) more than one channel of full-dynamic-range compression (the K-AMP design achieves similar results in a different way), (2) post-compression frequency-response shaping, and (3) adjustability of the compression in each channel, and of the equalization, to fit the amplified speech into the residual dynamic and frequency range of hearing of the individual patient. 


\title{
Session 4aSA
}

\section{Structural Acoustics and Vibration: Structure-Borne Noise Techniques}

\author{
Joseph M. Cuschieri, Cochair \\ College of Engineering, Florida Atlantic University, Boca Raton, Florida 33431-0991 \\ Gerard Carroll, Cochair \\ Carderock Division, Naval Surface Warfare Center, Bethesda, Maryland 20084-5000
}

Chair's Introduction-8:15

Invited Papers

8:25

4aSA1. Physical parameters of a welded t-joint that impact structural acoustics. J. Adin Mann, III, Matthew Camey, and Patricia Driesch (Aerosp. Eng. and Eng. Mech., 2019 Black Eng. Bldg., Iowa State Univ., Ames, LA 50011)

The influence of welded joints on the accuracy of analytical structural acoustic models is investigated. A beam with a T-shaped rib attached to it is studied. Welding a $t$-joint causes geometry variation, static stress, and material variations that are not currently included in computational models of the structural acoustics of a t-joint. Two techniques of processing the experimental data are used to show that the geometry and the static stresses caused by welding can have a significant impact on waves propagating past the joint and the resulting radiated sound power. Likewise experimental results show that the material variation caused by the welding of the joint has minimal impact on the wave propagation and sound radiation from the beam. The processing techniques are a phase-speed tracking filter and wave-number filtering. The phase-speed tracking filter shows an increase in the phase speed of propagating waves near the joint. There is also a reduction in the group delay of wave packets propagating past the joint and a change in the reflection coefficient of structural waves incident on the joint. The wave-number filtering is used to quantify the amount of far-field sound radiation from the area of the t-joint on the beam. Finally an analytic model that describes a rib as imparting a force and moment on a beam in reaction to the incident structural waves is modified to include the influence of the weld fillet geometry and static stress induced by welding. [Work supported by ONR, Dr P.B. Abraham Scientific Officer.]

4aSA2. The response and radiation of ribbed panels. J. Dickey and G. Maidanik (David Taylor Res. Ctr., Annapolis, MD 21402)

The dynamic response of a panel with an infinite number of regularly spaced line impedances (i.e., regularly ribbed), or a finite number which may be irregularly spaced and nonidentical, is a system which can be solved "exactly." Exact, that is, to within the approximations employed in developing the equations of motion for the panel and the ribs. Even though these approximations may preclude realistic modeling in some (usually high) frequency ranges, or with some configurations (e.g., wide ribs), these canonical models exhibit many characteristics of experimental and numerical data obtained on real and more complete structures. This makes them very useful and cost effective in identifying observed characteristics and evaluating noise control measures. Models which describe flexural or longitudinal waves on membranes or thin plates have been developed by the authors. These models are reviewed and used to describe the response and radiation of a panel into an adjacent fluid. The panel is driven either by a line drive parallel with the ribs or an incident plane wave. There are a host of phenomena which manifest themselves; some of which are: (1) pass and stop bands, (2) localization and de-localization, (3) aliasing, (4) free wave dispersion and the splitting of the dispersion curves due to fluid loading, (5) Bragg diffraction, and (6) radiation efficiency. Also presented is the effect on these phenomena of: (1) altering the strict periodicity or identity of the ribs, (2) the loss factor in the panel, and (3) the degree of fluid loading.

4aSA3. Cavity-backed simply supported plate for acoustic and structural response measurements. Yvan Champoux, Serge Brunet, and Alain Berry (GAUS, Mech. Eng. Dept., Univ. de Sherbrooke, Sherbrooke, PQ J1K 2R], Canada)

Originally, an acoustic cavity has been designed and fabricated to study active noise control strategies in a closed volume. It has a parallelepipedic shape of dimensions $0.868 \mathrm{~m} \times 1.15 \mathrm{~m} \times 1.0 \mathrm{~m}$ with walls made of $13-\mathrm{cm}$-thick reinforced concrete. The top is closed with a simply supported 6.63 -mm-thick aluminum plate. The plate support was carefully designed to meet the simply supported boundary conditions. The plate is excited by a point force and its structural response is measured with force and accelerometer transducers. The acoustic field inside the cavity is also measured using microphones. Comparisons between calculated and measured natural frequencies of the plate shows that for most of the modes found in the $0-500 \mathrm{~Hz}$ range, the discrepancy is less than $1 \mathrm{~Hz}$. Quadratic mean surface velocity and mean volume sound pressure have been calculated from frequency response functions measured for centered and off-centered point force excitations. Comparisons with calculated values shows very good agreement. The relative contribution of the various plate modes and cavily modes are well distinguishable. 
4aSA4. Experience predicting structure-borne noise within a prototype rectangular acoustical enclosure. Robert J. Bernhard and Nancy M. Franchek (1077 Herrick Labs., School of Mech. Eng., Purdue Univ., West Lafayette, IN 47907-1077)

Approximately 10 years ago a large rectangular enclosure was designed and constructed using a steel super-structure and sandfilled, double-panel plywood walls. The enclosure is approximately the size of a transportation vehicle passenger cabin ( $1.85 \mathrm{~m}$ by 1.05 $\mathrm{m}$ by $1.05 \mathrm{~m}$ ). The enclosure was built such that any of the walls could be replaced with more realistic structures such as vehicle panel constructions. The enclosure has been modified many times to accommodate various experimental apparatus. In this paper, the design of the enclosure will be described and some of the original data used to verify its characteristics will be shown. Recently, the enclosure has been modified to resemble structure (f) described by the Structure-borne Noise Committee of INCE-USA. One wall of the structure has been replaced by a simply supported panel. Experimental results have been compared to analytical and numerical predictions for both internal acoustic excitation and mechanical excitation of the panel. These results will be discussed. In addition, an effort has been made to design a small version of this enclosure which could be used for round robin testing. Preliminary results of this effort will also be discussed.

\section{0:05}

4aSA5. The French benchmark on computational methods and experiments in structural acoustics. C. Valor (Société Française des Mécaniciens (S.F.M.), Société Française d'Acoustique (S.F.A.). Paris, France)

The idea of creating a working group to perform benchmark on computational methods and experiments in structural acoustics originated as a conclusion of the congress "Prediction of the noise emitted by vibrating structures," held in March 1991. This group was effectively created in 1993, by S.F.M. and S.F.A. and is constituted by researchers working in laboratories, industries, and consulting companies. The name of the participants with their affiliation will be given at the oral presentation. In order to simplify the publications of this working group, a fictitious name (C. Valor) has been created and represents the participants. Each year several one-day meetings are organized, they are devoted to the comparison of the different results obtained on each benchmark case. The discrepancies are analyzed, leading to new calculations if necessary. When a common agreement is obtained by the working group, each participant having performed the results writes a scientific report collected by the working group. The final goal is to publish a book giving the synthesis of all the work produced by the group. During the presentation, the computational and experimental benchmark will be presented and examples of results will be discussed.

\section{0:30-10:40 Break}

\section{Contributed Papers}

\section{0:40}

4aSA6. Power transmission variance predictions in complex systems with dissipation. John Burkhardt and Richard Weaver (Dept. of Theor. and Appl. Mech., Univ. of Illinois, Urbana, IL 61801)

The eigenstatisties of damped complex systems are examined for the purpose of improving statistical response predictions such as those formulated in statistical energy analysis and statistical room acoustics. Both the statistical distribution of modal decay rates and the effect of dissipation on the intermodal correlation of the natural frequencies described by the gaussian orthogonal ensemble (GOE) of random matrix theory are explored. It is found that the modal decay rates are distributed according to a chi-square distribution whose degree depends on the distribution of damping in the systern and the wavelength of the disturbance. The intermodal correlations of the natural frequencies are found to be unaffected by the presence of moderate damping. Level repulsion (the absence of near degeneracies) and spectral rigidity (the near regularity of the spectrum) are found to conform to the prediction of the GOE provided the system is reverberant. The variance of the power transmission function for an irregularly shaped membrane is formulated using a GOE-lype natural frequency spectrum and chi-square distributed modal decay rates. Numerical simulations of membranes are performed which confirm the calculated effect of a distribution of decay rates on power transmission characteristics.

\section{0:55}

4aSA7. Influence of local static stress on structural acoustics: Experiment and modeling. Hareen K. Gangala and J. Adin Mann, III (Aerosp. Eng. and Eng. Mech., 2019 Black Eng. Bldg., Iowa State Univ., Ames, IA 50011)

An experimental setup was designed to locally introduce stresses in a steel beam. The far-field sound power radiated by the beam was experimentally calculated for various magnitudes of the induced stresses. As a model, a finite element code was developed for the equation of motion due to a modified theory of acoustoelasticity [Pao et al., J. Appl. Mech. 58, 11-17 (1991)]. The theory allows the static stress components to be additions to the elastic moduli of the body. The sound power radiated by a beam was calculated from the FEM model results for axial, transverse, shear, and combined distributions of the stress components. Experimental results showed that the sound power increased by up to $1 \mathrm{~dB}$ as the static stress was varied. Results from the FEM model showed that all the individual stress components contribute to the radiation and the combined effect was about a $1 \mathrm{~dB}$ increase in the radiated power. Both experimental and model results suggest that static stresses induced by discontinuities such as welding and bolting can play a role in the mechanisms of the sound radiation at a discontinuity in a structure. [Work supported by ONR.]

4aSA8. Effect of thickness variations on the sound radiation from beams. Julie C. Slaughter, J. Adin Mann, III, and Daniel O Adams (Aerosp. Eng. and Eng. Mech., 2019 Black Eng. Bldg., Iowa State Univ., Ames, LA 50011)

The effect that spatial thickness variations of a beam has on the beam's far-field sound radiation was studied. The steel beams that were used in the initial tests show that at low-frequency ranges $(500-1500 \mathrm{~Hz})$ the thickness variations acted as discontinuities along the beam that increased sound radiation. However, at higher frequency ranges $(2000-4000 \mathrm{~Hz})$ the thickness variations, when spaced at certain intervals, acted as global stiffness changes and decreased the sound radiation. This decrease occurred when the spacing of the variations corresponded to a structural wavelength. Composite beams with thickness variations have also been built and tested. The spatial thickness variations appear to have a similar effect on the sound radiation as in the steel beams. The two data processing techniques used to evaluate the influence of the spatial thickness variations were a phase-speed tracking filter to quantify wave propagation in the 
structure and a wave-number domain filter to quantify far-field radiation. Results from these tests will be used to help determine how quieter materials can be designed. [Work supported by NSF.]

\section{$11: 25$}

4aSA9. Structural vibration noise from open grid bridge decks. J. M. Cuschieri (Ctr. for Acoust. and Vib., Dept. of Ocean Eng., Florida Atlantic Univ., Boca Raton, FL 3343I) and M. Tournour (Sherbrooke Univ., Sherbrooke, Canada)

The contribution to the overall tonal component of the noise from the vibration of the grid section of an open grid bridge deck due to excitation by the interaction with the vehicle tires is investigated. Using vibration field measurements of the grid and laboratory measurements of the acoustic radiation efficiency, it is shown that the contribution to the overall noise level measured at the curb from the vibrations of the grid is insignificant. The main reason for this result is the poor acoustic radiation efficiency of the grid. Because of the large percentage of open area, the plate-like modes of the grid deck have very poor radiation efficiency. Similarly, the individual grid elements, based on their size and the frequency range where the predominant excitation from the tire takes place, are also very inefficient acoustic radiators. The significance of this result is that mitigation procedures that only reduce the vibration of the grid will not significantly reduce the overall radiated noise. [Work sponsored by FDOT.]

\section{1:40}

4aSA 10. Tire noise from open grid bridge decks. J. M. Cuschieri (Ctr. for Acoust. and Vib., Dept. of Ocean Eng., Florida Atlantic Univ., Boca Raton, FL 33431) and S. Gregory (General Dynamics, Groton, CT)

Having identified that the contribution to the overall noise from the grid vibrations is insignificant, the attention is focused on the noise radiated from the vibrations of the tire. Field measurements of tire vibrations are rather difficult, therefore laboratory measurements and analytic mod- eling were used to understand the contribution from the tire vibrations. The laboratory measurements of both vibration and radiated noise compare well with the estimates from the analytic modeling. Using an estimate of the interaction force between the tire and the grid, from the field vibration data, it is shown that the estimated radiated noise level is comparable to the field measured noise level at the curb. This leads to the conclusion that the noise from the tire vibrations is the dominant source, and mitigation procedures must consider ways of reducing the excitation of the tire. This conclusion is verified by sound level measurements performed with the contribution from the grid vibrations removed. [Work sponsored by FDOT.]

\section{1:55}

4aSA 11. Combined large area sensor-actuator tile for the NRL-ABC platform. Robert D. Corsaro and Brian Houston (Naval Res. Lab., Code 7130, Washington, DC 20375-5350)

A new research platform has been constructed for underwater studies of sensor/actuator coupling mechanisms. It consists of a 15-tile array of "ABC" tiles, where each tile contains a large area actuator, pressure sensor, and velocity sensor, where the latter is constructed by summing and integrating the outputs of four accelerometers. This paper presents details of the tile design and the predictive models used. Issues addressed include spatial sampling, near-field sensing, internal resonances, and both direct and extraneous coupling mechanisms, all of which can contribute to complicate the system transfer functions. Acoustic characteristics of the $A B C$ tile were evaluated in the NRL Large Pool Facility, both in the free field and when mounted on a backing structure, and these results are compared with predictions. Implications for local control of the actuator's surface are also discussed.

\title{
Session 4aSC
}

\section{Speech Communication: Potpourri (Poster Session)}

\author{
Sandra Gordon-Salant, Chair \\ Department of Hearing and Speech Sciences, University of Maryland, College Park, Maryland 20742
}

\section{Contributed Papers}

\begin{abstract}
All posters will be on display from 8:30 a.m. to 12:00 noon. To allow contributors an opportunity to see other posters, contributors of odd-numbered papers will be at their posters from 8:30 to 10:15 a.m. and contributors of even-numbered papers will be at their posters from 10:15 a.m. to 12:00 noon. To allow for extended viewing time, posters will remain on display until 8:00 p.m.
\end{abstract}

4aSC1. Lexical distinctiveness in lipreading: Effects of phonemic equivalence classes on the structure of the lexicon. Edward T. Auer, Jr. and Lynne E. Bernstein (Ctr. for Auditory and Speech Sci., Gallaudet Univ., 800 Florida Ave. N.E., Washington, DC 20002)

Speech perceived on the basis of viewing a talker's face affords less phonetic distinctiveness than acoustic speech. Effects of this reduced distinctiveness can be estimated in relation to the structure of the mental lexicon. Based on empirical measures of phonetic confusability, recoding rules can be defined for mapping fully specified phonological forms into lexical equivalence classes. For example, under the recoding rule that $/ \mathrm{b} /$ and $/ \mathrm{p} /$ are in the same phonemic equivalence class the words "bat" and "pat" map into the same lexical equivalence class. After applying a set of recoding rules to a large online lexical database, the resulting structure of the lexicon can then be studied quantitalively. One such measure of the recoding effects on the lexicon is percent information extracted (PIE) [D. M. Carter, Comput. Speech Lang. 2, I-11 (1987)]. Lexical statistics describing the results of applying sets of recoding rules derived from analyses of visual-phonetic confusability to a 30000 -entry lexicon will be pre- 
sented. Implications for the use of top-down lexical constraints in resolving bottom-up visual-phonetic ambiguity during lipreading will be discussed. [Work supported by NIH.]

4aSC2. The effects of featural priming on word recognition. $E$. Morrish (Nottingham Trent Univ., Nottingham NG11 8NS, UK)

The aim of this paper is to test the hypothesis that broad phonetic categories [V. Zue, Proc. IEEE 73, 1602-1615 (1985)] can act as a contact representation for lexical access. The results of this work will be considered in terms of implications for the neighborhood activation model. The technique used in this experiment was "phonetic priming" [A. Jongman and J. Sereno, Working Papers Cornell Phon. Lab. 7, 151-176 (1992)]. Subjects were asked to make a lexical decision about a target word which was preceded by a subphonemic prime (either an ambiguous fricative or an ambiguous stop). The prime either matched or mismatched the manner of articulation of the initial phoneme of the target. Reaction time results showed an interaction between initial and prime, so that when these matched, the subjects' reaction time was inhibited. Furthermore the neighborhood effect [Goldinger et al., J. Memory Lang. 28, 501-518 (1989)], that words from a low-density similarity neighborhood are recognized faster than words from a high-density neighborhood, was enhanced by the presence of the phonetically related prime. These results suggest that broad phonetic features may activate lexical candidates.

4aSC3. Multinomial models of speech perception. Court $S$. Crowther (UCLA Phon. Lab., Dept. of Linguistics, Los Angeles, CA 90024-1543) and William H. Batchelder (Univ. of California, Irvine)

It is often the case that two different acoustic cues can influence perception of both of two adjacent phonemes within a syllable. In such cases, it is of interest to determine whether the cues are evaluated independently; that is, does a listener's evaluation of one cue influence evaluation of the other cue? Another, related question is whether, as some researchers claim, phoneme decisions are dependent in the sense that, when cued by the same information, the decisions compete for the information. This study modeled data from a two factor, four category experiment in which $F 1$ offset frequency and vowel duration cue both voicing and vowel identity in CVC syllables. A family of multinomial processing tree models [e.g., D. M. Riefer and W. H. Batchelder, Psychol. Rev. 95, 318-339 (1988)], of which the fuzzy logic model of perception for the two factor, four category design is a special case, was developed and tested to explore the independence issues. This modeling approach is highly flexible and allows one to test a wide range of hypotheses regarding speech cue processing.

4aSC4. Talker variability and token variability in spoken word recognition: Effects on repetition priming in lexical decision and an explicit recognition memory task. Emily A. Lyons (Language Perception Lab., Dept. of Psych., Park Hall, SUNY, Buffalo, NY 14260)

Recent research (e.g., Goldinger, 1993) has suggested that representations of spoken words in memory may be veridical exemplars that encode instance-specific information, such as characteristics of the talker's voice. This account of spoken word recognition was examined in work presented at a recent meeting of the Acoustical Society (Luce and Lyons, 1994). Evidence was presented in support of the claim that exemplar-type representations are used in explicit recognition memory tasks, but that more abstract representations play a role in tasks such as lexical decision. However, the previous work did not make clear the explicitness of the information encoded in exemplar-type representations; specifically whether only gross factors such as talker variation are encoded. or whether within speaker variation is also encoded. The present research expands on these previous results by comparing the effects of talker variation and samespeaker token variation on a repetition priming and an explicit recognition memory task. [Work supported by NIDCD.]

4aSC5. Lexical influence on the perception and segmentation of speech: Evidence from the migration paradigm. Sven L. Mattys and Arthur G. Samuel (Dept. of Psych., SUNY at Stony Brook, Stony Brook, NY 11794-2500)

The present study addresses the issue of lexical influence on perception through a new paradigm based on the migration of linguistic units from one ear to the other when two stimuli are presented dichotically. For ex- ample, "dentast" and "kolbisk" could be presented dichotically, with subjects judging if "dentist" was presented. The migration of the vowel, leading to erroneous perception of a pre-specified target, occurred less often with word targets than with nonsense word targets. This result indicate that the lexical representation of a signal imposes some restriction on the acoustic-phonetic stage of processing of the signal. Furthermore, when the two items of the pairs were played binaurally rather than dichotically, the migration rate increased substantially, but the size of the lexical effect remained unchanged. However, the lexical effect disappeared when the migrating vowel was located in the stressed syllable of the stimuli. In this condition, the lexical resistance collapses and words become as susceptible to migrations as nonsense words. This second result may suggest that lexical access is initiated on strong syllables of words: when the stressed syllable is mispronounced, lexical access is impaired, eliminating the lexical effect. [Work supported by AFOSR, NIMH, and BAEF.]

4aSC6. Phonological and phonotactic influences on perception of two non-native vowel contrasts. Robert $A$. Avery and Catherine T. Best (Dept. of Psych., Wesleyan Univ., Middletown, CT 06459 and Haskins Labs., 270 Crown St., New Haven, CT 06511)

Listeners' native phonology constrains their perception of non-native phonetic distinctions. Although most non-native contrasts are discriminated poorly, recent findings that certain contrasts are discriminated well led to development of a "perceptual assimilation model" (PAM), which proposes that the perceptual assimilation of non-native phones to native categories predicts discrimination performance [e.g., Best et al., JEP:HPP 14, 345-360 (1988)]. The current study investigated native phonotactic influences on three assimilation patterns. Non-native contrasts that: (1) assimilate to two categories (TC) and show excellent discrimination; (2) assimilate equally to a single category (SC) and show poor discrimination; (3) assimilate as a category goodness difference (CG) and show good discrimination. American adults labeled and discriminated lip-rounding contrasts among Norwegian high front vowels $[/ \mathrm{j} /-/ \mathrm{y} / \mathrm{t} / \mathrm{t} / \mathrm{H} / \mathrm{y}]$ in CV's and CVC's. In both contexts, Norwegian $/ \mathrm{f} /$ of course was assimilated to English $/ \mathrm{i} / \mathrm{/} / \mathrm{t} /$ was assimilated to $/ \mathrm{u} /$. Although $/ \mathrm{y} /$ was assimilated equally to $/ f /$ or $/ \mathrm{i} /$ in CVCs, it was virtually always called $/ \mathrm{i} /$ in CVs, compatible with the English phonotactic constraint against final lax vowels. Thus, $/ 4 /-/ y /$ showed TC assimilation and good discrimination in both syllabic contexts. In CVs, $/ \mathrm{V} / \mathrm{-} / \mathrm{y} /$ showed $\mathrm{SC}$ assimilation and poor discrimination; in CVCs, it showed TC or CG assimilation and good discrimination.

4aSC7. Phonotactic and metrical influences on subjective ratings of spoken nonsense words. Michael S. Vitevitch, Paul A. Luce (Language Perception Lab., Dept. of Psych., SUNY, Buffalo, NY 14260), Jan Charles-Luce, and David Kemmerer (SUNY at Buffalo)

This research examined English speakers' intuitions about the phonological "goodness" of nonsense words. Subjects rated bisyllabic, CVCCVC nonsense words that varied in phonotactic probability and stress placement. Using a ten-point scale, subjects judged how English-like the nonsense words sounded. Although all nonsense words were phonotactically legal in English, subjects showed strong preferences for stimuli composed of highly probable phonotactic contributions. Moreover, subjects judged nonsense words with strong-weak stress patterns as constituting "better" sounding English words than nonsense words with weak-strong patterns. No interaction between phonotactic probability and stress was observed. These results will be discussed in light of recent findings regarding adults' (Auer and Luce, 1993) and infants' (Jusczyk, Luce, and Charles-Luce, 1994) sensitivity to the phonotactic configurations of spoken stimuli. [Work supported by NIDCD.]

4aSC8. The segmental representation of words as revealed by priming in a lexical decision task. James $R$. Sawusch and Nancy $J$. Palmer (Dept. of Psych., Park Hall, SUNY, Buffalo, NY 14260)

In previous studies, a priming task has been used to explore the nature of the segmental representation of words. The phonetic overlap between a prime and a target was varied and the speed of naming or lexical decision to the target examined. The previous results showed that when phonemes occurred in the same syllable position in prime and target, responses to the target were faster than for control trials where the prime and target had no phonemes in common. In contrast, when prime and target shared pho- 
nemes but the positions of the phonemes in prime and target were different, no facilitation of target responses was found. In a new series of studies, this result is further examined using new stimuli and a dichotic priming format. Results will be discussed in terms of their implications for the nature of the abstract, segmental representation that underlies word recognition and lexical access. [Work supported by NIDCD Grant DC00219 to SUNY at Buffalo.]

4aSC9. Are coronals different? The influence of the lexicon on coronal identification. Rochelle Newman, James R. Sawusch, and Paul Luce (Dept. of Psych., Park Hall, SUNY, Buffalo, NY 14260)

In previous studies of lexical effects on phoneme identification, robust effects have been reported for voicing series with noncoronal places of articulation (e.g., $/ \mathrm{b} /-/ \mathrm{p} /$ or $/ \mathrm{g} /-/ \mathrm{k} /$ ). These same effects have been much more variable, smaller, and often absent for coronal voicing series $(/ \mathrm{d} /-\mathrm{t} / \mathrm{t})$. Previously, results were presented suggesting that neighborhood density could have effects similar to those of lexical status in phoneme identification tasks for noncoronals. For coronals, neighborhood density did not have an effect, again paralleling the lexical results. In new studies, additional coronal stimuli have been tested. Series have used word-nonword series to examine the influence of lexical status and nonword-nonword series to examine the influence of lexical neighborhood (the number of words similar to the target). The new stimuli included $/ \mathrm{d} /-/ / /$ series where the initial stop is part of a consonant cluster. The results for $/ \mathrm{d} /-/ \mathrm{t} / \mathrm{series}$ in clusters show robust lexical influences on phoneme identification. These results will be discussed in terms of their implications regarding the status of coronals and the nature of consonants in clusters. [Work supported by NIDCD Grant DC00219 to SUNY at Buffalo.]

4aSC10. The acoustic characteristics of babble produced by infants with and without early onset otitis media with effusion. Susan Rvachew and Elzbieta B. Slawinski (Dept. of Psych., Univ. of Calgary, 2500 University Dr. NW, Calgary, AB T2N 1N4, Canada)

Prelinguistic speech development was studied longitudinally in two groups of infants: nine infants in the early onset group experienced their first episode of otitis media with effusion (OME) at or before the age of 6 months; nine infants in the late onset group experienced no OME before 9 months of age. All children were assessed at $6,9,12,15$, and 18 months of age. Each assessment consisted of an audiometric assessment and a recording of babble/speech produced by the infant. Acoustic analysis of the babble/speech samples revealed the following: all infants showed an increase in the frequency of canonical babble with age, when canonical babble is defined according to the acoustic criteria suggested by Oller [in B. Lindblom and R. Zetterstrom (Eds.) Precursors of Early Speech (1986)]; the mean canonical babble ratio was greater for the late onset group in comparison with the early onset group at all age levels; the vowels produced by some infants with recurring or chronic OME were characterized by a restricted range of second formant values. These findings suggest that the canonical babble ratio may be a useful measure for investigating the relationship between OME and speech delay in infants and young children. [Work supported by MRC.]

4aSC11. Effect of relative amplitude, presentation level, and vowel duration on perception of voiceless stop consonants by normal and impaired listeners. Mark S. Hendrick (Dept. Commun. Disorders, Univ. of Alabama, Tuscaloosa, AL 35487) and Walt Jesteadt (Boys Town Nall. Res. Hosp., Omaha, NE 68131)

In the present study, previous work on the influence of relative amplitude and presentation level in listeners with normal hearing and those with sensorineural hearing loss is extended to include (1) a comparison of relative amplitude manipulation in the presence of neutral formant transitions versus manipulation of both formant transition and relative amplitude and (2) the additional variable of vowel duration. Synthetic CV stimuli were used, and the amplitude of the burst relative to the vowel in the F4-F5 frequency range was varied across a $20-\mathrm{dB}$ range. In experiment 1 , some stimuli had neutral formant transition values and relative amplitude manipulations; other stimuli had both formant transition and relative amplitude manipulations. For stimuli in experiment 2, neutral formant transition values were used, relative amplitude was manipulated, and vowel duration ranged from 14 to $200 \mathrm{~ms}$. Results from experiment showed no significant difference between subject groups when only relative amplitude information was manipulated, but significant differences when both relative amplitude and formant transition information was present. Results from experiment 2 indicated that vowel duration influenced the use of relative amplitude by listeners with normal hearing, but did not have this effect for listeners with sensorineural hearing loss. [Work supported by NIH.]

4aSC12. Reading disability: A deficit in the processing of rapid spectral changes or in phonological coding? Maria Mody (Dept. of Otolaryngol., Kennedy Ctr., Albert Einstein College of Medicine, 1300 Morris Park, Bronx, NY 10461) and Michael Studdert-Kennedy (Haskins Labs., New Haven, CT 06511)

Reading-impaired children have been said to suffer from an auditory deficit, indexed by difficulties in processing formant transitions [P. Tallal, Brain \& Lang. 9, 182-198 (1980)]. Having established that poor readers $(n=20)$ made significantly more errors than good readers $(n=20)$ on discrimination between stop-vowel syllables, contrasting in initial $F_{2}$ and $F_{3}$ transitions $(/ \mathrm{ba} /$ and $/ \mathrm{da} /$ ), but not between nonspeech sinewave analogs of $F_{2}$ and $F_{3}$, the present study compared the same groups on identification of (i) a /sel-stel/ synthetic continuum, where $F_{1}$ transition cued the contrast, and (ii) two hybrid /su- $\int \mathrm{u} /$ continua, each biased toward one end $(/ \mathrm{s} /$ or $/(\mathrm{J})$ of a synthetic fricative series by the formant transitions of naturally spoken vowels. Poor readers did not differ from good readers in phoneme boundaries, but did exhibit shallower identification functions, significantly so on the fricative continua. The results do not support the hypothesized auditory deficit: Less consistent discrimination or identification by poor readers on speech tasks but not on the nonspeech control task, suggests phonological rather than auditory difficulties.

4aSC13. Interrelationships between ethanol intoxication and speech. Harry Hollien (Inst. for Advanced Study of the Commun. Process., Univ. of Florida, Gainesville, FL 32611) and Camilo A. Martin (Chemical Abuse Treatment Ctr., Veterans Admin. Med. Ctr., Gainesville, FL 32601)

The focus of this paper is on (1) establishing well-controlled procedures which will permit reasonably precise study of the effects of intoxication on speech and (2) assessing correlations between physiological and behavioral measures of intoxication. In the first instance, selection and exclusionary criteria will be discussed and their impact on the resulting experiments noted. Included will be (1) subject selection (familial history, background, health, gender, drug/medication status, drinking patterns), (2) dosage protocols (control over and monitoring of intoxication, modifications), and (3) procedural constraints (speech tasks, experimental controls). Some of the criteria and procedures employed proved satisfactory, others did not and had to be modified. Second, two aural-perceptual experiments (eight talkers, 106 auditors) will be reported; a scaling model was used in the first, three variations of a blind sort procedure in the second. Two patterns of subject behavior were found; one correlated well with the physiological measures of intoxication but the other did not. Moreover, the differences in the speech shifts did not appear directly related to subjects' drinking patterns. [Research supported by NIH.]

4aSC14. Shifts in fundamental frequency and articulation resulting from intoxication. G. Allan Alderman, Harry Hollien, Camilo Martin, and Gea DeJong (Inst. for Advanced Study of the Commun. Process., Univ. of Florida, 63 Dauer Hall, Gainesville, FL 32611)

Two groups of subjects were administered controlled doses of alcohol while breath alcohol concentration $(\mathrm{Br} A C)$ measurements were made at regular intervals. They were recorded reading a 30-s passage when they reached preset $\mathrm{BrAC}$ windows. Fundamental frequency measurements were calculated and compared for sober $(0.00 \mathrm{BrAC})$ and intoxicated $(0.12$ $\mathrm{BrAC}$ ) productions. The number of misarticulations occurring during the readings also were assessed. In the first study, subjects were grouped on the basis of whether they were rated as sounding intoxicated at $0.12 \mathrm{BrAC}$ (ratings were performed by 50 auditors using a 5-pt. scale). Subjects who sounded intoxicated were placed in one group, while those that did nol were placed in a second. The first group showed a consistent, but statistically nonsignificant decrease in $F 0$ as a result of intoxication; group 2's $F 0$ changes were not consistent. In addition, the first group showed a higher mean increase in misarticulations than did group 2. The second population was grouped by drinking level (heavy, medium, or light); none 
showed a statistically significant change in $F 0$. Moreover, misarticulations increased (nonsignificantly) as drinking level increased. The results will be correlated with data from other studies. [Research supported by NIH.]

4aSC15. Speaking rate and alcohol intoxication. Gea DeJong, Harry Hollien, Camilo Martin, and G. Allan Alderman (lnst. for Advanced Study of the Commun. Process., Univ. of Florida, 63 Dauer Hall, Gainesville, FL 32611)

Eighteen subjects were recorded at five different levels of intoxication: i.e., at BrAC levels of $0.00,0.04-0.05,0.08-0.09,0.12-0.13$, and 0.09 0.08 . Two of the speech tasks completed were: reading a standard passage and repeating diadochokinetic syllables as fast as possible. The effects of intoxication on speaking rate were measured by the time needed to read the rainbow passage and produce 20 utterances of "pataka," "shapupi," and "buttercup." The first of these reflects the subjects' ability to sustain motor speech and the second their motor speech competence. Although variation was observed among speakers, the overall tendency was for both the diadochokinetic test and the rainbow passage to increase in length (s) from sober $(\mathrm{BrAC}=0.00)$ to the intoxicated level $(\mathrm{BrAC}=0.12)$. Further, the aural perceptual experiments carried out in parallel suggest that listeners might use rate when judging the level of intoxication. That is, speakers judged as highly intoxicated at $0.12 \mathrm{BrAC}$ (ratings were performed by 50 auditors using a 5-pt. scale) exhibited a mean difference of $3.05 \mathrm{~s}$ between sober and intoxicated utterances, whereas those speakers who did not sound intoxicated showed a mean difference of $0.05 \mathrm{~s}$. [Research supported by NIH.]

4aSC16. Predicting speech improvement from clinical profiles in stutterers. Peter Howell (Dept. of Psych., Univ. College London, Gower St., London WCIE 6BT, England)

Procedures for assessing dysfluencies in stuttered speech have the limitation that they show poor inter-judge agreement. This constitutes a serious problem for, amongst other things, assessing how a stutterer's speech benefits from therapy. A critical review of alternative procedures that are available indicates that they do not offer any better prospect for assessing improvements due to treatment than do the traditional procedures. Several factors are identified which might lead to the limitation in agreement. A new procedure which overcomes some of the problems identified was devised and applied to a group of stuttering children before and after they received a 2-week intensive therapy course. The new procedure produced substantially higher levels of agreement than those reported in the literature. This procedure is used to measure the benefit each individual child achieved after treatment. Clinical review data on these children were then employed to see whether any aspect of the interview could have predicted the child's response to treatment. The child's general health and cognitive skills appear to be reliable indications as to treatment prognosis. [Work supported by MRC.]

4aSC17. Acoustic and perceptual effects of endoscopic nasal surgery. Marilyn Y. Chen (Res. Lab. of Electron. and Dept. of Health Sci. and Technol., MIT, Rm. 36-511, Cambridge, MA 02139) and Ralph Metson (Massachusetts Eye and Ear Infirmary, Boston, MA 02114)

The aim of this paper is to determine the effects of surgical alterations of nasal anatomy on the spectral characteristics and perceptual attributes of speech. Five patients who underwent endoscopic nasal surgery were recorded prior to surgery, and 1 week and 1 month after surgery. The utterances were six vowel types adjacent to nasal consonants and stop consonants. In addition to endonasal surgery enlarging the sinus openings, some patients had a correction of deviated septum, resection of a turbinate, or removal of polyps. Acoustic analysis was carried out for nasalized vowels, non-nasalized vowels, and the nasal consonants $/ \mathrm{m} /$ and $/ \mathrm{n} /$. Significant differences in spectral properties were observed in the consonants and nasalized vowels recorded before and after surgery. For example, changes were found in the prominence of extra peaks due to nasalization and the first formant bandwidth was narrower for the nasal consonants and nasalized vowels. All except one patient perceived a change in their speech post-operatively. Attempts were made to account for the speech changes by incorporating the anatomical alterations caused by surgery into a speech production model. [Work supported by NSF NIH, and LeBel fellowship.]

4aSC18. Intraspeech masking in hearing-impaired listeners. Van Summers and Marjorie R. Leek (Army Audiol. and Speech Ctr., Walter Reed Army Med. Ctr. Washington, DC 20307-500I)

Upward spread of masking (USM), the masking of an acoustic signal by energy at lower frequencies, tends to increase with presentation level and is more pronounced in listeners with sensorineural hearing impairment (HI listeners) than in normal-hearing (NH) listeners. At high presentation levels, HI listeners may experience a decrease in the cues available from higher frequency regions due to masking produced by high-amplitude first formant $(F 1)$ resonances. In the present study $\mathrm{HI}$ and $\mathrm{NH}$ listeners labeled synthetic consonant-vowel stimuli at moderate and high presentation levels. $F 1$ regions were attenuated by $0,6,12$, or $18 \mathrm{~dB}$ to test whether $F 1$ attenuation might reduce USM, making cues present at higher frequencies more available. Performance was tested in quiet and in broadband noise sufficient to mask initial consonantal bursts. For both groups of listeners, noise reduced labeling accuracy. For normal-hearing listeners, performance was not affected by presentation level or $F 1$ attenuation. While some $\mathrm{HI}$ listeners showed clear improvernents in labeling with $F 1$ attenuation and/or increases in presentation level, the pattern did not hold across the entire group. The results will be discussed in light of the severity and configuration of the individual hearing losses.

4aSC19. Acoustic and perceptual investigation of breathy voice. Ratree Wayland, Scott Gargash, and Allard Longman (Dept. of Modern Lang. and Linguistics, Cornell Univ., Ithaca, NY 14853)

Although breathy voice is typically characterized by an increase in spectral noise, it is notoriously difficult to devise a computational method to distinguish breathy from clear (modal) voice. The present study successfully makes use of an algorithm, originally developed to quantify aspects of pathological voice quality [G. de Krom, J. Speech Hear. Res. 36, 254-266 (1993)], which computes a harmonics-to-noise ratio (HNR). The algorithm calculates the harmonics-to-noise ratio using a comb filter defined in the cepstrum domain to separate the harmonics from the noise. Performance of the algorithm was tested on three speakers ( 2 male, 1 female) of Javanese producing a word list of 31 minimal breathy/clear word pairs. Results showed that the algorithm reliably distinguished breathy from clear tokens for all three speakers, with higher HNRs for clear than for breathy tokens. Moreover, accurate performance was obtained for nearly all frequency ranges investigated $(60-2000 \mathrm{~Hz}, 2000-$ $3000 \mathrm{~Hz}, 3000-5000 \mathrm{~Hz}$ ). A comparison to orher methods (such as $\mathrm{Hl}-$ H2) will also be presented. Finally:-perceptual rating experiments will be conducted to determine if the algorithm's performance correlates with perceived degree of breathiness.

4aSC20. Modeling pathologic vocal quality. Part I. Jody Kreiman and Bruce R. Gerratt (Div. of Head and Neck Surgery, UCLA School of Medicine, 31-24 Rehab Ctr., Los Angeles, CA 90024-1794)

Previous studies of pathologic vocal quality have not yielded a standard set of scales for describing disordered voices, possibly due to the limited number of voices studied. The present study used large sets $(n=80)$ of male and female voices, representing a broad range of diagnoses and vocal severities. Eight experts judged the dissimilarity of each pair of voices, and responses were analyzed using nonmetric individual differences multidimensional scaling. Results indicate that differences between listeners in perceptual strategy are so great that the fundamental assumption of a common perceptual space must be questioned. Group spaces revealed a single "feature" (severity of pathology). Spaces for individual listeners were characterized by clusters of voices which seemed structured by "family resemblances": Each voice in a cluster resembled others with rspect to some property, but the same property did not constitute the resemblance for all voice pairs. Further, voices did not appear consistently in the same clusters across listeners. In the absence of a common perceptual 
space - of a shared sense of how voices sound similar - valid perceptual scales cannot be defined. The results lead to the conclusion that standardization of perceptual labels for voice quality is theoretically impossible.

4aSC21. EMMA and $x$-ray microbeam comparison. Dani Byrd (Haskins Labs., 270 Crown St., New Haven, CT 06511-6695), Catherine P. Browman (Haskins Labs.), Louis Goldstein, and Douglas Honorof (Haskins Labs. and Yale Univ.)

In the past, much articulatory movement data have been obtained using the $x$-ray microbeam. Currently, however, magnetometer systems such as EMMA are becoming predominant. A unique data set, addressing concerns of consistency between these instruments is reported. A single speaker was recorded with both instruments reading the same utterances: "It's a [pV'CVp] again." Data from two $x$-ray microbeam (XRMB) runs were collected on the same day (pellets re-placed for the second run); 27 months later the parallel EMMA data were collected. Vertical movement of the lower lip, tongue tip, and tongue body during the VCV's was analyzed. Extrema positions (amplitudes) and distance and time between these positions (gestural displacements and durations) were obtained. The results demonstrate that the correlation between EMMA and XRMB runs is very high, almost as high as that between the two XRMB runs.

\begin{tabular}{|l|l|l|l|}
\hline$r$ values $(p<0.0001)$ & amplitude & displacement & duration \\
\hline XRMB-1 \& EMMA & 0.9898 & 0.8615 & 0.7570 \\
\hline XRMB-2 \& EMMA & 0.9852 & 0.8948 & 0.7210 \\
\hline XRMB-1 \& XRMB-2 & 0.9901 & 0.9107 & 0.7933 \\
\hline
\end{tabular}

Using further analyses of variance it was concluded confidently that the results obtained using EMMA for articulatory data collection do not differ substantially from those obtained for similar data collected at different time with the $x$-ray microbeam. [Work supported by NIH.]

4aSC22. An acoustic and articulatory study of anticipatory labial coarticulation. Marios Fourakis and Ying $\mathrm{Xu}$ (Dept. of Speech and Hear. Sci., Ohio State Univ., 110 Pressey Hall, 1070 Carmack Rd., Columbus, $\mathrm{OH} 43210$ )

The acoustic and articulatory aspects of anticipatory labial coarticulation were examined. One female native speaker of American English read a list containing ten repetitions of nonsense V1CV2 sequences where V1 was always [i], C was one of [s], []], [z], [3], and V2 was one of [i], [u], or [a]. The movement of upper and lower lip and jaw were recorded using the strain gauge apparatus developed by Barlow and colleagues, and the acoustic signal was also recorded. Short term spectra were taken of the fricative noise at the beginning and at intervals spaced at $0.25,0.50$, and 0.75 of the duration of the fricative. Two spectral peaks were identified at each point. It was predicted that the frequencies of those peaks would be affected by the nature of the following vowel and that the coarse spectral track would show a downward movement when the following vowel was [u]. Neither of these predictions obtained. This result is in agreement with the EMG data of Gelfer et al. [J. Acoust. Soc. Am. 86, 2443-2445 (1989)] but not with the acoustic data of, among others. Sereno et al. [J. Acoust Soc. Am. 81, 512-519 (1987)]. Results from a second speaker and from the analysis of the lip movement data will also be presented.

4aSC23. Correlating movement and acoustic measures of nasalization. Sharon Y. Manuel (Res. Lab. of Electron., Rm. 36-511, MIT, Cambridge, MA 02139) and Rena A. Krakow (Temple Univ., Philadelphia, PA 19122 and Haskins Labs., New Haven, CT)

Previous research using physiological instrumentation (e.g., Velotrace) has shown systematic effects of syllable structure and stress on velum movements [Krakow, in Phonetics and Phonology V (Nasals, Nasalization, and the Velum, edited by Huffmann and Krakow (Academic, San Diego, 1993), pp. 87-113]. However, what has not been investigated previously is the correspondence between the time-varying position of the velum in such data and the timing and magnitude of acoustically detectable nasalization. A new technique developed by Chen [J. Acoust. Soc. Am. 96, 3283 (A) (1994)] for identifying the spectral effects of nasal coupling was found to provide a valuable way of linking the acoustic and articulatory changes related to velopharyngeal port aperture. This paper reports on acoustic-toarticulatory mapping using acoustic data collected in concert with
Velotrace data for a number of different utterance types, including those with stressed versus unstressed syllables and those with syllable-initial versus syllable-final nasals. One consistent observation is that velum lowcring can be quite extensive before acoustic effects are observed. [Work supported in part by NIH.]

4aSC24. Use of a spiking neural model to simulate lip motor unit activity and movements in speech. Michael D. McClean and John L. Clay (Audiol. and Speech Ctr., Walter Reed Army Med. Ctr., Washington, DC 20307-5001)

In order to clarify the nature of EMG-kinematic relationships involved in speech production, a rudimentary computer model of the lower lip as a neuromechanical system was developed. The model consists of three muscles having forcing functions that act on a common point. Each muscle is treated as a set of single motor units with associated motoneuron spike activity and muscle-unit twitch forces. The temporal patterns of motoneuron spike trains, which serve as the system input, are based on single motor unit EMG recordings obtained during syllable repetition [M. McClean and J. Clay, Society for Neuroscience Abstracts 19, 229.15 (1993)]. System output is evaluated in terms of lower-lip movement through the midsagittal plane. Simulations produce realistic movement patterns when they are constrained by reasonable assumptions about motor unit intrinsic properties, muscle force angles, and relative timing of motoneuron spike trains. Initial applications of the model have focused on the mechanisms of speech rate control. Different rates of syllable production are established by the overall temporal pattems of motoneuron spike trains, and the effects of varying system parameters are evaluated at each rate.

4aSC25. Clarity versus effort in speech production: Initial results of a pilot study. Joseph Perkell, Melanie Matthies, Mario Svirsky, and Reiner Wilhelms-Tricarico (Speech Commun. Group, Res. Lab. of Electron., Rm. 36-511, MIT, Cambridge, MA 02139)

The goal of this research is to test the hypothesis that there is a trading relation between clarity and effort in speech production. Midsagittal-plane articulatory movements of points on the tongue body, tongue blade, lips and mandible (with an EMMA system) and the acoustic signal from a male talker in five speaking conditions: normal, clear, clear plus fast, fast, and slow, were recorded. Different CVC words were embedded in two positions in a carrier phrase. Analyses were performed as a function of speaking condition and position in the phrase. As an example, CV movements of a point on the tongue body for the word "cab" have the following characteristics. Movement duration was shorter for position two; in both positions, it followed the order: slow $>$ clear $>$ normal $>$ clear plus fast $>$ fast. The range of distance traveled was greater for slow and for clear, than the other conditions. Peak velocity was higher for fast and clear plus fast than the other conditions. Midsagittal plane location at movement end was less variable with the longer duration movements of the word in position one. Effort was higher for clear, clear plus fast and fast, than for normal or slow, in both word positions. [Work supported by N.I.D.C.D.]

4aSC26. The gestural and temporal organization of assimilation. Sidney A. J. Wood (Dept. of Linguistics, Helgonabacken 12, 22362 Lund, Sweden)

Ever'since Wang and Fillmore [J. Speech Hear. Res. 4, 130 (1961)] phoneticians have distinguished between nonpredictable language-specific speech habits and predictable universal articulatory constraints, the former being cortical and extrinsic to the vocal tract, the latter intrinsic to the vocal tract and the consequence of articulation mechanics. Assimilation came to be seen as an intrinsic vocal tract process, explained in terms of Öhman's model of coarticulation [J. Acoust. Soc. Am. 39, 151 (1966)] This contrasts with classical phonetics where assimilation is the result of preplanned reorganization of articulation. The work reported in this paper favors the classical view. Gestures involved in an assimilation are initiated earlier, delayed, or are held longer, than in nonassimilated situations. It is argued that this revision of gesture timing in relation to adjacent activity is an expression of preplanning of motor commands that takes place in the cortex where there is access to larger portions of the utterance (the subcortical motor structures have limited access and cannot advance or delay 
gestures). Examples of palatalization of Bulgarian apico-alveolar stops and Swedish palatovelar stops are presented. The data are analyzed from $x$-ray motion films by procedures described by Wood [J. Phon. 19, 281 (1991)].

4aSC27. Modeling pathologic vocal quality. Part II. Bruce R. Gerratt and Jody Kreiman (Div. of Head and Neck Surgery, UCLA School of Medicine, 31-24 Rehab Ctr., Los Angeles, CA 90024-1794)

The utility of acoustic measures of pathologic voice depends on their consistent correspondence to physiological states or to what listeners hear. Unfortunately, such consistent patterns of association have never emerged, despite many years' reseach. Attempts to map from acoustics to physiology may never produce unique associations, because many physiological configurations may produce equivalent acoustic signals. Similarly, studies seeking correlations between acoustic and perceptual measures will never produce consistent, replicable associations, because individual differences in perceptual strategy are so great that unique mappings do not exist, as argued in Part $\mathrm{I}$. It is believed that the difficulties in establishing acousticperceptual relations in voice research can be solved only by modeling quality as an interaction between a particular listener and voice, rather than as a property of the voices themselves. An analysis-by-synthesis method is described in which listeners construct a synthetic signal to match a particular natural voice. In this way, listeners' perceptions are defined objectively by the values of the synthesizer parameters they select. This approach explicitly links acoustic representations to perceived qualities, thus reducing concerns about utility and validity in both domains.

FRIDAY MORNING, 2 JUNE 1995

GRAND BALLROOM SOUTH, 7:55 A.M. TO 12:05 P.M.

\title{
Session 4aUW
}

\section{Underwater Acoustics: Transient Sounds: Their Measurement and Description}

\author{
Charles R. Greene, Chair \\ Greeneridge Sciences, Inc., 4512 Via Huerto, Santa Barbara, California 93110
}

Chair's Introduction-7:55

Invited Papers

8:00

4aUW1. Acoustic transients research at the Naval Research Laboratory. Steven Finette, Peter C. Mignerey, Robert L. Field, and Edward R. Franchi (Naval Res. Lab., Washington, DC 20375 and Stennis Space Center, MS 39529)

From 1988 to 1992 the Naval Research Laboratory conducted basic research in acoustic transients. This paper will summarize the key results of that research. First, the time-domain parabolic equation model was developed to predict the effects of the ocean environment on acoustic transient signals. Comparisons between modeled and measured ocean impulse responses will be reviewed and the ability of the model to predict signal feature distortion will be shown for data from an Atlantic, downward refracting, rangedependent environment. Second, the development of a multi-sensor deconvolution estimator which makes full use of an array aperture will be discussed. Use of the full array aperture ensures that no part of the impulse response spectrum drops out due to poor illumination. Multi-sensor deconvolution processors based on this principle have produced stable reconstructions of source time-series. Data will be shown that illustrate successful multi-sensor deconvolution that was obtained by a large, vertical, line-array in a deep Pacific environment for source-receiver separations of 1-2 convergence zones. Third, time-domain higher-order correlators were developed for the detection and time-delay estimation of short-time/broadband transients. The signal and environmental conditions required for higher-order processors to outperform conventional cross-correlators will be shown.

8:20

4aUW2. Transient signals-based sonar systems. James E. Barger (BBN Systems and Technologies, 70 Fawcett St., Cambridge, MA 02138)

The detectability of signals (echoes) depends only upon the energy and bandwidth of the signals, in a wide variety of different interference backgrounds. This fact is represented in a sonar equation that is both written in terms of these quantities, and that also differs from the more traditional form that incorporated signal power and time span. Beyond this, fluctuations in the transient signal's arrival times, time spreads, arrival angles, and angle spreads; all affect target localization processing. Since a transient signal has a shorter time span than does a longer waveform with equal energy, the effects of propagation-induced fluctuations between successive transient signals can be different than between successive long-waveform signals. The potential for these differences is discussed for four different causes of fluctuation; multipath, refraction, direct-path fluctuation, and reflected path fluctuation.

\section{8:40}

4aUW3. Quantitative calibration and characterization of marine seismic sources. J. Robert Fricke (Dept. Ocean Eng., Rm. 5-218, MIT, Cambridge, MA 02139)

In the mid '80s the offshore seismic exploration industry needed a standard procedure for describing transient seismic source signatures. The need was motivated by several issues including a need to specify performance criterion to the operations crew, a need to evaluate one source versus another for a particular seismic program, and the need to determine performance for new seismic source designs. Prior to that time, seismic source signatures were usually described in the time domain in terms of peak-to-peak pressure (bar-m) and peak-to-bubble ratio. Spectral characterization, if it existed, was relative to the peak value, which was usually set to $0 \mathrm{~dB}$. 
These measures do not provide quantitative descriptions of source energy or spectral energy density, hence a quantitative standard was needed and developed. The standard focused on a spectral, rather than a temporal, description of the seismic signature and allowed for a detailed analysis of the total source energy and the distribution of that energy as a function of frequency. This approach opened the way for a consistent and quantifiable description of the source signature. Using this description, the above mentioned needs regarding performance and evaluation could be addressed.

4aUW4. Metrics and standards for acoustic environmental assessments. Raymond C. Cavanagh and John S. Hanna (Science Applications Int'l. Corp., McLean, VA 22102)

Because of stringent legal requirements and growing public interest, researchers must win government approval for any sea test involving acoustic emissions which could injure or harass marine animals. The approval process requires extensive documentation on the animals in the test area and likely impact of the test. This documentation (Environmental Assessment or Environmental Impact Statement) then includes predictions of shock wave and acoustic field characteristics in terms relevant to animal harassment and injury metrics. At present, there is no "standard" approach to developing an EA or an EIS; each research program provides its own format, metrics, etc. This presentation considers issues of standardization for the EA and EIS processes. In particular, literature on animal effects provides a variety of empirical thresholds in various units (e.g., peak pressure, impulse), while source metrics have their own particular units (e.g., energy density at range, intensity). Finally, signal must be estimated with a model that correclly propagates the quantity of interest in complex environments. None of these steps has a standard approach. Examples are given of problems encountered and recommendations made for standardization. Special attention is given to measurement techniques for marine animal response to sound.

4aUW5. Fourier and direct time-marching methods for transient signals. Michael B. Porter and Zoi-Heleni Michalopoulou (Ctr. for Appl. Math. and Stat., New Jersey Inst. of Technol., Newark, NJ 07102)

The ocean normally vibrates in harmony with the hum of a narrow-band sound source. Thus, for modeling such signals, time can be removed from the wave equation reducing it to the simpler Helmholiz equation which in essence governs only the intensity of the hum. Computational ocean acoustics has most often focused on this latter case. Broadband signals such as pings, clicks, chirps, and gurgles require far more work. For researchers accustomed to narrow-band models the fastest approach is usually to simply add a frequency loop to their narrow-band codes. (Of course, the run time goes up in accordance with the number of frequencies required.) Others have advocated the development of new models that solve the wave equation directly in the time domain. These options are surveyed and illustrative examples are provided using both idealized test cases and measured data from a recent experiment in the Gulf of Mexico.

9:40-9:50 Break

\section{Contributed Papers}

9:50

4aUW6. Transient sounds in marine seismology. R. A. Stephen (Woods Hole Oceanogr. Inst., Woods Hole, MA 02543)

In marine seismology two types of transient sound sources are used. Controlled sources such as airguns or explosions are used in seismic reflection and refraction surveys to study geological structure below the seafloor. Naturally occurring earthquakes are also observed and they are used to study tectonic and regional and whole earth structure problems. In both cases three-component particle motion, either velocity or acceleration, is measured on or within the seafloor. However controlled source studies work in the VLF band $(1.0-100 \mathrm{~Hz})$ and earthquake studies generally run in the lower ULF band (0.001-5 Hz). A major advantage of transient sources versus harmonic sources is that multi-pathing can be distinguished by the time separation of phases. In fact, many schemes for inferring geological structure from transient seismic data are based solely on the arrival times of certain phases. Also time spread (coda or signalgenerated noise) can be observed directly as reverberation after the coherent arrivals. The polarization of the separated phases can be used to study propagation effects. Examples of quantitative data of both controlled and earhquake sources will be shown and discussed.

\section{0:05}

4aUW7. Low-frequency ambient noise: Long time series and statistics. Keith R. Curtis, Bruce M. Howe, and Shaun Leach (Appl. Phys. Lab., Univ. of Washington, Seattle, WA 98105)

Low-frequency ocean ambient noise spectra have been collected at U.S. Navy Pacific SOSUS sites during the last year as a background com- ponent of the Acoustic Thermometry of Ocean Climate (ATOC) Program. Preliminary results are presented. Three-minute averaged spectra were obtained every $5 \mathrm{~min}$; only the relative noise level as a function of frequency is presented because (at this time) unknown offsets prevent determination of absolute levels. One important statistic estimated is the probability as a function of frequency that the noise level exceeds a specified spectral level. [Work supported by the Strategic Environmental Research and Development Program through ARPA.]

\section{$10: 20$}

4aUW8. Are ANSI standards for deseribing airborne impulses applicable to underwater transients? Charles R. Greene (Greeneridge Sciences, Inc., 4512 Via Huerto, Santa Barbara, CA 93110-2324) and Frank T. Awbrey (San Diego State Univ., San Diego, CA 92187-4614)

ANSI standards exist for measuring and assessing airborne impulsive sounds. This paper reviews those standards and considers their applicability and utility in measuring and assessing underwater transient sounds.

10:35

4aUW9. The Bragg condition limitation on inversion of normal incidence reflection data. Kenneth E. Gilbert and Timothy J. Kulbago (Appl. Res. Lab. and the Graduate Program in Acoust., Penn State Univ., P.O. Box 30, State College, PA 16804)

A simple analytic expression is derived for the impulse response of a continuously stratified sediment. The expression, which neglects multiple scattering, allows a straightforward calculation of both the forward and 
inverse problem, but more importantly, it clearly establishes the "Bragg condition" as a fundamental limitation on the inversion of acoustic data dominated by single scattering. (For normal incidence backscatter, the Bragg condition states that an acoustic probe signal of wavelength $\lambda$ senses or "filters out" only the Fourier component of the impedance profile having wavelength $\lambda / 2$, i.e., $\lambda_{\text {madium }}=\lambda_{\text {accoustic }} / 2$.) The validity of the analytic expression is demonstrated by comparing it with exact numerical calculations for both the forward and inverse problem. In particular, it is shown that to obtain trends in impedance that occur over meters, requires a probe signal with wavelengths of approximately twice the desired trend distance. Many high-frequency inversions reported in the literature show trends with wavelengths that are orders of magnitude larger than any wavelength in the probe signal. Consequently, it is worthwhile to ask whether the long wavelength trends, which cannot be acoustically sensed by the short wavelength probe signals, are physically meaningful, or whether they are arifacts of arbitrary underlying assumptions in the signal processing method. [Work supported by NRL and ONR.]

\section{0:50}

4aUW10. Localization of sources of acoustic transients using a broadband matched-field processing technique. F. D. Tappert (Appl. Marine Phys., Univ. Miami, RSMAS, 4600 Rickenbacker Cswy., Miami. FL 33149) and L. Nghiem-Phu (MAC Systems Corp., Miami, FL 33155)

By matching, or correlating, a complex-demodulated (envelope) time series measured at a single hydrophone receiver to predicted envelope response functions obtained by back propagation using a full-physics broadband PE model, it is possible to remove multipath distortion effects and to delermine the location of the source of an acoustic transient with good resolution and high processing gain. This "semi-coherent" broadband matched-field processing (BMFP) technique is demonstrated in several deep water and shallow water situations. [Work supported by ONR and ARPA.]

\section{1:05}

4aUW11. Timespread modeling of propagating pulsed and transient underwater signals. Ellen M. Daley, Ruth E. Keenan, and Anthony I. Eller (Ocean Sci. Group, SAIC, 1710 Goodridge Dr., McLean, VA 22102)

Many Navy standard and other widely used underwater acoustic propagation models were developed through an evolutionary process to address applications featuring narrow-band or single-frequency cw signals. A consequence of this development process is that, although the models may handle the originally intended problem very well, they are not necessarily able to describe the propagation of high resolution pulses or transients, where time-domain or multi-frequency FFT approaches seem called for. This paper presents the results of an evaluation of several propagation models to characterize short duration signals. The models tested represent a variety of physics-based approaches: parabolic equation, rays and Gaussian beams, modes, and hybrid approaches. The evaluation criteria include an ability of the modeling system to accommodate real-world, in-situ environments, accuracy of the results in the context of intended applications, and reasonable execution time.

\section{1:20}

4aUW12. Theory and experiment of pulse propagation in shallow water. Renhe Zhang (State Key Lab. of Acoust., Chinese Acad. of Sci., Beijing 100080, People's Republic of China and School of Mech. Eng., Georgia Inst. of Technol., Atlanta, GA 30332) and Ye Zhu (State Key Lab. of Acoust.. Chinese Acad. of Sci., Beijing 100080, Pcople's Republic of China)

An experiment was conducted in shallow water with strong thermocline, located between the depths from 11 to $15 \mathrm{~m}$. Explosive sources were detonated at 7 and $25 \mathrm{~m}$, with receivers also at 7 and $25 \mathrm{~m}$. Bandlimited signals with $1 / 3$ oct bandwidth for different center frequencies and ranges were analyzed. The waveform structures are strongly dependent on source and receiver depths, center frequency, and range. A ray-mode theory including beam displacement was used to calculate the waveform structures and to invert for the bottom-loss versus grazing angle. The calculated waveform structures are consistent with the measured ones well.

\section{1:35-12:05}

\section{PANEL DISCUSSION}




\title{
Meeting of Accredited Standards Committee S12 on Noise
}

to be held jointly with the

\section{U.S. Technical Advisory Group for ISO/TC 43/SC1 Noise and ISO/TC 94/SC12 Hearing Protection}

\author{
D. L. Johnson, Chair S12
}

EG\&G Special Projects, Albuquerque Operations, Albuquerque, New Mexico 87119-9024

P. D. Schomer, Chair, US Technical Advisory Group (TAG) for ISO/TC 43/SC1, Noise (and Vice Chair S12) U.S. CERL, P.O. Box 4005, Champaign, Illinois 61820

H. E. von Gierke, Vice Chair, U.S. Technical Advisory Group (TAG) for ISO/TC 43/SC1, Noise 1325 Meadow Lane, Yellow, Springs, Ohio 45387

Elliott H. Berger, Chairman, U.S. Technical Advisory Group (TAG) for ISO/TC 94/SC12, Hearing Protection Cabot Safety Corporation, 7911 Zionsville Road, Indianapolis, Indiana 46268-1657

Standards Committee S12 on Noise. Working group chairs will report on their progress for the production of noise standards. The interaction with ISO/TC $43 / \mathrm{SCl}$ and ISO/TC $94 / \mathrm{SC} 12$ activities will also be discussed, with reference to the international standards under preparation. The Chairs of the respective U.S. Technical Advisory Groups (H. E. von Gierke and E. H. Berger) will report on current activities of these International Technical Subcommittees under ISO.

Scope of S12: Standards, specifications and terminology in the field of acoustical noise pertaining to methods of measurement, evaluation and control; including biological safety, tolerance and comfort and physical acoustics as related to environmental and occupational noise.

\section{Session $4 p A B$}

\section{Animal Bioacoustics: Recognition of Animal Sounds: Methods and Applications}

\author{
David K. Mellinger, Chair
}

Cornell Bioacoustics Research Program, 159 Sapsucker Woods Road, Ithaca, New York 14850-1999

\author{
Chair's Introduction-1:00
}

Invited Papers

1:05

4pAB1. Marine animal sound classification. Kurt M. Fristrup and William A. Watkins (Woods Hole Oceanogr. Inst., Woods Hole, MA 02543)

Software was developed to measure characteristics of marine animal sounds (AcouStat). These measurements included aggregate bandwidth, duration, amplitude modulation, frequency modulation, center frequency, and interactions among some of these variables. These measurements proved effective for describing and classifying sounds in several contexts: identifying species, quantifying the repertoire of a single species, and identifying individuals. Our experience during development suggests that removal of noise energy and use of robust statistical measures are necessary to ensure repeatable results with open water recordings of marine mammal species. 
4pAB2. Quantitative analysis of dolphin sounds. Peter L. Tyack (Dept. of Biol., Woods Hole Oceanogr. Inst., Woods Hole, MA 02543)

Most analyses of acoustic communication in animals lump sounds into qualitative categories. These are usually based upon aural impressions for human listeners or visual inspection of spectrograms. Quantitative analysis of acoustic features from beluga whale sounds raise serious questions about how discrete and robust the traditional categories of beluga calls are. Other categories, such as the contour of a dolphin whistle, use features that need not relate directly to absolute measure of time and frequency. Studies of vocal development and vocal imitation often benefit from a quantitative measure of similarity between sounds and putative models. Several different methods are compared to analyze whistle contours, including multivariate analysis of time-frequency features, dynamic time warping, and a signal compression approach. Ultimately, all such techniques need to be validated by studies of how each species perceives its own signals.

\section{1:55}

4pAB3. Comparison of the whistle structure of six species of dolphin. William E. Evans (Texas Inst. of Oceanogr., Texas A\&M Univ., P.O. Box 1675, Galveston, TX 77553), Wang Ding (Inst. of Hydrobiol., Chinese Acad. of Sci., People's Republic of China), and Bernd Würsig (Texas A\&M Univ., Galveston, TX 77553)

Spectral and statistical analyses were used to compare the whistle structure of six species of dolphin; Stenella longirostris, Stenella frontalis, Stenella attenuata, Lagenorhynchus obscurus, Tursiops truncatus, and Sotalia fluviatilis. A consistent pattern existed in the various coefficients of variation calculated for the different species. In general, the frequency variables had the lowest coefficients of variation (cv). The values of cv for maximum frequency were usually the lowest. Compared to other species Tursiops had relatively large coefficients of variation of the frequency variables indicating that the frequencies of Tursiops whistles were more diverse. The other five species had similar frequency ranges which had higher upper frequencies than Tursiops. The results of discriminate analysis indicated that here were significant differences between the whistle structures of the different species, and that these differences were related to taxonomic relations, body size, and habitat. The magnitude of the differences in whistle structure correlated with taxonomic relationships of the various species studied. The pelagic species emitted whistles in a relatively higher frequency range and greater frequency modulation than the coastal or riverine species.

\section{2:20}

4pAB4. Automatic detection and classification of nocturnal migrant bird calls. Harold Mills (Cornell Lab. of Ornithol., 159 Sapsucker Woods Rd., Ithaca, NY 14850)

Computer software was developed to detect the nocturnal flight calls of nine species of migrating warblers in digitized field recordings, and to classify the calls by species. The calls are frequency-modulated tones in the 5- to 9-kHz frequency band, and between 50 and $100 \mathrm{~ms}$ in duration. Detection was accompanied by locating temporal peaks in call band energy. Some false detections of insect calls were prevented by rejecting certain types of peaks. Classification is approached by tracking the frequencies of the calls over time and classifying the frequency tracks with an artificial neural network.

\section{Contributed Papers}

4pAB5. Bird flight call discrimination using machine learning. Andrew Taylor (Comp. Sci. and Eng., Univ. of NSW, Sydney 2052, Australia)

The development of a software system which can detect and identify the flight calls of migrating birds is reported. The system first produces a spectrogram using a DFT. Calls are detected in the spectrogram using an ad hoc combination of local peak-finding and a connectedness measure. Attributes are extracted both globally from the call and from a window moved incrementally through the call. Decision trees are then used to determine the bird species. These decision trees are induced from a training set using Quinlan's C4.5 system [J. R. Quinlan, C4.5: Programs for Machine Learning, Morgan Kauffman (1993)]. The system has been tested on a set of 138 nocturnal flight calls from nine species of birds [W. R. Evans, personal communication]. Some calls are faint, and interfering insect noise is present in others. Tenfold resampling was used to classify the calls unseen. Seventy-eight percent of calls were identified correctly, $4 \%$ incorrectly and $18 \%$ were placed in an "uncertain" category. Neural network-based classifiers are commonly used in this general domain and would likely produce similar accuracy, but use of symbolic machine learning offers two important advantages: Training time is linear in the number of examples and the resulting classifier is less opaque. Both significantly ease classifier construction.
4pAB6. Vocal learning in Budgerigars (Melopsittacus undulatus) using food reward. Kazuchika Manabe and Robert J. Dooling (Psych. Dept., Univ. of Maryland, College Park, MD 20742)

Budgerigars (parakeets) are small, highly social, Australian parrots capable of vocal learning throughout adulthood. These birds readily produce short (200 ms), whistled, frequency-modulated contact calls when separated from one another. In this experiment, birds were trained twice daily in $10 \mathrm{~min}$ sessions to produce or modify contact-call-like vocalizations using food reward. Calls were analyzed in real time using serial FFTs and each production was compared to a digitally stored "template." Call productions which exceeded a predetermined criterion of similarity were rewarded, while those below criterion were not. Results show that budgerigars can learn to modify the intensity and spectro-temporal pattern of their species-typical calls within several days. Aside from human language, bird vocalizations have provided the only other clear example of learning in the acquisition and maintenance of a vocal repertoire. While song learning in birds has led to a number of important insights into the neurobiology of learning, such learning typically occurs over a time frame of months to years. The present results demonstrating call learning over a period of several days more closely parallel the time course of other more common forms of vertebrate learning. [Work supported by NIH Grants DC00198 and MH00982.] 
4pAB7. Detection of sinusoidal spectral envelopes by budgerigars (Melopsittacus undulatus). Satoshi Amagai, Robert J. Dooling, Tracy L. Kidd (Psych. Dept., Univ. of Maryland, College Park, MD 20742), and Shihab Shamma (Univ. of Maryland, College Park, MD 20742)

Previous psychoacoustic measures of both critical ratios and critical bands in budgerigars indicate that the size of the auditory filters around $2-4 \mathrm{kHz}$ approach or are even smaller than those of humans. Accordingly, budgerigars should excel at spectral discrimination tasks involving channels around $3 \mathrm{kHz}$. This study examined the ability of budgerigars to discriminate spectrally rich sounds by using rippled noise stimuli. Three budgerigars were trained in a repeating background procedure using operant conditioning to discriminate between a complex stimulus with a flat spectrum and one that had a sinusoidal ripple imposed on its spectrum. Each stimulus was composed of 201 spectral components logarithmically spaced from $500 \mathrm{~Hz}$ to $10 \mathrm{kHz}, 100 \mathrm{~ms}$ in duration and sampled at $40 \mathrm{kHz}$. Ripple frequencies between 0.5 and 12 cycles/oct were tested using ripple amplitude steps of $1 / 2$ to $2 \mathrm{~dB}$. Overall levels of the stimuli were varied randomly from $55 \mathrm{~dB}$ to $65 \mathrm{~dB}$ SPL to remove intensity cues. Ripple amplitude detection thresholds for budgerigars were similar to those measured for humans with budgerigars slightly better at higher ripple frequencies. [Work supported by NIH Grants DC00198 and MH00982.]

\section{$3: 45$}

4pAB8. The use of very low and very high frequencies in the development of an acoustical deterrent for bird-aircraft collisions. M. Lenhardt, A. Ochs (Biomed. Eng. Prog., Virginia Commonwealth Univ., Richmond, VA 23298-0168), J. Genova, N. Castiglia (Raven, Inc., Alexandria, VA 22312), and M. Kelley (Wright-Patterson AFB, OH 45433)

Bird strikes have become an increasing problem at commercial and military airports. With the demand for reduced aircraft noise and increased speed, birds are presented with reduced auditory cues and less reaction time when confronted with planes. The major drawback to the use of acoustic deterrents is the rapid habituation. Caged sparrowhawks readily detected signals between 5 and $40 \mathrm{~Hz}$ with thresholds of detectability ranging from 76 to $64 \mathrm{~dB}$ SPL. No flying induced startles were noted, although the birds did try to localize the source. Habituation to lowfrequency stimuli occurred with as little as three presentations. Freeranging geese were presented with the same frequencies as substrate vibration along with sound pressure stimulation. In a limited sample (three geese) avoidance was noted. The application of low-frequency sound as substrate vibration on runways is promising. Alternatively, microwaves produce an auditory stimulus to the ear that can be altered by changes in frequency, pulse width, and overall power. Pulsed microwaves or microwaves modulated by low-frequency sound are potential sources alerting birds from a flying plane already carrying navigation radar.

\section{4:00}

4pAB9. Low-frequency auditory behavior in sea lampreys, primitive fish, and marine turtles. M. L. Lenhardt (Biomed. Eng. Prog., Virginia Commonwealth Univ., Richmond, VA 23298-0168 and Virginia Inst. of Marine Sci., Gloucester Point, VA 23062)

Sea lampreys (Petromyzon marinus) are one of only two species of living jawless fish. The brains and ears of lampreys are remarkably similar to that of fossil Ostracoderms, a group of fish that existed over 500 millions years ago. Jawed fish are derived from jawless fish which in turn radiated into primitive bony fish as gars (Lepisosteus) and teleosts and into the line leading to development of tetrapods. Marine turtles (Caretta $\mathrm{c}$. caretta; Lepidochelys kempi) are considered primitive in that they share many characteristics with stem reptitian stock from which birds and mammals also radiated. The three species responded behaviorally and electrophysiologically to very low frequencies in a similar fashion despite their obvious morphological differences. All appear to share an ability to detect substrate vibrations, suggesting that the primitive vertebrate ear was also a low-frequency vibration sensor. It is hypothesized that the first vertebrates evolved an otic organ for both equilibrium and vibration and each function was not separated until jawed vertebrates. In the primitive forms as in living lampreys, the brain must inhibit the ear function when detection of movement and gravity is needed.

\section{4:15}

4pAB10. Dynamic model of the goldfish peripheral auditory system. James J. Finneran and Mardi C. Hastings (Dept. of Mech. Eng., Ohio State Univ., 206 W. 18th Ave., Columbus, OH 43210)

In this study, a dynamic, lumped parameter mathematical model was developed for the peripheral auditory system of the goldfish (Carassius auratus). This model expands upon and couples existing models for the swimbladder (anterior chamber) and saccular otolith by including the swimbladder tunica interna and tunica extema, along with the Weberian apparatus. The viscoelastic properties of the tunica externa are based on data from Alexander [J. Exp. Biol. 38, 747-757 (1961)]. Model predictions correlate well with experimental data for swimbladder resonance frequency and damping and with audiograms obtained from behavioral conditioning experiments. Model results indicate that the Weberian apparatus has a major affect on auditory sensitivity throughout the audible frequency range of the goldfish. In addition, specific phase relationships between the Weberian, direct, and indirect contributions to saccular otolith motion are observed for changing source location. [Work supported by ONR Grant No. N00014-94-1-0337.]

\section{$4: 30$}

4pAB11. A computer model for simulation of undersea noise from marine mammals. Thomas J. Hayward and Richard M. Heitmeyer (Naval Res. Lab., Washington, DC 20375-5350)

A computer model for the simulation of the spatial and temporal distribution of acoustic signals generated by marine mammals is presented. The model incorporates information from existing references on the geographic distribution of marine mammal species. Spatial distributions of individuals of each species that may be present at a site are generated using a random point process model that incorporates a statistical representation of the spatial clustering characteristics of each species. Individual vocalizations for each species are simulated by randomly selecting sampled acoustic source signatures from either the Woods Hole Oceanographic Institution's SOUND database or from the NRL Dual Use Acoustics Center (DUAC) database. The acoustic source signatures are generated in random time patterns that emulate known vocalization repetition characteristics for each species. Sound propagation effects are incorporated by generating acoustic transfer functions using propagation models appropriate to the frequency band of each acoustic signature. The potential use of the model in the validation of marine mammal acoustic survey techniques is discussed. [Work supported by ONR.]

\section{$4: 45$}

4pAB12. Study of sound transmission in various types of stored grain for acoustic detection of insects. Robert Hickling, Wei Wei (Natl. Ctr. for Phys. Acoust., Univ. of Mississippi, University, MS 38677), and David W. Hagstrum (U.S. Grain Marketing Res. Lab., Manhattan, KS 66502)

In detecting insect infestations in bulk-stored grain using an array of acoustic sensors, it is important to know the range of sound transmission in grain. Preliminary tests [R. Hickling and W. Wei, Appl. Acoust. 44 (1995)] have shown that grain can be highly absorbing, depending on frequency, and this may limit the range at which insects can be detected. Tests were conducted with six different types of grain: hard and soft wheat, brown rice, soybeans, corn, and sorghum. The results were analyzed using soundtransmission theory in porous media. Transmission characteristics were determined and related to grain size and shape. Tests were conducted of the effect of grain depth. Data analysis provides a measure of the average spacing between grains. The relation of grain spacing to insect size is discussed. 


\title{
Session 4pEA
}

\section{Engineering Acoustics: Laboratory and Industrial Applications of Ultrasound}

\author{
Gerald V. Blessing, Chair \\ National Institute of Standards and Technology, Sound Building, Room A147, Gaithersburg, Maryland 20899-0001
}

Chair's Introduction-1:00

Invited Papers

1:05

4pEA1. Application of Green's function modeling to ultrasonic probing of a planar interface. Nelson N. Hsu (NIST, Gaithersburg, MD 20899)

The use of plane-wave ultrasound to probe interface properties based on angular spectrum analyses is common in the nondestructive testing of materials. Space-time domain Green's function modeling is an alternative approach to frequency domain modeling. it is better suited for the implementation of short duration pulses, small aperture transducers, and time-resolved pulse-echo methods. However, a computation based on the Green's function, though conceptually simple, is more involved than a computation based on the model of a plane wave of fixed frequency. Recently, an explicit Green's function for layered media has been derived. Efficient computer programs have also been developed for cases which can be easily tested experimentally and have immediate applications. As examples, comparison results between theory and experiment are reported here for three test configurations: (1) a solid plate on a half-space with different interface conditions, with a point step-function source and a point detector located on top of the plate; (2) a liquid/solid interface, with both the point source and point detector in the liquid; and (3) a line focus transducer probing a liquid/solid interface. Practical applications to laser ultrasound, simulated acoustic emission, and the acoustic microscope are illustrated.

4pEA2. Laser ultrasonic monitoring of steel microstructure at elevated temperatures. James B. Spicer (Dept. of Mater. Sci. and Eng., Johns Hopkins Univ., Rm. 102 Maryland Hall, Baltimore, MD 21218)

Laser ultrasonics as a remote, noncontacting ultrasonic testing method has been used extensively for materials characterization. However, the unique character of the laser source has not been exploited fully. This work on steel at elevated temperatures has made use of relationships which exist for the thermoelastic laser source in monitoring microstructural changes in steel. Ultrasonic monitoring of microstructural evolution in solids is performed by making measurements of ultrasonic velocity and attenuation. At elevated temperatures, both the ultrasonic velocity and the attenuation are affected independently of the material microstructure. Thermal expansion of the sample complicates the determination of velocity from ultrasonic data since an independent measurement of sample length is needed. Contacting transducers allow only intermittent determination of velocity and attenuation since continuous contact might adversely affect either the transducer or the material. In this study, the laser-ultrasonic source characteristics are used to address these issues related to the testing of materials at elevated temperatures. Results obtained for the testing of austenitic and martensitic stainless steels to temperatures in excess of $1273 \mathrm{~K}$ indicate that characteristic microstructural changes may be detected by analysis of the laser ultrasonic data. This analysis permits real-time, continuous monitoring of microstructural evolution.

$1: 55$

4pEA3. Ultrasonic resonance techniques for materials characterization and external stress measurement in cylinders and spheres. Ward Johnson and G. A. Alers (NIST, MS 853, 325 Broadway, Boulder, CO 80303)

Several applications have been explored for ultrasonic resonance measurements using electromagnetic-acoustic transduction on cylinders and spheres. Since the transduction is noncontacting, acoustic velocities and damping can be determined with great accuracy, and small changes in related material properties or the sample environment can be detected. The resonant frequencies of certain "axial-shear" modes in induction-hardened steel rods have been found to be highly correlated with case depth, providing a nondestructive measure of depth comparable in accuracy to destructive tests. Applied stress on an aluminum cylinder has been measured with automatic temperature compensation using the resonant frequencies of an axial-shear mode and a torsional mode, thus demonstrating the basis for an acoustic load cell. Elastic anisotropy in aluminum has been measured from the relative frequency splitting of axial-shear modes that would be degenerate in isotropic material. Also, electromagnetic-acoustic transduction has been incorporated in a system used to obtain basic data on the elastic constants and damping of metallic spheres at elevated temperatures. 
$2: 20$

4pEA4. Ultrasonic evaluation of dynamic strain, crack nucleation, and growth. D. Hazony, G. Welsch, S. Hailu (Case Western Reserve Univ., Cleveland, $\mathrm{OH} 44106$ ), and G. Halford (NASA Lewis Res. Ctr., Cleveland, $\mathrm{OH}$ 44135)

The method proposed is a further development of an earlier work described by Hazony et al. ["Ultrasonic monitoring of tensile, fatigue, or creep specimens in situ," J. Acoust. Soc. Am. 93, 2279(A) (1993)]. Two transducers are imbedded at the front and back faces of the specimens along the principal axis. These transmit and receive arbitrarily sharp stress pulses that probe both the volume and the surface of the specimen. The respective times of fight are sufficient for the monitoring of longitudinal and transversal strains. Moreover, intensity tracking of the various received acoustic signals also serves well for monitoring onset and growth of cracks. Sensitivity and calibration issues will be discussed and experimental data on high-strength low-alloy steel and other alloy specimens will be presented. The method lends itself to high precision measurements of material deformation and damage and may be used at high temperatures and in aggressive environments. [Work supported by ONR and TecSonics, Inc. of Twinsburg, Ohio.]

\section{2:35}

4pEA5. Effect of particle shape on viscous attenuation of ultrasound in concentrated clay suspensions. Wei Han and Hemant P. Pendse (Dept. of Chem. Eng., 5737 Jenness Hall, Univ. of Maine, Orono, ME 04469-5737)

Acoustic wave propagation in concentrated suspensions is analyzed using a modified coupled-phase modeling approach. The particle, size, shape, and orientation are taken into account for estimation of viscous drag coefficient of spheroid particles over wide ranges of frequency and particle concentration. The study deals with frequency-dependent and concentration-dependent ultrasound attenuation coefficient of clay suspensions, with particular attention to the effect of particle size and shape distributions. For clay suspensions, the major loss of acoustic energy results from the viscous dissipation arising from the relative particle-fluid motion. Predicted results of sound-speed and attenuation coefficient based on consideration of particle shape factors agree well with the measurements available in literature for frequencies of $0.10-5.0 \mathrm{MHz}$ and solid concentrations up to $40 \mathrm{vol} \%$. Experimental results of attenuation spectra of plate-shaped kaolin clay slurries with solid concentrations of 0.6 to 16.9 vol\% over 3 to $60 \mathrm{MHz}$ are obtained using a newly developed AcoustoPhor System 8000 . These attenuation spectra are interpreted in terms of realistic particle size and shape distributions. The effects of nonsphericity become dominant as particle concentrations and operating frequencies are increased. Realistic size-dependent shape factors are shown to explain the measured spectra.

\section{2:50}

4pEA6. Unified coupled-phase formulation for estimating complex wave-number spectra associated with ultrasound propagation through concentrated colloidal dispersions. Hemant P. Pendse and Wei Han (Dept. of Chem. Eng., 5737 Jenness Hall, Univ. of Maine, Orono, ME 04469-5737)

The unified coupled phase continuum (UCPC) approach is utilized to analyze sound propagation through colloidal dispersions consisting of solid particles or liquid droplets suspended in a fluid. Attention is focused on the case of submicron particles at moderate to high (5\% to $50 \%$ ) volume fractions under ultrasonic frequencies. The governing equations involve equations of state, continuity, momentum, and energy for the discontinuous and continuous phases, respectively. The viscous (thermal) coupling between the two phases is accounted through matching terms in the equations of momentum (energy) formulated separately for each phase. Expressions for the viscous and thermal coupling coefficients explicitly account for the effects of particle size, shape factor, orientation, as well as concentration and the sound frequency. Theoretical predictions of sound speed and attenuation coefficient spectra are compared with several available experimental measurements on a variety of colloids including aqueous suspensions of polystyrene latex, $\mathrm{TiO}_{2}$ or kaolin pigment, as well as a roluene in water emulsion and an oleic acid in nitrogen aerosol. The UCPC approach can successfully predict the attenuation spectra of concentrated colloids covering a wide range of relative magnitudes of viscous versus thermal contributions. Its applications for particle size and shape characterization will be discussed.

\section{3:05}

4pEA7. Liquid-borne sub-micron particle detection through acoustic coaxing. Sameer I. Madanshetty (Aerosp. and Mech. Eng., Boston Univ., 110 Cummington St., Boston, MA 02215)

A novel approach to the detection of liquid-borne submicron particles in ultraclean liquids (used in semiconductor manufacture) is described. The key concept is to coax the submicron particles to soft cavitate and to detect the ensuing transient bubble activity acoustically rather than the particle itself. The method, therefore, relies on facilitating acoustic microcavitation through acoustic coaxing. Liquid-borne microparticles do not, ordinarily, cause any cavitation when exposed to strong sound fields (of $1 \mathrm{MHz}$ ). If, however, a very weak, high-frequency auxiliary acoustic field (e.g., 30 $\mathrm{MHz}$ ) is added to this sound field, cavitation by the microparticles is readily facilitated. This technique of facilitating cavitation is termed "acoustic coaxing." Results of preliminary experiments indicate that even smooth spherical microparticles can be coaxed to cause cavitation. An explanation of the "acoustic coaxing effect" is offered. This novel method based on the acoustic coaxing of microcavitation promises to be a good basis for an on-line, real-time monitor of liquid-borne submicronic particulate presence. This method is not limited to small sensing volumes, and, unlike optical methods, it has an intrinsic, location-specific, signal enhancement at the source particle.

\section{3:20}

4pEA8. Investigation of accuracy of sound-speed measurements in solids: A novel method. Thomas M. Proctor (T-PROsic, 22901 Old Hundred Rd., Barnesville, MD 20838)

Speed of sound in solids has been measured by a number of different techniques over the past 5 decades. Unfortunately, consistent repeatability and accuracy have been a rare occurrence. Many error-producing problems of a fundamental nature have kept this experimental finding suspect at best. In liquids, the definition, $d l / d t$, for speed of sound can be used to check the accuracy of the method but, for solids, such incremental changes in path length are not possible. In the case of a plate, a combination of modifications to the procedure for the normal on-axis-plane wave experiment are possible. Over the past 15 years, a number of improvements and innovations have enhanced the field of acoustic emission. The development of a point contact receiving transducer and a repetitive impulsive point source in conjunction with developed Green's function theory for the plate geometry have allowed for a new way of doing the ultrasonic velocity measurement. This new approach will be discussed and error problems inherent in this technique will be examined. Results of change in length versus change in time of flight will be analyzed. [Work performed by contract to NIST.]

\section{3:35}

4pEA9. Determination of the elastic properties of composite plates using gas-coupled ultrasonics. Michael J. Anderson (Dept. of Mech. Eng., Univ. of Idaho, Moscow, ID 83843-1030), Christopher M. Fortunko (NIST, Boulder, CO 80303), and Edwin M. Odom (Univ. of Idaho, Moscow, ID)

A nonintrusive technique has been developed for measurement of the elastic moduli of reinforced polymer composite plates. Two ultrasonic transducers placed in a transmission configuration use air as the coupling medium to excite $A 0$ Lamb waves in the plate sample. The phase speed of 
the plate waves at a given frequency is inferred from the angle of incidence for maximum transmission. Use of a low-impedance couplant gives several advantages beyond the nonintrusive nature of the technique. First, the influence of the gas medium is entirely decoupled from the determination of stiffness moduli. Second, a low-impedance couplant gives a highquality factor, which enhances the potential precision of the technique. Gas coupled measurements of stiffness moduli on unidirectionally reinforced graphite/epoxy and glass/epoxy plates of thickness in the range $\approx 1-6 \mathrm{~mm}$ are presented. Plate waves were excited over the frequency range 100-200 kHz. Stiffness moduli $E_{11}, E_{22}, G_{12}$, and Poisson's ratio $\nu_{23}$ (fibers in the "l" direction) were obtained from the phase-speed/frequency data using least squares. Properties determined with the gas coupled technique are compared with static measurements, and with model predictions based upon the properties of the constituents and fiber-volume ratio.

\section{3:50}

4pEA10. The ultrasonic reflection from a flat-bottom hole. Martin Manley (Sound Bldg., Rm. A-147, NIST, Gaithersburg, MD 20899)

The reference for calibration of ultrasonic measuring equipment used to detect flaws in metal is the reflection from a set of flat-bottom holes in metal blocks. Early experimental observation as well as an early analysis using a far-field assumption suggested that the response should vary linearly with the area of the hole. Recently, the validity of this linear result has been called into question by new analyses as well as experiment. This paper presents a new analysis of the reflecled signal and evaluates the block set which is used for ASTM recommended practice E-127 in light of this analysis.

\section{4:05}

4pEA11. Lamb wave measurements for rapid monitoring of sheet metal. George A. Alers (NIST, MS 853, 325 Broadway, Boulder, CO 80303)

Lamb waves represent a very natural choice of interrogating energy for problems involving ultrasonic inspection of sheet and plate shaped materials. They have not enjoyed wide use in nondestructive evaluation because their excitation and detection by piezoelectric transducers require special mechanical supports and corrections for the acoustic loading of the sheet by the coupling medium must be made. By using properly designed electromagnetic acoustic transducers (EMATs), the transducer supports can be simplified, corrections for leaky waves can be eliminated, and very specific modes can be used to optimize a particular inspection process. Several examples of using special Lamb wave modes to inspect pipes and tubes in industrial environments will be presented, and their use for materials characterization in ferromagnetic metals will be emphasized.

\section{4:20}

4pEA12. Ultrasonic power output measurement by pulsed radiation pressure. Steven E. Fick (NIST, Gaithersburg, MD 20899)

Direct measurements of time-average spatially integrated output power radialed into reflectionless water loads can be made with high accuracy by exploiting the radiation pressure which causes an absorptive target intercepting the entirety of an ultrasound beam to experience a force proportional to the total beam power, which, in turn, is readily determinable as accurately as the radiation force can be measured in isolation from confounding forces arising from such effects as buoyancy, surface tension, and vibration. A radiation force balance (RFB) constructed at NBS in 1974 is the first and only RFB known to be designed to employ simultaneous mechanical and electrical signal processing based on temporal modulation of the incident ultrasound at a frequency well above those characteristic of confounding phenomena. Equipped with purpose-built electronics, the RFB is operated by manually equalizing the radiation force and a counterforce generated by an actuator calibrated against reference masses using de current as the transfer variable. Improvements made to the RFB during its one overhaul in 1988 have nearly halved its overall measurements uncertainty, and extended its capabilities to include measuring the output of ultrasonic systems with arbitrary pulse waveforms.

\section{$4: 35$}

4pEA13. Effects of structure sensitivity of linear and nonlinear elastic moduli in built-of-contact systems. V. Yu. Zaitsev (Inst. of Appl. Phys., 46 Uljanova str., Nizhny Novgorod 603600, Russia)

Acoustical properties of built-of-contact systems are rather interesting both from the viewpoint of general physical acoustics and applied problems. Such structures can often demonstrate rather unusual acoustic properties (in this sense they are similar to such a popular object in acoustics as bubbly liquid). On the other hand, built-of-contact systems correspond to real physical structures, e.g., grainy media and rough contacting interfaces. Manifestations of their structural changes in linear and nonlinear acoustic properties are important for the creation of new methods of acoustical diagnostics. The results of numerical simulation of nonlinear harmonic generation under the action of an external sinusoidal drive in a model built-of-contact system with different initial compression is presented. The model has allowed experimental explanation of observed "unusual" nonlinear elastic properties of real grainy materials, which cannot be explained by known theoretical models, which do not take into account nonideality of packing, i.e., the presence of intergrain contacts with significantly different initial compression. It was revealed that higher-order elastic parameters are much more sensitive to the material structure changes than the liner modulus. This fact is rather encouraging for the creation of new nonlinear acoustic structure diagnostics methods.

\section{4:50}

4pEA14. Nonlinear combinational sound scattering by discontinuity-like defects in solids and its possible usage for crack diagnostics. I. Yu. Belyaeva and V. Yu. Zaitsev (Inst. of Appl. Phys., 46 Uljanova str., Nizhny Novgorod, 603600, Russia)

One of the important problems in structural control of solids is the problem of discontinuity-like defects diagnostics. The main drawback of conventional linear diagnostic methods is the complicated differentiation between a weak "useful" signal originated due to a crack and the waves scattered by other inhomogeneities of the material, which are not interesting from the viewpoint of flaw detection. In view of this problem, new acoustic diagnostic methods based on measuring nonlinear acoustic characteristics have recently begun to attract the attention of researchers. The effect of acoustic waves scattering nonlinearly from a thin discontinuitylike defect in a solid is analyzed. The defect is considered as the interface between two surfaces covered with bulges characterized by different initial deformation. It is shown that the nonlinear scattering into combinationalfrequency waves is much more sensitive to the loading of the interface contacts compared with the scattering into the wave of fundamental frequency. The observation of nonlinear scattering by crack-type defects should allow one to distinguish cracks from other scatterers and can be used for creation of new methods of crack detection and positioning.

\section{5:05}

4pEA15. Theoretical analysis of dynamic characteristics of piezoceramic polymer composites with $2-2$ connectivity. $Q$. M. Zhang and Xuecang Geng (Intercollege Mater. Res. Lab., Penn State Univ., University Park, PA 16802)

A dynamic model of piezoceramic polymer composites with 2-2 connectivity is presented. It can be used to evaluate the dispersion curves for the thickness resonance and the stop band edge resonance, the effective velocity of the longitudinal wave, as well as the distributions of strain, stress, and the electric field in a composite. Based on these results, the electromechanical coupling factors are determined using two different methods according to the IEEE recommendation. It is shown that the thickness mode coupling factor $k_{1}$ obtained from the difference of the effective longitudinal velocities at the constant electric field and constan! electric displacement is in good agreement with the experimental values. It is also shown that, due to the composite structure, the piezoelectric shear coefficient $e_{\text {is }}$ has a strong effect on both the effective velocity of the longitudinal wave $v^{D}$ and $k_{t}$ even at the limit where the composite thickness is much larger than the period. On the other hand, $k$, calculated from 
the energy consideration is much higher than the experimental values and cannot account correctly for the effect of $e_{15}$ on $k_{t}$. From the model, the evolution of the thickness mode and the stop band edge resonance with the thickness of a composite and the modes coupling between the two can also be elucidated.

4pEA16. The effect of length on the acoustic attenuation performance of concentric expansion chambers. A. Selamet and $P$. M. Radavich (Dept. of Mech. Eng. and Appl. Mech., 120 W. E. Lay Automotive Lab., Univ. of Mich., Ann Arbor, MI 48109-2121)

Following an earlier analytical and computational work of Sahasrabudhe et al. [Noise Control Eng. J. 38(1), 27-38 (1992)], the present study investigate the effect of the length on the acoustic attenuation performance of concentric expansion chambers. Three approaches are employed here to determine the transmission loss: (1) a two-dimensional, axisymmetric analytical solution; (2) a three-dimensional computational solution based on the boundary element method; and (3) experiments on an extended impedance tube setup with nine expansion chambers fabricated with the fixed inlet and outlet duct, and chamber diameters, and varying chamber length to diameter ratios from $l / d=0.2-3.5$. The results from all three approaches are shown to agree well. The effect of multidimensional propagation is discussed in comparison with the classical treatment for the breakdown of planar waves. The study also provides a simple relation between the $l / d$ ratio of the expansion chamber and the number of repeating attenuation domes prior to the domination of higher-order modes.

FRIDAY AFTERNOON, 2 JUNE 1995

AUDITORIUM, 1:00 TO 5:00 P.M.

\title{
Session 4pPA
}

\section{Physical Acoustics: Nonlinear Acoustics}

\author{
Murray S. Korman, Chair \\ Department of Physics, U.S. Naval Academy, Annapolis, Maryland 21402
}

\section{Contributed Papers}

$1: 00$

4pPA1. The Tait equation and its connection with $B / A$ : An historical note. Robert T. Beyer (Dept. of Phys., Brown Univ., Providence, RI 01912)

In the $1880 \mathrm{~s}$, the Scottish physicist P. G. Tait set up an empirical equation to fit pressure-volume data for fresh and salt water, gathered on a research expedition of the H. M. S. Challenger ["Physics and Chemistry" of the Voyage of H. M. S. Challenger, Vol. II, part IV (1888)]. This equation, together with two or three variants of it, was the starting point of a number of researches over the next 70 years. In the 1950 s, it was put to use in still another form, and became the ancestor of the quadratic relationship of pressure and density used in nonlinear acoustics. The history of this development and the connection between the Tait equation and the ratio $B / A$ is set forth.

\section{$1: 15$}

4pPA2. General proof of the nonscattering of sound by sound in the lossless dispersionless case. Peter J. Westervelt (Dept. of Phys., Brown Univ., Box 1843, Providence, RI 02912)

Introducing the variable $\pi=\rho_{s} c_{0}^{2}+L+\Lambda V$ into Eq. (14) of Westervelt [P. J. Westervelt, J. Acoust. Soc. Am. 29, 200 (1957)], two equivalent dissipationless wave equations are obtained for the scattered variable $\rho_{s} c_{0}^{2}$ : Eq. (1) is $\square^{2}\left[\pi+2\left(\psi^{2}\right)_{.00}\right]=\Gamma V_{.00}$ and Eq. (2) is $\square^{2}\left[\pi-\Lambda\left(\psi^{2}\right)_{00}\right]=\Gamma T_{00}$ in which $\square^{2} \psi^{2}=L$ and $\Gamma=-(2+\Lambda)$. Outside the region of interaction $\pi=\rho_{s} c_{0}^{2}$ so the source for $\rho_{s} c_{0}^{2}$ can be either $\Gamma V_{, 00}$ or $\Gamma T_{, 00}$ and since, in general, $T \neq V, \rho_{s} c_{0}^{2}$ must be zero (except for unidirectional waves when $T=V$ in which case the well-known solution is still restricted to the interaction zone). As an example, consider two colliding waves for which the interaction energies satisfy $T_{12}=-V_{12}$ requiring $\rho_{s} c_{0}^{2}$ from Eq. (1) to be the negative of that from Eq. (2) which demands that $\rho_{s} c_{0}^{2}=0$. Within the interaction region the solution to either equation is $\left.\rho_{s} c_{0}^{2}=(\Lambda-2)\left(\phi^{+} \phi_{, 00}^{-}+\phi^{-} \phi^{+}, 0\right)-2 \phi_{, 0}^{+} \phi_{, 0}^{-}\right)$, where $\psi=\phi^{+}+\phi^{-}$the sum of right and left traveling waves. The scattered pressure $p_{s}=\rho_{s} c_{0}^{2}+\Lambda V$. For scattering to occur outside the interaction region, there must exist losses, as witnessed in the parametric array, or dispersion [see, e.g., P. J. Westervelt, "Scattering of Sound by Sound," in Finite Amplitude Wave Effects in Fluids, edited by L. Bjernd, Proc. 1973 Symp.
Copenhagen (IPC Science and Technology, London, 1974), or interaction with real sources [P. J. Westervelt, Virtual Sources in the Presence of Real Sources in Nonlinear Acoustics, edited by T. G. Muir (Proc. of Univ. of Texas at Austin Conference, Nov. 1969)].

$1: 30$

4pPA3. Scattering of sound by sound within the interaction zone: Approximate solutions. Peter J. Westervelt (Dept. of Phys., Brown Univ., Box 1843, Providence, RI 02912)

The exact second-order transient solution to the interaction of an arbitrary wave with a plane wave is given by Westervelt [P. J. Westervelt, J. Acoust. Soc. Am. 96, 3320 (1994)]. Let the arbitrary wave be $\chi\left(x^{0}-\hat{\mathbf{m}} \cdot \mathbf{r}\right)$, a plane wave traveling in the $\hat{\mathbf{m}}$ direction. In this case $\sigma=(1-\hat{\mathbf{n}} \cdot \hat{\mathbf{m}})^{-1} \chi$ and the solution to Eckart's equation becomes $\rho_{s} c_{0}^{2}=E_{12}$ $+(1-\hat{\mathbf{n}} \cdot \hat{\mathbf{m}})^{-1}(\Lambda+2 \hat{\mathbf{n}} \cdot \hat{\mathbf{m}})=\cos \theta \nabla^{2} \psi^{2}$, which is identical to Eq. (10) of Westervelt [P. J. Westervelt, J. Acoust. Soc. Am. 29, 934 (1957)] provided the substitutions $\psi^{2}=\frac{1}{2}\left(w_{1} w_{2}\right)^{-1} W_{12}$ and $\hat{\mathbf{n}} \cdot \hat{\mathbf{m}}=\cos \theta$ are made. It is asserted that this exact solution serves as an approximate solution to the far-field interaction of arbitrary sources. This is done by allowing $\hat{\mathbf{m}}$ and $\hat{\mathbf{n}}$ to be space dependent. As an example, the exact solution for the cardioid wave $\chi=(4 \pi r)^{-1}\left[\hat{\mathbf{n}} \cdot \hat{\mathbf{m}}\left(G_{.0}+G r^{-1}\right)-G_{.0}\right]$ interactions with a plane wave is obtained from $\sigma=-(4 \pi r)^{-1} \mathrm{G}_{0}$, where $G=G\left(x^{0}-r\right)$ and $\hat{\mathbf{m}}=\mathbf{r} r^{-1}$. In the far field of the cardioid source, $\sigma=(1-\hat{\mathbf{n}} \cdot \hat{\mathbf{m}})^{-1} \chi$, as in the plane wave-plane wave interaction, thus demonstrating the assertion.

\section{$1: 45$}

4pPA4. Nonlinear bubble oscillations and Bjerknes forces. Sean M. Cordry (Dept. of Phys., Univ. of Mississippi, University, MS 38677), Lawrence A. Crum, and Ronald A. Roy (Appl. Phys. Lab., Seattle, WA 98105)

The acoustic levitation of bubbles in standing waves has been considered by many authors. Calculations of the equilibrium levitation position have followed the example of Eller [A. Eller, J. Acoust. Soc. Am. 43, 170-171 (1968)] by considering linear radial pulsations whose amplitude is given by a linearized Rayleigh-Plesset equation. This procedure begins to fail as the bubble starts behaving in a nonlinear fashion. For bubbles experiencing nonlinear behavior, the radiation pressure (primary Bjerknes 
force) must be numerically calculated from the product of the instantaneous bubble volume and the pressure gradient of the sound field. Bubbles smaller than resonance size are normally attracted to the pressure antinode, but when nonlinear effects are considered, the bubbles can be repulsed by the pressure antinode for driving pressures above a certain threshold amplitude. Results of our calculations will be presented along with the implications for single bubble sonoluminescence. [Work supported by the office of Naval Research.]

\section{2:00}

4pPA5. Nonlinear elastic wave propagation along free surfaces of a thick plate. M. F. Hamilton, Yu. A. I''inskii, and E. A. Zabolotskaya (Dept. of Mech. Eng., Univ. of Texas, Austin, TX 78712-1063)

Nonlinear elastic wave propagation along free surfaces of a thick isotropic plate is investigated theoretically. The finite thickness of the plate introduces dispersion. In the linear approximation the solution is a superposition of symmetric and antisymmetric modes, and the wave field undulates along the surfaces of the plate. Nonlinearity is taken into account with the Hamiltonian formalism used to model Rayleigh waves of finite amplitude [Zabolotskaya, J. Acoust. Soc. Am. 91, 2569-2575 (1992)]. The resulting coupled spectral equations were integrated numerically to investigate harmonic generation and waveform distortion in an initially monochromatic wave generated on one side of the plate. Two different plate thicknesses were considered, 20 and 100 shear wavelengths. For the thicker plate, and with a shock formation distance much smaller than the dispersion length, the solutions resemble those for nonlinear Rayleigh waves in a half-space. For the thinner plate, and with the two length scales of the same order, propagation curves for the second harmonic component exhibit the "growth-decay cycles" that have been measured in experiments and discussed in a previous article [Shull et al., J. Acoust. Soc. Am. 94, $418-427$ (1993)]. [Work supported by NSF, Schlumberger, and the Office of Naval Research.]

\section{2:15}

4pPA6. Acoustic streaming at high Reynolds numbers in focused sound beams. M. F. Hamilton, Yu. A. Il'inskii, and E. A. Zabolotskaya (Dept. of Mech. Eng., Univ. of Texas, Austin, TX 78712-1063)

In experiments reported recently on acoustic streaming in focused sound beams [Starritt et al., Ultrasound Med. Biol. 15, 363 (1989), and Matsuda et al., Advances in Nonlinear Acoustics: 13th ISNA, edited by $\mathrm{H}$. Hobæk (World Scientific, Singapore, 1993), pp. 595-600], the Reynolds numbers for the streaming are of order 100 . To model such experiments, nonlinearity in the momentum equation for the streaming velocity should be taken into account. Previous comparisons of theory with measurements of acoustic streaming in focused beams are based on linear equations for the streaming velocity, which are normally valid only for Stokes flows characterized by Reynolds numbers less than unity [Lighthill, J. Sound Vib. 61, 391 (1978)]. The numerical results presented here were obtained with nonlinear inertia terms for the streaming velocity retained in equations that describe the paraxial region of the beam. The primary wave is assumed to be a focused Gaussian beam, with parameters corresponding to the experiments cited above. Inclusion of nonlinearity yields predictions of streaming patterns that are more localized in the focal region, and in closer agreement with experiment, than predictions based on linearized equations for the flow. [Work supported by Schlumberger, NSF, and the Office of Naval Research.]

\section{2:30}

4pPA7. Nonlinear waves through multicomponent fluid media with chemical reactions. Timothy S. Margulies (Natl. Ctr. for Phys. Acoust., Univ. of Mississippi, Oxford, MS 58677)

Finite-amplitude wave propagation has been investigated using balance and constitutive equations derived via continuum mixture theory for a multicomponent system such that simultaneous chemical reactions can occur. A multiple-time scale perturbation approach [T. Tanuiti and C.-C. Wei, J. Phys. Soc. Jpn. 24 (4), 941 (1968)] was used to develop a differential-integral equation for nonlinear wave propagation when diffusive motions can be neglected. The processes of dissipation (e.g., viscous and thermal), chemical relaxation, and nonlinear equation of state response influence the wave profile. Approximations, such as low-frequency and high-frequency expansions [J. Engelbrecht, Wave Motion 1, 65 (1979)] are examined. The low-frequency results in the classical Burgers' equation. Applications with calculations use information available in the literature for ocean and electrolytic solution environments. The case of slightly inhomogeneous media will also be discussed.

\section{2:45-3:00 Break}

\section{3:00}

4pPA8. An acoustic device for Prandtl number measurements in gases. Keith A. Gillis, Michael R. Moldover (NIST, Thermophys. Div., Bldg. 221/Rm. Al11, Gaithersburg, MD 20899), and James B. Mehl (Univ. of Delaware, Newark, DE 19716-2570)

An acoustic method is being developed for measurements of both the thermal and viscous diffusivities of gases using different sets of resonances of a single cavity. The ratio of the diffusivities, i.e., the Prandtl number, is a key parameter in determining the heat transfer from a solid to a moving fluid. Honeycomb inserts placed near the center $(z=L / 2)$ of a cylindrical cavity (length $L$ ) provide a large surface area with minimal blockage of longitudinal acoustic flow. Odd-order longitudinal modes, which have a velocity antinode at $z=L / 2$, have a strong viscous interaction with the insert. For even-order modes, which have temperature antinodes at $z=L I$ 2 , the thermal interaction dominates. Measurements of the resonance parameters of both types of modes are analyzed using a model which enables the viscous diffusivity to be determined from the odd modes and the thermal diffusivity from the even modes. Because the same insert is used for both the even and the odd modes, the ratio of the diffusivities is not sensitive to details of the geometry of the insert; thus, the Prandtl number can be determined more accurately than either diffusivity. [Work-supported by ONR.]

\section{3:15}

4pPA9. $A b$ initio calculations for helium, acoustics, and metrology. Michael R. Moldover (NIST, Thermophys. Div., Gaithersburg, MD 20899)

Lossy, gas-filled resonators are now being developed at NIST to measure the viscous and thermal diffusivities of gases. If these resonators become as well understood as the low-loss spherical resonators already developed at NIST, the uncertainty in the measured viscosity of a dilute gas will become less than $0.1 \%$, possibly limited by the difficulty of measuring the dimensions of a double-Helmholtz resonator (i.e., a Greenspan viscometer). The accuracy of the data from the acoustic viscometer will be tested by comparing experimental results for helium with the recent $a b$ initio result from quantum mechanical calculations: $\eta=(19.800 \pm 0.010)$ $\mu \mathrm{Pa}$ S at $298.15 \mathrm{~K}$. Similarly, the results for the Prandtl obtained from cylindrical acoustic resonators with inserts will be tested by comparison with the $a b$ initio result: $\operatorname{Pr}=\left(C_{p} \eta / \lambda M\right)=0.66419$. Helium-based gas thermometry (both traditional and acoustic) will benefit from the theoretical results for the second virial coefficient of helium. Perhaps pressure measurements in the 1- to 10-MPa region could be improved by combining the $a b$ initio results with highly accurate measurements of the dielectric constant of helium.

\section{3:30}

4pPA10. Recent progress and problems on study of forced standing solitons generated in Faraday wave experiment. Rongjue Wei (Inst. of Acoust., Nanjing Univ., Nanjing 210008, People's Republic of China)

From the simple Faraday wave experiment with rectangular tanks of variable length and width, or with insection of partitions, the standing or nonpropagating solitons will manifest a number of interesting nonlinear phenomena. These include parameters governing generation, stability, collision dynamics, reflection symmetry, chaotic behaviors, etc., of one or 
more solitons. Some of the not well-solved problems of the physics and mathematical formulations will be presented. [A brief summary of the work by the Nonlinear Acoustics Group I of Nanjing, supported by Chinese Nonlinear Sc. Foundation.]

\section{3:45}

4pPA11. Capillary bridge stability in an acoustic standing wave: Linearized analysis of passive stabilization with radiation pressure. Philip L. Marston (Dept. of Phys., Washington State Univ., Pullman, WA 99164-2814)

A liquid bridge between two solid surfaces, known as a capillary bridge, has applications in low gravity such as the solidification of floating zones. Long bridges are naturally unstable to a symmetric mode where a region adjacent to one end bulges while the opposite end thins. For a cylindrical bridge of radius $R$ and length $L$, the slenderness $S=L / 2 R$ has a natural (Rayleigh) limit of $\pi$ beyond which the bridge breaks. It is proposed here to stabilize dense bridges at a velocity antinode of a standing acoustic wave perpendicular to the bridge axis. Let $\left\langle p_{r}\right\rangle$ denote the angular average of the radiation presșure over the surface of a cylindrical bridge. It is necessary to select the acoustic frequency such that $\left\langle p_{r}\right\rangle$ increases with $R$ so that a bulge is automatically squeezed by the increased radiation pressure. Linearized analysis of the stability indicates $S$ may be extended beyond $\pi: S_{\max }$ depends on $q=\left(R^{2} / \gamma\right) d(p,\rangle / d R$, where $\gamma$ is the surface tension and $S_{\max }$ diverges as $q$ approaches unity. The analysis supports the possibility of passive acoustic stabilization of capillary breakup in low gravity. The one-dimensional inviscid slice approximation is used in the analysis of axisymmetric deformations. [Work supported by NASA.]

\section{4:00}

4pPA12. Capillary bridge modes driven with modulated acoustic radiation pressure. Scot F. Morse, David B. Thiessen, and Philip L. Marston (Dept. of Phys., Washington State Univ., Pullman, WA 99164-2814)

A liquid bridge between two solid surfaces is known as a capillary bridge. In the experiments to be described, the bridge liquid is a mixture of PDMS with a dense organic liquid. The bridge has the same density as the surrounding water bath such that its equilibrium shape between circular posts is a circular cylinder of length $L$ and radius $R$. Low-frequency modes of such a bridge are known to exist where surface tension provides the restoring force. Even neglecting viscosity, the modal characteristic equation is complicated by the boundary conditions. It is demonstrated that low-frequency modulation of an ultrasonic standing wave in the surrounding bath can be used to excite specific axisymmetric or nonaxisymmetric capillary modes. Coupling to each mode depends on the location of the bridge in the standing wave. The coupling is a consequence of the spacetime modulation of the radiation pressure as discussed previously for drops and bubbles [P. L. Martson, J. Acoust. Soc. Am. 67, 15-26 (1980)]. The predicted decrease of mode frequency-with increasing slenderness $S=L /$ $2 R$ is confirmed as is the vanishing of the frequency of the most unstable mode as $S$ approaches $\pi$. [Work supported by NASA.]

\section{$4: 15$}

4pPA13. An electromagnetic liquid shock wave generator for the production of a pulsed water jet. Bruce J. P. Mortimer (School of Elec. Eng., Cape Technikon, P.O. Box 652, Cape Town, 8000, South Africa) and Beric W. Skews (Univ. of the Witwatersrand, Johannesburg, 2050, South Africa)

Water jets can be either continuous or pulsed. Pulsed or discrete water jets have many advantages over continuous jets in certain applications. The production of discrete jets has mostly been due to a momentum transfer from a mass impacting a compression chamber. In this study the feasibility of using a novel adaptation of an electromagnetic source (EMAS) for the production of a pulsed water jet is investigated. This device consists of a high-voltage 4-8 F, 20-kV capacitor which is discharged through a flat pancake coil. An insulated metal disk is in close proximity with the coil.
Lorentz forces due to the eddy currents induced in the disk accelerate the disk away from the coil. The disk acceleration produces a shock wave in a liquid-filled cavity. A convergent reflector focuses the wave onto a nozzle. The reflection of the shock wave from the water-air interface produces a discrete water jet. A theoretical analysis of the electrical characteristics of the transducer is presented. A finite-element package was used to optimize the convergent reflector. Experimental results from a prototype generator are given including pressure measurements from a needle hydrophone and schlieren photographs of the water jet. [Work supported by FRD.]

\section{$4: 30$}

4pPA 14. Coherent enhancement of reflected signals in a parametric echo-sounder. Dimitri M. Donskoy (Davidson Lab., Stevens Inst. of Technol., 711 Hudson St., Hoboken, NJ 07030), Alexander M. Sutin, and Andrew I. Potapov (Inst. of Appl. Phys., Nizhnii Novgorod, Russia)

A parametric echo-sounder is used for sea sub-bottom profiling and target (cables, mines, etc.) finding. It generates a modulated highfrequency signal (pump signal). Due to the nonlinearity of water, part of the pump energy is converted into a low-frequency wave named the secondary signal. The high-frequency pump signal reflects mostly from a bottom surface and has high signal-to-noise ratio. The low-frequency secondary signal penetrates deeper under the bottom and reflects from subbottom layers and buried objects. However, the level of these reflected signals is low, often below noise level. In order to enhance the signal-tonoise ratio for these reflected secondary signals, the coherent average technique can be employed with the use of the reflected pump (high-frequency) pulses as reference signals. The feasibility of this approach is based on the following considerations. (1) The pump and the secondary signals are always correlated. (2) Average from one pulse to another does not depend on deviation in distance between the transducer and the bottom (due to the pitching, drifting, etc.) because the reference signal (reflected from the bottom pump signal) always follows this deviation. (3) The front of the pump signal is much sharper as compared with the front of the secondary signal. Therefore coherent averaging of the secondary signals can be done precisely. (4) For the "frozen" sub-bottom structure time delays between the first reflected signal and the all following reflected signals are fixed; therefore the coherent accumulation will take place for all of these signals. The proposed method was proved during feasibility field test performed at Barents Sea.

\section{$4: 45$}

4pPA15. Nonlinear vibro-acoustic method for diagnostics of cracks in construction materials. Aleksey $\mathrm{S}$. Korotkov and Alexander $\mathrm{M}$. Sutin (Inst. of Appl. Phys., Russian Academy of Sciences, 46 Uljanov St., 603600 Nizhny Novgorod, Russia)

New methods for the diagnostics of defects in objects made of construction materials, primarily of metals, are proposed. The basic concept of the proposed methods is simple: Materials containing defects have a much larger nonlinear response than materials with no ciefects. For materials with integrity, the nonlinear responses are up to several orders less as compared with the case of defected materials with defects. It is noteworthy to stress that these nonlinear effects are noticeable even at small strains far from a material damage threshold, thus making it rather attractive for nondestructive testing. The first experiments were performed employing samples shaped as rods made of steel used for fabricating parts of nuclear reactors. The samples were exposed to static stretching in order to introduce defects. Acoustic field modulation was measured by low-frequency vibrations and its increase (more than $30 \mathrm{~dB}$ ) in stretched samples was observed. The nonlinear interaction of the impact-produced vibrations with the acoustic field in steel samples with a crack was investigated. It was shown that the modulation of sound by such vibration can be used for cracks detection. The method was also used to test a welded joint in a large pipe line for a nuclear power station. 


\title{
Session 4pPP
}

\section{Psychological and Physiological Acoustics: Harvey Fletcher Memorial Session II}

\author{
Jont B. Allen, Cochair \\ AT\&T Bell Laboratories, Room 2D-553, 600 Mountain Avenue, P.O. Box 636, Murray Hill, New Jersey 07974-0636 \\ Roy D. Patterson, Cochair \\ MRC Applied Psychology Unit, 15 Chaucer Road, Cambridge, CB2 2EF, United Kingdom
}

Larry E. Humes, Cochair

Department of Speech and Hearing Science, Indiana University, Bloomington, Indiana 47405

\section{Invited Papers}

1:00

\begin{abstract}
4pPP1. Cochlear modeling since Fletcher. William M. Siebert '(Res. Lab. of Electron., Rm. 36-825, MIT, Cambridge, MA 02139)
In 1951, Harvey Fletcher published a comprehensive analysis of the macromechanical dynamic behavior of the cochlear partition in response to sound. Although not the first to derive the now-familiar one-dimensional long-wave differential equation, Fletcher's discussion is so clear and so careful that it had considerable impact at the time and remains today a model of elegant biophysical thinking. His results, moreover, matched the observations then available (primarily those of von Bekesy). The last 44 years, however, have seen the introduction of a variety of new experimental observations casting serious doubts on whether the cochlea can usefully be considered a linear passive system as Fletcher and his contemporaries assumed. And new analytical tools, notably the wide availability of extensive computational facilities, have substantially altered our idea of what constitutes a workable mathematical model. This paper will review the place of models of the Fletcher type in our current understanding of cochlear behavior.
\end{abstract}

$$
\text { 1:30 }
$$

4pPP2. The auditory filterbank: Fletcher's functional model of hearing. Roy D. Patterson (MRC Appl. Psych. Unit, 15 Chaucer Rd., Cambridge CB2 2EF, UK)

Fletcher [Rev. Mod. Phys. 12, 47-65 (1940)] summarized his observations on pitch, loudness, and masking in terms of "auditory patterns-spiral lines representing the cochlea with shaded regions showing neural responses to sinusoids. The auditory filterbank was a set of overlapping auditory patterns spanning the frequency range of hearing-a concept that has served as a functional model of auditory frequency analysis ever since. It has four main components: the filter shape, its bandwidth, the distribution of filters across frequency, and the detection criterion at the filter output. Fletcher identified these components and focused attention on them with his famous "band-widening" experiment in which a tone is masked by a variable width noise centered on the tone. The current paper reviews four areas of research spawned by the components of Fletcher's filterbank concept. The research has shown that the band-widening experiment is actually rather insensitive, and subject to a confounding which led to underestimation of filter bandwidth and overestimation of the detection criterion. But the more important assumptions about filter shape and distribution were proven essentially correct. As a result, current, computational, auditory filterbanks are surprisingly similar to Fletcher's original conception.

\section{2:00}

4pPP3. The excitation-pattern model according to Fletcher. S6ren Buus (Commun. and Digital Signal Process. Ctr., Dept. of Elec. and Comput. Eng. (409 DA), Northeastern Univ., 360 Huntington Ave., Boston, MA 02115-5096)

This talk reviews how Fletcher used excitation patterns to understand auditory processing. In the 1930's, Fletcher suggested that the relation between place of maximal excitation on the basilar membrane could be derived from measurements of critical bandwidths and frequency difference limens. He also showed that loudness and masking were closely related and that loudness for an arbitrary sound could be calculated on the basis of patterns of excitation integrated along the basilar membrane. Likewise, he suggested that discrimination of level differences between sounds could be understood on the basis of their excitation patterns. These concepts remain valid 60 years later.

\section{2:30}

4pPP4. Fletcher and the power spectrum model of hearing. Robert P. Carlyon (MRC Appl. Psych. Unit, 15 Chaucer Rd., Cambridge CB2 2EF, England)

Fletcher's famous band-widening experiment demonstrated that the masking of a pure tone by a continuous broadband noise was determined only by those components of the noise close to the signal frequency. This formed the basis of the pervasive "power spectrum" model of hearing, according to which the threshold of a signal of a given frequency is determined by the amount of masker energy passing through an auditory filter centered on that frequency. This rigorous prediction has aided the identification of additional forms of auditory processing, which have been revealed by exceptions to the power spectrum model. The interpretation of such 
findings, which include CMR, profile analysis, informational masking, and the overshoot effect, is greatly facilitated by the existence of a "default" model which accounts for a large amount of other data. In addition to reviewing these developments, new evidence will be presented demonstrating that the shape of a masker's excitation pattern can also be affected by the shape of its (temporal) envelope

\section{$2: 45$}

4pPP5. Evaluating human neural tuning curves from a mechanical model of the cochlea by relating them to psychophysical masking data. P. A. Garbes and J. B. Allen (Acoust. Res. Dept., Rm. 2D553, AT\&T Bell Labs, Murray Hill, NJ 07974)

Human neural threshold tuning curves are estimated by scaling the parameters of Allen's [J. Acoust. Soc. Am. 68, 1660-1670 (1980)] resonant tectorial membrane model of the cat cochlea. A way to evaluate the derived tuning curves using psychophysical data is developed, based on a psychophysical detection model which relates the physiological tuning curves to psychophysical masking data. A detection criterion, defined by a relationship among the bandwidth of the frequency tuning curves, expressed as an equivalent rectangular bandwidth (ERB), the width of the excitation patterns, expressed as an equivalent rectangular spread (ERS), and the psychophysical critical ratio, is explored and verified using cat data. The detection criterion is then used to test the derived human curves by making predictions of psychophysical masking and comparing the predictions to experimental data. The detection model may also provide a deeper understanding of the frequency resolving properties of the cochlea.

3:00-3:15 Break

3:15

4pPP6. Fletcher and loudness. Larry E. Humes (Dept. of Speech \& Hear. Sci., Indiana Univ., Bloomington, IN 47405)

In this presentation, some of the pioneering work performed by Harvey Fletcher and colleagues on the loudness of simple and complex sounds will be reviewed. The focus will be placed on the model and data presented by Fletcher and Munson [H. Fletcher and W. A. Munson, J. Acoust. Soc. Am. 5, 82-108 (1933)]. In this classic paper, the authors lay a solid foundation for the understanding of loudness which remains remarkably intact today in light of contemporary psychophysical and physiological knowledge. They provide thorough descriptions of several loudness phenomena, including loudness growth, monaural loudness additivity, and binaural loudness summation. Among other things, they demonstrate that loudness for a single tone grows as a function of the cube root of stimulus intensity, that loudness is additive for stimulus components in separate auditory filters or in separate ears, and that loudness of partially masked stimulus components is also additive once adjustments for partial masking have been made. Fletcher and colleagues were also interested in applying their model of loudness to hearing-impaired listeners. Their prior efforts in this area will be updated by the review of contemporary data on loudness growth and summation in the hearing impaired.

3:45

4pPP7. Fletcher's contributions to loudness measurement and theory. Rhona P. Hellman (Aud. Percept. Lab., Northeastern Univ., 360 Huntington Ave., Boston, MA 02115)

More than 70 years ago, Fletcher and his colleagues at Bell Telephone Laboratories initiated the first comprehensive investigations of loudness. Fletcher recognized that to improve the quality and efficiency of speech communication systems, one must know how the ultimate receiver, the human auditory system, processes and perceives sound. In his pioneering loudness studies, Fletcher devised innovative techniques for the measurement of loudness, uncovered the key parameters that modify loudness, and developed a model relating loudness to basic properties of the hearing mechanism. The achievements of Fletcher and his group demonstrated that loudness measurements can disclose (1) the relation between the spectral selectivity of hearing and sound intensity, (2) the effects of masking on loudness, (3) the principles of loudness additivity, (4) the dependence of loudness on spectral bandwidth, as well as (5) the functional integrity of the auditory system. These seminal discoveries not only inspired subsequent loudness studies, they also provided the empirical and theoretical foundation of contemporary loudness research and models. [Work supported in part by NIH Grant RO1 DC00084-18.]

4:00

4pPP8. Multichannel auditory perspectives, a historical view of Harvey Fletcher's forgotten contributions and their ramifications. Frederick J. Ampel (Technol. Visions S.A., 9512 W. 93rd St., Overland Park, KS 66212) and Ted Uzzle (Sound \& Video Contractor Magazine, Overland Park, KS 66212)

The history of sound transmission, recording and reproduction, and the number of channels those technologies may employ, is complex and convoluted. It has proceeded down numerous paths, and found many dead ends. Beginning with the work of Bell, Edison, and Clement Ader, the technical achievements and insights needed to produce successful and aurally accurate reproduction required much invention and enormous amounts of insight. How many channels? Sonic accuracy or artistic effect? Loudspeakers or headphones? Live or recorded? When Fletcher began his investigations in 1931, no one knew the answers. Regrettably, his significant contributions have been either lost to time or ignored. Without Fletcher much of what is known today about perception and sound would not have been possible. This paper will examine his contributions, remind one of the answers he found, show where they led, and bring them once again to the forefront. 
4pPP9. Harvey Fletcher and musical acoustics. William J. Strong, Irvin G. Bassett (Dept. of Phys. and Astron., Brigham Young Univ., Provo, UT 84602), and William M. Hartmann (Michigan State Univ., East Lansing, MI 48824)

Although the bulk of his work was concerned with various aspects of speech and hearing in communication, Harvey Fletcher had a longtime interest in musical acoustics. This interest is evidenced in his papers on perceptual aspects of musical tones, which stressed the complex interdependences of the percepts of loudness, pitch, and timbre on the physical parameters of intensity, frequency, and spectrum. However, his active involvement in musical acoustics research came to fruition only after he was released from other research, administrative, and teaching responsibilities. In conjunction with others, he explored perceptual aspects of piano, organ, violin, and percussion tones. His approach was that of analysis of natural tones, followed by synthesis of these tones based on the analysis results. Real and synthetic tones were presented to listeners who were asked to judge the tones as being real or synthetic. When listeners were unable to distinguish between the two, the synthetic tones were considered to contain all of the essential ingredients of the real tones. Fletcher's papers in which this work was described will be reviewed and samples of his real and synthetic tones will be presented.

\title{
Session 4pSAa
}

\author{
Structural Acoustics and Vibration: Radiation Transmission and Coatings
}

\author{
J. Stuart Bolton, Chair \\ Purdue University, 1077 Ray W. Herrick Laboratories, West Lafayette, Indiana 47907-1077
}

\section{Contributed Papers}

\section{1:15}

4pSAa1. Estimating acoustic radiation from a Bernoulli-Euler beam using shaped polyvinylidene fuoride film. Brian L. Scott and Scott D. Sommerfeldt (Grad. Prog. in Acoust. and Appl. Res. Lab., Penn State Univ., P.O. Box 30, State College, PA 16804)

A number of methods exist for determining far-field sound radiation from structures. Among such prediction techniques are purely analytical models and use of discrete sensors placed directly on the structure or in the surrounding fluid media. Analytical methods suffer from a lack of ability to model the structure completely and adapt to unforeseen system changes. Discrete sensors on the beam may require a large number of transducers to obtain an accurate estimate of the radiation and may also suffer from aliasing problems. Far-field sensors are often not suitable due to environmental constraints. An alternative method is the use of shaped distributed sensors on the structure, which act as low-pass filters in the wave-number domain. The use of this type of sensor helps to isolate the radiating portion of the wave-number spectrum and is also beneficial in reducing the number of sensors required to avoid aliasing. This work presents numerical results of using shaped polyvinylidene fluoride (PVDF) film to predict the farfield radiated sound. These results are compared to the theoretical results and to the results obtained when discrete sensors mounted to the structure are used.

\section{$1: 30$}

4pSAa2. Strategies for using strain sensing in active control of acoustic radiation. Patrice Masson, Alain Berry, and Jean Nicolas (G.A.U.S., Mech. Eng. Dept., Univ. of Sherbrooke, Sherbrooke, PQ J1K 2RI, Canada)

Sensing approaches used in active structural acoustic control (ASAC) are mainly concerned with monitoring acceleration, velocity or displacement of the structure, typically by means of accelerometers or PVDF, thereby eliminating the need for far-field acoustic sensor(s). The radiation of the structure is estimated from this information, either directly or from a model of the radiating structure. The direct approach has the advantage of not being dependent on the accuracy of any model. A new sensing strategy is herein presented, in both direct- and model-based approaches, where the estimation of acoustic radiation involves monitoring the strain field of the structure. Such information is directly given by fiber optics sensors, for example, so that signiticant gain is expected at this level. Two approaches are presented using strain information. The first formulation is obtained by integrating by parts the Rayleigh's formula. Dependence on boundary conditions then appears and has to be dealt with. The second formulation utilizes a finite-difference scheme in order to estimate, from the strain information, the displacement field in Rayleigh's formula. Simulations are performed using a given displacement field and the two formulations are compared in order to evaluate their effectiveness for use in active control schemes.

\section{1:45}

4pSAa3. The acoustic characterization of a new reverberant water tank. Richard Weyer, Dean Capone (Appl. Res. Lab., Penn State Univ., P.O. Box 30, State College, PA), and Richard Szwerc (Penn State Univ., State College, PA 16802)

A reverberant water tank has been constructed at the Pennsylvania State University. The background noise, diffusivity of the sound field, and the reverberation time in the tank have been measured as a function of frequency in one-third octave bands, from $80 \mathrm{~Hz}$ to $63 \mathrm{kHz}$. The techniques used to characterize the tank are presented. The characteristics of the tank are compared to other known water tanks. It is demonstrated that the tank is suitable for the experimental determination of the radiation efficiency of a structure by comparing data obtained in this tank with previously presented data for a flat plate. [Work supported by ONR.] 
driven on the side opposite the blanket by an incident acoustic wave. The

4pSAa4. Shape optimization of foam liners in double panel systems. Y. J. Kang and J. S. Bolton (1077 Ray W. Herrick Labs., School of Mech. Eng., Purdue Univ., W. Lafayette, IN 47907-1077)

Although elastic porous materials such as foams are widely used for passive control of aircraft and automobile interior noise treatments, numerical models of these materials have only recently became available [ $\mathrm{Y}$. J. Kang and J. S. Bolton, J. Acoust. Soc. Am. (1995)]. In the work described here the procedures of optimal design were combined with elastic porous material finite elements to optimize the shape of the foam liners. Specially, the surface contour of the foam lining was adjusted to minimize the sound transmission through a foam-lined double panel structure system in a waveguide. This model is a simple representation of an aircraft fuselage segment. The foam finite element was based on a complete elastic porous material theory that accounts for the three wave types known to be significant in foams. The procedures for coupling the foam finite element with adjacent acoustical and structural elements will also be presented. In the example problems, a plane wave is assumed to impinge on the incident surface of the double panel structure. The shape and material parameters of the foam liners were used as design variables, and the performance measure was the frequency-averaged sound transmission loss in the frequency bands of interest, e.g., the speech interference range.

\section{$2: 15$}

4pSAa5. Tailoring material properties of acoustic blankets for reducing radiated noise. Courtney B. Burroughs (Grad. Prog. in Acoust., Penn State Univ., State College, PA 16804)

The development and application of a mathematical model that considers the reduction in acoustic radiation produced by varying the material properties of a viscoelastic blanket in the direction parallel to the surface of the blanket are presented. The blanket covers an elastic plate which is model is used to compute the spatial distribution of the pressure on the top surface of the blanket. The total acoustic radiated power, normalized to the magnitude of the pressure incident on the plate, is computed from the surface pressure. Examples of the effectiveness of homogeneous and nonhomogeneous blankets in reducing acoustic radiation are presented. The effects of damping in the blanket are also examined.

\section{$2: 30$}

4pSAa6. The use of structural coatings to attenuate transmitted flexural wave-induced vibration. Richard F. Keltie (Ctr. for Sound and Vib., Dept. of Mech. and Aerospace Eng., Box 7910, North Carolina State Univ., Raleigh, NC 27695)

This study examined the used of a compliant coating on a plate to attenuate the transmitted structure-borne sound at the interface between the coating and the ambient acoustic medium. In order to gain physical insight into the problem, the layer was modeled as a fluid with dissipation. Although realistic layers are likely to be visco-elastic materials, the fluid layer approach has been used to provide for a tractable and useful analytical model. The performance of the layer model was evaluated by calculating the displacement field at the layer/medium interface in response to structural excitation. The 3-D wave-number-frequency response amplitude spectrum of the dynamic compliance was examined. This provided for the identification of the dominant processes determining the layer response. A simple physical interpretation of these results was shown to involve the dispersive characteristics of plate waves and acoustic waveguides. It was shown that the structural effects of the plate could be eliminated from the analysis, resulting in a model involving only the layer itself. The subsequent performance of the layer as a displacement (or velocity or acceleration) attenuator or amplifier was then examined. [Work supported in part by Naval Undersea Warfare Center, New London, CT.]

FRIDAY AFTERNOON, 2 JUNE 1995

MEETING ROOMS 12, 13, 14, 3:15 TO 5:15 P.M.

\title{
Session 4pSAb
}

\section{Structural Acoustics and Vibration: Plates and Shells I}

\author{
Jerry H. Ginsberg, Chair \\ School of Mechanical Engineering, Georgia Institute of Technology, Atlanta, Georgia 30332-0405
}

Contributed Papers

3:15

4pSAb1. Vibration and sound radiation of Mindlin plates including in-plane motion, stiffened by thin walled beams. C. Locqueteau and A. Berry (GAUS, Dept. of Mech. Eng., Univ. of Sherbrooke, Sherbrooke, Quebec J1K 2R1, Canada)

Stiffened panels such as those encountered in marine structures may exhibit in-plane motion in the panel due to the coupling with the deformation of the stiffeners. Most of the published papers on Mindlin stiffened plates do not take into account the in-plane motion of the panel. This paper presents a vibroacoustic model of finite, stiffened panel including flexion, shear, and in-plane motion of the panel, and flexion, torsion, and warping of the thin-walled beam stiffeners. The vibration problem is solved through a Rayleigh-Ritz approach, both for the fluid-coupled and the uncoupled cases. The numerical results are compared to recently published data. The effects of shear deformation and in-plane deformation of the panel are systematically investigated in terms of the panel response and sound radiation, and the results are compared to a simpler model retaining only pure flexural deformation of the panel and stiffeners. [Work supported by Defense Research Establishment Atlantic, Contract OSC92-01908.]
3:30

4pSAb2. A modal/spectral analysis of mass distribution effects in a fluid-loaded plate. John Babish and Jenry H. Ginsberg (School of Mech. Eng., Georgia Inst. of Technol., Atlanta, GA 30332-0405)

Feit and Johnson [J. Acoust. Soc. Am. 90, 2309(A) (1991)] showed that the signal scattered by a plate is greatly affected by the spatial distribution properties of an attached mass. Ginsberg et al. [ASME Proc., paper 93WA/NCA-20 (1993)] used the surface variational principle (SVP), which describes the surface pressure and displacement as a set of interacting waves, to examine a fluid-loaded plate with attached point mass in an infinite baffle. In their analysis the mass distribution was represented as a Fourier series in an effort to determine how coarse the model could be, and still accurately represent the direct point mass solution. Their conclusion was that a series length of eight terms is adequate to describe the system behavior in the frequency range $k L \leqslant 3$, but a series of fewer than six terms is substantially inaccurate. The current work uses the in-vacuo modes of the spectrally smoothed mass-plate system as Ritz functions for displacement. The solution in terms of these modes is compared to the results 
obtained from the Fourier series representation, in order to identify why a critical number of terms is required for the spectral description of mass distribution.

\section{3:45}

4pSAb3. Attenuation of waves in plates and bars using a graded impedance interface. Chandrasekar Vemula, Andrew N. Norris (Dept. of Mech. Eng., Rutgers Univ., Piscataway, NJ 08855), George Cody, and Gerry V. Storch (Exxon Res. and Eng., Annandale, NJ 08801)

A new method is proposed for allenuation of reflected energy at the edges of plates and bars, using a graded impedance interface. A standard approach is to embed the plate edges in sand. However, it has been verified that a graded impedance interface at the edges is far more effective in damping the radiating structural energy. $I t$ is well known that impedance mismatch causes complete reflection of energy at the free edge of a plate. Hence, it seems reasonable to expect a reduction in rellected energy when impedance is varied gradually. Experimental results for 1-in. plates indicate that at most $30 \%$ of the energy is damped for frequencies above $2 \mathrm{kHz}$ when sand is used, whereas as much as $60 \%-80 \%$ of the energy is damped between 2 and $10 \mathrm{kHz}$ using a graded impedance. Experiments on bars also give similar results. A theoretical formulation for a bar with graded impedance interface at the edges is presented, using both the Kirchhoff and Mindlin theories. The Mindlin theory predicts the level of energy damping that is observed in experiments, while the Kirchhoff theory predicts much lesser energy damping. The shear effects are modeled more accurately in the Mindlin theory and they seem to be important in this problem.

\section{4:00}

4pSAb4. A perturbation solution for forced response of systems displaying eigenvalue veering and mode localization. Hoang Pham and Jerтy H. Ginsberg (School of Mech. Eng., Georgia Inst. of Technol., GA 30332)

Various aspects associated with eigenvalue veering, such as extreme parameter sensitivity and localization of response, are of paramount importance for predicting the dynamic response of lightly coupled systems having nearly periodic components. This paper presents a perturbalion solution that simplifies analysis of forced response of continuous systems whose free vibration eigensolutions display eigenvalue veering and mode localization. In this investigation, the classic two-span beam with a strong torsional restraint at the intermediate pin support provides a prototype for developing the perturbation solution. Offset of the pin from the center position destroys periodicity of the two-span system, whereas the strength of the torsional spring relates the extent of interspan decoupling. A study by Chen and Ginsberg [J. Vib. Acoust. 114, 141 (1992)] established a relationship between the eigenfunctions and the eigenvalues for different values of the pin offset. The previous findings are merged with a Ritz expansion to formulate the forced response. The adequacy of the solution is determined by comparisons to results of baseline "Sturm-Liouvilletype" analyses. Flexibility of the perturbation solution is assessed in various case studies of lightly coupled two-span systems.

\section{4:15}

4pSAb5. Sensitivity analysis of structural eigenfrequency to plate design parameters by using neural network. Z. Q. Yu, L. H. Yam, and T. P. Leung (Dept. of Mech. \& Marine Eng.. Hong Kong Polytech. Univ., Hung Hom, Kowloon, Hong Kong)

The dynamic characteristics of plate structure can be improved by arranging the natural frequencies of the system in specified ranges. The sensitivities of structural dynamic response to design parameters can provide the essential gradient information for deriving efficient optimization procedures. They are used to form an approximate problem for solving the original structural optimization problem, while the sensitivity of geometric parameters to frequency can provide the essential gradient information for deriving efficient design procedures. In this paper, first the training set of neural network is generated by the finite-element method; second, the original design of a cantilever rectangular plate with multifrequency con- straints is obtained by using the neural network; third, the sensitivities of the plate eigenvalue to design parameters (i.e., $\partial f / \partial R_{i}$ ) around the point of original geometric design are obtained by using the trained neural network. Finally, the sensitivities of design parameters to eigenvalue (i.e., $\partial R / \partial f_{i}$ ) are also oblained by using the trained neural network. The influence of frequency on geometric parameters, as well as the influence of geometric parameters on frequency, is also discussed.

\section{4:30}

4pSAb6. Experiments on turbulent boundary layer/flexible structure/sound interactions in accelerated motion. L. Maestrello (NASA Langley Res. Ctr., Mail Stop 463, Hampton, VA 23681-0001)

Measurements are made of the response and acoustic radiation of a panel structure mounted in a wind tunnel and forced by a subsonic turbulent boundary layer and pure-tone sound. Measurements are made at accelerated speed because the resultant response exhibits a variety of behaviors different from data at constant speed. The structure exhibits broadband response with superimposed pure tone and its harmonics over the band, a nonlinear, nonstationary, and non-Gaussian process. The mechanism by which harmonics are generated becomes clear as the amplitudes of the fundamental and low-frequency band reduce with increasing level of the harmonics-a manifestation of energy transfer. Active control of the panel response using a time-invariant system recovers losses in relative stability as it regains partial reduction of the harmonics.

\section{$4 \cdot 45$}

4pSAb7. Observations of vibration-induced fatigue in steel using modal analysis. Sevag H. Arzoumanian, Douglas P. Koehn, and David C. Swanson (Appl. Res. Lab., Penn State Univ., P. O. Box 30, State College, PA 16804)

The acceleration to force transfer functions across a shaker-excited thin band under tension with a deep notch cut through its center is examined up to the point of fatigue-induced failure. An analytical model is developed to track the changes in modal properties evident in the experimental results as a function of band parameters. The progression of the structure toward failure is documented by plots of rolational stiffness and crack length as a function of fatigue time.

\section{5:00}

4pSAb8. Vibration analysis of triangular plates using the ray-tracing technique. R. Gunda, S. M. Vijayakar, and R. Singh (Acoust. and Dynam. Lab., Dept. of Mech. Eng., Ohio State Univ., 206 W. 18th Ave., Columbus, $\mathrm{OH}$ 43210-1107)

A new ray-tracing technique is proposed for the dynamic analysis of thin plates in flexure. This approach is based on the fundamental solution to the plate biharmonic equation and suitably placed image sources which simulate waves reflected from the plate edges. In this paper, this method is applied specifically to a class of triangular plates with certain aspect ratios. Since exact eigensolutions for some plates with simply supported boundary conditions are known, these cases are analyzed first. The method of images is also being extended or arbitrary polygonal plates where all the image sources may not be seen by all points inside the plate unlike the previous cases. Consequently, the image sources have to be selectively chosen depending on the excitation and observation points. Preliminary results, when compared with exact solutions and finite-element calculations, indicate that this method is capable of accurately predicting harmonic response. Another advantage of this technique is that the solution can be obtained within a spectral bandwidth. Convergence sludies show that the error norms rapidly decrease with an increase in frequency and damping. Hence, this method is computationally efficient in the highfrequency regime. [Work supported by the Army Research Office.] 


\title{
Session $4 \mathrm{pSC}$
}

\section{Speech Communication: Prosody}

\author{
Sally G. Revoile, Chair \\ Center for Auditory and Speech Sciences, Gallaudet University, Washington, DC 20002
}

\section{Contributed Papers}

4pSC1. Some phrase-level influences on the glottal waveform. Kenneth N. Stevens (Res. Lab. of Elec. and Dept. of Elec. Eng. and Comput. Sci., MIT, Cambridge, MA 02139)

The prosody of an utterance is often described in terms of the locations of attributes such as pitch accents, boundary tones, and vowel reduction. These prosodic descriptions are usually represented in the sound by changes in fundamental frequency, duration, and other temporal and spectral attributes of the segments. This paper presents data showing that the amplitude and spectrum of the glottal pulses, as well as their frequency, undergo modification depending on locations of pitch accents and boundary tones and depending on vowel reduction. The following results were obtained: (1) Reduced vowels tend to have significantly lower amplitude (7 to $13 \mathrm{~dB}$ ) than nonreduced vowels, together with an increased first-formant bandwidth and an increased tilt in the source spectrum; (2) a nonreduced vowel has a somewhat reduced amplitude (by about $6 \mathrm{~dB}$ ) when it follows a nuclear pitch accent; and (3) a nonreduced vowel in utterance-final position in a declarative sentence shows a decreased amplitude of the firstformant peak, an increased open quotient, and an increased spectrum tilt. Production mechanisms that could account for these changes include modifications in subglottal pressure, adjustments of the glottal configuration, and influences of constrictions in the supraglottal airway. [Work supported in part by a grant from NIDCD.]

\section{$2: 45$}

4pSC2. Effects of stress, vowel duration, and vowel height on the timing of pitch peaks in Czech. Christine Bartels (Linguist. Dept., South College, Univ. of Massachusetts, Amherst, MA 01003)

Czech is described as having word stress fixed on the first syllable, independent of vowel quantity. The pitch peak of a word generally falls on that first, stressed syllable. However, in some environments, the pitch peak shifts to a later syllable, leading to the perception that this later syllable is more prominent. This study investigated the phonetic factors conditioning such divergent placement of stress and pitch peak in Czech. Native speakers produced contextually embedded trisyllabic nonsense words with highpitch accents. Vowel height and quantity in the first and second syllable were independently varied to assess their effect on syllable pitch and the timing of the pitch peak. Whether a syllable was stressed constituted the third independent variable in the analysis. To determine whether stress remained on the first syllable, the timing of stress-related properties, i.e., amplitude and syllable lengthening, was examined. Results showed that a high vowel in either syllable tended to raise the pitch, but the second vowel could only attract the pitch peak if the first vowel was short and low. A long first vowel prevented accent shift to the second syllable. Stress-related properties remained largely confined to the first syllable, although the amplitude maximum occasionally shifted to the second syllable, if long.
3:00

4pSC3. Global durational patterns in spontaneous speech. Douglas O'Shaughnessy (INRS-Telecommun., 16 Place du Commerce, Verdun, PQ H3E 1H6, Canada)

Most analysis of speech examines carefully read texts. Natural spontaneous speech differs in several ways, having interruptions and large changes in speaking rate. The acoustic phenomena studied here concern the global speaking rate of spontaneous speech, how it varies for both fluent and disfluent speech. Such a model should find application in automatic speech synthesis and recognition. There is a tendency for speakers to adopt a specific articulation rate in fluent speech, especially for brief monosyllabic function words; stressed content words show greater variability, especially in prepausal situations. The greatest variability, however, occurs in disfluent speech, which have extremes in slow and fast speech. A large amount of the variability among word durations can be accounted for by: the number of phonemes per word, whether it is a function word or a content word, and whether the word forms part of a common sequence of words. Words at the start of a syntactic unit tended to be shorter than those at the end of the unit. The first time a content word was introduced into a discourse, it was stressed; repeated occurrences of such a word in the ensuing discourse had shortened durations. [Work supported by NSERCCanada.]

\section{3:15}

4pSC4. Acoustic and kinematic evidence for difference in language timing structure. Margaret H. Dunn (Haskins Labs., 270 Crown St., New Haven, CT 06511)

Proposed differences in the timing organization of Italian and Finnish are examined from the perspectives of simultaneous acoustic duration and bilabial movement (selspot tracked) collected from four native speakers of each language. All utterances were disyllabic, shaped CVC(C)V for Italian and $\mathrm{CV}(\mathrm{V}) \mathrm{C}(\mathrm{C}) \mathrm{V}(\mathrm{V})$ for Finnish. Italian, generally believed to be syllabletimed, shows regular acoustic intervals between vowels implemented by shortening of the initial vowel in closed syllables. Movement data shows that shortening is achieved by early initiation of clusters and geminate consonants, truncating the initial vowel. Finnish, generally described as mora-timed, shows increments of acoustic duration of disyllabic utterances with the addition of moras, either to vowels or to medial consonants. An apparent constraint on syllable duration leads to acoustic shortening of both initial vowels and intervocalic consonants in the longest, utterances. Movement data show that increases in quantity affect both medial consonant and preceding vowel portions of utterances. It is proposed that syllable-timing works directly from vowel to vowel, while mora-timing allows increase in duration of disyllabic utterances, adjusting both vowel and consonant durations to fill out or limit the possible intervals between vowels. [Work supported by NIH Grant DC 00121 to Haskins Laboratories.] 


\section{3:30-3:45 Break}

\section{3:45}

4pSC5. Importance of tonal envelope cues in Chinese speech recognition. Qian-Jie Fu (Dept. of Biomed. Eng., Univ. of Southern California and House Ear Inst., 2100 West Third St., Los Angeles, CA 90057), Fan-Gang Zeng, Robert V. Shannon, and Sigfrid D. Soli (House Ear Inst., Los Angeles, CA 90057)

Temporal waveform envelope cues provide significant information for English speech recognition, and, when combined with lip reading, could produce near-perfect consonant identification performance [Van Tasell et al., J. Acoust. Soc. Am. 82, 1152-1161 (1987)]. Tonal patterns are important for Chinese speech recognition and can be effectively conveyed by temporal envelope cues [D. H. Whalen and Y. Xu, Phonetics 49, 25-47 (1992)]. This study investigates whether tones can help Chinese-speaking listeners use envelope cues more effectively than English listeners. The speech envelope was extracted from broad frequency bands and used to modulate a noise of the same bandwidth. Mandarin vowels, consonants, tones, and sentences were identified by ten native Chinese-speaking listeners with 1, 2, 3, and 4 noise bands (or channels). The results showed that recognition of vowels, consonants and sentences increases dramatically with the number of channels, a pattern similar to that observed in English speech recognition. However, tones were consistently recognized at about $80 \%$ correct level independent of the number of channels. This high level of tone recognition produced a significant difference in open-set sentence recognition between Chinese $(11 \%)$ and English $(1 \%, p<0.01)$ for the one channel condition where no spectral information is available.

\section{4:00}

4pSC6. Demarcating prosodic groups with articulation. Cecile Fougeron (Phon. Lab, Dept. of Linguist., UCLA, Los Angeles CA 90095-1543 and Inst. de Phonet., Paris Sorbonne Nouvelle, Paris, France), Patricia A. Keating (UCLA, Los Angeles, CA)

It is hypothesized that phrasal position affects articulation, and that what looks like overall declination of articulatory displacement could arise from local enhancements or reductions at particular phrasal positions. To test this hypothesis, linguopalatal contact was measured for both the consonant $/ \mathrm{n} /$ and the vowel $/ \mathrm{o} /$ in reiterant speech. Utterances were based on arithmetic statements like " $(1+1) \times(1+1)$ " [reiterant "(no no no) no (no no no)"], where stress and phrasing were varied by choice of numerals and location of parentheses. For the one speaker analyzed, there was no overall downtrend in contact of $/ \mathrm{n} /$ through any sentence type when all syllablesstressed, word-initial, or phrase-initial-were compared. Instead, word-, phrase-, and sentence-initial $/ n /$ 's have cumulatively more contact than medial and final ones. It is proposed that greater initial contact contributes to marking prosodic boundaries by enhancing the consonant-vowel contrast. Also enhancing the $\mathrm{CV}$ contrast is the more open position for phrasefinal /o/'s. As a result, final edges of domains are marked by a greater articulatory distance from final $/ \mathrm{n} /$ to $/ \mathrm{o} /$, and initial edges of domains are marked by a greater articulatory distance from final $/ o /$ to initial $/ \mathrm{n} /$.

\section{4:15}

4pSC7. Intelligibility of Arab's production of English intonation. Fares Mitleb (Dept. of English, Yarmouk Univ., Irbid, Jordan)

This paper was intended to test the intelligibility of English intonation spoken by Arabs learning English as a foreign language. Two native British speakers heard English sentences produced by two native British speakers and six Arabs to decide whether a sentence is said with a falling or rising tune. Findings indicated that most Arabs confused the two English tunes to a significant level. They showed insensitivity to the two intonation patterns, a situation that might have resulted from the priority given to the teaching of segmental units of the target language. Therefore, the question of priority to at least the incorporation of intonational information and other suprasegmentals in the teaching process right from the outset is reconsidered. [Research supported by Yarmouk University.]

\section{$4: 30$}

4pSC8. Acoustic phonetic characteristics of internal open juncture by Mexican Spanish readers. Helen E. Karn (Dept. of Linguist., Georgetown Univ., Washington, DC 20057-1068) and Grace $H$. Yeni-Komshian (Univ. of Maryland, College Park, MD)

Internal open juncture refers to a set of two or more phrases which contain the same sequence of phonemes, but differ in their prosody, meaning, and orthography. In this study, ten adult native speakers of Mexican Spanish read three sets of phrases. Each set of phrases contained two words and three syllables with the sequence (la)\#(sa), 〈las)\#(a), and (las) \#(sa), as in the phrases la sabes "you know it," las aves "the birds," and las sabes "you know them." The phrases were read both in isolation and embedded in sentences (initial, medial, and final positions). Acoustic phonetic measurements were made of: (1) the duration of pauses (when present) between the first orthographic word and the second, (2) the duration of $\langle s\rangle$ and $(s s\rangle$, and (3) the average duration, fundamental frequency, and amplitude of the first syllable versus the second. Preliminary results suggest that internal open juncture in Mexican Spanish is more salient in phrases read in isolation than embedded in sentence contexts and that pause duration is the most consistent indicator of internal open juncture. 


\title{
Session 4pUW
}

\section{Underwater Acoustics: Bottom Scattering}

\author{
Peter M. Ogden, Chair \\ Naval Research Laboratory, Code 7142, 4555 Overlook Avenue, S.W., Washington, DC 20375-5350
}

Chair's Introduction-1:00

\section{Contributed Papers}

$1: 05$

4pUW1. Bottom backscattering strengths at low grazing angles using adaptive beamforming. Peter $M$. Ogden and Fred T. Erskine (Naval Res. Lab., Code 7142, 4555 Overlook Ave., SW, Washington, DC 20375-5350)

Bottom backscattering strengths have been measured between 70 and $1000 \mathrm{~Hz}$ using broadband, omnidirectional SUS charges as sources and a horizontal line array as a receiver in the Critical Sea Test (CST) series of at-sea tests. When conventional beamforming is used to form beams in this geometry, bottom fathometer returns often contaminate the backscattering results for grazing angles below about $30 \mathrm{deg}$. The use of an adaptive beamformer $(\mathrm{ABF})$ combined with examination of near-endfire beams allows scattering strengths to be reliably determined down to considerably lower grazing angles in many cases, over the entire range of frequencies measured. The ABF technique and its parameters and limitations will be discussed briefly. Examples of low-grazing-angle backscattering-strengths results will be shown for a variety of sites visited during various CST tests. [Work supported by ONR.]

\section{$1: 20$}

4pUW2. Shallow water bottom scattering strength at low frequencies. Peter G. Cable (BBN Systems and Technol., Union Station, New London, CT 06320-6147), Mike Steele, and James O'Connor (BBN Systems and Technol., Arlington, VA 22209)

Determinations of acoustic scattering strength for sand bottoms have been made at several shallow water sites under downward refracting sound propagation conditions in the frequency band $50 \mathrm{~Hz}$ to $1 \mathrm{kHz}$. The measurements have been made using explosive sources detonated at mid-water depth and received on two collocated, bottom-mounted hydrophone line arrays, with one array in vertical and the other in horizontal orientation. The major focus of this work has been the variation of scattering strength with frequency and with bottom grazing angle. For the scattering strength measurements, the presence of multipaths in shallow water prevents a direct path scattering geometry from being used at low frequencies and, consequently, scattering strength must be extracted from the full reverberation field. Two techniques for obtaining scattering strength integrated over grazing angle and for using the vertical array to examine the angular dependence of the scattering strength will be described. The results will be compared with other determinations of scattering strength for shallow water sand bottoms and will be discussed in terms of the role of scattering from structure within the bottom sediment.

\section{$1: 35$}

4pUW3. Preliminary comparisons between bistatic reverberation from an ocean ridge and wavelength-scale geomorphology. Nicholas C. Makris, Lilimar Avelino, and Richard Menis (Naval Res. Lab.. Washington, DC 20375)

Bistatic reverberation measured from a highly lineated ridge in the mid-Atlantic during the ARSRP Main Acoustics Experiment of 1993 was compared with bottom slope orientation determined from $200-\mathrm{m}$ resolution Hydrosweep bathymetry in a previous abstract [Makris et al., J. Acoust.
Soc. Am. 96, 3329(A) (1994)]. It was found that prominent retums register with extended scarps facing the source and receiving array. These scarps typically extend for lengths greater than $1 \mathrm{~km}$ and have widths that are less than $1 \mathrm{~km}$. However, the hydrosweep bathymetry is not of sufficient resolution to determine the fine-scale geomorphology of the scarps, and leads to slope estimates $\left(20^{\circ}\right.$ to $\left.30^{\circ}\right)$ that are a severe underestimate. Fine-scale bathymetry of $5-\mathrm{m}$ resolution recently obtained for the same ridge shows that the scarps returning prominent reverberation generally contain cliff faces with slopes between $60^{\circ}$ to $90^{\circ}$. However, the separation between these cliff faces is typically less than the roughly 500-m cross-range resolution of the towed-array imaging system. An attempt is made to resolve individual cliff faces with remote returns measured at $1 / 2 \mathrm{CZ}$ by exploiting the much higher range resolution of the towed-array imaging system (which approaches roughly $10 \mathrm{~m}$ ) and examining differently oriented bistatic measurements of the same sites.

\section{$1: 50$}

4pUW4. Backscatter from midfrequency $(325-1375 \mathrm{~Hz})$ LFM signals at the Mid-Atlantic ridge. J. R. Preston (Appl. Res. Lab., Penn State Univ., P. O. Box 30, State College, PA 16804)

In July 1993 SACLANTCEN participated in an experiment for the Acoustic Reverberation Special Research Program (ARSRP). The primary objective was to take high-resolution measurements to shed light on the detailed physical processes dominating the low-frequency scattering from rough topographic features and from deep sediment pond areas. A very detailed set of monostatic and bistatic scattering experiments were conducted (using lower frequencies from $200-375 \mathrm{~Hz}$ ) just west of the MidAtlantic Ridge near $26^{\circ} \mathrm{N}$ and $47^{\circ} \mathrm{W}$. New results are presented which show higher frequency monostatic scattering from selected bathymetric features using LFM signals. The receivers were horizontal arrays of 128 elements spaced at $0.5,1$, and $2 \mathrm{~m}$. Source/receiver depths were $\approx 130$ and $450 \mathrm{~m}$, respectively. Received reverberation levels are presented as functions of the estimated scatterer position on area bathymetry maps. Inferences for monostatic bottom scattering mechanisms are given using twoway FEPE parabolic equation propagation models from Collins to interpret the data. Some comparisons of measured and modeled reverberation are also presented.

\section{2:05}

4pUW5. Pulse length dependence in the CST bottom reverberation measurements. Sean M. Reilly (Tracor Appl. Sci., Inc., Anal. and Simul. Dept., 35 Thomas Griffin Rd., New London, CT 06320), Raymond J. Christian, John B. Chester, and Mark J. Vaccaro (Naval Undersea Warfare Ctr., New London, CT)

The Critical Sea Test (CST) is an empirical Navy program which collects low-frequency $(100-1000 \mathrm{~Hz})$ environmental acoustics measurements in order to provide a diverse, high-quality database for sonar performance prediction. During the course of its experiments in direct path bottom reverberation, CST has observed frequency, grazing angle, and 
waveform behaviors which differ significantly from those assumed in commonly available bottomscattering strength models. For empirical programs, deviations from standard models are significant because they limit the ability to extrapolate survey measurements into sonar performance scenarios. The most controversial aspect of the coherent source experiments has been the observation of a pulse length dependence in the scat lering strength measurements beyond the linear term nomally associated with ensonified area. This paper discusses CST's bottomscattering strength measurement technique and summarizes the pulse length "anomaly" results for a variety of geographic areas. Because these results run counter to standard modeling assumptions, this paper will also discuss the data quality procedures used to reach these conclusions. [Work supported by SPAWAR.]

\section{2:20}

4pUW6. Preliminary study of the semi-empirical geoacoustic (SEGA) model for bottom reverberation. Sean M. Reilly (Tracor Appl. Sci., Inc., Anal. and Simul. Dept., 35 Thomas Griffin Rd., New London, CT 06320)

Standard bottom reverberation models such as Lambert's rule and perturbation theory assume that scattering from the rough water/bottom interface is the dominant mechanism at all sonar frequencies. However, direct path bottomscattering strength measurements by the Critical Sea Test (CST) program in the 100 - to $1000-\mathrm{Hz}$ frequency range suggest that volume scattering from within the sediments may be the dominant reverberation mechanism in many applications. The CST program has been actively investigating a number of new bottom reverberation models which include scattering from within the sediments [Mourad et al., APL/UW Tech. Rep. No. 9107 (1991); Holland et al., J. Acoust. Soc. Am. 96, 3247(A) (1994)]. Using a combination of elements from these prior techniques, the SemiEmpirical Geoacoustic (SEGA) model for botrom reverberation is used to extrapolate geoacoustic survey measurements into general-purpose reverberation predictions. This presentation summarizes the results of a preliminary 3-month study which explored the viability of this new model using direct path bottom reverberation measurements from CST-5. [Work supported by SPAWAR.]

\section{2:35}

4pUW7. Bottom loss measurements and analysis in the eastern Mediterranean sea. Dmitry Chizhik (Naval Undersea Warfare Cur., New London, CT 06320)

Wideband bottom loss measurements were conducted at two sites in the eastern Mediterranean. The data were processed incoherently to determine bottom loss in one octave bands at center frequencies ranging from 200 to $4000 \mathrm{~Hz}$ and spanning grazing angle range of $10^{\circ}-90^{\circ}$. Geological literature indicated that the bottom at Herodotus abyssal plain location was a "fast" turbidite bottom, while the Nile cone site was characterized by a "slow" silty-clay bottom. The bottom loss model was formulated for each site and was found to be in good agreement with the data. A geometric ray model is often used for bottom loss prediction which includes the refracted (diving) wave arrival at the proper grazing angle. This "approach works well at high frequencies and high grazing angles, but gives erroneously low bottom loss values at low frequencies and low grazing angles. A hybrid ray/plane wave model was developed to account for the additional loss due to penetration of the wave into the sediment in excess of that predicted by the geometric ray theory. This correction is most important for "slow" bottoms at low grazing angles and low frequencies, as demonstrated by comparison to data.

\section{2:50-3:05 Break}

\section{3:05}

4pUW8. Further results from modeling deep-ocean reverberation from the Mid-Atlantic ridge. Stanley A. Chin-Bing (Naval Res. Lab., Stennis Space Center, MS 39529-5004) and Joseph E. Murphy (Univ. of New Orleans, New Orleans, LA 70148)

Results comparing computer model simulations and reverberation data [Chin-Bing and Murphy, J. Acoust. Soc. Am. 96, 3328(A) (1994)] have been reported previously. The reverberation data were taken from the MidAtlantic ridge region during the 1993 Acoustics Experiment as part of the ONR Acoustic Reverberation Special Research Program (ARSRP). A representation of the rough, range-dependent ocean sediment and sub-bottom bathymetry of the region was obtained from geomorphological models (the Webb-Jordan sediment distribution model and the Goff-Jordan fractal seafloor/basement model). A modified version of the Collins FEPE2WAY model was used for the long-range reverberation predictions. Our model simulations compared favorably with $210-$ to $290-\mathrm{Hz}$ bandwidth reverberation data gathered from "site A, seg 076." In this continuing study of site A, seg 076, the FFRAME and SAFE models are being used to study the influence of heavily sedimented regions on reverberation. New modeling results will be presented and compared with the measured reverberation. [Work supported by Office of Naval Research, Acoustic Reverberation SRP, and a High Performance Computing DoD Shared Resource Center grant.]

\section{3:20}

4pUW9. Effects of azimuthal coupling on bistatic reverberation. Kevin B. Smith (Dept. of Phys., Code PH/Sk, Naval Postgrad. School, Monterey, CA 93943), William S. Hodgkiss (Scripps Inst. of Oceanogr.. La Jolla, CA), and Frederick D. Tappert (Univ. of Miami, Miami, FL)

The University of Miami PE (UMPE) model [K. B. Smith and F. D. Tappert, MPL Tech. Memo. 432 (1993)] has been upgraded to include azimuthal coupling, making it a fully three-dimensional forward propagation model. By incorporating Tappert's PE reverberation (PEREV) model [F. D. Tappert, J. Acoust. Soc. Am. Suppl. 188, S84 (1990)], the effects of azimuthal coupling on bottom reverberation can be examined. During the July 1993 ONR-ARSRP main acoustics cruise, extensive bistatic data were recorded. using bathymetric data from the ARSRP Natural Laboratory, predictions of bistatic reverberation are made with and without azimuthal coupling. Differences between these two calculations'provide quantitative evidence of azimuthal coupling effects. Comparisons with measured data will also be presented. [Work supported by ONR, Code 321OA.]

\section{3:35}

4pUW10. The influence of sediment scattering and variability on acoustic intensity including data comparisons. Keith $J$. Howell, Melvin J. Jacobson, William L. Siegmann (Rensselaer Polytech. Inst., Troy, NY 12180-3590), and William M. Carey (Adv. Res. Projects Agency, Arlington, VA 22203-1714)

Previous work [Howell et al., J. Acoust. Soc. Am. 95, 2928 (1994)] has shown that statistics of multimodal intensity could be matched using a three-layer isospeed model that included effects of scattering. To more accurately estimate scattering effects on intensity over a broad frequency range, additional boltom information must be included. In this paper, an analytic treatment of a shallow channel consisting of $n$ isospeed layers is developed for a wide-angle PE propagation model. A scattering layer is introduced at a horizontal water-bottom interface to model effects of attenuation due to interface and sediment scattering. The local mean of intensity is determined and its sensitivity to parameters such as the number of layers, frequency, scattering layer thickness, and correlation length of inhomogeneities is investigated. The standard deviation of intensity is also examined. Comparisons are made between intensity statistics for the multilayer models and depth-dependent profile models consistent with data 
from a New Jersey Shelf experiment. Additional emphasis is directed toward the number of isospeed layers needed to match experimental intensity over a range of frequencies. [Work supported by ONR.]

\section{3:50}

4pUW11. New multiple scatter model of the ocean sediment. Dennis J. Yelton and Nicholas P. Chotiros (Appl. Res. Labs., Univ. of Texas, Austin, TX 78713-8029)

Reflection, transmission, and scattering properties of an inhomogeneous Biot medium were studied via numerical simulation. The inhomogeneous medium was bounded by a semi-infinite water medium on the source side, and a semi-infinite Biot medium of equivalent average properties on the other side. The inhomogeneous medium was simulated as a randomly layered Biot material. Each layer represented a granular material of particular grain size. By setting the thickness of each layer equal to a random grain size, a randomly stratified granular medium was simulated. Lateral variations in grain size were simulated by performing a coherent ensemble average of results from several realizations of the randomly stratified medium. The free parameters were the grain size mean and standard deviation. Other parameters were chosen to represent water-saturated sand. Reflected and transmitted signals were computed for a 1-MHz normally incident plane wave. Coherent and random components of the reflected signal were calculated. The random component was related to the scattering strength of the medium. It was found to increase with both the mean and the standard deviation of the grain-size distribution. The results are consistent with previous experimental results [Nolle, J. Acoust. Soc. Am. 35, 1394-1408 (1963)]. [Work supported by ONR code 1125OA.]

\section{$4: 05$}

4pUW12. High-frequency acoustic penetration into seafloor sediments at subcritical grazing angles due to roughness at the water-sediment interface. Eric I. Thorsos (Appl. Phys. Lab., Univ. of Washington, 1013 NE 40th St., Seattle, WA 98195)

Acoustic penetration into sediments at low grazing angles is of interest for buried mine detection. For sandy sediments the critical angle can be in the $25^{\circ}-30^{\circ}$ range, which suggests that penetration at lower grazing angles might be quite limited. It will be shown, however, that when the sediment roughness is modeled to be consistent with the occurrence of sand waves, then significant penetration into the sediment can occur at subcritical grazing angles due to scattering at the rough interface. An integral equation method is used to obtain acoustic fields near the surface to illustrate the degree of penetration. A 1-D surface model is used, and the sediment is modeled as a fluid. For a given surface profile, the acoustic penetration is found to increase as the frequency increases. When the frequency is reduced to bring the roughness into the perturbation theory region, acoustic penetration is much reduced. [Work supported by ONR.]
4pUW13. Effects of bottom type variability on modeled backscattered levels of a high-frequency sonar. Kristen D. Savage and Roger W. Meredith (Naval Res. Lab., Code 7174, Stennis Space Center, MS 39529)

Results investigating the effects of variable bottom composition on modeled high-frequency backscattered levels are presented for a typical shallow water, range-dependent environment. The modeled environment consisted of a single sound-speed profile, a flat sea bottom with rangedependent bottom composition, and a benign sea surface. Coarse and fine grain sandy areas were partitioned in range to create range dependence. Bottom backscattering and reflection loss for each partition were obtained from the recent University of Texas high-frequency ocean bottom backscatter model [N. P. Chotiros and F. A. Boyle, J. Acoust. Soc. Am. 96, 3264(A) (1994)]. Differences of $5 \mathrm{~dB}$ were discernible between the finecoarse-fine sand bottom and a range-independent fine sand bottom for ranges $<500 \mathrm{~m}$. The trend and structure of the backscattered levels were nearly identical for both bottom types. Differences of $42 \mathrm{~dB}$ for ranges $<500 \mathrm{~m}$ were visible in the modeled backscattered level of the finecoarse-fine sand bottom and the coarse-fine-coarse sand bottom. The trend and structure of the backscattered levels were also different. In all cases, surface reverberation had a strong impact on the backscattered levels, trends, and structures. [Work supported by MCM Tactical Environmental Data Systems (MTEDS) under Program Element 62435N.]

\section{4:35}

4pUW14. Acoustic scattering by marine sediments with irregularities of different types. Anatoliy N. Ivakin (N. N. Andreev Acoustics Inst., Shvernika 4, Moscow 117036, Russia)

There are two different types of irregularities giving the main contributions to bottom scattering: volume inhomogeneities of the sediment and roughness of its surface and interfaces. The problem of distinguishing and/or separating these contributions is considered using differences in frequency-angular dependencies of the scattering coefficient and analysis of the spatial autocorrelation function of the scattered field. The volume inhomogeneities of the sediment in turn can be considered as one of two types: spatial fluctuations of density and fluctuations of sound velocity. For water-saturated sediments (without gas), density fuctuations are usually stronger and their contribution to the total volume scattering is much more than that due to sound velocity fluctuations. But even low concentrations of gas bubbles can alter acoustic properties of sediments considerably, reducing the effective (average) sound velocity and enhancing fluctuations of sound velocity, with virtually no effect on the density. As a result, some specific features appear in sound scattering, and these can be used for remote acoustic characterization of sediments and determination of the type of bottom irregularities. [Work partially supported by ONR.] 


\title{
Meeting of Accredited Standards Committee S3 on Bioacoustics
}

to be held jointly with the

\section{U.S. Technical Advisory Group (TAG) Meetings for ISO/TC 43 Acoustics, IEC/TC 29 Electroacoustics, and ISO/TC 108/SC4 Human Exposure to Mechanical Vibration and Shock}

\author{
T. A. Frank, Chair S3
}

Penn State University Speech \& Hearing Clinic, 110 Moore Building, University Park, Pennsylvania 16802

R. F. Burkard, Vice Chair S3

Boston University Dept. of Communication Disorders, 635 Commonwealth Avenue, Boston, Massachusetts 02215

P. D. Schomer, Chair U.S. Technical Advisory Group (TAG) for ISO/TC 43, Acoustics

U.S. CERL, P.O. Box 4005, Champaign, Illinois 61820

H. E. von Gierke, Vice Chair U. S. Technical Advisory Group (TAG) for ISO/TC 43, Acoustics and ISO/TC 108/SC4, Human Exposure to Mechanical Vibration and Shock

1325 Meadow Lane, Yellow Springs, Ohio 45387

V. Nedzelnitsky, U.S. Technical Advisor (TA) for IEC/TC 29, Electroacoustics

National Institute of Standards and Technology (NIST), Building 233, Room A149, Gaithersburg, Maryland 20899

\footnotetext{
Standards Committee S3 on Bioacousties. The current status of standards under preparation will be discussed. In addition to those topics of interest, including hearing conservation, noise, dosimeters, hearing aids, etc., consideration will be given to new standards which might be needed over the next few years. Open discussion of committee reports is encouraged.
}

The international activities in ISO/TC 43 Acoustics, and IEC/TC 29 Electroacoustics, and ISO/TC 108/SC4 Human Exposure to Mechanical Vibration and Shock, will also be discussed. The Chairs of the U.S. Technical Advisory Groups for ISO/TC 43 (H. E. von Gierke), and IEC/TC 29 (V. Nedzelnitsky), will report on current activities of these Technical Committees and Subcommittees.

Scope of S3: Standards, specifications, methods of measurement and test, and terminology in the fields of mechanical shock and physiological acoustics, including aspects of general acoustics, shock, and vibration which pertain to biological safety, tolerance, and comfort. 


\section{Meeting of Accredited Standards Committee S1 on Acoustics}

to be held jointly with the

\section{U. S. Technical Advisory Group for ISO/TC 43 Acoustics and IEC/TC 29 Electroacoustics}

G. S. K. Wong, Chair S1, Institute for National Measurement Standards (INMS) National Research Council, Ottawa, Ontario KIA OR6, Canada

R. W. Krug, Vice Chair S1

Cirrus Research, Inc., 6423 West North Avenue, Suite 170, Wauwatosa, Wisconsin 53213

P. D. Schomer, Chair U. S. Technical for ISO/TC 43, Acoustics

U.S. CERL, P.O. Box 4005, Champaign, Illinois 61820

H. E. von Gierke, Vice Chair U. S. Technical Advisory Group (TAG) for ISO/TC 43, Acoustics 1325 Meadow Lane, Yellow Springs, Ohio 45387

V. Nedzelnitsky, U. S. Technical Advisor (TA) for IEC/TC 29, Electroacoustics

National Institute of Standards and Technology (NIST), Building 233, Room A149, Gaithersburg, Maryland 20899 Standards

Standards Committee S1 on Acoustics. Working group chairs will report on their preparation of standards on methods of measurement and testing, and terminology, in physical acoustics, electroacoustics, sonics, ultrasonics, and underwater sound. Work in progress includes measurement of noise sources, noise dosimeters, integrating sound-level meters, and revision and extension of sound level meter specifications. Open discussion of committee reports is encouraged.

The international activities in ISO/TC 43 Acoustics, and IEC/TC 29 Electroacoustics, will also be discussed. The chairs of the respective U. S. Technical Advisory Groups for ISO/TC 43 (H. E. von Gierke), and IEC/TC 29 (V. Nedzelnitsky), will report on current activities of these Technical Committees.

Scope of S1: Standards, specifications, methods of measurement and test and terminology in the field of physical acoustics including architectural acoustics, electroacoustics, sonics and ultrasonics, and underwater sound, but excluding those aspects which pertain to biological safety, tolerance and comfort.

\section{Session 5aMU}

\section{Musical Acoustics: General Topics}

Uwe J. Hansen, Chair

Department of Physics, Indiana State University, Terre Haute, Indiana 47809

Chair's Introduction-8:25

\section{Invited Papers}

8:30

5aMU1. Interaction of lips and mouthpiece in a brass instrument. Gabriel Weinreich and Fang-Chu Chen (Randall Lab. of Phys., Univ. of Michigan, Ann Arbor, Ml 48109-1120)

The difference between the frequency of a played note on a brass instrument and the frequency of the resonator (defined as the location of the maximum of its input impedance at the mouthpiece) has been experimentally investigated. To avoid the complexity of the lips interacting simultaneously with many modes, a Helmholtz resonator with an attached "mouthpiece" whose diameter is that of a trumpet was used. By having a loudspeaker as one of the walls of the resonator, and driving it with a signal derived from an attached 
microphone, it was possible to adjust both the resonant frequency and the $Q$. The input impedance is measured (without the player's lips leaving the mouthpiece) by comparing the passive response of the resonator with and without a known increase in volume. In the considerable majority of cases, the playing frequency is higher than the resonator frequency, indicating that the lips "beat outwards." Possible error sources will be discussed. [Work supported by NSF.]

9:00

5aMU2. Acoustics of a bell with a tube on its head. Tong Chen (Inst. of Acoustics, Acad. Sinica, 100080 Beijing, People's Republic of China)

Temple bells in Korea are quite special as compared with bells in other countries. The bell has a tube on its head and a pit or cavity ("rumbling structure") under it. The acoustical behavior of the bell and its pit has been modeled as a cylindrical cavity with an air gap. The air gap changes the mode frequencies and damping factors of the cavity; the damping factor increases sharply with increase in length of the air gap, especially for some modes. In real cases, the air gap between the bell and the rumbling structure is limited to 0.3-0.4 $\mathrm{m}$. The tube on the bell is effective for radiation of sound only when its length is nearly an integer times the half-wavelength of a cavity mode. It is reasonable to adjust the tube length for the most prominent partial in the bell sound, which determines the strike note. The acoustic mode frequencies can be tuned by adjusting the depth of the pit and the length of the tube to improve the timbre of the bell sound.

\section{9:30}

5aMU3. Musical pitchness evaluation through pitch extraction. Alexandre Galembo (47 Furstadtskaia St., \#17, 191123 St. Petersburg, Russia)

Timbre and pitch are different properties of sound according to the ASA definition, but they are closely connected in the sounds from musical instruments. An interesting property is the "pitchness" (or "pitch strength") which refers to the distinctiveness of pitch in the sound. This property was investigated for piano notes, in particular in the bass and treble. In these ranges, the degree of pitchness is an important factor in determining instrument quality. Pitch extraction methods were used to calculate a noise factor in the treble, and an inharmonicity factor in the bass. New methods for judging the sound quality in the treble, and for measuring and evaluating inharmonicity of bass strings are proposed. [Work supported by "Red October" piano factory, St. Petersburg, Russia, and Swedish Institute, Stockholm, Sweden.]

\section{Contributed Papers}

\section{0:00}

5aMU4. An efficient time-domain model for the piano using commuted elements. Julius O. Smith and Scott A. Van Duyne (Ctr. for Comput. Res. in Music and Acoust. (CCRMA), Dept of Music, Stanford Univ., Stanford, CA 94305)

A new time-domain model for the piano is proposed which is extremely efficient for synthesizing piano sounds in hardware or software. The model includes multiple coupled strings, a nonlinear damped-spring hammer model, and a linear soundboard and enclosure component which can have arbitrarily large order at very low cost. Simplifications based on the commutativity of linear, time-invariant systems greatly reduce computational complexity [Comput. Music J. 74-91 (Winter 1992); Proc. International Computer Music Conference, Tokyo, pp. 56-71]. The hammerstring interaction is highly nonlinear and therefore does not commute with other components, in principle. However, by introducing a very mild approximation having little or no impact on the sound, commutativity can be achieved, leading to the enormous computational savings. This presentation will review the derivation of the piano synthesis model with special emphasis on the nonlinear hammer component. In its present form, a complete, two-key piano can be synthesized in real time on a single Motorola DSP56001 signal processing chip with $8 \mathrm{~K}$ words of static RAM and a clock rate of $25 \mathrm{MHz}$

\section{0:15}

5aMU5. A linear filter approximation to the hammer/string interaction for use in a commuted synthesis piano model. Scott A. Van Duyne and Julius O. Smith, III (Ctr. for Comput. Res. in Music and Acoust., Dept. of Music, Stanford Univ., Stanford, CA 94305)

In commuted synthesis of string instruments, the soundboard/body resonator is commuted to the excitation point and replaced by its own impulse response [Smith and Van Duyne, elsewhere in this session]. Hence, the highly nonlinear hammer/string interaction must be replaced by a commutable linear filter. Using the wave digital hammer computational model of the piano hammer [J. Acoust. Soc. Am. 96, 3300(A) (1994)], it was observed that the force pulse of a hammer striking an infinite string was qualitatively similar to the impulse response of a second-order filter with two real poles. Hence, good second- and higher-order filter designs based on physical data were possible. However, multiple humps may appear in the bammer force pulse on a terminated string due to returning string waves. It was observed that the magnitude spectra of the single hump spectrum and the multiple hump spectrum were similar in bandwidth, differing only in a slight ringing in the lower spectrum due to the lowpassed combing effect of the returning string waves. Therefore, an equalization filter was designed to summarize this combing effect by fitting a bank of parallel second-order sections to the complex ratio spectrum. Excellent linear piano hammer simulations were produced.

\section{0:30}

5aMU6. A passive nonlinear digital filter design which facilitates physics-hased sound synthesis of highly nonlinear musical instruments. Scott A. Van Duyne and John R. Pierce (Ctr. for Comput. Res. in Music and Acoust., Dept. of Music, Stanford Univ., Stanford, CA 94305)

Nonlinearities, small or large, favorably affect the sounds of many musical instruments. In gongs and cymbals, nonlinearities cause the passive transfer of energy from lower frequency modes to higher frequency modes after the instrument has been struck. While many spectral modifications can be achieved by the inclusion of memoryless nonlinearities (such as square-law or table look-up) within the resonant loops of physicsbased digital sound synthesis algorithms, energy conservation cannot be achieved without compensating amplitude scaling. In fact, the greater the nonlinear effect desired, the more difficult it is to maintain passivity. Yet, for gongs and cymbals, a very large nonlinear effect is required. A computationally efficient nonlinear digital filter has been designed based on a physical system constructed from passive lossless elements only. This filter may be incorporated into any physical modeling algorithm (such as Karplus-Strong, digital waveguide, or 2D digital waveguide mesh) where traveling waves are being computed. System loss is decoupled from the 
nonlinear effect, and may be designed independently. Further, system energy spreads locally in the spectrum, as is found in real musical instruments; the rate and spectral region of energy spreading is controllable. Promising gong sounds have been produced.

\section{0:45}

5aMU7. Horn reflectance update. David Berners and Julius O. Smith, III (Ctr. for Comput. Res. Music and Acoust., Stanford Univ., Stanford, CA $94305-8180$ )

The flared horn is modeled according to Webster's equation. A change of variables transforms the equation into the form of the one-dimensional Schrödinger wave equation. The Schrödinger form facilitates specification of arbitrary axisymmetric wavefronts for the acoustic disturbance within the hom. To provide a physically motivated choice of wavefront shape, Poisson's equation is solved inside the horn subject to the boundary condition that the nornal component of the potential gradient is zero at the boundary of the horn. Since the disturbance within the horn must satisfy the wave equation, the velocity potential satisfies Poisson's equation when viscous effects and losses are ignored. Physical data from brass instrument bells are used to model musical horns using the Poisson solution, and results are compared to those obtained by traditional models which assume spherical wavefronts. Results are also compared to acoustic measurements.

\section{1:00}

5aMU8. Equivalence of finite difference approximation and digital waveguide modeling for lossless, nondispersive media in one to three dimensions. Julius O. Smith and Scott A. Van Duyne (Ctr. for Comput. Res. in Music and Acoust. (CCRMA), Dept. of Music, Stanford Univ., Stanford, CA 94305)

The finite difference approximation method is commonly used to convert a differential equation into a recursive computation for computer simulation of an acoustic medium. Less well known is the digital waveguide modeling approach to the same problem, which is based on simulating the propagation of sampled traveling waves in the medium, and which implements losses and dispersion using digital filters applied to the traveling waves [Comput. Music. J., 74-91 (Winter 1992)]. It turns out the two methods are equivalent in rectilinear coordinates in one, two, and three dimensions, in the lossless, nondispersive case, provided the spatial sampling interval is chosen to be a specific constant ( $c$ in the one-dimensional case) times the temporal sampling interval. Since the digital waveguide simulation technique requires far less computational effort, it can be used to both accelerate and increase the accuracy of numerical simulations of acoustic media.

\section{1:15}

5aMU9. Qualitative and quantitative nonlinear dynamics of clarinet tones and multiphonics. Teresa D. Wilson and Douglas H. Keefe (Systematic Musicol. Program, School of Music DN-10, Univ. of Washington, Seattle, WA 98195)

Nonlinear dynamics offers a nontraditional but useful viewpoint from which to study tone production in the clarinet, and its applications have not been fully explored. A qualitative and quantitative analysis using nonlinear dynamics was done on pressure time series for single (monophonic) and multiphonic tones performed by a professional clarinetist. The pressure was measured by a piezoresistive pressure transducer inserted flush with the inner mouthpiece wall $4.5 \mathrm{~cm}$ from the tip. Phase portraits and Poin- care sections were produced, and pressure spectra, correlation dimensions $D$, and Lyapunov exponents were calculated. The multiphonic spectra were biperiodic and phase-locked. For single tones $D=1.0-1.1$, and for multiphonics $D=2.2-2.5$. The largest exponent for both single tones and multiphonics was small and positive; for example, the information loss was 1 bit per 44 periods for the written note $E_{3}$ and 1 bit per 68 periods for $\mathrm{E}_{5}$. The slightly positive Lyapunov exponent and the values $D>1$ for single tones, and $D>2$ for multiphonics, may be interpreted as the information loss due to slight fluctuations of a performer who maintains a steady tone. This result represents a significant difference between human performance and physical modeling of musical tone production.

\section{1:30}

5aMU10. Sound spectra from air-driven American organ reeds. James P. Cottingham, Ben L. Colson, Scott T. Wilson (Phys. Dept., Coe College, Cedar Rapids, IA 52402), and Keelyn W. Quigley (Coe College, Cedar Rapids, IA 52402)

The "American" reed organ is distinguished from the "European" harmonium by the use of a partial vacuum to draw air past the free reeds rather than the use of a pressure bellows to drive compressed air through them. A sample of reeds from three instruments has been studied in the laboratory using a specially constructed windchest. Spectral analyses of near field sound produced by air-driven reed vibrations were obtained. Measurements were made of the variation with pressure of the frequency and amplitude of the fundamental as well as the spectral components. Relationships between certain aspects of reed design and the spectrum of the resulting sound can be compared with some "conventional wisdom" found in the literature on the instrument. Results are also compared with spectra obtained when these reeds are played in the instruments and with averaged sound spectra from the instruments. It is found that the frequency remains essentially constant over a very wide pressure range, and that the sound spectrum from a given reed, although modified somewhat by its surroundings in the actual instrument, is recognizably the main determinant of the sound spectrum radiated from the instrument.

\section{$11: 45$}

5aMU11. Discrimination of musical chord components. Barbara E. Acker and Richard E. Pastore (Dept. of Psych., SUNY, Binghamton, NY 13902)

The current study uses an accuracy version of the Garner paradigm to evaluate the nature of the roles played by the $E$ and $G$ frequencies in the perception of root position $C$ major triads. Triads differed in the $E$ and/or $\mathrm{G}$ frequencies, relative to an equal-tempered triad (prototype, or $\mathrm{P}$ set) or an out-of-tune triad (nonprototype, or NP set). Thus, the $P$ stimuli were more representative of major chords than the NP stimuli. Significant redundancy gains and interference effects were found for both frequencies in both contexts, thus demonstrating the components to be integral, as might be expected for tuning based upon intervals. The amount of redundancy gain found was dependent on the type of correlation, with the negatively correlated stimuli experiencing a greater redundancy gain than the positively correlated stimuli. Finally, better overall discrimination was found in the P context, thus replicating previous work [Acker, Pastore, and Hall, Percept. \& Psychophys. (in press)] showing that a prototype functions as a perceptual anchor. In addition, major chord prototypes appear to be located in the physiologically motivated area of just temperament, as opposed to the more experientially defined area of equal temperament. [Work supported by AFOSR.] 


\title{
Session 5aNS
}

\author{
Noise: Community Noise and Flow Noise \\ Louis C. Sutherland, Chair \\ 27803 Longhill Drive, Rancho Palos Verdes, California 90274
}

Chair's Introduction- $-8: 00$

\section{Contributed Papers}

8:05

5aNS1. Modeling urban noise pollution as an architectural acoustics problem. Gregory A. Miller (Mech. Eng. Dept., The Cooper Union for the Advanced of Science and Art, Cooper Square, New York, NY 10003-7183) and Daniel R. Raichel (The Cooper Union and the Graduate Center of the City University of New York)

The problem of urban noise pollution can almost be considered as an architectural acoustics problem. City blocks are treated in a manner mathematically analogous to the acoustics of rooms. Six New York city blocks were selected-chosen for their varying geometries, materials, and traffic patterns-and measurements of $L_{\mathrm{eq}}$ were taken during one-hour periods using a type 1 sound level meter. This is an experiment in progress with even more data to be accumulated for further refinement of the theory. The sound levels produced by various sources in these blocks are found by measuring average traffic flow, pedestrian traffic, etc. Predicative noise level equations (based on the sound level from a point source) are being generated on the basis of the physical characteristics of each block, and will be reconciled with experimental data to formulate characteristic equations that should accurately predict the sound pressure levels which observers are subjected to. It is anticipated that the alteration of the absorption coefficients of the structures lining the blocks will constitute the major factor within the reconciliation. The results of this experiment can in time be used to encourage wiser materials choices and more careful planning in future urban development.

\section{8:20}

5aNS2. Noise barriers with random edge profiles. Steve Ho, llene J. Busch-Vishniac, and David T. Blackstock (Dépt. of Mech. Eng., University of Texas, Austin, TX 78712-1063)

In nornal design the top edge of a noise barrier is straight. As a result, noise diffracted from the barrier edge seems to come from a string of highly correlated point sources, that is, a straight line source. The coherence of the diffracted sound therefore limits the effectiveness of the barrier. One way to spoil the coherence of the diffracted sound, and thus increase the barrier's insertion loss, is to vary the barrier height by making the top edge irregular instead of straight. The radiations from the point sources at the edge then are not well correlated. We have conducted preliminary experiments using physical scale models with random edge profiles. The spacing between height transitions and the height variation maxima are scaled to the wavelength at which the sound source (a spark) has a peak in its spectrum. Results to date show significant improvement (3-8 dB) for a barrier with a random edge profile compared to one of the same average height with a straight edge. [Work supported by TxDOT.]

\section{8:35}

5aNS3. Texas highway noise barriers-The good, the bad, and the ugly. Ilene Busch-Vishniac, Steve Ho (Dept. of Mech. Eng., Univ. of Texas, Austin, TX 78712), Ron Peron, and Michael McNemey (Univ. of Texas, Austin, TX)

Until recently, Texas has managed to avoid extensive use of highway noise barriers for roadway noise mitigation in residential areas. In recognition of the growing noise problem, particularly in the urban areas of Texas, the Texas Department of Transportation (TxDOT) is supporting a 3-year project aimed at producing a highway barrier design guide. In addition to acoustical performance, the design guide will consider issues such as barrier support and aesthetics. This talk will review the barrier project, concentrating on the first phase of the work which has been to document and evaluate the existing barriers in Texas. [Work supported by TxDOT.]

\section{8:50}

5aNS4. Sonic boom as possible mechanism of the low-frequency hum. Victor V. Krylov (Ctr. for Res. into the Built Environment, Nottingham Trent Univ., Burton St., Nottingham NGl 4BU, UK)

The problem of disturbing low-frequency noise, also called lowfrequency hum, has been known for at least two decades. However, in many aspects the nature of low-frequency hum still remains a mystery (see, e.g., "The Independent" of 22 June 1994). It may be possible that in some cases the sources of this hum are underground gas or petrol pipes where turbulent flows of gas or liquid generate sound waves of high amplitude propagating in a pipeline as in a waveguide. The velocities of sound $C_{0}$ inside the pipes ( $450 \mathrm{~m} / \mathrm{s}$ for methane) sometimes may be higher than the velocities of Rayleigh surface waves $C_{R}$ in the ground at the frequencies of interest $\left(5-50 \mathrm{~Hz}\right.$ ). Typical values of $C_{R}$ are $300-600 \mathrm{~m} / \mathrm{s}$. If so, i.e., if $C_{0}>C_{R}$, then ground Rayleigh waves might be effectively generated by sound wave propagating inside the pipes, the mechanism of generation being similar to that of a sonic boom from supersonic jets. Preliminary calculations show that central frequencies of generated Rayleigh wave ground vibration spectra depend on depth of the pipe and are in the low-frequency range. The amplitudes of generated ground vibration velocity due to sound waves propagating in gas pipes buried at a depth of $2 \mathrm{~m}$ can be around $70 \mathrm{~dB}$ (relative to $10^{-9} \mathrm{~m} / \mathrm{s}$ ). This may be enough to annoy some people both because of direct impact of vibrations and due to generated structure-borne noise.

\section{9:05}

5aNS5. Environmental vibrations associated with high-speed trains. Victor V. Krylov (Ctr. for Res. into the Built Environment, Nottingham Trent Univ., Burton St., Nottingham NG1 4BU, UK)

The dramatic revival of railways in Europe to become one of the most advanced and fast developing branches of transportation technology may be compared with the space technology breakthrough of the 1960s. The reason is high speeds achievable by the most advanced modern railway 
trains, e.g., French TGV trains for which a maximum speed of more than $515 \mathrm{~km} / \mathrm{h}$ was recorded in May 1990. Unfortunately, the increased speed of railway communications is likely to raise levels of associated environmental noise and vibration far beyond those significant even for conventional railways. A review is given of recent progress in the theoretical investigation of ground vibrations generated by high-speed railway traffic, with emphasis on problems associated with superfast trains, i.e., trains traveling at speeds close to or greater than $300 \mathrm{~km} / \mathrm{h}$. Significant increase in ground vibration levels (more than $70 \mathrm{~dB}$ ) is predicted for superfast trains if they travel at speeds higher than Rayleigh wave velocity in the ground [V. V. Krylov, J. Phys. IV, C5 4, 769-772 (1994)]. Attention is paid to establishing relations between parameters of the problem, e.g., geometrical dimensions of track and train, layered structure of the ground, train speed, etc., that could result in direct reduction in ground vibration generation efficiency.

\section{9:20}

5aNS6. Application of computational fluid dynamics (CFD) to automobile wind noise source minimization. Keng D. Hseuh, Sanjay Abhyankar, Sanjeeva Addala, Anant Kamat, Chun Wu, and Mike P. Haffey (Vehicle Wind Noise \& Road NVH Dept., Core \& Adv. Veh. System Eng., MD 45-Adv. Eng. Ctr., Ford Motor Co., 20000 Rotunda Dr., Dearborn, MI 48121-2053)

Wind noise is becoming a critical comfort requirement to customers due to greatly reduced powertrain and road noise, particularly at highspeed driving conditions. The physical phenomenon generally can be categorized into: sources (A-pillar vortex and mirror wake) and path (door/ window weatherstrip leakage). Two different vehicle greenhouse geometries that produce attached- and vortex-flow, respectively, were used in this A-pillar vortex minimization case study to correlate the CFD predicted A-pillar vortex size and sideglass pressure distribution $(C p)$ with measurements from wind tunnel testing. A-pillar vortex and pressure distribution have been shown to be key factors affecting wind noise excitation on sideglass and shape-induced aerodynamic suction force on door/ window (which is required in designing door/weatherstrip to prevent aspiration leaks through weatherstrip under high-speed condition), respectively. Results demonstrate that the CFD can successfully predict the effects of geometry changes on A-pillar vortex size and $C p$ within $>80 \%$ accuracy under various speeds/yaw angles. This study confirms that CFD can be applied with confidence as an upfront engineering tool to optimize vehicle greenhouse geometry for minimum wind noise excitation in a cost and time effective manner.

\section{9:35}

5aNS7. Numerical and analytical investigation of unsteady lift and sound due to airfoil-wake interaction. Paul $R$. Moran, Donald $E$. Thompson, and David Swanson (Acoust. Program, Appl. Res. Lab., Penn State Univ., P.O. Box 30, State College, PA 16804)

The passage of an airfoil through a wake is a dominant source of noise in low Mach number flows. In order to examine this phenomenon, two prediction schemes were used. The first was a thin airfoil analysis used to predict the unsteady lift response to sinusoidal transverse and longitudinal gusts [H. Naumann and H. Yeh, ASME J. Eng. Power 12, 1-10]. The strengths of these gusts are determined from the Fourier coefficients of the wake profiles. The sound was then determined from the unsteady force using a concentrated force radiation mechanism [N. Curle, Proc. R. Soc. London Ser. A 231, 451-460 (1955)]. The second method used an incompressible Navier-Stokes equation to predict the mean flow and unsteady pressure due to the passage of the wake past an airfoil. The resulting sound was then determined with a Kirchhoff solver. The pressure and sound results from both of these methods were compared with experimental data. [H. Fujita and L. S. G. Kovásznay, AIAA J. 12, 1216-1221 (1974).]

5aNS8. Sound due to flow through a nozzle with time-dependent throat area, Paul R. Moran and David Swanson (Acoust. Program, Appl. Res. Lab., Penn State Univ., P.O. Box 30, State College, PA 16804)

An unsteady compressible Euler code was developed for flow through a nozzle. Method of characteristic boundary conditions were used to permit the unsteady pressure to be nonzero downstream of the exit boundary. The flow is perturbed by modulating the cross-sectional area in the midsection of the nozzle. The far-field unsteady pressure was determined for both steady and unsteady inlet velocities and compared to experimental results.

\section{0:05}

5aNS9. An experimental and computational study of fundamental reactive silencers in the induction system of firing spark-ignition engines. A. Selamet (Dept. of Mech. Eng. and Appl. Mech., 120 W. E. Lay Automotive Lab., Univ. of Michigan, Ann Arbor, MI 48109-2121) and J. M. Novak (Ford Motor Company, Dearborn, MI 48121)

The present study investigates the performance of fundamental reactive silencers such as expansion chambers and Helmholtz resonators in the presence of both high-amplitude pressure waves and oscillating fluid flow. The silencers are installed in the induction system of a Ford 3.0-Liter V6 Vulcan engine in a dynamometer test facility. The experiments with the firing engine have been conducted with speeds ranging from 1000 to 5500 $\mathrm{rpm}$. Measurements including the mean flow rate, the temperatures, and the absolute dynamic pressures of the induction air before and after the silencer with fast-response, piezoresistive transducers facilitate the calculation of acoustic and flow performance of these elements. A nonlinear computational fluid dynamics method is then employed in the time domain for the prediction of noise reduction and insertion loss characteristics of these silencers. The study is concluded with comparisons of these predictions to the experimental results from the engine dynamometer facility. 


\title{
Session 5aPA
}

\section{Physical Acoustics: Surface Waves and Inhomogeneous Media}

\author{
Donald W. Brill, Chair \\ Physics Department, U. S. Naval Academy, Annapolis, Maryland 21402
}

Chair's Introduction-7:45

Contributed Papers

7:50

5aPA1. Surface acoustic wave investigation of phase transitions in $\mathrm{YBa}_{2} \mathrm{Cu}_{3} \mathrm{O}_{-5}$ films. J. Feller, C. Hucho, R. Gaffney, M. J. McKenna, B. K. Sarma, and M. Levy (Dept. of Phys., Univ. of Wisconsin, Milwaukee, WI 53201)

Pulsed surface acoustic waves (SAW's) at $165 \mathrm{MHz}$ are used to study the superconducting transition and an apparent structural phase transition in films of $\mathrm{YBa}_{2} \mathrm{Cu}_{3} \mathrm{O}_{7-\delta}(\mathrm{YBCO})$. The structural phase transition occurs in the temperature range $200-230 \mathrm{~K}$ and is evidenced by large changes in SAW attenuation and velocity. Small changes are also seen at the superconducting transition temperature. These changes are markedly different when a conventional conductor (e.g., copper) is brought into close proximity to the YBCO film. The results of this investigation are in agreement with the hypothesis that YBCO is piezoelectric below the $200 \mathrm{~K}$ transition. [This work was supported by the Office of Naval Research, with C. Hucho supported by Deutsche Forschungsgemeinschaft.]

\section{8:05}

5aPA2. Acoustic resonances in square cross-sectional rods (preliminary results). Jerome Beige and Michel De Billy (G. P. S. Univ. Paris VI and VII, Tour 23, 2 Place Jussieu, 75251 Paris Cedex 05, France)

As it was observed, it is shown that for particular frequencies, a square cross section normally insonified gives rise to resonance phenomenon. This is illustrated by using two different acoustic approaches: a dynamic visualization of the pressure scattered by a square bar and a measurement of the reradiated acoustic pressure in a plane normally oriented to the axis of a square rod. Different structures of the angular patterns are observed according to the nature of the excited guided mode. The experiments were performed on specimens of different materials such that the value of the product of the frequency times the width of the rod is less than $28 \mathrm{MHz}$ $\mathrm{mm}$. A theoretical approach based on the Fraser's work [W. B. Fraser, Int. J. Solids Structures 5, 379-397 (1969)] will be proposed.

\section{$8: 20$}

5aPA3. Acoustic microscopy and dispersion of leaky Rayleigh waves on randomly rough surfaces: A theoretical study. Claudio Pecorari and G. A. D. Briggs (Dept. of Mater., Oxford Univ., Oxford OX1 3PH, UK)

A theoretical investigation of the dispersion of leaky Rayleigh waves propagating along one-dimensional, rough, fluid-solid interfaces was carried out by simulating the measurement process of a line-focus acoustic microscope. The interface profiles were described in terms of their rms, also known as the roughness of the profile, autocorrelation length, and autocorrelation function. Theoretical $V(z)$ curves were obtained and analyzed to yield values of the phase velocity of the Rayleigh waves. The reflectivity of the interfaces was calculated by using a second-order perturbation approach in the profile roughness. The dependence of the Rayleigh wave velocity on the profile and material parameters was examined.
Significant variations of the phase velocity were found for values of the roughness which are small compared to the shortest of the wavelengths involved in the scattering. Similarly, the dispersion relations showed considerable sensitivity to changes in mechanical properties typical of materials of engineering interest. In the low-frequency range, on the other hand, simulations illustrated the dispersion of Rayleigh waves to be rather insensitive to the spectral content of the profile. [Work performed within the LINK Project on Characterization of Surface and Sub-Surface Damage.]

\section{8:35}

5aPA4. Relation between the damping coefficient and the excilation coefficient of leaking Lamb waves. M. Ech Cherif El Kettani, F. Luppé, J. M. Conoir, and J. Ripoche (L.A.U.E., U.R.A. C.N.R.S. 1373, Univ. du Havre, pl. R. Schuman, 76610 Le Havre, France)

The relation between the leaking ability and the excitation coefficient of a propagating Lamb wave on an immersed plate is studied. The more the Lamb wave radiates during its propagation, the more strongly it is expected to be excited from the incidence of an ultrasonic bearn at the appropriate angle. The excitation coefficient of a given Lamb wave at a given frequency-thickness product is obtained from a residue calculation. It is numerically achieved from the exact formulation of the reflection coefficient, and analytically performed with the help of the resonance scattering theory approximations. Both methods exhibit clearly the proportionality of the excitation coefficient with the normalized damping coefficient. The direct measurement of the excitation coefficient is not possible because of the interference phenomenon of the Lamb wave radiation with the specularly reflected beam. Consequently, the measurements are performed far away from the interference area. The linearity of the pressure amplitude (in $\mathrm{dB}$ ) with the propagation distance allows the direct determination of the damping coefficient, as well as the extension of the experimental data to the interference area. The excitation coefficient is herefrom deduced. The results confirm the theoretical ones. [Work supported by D. R. E. T., France.]

\section{8:50}

5aPA5. Geometrical theory of diffraction applied to Scholte wave diffraction. H. Dutlo, A. Tinel, and J. Duclos (Lab. d'Acoustique Ultrasonore et d'Electronique, U.R.A. C.N.R.S 1373, Université du Havre, Place R. Schuman, 76610 Le Havre, France)

The geometrical theory of diffraction (GTD) has been established by Keller in order to describe the interaction of a bulk wave with a dihedral edge. It was experimentally verified that Keller's laws were also valid to describe the interaction of a Scholte wave with the edge of an elastic dihedral. When the Scholte wave met the dihedral at oblique incidence, the diffracted energy was actually on Keller's cone the axis and generatrix of it were respectively the dihedral edge and the direction of the incident Scholte wave. It was assumed that incident energy spread among a bulk wave in the direction of the incident Scholte wave as well as reflected and transmitted surface waves (Scholte and Rayleigh waves). The direction of emission of these waves was calculated as a function of dihedral and 
incidence angles. This calculus showed that Rayleigh waves did not exist if the incidence angle was higher than the Rayleigh angle. Experimental results were obtained for duraluminum dihedrals of various angles ranging from 30 to $90 \mathrm{deg}$ and for incidence directions between 0 and $45 \mathrm{deg}$. Diffracted energy was only noted on Keller's cone. [Work supported by D.R.E.T.]

\section{9:05}

5aPA6. Acoustic scattering from finite length cylinder bounded by two spherical endcaps. J. Carnet, ${ }^{\text {a) }}$ J. Dujardin, D. Décultot, and G. Maze (LAUE URA CNRS 1373, Université du Havre, Place Robert Schuman, 76610 Le Havre, France)

The acoustic scattering from finite cylindrical objects endcapped by two hemispheres is studied. To explain the experimental resonance spectra when the abject is parallelly insonified to the axis, a condition of phase matching is written on the meridian circumference. To apply this condition, it is necessary to know the phase velocity of surface waves along the different parts of the object. To verify this hypothesis, resonance identifications are realized. The identification diagrams are not easy to interpret. The lobe number is not equal to the antinode number, and these lobes do not have the same amplitude. An integral method will permit the knowledge of the acoustic pressure in the far field if the profile of the vibration state along the meridian line is sinusoidal. A good agreement is obtained between the theoretical and experimental studies. To confirm this hypothesis, a finite element method is developed. A 2-D problem is considered. A meridian plane and the water around the object is meshed. A sinusoid burst with several periods insonifies the object. The vibration amplitude of the surface of the object is determined in every way. The results confirm the hypothesis used in the integral method and allows the validation of the

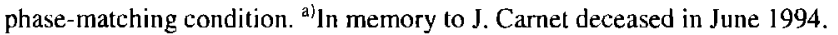

\section{9:20}

5aPA7. Acoustic scattering from a circular cylindrical shell excited by a short pulse in oblique incidence: Helical waves. G. Maze, J. M. Conoir, F. Léon, and D. Décultot (Laboratoire d'Acoustique Ultrasonore et d'Électronique URA CNRS 1373, Université du Havre, Place Robert Schuman, 76610 Le Havre, France)

The theoretical and experimental spectra, obtained from an infinite pipe insonified with a plane wave in oblique incidence, show resonances which are related to three types of helical waves: the circumferential waves ( $A_{i}$ or $S_{i}$ waves), the transversal guided waves ( $T_{i}$ waves), and the Scholte wave ( $A$ wave). The resonances of the Scholte wave are detected in a frequency window and their frequency slowly increases when the incidence angle increases, whereas the ones of the other waves increase towards infinity when the ineidence angle tends towards the transversal critical angle. At oblique incidence, the experimental results obtained with the MIIR show resonances which are related to the helical waves $S_{0}, T_{0}, T_{1}$, and $A$. This method uses a long pulse with many sinusoid periods, a steady state takes place in a part of the infinite shell. In this presentation, the excitation is a short pulse and the scattered echoes are detected when the helical wave emission is in front of the receiver. Between each echo, the helical wave has covered one step of the helix $(L=2 \pi a / \cos \gamma, a$ is the outer radius of cylinder, $y$ is the angle of the helix). This method allows us to calculate the group velocity of the helical waves.

\section{9:35}

5aPA8. Lamb waves of two elastic plates coupled by a thin water film. M. Rousseau, F. Coulouvrat (Laboratoire de Modélisation en Mécanique, Université Pierre et Marie Curie et C.N.R.S. (URA 229) Case 162, 4, place Jussieu, F-75252 Paris Cedex 05, France), O. Lenoir, H. Khelil, and J. L. Izbicki (Université du Havre and C.N.R.S. (URA 1373) F-76610 Le Havre, France)

This experimental and theoretical study investigates the guided waves that propagate along a multilayered structure, composed of two identical, infinite, aluminum plates coupled by a thin water film. The related dispersion equation is written under a closed form. Solutions are calculated numerically to draw the dispersion curves. The low- and high-frequency limits are calculated analytically. Special attention is devoted to the determination of the cut-off frequencies. An asymptotic analysis shows that, in most cases, a doubling of the classical Lamb waves happens. The small deviation of the cut-off frequencies from the classical ones has been evaluated. An additional, single mode is also outlined; its cut-off frequency is smaller than the Lamb waves ones and depends heavily on the water film thickness. The experiments have been performed on 3- and 5-mm aluminum plates insonated at normal incidence by short pulses. The reflected signal is then FFT-processed; depending on whether the specular echo is removed or not, a resonance or a backscattered spectrum is obtained. The doubling of the Lamb waves, and the existence of an additional lowfrequency mode, have been thus confirmed. For a structure composed of three aluminum plates, the experiment now outlines families of three models, each family being closely related to one classical lamb wave.

\section{9:50-10:05 Break}

\section{0:05}

5aPA9. Acoustics of fluid conveying elastic structures. Michael A. Grinfeld and Andrew N. Norris (Dept. of Mech. and Aerosp. Eng., Rutgers Univ., Piscataway, NJ 08855-0909)

Acoustic waves are considered that are propagating in a layered structure consisting of elastic layers with ideal fluid moving between them. This is a simple model of a fluid-filled porous medium. First discussed is the dependence of the dispersion curves on the relative velocity of the fluid and on the material and geometrical parameters of the constituents. Dispersion relations for a periodic layering are derived explicitly. Different asymptotic limits can be obtained, which reduce to known solutions for interfacial acoustical wave motion in the presence of flow. The main focus of this talk is the possibility of instabilities triggered by the moving fluid in the layered system. The role of such acoustical instabilities in nonlinear dynamics of poroelastic fluid-filled media is discussed. This effect can be viewed as a generalized futter phenomenon, made possible by the permeability of the pore space. [Work supported by ONR.]

\section{$10: 20$}

5aPA 10. Observation of seismic wave propagation in particulate composite material with various inclusion sizes and concentrations. Seiji Nakagawa, Neville G. W. Cook (Dept. of Mater. Sci. \& Mineral Eng., Univ. of California, Berkeley, CA 94720), K. T. Nihei, and L. R. Myer (Lawrence Berkeley Laboratory, Berkeley, CA 94720)

Two-phase particulate composite samples with inclusions of varying size and concentration were made with cement and sand or pebbles. The velocities of $P$-wave $(60-\mathrm{kHz})$ and $S$-wave $(150-\mathrm{kHz})$ pulses and the attenuation of $S$-wave pulses were examined. The results of the tests suggested that the velocities were consistent with the results of effective media models which were inclusion size independent. However, the frequencydependent attenuation of the $S$ wave was strongly affected by the size of the inclusions. For inclusions sized on the order of $1 / 10$ of the wavelength, the waves showed decreasing attenuation with increasing concentration, while for the sizes of about $1 / 4$ and $1 / 1$ of the wavelength, the waves showed maximum attenuation at volumetric concentrations of about $30 \%$ and $70 \%$, respectively. Many aspects of the observed wave behavior for moderate concentrations were similar to numerical simulations of 1-D wave propagation in a layered media, where the matrix and inclusions were represented by alternating layers with different seismic properties and stochastically varied thicknesses. [Work supported by NSF.]

\section{0:35}

5aPA 11. Flow resistance of fibrous materials. Viggo Tarnow (Dept. of Appl. Eng. Design and Production, Danish Technical Univ., Bygning 358, DK 2800, Denmark)

It is of interest to be able to compute the acoustical properties of fibrous materials from the fiber density and thickness of materials such as glass wool. These properties are mainly determined by the air flow resis- 
tance of the fibrous material. Theoretical calculations of air flow resistance will be presented and compared with measurements on glass wool.

\section{0:50}

5aPA12. Guided sound propagation in three-dimensional inhomogeneous moving nonstationary fluid. Oleg A. Godin (NOAA/ Atlantic Oceanogr. and Meteorol. Lab., 4301 Rickenbacker Cswy., Miami, FL 33149)

Sound propagation in a multicomponent fluid, parameters of which including flow velocity as well as shape of boundaries are smooth and slowly varying functions of time and two horizontal coordinates, is considered. No limitations are imposed on the Mach number and the fluid parameters dependence on the vertical coordinate other than the assumption that sound/flow synchronism points are absent. All the processes in the fluid are assumed to be adiabatic. The method of two-scale expansions is used to construct asymptotic development of solutions of the set of hydrodynamic equations linearized with respect to the wave's amplitude. The well-known "vertical modes-horizontal rays" approach is generalized to cover acoustic waves in nonslationary moving media. An adiabatic invariant is found that governs the amplitude variation of a normal mode along the corresponding space-time horizontal ray. Physical meaning of the adiabatic invariant is analyzed. Results are compared to those of recent studies of guided propagation in moving time-independent [O. A. Godin, DAN SSSR 320, 204-208 (1991)] and unstationary motionless fluid [A. V. Aref'ev and V. S. Buldyrev, Akust. Zh. 40, 205-211 (1994)]. The importance of a consistent account of medium's motion and its time dependence is demonstrated. [Work supported by RBRF and NRC.]

\section{1:05}

5aPA13. Reciprocity-type relations for waves in a moving inhomogeneous fluid. Oleg A. Godin (NOAA/Atlantic Oceanogr. and Meteorol. Lab., 4301 Rickenbacker Cswy., Miami, FL 33149)

The reciprocity principle is known to be invalid for acoustic waves in a moving fluid due to differences in up- and down-flow wave propagation velocities. A variation of the reciprocity principle, a flow reversal theorem (FRT), which states symmetry of some field quantity with respect to interchange of the source and receiver positions and the simultaneous reversal of flow, was considered by many authors during the last four decades. It was proven under that or other specific assumptions about fluid parameters and/or flow velocity space dependence or in a framework of some asymptotic representations of the acoustic field. A simple but rather general proof of the FRT will be presented which is valid for sound as well as acoustic-gravity waves in an arbitrary three-dimensional inhomogeneous moving fluid with time-independent parameters provided divergence of the flow velocity is zero. The fluid may be unbounded or have pressurerelease, rigid, or impedance boundaries. The key point in the approach is the choice of a set of dependent variables in hydrodynamic equations for describing the wave field. An implication of the FRT on wave action conservation for $\mathrm{cw}$ sound in moving fluid is considered. Some possible FRT applications are indicated. [Work supported by NRC.]

\section{1:20}

5aPA14. Measurements of Biot type I and type II waves in an air-filled packing of sand. Craig J. Hickey and James M. Sabatier (Natl. Ctr. for Phys. Acoust., Univ. of Mississippi, University, MS 38677)

The interpretation of acoustic and seismic measurements in porous materials are usually carried out using the Biot theory. This theory predicts the existence of two $P$ waves (referred to here as type $I$ and type II) and one $S$ wave. The type I $P$ wave is predicted to have small dispersion and attenuation. The type II $\boldsymbol{P}$ wave is predicted to be dispersive and highly attenuating. An experiment is carried out whereby air borne sound is incident upon an air-filled packing of sand. For such a configuration, a large proportion of the transmitted energy is partitioned to the type II $P$ wave. However, due to the high attenuation of the type II $P$ wave this energy should decay rapidly near the surface. Measurements using a probe microphone apparatus, in-situ microphones, and in-situ geophones are obtained in the frequency range of $500-3000 \mathrm{~Hz}$. The in-situ microphones and geophones extend to a depth of $40 \mathrm{~cm}$ below the surface in an attempt to detect the Type I $P$ wave. Attenuation and phase velocity of the air-filled packing of sand is determined as a function of depth from these measurements. [Work supported by USDA.]

\section{1:35}

5aPA15. Simulation of wave propagation through an inhomogeneous turbulent medium with the aid of an integral formulation. Hirobumi Kaneko (130C Olde Derby Rd., Norwood, MA 02062)

A propagation simulation algorithm is described whereby, at the beginning of step $n$, the pressure associated with a progressive wave is specified as a function of time and lateral position along a planar surface, $x=x_{n}$. The algorithm for step $n$ yields the pressure as a function of time and lateral position at $x=x_{n+1}$. The basic mathematical formulation employs an integral equation that predicts the waveforms at arbitrary positions to the right of the first surface. The medium between the point and the surface, $x=x_{n}$, is turbulent. The integral over the surface involves the Green's function for a point source in the actual medium. Although the exact Green's function is extremely difficult to compute, it is argued that a good approximation for moderate step sizes, $\left|x_{n-1}-x_{n}\right|$, results if the Green's function is taken to be that which results from a ray-acoustics approximation. Moreover, because the first caustic for a point source Green's function is not encountered for a distance considerably longer than the distance between successive caustics for a nominally parallel group of rays within a ray tube, the difficulties of explicitly taking caustics into account is circumvented with this algorithm. 


\title{
Session 5aPP
}

\section{Psychological and Physiological Acoustics: Cortical Representation and Processing of Auditory Stimuli}

\author{
Eric D. Young, Chair \\ Johns Hopkins School of Medicine, 505 Traylor Building, 720 Rutland Avenue, Baltimore, Maryland 21205
}

\author{
Chair's Introduction-8:30
}

Invited Papers

5aPP1. Functional organization and reorganization of primary auditory cortex. Christoph E. Schreiner (Coleman Mem. Lab., W. M. Keck Ctr. for Integrative Neurosci., Dept. of Otolaryngol., Univ. of California, San Francisco, CA 94143-0732)

Neurons in primary auditory cortex (AI) of cats and monkeys have a wide range of functional properties that are related to the coding of basic attributes of simple and complex signals. In particular, cortical neurons have been found to be sensitive to the bandwidth of the signal, the spectral energy distribution of broadband stimuli, the overall intensity of the signal, and the temporal sequence of signal elements, in addition to their frequency selectivity and sound localization sensitivity. These response properties appear to be nonuniformly distributed across AI and, thus, create spatially and functionally distinct subregions that may constitute parallel auditory processing streams. Studies with behaviorally trained animals suggest that these functional cortical organizations are not fixed but can undergo changes. This representational plasticity results in more detailed representations of portions of the animal's acoustic environment that have high behavioral relevance. An understanding of the creation, relationships, and interconnections of these different coding properties is essential for the comprehension of the neural code of communication sounds such as speech. [Work supported by the Office of Naval Research, the Human Frontier Science Project, and the Coleman Fund.]

5aPP2. Representation of the acoustic spectrum in the primary auditory cortex. Shihab A. Shamma (Elec. Eng. Dept. and Inst. for Systems Res., Univ. of Maryland, College Park, MD 20742)

A fundamental goal in auditory cortical physiology has been to understand how the spectral profile is represented in the firing rate of cortical cells. Recent experimental findings shed light on two basic properties of this representation in AI: (1) Responses to sounds with broadband spectra (such as speech and most environmental sounds) superimpose linearly. Thus if a complex arbitrary spectral profile is viewed as composed of elementary spectral profiles, then AI responses to such a profile can be reconstructed from the sum of the responses to these simpler elementary profiles. This seems to be true both for stationary and dynamic spectra. (2) AI units are rather selective to the spectral and temporal parameters of the acoustic profile. Specifically, when tested with elementary spectral profiles that are sinusoidally shaped against the logarithmic frequency axis (or so called rippled spectra), and that slide against this axis at various velocities, $\mathrm{AI}$ units are found to be tuned around different ripple densities, ripple phases, and ripple velocities. The above two findings suggest that AI performs a Fourier-like analysis of the spectral profile into a composite of weighted sinusoidally shaped spectra (ripples). Such an analysis is analogous to that of visual scenes found in the primary visual cortex (VI).

5aPP3. Processing of complex sounds in rhesus monkey auditory cortex. Josef P. Rauschecker (Lab. of Neuropsych., NIMH, Bldg. 49, Rm. 1B80, Bethesda, MD 20892-4415)

Several auditory fields surrounding primary auditory cortex (AI) have been described in the rhesus monkey. Most recently, three areas have been identified in the so-called auditory belt region on the lateral surface of the superior temporal gyrus [Rauschecker $e t$ al., Science (1995) (in press)]. Functional identification of these lateral areas (AL, ML, and CL) was based on single unit responses to bandpassed noise (BPN) bursts, which constitute a class of superior stimuli for neurons in this region. Best center frequency (BFc) of the BPN bursts varies systematically along a rostro-caudal axis, revealing three cochleotopic maps. Neurons in the lateral areas also display tuning to a "best bandwidth" of the noise bursts, which varies along a medio-lateral axis, orthogonal to the BFc axis. Digitized vocalizations from the rhesus monkeys' own repertoire were finally used for presentation during single-unit recording. In many instances, neurons responded even better to the monkey calls than to noise bursts or other synthetic stimuli. It appears that the lateral belt areas of macaque auditory cortex could form an important step in the preprocessing of species-specific communication sounds, somewhat equivalent to superior temporal areas in humans that have been implicated in phonological processing. 
5aPP4. Elevation sensitivity in the cat's thalamo-cortical auditory system: Role of monaural spectral and binaural disparity cues. Thomas J. Imig, Pierre Poirier, and Frank K. Samson (Dept. of Physiol., Kansas Univ. Med. Ctr., 3901 Rainbow Blvd., Kansas City, KS 66160-7401)

High-frequency neurons in auditory cortex (AI) and medial geniculate (MGB) of anesthetized cats are differentially sensitive to monaural and binaural directional cues present in broad-band noise. Binaural directional (BD) cells depend upon binaural stimuli for azimuth sensitivity, whereas monoaural directional (MD) cells are sensitive to the direction of monaural stimuli. In the MGB, neurons' spatial receptive fields (SRFs) were obtained using noise bursts presented throughout the frontal hemifield and sound pressure levels that varied over an $80-\mathrm{dB}$ range. BD cells were broadly tuned to elevation. Those that received excitatory input from one ear and exhibited binaural inhibition or mixed interactions had SRFs that extended throughout one lateral hemifield. Predominantly binaural cells exhibited strong binaural facilitation and had SRFs that formed a vertical band in front of the head. MD cells' SRFs varied considerably, some were focal areas restricted in azimuth and elevation. Others consisted of multiple areas that in some cases showed level-dependent changes in location. The elevation sensitivity of MD cells was nearly identical under monaural and binaural conditions showing that it derived from monaural spectral cues. AI units showed similar response properties. [Work supported by NIDCD.]

\title{
11:30
}

5aPP5. Cortical codes for sound location. Iohn C. Middlebrooks (Depts. of Neurosci. and Otolaryngol., Box 100244, Univ. of Florida, Gainesville, FL 32610), Ann Clock Eddins (Indiana Univ., Bloomington, IN 47405), Li Xu, and David M. Green (Univ. of Florida, Gainesville, FL 32611)

The responses of auditory cortical neurons carry information both in the number of spikes within a burst and in the timing of those spikes. The capacity of single neurons to encode sound locations was studied in areas Al and AES of $\alpha$-chloralose-anesthetized cats. The spike counts of about half of studied neurons were modulated by more than $50 \%$ as a noise source was varied in azimuth. Nevertheless, the "best areas" of most units, using a 50\%-of-maximum-response criterion, were larger than $180^{\circ}$. Temporal spike patterns of single neurons could be classified according to sound source azimuth, using an artificial neuron network. The classification of temporal patterns consistently surpassed a maximum-likelihood classification of spike counts. The spike patterns of single neurons could successfully encode locations throughout $360^{\circ}$ of azimuth. The localization performance of any single neuron was substantially worse than that of an awake, behaving cat. When responses were averaged across multiple trials, however, performance improved monotonically with increases in the number of trials, suggesting that the information carried out by the temporal spike patterns of multiple neurons could account for a cat's localization behavior.

SATURDAY MORNING, 3 JUNE 1995

MEETING ROOM 16, 9:00 TO 11:30 A.M.

\section{Session 5aSA}

\section{Structural Acoustics and Vibration: Plates and Shells II}

\author{
Courtney B. Burroughs, Chair \\ Applied Research Laboratory, Pennsylvania State University, P. O. Box 30, State College, Pennsylvania 16801
}

\section{Contributed Papers}

9:00

5aSA1. Transmission of turbulent boundary layer pressures through thin and thick shells. Jack Cole (Cambridge Acoust. Assoc., Inc., 200 Boston Ave., Ste. 2500, Medford, MA 02155-4243)

Turbulent flow along a structural boundary acts as a source of noise to the interior space. Characteristics of the transmitted noise are determined not only by the turbulent flow field but also by the transmission characteristics of the bounding structure. In low-speed flow applications, the spatial scale of the turbulence can be smaller than the structural wall thickness. Under this circumstance, thickness effects are important in modeling the wall transmissibility. These effects are examined using the elas- ticity solution for the axisymmetric response of cylindrical shells. Results are presented for steel-like and rubber-like wall materials and compared with results from thin shell theory.

\section{9:15}

5aSA2. Frequency domain assessment of the doubly asymptotic approximation. Jerry H. Ginsberg (School of Mech. Eng., Georgia Inst. of Technol., Atlanta, GA 30332-0405)

The most widely implemented technique for modeling fluid-structure interaction effects associated with shock response is the doubly asymptotic approximation (DAA), which has been developed in a variety of versions. Analytical validations of this method have thus far only been performed 
for spherical and infinitely long cylindrical shells. The present work uses Nicholas-Vuillierme's derivation [Numerical Techniques in Acoustic Radiation, edited by R. J. Bernhard and R. F. Keltie (ASME-NCA, Vol. 6 , 7-13 1989)] of the frequency-domain version of DAA as the basis for examining the accuracy and limitations of DAA for a slender hemi-capped cylindrical shell. The basic concept is to use DAA to determine the wet surface impedance matrix relating surface pressure and velocity variables, whose values are compared to those obtained from the surface variational principle (SVP) using the same set of basis functions. After such comparison is made, the alternative wet surface impedances are used to predict the structural response of the hemi-capped cylindrical shell to a ring force. Assessments for frequencies in the range $k a<10$ for a shell whose length to diameter ratio is $L / 2 a=6$ indicate that DAA fails to recognize effects associated with transition from supersonic to subsonic structural waves. The implications of this shortcoming for structural response are discussed. [Work supported by the Office of Naval Research, Code 1222.]

5aSA3. Spiked volleyballs, dribbled basketballs, and kicked footballs: Excitation of the fundamental breathing mode in inflated sport balls. David G. Browning (Browning Biotech, 139 Old North Rd., Kingston, RI 02881), Robert H. Mellen (Kildare Corp., New London, CT 06320), Robert J. Schneck, and Stephanie Milbradt (Univ. of Rhode Island, Kingston, RI 02881)

The analyses of the sound from a hand-struck or "spiked" volleyball, a "dribbled" basketball, and a place-kicked football show that in each case the fundamental "breathing" vibrational mode is excited in these inflated sport balls. Under regulation conditions the resonant frequency of a volleyball was found to be approximately $150 \mathrm{~Hz}$; a basketball, which is larger but has a higher inflation pressure, was found to have a slightly higher frequency. Due to the shell material these vibration's are quickly damped. The results support the theoretical work [P. M. Morse and $\mathrm{H}$. Feshbach, Methods of Theoretical Physics (McGraw-Hill, New York, 1953), p. 1469] for a hollow, flexible sphere. It was found that "good" hits strongly excite this mode, "poor" ones do not. Acoustic analysis may be an important tool in determining optimum performance.

\section{9:45}

5aSA4. An approximation to the local acoustic impedance of a cylindrical shell with hemispherical endcaps. Michael J. Utschig, Jan D. Achenbach, and Takeru Igusa (Dept. of Civil Eng., Northwestern Univ., Evanston, IL 60208)

A method has been developed to approximate the local radiation impedance of a cylindrical shell with hemispherical endcaps. This approximation greatly reduces the effort required to calculate the acoustic impedance as compared to an approach using a variational method. The approximate method uses the exact acoustic impedances of several separable geometric shapes to approximate the acoustic impedance of a complex structure that is a composite of those shapes. The shell is broken into three components: two hemispheres and a finite cylinder. These components are parts of regions that are separable in an orthogonal coordinate system. It is assumed that the acoustic impedance of the components is the same as the acoustic impedance calculated exactly for the separable shapes. Furthermore, it is assumed that the local acoustic impedance of the original complex shape is the same as that of corresponding points on the simple components. The accuracy is improved when the interaction between neighboring components is considered. The acoustic impedance and surface pressure generated by a load applied in the center of the complex shape is compared with that calculated using a variational fornulation.
10:00

5aSA5. The acoustical background for elastic shells of nonspherical objects. M. F. Werby (Naval Res. Lab., Stennis Space Center, MS 39529) and N. A. Sidorovskaia (Univ. of New Orleans, New Orleans, LA 70148)

The acoustical background for elastic shelis which allows one to isolate elastic shell resonance's was first presented at an ASA conference in 1989 for spherical shells. A formulation has been developed that is based on the $T$-matrix method general for nonspherical shells. An outline of the formulation is presented and it's use in isolating resonance's for elastic spheroidal shells is presented. [Work sponsored by NRL and the Office of Naval Research.]

\section{0:15-10:30 Break}

\section{0:30}

5aSA6. A new analytical approach for radiation problem from finite cylindrical piezoceramic shell. Victor $T$. Grinchenko (Dept. of Hydrodyn. Acoust., Inst. of Hydromech., Natl. Acad. of Sci., Kiev, 252057, Ukraine)

The objective of this paper is to present a complete analytical solution for a finite piezoelectric cylinder radiation problem. Three aspects of the problem are discussed. (1) Development of a theory of thin piezoceramic shells with hypotheses for electric field components that are equivalent to the classical Love's mechanical ones. (2) Explanation of a new analytical approach to solve a coupled elastic-electric-fluid interaction problem. The exact solution for the acoustic potential function is given in terns of an infinite series of partial solutions of the Helmholtz equation in cylindrical and spherical coordinate systems. The associated coefficients are the solution of an infinite linear system. A new approach to truncation of this system which takes into account the sharp edge effects, is developed. An estimation of the calculation accuracy of the method is presented. (3) Numerical implementation of the analytical solution for different values of key parameters gives a ground to elucidate a complex exchange of energy between electric generator, elastic structure, and surrounding fluid. Modification of eigenforms of the shell due to radiation in water is described.

\section{0:45}

5aSA7. Dispersion of longitudinal waves in periodic septate liquid-elastic waveguides. L. Sheiba (EG\&G WASC, Inc., 1396 Piccard Dr., Rockville, MD 20850)

The propagation of longitudinal waves in a composite liquid-elastic waveguide is analyzed. The waveguide is a periodic structure consisting of alternating liquid-elastic cylinders joined by rigid septa. The elastic material (Lamé constants: $\mu \ll \lambda$, Poisson relation $\sigma \Rightarrow 0.5$ ) and the liquid material $(\mu=0, \sigma=0.5)$ are assumed to have low compressibility, and the septa are rigid and weightless. Consequently, the boundary between the cylinder and the septum's radial displacement is absent, and the axial displacements are planar. The admittance matrix $\mathbf{Y}$ of the unit cylinder is initially constructed within a framework of the hypothesis of plane cross sections, neglecting strains induced by hydrostatic stress. Hydrostatic stress waves are approximately included by adding to the unit cylinder admittance matrix correction $\Delta \mathbf{Y}$ associated with the strains of hydrostatic stress in the cylinder. The transfer matrix of the liquid-elastic waveguide and its elements, expressed through the waveguide sections elastic parameters, have been obtained. It has been shown that damping in a waveguide with a periòdic structure is much greater in comparison with a regular waveguide, even when the latter is made with a high-loss material. 
5aSA8. Electroelastic modeling of the motions of ceramic bars under large driving voltages. M. C. Dökmeci (Istanbul Tech. Univ.-Teknik Üniversite, P.K. 9, Taksim, 80191, Istanbul, Turkey) and G. Aşkar Altay (Boğaziçi Univ., Bebek, Istanbul)

In relation to electroceramic media subject to large driving voltages, this paper presents the one-dimensional nonlinear equations of a cylindrical ceramic bar. The field variables of the ceramic bar are all expanded in the power series expansions of its cross-sectional coordinates. By means of a unified variational principle, which is formulated through Hamilton's principle and Legendre's transformation [e.g., M. C. Dökmeci, IEEE Trans. UFFC 35, 775-787 (1988)] together with the series expansions of field variables, the one-dimensional electroelastic equations are systematically derived in both differential and variational forms. They are capable of predicting the extensional, flexural, and torsional as well as coupled motions of a ceramic bar at low and high frequencies. By a proper truncation of the series expansions, the electroelastic equations incorporate as many higher-order effects as deemed desirable in any case. Special cases are investigated [cf. M. C. Dökmeci, Int. J. Solids Struct. 10, 401-409 (1974) and Proceedings of the 40th Annual Symposium on Frequency Control (IEEE, New York, 1986), pp. 168-178]. The uniqueness is examined in solutions of the linearized electroelastic equations. [Work supported in part by The Scientific and Technical Research Council of Turkey.]
5aSA9. Investigation of the vibroacoustical characteristics of cylindrical shells. Rostislav A. Dudnik and Andrei B. Kolpakov (Dept. of Phys., Inst. Arch. Civil Eng., 65 Il'inskaya St., 603600 N.-Novgorod, Russia)

Experimental and theoretical investigations of the influence of inhomogeneity upon the oscillations and radiation of thin cylindrical shells are executed. The inhomogeneity is presented by either one additional mass $\left(m_{0}\right)$ fastened (at $\Phi=180^{\circ}$ ) on the shell surface, or two identical inertia masses $\left(m_{1}=m_{2}=m_{0} / 2\right)$ which are fastened at $\Phi= \pm \Phi_{1}$. The impact of such division of a unit inhomogeneity on oscillating velocity distribution as well as on the pressure radiated levels and directionality diagrams of the relevant shell models is analyzed for different quantities of summary mass $\left(m_{0}\right)$. The execution analysis is limited by the investigation of azimuth lower-frequency modes. It was shown that the considered distribution of inhomogeneity makes it possible to control the radiation level of the shell in certain frequency ranges. The investigation of a structure of near and far acoustical fields radiated by these shells at various distances from the radiating surfaces was conducted also. It was shown that as this distance increases, the number of the mode providing the largest contribution to the complete field decreases. Corresponding experimental results are presented.

SATURDAY MORNING, 3 JUNE 1995

RENAISSANCE WEST B, 9:00 A.M. TO 12:00 NOON

\title{
Session 5aSC
}

\section{Speech Communication: Articulatory Models and Measurements}

\author{
Maureen L. Stone, Chair \\ Division of Otolaryngology, HNS, Department of Surgery, University of Maryland, School of Medicine, Baltimore, \\ Maryland 21201
}

Contributed Papers

9:00

5aSC1. Is intra-articulator speech coarticulation planned? David J. Ostry (McGill Univ., Montreal, PQ H3A 1B1, Canada) and Vincent L. Gracco (Haskins Labs., New Haven, CT 06511)

Does the nervous system take account of upcoming phonetic context when planning successive movements of a speech articulator? The kinematics of intra-articulator coarticulation are readily measurable in empirical studies and may appear to be centrally controlled on the basis of kinematic changes which arise in response to upcoming phonetic segments. However, without explicit models of speech articulators, measured kinematic effects correctly attributable to central planning cannot be distinguished from the kinematic patterns which are due to dyrranics and are not represented in the underlying control. In the present paper, this question is addressed by comparing the results of empirical and modeling studies of jaw motion. The simulated kinematics of sagittal plane jaw rotation and horizontal jaw translation are compared to empirical studies in which subjects produce CVC sequences at a normal rate and volume. The jaw motion simulations show that even when control signals underlying the initial CV transition are fixed, "anticipatory" kinematic patterns vary in amplitude and duration as a function of upcoming context. This suggests that unplanned effects due to articulator dynamics must be accounted for before drawing conclusions about the role of central control in intraarticulator coarticulation. [Work supported by NIH Grant CD-00594.]
9:15

5aSC2. Recovery of task-dynamic parameters with a mismatched articulatory model. Richard S. McGowan and Mindy Lee (Haskins Labs., 270 Crown St., New Haven, CT 06511)

A method for recovering task-dynamic parameters from speech acoustics has been proposed [see McGowan, Speech Commun. 14, 19-48 (1994)]. In the series of tests to be described here, there were mismatches imposed between the task dynamics that produced the speech data and the task dynamics used in the analysis-by-synthesis procedure for recovery. One of the mismatches was in the articulator weights used in specifying how much of each articulator to use in attaining construction goals. Another mismatch was in the allowed relation between activation interval and the natural frequency of the constriction dynamics (natural frequency constraint). Bilabial and velar places of articulation (constriction) were tested, and the first three formant trajectories were used as data. When there was articulator weight mismatching, it was found that articulators would compensate so that the recovered constriction trajectories were similar to those of the data producing trajectories. It appeared to be more difficult to overcome mismatches in the natural frequency constraint. Also, the nonuniqueness of the recovered task dynamics is exhibited when there is not sufficient acoustic data. [This work was supported by NINDCD Grant 01247 to Haskins Laboratories.] 
responding acoustics than coefficients from either the nonorthogonal twofactor solution or the orthogonally constrained three-factor solution. These three factors also correspond qualitatively to the three nonorthogonal factors extracted from Icelandic $x$-ray film vowel data [Jackson, J. Acoust. Soc. Am. 84, 124-143 (1988)]. Thus the current English solution contradicts Jackson's distinction (based on Harshman et al.'s two-factor English vowel solution) between language-independent and language-specific vowel articulation primes. This low-dimensional, potentially crosslinguistic representation could benefit speech recognition, coding, or synthesis applications in which an acoustically correlated vowel tongue shape parameterization is required. consonant is one of the set $/ p, b, t, d, k, g /$ and the second vowel one of $/ i, a, b /$. A magnetometer system was used to track vertical, and horizontal movements of receivers placed on the lips and the jaw, and on four points on the tongue. Tangential velocity was used to define movement onsets and ofisets. Movement amplitude was calculated as the path of the receiver from movement onset to offset. Preliminary results from two subjects suggest the possibility that the effects of consonant voicing on movement kinematics vary for different articulators. Tongue body movements towards consonantal closure had consistently higher velocity. larger amplitude and longer duration for voiced than for voiceless velar stops. Tongue tip and lip and jaw closing movements showed less robust differences between voiced and voiceless alveolar and labial stops. [Work supported by NIH.]

\section{9:45}

5aSC4. Articulatory activity and aerodynamic variation during voiceless consonant production. Laura L. Koenig, Anders Löfqvist, Vincent L. Graceo, and Richard S. McGowan (Haskins Labs., 270 Crown St., New Haven, CT 06511)

The production of voiceless consonants is associated with changes in oral air pressure which reflect the time course and coordination of the requisite laryngeal and supralaryngeal articulations. The present experiments were conducted in order to characterize more precisely the relationships among glottal and supraglottal activities and the resulting aerodynamic changes in the vocal tract. In the first experiment, simultaneous recordings were made of glottal articulation (obtained via transillumination), two-dimensional lip and jaw movements, and air pressure variation during the production of voiceless labial consonants. In the second experiment, two-dimensional motion of the jaw and multiple points on the longue were obtained along with air pressure during the production of voiceless lingual consonants. Analysis focused on the time-varying changes in the pressure waveform and the accompanying articulatory motion. The data suggest that the overall time course of pressure variation depends largely on the timing of the laryngeal devoicing gesture. However, details in the pressure waveforms showed a close correspondence to the time course of supraglottal articulations. Taken together, results suggest that laryngeal and supralaryngeal timing is highly constrained, and that different articulators contribute uniquely to producing pressure variations within the vocal tract. [Work supported by NlH.]

\section{0:00}

5aSC5. Three acoustically predictable factors underlying vowel tongue shapes. David A. Nix and George J. Papcun (Los Alamos Natl. Lab., CIC-3, MS B265, Los Alamos. NM 87545)

To obtain a low-dimensional, speaker-independent parameterization of vowel tongue shapes, the three-mode factor analysis procedure PARAFAC [Harshman et al., J. Acoust. Soc. Am. 62, 693-707 (1977)] was applied to $\mathrm{x}$-ray microbeam tongue measurements of ten English vowels spoken by two male and two female subjects in seven different $/ \mathrm{CVC}$ contexts. PARAFAC reliably extracts three speaker-independent, nonorthogonal factors. The resulting speaker-independent factor coefficients cluster by vowel in three-dimensional articulatory space. In two-dimensional projections, they qualitatively reflect the traditional vowel quality chart. A multi-layer perception (neural network) independently conroborates this solution: these tongue shape coefficients are significantly more predictable from the cor-

\section{0:15-10:30 Break}

\section{0:30}

5aSC6. Evaluating a topographical mapping from speech acoustics to tongue positions. John Hogden and Michelle Heard (Los Alamos Natl. Lab., MS B256, Los Alamos, NM 87545)

The continuity mapping algorithm-a procedure for learning to recover the relative positions of the articulators from speech signals-is evaluated using human speech data. The advantage of continuity mapping is that it is an unsupervised algorithm; that is, it can potentially be trained to make a mapping from speech acoustics to speech articulation without articulator measurements. The procedure starts by vector quantizing short windows of a speech signal so that each window is represented (encoded) by a single number. Next, multidimensional scaling is used to map quantization codes that were temporally close in the encoded speech to nearby points in a continuity map. Since speech sounds produced sufficiently close together in time must have been produced by similar articulator configurations, and speech sounds produced close together in time are close to each other in the continuity map, sounds produced by similar articulator positions should be mapped to similar positions in the continuity map. The data set used for evaluating the continuity mapping algorithm is comprised of simultaneously collected articulator and acoustic measurements made using an electromagnetic midsagittal articulometer on a human subject. Comparisons between measured articulator positions and those recovered using continuity mapping will be presented.

\section{0:45}

5aSC7. Across session temporal stability of the lip-jaw complex in bilabial closure. Peter J. Alfonso (Dept. of Speech and Hear. Sci., Univ. of Illinois at Urbana-Champaign, 901 South Sixth St., Champaign, IL 06510 )

Movements of the tongue, lips, and jaw were transduced by electromagnetic midsagittal articulography. A single session included 20 repeticions of $/ \mathrm{pap} / \mathrm{htat} /$, and $/ \mathrm{sas} /$ imbedded in a carrier phrase at normal, slow, and fast speech rates. Seven talkers completed three sessions at I week intervals. Reported here are across session comparisons of temporal ordering and relative timing of the movement of the lips and jaw for labial closure at normal rates. The predominant sequence patterns are those in which either upper or lower lip movement occurs first and jaw movement occurs last. Across-subject comparisons show that either lip lead sequence is equally likely to occur. Within-subject across-session comparisons show no clear preference for either lip lead sequence for some subjects, and reversals in dominate lip lead sequences for other subjects. Across-session stability of temporal order is relared to interarticulator relative time: Subjects who demonstrate tight coupling of the upper and lower lips have a higher probability of producing both lip-lead sequences, whereas subjects who demonstrate longer interarticulator relative timing produce consistent 
sequence patterns across sessions. Temporal measures representing the central tendencies of the complete set of session closure gestures are most stable across sessions. [Work supported by NIH DC-00121 to Haskins Laboratories and University of Illinois research grant.]

\section{1:00}

5aSC8. Three-dimensional visualization of humân jaw motion in speech. Thierry Guiard-Martigny (Inst. de la Commun. Parlée, Grenoble Cedex 9, France) and David J. Ostry (McGill Univ., Montreal, PQ H3A IB1, Canada)

With the development of precise 3-D motion measurement systems and powerful computers for 3-D graphical visualization, it is possible to record and fully reconstruct jaw motions. In this paper, a video demonstration of a visualization system for displaying 3-D jaw movements in speech will be presented. Kinematic records of jaw motion and the corresponding speech signal are recorded using an optoelectronic measurement system. The three orientation angles and three positions which describe the motion of the jaw as a rigid skeletal structure and derived from the empirical measurements. These six kinematic variables, which account fully for jaw motion kinematics, are used to drive a real-time 3-D animation of a skeletal jaw and upper skull. The visualization software enables the user to view jaw motion from any orientation and to change viewpoint in the course of an utterance. Selected portions of an utterance may be re-played and the speed of the visual display may be varied. The user may also display, along with the audio track, individual kinematic degrees of freedom or several degrees of freedom in combination. The system is now being integrated into a 3-D audio-visual articulatory speech synthesizer. [Work supported by NIH Grant DC-00594.]

\section{$11: 15$}

5aSC9. Functional data analyses of lip motion. J. O. Ramsay (Dept. of Psych, McGill Univ., 1205 Dr. Penfield Ave., Montreal, PQ H3A IB1, Canada), K. G. Munhall (Queens University, Kingston, ON, Canada), V. L. Gracco (Haskins Labs., New Haven, CT 06511), and D. J. Ostry (Montreal, PQ H3A 1B1, Canada)

The yocal tract's motion during speech is a complex patterning of the movement of many different articulators according to many different time functions. Central issues are the accurate description of the shape of the vocal tract and determining how each articulator contributes to this shape. Techniques are described that provide useful tools for describing multivariate functional data such as the measurement of speech movements. The choice of data analysis procedures has been motivated by the need to partition the articulator movement in various ways: movement start- and end-effects separated from shape effects, effects due to different syllables, and the splitting of within-sensor variation from between-sensor overall variation. The techniques of functional data analysis seem admirably suited to the analyses of phenomena such as these. Familiar multivariate procedures such as analysis of variance and principal components analysis have their functional counterparts, and these reveal in a way more suited to the data the important sources of variation in lip motion. Finally, it is found that the analyses of acceleration were especially revealing in considering the character of possible control mechanisms. [Work supported by NIH Grant DC-00594.]
5aSC10. Orofacial and finger force control in normal subjects. Michèle Gentil (INSERM U 318, CHU, Clinique Neurologique, BP 217, 38043 Grenoble Cedex, 9, France), Abdelkader Rezali, Claire Lise Tournier (Clinique Neurologique, Grenoble, France), Pierre Pollak, and Alim L. Benabid (Univ. Joseph Fourier, 38043 Grenoble Cedex 9, France)

The relationship among several parameters of the ramp-and-hold force contraction and target force level was quantified for the upper lip, lower Iip, and tongue on the one hand, and right and left index on the other hand, in 12 normal subjects ( 6 males and 6 females). Using visual feedback, subjects produced ramp-and-hold compression lip, tongue, and index forces as rapidly and accurately as possible to end-point target levels ranging from 0.25 to 2 newtons, these fine force occurring within physiologic levels presumably involved in speech production. Given the special anatomic and physiologic characteristics of the orofacial system, the purpose of this study was to compare lip, tongue, and finger force control in order to determine whether the motor control is uniform in both systems, that of the speech production and that of the limb, and within subsystems of speech production. Consequently, can be pathophysiology of a movement disorder in its stereotypic form be considered across limb and speech systems? It seems likely that measures of orofacial force control provide useful insights into the fundamental motor control problem of any individual with dysarthria.

\section{1:45}

5aSC11. A computational model using formant space planning of articulator movements for vowel production. Frank $\mathrm{H}$. Guenther (Dept. of Cognitive and Neural Systems, Boston Univ., 111 Cummington St., Rm. 244, Boston, MA 02215) and Dave Johnson (Boston Univ., Boston, MA)

It is often hypothesized that articulator movements are planned within a coordinate frame whose variables correspond to key vocal tract constrictions [e.g., E. Saltzman and K. G. Munhall, Ecol. Psych. 1, 333-382 (1989)]. However, recent evidence suggests that speakers may utilize a more acoustic-like space for planning vowel movements [J. Perkell et al., J. Acoust. Soc. Am. 93, 2948-2961 (1993)]. Previous work has verified the capacity of a computational speech production model called DIVA to explain a wide range of experimental data using a constriction planning space. The current work extends the model to allow formant space planning of vowel movements. The model learns target regions for $F 1$ and $F 2$ for each vowel during a babbling cycle. A mapping between desired formant changes and articulator movements that achieve these changes is also learned. After babbling, the model successfully reaches all vowel targets from any initial vocal tract configuration, even in the presence of constraints such as a blocked jaw, and the resulting synthesized vowels are easily recognizable. Although vowel targets specify only formant ranges with no articulatory information, articulator configurations used by the model to produce vowels are similar to human configurations. [Work supported by AFOSR F49620-92-J-0499.] 


\title{
Session 5aUW
}

\section{Underwater Acoustics: Sea-Surface Scattering}

\author{
Guillermo C. Gaunaurd, Chair \\ Naval Surface Warfare Center, Code 684, White Oak, Silver Spring, Maryland 20903-5640
}

Chair's Introduction $-8: 30$

\section{Contributed Papers}

8:35

5aUW1. Shallow water propagation in the presence of a rough sea surface and the associated bubble clouds. Guy V. Norton (Naval Res. Lab., Stennis Space Center, MS 39529-5004), Jorge C. Novarini (Planning Systems, Inc., Slidell, LA 70458), and Richard S. Keiffer (Naval Res. Lab., Stennis Space Center, MS 39529-5004)

Propagation models in underwater acoustics usually incorporate the sea-surface roughness as a perturbation, (i.e., a loss mechanism through an additional attenuation factor) based on coherent loss in the specular direction. In addition, scattering kernels are generally derived assuming a homogenous medium underlying the sea surface, an assumption incompatible with a realistic environment. Using a numerical model [Norton et al., J. Acoust. Soc. Am. 95, 3018(A) (1994)] that combines a high-fidelity parabolic equation propagation model with the conformal mapping technique to handle surface roughness in a marching algorithm developed by Dozier [L. B. Dozier, J. Acoust. Soc. Am. 75, 1415-1432 (1984)], forward propagation under a rough surface can be modeled in a mathematically consistent way. The technique is applied to the problem of shallow water propagation in the presence of an inhomogeneous bubble distribution. The ocean environment consists of bubble plumes in different stages of development. The effect of the bubbles are introduced through the complex index of refraction. The model is exercised for frequencies between 20 and $40 \mathrm{kHz}$. The combined effect that the rough surface and the inhomogeneous environment has on amplitude and phase fluctuations and on transmission loss is examined. [Work supported by ONR.]

\section{8:50}

5aUW2. The influence of bubble clouds on surface scattering strengths. Michael Nicholas, Peter M. Odgen, and Fred T. Erskine (Naval Res. Lab., Code 7142, 4555 Overlook Ave. S.W., Washington, DC 20375-5350)

One of the most comprehensive sets of surface scattering strength measurements was made during the CST experiments between August 1988 and May 1993, covering a frequency range from $\sim 70$ to $\sim 1500 \mathrm{~Hz}$. An extensive set of environmental measurements (including bubble cloud measurements) collected on several of these experiments make this a unique data set for examining the effect of sub-surface bubble clouds on surface backscattering strength. Analysis of these data have shown that wind speed, while the most important environmental descriptor, cannot by itself explain some important differences in the surface scattering strengths measured for the different CST experiments. These differences appear to be linked to the sub-surface bubble clouds which, in tum, are influenced by the dynamics of the upper layers of the ocean. Latest results from the continuing analysis of the surface scattering and bubble cloud data will be presented which illustrate the influence of the bubble clouds and indicate how the stability of the upper layers of the ocean play a role in distributing those bubble clouds. [Work supported by ONR.]
9:05

5aUW3. Sound scattering by a single air bubble and by a cloud of air bubbles near the sea surface. G. C. Gaunaurd and H. Huang (Naval Surface Warfare Ctr., White Oak Detachment, Silver Spring, MD 20903-5640)

Sound scattering by an air bubble in a boundless fluid is an old classical problem [i.e., R. Y. Nishi, Acustica 33, 65-74 (1975)]. If the air bubble is near, and strongly interacting with the surface of a liquid halfspace, then the scattering cross section (SCS) of the bubble is quite different from its value far away from the boundary. The exact solution for this scattering problem is given which is valid for any incidence direction of the (plane) sound waves, and for any bubble depth, obtained by the method of images. This benchmark solution is found by means of the addition theorems for the spherical wave functions. The resulting SCS contains contributions from the interface, the bubble, and from its image, and it is expressible in terms of coupling coefficients containing products of Wigner 3-j symbols. The formulation is illustrated with many computed plots and it is finally extended to the case of a round, low-concentration cloud of equal size bubbles, just beneath the sea surface. This generalization is possible by replacing the individual bubble properties by those of an "effective medium" describing the bubble cloud just as we found them earlier [i.e., J. Acoust. Soc. Am. 85, 54I-554 (1989)]. [Work supported by NSWC's IR Program.]

\section{9:20}

5aUW4. Deriving scattering strengths from nonstationary time-series data: A comparison of low-frequency surface-backscattering strengths using both impulsive and coherent sources. Roger $\mathrm{C}$. Gauss, Peter M. Ogden (Naval Res. Lab., Washington, DC 20375-5350), John B. Chester (Naval Undersea Warfare Ctr., New London Detachment, New London, CT 06320), and Joseph M. Fialkowsi (Planning Systems, Inc., McLean, VA 22102)

The derivation of scattering strengths from acoustic field data involves applying the active sonar equation to the measured reverberation response. However, due to the typical nonstationarity observed in the direct-path reverberation time series (e.g., $10 \mathrm{~dB} / \mathrm{s}$ ), the choice of appropriate corresponding grazing angles becomes more problematical as the duration of the transmitted signal increases. Direct-path measurements of lowfrequency $(200-1000 \mathrm{~Hz})$ acoustic surface scattering were made in the Gulf of Alaska in the February of 1992 using interleaved SUS and pulsed waveforms ( $\mathrm{cw}, \mathrm{FM}$ ). Backscattering strengths were derived for pulse lengths varying between $50 \mathrm{~ms}$ and $2.4 \mathrm{~s}$. A comparison of results revealed that scattering strengths have a nontrivial dependence on signal type and pulse length, and on the signal-processing methodology used in their derivation. General implications for deriving scattering strengths from nonstationary time-series data will be discussed. [Work supported by ONR (NRL).] 
5aUW5. Low-frequency sea surface scatter and horizontal bistatic angle dependence utilizing short coherent pulses. John B. Chester and Raymond J. Christian (NUWC Div. Newport, New London Detachment, New London, CT 06320)

A comprehensive low-frequency $(<1000-\mathrm{Hz})$ low grazing angle $\left(<30^{\circ}\right)$ sea surface acoustic scatter experiment was conducted during February/March 1992 in the Gulf of Alaska. During data collection, wind speeds ranged from 3-17.5 m/s and sea states from 2-6. cw waveforms less than $500 \mathrm{~ms}$ in length at $250,380,900$, and $935 \mathrm{~Hz}$ were transmitted by three collinear vertical towed source arrays. Surface scattered energy was received in 17 cosine-spaced beams by a horizontal source-ship-towed line array. This experimental geometry allowed measurement of surface scattering strength as a function of mean grazing angle, frequency, and horizontal bistatic angle. Analysis of these measurements in terms of these dependencies is presented along with comparison to current surface scattering strength model predictions.

\section{9:50}

5aUW6. On the correlation of ambient noise levels and other parameters with backscattering strengths observed during the acoustic surface reverberation experiment (ASREX). Neil J. Williams, Hien B. Nguyen, Charles L. Monjo, and Harry A. Deferrari (Appl. Marine Phys., RSMAS, Univ. of Miami, 4600 Rickenbacker Cswy., Miami, FL 33149)

Preliminary results from a surface reverberation experiment performed in the North Atlantic during the winter of 1993-94 indicate that ambient noise levels may prove to be a convenient and accurate indicator of surface backscattering strengths. Backscattered energy from coherent sources ranging in frequency from 100 to $800 \mathrm{~Hz}$ were observed over a three month period. Both the backscattered returns and ambient noise were recorded by a 64-element vertical array while simultaneous measurements were made of a variety of environmental parameters, including: wind speed and direction, wave spectra, air and sea temperature and current fields, bubble plumes, etc., by investigators from SIO, WHOI, and IOS/ $\mathrm{BC}$, Canada. The nature of the data set lends itself to time series analysis techniques. Multivariate analysis of backscattering strengths as a function of ambient noise and various other environmental parameters will be presented and discussed. [Work supported by ONR.]

\section{0:05-10:20 Break}

\section{$10: 20$}

5aUW7. Backscattering from the ocean surface: Observations from the acoustic surface reverberation experiment (ASREX) December '93-March '94. Charles L. Monjo, Hien B. Nguyen, Neil J. Williams, and Harry A. Deferrari (Appl. Marine Phys., RSMAS, Univ. of Miami, 4600 Rickenbacker Cswy., Miami, FL 33149)

Preliminary results from a surface reverberation experiment that was performed in the North Atlantic Ocean during the winter of 1993-94 will be presented. Observations of acoustic backscatter from the ocean surface were made from a moored vertical array at frequencies ranging from 100 to $800 \mathrm{~Hz}$ at 12 -min intervals during a 3-month period. Simultaneous measurements were made by other investigators (from SIO, WHOI, and IOS-BC, Canada) of a variety of environmental parameters, including wind speed and direction, wave spectra, air and sea temperature and current fields, and the presence and nature of bubble clouds were observed with devices that detected anomalies in the near surface sound speed and using high-frequency side scan sonars. Variation of backscattering strengths with changes in the environment will be presented and comparisons with results from other recent observations [P. M. Ogden and F. T. Erskine, J. Acoust. Soc. Am. 95, 746-761 (1994)] will be made. [Work supported by ONR.]
5aUW8. Effect of surface height distributions on underwater acoustic backscattering statistics. Kyle M. Becker (Graduate Program in Acoust., Penn State Univ., Appl. Res. Lab., Student Area, P.O. Box 30, State College, PA 16804)

A problem of interest to ocean environmental acousticians is understanding the relationship between ocean surface characteristics and acoustic backscattering statistics. Recent experimental work has focused on determining surface properties which cause backscattering strength statistics to deviate from exponential. Using the nomenclature of Ogilvy (Theory of Wave Scattering from Random Rough Surfaces, 1991) several scattering surfaces have been fabricated. Surfaces were modeled using a moving average technique with prescribed distribution functions and correlation functions $C(\mathbf{R})$. Representing surface elevation, $h$, as a continuous random process, the moving average process of order $N$ is given as $h_{n}=\sum_{i=0}^{N} w_{l} u_{n-1}$. Statistical properties of $h$ are determined by the random uncorrelated variables, $u_{n}$, and the choice of weights, $w_{l}$. Weights are determined by the choice of $C(\mathbf{R})$ and corresponding correlation length, $\lambda_{0}$, or distance over which $C(\mathbf{R})$ decays by $1 / e$. Isotropic and anisotropic surfaces have been constructed for both Gaussian and non-Gaussian surface height distributions. Acoustic backscattering results are presented for three cases: case I-log-normal height distribution in $x$ and $y$, equal correlation lengths; case II-Gaussian height distribution, correlation length in $y$ equal to ten times that of $x$; case III-Gaussian height distribution in $x, \log$-normal distribution in $y$, equal correlation lengths.

5aUW9. Numerical studies of the small slope approximation for rough surface scattering using a Pierson-Moskowitz spectrum. Shira L. Broschat (School of Elec. Eng. and Comput. Sci., Washington State Univ., Pullman, WA 99164-2752) and Eric I. Thorsos (Univ. of Washington, Seattle, WA 98105)

The small slope approximation (SSA), introduced by Voronovich in the mid-1980s [A. G. Voronovich, Sov. Phys. JETP 62, 65-70 (1985)], has been shown to be a very promising method for modeling wave scattering from rough surfaces. The theory gives a systematic series which is manifestly reciprocal at each order, reduction to perturbation theory is intrinsic to the formulation, and reduction to the Kirchoff approximation occurs under the appropriate conditions when the first two terms of the series are retained [E. I. Thorsos and S. L. Broschat, J. Acoust. Soc. Am. 97, 20822093 (1995)]. In this paper numerical results are presented for scattering strengths for one-dimensional, pressure release surfaces satisfying a Pierson-Moskowitz power law spectrum. Results are given for incident angles varying from grazing to normal over the full angular range of scattering. Comparisons with Monte Carlo integral equation results show that the SSA is extremely accurate over a large range of scattering angles including low forward grazing angles.

\section{1:05}

5aUW10. A physically motivated model for sea surface backscattering in the low to moderate frequency regime. Richard S. Keiffer (Naval Res. Lab., Code 7181, Stennis Space Center, MS 39529) and Jorge C. Novarini (Planning Systems, Inc., Slidell, LA 70458)

Based on the refractive properties of the bubble clouds and the background (uniform) bubble layer and resonant scattering at the sea surface, a simple model is presented to estimate the backscattering from the ocean surface for frequencies below $1 \mathrm{kHz}$. This new model yields a dependence on the three fundamental variables, wind speed, grazing angle, and frequency, that is in general agreement with trends observed in reverberationderived backscattering data. [Work supported by ONR.] 
5aUW11. Modeling sea surface scattering with a finite difference method. R. A. Stephen (Woods Hole Oceanogr. Inst., Woods Hole, MA 02543)

A numerical scattering chamber based on the finite difference solution of the two-way elastic wave equation is applied to the sea surface scattering problem. For a reference model, the method gives excellent agreement in amplitude and phase with the solution obtained by an integral equation method [Eric Thorsos, personal communications]. This problem demon- strates a number of issues in numerical modeling of wave scattering: (1) the spreading of Gaussian beams (even in homogeneous media) has implications for (i) defining the surface footprint or scattering area and (ii) for generating approximate Gaussian beams by tapered vertical arrays; (b) the treatment of a rough, free, fluid surface in a Cartesian grid scheme; (c) solving continuous wave (cw) problems in the time domain; (d) diffraction of waves from roughness elements with scale lengths on the order of a wavelength; (e) multiple scattering effects between roughness elements; and (f) omni-directional scattering including low angle backscatter. [Work supported by Office of Naval Research.]

\title{
Session 5pBV
}

\section{Bioresponse to Vibration and to Ultrasound and Physical Acoustics: Biomolecular Acoustics}

\author{
Armen Sarvazyan, Chair \\ Department of Chemistry, Rutgers University, P. O. Box 939, Piscataway, New Jersey 08855
}

Chair's Introduction-1:00

Invited Papers

1:05

5pBV1. Ultrasonic studies of phase transitions in lipid membranes and protein molecules. Dmitri P. Kharakoz (Inst. of Theoret. and Exp. Biophys., Russian Acad. Sci, 142292 Pushchino, Moscow, Russia)

This paper reviews phase transitions in the lipid membranes and protein molecules studied by means of ultrasonic (sound velocity and absorption) and related methods (calorimetric, volumetric, and others). Peculiarities of phase transitions in small-dimension systems in an aqueous environment (a finite width of the transition zone, probability of critical nuclei formation, hysteresis, hydration changes, etc.) are discussed. A comparison of ultrasonic and calorimetric methods showing their complementarity in the studies of kinetics of phase transitions is presented. Ultrasonic data on the melting of lipid vesicles in aqueous dispersions are reviewed. Recent achievements in the ultrasonic study of the kinetic mechanism of creation and growth of gel nuclei within the parent liquid-crystalline phase are reported. Ultrasonic studies of the protein/lipid interactions are reviewed and perspectives are outlined. Native to molten globule state transition in globular proteins is considered (volume and compressibility changes, acoustic relaxation, protein interior hydration). The differences in the behavior of the phase transitions in membranes and in protein molecules are discussed. New questions for further ultrasonic studies of the phase transitions are formulated.

$1: 35$

5pBV2. Molecular acoustics in thermodynamic characterization of proteins. Tigran V. Chalikian (Dept. of Chemistry, Rutgers Univ., Piscataway, NJ 08855)

Processes such as conformational phase transitions, ligand binding events, and enzymatic catalysis alter the structure of proteins as well as the nature of their solvent accessible surface. Consequently, these processes result in corresponding changes in the sound velocity of the protein solution. As is shown by the example of two typical globular proteins, cytochrome $c$ and $\alpha$-chymotrypsinogen $A$, the transition from the native state to the molten globule state is accompanied by an increase in sound velocity, while the native state-unfolded state transition is accompanied by a decrease in sound velocity. The interpretation of the macroscopic sound velocity data for the different phase states of proteins in terms of microscopic structural and hydrational differences is a very sophisticated problem. One of the ways of attempting to solve the problem of distinguishing between the contributions of protein structure and hydration to the sound velocity is to search for correlations between the structural crystallographic data of a large number of globular proteins and their ultrasonic velocimetric characteristics.

5pBV3. Macromolecular absorption: The roles of molecular size, structure, solvation, and solute interactions. Frederick W. Kremkau (Ctr. for Med. Ultrasound, Bowman Gray School of Medicine, Wake Forest Univ., Winston-Salem, NC 27157-1039)

Attenuation of ultrasound in tissues is due primarily to macromolecular absorption. Biomacromolecules have higher absorptions than the constituent molecules of which they are made [F. W. Kremkau and W. Cowgill, J. Acoust. Soc. Am. 76, 1330-1335 (1984)]. Absorption dependencies on structure [F. W Kremkau and R. W Cowgill, J. Acoust. Soc. Am. 77, 1217-1221 (1985)] and solvation [F. W. Kremkau, J. Acoust. Soc. Am. 83, 2410-2415 (1988)] are probably interrelated. Underlying absorption processes are enhanced or new processes are added under conditions of high concentration, aggregation, or cross linking [F. W. Kremkau et al., J. Acoust. Soc. Am. 53, 1448-1451 (1973)]. 


\section{2:35-2:50 Break}

\section{2:50}

5pBV4. Major accomplishments in development of acoustic instrumentation for biomolecular studies. Armen P. Sarvazyan ${ }^{\text {a) }}$ (Dept. of Chem., Rutgers Univ., New Brunswick, NJ 08903)

A great potential of acoustics in the thermodynamic characterization of biomolecular systems, studies of conformational transitions and hydration of proteins and nucleic acids and drug-biopolimer interactions has been revealed in the last decade mainly due to advances in the instrumentation for ultrasonic measurements in small volumes of liquids. The methods and devices reviewed in this talk are based upon the fixed path interferometer method, also known as a "resonator method." It is the only method which can provide measurements of ultrasound velocity and attenuation in the sample volume of the order of 10-100 microliters with sufficient precision. The requirement of small sample volume is a major precondition for applicability of the method to the most of the problems in biomolecular studies. Different types of resonators will be described in detail including a new type of micro-volume cylindrical standing wave resonators with a chamber volume of about 3 microliters. The electronic circuits for high precision measurement using acoustical resonators are usually based on the phase-locked loop which provides the oscillation in the system at the chosen resonance.

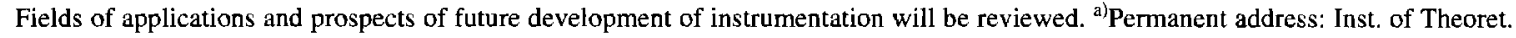
and Exp. Biophys., Russian Acad. of Sci.

\section{Contributed Papers}

$3: 20$

5pBV5. Passive acoustic fetal heart rate monitoring with a parallel redundance approach. Stephen A. Zahorian, Zongyao Zhou, and Charles Brewton (Dept. of Elec. Eng., Old Dominion Univ., Norfolk, VA 23529)

Acoustic methods for monitoring fetal heart rate are potentially advantageous over ultrasound methods, since, eventually, long-term low-cost monitoring would be possible for high risk mothers, with no concern for fetal damage due to the monitoring device. However, sensor design and signal processing requirements are very demanding in this low signal-tonoise ratio environment. The present approach is patterned after that by Zuckewar $e$ t al. [IEEE Trans. Biomed. Eng. 40-9, 963-969 (1993)] using a third-generation sensor belt, and enhanced signal processing strategies. In the new method, each of seven differential sensor signals is scanned continuously and processed in parallel with a PC-hosted DSP system. The processing of each channel includes FIR bandpass filtering, Teager energy calculations, autocorrelation, and "intelligent" peak picking. "Candidates" for fetal heart rate are computed every $0.5 \mathrm{~s}$ and evaluated with a figure of merit which incorporates both measures of peak quality and continuity constraints. Clinical testing indicates that the new method of signal processing is able to reliably track fetal heart rate for most fetuses with a gestation age of at least 30 weeks. The multiple-channel approach results in a significant improvement over a single-channel method.

\section{$3: 35$}

5pBV6. Effects of acoustic cavitation on luminescent bacteria. E. Carr Everbach, Inder R. S. Makin, and Amy Cheng Vollmer (Dept. of Eng. and Biol., Swarthmore College, Swarthmore, PA 19081-1397)

The interaction of intense ultrasound with bacteria has implications for improving fundamental understanding in biology and bioacoustics, as well as providing possible applications in water purification and medicine. An investigation will be presented of the effects of acoustic cavitation on
E.coli bacteria that have been genetically engineered to emit visible light when subjected to physical or chemical stress. Genetic cloning techniques allow the placement of the lux gene, derived from luminescent marine microorganisms, at specific locations in bacterial DNA. Each placement location yields light production in proportion to the repair mechanism employed by the bacteria in response to specific kinds of stress (oxidative damage, DNA damage, membrane damage, protein damage, thermal damage, etc.). A 20-MHz passive acoustic detector [abstract, Huertas et al., J. Acoust. Soc. Am. 95, 2856 (1994)] was used to quantify cavitational activity when bacteria were exposed to 1-MHz pulsed ultrasound. By comparing a measure of cavitational activity with light output from exposed bacteria that were presensitized to different kinds of damage, cavitational effects on both bacteria and mammalian cells can be better understood.

\section{3:50}

5pBV7. Intestinal hemorrhage produced by pulsed ultrasound or lithotripter fields. Diane Dalecki, Sally Z. Child, Carol H. Raeman, and Edwin L. Carstensen (Dept. of Elec. Eng. and the Rochester Ctr. for Biomed. Ultrasound, Univ. of Rochester, Rochester, NY 14627)

The threshold for intestinal hemorrhage in mice was determined using a piezoelectric lithotripter. Mice were exposed to 200 lithotripter pulses with a PRF of $\sim \mathrm{I} \mathrm{Hz}$. The amplitude of exposure was determined by either varying the position of the animal relative to the focus or by varying the voltage used to charge the lithotripter. Threshold for intestinal hemorrhage occurred at a peak acoustic pressure of 1-2 MPa. At threshold, the lithotripter waveform was nearly sinusoidal. Thresholds for intestinal hemorrhage were also determined for exposure to pulsed ultrasound at frequencies of $0.7,1.1,2.4$, and $3.6 \mathrm{MHz}$. Intestines were exposed at 3-4 sites for 5 min per site using focused sources operating with a $10-\mu$ s pulse length and $100 \mathrm{~Hz}$ PRF. The threshold at $1.1 \mathrm{MHz}$ was $\sim 1.5 \mathrm{MPa}$ and thresholds increased with increasing frequency. Temperature rises of $1-2{ }^{\circ} \mathrm{C}$ were measured in the intestine during exposure at the highest exposure conditions. Both the frequency dependence of intestinal hemorrhage and the limited heating are consistent with a cavitation mechanism of action. 
Session 5pMU

\author{
Musical Acoustics and Noise: Acoustic Ecology \\ Fred Lipsett, Chair
37 Oriole Drive, Gloucester, Ontario KIJ 7E8, Canada
}

Chair's Introduction-12:55

Invited Papers

$1: 00$

5pMU1. Sound in context: Acoustic communication and soundscape research at Simon Fraser University. Barry Truax (School of Commun. \& School for the Contemporary Arts, Simon Fraser Univ., Burnaby, BC V5A 1S6, Canada)

Research activities at Simon Fraser University over the past 25 years that bridge environmental acoustics and music are summarized, including soundscape studies, acoustic communication, soundscape composition, and the granulation of sampled sound. Gaps in traditional disciplinary approaches in dealing with environmental sound are identified, and an acoustic communicational model is proposed as an interdisciplinary alternative. Sound examples drawn from the author's compositions, and the work of R. Murray Schafer, Hildegard Westerkamp and the World Soundscape Project will be included.

\title{
1:30
}

5pMU2. Community noise: The mainstream approach. Kenneth J. Plotkin (Wyle Labs., 2001 Jefferson Davis Hgwy., Ste. 701, Arlington, VA 22202)

Community noise analysis and control has been a concern for centuries. The introduction of jet commercial aircraft in the 1950s escalated it to a widespread practice with widely accepted quantitative rules. The rules have their technical basis in scientific studies ranging from laboratory psychoacoustic experiments through social attitudinal surveys. Implementation is in the form of land use planning and noise control regulations. A review is presented of the current practice, its successes, and its limitations.

\section{2:00}

5pMU3. Acoustic hygiene: The importance of protecting ears, lungs, and other organs from acoustic insult. Michael A. Zagorski (Dept. of Psych., Memorial Univ. of Newfoundland, St. John's, NF, Canada)

While the importance of controlling noise is widely accepted, the major concern has been with annoyance. The public is not generally aware of the potential severity of even a few noise exposures. Most people do not realize the extent to which they use acoustic cues in daily life, nor the extent of debilitation associated with recruitment or loss of binaural abilities. Similarly, most do not realize that a flyover producing short-term noise measurement of a little over $130 \mathrm{dBA}$ can have the same effect on the lung as $2 \mathrm{lbs}$. of TNT exploding a meter or two from the body. It is argued here that to promote acoustic hygiene the public must be made aware that hearing loss is a complex and serious handicap that is not generally corrected with hearing aids. To do this emphasis must be placed on the importance of acoustic cues in everyday activities even without speech, the handicap produced by not being able to localize, the difficulty of hearing in crowds, and the annoyances of hearing aids. A number of methods for illustrating the effects of hearing loss and the value of normal hearing will be presented and demonstrated. [Work partially supported by the National Research Council of Canada.]

$$
2: 30
$$

5pMU4. From a "sound observatory" to a "sound city" masterplan. Andres Bosshard (Wildbachstr. 62, CH 8008 Zürich, Switzerland)

"Sound sculptures," "sound installations," and "telematic media network links" can be considered as observatories as soon as they look for resonances, reverberations, and other spatial qualities of the actual site and if they are composed to look for possible interaction with the ongoing stream of sounds of the real environment. Time structures of human perception and behavior help to find a basis for "sound perspectives" and "territorial sound fields" and "tidal sound spheres," which can be stimulated, simulated, and modified by advanced electroacoustic networks. The "K̈langturm" in St. Pölten, Austria and the "Adi Srvya" sound gallery in New Delhi, India are two house-sized musical instruments under construction; both will be opened in fall 1996. Field research of the indigenous "Santal" population in West Bengal, India, shows very sophisticated and complex "natural" sound architectures for entire towns. The acoustic tuning of the "Santal" villages is a basic model for an acoustic and electronic study to prepare a "sound city" masterplan. 


\title{
Contributed Papers
}

3:00

5pMU5. Understanding individual differences in response to environmental noise: An essential step in determining health risk. Susan L. Staples (58 Spongia Rd., Stone Ridge, NY 12484)

Evidence is reviewed indicating that psychological and social factors render people more or less susceptible to stress-related health effects from noise. Factors such as appraised meaning, personality traits, and perceived control are considered in terms of their relationship to physiological variables and in terms of the mechanisms underlying adverse effects. The limitations of epidemiological and dose-response methods for determining and understanding health effects are discussed. Modifications in these approaches are suggested to clarify the role of intervening psychological factors, to understand the physiological mechanisms implicated, and to delineate public health risks.

\section{3:15}

5pMU6. Acoustical ecological risk. Timothy S. Margulies (Johns Hopkins Univ., Baltimore, MD 21218)

Risk-based prioritization and decision making has been implemented by the U. S. EPA in several areas to assure quality and consistency of risk and environmental assessment and to identify areas to reduce uncertainty. This talk will review the elements of risk assessment and risk management and will compare the paradigms for considering primarily ionizing radiation and mechanical radiation to assess existing and projected risks to human health and the environment. Treatment of uncertainty propagation in model parameters, such as latin hypercube Monte Carlo sampling and by adjoint methods, as well as decision-making approaches including statistical hypothesis testing will be discussed for comparing alternatives to control unwanted sounds.

\section{3:30}

5pMU7. Characterization of the sound quality of vacuum cleaners. Guyot Frédérique, Piron Christine, Castellengo Michèle, and Fabre Benoit (Lab. d'Acoust. Musicale, Univ. Pierre et Marie Curie, Tour 66 Case 161, 4 place Jussieu, 75252 Paris Cedex 05, France)

The intensity level is not the only parameter responsible for noise disturbance. The basis of the perceived world is the categorization process which permits recognition and qualitative appreciation. This study is based on Rosch's categorization concept. [E. Rosch, Cognitive Psychol. 7, 573,605 (1975)]. In order to estimate the pleasantness features of a set of 23 vacuum cleaner noises and to characterize the prototype of "the pleasant aspirator noise," a free categorization method was adopted. The subjects are asked to group, in a free number of categories, the noises according to the comfort sensation they evoke. By analyzing the results the role played by the typicality and the gradation phenomenon in the structures relative to these noises quality can be estimated. Finally, the organization of these categories is analyzed by estimating on one hand the relative importance of parameters coming exclusively from noises physical content, and on another hand, the significance given to the stimuli by each subject.

\section{Session 5pPAa}

\section{Physical Acoustics: Photoacoustics}

\author{
W. Patrick Arnott, Chair \\ Atmospheric and Energy and Environmental Engineering Centers, Desert Research Institute, University of Nevada, \\ P. O. Box 60220, Reno, Nevada 89506
}

\section{Invited Papers}

5pPAa1. Resonant photoacoustic measurements of optical absorption in solids. Wei-li Lin (Dept. of Phys., Penn State Univ., University Park, PA 16802)

For glasses and crystals the optical absorption near the infrared is much lower than that in the visible. While the previous noncontact resonant photoacoustic (PA) measurement is several orders of magnitude more sensitive than that of conventional PA in the visible for a piezoelectric sample, it was further developed to apply to nonpiezoelectric materials in the near infrared. The noncontact interdigital capacitor transducers were replaced by lithium niobate disks and PVDF films. By supporting the sample between transducers only at its comers, the quality factor has been improved and is comparable to that of a noncontact system, while the separate supports and the precise positioning of the sample are avoided. An optical absorption coefficient on the order of $10^{-7} \mathrm{~cm}^{-1}$ at $1.06 \mu \mathrm{m}$ has been measured with a 20 signal-to-noise ratio. Recent results on single crystals of quartz and calcium fluoride, using different piezoelectric transducers, will be discussed. [Work supported by NSF Grant No. DMR-9000549 and by the Office of Naval Research.]

$12: 55$

5pPAa2. Resonant photoacoustic measurements of optical absorption in gases. Wayne M. Wright (Dept. of Phys., Kalamazoo College, Kalamazoo, MI 49006)

The use of the photoacoustic (or optoacoustic) effect in spectroscopy gives a valuable tool for examining weak optical absorption features of gases. It is inherently a more sensitive detector of light absorption than other techniques, which attempt to measure a small attenuation of an intense beam. Instead, absorption by the gas of periodically interrupted optical energy, from either a tunable or fixed-wavelength laser, results in the production of sound at the same frequency. Device optimization has followed a number of 
different paths. The particular approach emphasized here uses a cylindrical gas cell, driven at its 1 st azimuthal acoustic resonance, to increase signal amplitude. Experimental concerns include enhancement of the signal relative to acoustic and electronic noise. Examples are presented of what can be learned through application of this technique. These include results for the absorption of visible light by nitrogen dioxide and aerosol particles, as well as from a study of the profiles of oxygen absorption lines in the infrared region.

$1: 20$

5pPAa3. Transient grating studies of nonlinear photoacoustic effects in particulate solutions. G. J. Diebol, H. X. Chen, Y. N. Cao, and M. B. Zimmt (Dept. of Chem., Brown Univ., Providence, RI 02912)

Experiments where transient grating signals are recorded from irradiation or particulate matter are reported. A relatively low power, picosecond laser produces transient grating signals in reverse micellar solutions that have a unique time dependence, and which can be explained by heat diffusion from the interior of the micelle to a surrounding organic solvent. A 16-ns-long, high-power pulse from a $Q$-switched ND:YAG laser irradiating a gold sol produces a diffracted light signal that is explainable in terms of a thermally generated photoacoustic effect. Particulate carbon, on the other hand, gives transient grating signals that appear to possess an overtone of the usual thermally generated fundamental frequency. The effect is nonlinear in its dependence on the laser intensity, and is dependent on the time of irradiation of the solution. A second characteristic of the effect is that a pronounced noise is generated by the solution on firing the laser. It is believed that the effects can be attributed to endothermic chemical reactions at the surface of the carbon that act to unbalance the thermal and acoustic modes producing an acoustic density that oscillates about the ambient. Since the diffracted light signal is proportional to the square of the acoustic density, what appears to the eye as an overtone is seen in the recorded signal.

\section{Contributed Papers}

\section{$1: 45$}

5pPAa4. Forward and backward projection of transient acoustic field functions obtained from schlieren and fiber-optic measurements. Jie Sun, Peter R. Stepanishen (Dept. of Ocean Eng., Univ. of Rhode Island, Kingston, RI 02881), Gregory Clement, Ruiming Liu, and Stephen V. Letcher (Univ. of Rhode Island, Kingston, RI 02881)

A combination of a schlieren system and a polarimetric fiber-optic detection system is used to obtain line integrals of transient acoustic fields a distance $z$ in front of a planar source. The $x, y$ dependence is obtained from a tomographic reconstruction algorithm. The signal is then forward projected away from the source or back-projected toward the source using wave vector/time domain and frequency methods. Numerical results will be presented to illustrate the projection methods for a number of different transient signals from planar sources with separable and nonseparable space/time velocity distributions. Experimental results for selected source distributions from pulsed ultrasonic transducers will then be presented and discussed.

\section{2:00}

5pPAa5. Modeling of optical microphone performance. Nykolai Bilaniuk and André Delâge (Inst. for Microstructural Sci., Natl. Res. Council of Canada, Ottawa ON K1A 0R6, Canada)

A number of modulation principles and an even larger variety of specific implementations may be considered for all-optical microphones. The procedures for designing optical microphones described in this paper include three principal steps. First the mechanical response of a membrane to an acoustic field is modeled by an analytical technique [A. Hu et al., J. Acoust. Soc. Am. 91, 3049-3056 (1992)], and is used to obtain the magnitude of the membrane deformation and its frequency response. Next the behavior of the optical path under this flexure is studied using the beam propagation method. Predictions of the optical sensitivity (depth of modulation versus membrane displacement), and optical insertion loss are thus obtained. Third the signal-to-noise performance of the device is evaluated by representing all noise sources as equivalent acoustic noise fields at the microphone input. Applications of this methodology to optical microphone designs under consideration are described.

\section{2:15}

5pPAa6. Signal-to-noise ratio of thermoacoustic enhanced photoacoustic spectrometers. W. Patrick Amott, Hans Moosmüller, Robert E. Abbott, and Michael Ossofsky (Atmospheric and Energy \& Environmental Eng. Ctrs., Desert Res. Inst., Univ. of Nevada, P. O. Box 60220, Reno, NV 89506)

The quality factor, $Q$, of plane or radial wave resonators can be increased and controlled using thermoacoustics. When such a resonator is used in photoacoustics, a laser beam passes through it and sound is produced when light absorption occurs. Sound amplitude is proportional to the coefficient for light absorption, to the resonator $Q$, and to the laser beam power. Traditionally, resonator dimensions have determined both the coupling strength of the laser beam to a particular mode and the quality factor. High coupling strength is desirable, but implies use of a narrow resonator where most of the interior is filled with laser. On the other hand, high $Q$ traditionally implied need for a wide resonator. Thermoacoustics can be used to increase the $Q$ of the narrow resonator to optimize the acoustic signal. Our theory and measurements show that the thermoacoustically enhanced photoacoustic spectrometer can be viewed as an analog narrowband amplifier for sound where the resonance frequency is $f_{0}$, the bandwidth is $f_{0} / Q$, the gain is $Q$, and the signal-to-noise ratio for discrimination against Gaussian acoustic noise in the resonator is $Q^{1 / 2}$. [Work supported by the EPA, ONR.] 


\title{
Session 5pPAb
}

\section{Physical Acoustics: Thermoacoustics, Bubbles, and Droplets}

\author{
Paul E. Barbone, Chair \\ Department of Aerospace and Mechanical Engineering, Boston University, Boston, Maśsachusetts 02215
}

\section{Contributed Papers}

$2: 45$

5pPAb1. Numerical study of a simplified model of nonlinear processes in thermoacoustic engines. A. Prosperetti and $M$. Watanabe (Dept. of Mech. Eng., Johns Hopkins Univ., Baltimore, MD 21218)

A simplified one-dimensional model of thermoacoustic devices, obtained by integrating the governing equations over the tube cross section, was described earlier. Here the results of a numerical exploration of the model as applied to a prime mover are described. The effects of temperature distribution, wall drag, heat transfer, and cross-sectional area variations are explored and compared with available experimental results. Some initial results for the heat pump configuration will also be presented. [Work supported by the Office of Naval Research.]

\section{3:00}

5pPAb2. Visualization of oscillating temperature and flow fields in the stack region of a thermoacoustic refrigerator model. C. Herman, M. Wetzel, and M. Volejnik (Dept. of Mech. Eng., Johns Hopkins Univ., Baltimore, MD 21218-2686)

In order to study the oscillating temperature and flow fields in the stack region, a model of a thermoacoustic refrigerator is built. The model allows the application of visualization techniques such as holographic interferometry, thermochromatic liquid crystals, and smoke injection. In the first experiments, the correct operation of the thermoacoustic refrigerator is verified. Using the Kundt's dust tube method, the propagation of longitudinal plane-wave fronts is analyzed. Furthermore, the distortion due to the presence of higher harmonics in the resonance tube is kept small. This is verified by applying the Fourier transform to a pressure signal at several positions within the resonance tube. The focus of current investigations is to apply the above visualization techniques in the stack region and its neighborhood. These measurements provide information which can be used to determine requirements for the design of heat exchangers. An improved design of the heat exchangers and a better understanding of heat transfer processes and flow phenomena are key issues to improve the efficiency of thermoacoustic refrigerators. [Work supported by the Office of Naval Research; Martin Wetzel is also supported by a scholarship from DAAD.]

\section{3:15}

5pPAb3. Plane- and radial wave thermoacoustic engines with variable plate separation. Jay A. Lightfoot, Richard Raspet, Henry E. Bass (Dept. of Phys. and Astron., Univ. of Mississippi, University, MS 38677), and W. Patrick Amott (Univ. of Nevada, Reno, NV 89506)

Rott developed the theory of thermally driven acoustic oscillations in tubes with variable cross section. Bennett, in her dissertation, considered plane-wave thermoacoustic refrigerators with variable plate separation and found them to have higher COP's than those with parallel plates. This principle has application to the work in radial wave thermoacoustics, since variable plate separation is a natural product of radial vertical-plate engines with constant plate thickness. Bennett appears to have overlooked a term in the wave equation. An analysis is presented including this term to study two important questions: What is the optimal slope, and even more basic, which side (hot or cold) should have the greater plate separation? [This work was supported by the Office of Naval Research.]

\section{3:30}

5pPAb4. The effect of phase of the acoustic wave on the efficiency of thermoacoustic refrigerators. James Brewster and Richard Raspet (Dept. of Phys. and Astron., Natl. Ctr. for Phys. Acoust., Univ. of Mississippi, University, MS 38677)

An analytic approach to the study of thermoacoustics and application of this technique to the optimization of a refrigerator driven by an arbitrary acoustic wave will be presented. It will be shown that although a standing wave driven cooler has close to optimum efficiency, the efficiency can be improved by the addition of a traveling component to the wave field flowing in the direction of negative temperature gradient. The value of the acoustic impedance in the gas bordering the cold end of the stack is fixed to an arbitrary value. Application of the continuity of pressure and velocity is combined with the wave equation to yield the value of the pressure wave function and its first two derivatives at the cold end of the stack. Knowledge of these allows the direct calculation of the efficiency of refrigeration and the second derivative of the temperature distribution. [Work supported in part by Office of Naval Research.]

\section{$3: 45$}

5pPAb5. Numerical simulation of gas cavity response to shock waves. Zhong Ding and S. M. Gracewski (Dept. of Mech. Eng., Univ. of Rochester, Rochester, NY 14627)

An understanding of cavitation phenomena including the response of a gas cavity in liquid to a time-dependent pressure wave is important in a large range of situations. In the paper, the response of a gas cavity in water to shock waves is analyzed using the arbitrary Lagrangian-Eulerian technique. An axisymmetric model is used in the numerical simulation of a gas cavity response to both weak shocks $\left(p / p_{0} \leqslant 300\right.$, with $\left.p_{0}=0.1 \mathrm{MPa}\right)$ and strong shocks $\left(p / p_{0}\right.$ ranges from 5000 to 20000$)$. An artificial viscosity to capture the shock was developed. A simple, stable, and adaptive mesh generation technique was designed to generate finer meshes at higher pressure gradients. The shock propagation, rarefaction, and transmission were analyzed over the entire computational domain. When impacted by a weak shock, a gas cavity will collapse almost spherically creating a large pressure within the gas and the surrounding liquid due to the rapid compression of the gas. When impacted by a strong shock, a gas cavity will collapse with a jet penetrating the opposite side of the interface with a speed as high as $2000 \mathrm{~m} / \mathrm{s}$.

\section{4:00}

5pPAb6. A new kind of multiple-droplet levitator. Yuren Tian and Robert E. Apfel (Ctr. for Ultrasonics and Sonics, Yale Univ., New Haven, CT 06520-8286)

Many different kinds of levitators are used to study liquid drops. In most of them, only a single drop can be levitated. Under some circumstances, however, it is desired to levitate multiple drops simultaneously. An acoustical levitator has been modified for the purpose of multiple-droplet 
levitation. During the experiments, a liquid is poured on the surface of the acoustic transducer and is ultrasonically atomized. By applying a static electric field on the surface of the liquid, the atomized drops are electrically charged. After the drops are dispersed into the levitator, they can be contained together and levitated by acoustic radiation force. Coalescence among the drops is prevented by the forces of electric repulsion. The separation distance between the drops, the droplet size, and the number of levitated drops can be controlled by the intensity of the acoustic and electric fields. Preliminary experimental results will be reported, including the observation of mass transfer among the levitated drops. [Work supported by NASA through JPL, contract 958722 .]

\section{4:15}

5pPAb7. Nonlinear acoustics of bubbly liquids: Traveling waves in a quadratic approximation. Daniel Goldman, Ali Nadim, and Paul E. Barbone (Dept. of Aerosp. and Mech. Eng., Boston Univ., 110 Cummington St., Boston, MA 02215)

An important aspect of underwater acoustics is the effect of trapped clouds of air bubbles. Recently, an approach to wave propagation in dilute bubbly liquids was described in which the Rayleigh-Plesset equation is used to derive a nonlinear equation of state for the gas bubble-liquid mixture [Nadim et al., Bull. Am. Phys. Soc. 39, 1976 (1994)]. In this formulation, the pressure in the mixture depends in a highly nonlinear way on the local density and its first and second time derivatives. Linearization leads to the standard acoustic equation for bubbly liquids. Here the quadratic approximation to the nonlinear equation of state is examined, along with the related nonlinear wave equation. The high- and low-frequency limits are then analyzed, and traveling wave solutions are sought in each case. Comparison is made with existing results for nonlinear waves in bubbly media.

4:30

5pPAb8. Feasibility of low-frequency single-bubble sonoluminescence. Robert E. Apfel, Tao Shi, Joseph Jankovsky, Jeffrey Ketterling, and Xiaohui Chen (Dept. of Mech. Eng., Yale Univ., New Haven, CT 06520-8286)

The potential to perform single-bubble sonoluminescence (SBSL) at low frequencies is motivated by the payoff of greatly enhanced energy concentration during collapse. Yet it is also known that bubbles undergoing such catastrophic collapse tend to be unstable. Experimental apparatus has been designed and computer simulations have been performed to test the feasibility of low-frequency, single-bubble sonoluminescence. The experimental apparatus consists of a cylindrical cell that is driven by an aluminum, half-wavelength resonator with fundamental resonance of less than $15 \mathrm{kHz}$. The cell is designed to be pressurized up to 5 atmospheres to allow levitation without significant spurious cavitation in the liquid. To complement this experimental work, our computer simulations of this phenomena are continuing [T. Shi and R. Apfel, J. Acoust. Soc. Am. 96, 3253 (1994)] in order to follow the shape distortion of collapsing bubbles for varying parameters, including acoustic frequency. The basic characteristics of our low-frequency resonator and the results of experimental work and computer simulations will be reported.

\title{
Session 5pPP
}

\section{Psychological and Physiological Acoustics: Binaural and Spatial Hearing; Cochlear and Auditory Nerve Function}

\author{
Kim S. Abouchacra, Chair \\ U.S. Army Research Laboratory, AMSRL-SD-HR, Building 520, Room 39, Aberdeen Proving Ground, Maryland 21005-5425
}

\section{Contributed Papers}

1:15

5pPP1. A dynamic, psychophysical model of adaptation in localization experiments. Barbara Shinn-Cunningham (Res. Lab. of Electron., MIT, 77 Massachusetts Ave., Cambridge, MA 02139)

Previous psychophysical models have described how resolution depends upon the range of physical stimuli employed in a given experiment; however, these models generally assume that subject performance is stable over time. The current work extends the context-coding model of Durlach and Braida [J. Acoust. Soc. Am. 46, 372-383 (1969); Braida and Durlach, J. Acoust. Soc. Am. 51, 483-502 (1970)] to account for changes in subject performance that occur when feedback is used to retrain subjects during the course of an experimental session. In the current model, observed changes in performance are accounted for by assuming a single exponential adaptation process. This process, which describes the adaptive state of the subject, determines the decision criteria and context (effective stimulus range) used in the model at a given point in time. From the dynamic decision criteria and effective range, and knowledge of the underlying sensitivity to localization cues, the model predicts mean response, bias, and resolution. The model predictions are in good agreement with results from a relatively large body of experiments, including experiments in which the number and range of stimuli were varied.
$1: 30$

5pPP2. Model simulations of masked thresholds for tones in dichotic noise maskers. Inga Holube, $\mathrm{H}$. Steven Colburn (Dept. of Biomed. Eng., Boston Univ., 44 Cummington St., Boston, MA 02215), Steven van de Par, and Armin Kohlrausch (Inst. for Perception Res. (IPO), Eindhoven, The Netherlands)

The study of masked thresholds in dichotic noise maskers is important for understanding the processing in binaural hearing. To simulate these thresholds a psychoacoustically motivated perception model was used [T. Dau et al. (1995). "A quantitative model of the "effective" signal processing in the auditory system: I. Model structure," submitted to J. Acoust. Soc. Am.]. This model, which has been successfully applied to several monaural psychoacoustical experiments, was extended by an additional binaural processing unit. It consists of a filterbank, half-wave rectifier, low-pass filter, and adaptarion loops, which model the temporal processing. The binaural processing unit detects the interaural correlation and makes decisions based on the difference between the signals from both ears. Masked thresholds in the NoS $\pi$ and $\mathrm{N} \pi$ So configurations, obtained as a function of noise masker frequency and bandwidth, were simulated and compared to new experimental measurements. The dependence on interaural delay and interaural decorrelation of the noise masker was also modeled and compared to data in the literature. In general, model simula- 
tions agree well with the main features seen in the measurements. [Work supported by DFG (Ho 1627/1-1) and by NIDCD (Grant DC00100).]

\section{$1: 45$}

5pPP3. The index of interaural envelope correlation: Normalized cross-covariance or normalized cross correlation? Leslie R. Bernstein and Constantine Trahiotis (Ctr. for Neurological Sci. and Surgical Res. Ctr., Dept. of Surgery, Univ. of Connecticut Health Ctr., Farmington, CT 06030)

This study principally evaluated whether the normalized cross covariance (Pearson product-moment correlation) or the normalized cross correlation describes discriminability of changes in interaural disparities conveyed by the stimulus enveloped. In a four-interval, two alternative task, listeners detected which interval contained a $4-\mathrm{kHz}$ tone added antiphasically to diotic, 200-Hz-wide, noise (NoS $\pi$ ). The "nonsignal" intervals contained the tone added homophasically (NoSo). Discriminability $\left(d^{\prime}\right)$ was measured as a function of $S / N$ for values between -30 and $+30 \mathrm{~dB}$ (really!). For all S/N's, overall level was $70 \mathrm{~dB}$ SPL. Listeners' performance was very well accounted for by the normalized cross correlation but not the normalized cross covariance. Additionally, listeners were tested in a "direct" discrimination task where changes in envelope correlations $(\Delta \rho)$ were produced by "mixing" two independent Gaussian noises. Although $\Delta \rho$ 's at threshold $\left(d^{\prime}=1.0\right)$ obtained from the two tasks were similar, the psychometric functions obtained with the direct discrimination task were more steep. Discussion will include how the normalized cross correlation of the envelope accounts for classic data concerning discriminability of interaural time differences at high frequencies as a function of depth of modulation. [Work supported by NIH DC02103.]

\section{2:00}

5pPP4. Precedence and plausibility. William A. Yost and Sandra J. Guzman (Parmly Hear. Inst., Loyola Univ. Chicago, 6525 N. Sheridan Rd., Chicago, IL 60626)

The "Clifton effect" [R. K. Clifton, J. Acoust. Soc. Am. 82, 18341835 (1987)] was studied in a sound-deadened room with seven loudspeakers. One loud speaker produced a source click while other loudspeakers produced delayed copies simulating echoes (delays: 2-30 ms). Each combination of source and echoes is one click event and was presented as a train of click events (1-20 click events). A train was presented to listeners who made two judgments for the LAST click event presented: (1) The number of loudspeakers which produced sounds for the last click event, and (2) the loudspeaker location for each perceived source. "Catch trials" were introduced to make sure listeners used all possible responses and were able to locate the loudspeaker sources. When more than 10 click events were presented, a switch in conditions was introduced between the 10th and 11th click event. If the switch was plausible for a natural source and its echoes, responses indicated that listeners processed delayed clicks as echoes. If the change was implausible, then responses after the switch changed indicating listeners processed all clicks as if they were sources rather than echoes. [Work supported by NIH and AFOSR.]

\section{2:15}

5pPP5. Lateralization of high-frequency FM tones and frequency sweeps. Kourosh Saberi (Psychoacoust. Lab., Dept. of Psychol., Univ. of Florida, Gainesville, FL 32611)

Lateralization thresholds were measured in three experiments. In experiment I, thresholds measured for sinusoidal FM with carriers of 3 or 4 $\mathrm{kHz}$ and modulation rates from 50 to $800 \mathrm{~Hz}$ were comparable to those measured for sinusoidal AM stimuli. Lowest thresholds (at $71 \%$ correct) were about $100 \mu \mathrm{s}$, obtained at modulation rates of 300-350 Hz. In experiment II, a 300-ms tone was linearly swept in frequency from 2 to 5 $\mathrm{kHz}$. The slope and intercept of the time-frequency function were randomized by $10 \%$ on each observation. Lateralization thresholds were about 50 $\mu \mathrm{s}$. Unequal time-frequency slopes at the two ears produced a sense of motion. In experiment III, a sinusoidal FM was presented to one ear and a sinusoidal AM to the other ear. When the FM and AM had the same modulation rate $(250 \mathrm{~Hz})$, a single image was perceived. Observers were sensitive to the interaural modulation phase with near perfect discrimination of homophasic from antiphasic conditions. Results are discussed in terms of an FM-to-AM transduction mechanism. [Work supported by NIH and AFOSR.]

\section{$\mathbf{2 \cdot 3 0}$}

5pPP6. The segregation of SAM 4-kHz targets from SAM 2-kHz distractors on the basis of interaural envelope delay. R. H. Dye (Parmly Hear. Inst., Loyola Univ., 6525 N. Sheridan Rd., Chicago, IL 60626)

A stimulus-classification paradigm was used to examine the extent to which judgments of the laterality of $4-\mathrm{kHz}$ targets, sinusoidally amplitude modulated at $200 \mathrm{~Hz}$, were influenced by $2-\mathrm{kHz}$ distractors that were modulated at rates ranging from 50 to $400 \mathrm{~Hz}$. On each trial, the target was presented with one of ten envelope delays (ranging from -250 to $+250 \mu \mathrm{s}$ in $50-\mu \mathrm{s}$ steps), as was the distractor. Each test interval was preceded by a diotic presentation of the target alone. The duration of the signals was 200 ms. During a block of 100 trials, each combination of target-distractor delay was presented once. The relative salience of the envelope delays carried by the target and the distractor was assessed by the slope of the best linear boundary between left and right responses. Two listeners gave increasing weight to the target as the difference between the target and distractor modulation frequencies increased, but weighed that target and distractor equally when both were modulated at $200 \mathrm{~Hz}$. Two other listeners gave increasing weight to the $2-\mathrm{kHz}$ distractor as modulation frequency increased, as though its relative salience increased with "number of looks." These data will be compared to measures of binaural interference that have been obtained for similar stimuli. [Work supported by NIH.]

\section{$2: 45$}

5pPP7. Precision of sound localization measured by a reaching task. Daniel H. Ashmead, Xuefeng Yang, Robert Wall, and Kiara Ebinger (Dept. of Hear. \& Speech Sci., Vanderbilt Univ. Med. Ctr., Nashville, TN 37232-8700)

This study validates a reaching measure of sound localization for subsequent application to children. Seven adults reached for broadband sound sources while hand position was measured to within $2 \mathrm{~mm}$. Sounds came from 18 regions in frontal reaching space, with the loudspeaker moved away just after stimulus offset. In the "visual" condition subjects watched until the sound ended, then closed their eyes and reached. In the "auditory" condition subjects were blindfolded. Precision of sound localization was estimated by comparing variability in the visual and auditory conditions: $s=\sqrt{s_{A}^{2}-s_{V}^{2}}$. Estimates were computed for horizontal angle, vertical angle, and distance for each target location. Results agreed reasonably well with conventional measures. For targets straight ahead at ear level, horizontal $s=2.6^{\circ}$, vertical $s=5.0^{\circ}$, and distance $s=9.5 \%$. Systematic variations occurred across target locations. This reaching task is a rapid, naturalistic way of measuring three-dimensional sound localization. [Work supported by DOE and NIH.]

\section{3:00}

5pPP8. Virtual auditory reality reduced to the bare essentials. William Morris Hartmann and Andrew Wittenberg (Michigan State Univ., Dept. of Phys., East Lansing, MI 48824)

Successful imaging of real sound sources by headphones can be done by measuring free-field head-related transfer functions (HRTF) using small microphones in the ear canals and then inverse filtering by the headphone response. A simpler alternative to this standard procedure is described where a listener, with small microphones in the ear canals, wears open-air headphones throughout the experiment. A synthesized vowel is played, first from a loudspeaker and then from the headphones. The headphone signal is adjusted so that the amplitudes and phases of the harmonics measured with the small microphones are the same as those found when the loudspeaker is sounding. The technique is successful in that listeners cannot distinguish between real and virtual sources. It lacks the flexibility 
of the HRTF technique, but it allows an experimenter to study the most interesting psychoacoustical questions about sound localization and externalization using an experimental technique that is simpler and probably more reliable. [Work supported by the NIDCD and by the NSF Research Participation for Undergraduates Program.]

\section{3:15-3:30 Break}

5pPP9. Cochlear acoustic reflectance and traveling wave delay. Barry P. Kimberley (Dept. of Surgery, Univ. of Calgary, 3330 Hospital Dr. N. W., Calgary, Alberta T2N 4NI, Canada), Greg Shaw (Univ. of Calgary), Christopher Shera (Eaton Peabody Labs.), and Jont B. Allen (Bell Labs.)

Inner ear acoustic impedance and reflectance, SFOAEs, DPEs, and PTTs were measured with a calibrated probe situated in the ear canal of normal-hearing subjects. The use of a calibrated probe allowed for the conversion of the SFOAE recordings into reflectance. In a number of subjects, low stimulus levels results in rippling of the reflectance. The phase slope of the acoustic reflectance was found to be a linear over a narrow range of frequencies. This slope is taken as equivalent to the traveling wave delay to the corresponding frequency(ies). Traveling wave delay estimates (reflectance phase slope) however did not change as stimulus level ranged from 50 to $10 \mathrm{~dB}$ SPL. This is in contrast to previous traveling wave delay estimates using DPEs where delay increased with a decrease in stimulus level [Kimberley et al., J. Acoust. Soc. Am. 94, 1343-1350 (1993)]. The discrepancy between DPE-based and SFOAE-based estimates of traveling wave delay is discussed.

\section{3:45}

5pPP10. Modeling distortion product otoacoustic emission fine structure in humans. Carrick L. Talmadge, Amold Tubis, Pawal Piskorski (Dept. of Phys., Purdue Univ., West Lafayette, IN 47907), and Glenis R. Long (Purdue Univ., West Lafayette, IN 47907)

The presence of fine structure in distortion product otoacoustic emissions (DPOAEs) in humans and other species provides important clues concerning the underlying mechanisms which give rise to this structure. Recent experimental findings of our group indicate that (i) synchronous evoked emissions, hearing threshold microstructure, and the fine structure of DPOAES in each ear appear to correspond, (ii) the fine structure of higher-order DPOAEs, i.e., $f 1-n(F 2-f 1), n>2$ depends on the DPOAE frequency, and (iii) DPOAEs with $n<-1$ do not have wellformed fine structure. These data will be used to contrast and compare two distinct models of DPOAE generation, one in which the fine structure arises from the interactions in the strong overlap region of the $f 1$ and $f 2$ activity patterns (near the $f 2$ tonotopic place), and one in which the fine structure arises from reflections of the distortion product near its tonotopic location. The data strongly favor the later model.

4:00

5pPP11. Fine structure of $2 f_{1}-f_{2}$ acoustic distortion product: Effect of $L 1 / L 2$ ratio. Ning-ji $\mathrm{He}$ and Richard A. Schmiedt (Dept. of Otolaryngol. and Commun. Sci., Medical Univ. of South Carolina, 171 Ashley Ave., Charleston, SC 29425-2242)

The fine structure of the $2 f_{1}-f_{2}$ acoustic distortion product (ADP) was measured in a group of normal-hearing subjects with different combinations of primary levels $(L 1$ and $L 2)$. The frequency ratio $\left(f_{2} / f_{1}, f_{2}>f_{1}\right)$ was 1.2 , and the $f_{2}$ frequency was swept from 1781 to $2300 \mathrm{~Hz}$ in $\frac{1}{32}$ octave steps. In condition $1, L 1$ was fixed at $50 \mathrm{~dB}$ SPL while $L 2$ varied from 30 to $75 \mathrm{~dB}$ SPL in S-dB steps. In condition 2, L2 was fixed at $50 \mathrm{~dB}$ SPL and $L 1$ was vaned as in condition 1 . For a fixed $L 1$, an upward frequency shift was evident in the ADP fine structure as the $L 2$ level increased, whereas for fixed $L 2$, a downward frequency shift in the ADP pattern was observable with an increasing $L 1$. These results support a vector sum computer model for the ADP fine structure [Sun et al., J. Acoust. Soc. Am. 96, 2166-2174, 2175-2183 (1994)], suggesting that ADP fine structure is largely generated in the overlap area of the traveling waves for the primary tones, and the effects of reemissions from the $2 f 1-f 2$ area are minimal. A large variance was observed in the best $L 1 / L 2$ ratio, ranging from -7.5 to $20 \mathrm{~dB}$ both across and within subjects. [Work supported by NIDCD.]

\section{4:15}

5pPP12. Pure-tone thresholds and cubic distortion product otoacoustic emissions in the chinchilla following carboplatin treatment. Brenda M. Jock, Kristen-Lyn Petriello, Lori G. Aldrich, Ann R. Johnson, Roger P. Hamernik, and William A. Ahroon (Auditory Res. Lab., State Univ. of New York, Plattsburgh, NY 12901)

In the chinchilla, carboplatin has an unusual ototoxic effect on the sensory epithelium of the cochlea [Takono et al., Hear. Res. 75, 93-102 (1994)]. Twelve chinchillas were treated with a single IP or IV injection ( 50 or $75 \mathrm{mg} / \mathrm{kg}$ ) of carboplatin. Baseline auditory evoked potential audiograms and cubic distortion product otoacoustic emissions (3DPE) were obtained on each animal. Threshold and 3DPE functions were also acquired at regular intervals between one hour and 30 days post-injection. The sensory epithelium of the cochlea was evaluated using the surface preparation method. Anatomical analysis indicated that the carboplatin caused relatively severe but scattered losses of inner hair cells (JHC) throughout most of the cochlea. The outer sensory cell population was intact and the cells appeared normal at the level of the light microscope. Despite the IHC pathology, which also included vacuolization in the area of the IHCs, evoked potential thresholds, measured at the level of the inferior colliculus were very near normal and 3DPE functions showed very little change. [Work supported by Bristol-Myers Oncology Division and SUNY-Plattsburgh.]

\section{4:30}

5pPP13. Spontaneous and click-evoked otoacoustic emissions of the frog inner ear: Spectral characteristics and temperature dependence. Bert Maat, Pim van Dijk, and Hero P. Wit (Inst. of Audiol., ENT Dept. Univ. Hospital Groningen, P.O. Box 30.001, 9700 RB Groningen, The Netherlands)

Spontaneous otoacoustic emission (SOAE) and click-evoked otoacoustic emission (CEOAE) measurements were performed in frogs (Rana esculenta). The SOAE and CEOAE measurements followed each other repeatedly, while the frog's body temperature was changed at a rate of approximately $0.1^{\circ} \mathrm{C} / \mathrm{min}$. In some sessions, only SOAE measurements were performed. The SOAE and CEOAE spectra were closely related: The spectra of CEOAE contained the same peaks as those observed in the related SOAE spectrum. It seems that click evoked emissions in the frog are synchronized spontaneous emissions. The click-evoked responses show a nonlinear compressive amplitude growth with the stimulus intensity. As function of temperature, various emission modes, and complex amplitude patterns were observed for both types of emissions.

\section{4:45}

5pPP14. Enhanced cochlear responses after sound exposure. Yvonne M. Szymko and Jozef J. Zwislocki (Inst. for Sensory Res., Syracuse Univ., Syracuse, NY 13244)

Hensen's cell altemating potentials were recorded in the gerbil cochlea by means of the approach developed previously in this laboratory. An intensity series of magnitude and phase transfer functions (TFs) and cochlear microphonic (CM) TFs from $125 \mathrm{~Hz}$ to $16 \mathrm{kHz}$ were obtained at 40-90 dB sound pressure level (SPL) by means of frequency sweeps. Subsequently, the same frequency sweeps at either 80,90 , or $100 \mathrm{~dB}$ SPL were delivered to the ear for 10-40 min. CM, Hensen's cell TFs, and EP were monitored periodically post-exposure. Hensen's cell responses showed enhancement within $40-50 \mathrm{~min}$ post-exposure and a phase lead. There was no correlation between changes in peak response and changes in receptor potential or EP. The response enhancement and its associated phase lead is explained with the help of Zwislocki's cochlear model [Mechanics and Biophysics of Hearing, edited by P. Dallos (Springer-Verlag, 
Berlin, 1990)]. Since Hensen's cells cannot generate receptor potentials by themselves, and their AC potentials have been shown to be directly proportional to those of OHCs, the measured enhancement appears to reflect enhanced $\mathrm{OHC}$ responses. [Work supported by NIDCD.]

\section{5:00}

5pPP15. Representation of sinusoid "ramps" and "damps" in auditory-nerve fibers of the goldfish. R. Fay, M. Chronopoulos (Parmly Hear. Inst., Loyola Univ. Chicago, 6525 N. Sheridan Rd.. Chicago, IL 60626), and Roy Patterson (MRC Appl. Psychol. Unit, Cambridge CB2 2EF, England)

Earlier, behavioral data was presented showing that goldfish perceive repeated, asymmetrically shaped $400-\mathrm{Hz}$ tone bursts (ramps and damps) qualitatively as humans do: Ramps are more tonelike than damps [R. Fay and R. Patterson, J. Acoust. Soc. Am. 95, 2941 (A) (1994)]. Ramps have slow rise and relatively fast fall envelopes. Damps are ramps played backward. Single auditory-nerve fibers were recorded in response to the same ramp and damp stimuli used in the behavioral studies. Spike times were recorded for two presentations of six, 3-s stimuli: Ramps with 25-, 35-, and 45-ms rise times and 5-ms fall times, and these stimuli played backwand (damps), presented at several levels. Tuning was described using REVCOR analysis and by spike count functions of frequency at several levels. Responsiveness was defined using the coefficient of synchronization $(R)$ over the carrier period, the number of spikes evoked $(N)$, and the statistic $Z$ where $Z=R^{2} N$. For most fibers, $R, N$, and $Z$ were greater for ramps than for the corresponding damps. This neural behavior is quantitatively similar to the behavioral results. The differential perception of ramps and damps is represented within individual primary afferents. The origins of these physiological differences will be discussed. [Work supported by the NIDCD.]

\section{5:15}

5pPP16. Polynomial correlation used to estimate the degree of nonlinearity of the frog inner ear system. Pim van Dijk (Inst. of Audiol. (KNO), Univ. Hospital Groningen, P.O. Box 30.001, 9700 RB Groningen, The Netherlands)

The nerve fiber response to Gaussian noise was measured in the American bullfrog (Rana Catesbeiana). By cross correlating the stimulus and the response, Wiener kemels of the frog inner ear system were obtained. The first- and second-order Wiener kernels were fully calculated, while for the 3rd- to 10th-order kernels only the diagonals (also known as polynomial correlation functions) were computed. The data for fibers above $500 \mathrm{~Hz}$ could be described by a BPNL sandwich model. A new least-squares method was used to determine both filter functions in the model from the Wiener kernels. Preliminary analysis showed that response components could be found in kernels up to the order of 5. This suggests that the degree of the nonlinearity of the frog inner ear is at most 5 .

5pPP17. Binaural lateralization and discrimination in MS and CVA patients. Vered Aharonson, Miriam Furst (Dept. of Elec. Eng.-Systems, Faculty of Eng., Tel Aviv Univ., Tel Aviv, Israel 69978), and Amos D. Korczyn (Sackler School of Medicine, Tel Aviv Univ., Tel Aviv, Israel 69978)

Most previous studies on MS patients' binaural performance reported similar performance according to intensity and time cues, or abnormality only for interaural time differences. In this study, combined lateralization and discrimination tests on both MS and CVA patients detected other phenomena. Lateralization and discrimination ability were measured during the same session for each patient, with clicks, high, and low narrowband noises. Lateralization tests arbitrarily presented interaural time and intensity changes in 9 levels scale across the head. Adaptive 2I2AFC procedure determined jnd's. Ten CVA and 5 MS patients performed abnormally in some of the rests, and can be crudely divided into three groups: The first group performed normally in response to clicks and low frequencies, and failed only in high frequencies stimuli, in both lateralization and jnd. The second group performed abnormally in both tests and for all stimuli. The third group had normal jnd's for all stimuli. However, for some stimuli, those patients failed in the lateralization tasks, only in one of the interaural changes-either time or intensity. These results indicate that criteria used for jnd tasks are not applicable in lateralization and that time and level differences are estimated independently along the auditory pathways.

\title{
Session 5pSA
}

\section{Structural Acoustics and Vibration: Fuzzy Structures}

\author{
Victor W. Sparrow, Chair \\ Graduate Program in Acoustics, Pennsylvania State University, University Park, Pennsylvania 16802
}

\section{Contributed Papers}

1:00

5pSA1. An asymptotic analysis of the added damping effect of a distribution of fuzzy attachments with steady state excitation. Daniel A. Russell and Victor W. Sparrow (Graduate Prog. in Acoust., Penn State Univ., University Park, PA 16802)

In the current state of fuzzy structure theory, it is recognized that the primary effects of a fuzzy substructure are a frequency-dependent effect added damping and a frequency-dependent effective added mass. In this paper the fuzzy substructure is modeled, in accordance with current practice, as a system of independent 1-DOF oscillators whose masses are distributed with respect to natural frequency. The exact form of the distribution is shown to be relatively unimportant as long as it is smooth and continuous. The effects of the system of attachments, in response to steady-state excitation of the master structure to which they are attached, are obtained through an analysis of the input mechanical impedance. The real part of this impedance represents the effective added damping. Two asymptotic limits are investigated. In the first limit, the number of attachments becomes very large $(N \rightarrow \infty)$, and the impedance sum approaches an asymptotic integral. In the second limit, the damping ratio of the attachments becomes very small $(\zeta \rightarrow 0)$, and the asymptotic integral reduces to a simple algebraic expression. Comparison of the impedance sum and the asymptotic expression, in light of the product $N \zeta$, suggests that the simple 


\section{$1: 15$}

5pSA2. Average response of an infinite plate on a random elastic foundation. Joseph A. Turner and Richard L. Weaver (Dept. of Theor. and Appl. Mech., 104 S. Wright St., Univ. of Illinois, Urbana, IL 61801)

The average response of infinite thin plates with attached random impedances is examined. The added impedance, which represents typical heterogeneities that might oceur on complex shells, provides light coupling between the three propagation modes. The problem is formulated in terms of the Dyson equation which governs the mean plate response. It is solved within the assumptions of the Keller (smoothing) approximation which is valid when the heterogeneities are weak. Scattering attenuations are derived for each propagation mode. The specific case of delta-correlated springs provides a simple intuitive result for a statistically homogeneous plate. The attenuation of one wave type due to coupling to another is shown to be proportional to the modal density of the other wave type. Thus the attenuation of extensional and shear waves is predominantly due to mode conversion into flexural waves and is proportional to the large modal density of flexural waves. The flexural degrees of freedom serve as a sink for the energy of the membrane modes and constitute for them an effective fuzzy structure. A similar result is expected for the more complicated submerged shell case. A discussion of diffuse energy transport on such a plate will also be presented. [Work supported by ONR.]

\section{$1: 30$}

SpSA3. Vibrational response of a simple oscillator attached to an elastic structure. Douglas M. Photiadis, J. A. Bucaro, and Brian H. Houston (Naval Res. Lab., Washington, DC 20375-5350)

The effect of many internal degrees of freedom on the vibration of elastic structures has been of great recent interest; several theories have predicted damping effects arising from associated internal resonances. As an initial effort to explore this phenomena experimentally, the behavior of a single such oscillator attached to ribbed cylindrical shell section has been measured. The single oscillator has been found to give rise to a fairly broadband resonance structure, with a bandwidth that paradoxically increases with decreasing frequency. These results, along with the physical interpretation and modeling, will be discussed.

\section{$1: 45$}

5pSA4. Eigenstatistics in rectangular membranes with point scatterers. Richard L. Weaver (Dept. of Theor. and Appl. Mech., Univ. of Illinois, 104 S. Wright St., Urbana, IL 61801)

Conventional wisdom holds that a finite reverberant system with chaotic ray trajectories will have, at high frequencies, eigenvalue statistics identical to those of the Gaussian orthogonal ensemble of random matrices (GOE). It also holds that a nonchaolic system will have simple Poissonian statistics. Recent experiments on the eigenvalues of elastic blocks with angled cuts, recent calculations of the eigenfrequencies of membranes with staircases like jagged boundaries and the eigenfrequencies of a rectangular domain with a single isotropic point scatterer have, however, found GOE statistics even in these quasi-integrable systems-even though all rays in such systems are nonchaotic. In this work the rectangular domain with isotropic point scatterers is studied further. It is shown that the long-range level repulsion in this system is not in precise accord with the predictions of the GOE, nor is the long range spectral rigidity. GOE does, though, correctly describe the short range statistics. A quantitative prediction for the range in which GOE applies is advanced based upon the lifetime of a ray against mixing - -i.e., based upon the cross section of the scatterer. This prediction is corroborated by numerical calculations of the eigenfrequencies. [Work supported by NSF.]
5pSA5. Coupling between flexural and membrane wave types due to multi-degrees of freedom and rotational fuzzy attachments. Daniel A. Russell and Victor W. Sparrow (Graduate Prog. in Acoust., Penn State Univ., University Park, PA 168̈02)

In current fuzzy structure theory, the fuzzy substructure is generally modeled as a system of independent I-DOF oscillators attached to the master structure. Several assumptions are usually made. Firstly, while the attachments are applied to one side only of the master structure (plate or shell), the effects of asymmetric loading are ignored. In this paper, it is shown that the asymmetric loading may provide a coupling mechanism between flexural waves and membrane waves in plates and shells, thus altering the predicted added damping effect of the fuzzy substructure. Secondly, until now the motion of the attachments is usually restricted to only one translational direction. This paper considers the possibility of coupling between wave types in plates and shells when the fuzzy attachments are allowed to move in three translational directions, as well as rotational motion about their attachment points. Furthermore, the effects of these new attachment motions are analyzed using a wave impedance/ dispersion relation approach. [Work supported by ONR.]

\section{2:15}

5pSA6. Response of a fuzzy structure in terms of the impulse response function. G. Maidanik and J. Dickey (CD/NSWC, Code 703, Bethesda, MD 20084)

A master structure is defined in terms of a known and proper impulse response function $g_{x}\left(\mathbf{x} \mid \mathbf{x}^{\prime}, \omega\right)$, where $\mathbf{x}$ is the spatial variable that spans the master structure and $\omega$ is the frequency variable. The response $v_{x}(x, \omega)$ of the master structure to the external drive $p_{e}(x, \omega)$ is stated in the form $v_{\infty}(\mathbf{x}, \omega)=\int g_{x}\left(\mathbf{x} \mid \mathbf{x}^{\prime}, \omega\right) d \mathbf{x}^{\prime} p_{e}\left(\mathrm{x}^{\prime}, \omega\right)$, where $d \mathbf{x}$ is an elemental "volume" in the $x$ domain. An ensemble of appendages is attached to the master structure. The ensemble and its attachment configures an appended master structure. The response $v(x, \omega)$ of the appended master structure is stated in the form $v(\mathbf{x}, \omega)=\int g_{x s}\left(\mathbf{x} \mid \mathbf{x}^{\prime}, \omega\right) d \mathbf{x}^{\prime} p_{e}\left(\mathbf{x}^{\prime}, \omega\right)$, where $g_{\times s}\left(\mathbf{x} \mid \mathbf{x}^{\prime}, \omega\right)$ is the impulse response function of the appended master structure and it is assumed that the external drive remains unchanged. In the preceding equation it is implied that $g_{x \boldsymbol{s}}\left(\mathbf{x} \mid \mathbf{x}^{\prime}, \omega\right)$ is properly derived; indeed, without this propriety the equation is meaningless. An ensemble of configurations defines a master structure that is variously appendaged. When the ensemble of configurations is statisticalized, a fuzzy structure is defined. Using this equation and designating statistical averaging over configurations by angular brackets, one obtains the response $(v(\mathbf{x}, \omega))$ of a fuzzy structure in the form $\langle v(\mathbf{x}, \omega)\rangle=\int\left\langle g_{x S}\left(\mathbf{x} \mid \mathbf{x}^{\prime}, \omega\right)\right\rangle d \mathbf{x}^{\prime} p_{e}\left(\mathbf{x}^{\prime}, \omega\right)$. In $\left\langle g_{\times S}\left(\mathbf{x} \mid \mathbf{x}^{\prime}, \omega\right)\right\rangle$ only statistical variations in the properties of the appendages and their attachments are involved; a proper $g_{x s}\left(\mathbf{x} \mid \mathbf{x}^{\prime}, \omega\right)$ is not committed to $v(\mathbf{x}, \omega)$ and $p_{e}(\mathbf{x}, \omega)$. Thus it is argued that the last equation is an acceptable solution to the response $\langle v(x, \omega)\rangle$ of a fuzzy structure.

\section{2:30}

5pSA7. Prediction of scattering cross sections using averaged models. Douglas M. Photiadis and David Hughes (Naval Res. Lab., Washington, DC 20375-5350)

The presence of internal structure can greatly alter the acoustic behavior of elastic structures. Even in relatively simple systems, it is necessary to employ approximate models, effectively averaging out unwanted detail. Some phenomenological aspects can be easily obtained in this way provided the "base" structure is not too complicated. For example, the locus of peaks in the scattering cross section as a function of frequency and angle can often be predicted in a deterministic manner, but estimating the actual scattering levels requires more sophisticated modeling. One approach to this modeling problem is to employ a "fuzzy" structures paradigm in which a Neumann series involving the random aspects of the internal structure is averaged. A more sophisticated approach involves directly constructing and approximating equations for the desired averages; employing the Dyson and Bethe-Salpeter equations of random media theory. 
These techniques have been applied to predict the scattering cross section from an irregular ribbed structure. The results will be discussed and compared.

\section{2:45}

5pSA8. Electrodynamic reciprocity method for vibration standards. Li-Feng Ge (Anhui Bureau of Tech. Supervision, 30-Ma An Shan Rd., Hefei, Anhui 230001, People's Republic of China)

In a previous paper, a reciprocity calibration formula different from the Levy-Bouche's is derived [L.-F. Ge, Proceedings of the XIII IMEKO World Congress, 2606-2610 (1994)]; the latter has been regarded as a standard method [ISO 5347-0, 1987; ISO/DIS 5347-20, 1990]. The new formula is $S=(R T / j \omega)^{1 / 2}\left(\mathrm{~V} / \mathrm{ms}^{-2}\right)$, where $T$ is the mean of the transfer admittance ratio, $T_{n}=M_{n} /\left(Y_{n}-Y_{o}\right)$ and $R$ is the voltage ratio for the typical three-transducer-two-experiment procedure, in which an electrodynamic transducer serves as reciprocal one. This method is suited to the low- and mid-frequency ranges, and most accurate at $100-1000 \mathrm{~Hz}$. This paper reveals that a coefficient of variation, i.e., ratio of standard deviation of $T_{n}$ to its mean value, can be viewed as a reciprocity index of such an electrodynamic calibration system including two transducers and a solid mass medium. An index smaller than $0.5 \%$ indicates that the system is better reciprocal, and can be calibrated more accurately. At frequencies higher than about $3000 \mathrm{~Hz}$, nonlinear problems, such as stress wave effect and resonance, become pronounced, and should be considered; a better way would be to apply other more available methods, such as the laser interferometry and piezoelectric reciprocity method.
5pSA9. Free vibration of liquid-filled composite shells of revolution. Z. C. Xi, L. H. Yam, and T. P. Leung (Dept. of Mech. and Marine Eng., Hong Kong Polytech. Univ., Hung Hom, Kowloon, Hong Kong)

As composite materials are increasingly used in aerospace, marine, civil power generating, petrochemical, and chemical industries, the interaction of fluid and composite shells of revolution has been an active subject in the area of structural dynamies. In this paper, a semianalytical procedure was presented for predicting free vibration characteristics of liquid-filled composite shells of revolution. A new unaxisymmetric conical frustum element was derived based on the first-order shear deformation theory. The element considers both the displacement in the circumferential direction and the rotation around the meridian of the shell. The coupling between symmetric and antisymmetric modes is taken into account in the formulation. Reduced integration was employed to avoid "shear locking." Numerical examples were given for free vibrations of liquid-filled laminated shells of revolution with various types of boundary conditions. Comparisons of the results predicted by the present paper with those available in the literature were made. The effects of the liquid depth, the ratio of mass density of the liquid to that of the shell, circumferential wave number, thickness to radius ratio, ply angle, and stacking sequence on natural frequency of wet shells were discussed. [Work supported by RC, HK Poly U.]

\title{
Session 5pSC
}

\section{Speech Communication: Consonants, Vowels, and Voice (Poster Session)}

\author{
Peggy B. Nelson, Chair \\ Center for Auditory and Speech Sciences, Gallaudet University, Washington, DC 20002
}

\section{Contributed Papers}

\begin{abstract}
All posters will be on display from 1:00 a.m. to 5:00 p.m. To allow contributors an opportunity to see other posters, contributors of odd-numbered papers will be at their posters from 1:00 to 3:00 p.m. and contributors of even-numbered papers will be at their posters from 3:00 p.m. to 5:00 p.m. To allow for extended viewing, posters will be on display beginning at 8:00 a.m.
\end{abstract}

5pSC1. Interactions between acoustic dimensions contributing to the perception of voicing. Michael D. Hall, Katharine Davis, and Patricia K. Kuhl (Dept. of Speech and Hear. Sci., WJ-10, Univ. of Washington, Seattle, WA 98195)

It is well known that several dimensions contribute to the classification of stop consonants as voiced or voiceless. Three Gamer speeded classification experiments evaluated the nature of perceptual interactions between such dimensions. Specifically, the experiments examined the potential integrality between pairs of three critical dimensions-voice onset time (VOT), aspiration amplitude, and fundamental frequency $(F O)$ at the onset of voicing. Only within-category $(\mathrm{ka} /)$ syllable stimuli were used to eliminate phonetic classification as a basis for subjects' responses. Evidence was found for the asymmetric separability of VOT and $F 0$; while variability along $F 0$ significantly influenced VOT classification speed, variability along VOT did not similarly influence $F 0$ classification. A similar pattem of asymmetric separability for $F 0$ and aspiration amplitude was demonstrated, where aspiration variability did not interfere with $F 0$ classification. In contrast, VOT and aspiration amplitude were completely integral, with variability along either dimension influencing classification speed along the other dimension. Furthermore, performance accuracy reflected a time- intensity trade-off between perception of VOT and aspiration. The results indicate that $F 0$ and VOT/aspiration amplitude may contribute to two distinct sets of perceptual analyzers. Implications for phoneme perception will be discussed. [Work supported by NIH.]

5pSC2. Prediction of obstruent spectra from the slope of preceding and following second formant transitions. Gary Weismer, Vidya Guhan, and Ray D. Kent (Dept. Commun. Disord. and Waisman Ctr., Univ. of Wisconsin-Madison, 1500 Highland Ave., Madison, WI 53705-2280)

There is evidence that the slope of second formant $(F 2)$ transitions provides an index of the severity of speech mechanism involvement in motor speech disorders [Weismer et al., J. Acoust. Soc. Am. 91, 10851098 (1992)]. From an articulatory perspective, the slope of the $F 2$ transition is an ordinal index of the rate of change in vocal tract configuration. It was hypothesized that, for single words, these $F 2$ slopes would predict quantitative spectral characteristics of adjacent obstruents, under the assumption that the rate of change in vocal tract configuration during a vocalic segment would influence the articulation of a preceding or following obstruent. Quantification of obstruent spectra was achieved by means 
of spectral moments, which were regressed on $F 2$ transition slopes for a group of normally articulating men and a group with amyotrophic lateral sclerosis, a degenerative motoneuron disease typically associated with dysarthria. Results partially confirmed the hypothesis for both groups of subjects; the findings will be discussed in terms of separate treatment of vocalic and obstruent segments in a model of speech intelligibility. [Work supported by NIH.]

5pSC3. Acoustic-to-articulatory mapping of fricatives. Edward L. Riegelsberger and Ashok K. Krishnamurthy (Dept. of Electr. Eng., Ohio State Univ., 2015 Neil Ave., Columbus, $\mathrm{OH}$ 43210)

This research considers the acoustic-to-articulatory mapping of fricative speech. By incorporating additional knowledge about fricative production, perception, and dynamics into the mapping scheme, improved performance may be achieved over standard acoustic-to-articulatory mapping techniques. A hybrid time-frequency domain articulatory synthesizer and numerical optimization techniques are applied to sleady-state, unvoiced fricatives. The work differs from the fricalive inverse mapping experiments of Sorokin [V. Sorokin, Speech Commun. 14, 249-262 (1994)] and Shirai [K. Shirai and S. Masaki, Speech Commun. 2, III-114 (1983)] in that a fricative-specific linked codebook is used to initialize optimization and amplitude sensitive distance measures are used. The fricative codebook produces starting configurations for numerical optimization based on their spectral characteristics and their ability to match frication source characteristics for a given lung pressure and glottal configuration. The use of amplitude sensitive spectral distance measures is necessary to account for the interaction between the tract and the flowdependent frication source. Examples of acoustic-to-articulatory mapping are given that compare fricative articulatory models and fricative spectral distance measures for the inverse mapping scheme. Acoustic-toarticulatory performance is evaluated in terms of spectral distance and clustering ability. [Work supported by the AFOSR.]

5pSC4. Discrimination of synthetic /ba-wa/ by budgerigars (Melopsittacus undulatus). Michael L. Dent, Elizabeth F. Powell, Alisa Pierce, and Robert J. Dooling (Dept. of Psychol., Univ. of Maryland, College Park, MD 20742)

The perception of speech sounds by animals provides important comparative data for understanding the perception of speech by humans. Using operant conditioning procedures, three budgerigars (Melopsittacus undulatus) were trained and then tested on their ability to discriminate among tokens of synthetic /ba-wa/ speech continua. A "short" (120 ms) and a "long" ( $320 \mathrm{~ms})$ continuum were constructed, each consisting of 10 stimuli. For both the long and the short /ba-wa/ stimuli, budgerigars showed a marked improvement in discrimination near the human phonetic boundary. Similar to humans, budgerigars were significantly less sensitive to transition changes in the long syllables compared with the short syllables, suggesting that they may be susceptible to backward masking by the syllable of longer length. Two of the three budgerigars also showed a shift in their phonetic boundaries with changes in syllable length. These data extend the evidence of similarities between birds and mammals in the perception of speech sound contrasts. [Work supported by NIH Grants DC00198 and MH00982 to RJD.]

5pSC5. Measurement for measurement's sake: Phonologically irrelevant stop voicing durations. Leigh Lisker (Haskins Labs., 270 Crown St., New Haven, CT 0651I)

Durational differences between voiced and voiceless stops have been regularly reported, and phonological value has been attributed to some of them, i.e., VOT, closure duration (CD), and voicing-gap duration (VGD). Thus English /p,t,k/ and /b,d,g/can sometimes be separated by all three measures, and these are then acoustico-articulatory properties of the voicing feature. But VOT also separates subphonemic (allophonic) variants of $/ p, t, k /$ aspirated $\left[\mathrm{p}^{\mathrm{h}}, \mathrm{t}^{\mathrm{h}}, \mathrm{k}^{\mathrm{h}}\right]$ and unaspirated $[\mathrm{p}, \mathrm{t}, \mathrm{k}]$, and it is customary to regard this difference as phonologically irrelevant. Furthermore, CD cues voicing state only when accompanied by a voicing gap, when a short VGD is effectively (perceptually?) equivalent to one of zero duration, so the voicing gap may be "dismissed" as a consequence of pressure equalization rather than a linguistically motivated laryngeal maneuver. Elsewhere the value of $C D$ is more prosodic (moraic) than segment-identifying, distin- guishing, e.g., We play dominoes. from We played dominoes. Moreover. some VOT, $C D$, and VGD differences have little linguistic or even perception function. Thus /p, I.k/ show durational differences, but these cannot readily be shown to affect stop place perception. [Work supported by NIH Grant No. HD-01994.]

5pSC6. Measuring speaker normalization of fricatives using three methods. Keith Johnson, Shuhui Peng, and Elizabeth Strand (Dept. of Linguistics, Ohio State Univ., 222 Oxley Hall, 1712 Neil Ave., Columbus, OH 43210-1298)

Speaker normalization effects using continua from "sue" to "shoe" and three experimental tasks: Identification, goodness ratings, and direct prototype estimation are compared. The continua were formed by concatenating synthelic fricative noises ranging from [s] to [S] with the vowel portion of "sue" or "shoe" produced by a male and a female speaker. Speaker normalization was measured in the identification task as a boundary shift between responses to the "male" stimuli and the "female" stimuli. Comparison of category boundaries was also used to measure the speaker normalization effect in the goodness rating paradigm, where a stimulus was considered to be in the " $s$ " category when the "goodness as [s]" rating was higher than the "goodness as [S]" rating. Speaking normalization in the prototype estimation procedure was measured as the shift in the average estimate of the [s] or [5] prototype. Both measures of category boundary shift showed substantial speaker normalization effects, while the magnitude of the effect in the prototype eslimation task, though significant, was three to four times smaller. However, this smaller effect closely mirrors the magnitude of the acoustic difference usually found between male and female fricative. [Work supported by NIH.]

5pSC7. Testing the importance of talker variability in non-native speech contrast training. James S. Magnuson, Reiko A. Yamada, Yoh'ichi Tohkura (ATR Human Info. Process. Labs., 2-2 Hikaridai, Seika-cho, Soraku-gun, Kyoto, 619-02, Japan), and Ann R. Bradlow (Indiana Univ., Bloomington, IN 47408)

In contrast to results of training with stimuli produced by 5 talkers, Lively et al. [J. Acoust. Soc. Am. 94. 1242-1255 (1993)] reported that Japanese adults trained to perceive English $/ r /$ and $/ /$ with stimuli produced by a single talker failed to improve from pretest to post-lest, or to generalize to novel stimuli. That study was extended by training 5 groups of subjects each with a different talker, and by examining the retention of learning after 3 and 6 months. The previous results were partially replicated: Although all subjects showed significant learning during training, subjects in 3 of the 5 groups did not show significant improvement in a pretest-post-test comparison, did not generalize well to new stimuli, and did not show good retention in 3- and 6-month follow-up tests. Subjects in two of the five groups improved significantly from pretest to post-test, generalized well to new stimuli, and showed retention comparable to that of subjects trained with multiple talkers. The results indicate that while multiple-talker training leads to consistently good results, training with stimuli produced by only one talker may fail to promote generalization to new stimuli and talkers under certain conditions.

5pSC8. Perceptual training on Hindi dental and retroflex consonants by native English and Japanese speakers. John S. Pruitt (Dept. of Psych., Univ. of South Florida, Tampa, FL 33620)

Previous research has shown that both native English and native Japanese speakers have great difficulty distinguishing the dental and retroflex stop-consonants of the Hindi language. However, Japanese speakers perform much better than English speakers-particularly on voicedunaspirated consonants. This differential performance is thought to be due to contrastive experience that Japanese have with $/ \mathrm{d} /$ versus flapped $/ \mathrm{r}$. (While English does contain a flapped $/ r /$, it is not contrasted with $/ \mathrm{d} /$.) This research further compared English and Japanese speakers' ability to distinguish the Hindi contrast by providing laboratory training and generalization tests for both language groups. Twenty native speakers from each language group were pretested and post-tested on voiced-aspirated, voicedunaspirated, voiceless-aspirated, and voiceless-unaspirated dental and retroflex consonant-vowel syllables produced by 2 native Hindi speakers in 3 vowel contexts $(/ \mathrm{a} /, / \mathrm{e} /, / \mathrm{o} /)$. Training consisted of 12,30 -min sessions in which only voiced-aspirated tokens produced by 6 Hindi speakers in 2 
vowel contexts $(/ \mathrm{a} /$ and $/ \mathrm{o} /)$ were presented in a self-paced, self-selected fashion. While Japanese speakers performed at much higher levels than English speakers, both groups improved markedly and showed generalization to new speakers, new vowel contexts, and new voicing/manner contexts. [Work supported by NIDCD and Applied Telecommunications Research, Kyoto, Japan.]

5pSC9. Visual feedback training for a nasal/non-nasal distinction. Anna Marie Schmidt (School of Speech Pathol. \& Audiol., Kent State Univ., P.O. Box 5190, Kent, OH 44242)

Native speakers of some Chinese dialects do not make a distinction between $/ \mathrm{n} /$ and $/ 1 /$. This lack of a phonemic distinction may be a problem when these speakers learn English and the distinction is often difficult to teach. Three native Chinese speakers from Shanghai who had been in the US less than 2 years participated in a training experiment using a nasometer to provide visual feedback. Production was trained in initial position in minimal pairs using initial $/ \mathrm{n} /-/ 1 /$ and $/ \mathrm{sn} /-/ \mathrm{s} / /$ pairs. Subjects' perception of the distinction in English minimal pairs was tested prior to training and post-training using both same/different and categorization tasks.

5pSC10. Effects of perceptual learning on the production of a non-native contrast. Reiko A. Yamada, Yoh'ichi Tohkura (ATR Human Info. Process. Res. Labs., 2-2, Hikaridai, Seika, Soraku, Kyoto, 619-02, Japan), Ann R. Bradlow, and David B. Pisoni (Indiana Univ., Bloomington, IN 47408)

Previous research has shown a significant correlation between the perception and production of English $/ \mathrm{r} / \mathrm{and} / \mathrm{l} /$ by Japanese speakers [Yamada et al., Proc. ICSLP94, 2023-2026 (1994)]. The present study further investigated this perception-production link by examining the transfer of training in perception to production of a non-native contrast. Twelve monolingual Japanese speakers were trained to perceive the English $/ \mathrm{r} /-\pi /$ contrast using a high-variability training program [Lively et al., J. Acoust. Soc. Am. 96, 2076-2087]. Recordings were also made of the trainees' productions of English $/ \mathrm{r} /-\mathrm{f} / \mathrm{m}$ minimal pairs before and after the perceptual training. These pretest and post-test recordings were then evaluated perceptually by American listeners who were presented with pairs of tokens in an A-B test format. As expected, subjects' accuracy on the perceptual task improved by about $16 \%$ after 45 training sessions. More importantly, subjects' productions at pretest and at post-test were distinguishable by American listeners. More post-test productions were judged as "better"' tokens of English / $/ \mathbf{l}$ and /// than pretest productions. Implications for the perceptionproduction link in the acquisition of novel phonetic contrasts will be discussed. [Work supported by NIH and ATR.]

5pSC11. An articulatory and perceptual study of Tamil liquids. Joyce McDonough and Keith Johnson (Dept. of Linguistics, Ohio State Univ., 222 Oxley Hall, 1712 Neil Ave., Columbus, OH 43210-1298)

In Tamil there are 5 liquid consonants. They are the lateral approximation [1], the retroflex lateral approximant [l], the alveolar tap [ $\mathrm{c}$ ], the retroflex tap $[r]$, and an obscure segment that has been variously described. This paper presents electropalatography data from one speaker and perceptual data from five listeners exploring the articulatory and acoustic dimensions of the phonetic space for liquids in Tamil. The dimensions which are explored include duration (distinguishing flaps and approximants), dynamic tongue movement (distinguishing retroflex and nonretroflex), and constriction location/spectral shape (distinguishing [1] and the obscure liquid). Preliminary analyses suggest that the obscure liquid of Tamil is an apicopalatal central approximant. However, unlike other segments in Tamil which require tongue tip backing (the retroflexes) there is no evidence of dynamic tongue movement during the closure and some suggestion of acoustic zeros, perhaps from a lateral opening. Additional perception data designed to clarify this picture will be presented.

5pSC12. Acoustic analysis of Italian [r] and [1]. Francesca Argiolas, Federico Macri, and Maria-Gabriella Di Benedetto (Dept. INFOCOM, Università degli Studi di Roma 'La Sapienza', via Eudossiana 18, 00184 Rome, Italy)

Acoustic analysis of Italian [r] and [1] was carried out. Speech materials consisting of prestressed VCV syllables produced by 3 male and 3 female native speakers of Italian formed the basis of the present study.
Each syllable was repeated 3 times by each speaker. The vowel in the syllable was $[i, a, u]$ and the consonant was $[r]$ or $[1]$ in geminated and nongeminated form, leading to 216 syllables. Confirming the results of a previous study on Italian stop consonants [R. Rossetti, J. Acoust. Soc. Am. 95, 2874 (1994)] results of the present analysis showed that the first vowel duration was systernatically shortened in the geminated consonants. The data also showed that in the present case the duration of the consonant as well as, in most cases, of the VC and CV transitions were lengthened in the geminated form. Spectral analysis showed that there is little or no effect on the spectral properties of the consonants due to the presence of gemination. However, [r] in geminated form is in a few cases devoiced. Informal perceptual tests indicate that $[\mathrm{r}]$ can be automatically generated from [1] by introducing a silent portion within the stationary portion of [1]

5pSC13. A connection between coarticulation and variable rule application: Coda r's in Brooklyn English. Kenneth de Jong (Dept. of Linguistics, 322 Memorial Hall, Indiana Univ., Bloomington, IN 47403) and Elizabeth C. Zsiga (Georgetown Univ., Washington, DC 20057)

Ohala $(1975,1981)$ has proposed that sound changes are caused by listeners misperceiving coarticulatory effects. This paper examines variable " $r$-dropping" in Brooklyn English, and discusses the coarticulatory exigencies that may encourage speakers toward weakened forms. It is argued that production strategies do play a role in driving sound change apart from creating misperceptions. Recordings were made of speakers of Brooklyn and other dialects as part of the development of a larger multidialect database (Hertz et al., 1994). Analyses of nuclear r's (as in bird, and burl) show that neighboring l's both lower the second formant and raise the third formant, obscuring the $r$. Analyses of coda r's in " $r$-ful" speakers also show following coronals raise the r's third formant. Analyses of three Brooklyn speakers show one consistently produces coda r's, one never does, and a third does so variably as evident in a bimodal distribution of formant patterns. The variable speaker produced r-less tokens particularly in codas which contained coronals, especially 1 . This pattern suggests that coarticulatory influences affect the application of variable rules. Thus coarticulation seerns to exert pressure within a speaker toward a changed form. [Work supported by the NIDCD.]

5pSC14. Time-variation in Mandarin voiceless sibilant spectra. $Y_{i}$ Xu (Res. Lab. of Electron., Rm. 36-513, MIT, Cambridge, MA 02139)

The three Mandarin sibilant fricatives, [s], [s], and [c], were studied using a combination of time averaging and ensemble averaging as described by Xu and Wilde [J. Acoust. Soc. Am. 96, 3230 (A) (1994)]. Ten native speakers of Beijing Mandarin ( 5 male and 5 female) produced these fricatives followed by both unrounded and rounded high vowels. Timeaveraged spectra were obtained by averaging 13 consecutive 8-ms FFT windows over 20 -ms intervals. Token-ensemble-averaged spectra were then obtained by averaging over the time-averaged spectra of 10 repetitions of each sentence produced by each speaker at time-normalized locations in the utterances. Further ensemble-averaging was also performed over the male and female speakers separately to obtain group-ensembleaveraged spectra. Preliminary examination of both token-ensembleaveraged and group-ensemble-averaged spectra reveals that (a) Mandarin sibilants have substantial time variation in their noise spectra, indicating continuous articulatory movement throughout the production of the frication; and (b) all three sibilants manifest substantial spectral variation due to anticipatory coarticulation with the following vowel. This kind of variation is evident from the very beginning of the frication noise, indicating that the extent of the anticipatory coarticulation may go beyond the beginning of the frication. [Work supported by NIH.]

5pSC15. Individual differences in the glottalization of vowel-initial syllables. Laura Dilley and Stefanie Shattuck-Hufnagel (Speech Commun. Group, Res. Lab. of Electron., MIT; Cambridge, MA 02139)

Speakers of American English often glottalize the onset of a vowelinitial syllable, and recent work has shown that this is particularly likely to occur when the syllable is located at prosodically significant locations, such as the beginning of an intonational phrase, or at a pitch accent [Pierrehumbert and Talkin Labphon II, 1992; Dilley et al., J. Acoust. Soc. Am. 95, 2978 (A) (1994)]. Analysis of speech read by 4 different professional radio news announcers and 4 nonprofessional speakers shows (a) the im- 
portance of prosodic structure in determining the occurrence of glottalization holds across speakers, (b) speakers are differentially likely to glottalize overall, and (c) speakers are differentially likely to glottalize in different prosodic contexts. For example, the $4 \mathrm{FM}$ radio newscasters showed overall glottalization rates of $41 \%, 38 \%, 22 \%$, and $7 \%$, and some speakers were more likely to glottalize reduced vowels than others were. The implications of these patterns of variation for speech recognition and synthesis, as well as for models of speech production planning, will be discussed. [Supported in part by a grant from NIDCD.]

5pSC16. Articulatory modeling of so-called advanced tongue root vowels. Sean Fulop, Ethelbert Kari, and Peter Ladefoged (Phon. Lab., Linguistics Dept., UCLA, Los Angeles, CA 90024-1543)

Many West African languages have two sets of vowels that are said to differ by one having an advanced tongue root [ + ATR], and the other a retracted tongue root [-ATR]. The [+ATR] set may also have an enlarged pharynx and a lowered larynx. Words (roots) in these languages must contain vowels that are all of one set or the other. To test whether there is a constant articulatory difference between the two sets of vowels in Degema, a Delta Edoid language spoken in Nigeria, DAT recordings were made of $\mathbf{8}$ speakers. For $\mathbf{2}$ of the speakers, frontal and lateral videos were made of the lip aperture. The frequencies, amplitudes and bandwidths of the first 3 formants of the 5 pairs of vowels were determined from LPC analysis. These data were modeled using an articulatory synthesizer. After an appropriate set of vocal tract shapes had been determined for the [-ATR] set of 5 vowels, articulatory perturbations such as tongue root advancement, pharynx enlargement, and larynx lowering, were applied to the modeled [-ATR] vocal tract shapes to generate the [+ATR] set. A variety of articulatory gestures seem to be needed to convert vowels from one set into the other.

5pSC17. Perception of vowel length in Arabic. Abdulla A. AJ-Bannai ${ }^{\mathrm{a})}$ (Dept. of English Language and Literature, United Arab Emirates Univ., P.O. Box 671612, Al-Ain, U.A.E.)

This study investigates the acoustic correlates of distinctive vowel length in Arabic, and examines the extent to which these cues might figure in the phonological distinction of vowel length in Arabic. The perceptual experiment determines the accuracy with which vowel duration contrast in spoken Arabic can be auditorily represented. The method was to create sets of stimuli by adding or removing a variable numbers of the excised vowel pitch period in the data selected. These stimuli were presented to native speakers of Arabic in a word identification task. The perceptual accuracy of vowel duration as determined from the slopes of the identification curves revealed that relative vowel duration is the dominant acoustic cue in the phonological distinction of vowel length in Arabic. But spectral cues such as steady-state formant frequency, level of intensity play a significant secondary role in the discrimination of vowel contrast when the relative duration is maximally ambiguous between short versus long vowels. ${ }^{\text {a) }}$ Send notice to: P.O. Box 5837, Sharjah, U.A.E.

5pSC18. The relation between dialect attribution and vowel judgments. Alice Faber (Haskins Labs., 270 Crown St., New Haven, CT 06511), Lawrence Brancazio (Univ. of Connecticut and Haskins Labs.), and Catherine T. Best (Wesleyan Univ. and Haskins Labs.)

A spoken word's phonetic form provides information not only about the speaker's intended message but also about the speaker's language and/or dialect background. The form [dak] may reflect southern U.S. dike, Boston dark, or Chicago dock. Someone who makes an incorrect dialect attribution may misinterpret statements about the [dak]. Anecdotal reports of such misunderstandings abound (e.g., Labov, 1994). In an experimental investigation, it was assessed whether listeners' labeling of isolated words is systematically influenced by correct versus incorrect attribution of speaker dialect. Words that are incorrectly assigned to their own dialect should be mislabeled, in ways predictable from the relative positions of vowel nucler in the vowel spaces of the iwo dialects. Stımul were tokens of heed, hayed, head, hood, hoed, HUD, produced by Utah and Connecticut women. Discriminant analyses trained on the Connecticut productions correctly classified Utah heed, hayed, and head; Utah hoed was often classified as Connecticut hood, and Utah hood and HUD were classified as Connecticut head. Connection listeners answered two questions about each token: i. What word is the speaker saying? and, ii. Does she speak the same dialect you do? Their judgments were generally consistent with the discriminant analysis classifications.

5pSC19. Perceptual assimilation of North German vowels to American English categories. Sonja A. Trent, Brett H. Fitzgerald, Susan M. Crouse, and Winifred Strange (Dept. of Commun. Sci. \& Disord. and Psychol., Univ. of South Florida, 4202 E. Fowler Ave., Tampa, FL 33620-8150)

Perceptual assimilation of $\mathrm{NG}$ vowels to $\mathrm{AE}$ categories was assessed with stimuli in which the vowels occurred in $/ \mathrm{hVp} /$ syllables spoken in citation form and in the sentence, "Ich habe $/ \mathrm{hVp} /$ gesacht." Subjects were presented multiple tokens of 14 NG vowels produced by 4 male talkers. They selected the AE vowel category to which the NG vowel was most similar and rated its goodness-of-fit on a 7-point scale from "English" (7) to "foreign-sounding" (1). "Identical" vowels (transcribed as the same across languages) did not necessarily assimilate to the predicted category. "New" front rounded vowels $/ y, Y, \phi, \propto /$ assimilated to back rounded $A E$ vowels. Perceptual goodness-of-fit was not always predicted from the phonetic description or acoustic similarity in $F 1 / F 2$ space [B. H. Fitzgerald et al., J. Acoust. Soc. Am., this session]. Differences in modal identification response and goodness-of-fit were noted across citation versus sentence context and across individual speakers. Assimilation on the basis of intrinsic duration information was more consistent for syllables presented in sentences. These results have implications for studies of cross-language similarity which use citation-form stimuli. [Work supported by NIDCD.]

5pSC20. Acoustic specification of North German vowels produced in citation and sentence contexts. Brett H. Fitzgerald, Sonja A. Trent, Janet W. Stack, Xiange Ling, and Winifred Strange (Dept. of Commun. Sci. \& Disord. and Psychol., Univ. of South Florida, 4202 E. Fowler Ave., Tampa, FL 33620-8150)

As part of a larger study of the effects of speech style and consonantal context on the acoustic specification and cross-language perceptual similarity of vowels, this study examined differences in acoustic parameters of North German (NG) vowels in $\mathrm{hVp}$ syllables produced in citation-form (as lists) and in the carrier sentence "Ich habe-gesacht." Two instances of each of 14 vowels produced by 4 male speakers (Kiel dialect) spoken in each context were analyzed. Vowel formant frequencies (measured at syllable midpoint) showed some undershoot of $F 1$ in sentence context relative to citation context; $F 2$ undershoot was minimal. Duration differences between spectrally similar tense-lax pairs varied as a function of vowel height in both citation and sentence contexts. Overall, tense-lax duration differences were reduced in sentence context relative to citation form. Individual differences across speakers in both $F 1 / F 2$ targets and intrinsic durations were noted. Distances in $F 1 / F 2$ space could not account for perceptual assimilation patterns of $\mathrm{AE}$ listeners [see $\mathrm{S}$. Trent $e t$ al., J. Acoust. Soc. Am., this session]. These results have implications for conceptions of phonetic prototypes used to account for cross-language similarity. [Research supported by NIDCD.]

5pSC21. Acoustic correlates of Deg Xinag/Ingalik Athabaskan vowels. Alice Taff (Dept. of Linguistics, Univ. of Washington, GN-40, Seattle, WA 98195)

There has been controversy [A. Jerue et al., Deg Xinag Dindlidik. ANLC: Fairbanks (1993)] over the classification of the vowel in the second person plural morpheme (2p) in Deg Xinag/Ingalik as either $/ o /$ or $/ u /$. Acoustic measurements were undertaken towards resolving the issue. $F \mathbf{I}$, $F 2, F 3$, and duration were measured for each of the five vowels in the system, /a,e,o,u,a/. Contexts were limited to coronal and uvular. $F 1$ and $F 2$ means for data from two speakers indicate that $2 \mathrm{p}$ is more like $/ \mathrm{w} /$ than $\mathrm{lo}$. Vowel length data supports this claim.

5pSC22. Waveform dynamics and the perceptual segregation of concurrent vowels. Peter Assmann (School of Human Develop.. Univ. of Texas, Box 830688, GR 41, Richardson, TX 75083)

When two synthetic vowels are presented concurrently, listeners identify the vowels more accurately if they differ in fundamental frequency $\left(F_{0}\right)$ or if one of them is preceded/followed by a gliding (versus static) formant pattern. Previous experiments have shown that gliding formants 
generally do not help listeners identify the vowel to which they are linked; instead, they make the vowel without transitions easier to identify. One explanation is that the formant transition region provides a brief interval during which the competing steady-state vowel is perceptually more prominent. This interpretation is supported by two computational models that perform a filter bank analysis, process the waveform in each filter channel using a sliding temporal window, and determine which region of the signal provides the strongest evidence of each vowel. Model A computed the energy in each channel at successive time intervals to generate running excitation patterns. Model B used a temporal analysis to generate running autocorrelation functions, and included a further stage to partition the channels based on periodicity cues. Both models predicted effects of $F_{0}$ and gliding formants, but model $\mathrm{B}$ provided better predictions of the pattern of listeners' identification responses. Identification of concurrent vowels appears to benefit from an analysis of the composite waveform using a sliding temporal window, combined with a form of $F_{0}$-guided source segregation.

5pSC23. About the fourth angle of the "vowel triangle." René Carré (ENST., 46 rue Barrault, Paris, France)

The vowel triangle can be derived from the acoustic theory [R. Carre et al., J. Acoust. Soc. Am. 95, 2924 (A) (1994)]. The area function was deformed in order to increase or decrease $F 1$ or $F 2$. In this new study, the acoustic criterion is to increase (or decrease) both $F 1$ and $F 2$ or to increase $F 1$ (or $F 2$ ) and decrease $F 2$ (or $F 1$ ). At the end of the deformation process, the area functions produce the most "compact" ( $F 1$ and $F 2$ close as in /a/) or "diffuse" ( $F 1$ far from $F 2$ as in $/ / /)$ or "grave" ( $F 1$ and $F 2$ both low as in $/ \mathrm{w} /)$ sounds. But what about an "acute" vowel $(F 1$ and $F 2$ both high) which could correspond to the fourth angle? It can be automatically converged to a pattern where $F 1$ is around at $1000 \mathrm{~Hz}$ and $F 2$ at $2000 \mathrm{~Hz}$. But in this case the ratio between the maximum value of the cross-sectional area divided by the minimum value becomes around 80 . This ratio is only 20 (between 0.5 and $10 \mathrm{~cm}^{2}$ ) for the "compact" versus "diffuse" axis (from $/ \mathrm{a} /$ to $/ \mathrm{i} /$ ). Thus reaching this fourth angle is too much costly in deformation amplitude and thus poorly efficient.

5pSC24. Does the perceptual magnet effect hold for the [1] category? Joan E. Sussman (Dept. of Commun. Disord. and Sci., State Univ. of New York at Buffalo, 122 Park Hall, Buffalo, NY 14260) and Brian S. Gekas (SUNY at Buffalo, Buffalo, NY 14260)

Recent speech perception investigations [J. E. Sussman and V. J. Lauckner-Morano, J. Acoust. Soc. Am. 97, 539-562 (1995); P. Iverson and P. K. Kuhl, J. Acoust. Soc. Am. 97, 563-572 (1995)] support a "perceptual magnet effect" [P. K. Kuhl, Percept. Psychophys. 50, 93-107 (1991)], i.e., poor discrimination around a best exemplar compared with discrimination around a poor example of the category. To date, the magnet effect has been observed using the vowel [i] or consonant categories. A magnet effect might be expected because a range of good [i] tokens around a "best" exemplar would exist. However, a magnet effect may not occur for vowels with smaller and more variable categories. Each of 10 listeners rated [ib] stimuli as the $[\mathrm{I}]$ in "bib" or not the [I] in "bib." Then, each listener heard the tokens he/she had labeled as [1] at least $50 \%$ of the time and chose a "best [I]." Results show that [1] has a smaller range (45-60 mels) around a best exemplar than [i]. Subjects chose different tokens as their "best" exemplar and varied in the number of tokens located in their [1] categories. Finally, results were compared to two sessions of a Change/No-change discrimination task: One with the "best [1]" $s$ the fixed standard, the other with a poorer example of [I] as the fixed standard for measurement of a magnet effect.

5pSC25. Generalization in the vowel space. Michelle Aldridge (Callier Ctr. for Commun. Disord., Univ. of Texas, 1966 Inwood Rd., Dallas, TX 75235)

Vowels may be represented by a three-dimensional volume of space. The axes of the volume may be defined in articulatory terms (front-back, high-low, rounded-unrounded) or in physical terms $(F 1, F 2, F 3)$. Shepard [Science 237, 1317-1323 (1987)] plotted data of Peterson and Barney [J. Acoust. Soc. Am. 24, 175-184 (1952)] to show that generalization in the vowel space is an instance of his universal law of generalization, an exponential decay function with physical measures on the $x$ axis. This theory is contradicted by the magnet effect [P. Kuhl, Percept. Psychophys. 50, 93-107 (1991)] which indicates that some areas of the vowel space are perceptually shrunken. Experiments with naive adult subjects were executed. Generalization, ABX, and discrimination experiments were performed using eight cardinal vowels plus intermediate steps between selected vowels. Results, indicating both Shepard and Kuhl are partially correct, are best represented by Gluck's scheme [Psych. Sci. 2, 50-55 (1991)] with the data indicating that it is the distinctive features of vowels that are perceived categorically. There was little evidence of languagespecific effects for adults. Pilot work with newborns indicate that they organize the vowel space like adults.

5pSC26. Psychophysical procedure and the perceptual magnet effect: Comparisons of fixed and roving $A X$ discrimination of $/ \mathrm{i}$. Paul Iverson and Patricia K. Kuhl (Dept. of Speech and Hear. Sci., WJ-10, Univ. of Washington, Seattle, WA 98195)

Recent experiments by Iverson and Kuhl [J. Acoust. Soc. Am. 97, 553-562 (1995)] have demonstrated that the perception of $/ \mathrm{i} /$ is influenced by category goodness. Listeners exhibit a perceptual magnet effect characterized by high sensitivity to acoustic differences near poor exemplars of /i/ and low sensitivity near excellent exemplars of /i/. The present study examines whether this effect is influenced by psychophysical procedures. Listeners were asked to discriminate pairs of stimuli from an $/ \mathrm{i} /$ to $/ \mathrm{e} /$ continuum, and the task was varied in two ways: (1) Each block of trials had either one pair of tokens (fixed discrimination) or pairs of tokens from the entire stimulus range (roving discrimination), and (2) the acoustic difference between each pair of tokens was either 30 or 60 mels. The results demonstrated that the peak in discrimination at the $/ \mathrm{i} /$-le/ boundary diminished with fixed discrimination tasks and 30-mel differences between tokens, supporting previous findings [Macmillan et al., J. Acoust. Soc. Am. 84, 1262-1280 (1988)]. However, the magnitude of the perceptual magnet effect seemed less influenced by these manipulations. The results suggest that distortions of sensitivity at boundaries and within phonetic categories may arise from different mental processes.

5pSC27. An investigation of the perceptual magnet effect in adults. Susan C. Renda, John W. Hawks, and Richard Klich (School of Speech Pathol. and Audiol., Kent State Univ., Kent, $\mathrm{OH}$ 44242)

Three experiments were undertaken to investigate the validity and strength of the perceptual magnet effect in adults. First, the original perceptual magnet experiment [P. K. Kuhl, Percept. Psychophys. 50, 93-107 (1991)] was directly replicated with adults, using Kuhl's stimulus parameters. These subjects were then tested with the AAXX protocol used to test monkeys in Kuhl (1991). Generalization scores were computed for subjects under both conditions and compared. A second group of subjects rated a large number of $/ K Y /$ vowel tokens in the next experiment. Average ratings were computed and a prototype (P) and nonprototype (NP) were chosen based on them. Variants of each were synthesized as in Kuhl (1991). Subjects rated the goodness of each variant on a scale from 1 to 7. In addition they assigned a 0 to any token perceived as not belonging to the iv category. The subjects then participated in the perceptual magnet experiment. Data from trials containing stimuli assigned 0 were excluded from analysis. In the third experiment, a large number of /EH/ vowel tokens were rated and $\mathrm{P}$ and NP were chosen. Variants were synthesized and rated and the perceptual magnet effect experiment was again conducted using these tokens. Results and implications of these experiments will be discussed.

5pSC28. Production of highly similar yowels by language-impaired children. June Stealy, Rachel E. Stark (Dept. of Audiol. and Speech Sci., Purdue Univ., West Lafayette, IN 47907), and John M. Heinz (Kennedy-Krieger Inst., Baltimore, MD 21202)

It has been shown that language impaired (LI) children have greater difficulty than language normal (LN) children in identifying the synthesized vowels $/ \varepsilon / \mathrm{vs} / œ /$, but not $/ \mathrm{O} / \mathrm{vs} / \mathrm{i} /$, when the members of test pairs are of equal duration ( 240 to $40 \mathrm{~ms}$ ) [R. E. Stark and J. M. Heinz, J. Acoust. Soc. Am. 91, 2360 (A) (1992)]. It was concluded that, when deprived of the durational cues present in natural speech, LI children were less well able than $L N$ to rely upon subtle spectral cues. In the present study, children in both groups ( $11 \mathrm{LI}, 9 \mathrm{LN}$ ) were asked to imitate the 
synthesized vowels $/ \varepsilon /$ and $/ œ e /$ Their responses were recorded, transcribed, and submitted to spectral analysis. Responses of individual children that were given to either $/ \varepsilon /$ or $/ œ /$ were scored as correct, those that were given to both vowels indiscriminately were not. The scores of the two groups were not significantly different. However, the LI children's responses were transcribed as more variable, and occupied a greater range within $F_{1}-F_{2}$ plots, than those of the $\mathrm{LN}$ children. The results support the hypothesis that Ll children have "fuzzy" phonological categories. [Work supported by $\mathrm{NIH}$.]

5pSC29. Timing effects of postvocalic voicing and distinctive vowel length. Dawn M. Behne, Bente Moxness (Univ. of Trondheim, 7055 Dragvoll, Norway), and Peter E. Czigler (Umeå Univ., 90187 Umeå, Sweden)

Syllable-internal timing can be affected by a variety of factors, including postvocalic voicing and distinctive vowel length. Both factors have been found to inversely affect vowel duration and the duration of a postvocalic consonant, resulting in similar rhyme-intemal timing patterns. Is there any indication that syllable-internal timing uniquely characterizes distinctive vowel length and postvocalic voicing? Three experiments have been carried out investigating combinations of postvocalic voicing and distinctive vowel length in English, Norwegian, and Swedish. For each language a controlled set of CVCs was selected in which the final consonants were either voiced or voiceless. In addition, Norwegian and Swedish CVCs were selected which had distinctively short or long vowels, and for English, words were chosen which contained inherently short and long vowels. The target items were recorded in carrier sentences and durations of the initial consonant, the vowel, and final consonants were measured. Results demonstrated the expected inverse timing relations within the rhyme associated with postvocalic voicing and distinctive vowel length. In addition, prevocalic consonant duration was consistently distinct for conditions of postvocalic voicing compared to distinctive vowel length. Findings for English, Norwegian, and Swedish will be discussed in terms of syllable-intemal timing.

5pSC30. Laxness of voice quality integrates with $F 1$ (usually, but not always, negatively). Laura Walsh, Christine Bartels, John Kingston (Linguistics Dept., Univ. of Massachusetts, Amherst, MA 01003), and Neil A. Macmillan (Brooklyn College, Brooklyn, NY 11210)

Both lax voice and advanced tongue root typically concentrate energy at low frequencies: Laxing the voice increases the relative prominence of the first harmonic, and advancing the tongue root lowers $F 1$. A previous study [Thorburn et al., J. Acoust. Soc. Am. 95, 287I (A) (1994)] using the Gamer paradigm found that these acoustically similar effects integrated perceptually for vowels in CVC context. In this study, the range of voice quality was extended to include tensor values. Across the entire set of voice qualities sampled in the two experiments, laxness integrated negatively with $F 1$ at the lax and tense ends of the continuum but positively at intermediate values. This pattem of mean integrality is distinct from the additional finding of variance infegrality, that is, greater uncertainty in judging voice quality when $F 1$ varied than when it did not (and similarly for judgments of $F 1$ ). According to a model of Durlach et al. [Percept. Psychophys. 46, 293-296 (1989)], the variance-integrality effects can be attributed to a sensory rather than a context-coding source. [Work supponted by NSF and NIH.]

5pSC31. Perceptual normalization of synthesized vowels following natural speech and breath sounds. Sonya $M$. Sheffert and D. H. Whalen (Haskins Labs., 270 Crown St., New Haven, CT 06511)

The identification of synthetic vowels relies in part on a precursor phrase, suggesting that normalization is taking place. Breath intake sounds, which can also precede speech, may also give information. This study examines both speech and breath precursors. Two adult speakers (one male, one female) produced the phrase "The next one is." They also produced audible breath sounds before saying the words "bead," "bad," or "bud." Two 5-member continua were created, one from "bid" to "bed," another from "bad" to "bud," based on these words produced by each speaker. Formants were averages (across the two speakers) for the natural productions; $F 0$ was 160 , resulting in gender neutral tokens. The gender of precursor speech and breath before each continuum item were varied. The vowel information in the breaths also favored one end of the continuum or the other. Two results suggest that, under some circumstances, vowel identification was affected by breath precursors: (1) There were more $/ / /$ responses on the "bid-bed" continuum following the female breaths; (2) there were more $/ æ /$ responses following the male and female $æ$-breath. Paradoxically, natural speech preeursors shifted identification toward the opposite gender's formant space. Another test suggests that these vowels identification shifts did not arise postperceptually.

5pSC32. Intersyllabic regulation of $F 0$ and SPL with and without auditory feedback. Jane Wozniak, Harlan Lane, Joyce Manzella, Joseph Perkell, Melanie Matthies, Mario Svirsky, Michael O'Connell, and Clay Mitchell (Res. Lab. of Electron., Rm. 36-51 1, MIT, Cambridge, MA 02139)

Syllable-to-syllable fluctuation of $F 0$ and SPL were measured in readings of a passage by 4 post-lingually deafened adults, recorded before and after they received cochlear implants, and one adult with neurofibromatosis-2 (NF2), who was initially profoundly deaf in one ear and had a mild to moderate hearing loss in the other (aided). Three of the 4 cochlear implant users reliably reduced syllable-to-syllable fluctuation in $F 0$ and SPL following the activation of their speech processors. The fourth speaker began with and maintained the regularity achieved by the others post-activation. In recordings made following the onset of bilateral profound deafness, the NF2 subject showed increased syllable-to-syllable fluctuation in $F 0$ and SPL. Results from another experiment [M. A. Svirsky et al., J. Acoust. Soc. Am. 92, 1284-1300 (1992)], in which multiple repetitions of vowels in an $/ \mathrm{hVd} /$ context were produced by cochlear implant users with their processors turned off and on, suggest that some subjects showed less token-to-token variability in $F 0$ and SPL with their processors turned on. The present results indicate that auditory feedback may also constrain the syllable-to-syllable variability of $F 0$ and SPL contours. [Work supported by NIDCD.]

5pSC33. A qualitative study of mechanisms of jitter-induced shimmer in the voice. Darrell Wong (WJ Gould Voice Res. Ctr., Denver Ctr. for the Performing Arts, 1245 Champa St., Denver, CO 80204), Robert Lange (WJ Gould Voice Res. Crr.), Ingo R. Titze (Univ. of Iowa), and Chwen Geng Guo (WJ Gould Voice Res. Ctr.)

Measurements of jitter and shimmer are typically applied to microphone signals to discem the level of perturbations in the oscillatory behavior of the vocal folds. Shimmer, the average cycle-to-cycle change in amplitude, is known to linearly increase as the extent of amplitude modulation of tissue displacement increases. Behavior under frequency modulation (FM), however, is nonlinear. This nonlinearity is often attributed to time aliasing, the superposition of jittered impulse responses (assuming the source-filter model of phonation). As a consequence, whenever jitter is present, any measurement of shimmer cannot be solely attributed to amplitude modulation of the vocal fold issue. The present study attempts to characterize other sources of FM-induced shimmer by examining points earlier in the voice production process. An interactive computer simulation of the vocal fold and vocal tract system is used under conditions of FM $\frac{1}{2}$ subharmonic modulation of the tissue displacement. It appears that, in addition to time aliasing. shimmer may be generated from FM-induced perturbations in mucosal wave propagation and tissue displacement. The paper qualitatively describes and illustrates these jitter-induced shimmer mechanisms.

5pSC34. Exit jet particle velocity in the in vivo canine laryngeal model. Steven Bielamowicz, Gerald S. Berke, Jody Kreiman, and Bruce R. Gerratt (UCLA Div. of Head and Neck Surgery, UCLA School of Medicine, CHS 62-132, Los Angeles, CA 90024)

This study extends the previous work on exit jet particle velocity in an in vivo canine laryngeal model of phonation [G. S. Berke et al., J. Voice 3. 306-313 (1989)]. In both the previous and the current study, a hot-wire anemometer was used to measure air particle velocity in the midline of the glottis at anterior, intermediate, and posterior positions. Simultaneous measurements of subglottal pressure, EGG, and PGG were also obtained. In the previous study, these measures were obtained with constant levels of recurrent laryngeal nerve (RLN) and superior laryngeal nerve (SLN) stimulation. In the current study, exit jet particle velocity profiles were 
obtained across multiple levels of RLN and SLN stimulation. Exit jet particle velocity was also measured at midline and off-midline positions with constant levels of RLN and SLN stimulation. The time-varying features of exit jet particle velocity as a function of subglottal pressure and glottal vibratory events will be presented.

5pSC35. Glottal characteristics of female speakers-acoustic, physiological, and perceptual correlates. Helen M. Hanson (Div. of Appl. Sci., Harvard Univ., Cambridge, MA 02138 and Res. Lab. of Electron., Rm. 36-579, MIT, Cambridge, MA 02139)

A previous study of vowels produced by female speakers showed substantial individual differences in acoustic parameters related to glottal characteristics [K. N. Stevens and H. M. Hanson, in Vocal Fold Physiology: Voice Quality Control (Singular, San Diego, 1995)]. Based on measurements taken on the speech signal or spectrum, inferences were made about glottal configuration during phonation and the nature of the glottal pulse. Subjects fell into two groups based on the acoustic measurements, group 1, assumed to have abrupt glottal closure and group 2, assumed to have nonsimultaneous closure. Results of a listening test have shown that members of group 2 are perceived to be breathier than members of group 1 [H. M. Hanson, Proc. ICASSP-95 (in press)]. The current study extends the earlier work in several ways. Physiological measurements have been made for four subjects, including inversed filtered airflow recordings and glottal images obtained by fiberscopy. Also, listening tests using synthesized speech have been carried out to study the role of glottal characteristics in speaker discrimination, and the usefulness of the acoustic measures for guiding the synthesis of natural sounding speech. The physiological and perceptual data are found to be consistent with the earlier interpretations of the acoustic measures of glottal characteristics.

\title{
SATURDAY AFTERNOON, 3 JUNE 1995
}

GRAND BALLROOM SOUTH, 1:00 TO 4:50 P.M.

\section{Session 5pUW}

\section{Underwater Acoustics: Target Scattering}

\author{
Christopher Feuillade, Chair \\ Naval Research Laboratory, Stennis Space Center, Mississippi 39529-5004
}

Chair's Introduction-1:00

\section{Contributed Papers}

\section{1:05}

5pUW1. Bistatic scattering of underwater sound from a porous solid sphere: A comparison of theory and experiment. Martin $E$. Pace, Theodore W. L. Huskey, Steven R. Baker (Phys. Dept., Naval Postgraduate School, Monterey, CA 93943-5000), Steven G. Kargl (Univ. of Washington, Seattle, WS 98105), and Raymond Lim (Coastal Systems Station, Panama City, FL 32407-7001)

Measurements were made of the bistatic scattering of underwater sound from a porous solid sphere. Two sample spheres were employed composed of bonded glass beads of 100- and 500-micron nominal grain diameter, respectively. The diameter of each sphere was approximately 6.8 $\mathrm{cm}$. The scattering into the rearward hemisphere was measured over the frequency range 13 to $150 \mathrm{kHz}$, corresponding to values of $k a$ from approximately 2 to 20 . Over this frequency range the viscous penetration depth varies from approximately 5.0 to 1.5 microns, so that the water in the pores can move freely. The experimental results were compared to numerical computations based on a theoretical Biot model [S. G. Kargl and R. Lim, J. Acoust. Soc. Am. 94, 1527-1550 (1993)]. The physical parameters required by the model were obtained by independent measurements on the sample spheres and on similar cylindrical samples. Reasonably good agreement was found between the experimental and theoretical results for the 100-micron sphere, especially at the lower frequencies, except for a consistent deficit in the measured backscatter. Agreement between the results for the 500-micron sphere was poor, probably due to sample inhomogeneity.

\section{$1: 20$}

5pUW2. Scattering of a transient plane compression wave by a spherical inclusion in a Biot medium. Hans Schantz, Morris Stern, and Justin Beres (Appl. Res. Lab., Univ. of Texas, P.O. Box 8029, Austin, TX 78713-8029)

The scattering of an arbitrary transient plane wave by a spherical inclusion in a Biot medium is modeled, by applying the single frequency, steady-state solution of Zimmerman and Stern [J. Acoust. Soc. Am. 94,
527-536 (1993)] to the discrete Fourier components of the transient signal, and assuming linear supersposition. The solution of Zimmerman and Stern is an analytical series solution. Its accuracy is mainly determined by computation errors and the convergence of the series. The incident pulse can be any combination of Biot fast and low waves. The theoretical results for certain cases are shown. They are also compared to experimental measurements. [Work supported by Naval Research Laboratory, Stennis Space Center.]

\section{1:35}

5pUW3. Multiple scattering from submerged bodies with dissimilar acoustical properties. Part I. Local impedance models. Gabriella Turek (Marine Phys. Lab., Scripps Inst. of Oceanogr., La Jolla, CA 92093-0238), Peter H. Rogers, and Aldo A. Ferri (Georgia Inst. of Technol., Atlanta, GA 30332-0405)

A numerical solution for a two-body acoustical multiple scattering problem based on the analytical infinite series solution of the wave equation was developed. FORTRAN 77 codes implementing this solution were written which are capable of simulating the case of two spheres of arbitrary radius and distinct material properties subject to an acoustic plane wave of arbitrary incidence. Far-field solutions involving permutations of the two "degenerate" boundary conditions (pressure release and rigid) were compared to solutions obtained with the combined Helmholtz integral equation formulation problem (CHIEF) program. Surface pressures and velocities were also calculated. [Work supported by ONR.]

\section{$1: 50$}

5pUW4. Multiple scattering from submerged bodies with dissimilar acoustical properties. Part II. Experimental results. Gabriella Turek (Marine Phys. Lab., Scripps Inst. of Oceanogr., La Jolla, CA 92093-0238), Peter H. Rogers, Aldo A. Ferri, and Gary W. Caille (Georgia Inst. of Technol., Atlanta, GA 30332-0405)

In Part 1 [Turek et al.] a numerical solution for a two-body acoustical multiple scattering problem based on the analytical infinite series solution of the wave equation was presented. Surface and far-field solutions involv- 
ing permutations of the two "degenerate" boundary conditions (pressure release and rigid) were compared to solutions obtained with the combined Helmholtz integral equation formulation problem (CHIEF) program. In this paper the case in which one of the spheres is fully elastic and the other pressure release is modeled and the results verified experimentally.

\section{2:05}

5pUW5. Modeling low frequency scattering from small schools of fish. C. Feuillade, R. W. Nero, and R. H. Love (Naval Res. Lab., Stennis Space Center, MS 39529-5004)

A low-frequency scaltering model for small to moderately sized fish schools has been developed. The model uses a mathematical formalism based upon the harmonic solution of sets of coupled differential equations, and incorporates a verified swimbladder scattering "kernel" for an individual fish. All orders of multiple scattering interactions between the fish are included, and the aggregate scattering field calculated by coherent summation. Application to ensembles of closely spaced fish indicates significant deviations from the returns expected when incoherent scattering is dominant. The peak target strength is reduced and the resonance frequency is shifted due to multiple scattering. The target strength also varies strongly with frequency as a result of interference effects. For widely dispersed ensembles, the model reproduces the results of incoherent scattering. For larger ensembles the model predicts target strength values near the main resonance which diminish sharply with depth. The effects of a school on the scattering of any single individual within it become greater as the school size increases. By reducing the viscous damping in the scattering kernel, the model can also describe scattering from small bubble clouds.

\section{2:20}

5pUW6. Acoustic wave scattering from infinite cylindrical clad rods. Farhang Honarvar (Dept. of Mech. Eng., Univ. of Toronto, Toronto, Ontario M5S 1A4, Canada) and Anthony N. Sinclair (Univ. of Toronto, Toronto, Ontario M5S 1A4, Canada)

This paper considers the scattering of an infinite plane acoustic wave obliquely incident on an infinite clad rod. Comparing the form functions of the scattered pressure for the clad rod and a simple rod made of the cladding material, it is observed that the presence of the core will affect the lower mode resonances while the higher modes are not affected. The ratio $a / b$, where $a$ and $b$ are the radii of the core and the clad rod, respectively, is an indication of the boundary between the lower and higher modes. Additional resonances for this case are due to the surface waves propagating on the core/cladding interface. These resonances are highly dependent on the quality of the bond between the core and the cladding. Hence they could be used as key parameters in evaluation of the interfacial bond quality. A finite value of bond rigidity is used in order to model the core/ cladding boundary. Using this approach the quality of the interfacial bond in the model can range for perfect bonding to complete debond depending on the values of the boundary stiffness constants.

\section{2:35-2:50 Break}

\section{2:50}

5pUW7. Low-frequency volume scattering measurements from the central Mediterranean. Mark J. Vaccaro (Naval Undersea Warfare Ctr., New London, CT 06320)

Broadband $(200-1500 \mathrm{~Hz})$ measurements of direct path volume scattering were made in May of 1991 in the central Mediterranean's Ionian Sea and adjacent Medina Bank. Acoustic sources used in these measurements were explosive charges detonated several hundred meters below a vertical line array (VLA) receiver. Reverberation was processed from the upward looking end-fire beam of the VLA yielding highly resolved scattering strengths in depth and frequency for the upper $400 \mathrm{~m}$ of the water column. Analysis of the spectral characteristics of the volume scatter data reveals that the dominant scattering emanales from diffuse, broadband strata which are confined to the thermocline region of the water column. The applicability of classic swimbladder scattering models to describe these layers is presented in addition to the spatial variability observed in volume scatter from deep (Ionian Sea) to shallow (Median Bank) ocean areas.

3:05

5pUW8. Predicted target strength analysis using computer techniques. Gerard P. Gay (Mech. Eng. Dept., The Cooper Union, 51 Astor Place, New York, NY 10003) and Daniel R. Raichel (City University of New York)

Target strength was predicted for various geometric objects by a computer ray tracing algorithm. The ray tracing theory assumes that the properties of sound at high frequencies, entailing wavelengths which are small compared with the size of the target objects, are analogous to light properties. This is a preliminary experiment still in progress. Four simple objects: Sphere, cylinder, cube, and disk, and two compound objects: Cylinder with spherical ends, I-beam frame structure, were investigated. The objects were created in an object-oriented ray tracing software package and rendered at various orientations to describe the reflective properties. A separate program was written to decode the ray traced images of the objects and determine their target strength. Current work will yield results to be compared with the empirical high-frequency target strength analysis results obtained at Naval Surface Warfare Center for Naval Research Labs and at Cooper Union's Acoustical Research Center.

5pUW9. A generalized wave-number method for solving waveguide scattering problems. John A. Fawcett (SACLANT Undersea Res. Ctr., Viale San Bartolomeo, 400, 19038 La Spezia, Italy)

A general plane-wave decomposition method for solving scattering problems for objects in a possibly range-dependent oceanic waveguide is presented. The waveguide's interfaces and objects within the waveguide are characterized by plane-wave scattering matrices. The total response of the medium can be computed by combining these matrices using an invariant embedding approach. This operator formalism is independent of the method used to solve the individual free-space objector interface scaltering problems. The method is derived from the theory of generalized reflection operators [B. L. N. Kennett, Wave Motion 6, 419-429 (1984)] and is also closely related to some $T$-matrix formulations [G. Kristensson and S. Ström, J. Acoust. Soc. Am. 64, 917-936 (1978)]. The generalized wave-number method is used to compute scattering from a cylinder in perfect and penetrable waveguides. The case of a bottom sloping interface is also considered. For some of the examples, a comparison is made with the results from other computational techniques.

\section{3:35}

5pUW10. Sound scattering by a fluid-filled cylindrical shell in water. Gregory Kaduchak and Charles M. Loeffler (Appl. Res. Labs., Univ. of Texas, P.O. Box 8029, Austin, TX 78713-8029)

Previous calculations and observations of backscattering by cylindrical shells in water usually involve shells which are either empty or subject to extremely light interior loading conditions such as air. Typical echo signatures display distinct contributions described by specular reflection and guided waves launched along the shell structure. The present research analyzes the backscattering effects as a consequence of filling the interior cavity with a higher impedance fluid such as water. Energy transferred into the cavily couples into radiation mechanisms which drastically increase the fine structure in the backscattering form function. Dispersion curves derived from full 3-D elasticity theory via the Watson transform methodology display a complicated mode structure which is a combination of the guided waves found on a cylindrical shell in vacuum and the normal modes of a fluid-filled cylindrical cavity satisfying rigid boundary conditions. The radiation damping of the associated curves yields insight into mechanisms of 
energy transfer to and from the internal cavity through the guided Lamb waves traveling along the shell. The cylindrical shells studied are excited by plane waves at normal incidence and have radius to thickness ratios of $5 \%$ and $10 \%$

\section{3:50}

5pUW11. A perturbative technique to compute scattering of interface waves from pointlike obstacles. Eric Smith (Appl. Res. Labs., Univ. of Texas, P.O. Box 8029, Austin, TX 78713-8029)

A technique is presented to compute a scattering amplitude for waves from a pointlike inhomogeneity in an elastic medium with boundary, into interface as well as bulk modes. The particular system considered is a half-space of fluid overlying a half-space of a liner elastic solid, with the obstacle placed in the solid very near the boundary. The object of the calculation is to construct a perturbation theory in layered media in which the scattered wave respects the boundary conditions at each perturbative order. This can then be used to estimate and qualitatively analyze the significant features of such scattering, and to produce a well-defined yet simple algorithm for approximating exact solutions. [Work supported by the U.S. Navy, Office of Naval Research.]

\section{4:05}

5pUW12. Extraction of target characteristics from the response of a ribbed cylindrical shell in a shallow water environment. Angie Sarkissian, David H. Hughes, and Louis R. Dragonette (Naval Res. Lab., Washington, DC 20375-5350)

Bistatic and monostatic target strength values of a ribbed cylindrical shell contain various scattering phenomena such as Bloch waves, rib resonances, specular reflections and other effects due to waves traveling on the surface of the shell. When the target is placed in a shallow water environment, the response is further cluttered by the presence of the various multipaths that a signal travels in a bounded medium. By using a vertical line array of sources and receivers, the target response may be extracted. A vertical array of sources is used to suppress certain shallow water modes in the incident field. A receiver array is used to remove certain shallow water modes from the scattered field. Time-frequency analysis is a final filter for selecting the modes of interest for which the target response is extracted. The extracted target response is correlated against the free field target characteristics previously determined by time-frequency synthesis techniques.
5pUW13. Modeling of backscattering by bottom volumetric inhomogeneities. Dajun Tang (Dept. of Appl. Ocean Phys. \& Eng., Woods Hole Oceanogr. Inst., Woods Hole, MA 02543)

It has been recognized that when a sound wave encounters the ocean bottom, part of the energy will be scattered by volumetric inhomogeneities in the bottom. To simplify the mathematics, previous efforts in modeling the volumetric scattering process invoke some forms of plane-wave approximation to either or both of the forward and backward propagating fields. Since the ocean bottom has different acoustic properties from that of the water column, particularly since it often has a more complicated structure, introduction of the plane-wave approximation casts clouds on the validity of the modeling results. Here an approach is presented that uses the exact forward and backward propagating fields and calculates the backscattering strength numerically in the spatial domain. The bottom is assumed to be generally layered with superimposed random scatterers which have given correlation functions. Finally the validity of this model and its range of applicability will be discussed.

\section{$4: 35$}

5pUW14. Using matched-field processing technique to map the forward-scattered signal. Yung P. Lee (Sci. Applications Int'l. Corp., 1710 Goodridge Dr., MS T1-3-5, McLean, VA)

Matched-field processing combines an acoustic propagation model with matched-filter processing to localize a source in three-dimensional space. It has been shown to be effective in discriminating passive sources both in range and depth by exploiting the vertical structure of the signal. Reverberation, especially the surface-scattered energy, is the primary concern for active systems. Surface scattering occurs near the ocean surface. Since matched-field processing can discriminate in depth, it may potentially provide significant clutter suppression for active systems. In a sea test, a vertical array was deployed and a $\mathrm{cw}$ source was moored from a stationary platform approximately $18.6 \mathrm{~km}$ away from the array. The forward-scattered energy was spectrally separated from the carrier and then MFP was performed to examine the spatial distribution of the scattered energy. MFP successfully maps the scattered energy near the surface. Simulations was done by generating realization of an ocean surface with randomly distributed surface waves and a modified FEPE was used to propagate signals to a vertical array. Results of this simulation show that MFP also successfully maps the simulated scattered energy near the surface. [Work supported by U.S. Navy.] 